\title{
Abstracts of the 27th Congress of the World Society of Cardiovascular and Thoracic Surgeons (WSCTS)
}

Astana, Kazakhstan. 01-03 September 2017

Published: 15 December 2017

\section{Oral abstracts \\ Topic: Anesthesia and Intensive Care}

\section{1}

Cost effectiveness of using thrombelastography (TEG) in patients undergoing open heart surgery

Ali Bayoumi ${ }^{1}$, Khaled Al Khamees ${ }^{2}$, Ibrahim M. Yassin ${ }^{3}$, Hatem Al Taher ${ }^{4}$

Mustafa Al Refaei ${ }^{5}$, Farouk M. Oueida ${ }^{2}$, Khalid A. Eskander ${ }^{2}$

${ }^{1}$ Laboratory Department, Saud Al Babtin Cardiac Center SBCC, Al

Dammam, Saudi Arabia; ${ }^{2}$ Cardiac Surgery Department, Saud al Babtin

Cardiac Center, Al Dammam, Saudi Arabia; ${ }^{3} T a n t a$ University Hospitals,

Tanta, Egypt; ${ }^{4}$ Anaesthesia Department, Saud al-Babtin Cardiac Center

SBCC, Al Dammam, Saudi Arabia; ${ }^{5}$ Cardiology Department, Saud al-

Babtin Cardiac Center SBCC, Al Dammam, Saudi Arabia

Correspondence: Ibrahim M. Yassin

Journal of Cardiothoracic Surgery 2017, 12(Suppl 1):01

\section{Objective}

Thrombelastography (TEG) was recently introduced to our cardiac center. We hypothesized that the TEG- guided treatment reduces the transfusion of allogeneic blood and blood products and its wellknown hazards, the hospital stay and consecutively reduces the overall cost per patient. We thought to evaluate retrospectively the economic impact of (TEG) guided transfusion therapy in cases of non-surgical bleeding after open heart surgery.

Methods

After obtaining institutional research ethics committee (REC) approval, Retrospective enrolment of randomized consecutive 50 adult patients ( $>18$ Years) of postoperative non surgical bleeding after introduction of TEG in our center, from sept.2013 to Jan.2016 (TEG Group). They were compared to similar matched 50 patients group done before, from June 2011 to sept.2013 (Non-TEG Group). All relevant data for patients were collected and compared in terms of number and cost.

Results

Statistically non-significant difference $(\mathrm{P}=0.8943)$ was founded between two groups regarding the number and cost of transfused red cell concentrate (RCC). The number and cost of transfused fresh frozen plasma (FFP) and platelets (Plts) to patients in TEG group were reduced with a highly significant statistical difference $(P=0.0016)$ and $(P=0.0018)$ respectively. The overall cost of hospital stay, blood and blood products transfusion and TEG test was lower in patients of TEG group by average of 1924 US\$ per patient. There was no statistically significant difference regarding the cost of hospital stay between both groups $(P=0.5906)$.
Conclusions

TEG-guided transfusion reduces the number and cost of transfused FFP and Plts in patients with post cardiac surgery bleeding, but it does not affect the hospital stay.

\section{Topic: Anesthesia and Intensive Care}

02

Combined cardiac surgery and oncosurgery - is it feasible when necessary?

Abishek N. Raychura, Gaurav G. Goel, Jatin Desai, Dipayan Nandy

Sterling Hospital, Vadodara, Vadodara, India

Correspondence: Abishek N. Raychura

Journal of Cardiothoracic Surgery 2017, 12(Suppl 1):O2

\section{Background}

To study feasibility and safety of combined cardiac surgery and oncosurgery when necessary.

Methods

We report three cases of combined cardiac surgery and oncosurgery done in our institution. In all three patients oncosurgery was urgent due to possibility of malignancy spread, but underlying cardiac condition like critical CAD and critical valvular stenosis resulted in excess risk in such surgeries. Treating cardiac condition first would have meant postponement of oncosurgery and post-operative radio/chemotherapy by at least few weeks and additional problems like antiplatelet/anticoagulant therapy due to post cardiac surgery state. Our first patient was 56 years female, hypertensive, diabetic with carcinoma breast but found to have critical triple vessel CAD on evaluation. OPCAB followed by MRM was done under same anaesthesia. Second patient was 45 years female who presented with carcinoma ovary. She was detected to have critical mitral stenosis on preoperative evaluation. Mitral valve replacement followed by Salpingo-Oophorectomy was done under same anaesthesia. Third patient was 72 years male who developed carcinoma tongue. Patient had history of unstable angina and on evaluation was found to have critical triple vessel CAD. Here also CABG followed by hemiglossectectomy and modified radicle neck dissection was done under same anaesthesia. Cardiac surgery was done first in all cases to avoid heparinisation related bleeding in oncosurgery field. Meticulous attention was paid to correcting fluid deficits, acid base and electrolyte disturbances, maintaining adequate hematocrit and hypothermia avoidance in perioperative phase.

Results

In all three patients, perioperative course was uneventful. There was no excessive bleeding during oncosurgery or post operatively. Postoperative wound healing was also adequate. 


\section{Conclusion}

Combined cardiac surgery with oncosurgery is safe and advantageous due to avoiding delay in oncosurgery and radio/chemotherapy. Similarly cardiac surgery also does not get delayed due recovery from oncosurgery/radio-chemotherapy.

\section{Consent for publication}

Written informed consent to publish was obtained from the patients involved in this study.

\section{Topic: Heart Failure}

$\mathrm{O} 3$

10-year outcomes after pulmonary thromboendarterectomy

Alexander Edemskiy ${ }^{1}$, Alexander Chernyavskiy ${ }^{1}$, Natalya Novikova ${ }^{1}$

Alexandra Tarkova', Mikhail Chernyavskiy ${ }^{2}$

${ }^{1}$ Siberian Federal Biomedical Research Center, Novosibirsk, Russian

Federation; ${ }^{2}$ Federal Almazov North-West Medical Research Centre, St.

Petersburg, Russian Federation

Correspondence: Alexander Edemskiy

Journal of Cardiothoracic Surgery 2017, 12(Suppl 1):O3

\section{Objective}

Pulmonary thromboendarterectomy (PTE) is the main treatment option for patients with chronic thromboembolic pulmonary hypertension (CTEPH). The true prevalence of CTEPH remains unknown, especially in EMEA countries. We present 10-year outcomes of surgical treatment in patients with CTEPH.

Methods

Between 2004 and 2016252 patients underwent PTE for CTEPH at our institution. 10-year outcomes after surgery were assessed in 160 (63.5\%) patients. These patients underwent clinical (6MWD, Borg scale, sf-36 questionnaire) and instrumental evaluation (echocardiography, VQ-scintigraphy, right heart catheterization).

Results

Mean patient age at surgery was 48.5 [40;58.25] years. 6MWD in creased from 200 [197;202] m before surgery to 450 [440;460] $\mathrm{m}$ in long-term follow-up, $(p<0.001)$. Borg scale index decreased from 7 [6;8] to $1[1 ; 2],(p<0.001)$. There was a significant reduction in mean pulmonary pressure from $37[32 ; 40]$ to $29[28 ; 31] \mathrm{mmHg}(p<0.001)$ and pulmonary vascular resistance from $830[621.2 ; 1380]$ to 513 [482; 682] dyn.sec $\cdot \mathrm{cm}^{-5}(p=0.621)$ in long-term follow-up. We also noticed significant elevation of cardiac output from $3.68[2.8 ; 4.1]$ to 4 [3.2;4.4] $\mathrm{I} / \mathrm{min}$ in the whole group $(\mathrm{p}<0.001)$. According to VQscintigraphy we noticed statistically significant reduction of lung perfusion deficit from 43.4\% [37.85;41.9] to $10.8 \%$ [10.9;12.3], $(p<0.001)$. Overall mortality was in $13(7,69 \%)$ cases. There were 9 in-hospital deaths $(5.32 \%)$ due to reperfusion pulmonary edema $(n=4)$, multiorgan failure $(n=3)$ and pulmonary bleeding $(n=2)$. In 10-years followup period mortality was registered in $4(2.37 \%)$ cases due to residual pulmonary hypertension and progressive right hear failure.

\section{Conclusions}

Despite the increased perioperative risk and mortality PTE should be considered to patients with CTEPH as the first treatment option. Keeping increased perioperative risk and mortality in mind, significant pressure reduction and improved functional outcome can be achieved in the majority of these patients.

\section{Topic: Heart Failure}

\section{4}

Surgical ventricular restoration in cases of ischemic dilated cardiomyopathy: outcome at 15 years in 62 patients

Guglielmo Stefanelli', Fabrizio Pirro', Luca Weltert', Alina Alaru', Davide Trevisan", Paolo Giovanardi ${ }^{3}$

${ }^{1}$ Hesperia Hospital, Modena, Italy; ${ }^{2}$ European Hospital, Rome, Italy; ${ }^{3}$ University Hospitals, Modena, Italy

Correspondence: Guglielmo Stefanelli

Journal of Cardiothoracic Surgery 2017, 12(Suppl 1):04

\section{Background}

Aim of the study was to analyze the long term results (15 years) after left ventricular restoration (SVR) in cases of dilated ischemic cardiomyopathy.

Methods

Between March 2002 and September, 2015, 61 patients affected by ischemic dilated cardiomyopathy received a SVR at our institution, along with surgical myocardial revascularization. The STICH Trial criteria have been used as indication to SVR.The patients underwent SVR using a classical DOR operation, or, more recently adopting a different surgical technique, with the aim of reshaping the left ventricle also at equatorial level. A reduction of left ventricular end systolic volume greater than $50 \%$ was obtained in almost all cases $(p<0,05)$. A mitral valve repair was associated in $58 \%$ of cases.

Results

Total early-in hospital mortality was 3,4\%, (0\% in the last 31 cases). Reduction of LVED diameter, improvement of EF and NYHA class after surgery and at last follow-up were statistically significant $(p<0,05)$. Residual mitral incompetence. at discharge was absent or trivial in 56 patients $(96,6 \%)$, mild in $2(3,5 \%)$. Late mortality was $44 \%$ (25 pts) (mean follow up time: 7,6 yrs.), with a cardiac mortality of $20 \%$. Pre-operative severe mitral incompetence, renal failure and elevated EuroScore were strong predictors of worse outcome $(p<0,05)$. Freedom from rehospitalization for heart failure at last follow-up was $83 \%$ for the entire group of survivors.

Conclusions

Patients affected by ischemic dilative cardiomyopathy, in our experience, have a satisfactory short and intermediate-time outcome after SVR, particularly if accomplished by using a surgical protocol addressing both the volume and the shape of the left ventricle.

\section{Topic: Heart Failure}

05

Bridge to transplant in patients with cardiogenic shock: ECLS or BVAD?

Sandro Sponga, Veronica Ferrara, Andrea Lechiancole, Massimo Maiani, Chiara Nalli, Guzzi Giorgio, Ugolino Livi

Udine University Hospital, Udine, Italy

Correspondence: Sandro Sponga

Journal of Cardiothoracic Surgery 2017, 12(Suppl 1):O5

\section{Objective}

ECLS (extracorporeal life support) is considered the gold standard to treat patient affected by cardiogenic shock because of reduced invasiveness and rapidity of implantation. However, biventricular assist devices (BVADs) allow a long-term support and better patient stabilization before transplantation. This study evaluates the outcomes of ECLS and BVAD as bridge to transplant in patients with cardiogenic shock.

Methods

Since 1998, 133 patients with cardiogenic shock have been treated. Patients affected by myocardial infarction, cardiac arrest, respiratory failure and with transplant contraindications were excluded from this study. Forty seven patients treated with ECLS and 28 with BVAD were enrolled. Before the implant, patients with ECLS had worse acidosis (lactate $6.3 \pm 5.6$ vs. $3.5 \pm 2.9 \mathrm{mmol} / \mathrm{L}, \mathrm{p}=0.03$ ) and a tendency for greater need of mechanical ventilation ( $37 \%$ vs $15 \%, p=0.05$ ). While they did not differ by renal or liver function and other laboratory data.

Results

The peri-implant mortality was $21 \mathrm{vs} 11 \% \mathrm{p}=0.19$. After an average support of $9 \pm 8$ vs $47 \pm 68$ days $p<0.01$, in $9 v$ vs $11 \%, p=1$ weaning was possible and $64 v s 57 \% p=0.57$ underwent transplantation, respectively. Thirty-day mortality after transplantation was $22 \mathrm{vs} 18 \%$ $p=0,53$. After transplantation, patients treated with ECLS were more frequently affected by acute renal failure $(50 \%$ vs $6.7 \%, p=0.006)$ 
requiring dialysis $(39.3 \%$ vs $6.7 \%, p=0.03)$ and respiratory failure $(25 \%$ vs $0 \%, P=0.04$ ). Survival and long term complications were similar.

\section{Conclusions}

ECLS and BVAD are both effective as bridge to transplant in patients with cardiogenic shock. BVADs permits longer support and get to transplantation in more stable conditions, reducing perioperative complications.

\section{Topic: Heart Failure}

06

Does novel cardiac biomarker can predict cardiac-related complication after open heart surgery

Vadim Shumavets, Marina Kaljadka, Irina Russkih, Ihar Andraloits, Marina

Busjkevich, Alena Lisjonok, Yuory Ostrovski

Belarus Centre Cardiology, Minsk, Belarus

Correspondence: Vadim Shumavets

Journal of Cardiothoracic Surgery 2017, 12(Suppl 1):06

\section{Background}

Our study focused on prognostic capacity of novel cardiac biomarker in patients with severe compromised ischemic and non-ischemic left ventricle (LV) to predict cardiac-related complication after open heart surgery.

Methods

73 patients with severe depressed LV function (EF < 35\%) were included in pilot prospective study. Cardiomyopathy developed due to CAD in 51 patients or was idiopathic in 22 patients (mean age $62,2 \pm 4,9$ and $44,4 \pm 9,9$ years). Patients underwent either combined CABG with mitral valve procedure (49 patients) or isolated mitralvalve repair or replacement (24 patients) consequently. Blood samples for measurements of cardiac biomarkers (sST2, NT-proBNP, hsCTnl and (RP) were collected preoperatively, on $1^{\text {st }}, 7^{\text {th }}$ and $30^{\text {th }}$ postoperative days. The primary end point was complicated postoperative period due to cardiac-related events (duration of isotopes more then $24 \mathrm{~h}$, intra-aortic balloon pump, temporary VAD or hospital mortality).

Results

Cardiac-related complications were observed in 27 patients (37\% of cases) during postoperative period. Preoperatively only level of sST2 was significantly higher in patients with cardiac-related complications during hospital stay $(86,9(49,4-113,1)$ vs. $25,3(19,8-35,8)$ respectively, $\mathrm{p}=0,001)$. While no difference were found in NT-proBNP (2000 (427$6577)$ vs. 1200 (870-2169), $\mathrm{p}=0,422)$ and hs-cTnl $(0,015(0,005-0,035)$ vs.0,01 $(0,005-0,019), p=0,522)$ between patients with complicated or not postoperative period. AUC in ROC-analysis was also highest for preoperative sST2 level - 0,852 (95\% Cl 0,691-1,014, $p=0,02)$. The best cut-off value of the preoperative sST2 level was $45 \mathrm{ng} / \mathrm{ml}$ with $81,81 \%$ sensitivity and $93,75 \%$ specificity. sST2 level higher $45 \mathrm{ng} / \mathrm{ml}$ was identified as independent predictors for cardiac-related complication after heart surgery (OR - 5,345 $(95 \% \mathrm{Cl} 3,6-9,78, \mathrm{p}=0,01)$.

\section{Conclusions}

These results demonstrated that preoperative level of SST2 compared with NT-proBNP and hs-cTnl can be used to identify patients with depressed LV function at increased risk of postoperative complicated period.

\section{Topic: Heart Failure}

\section{7}

Left ventricular assist device-specific infections: prevention,

treatment and outcome - serbian experience

Svetozar Putnik, Dusko Terzic, Emilija Nestorovic, Ana Janjic,

Mina Zlatkovic, Miljko Ristic

Clinical Center of Serbia, Belgrade, Belgrade, Serbia

Correspondence: Svetozar Putnik

Journal of Cardiothoracic Surgery 2017, 12(Suppl 1):O7

\section{Objective}

Left ventricular assist device (LVAD) implantation has become an effective treatment option for patients with severe heart failure. LVAD specific infections are the leading cause of morbidity and the second most common cause of death after the first six months of the device implantation. This is also the most common cause of readmissions in hospital. The aim of this study is to report our single-center experience concerning prevention, treatment and outcome for the patients with LVAD specific infections.

Methods

Overall, 56 patients received LVAD at our center between 2013 and 2017. All patients were administered with antibiotic prophylaxis treatment for two weeks after surgery and a daily treatment of dressing the wound on the cable exit site.

Results

The median age of CF-LVAD was 51, the youngest patient was 16 years old, the oldest was 67 years old. The median duration of LVAD support was 10 months. Five patients develop VAD specific infections (8\%). Four patients developed driveline infections, and one patient develop pump pocket infection. Median time from VAD implantation to DLI was 126 days. Trauma to the driveline has been associated with DL infection in 3 of 4 causes. Pseudomonas aeruginosa was the most frequent infecting organism, causing 3 of $4 \mathrm{DL}$ infections. Two patients were treated with surgical driveline repositioning with extensive debridement of the wound. One patient underwent heart transplantation. One patient has a chronic infection that was conservatively treated with regular wound toilet. Patient with pump pocket infection was treated with chronic suppressive antibiotic therapy.

Conclusions

Considering the clinical complexity, prevention and treatment of LVAD specific infections presents a serious therapeutic challenge. With the development of surgical techniques and developing new devices, it is possible to reduce the rate of morbidity and increase survival rate in patients with implanted LVAD.

\section{Topic: Heart Failure}

\section{8}

Surgical right ventricle restoration after endomiocardial fibrosis resection

Marco Turrisi, Giuseppe M. Raffa, Chiara Minà, Michele Pilato

IRCCS-ISMETT (Istituto Mediterraneo per i Trapianti e Terapie ad

altaspecializzazione), Palermo, Italy

Correspondence: Giuseppe M. Raffa

Journal of Cardiothoracic Surgery 2017, 12(Suppl 1):O8

\section{Objective}

Surgery increases survival and quality of life, when compared to medical therapy, but must be performed before irreversible cardiac and hepatic damage occurs.

Methods

62 years black old man from Ghana on permanent AF was admitted to our institute because of several episodes of heart failure (NYHA III). Endomyocardial fibrosis diagnosis was made in 1996 with endomyocardial biopsy. Pre-operative $\pi$ echocardiogram showed obliteration and retraction of the right ventricle apex with dilation of its basal portion $(62 \mathrm{~mm})$, severe tricuspid regurgitation and tricuspid annulus enlargement and moderate systolic dysfunction (TAPSE $7 \mathrm{~mm}, \mathrm{~S}^{\prime} 0.07$ $\mathrm{m} / \mathrm{s})$; dilation of RA and IVC $(22 \mathrm{~mm})$. The Cardiac MRI images show right ventricle outflow tract and right atrial enlargement and, right ventricle apical segments obliteration. Surgery was carried out by full sternotomy, aorto-bicaval cannulation and selective antegrade cold blood cardioplegia. Surgical steps included 1) right ventricle fibro calcific endomiocardial tissue resection; 2) right ventricle wall and papillary muscles unbridling; 3 ) tricuspid valve anuloplasty with $36 \mathrm{~mm}$ flexible ring; 4) repair of the anterior leaflet of tricuspid valve, right atrial reduction and left appendage amputation.

Results

Cardiopulmonary bypass and aortic cross clamp time were 122 and $80 \mathrm{~min}$, respectively. ICU stays were 3 days. Postoperative course was uneventful and the patient was discharged home after 14 days. Postoperative echocardiogram showed reduction of RV dimensions (basal diastolic diameter, $50 \mathrm{~mm}$ ) with mild improvement of longitudinal function (TAPSE $11 \mathrm{~mm}$ ); tricuspidal valve well-functioning with 
regular transvalvular flow (Mean Gradient $2 \mathrm{mmHg}$ ) and mild residual regurgitation. At 3 months follow up we recorded a NYHA II.

\section{Conclusions}

A surgical technique for the relief of right ventricular trabecular cavity obliteration was described and results seem to be encouraging in terms of restoration of both structural and functional changes of the right ventricle.

\section{Consent for publication}

Written informed consent to publish was obtained from the patient involved in this study.

\section{Topic: Coronary Artery Disease}

\section{9}

- The medical aspect of rehabilitation of patients with coronary heart disease after coronary artery bypass grafting

Nurali Shakirov, Anar Tursynbekova, Yerkebulan Magauin,

Ainash Issayeva, Almaz Kiyabayev, Zeinat Ismailova

Sema Hospital, Almaty, Kazakhstan

Correspondence: Nurali Shakirov

Journal of Cardiothoracic Surgery 2017, 12(Suppl 1):09

\section{Objective}

To assess the efficiency of cardiac rehabilitation and drug therapy for cardiac patients at the outpatient stage after CABG, at the Sema Hospital.

Methods

The study included 100 patients who were in the rehabilitation department of Sema Hospital after CABG during the period of 20142017. The age of patients ranged from 37 to 79 years (an average of 59 years). The group consisted of 86 men and 14 women. All patients had a history of hypertension, 63 patients had one or more Mls in the past, and 3 patients had Mls associated with surgery (MI type 5). The number of bypasses ranged from 1 to 4 (mean 2.9). Mammary CABG was performed in 81 patient, auto venous CABG was performed in 83 patients, auto arterial CABG was performed in 6 patients. The operation with the use of cardiopulmonary bypass and cardioplegia was performed in 96 patients, surgery on a beating heart was performed in 4 patients.

Results

A pain syndrome in the chest, due to sternotomy and allocation of the internal thoracic artery - 42\%; aggravation of osteochondrosis with pains of radicular nature - 17\%; post venous ectomy syndrome (lymphatic edema of the limb) - 33\%. Dissection of the chest leads to an imminent breathing mechanics disorder, most significantly manifested in the early period after the operation. Expressed disorders of the function of external respiration are observed in $40 \%$ of patients. One-third of them have restrictive changes with a decrease in the vital capacity of the lungs, one-third of the changes are obstructive and one-third have mixed obstructive-restrictive disorders with a decrease in maximum ventilation of the lungs.

\section{Conclusions}

Comprehensive rehabilitation effects, including medication and nondrug methods, with a proven effect on the prognosis, should begin at the inpatient stage and continue at outpatient and outpatient rehabilitation stages.

\section{Topic: Pediatric Cardiology}

\section{0}

\section{Clinical spectrum of DCM caused by LMNA mutations}

Elena Zaklyazminskaya', Anna Shestak', Sergey Mironovich', Alexandre Polyakov², Olga Blagova ${ }^{3}$, Natalia Kotlukova 4 , Sergey Dzemeshkevich

${ }^{1}$ Petrovsky Russian research Centre of Surgery, Moscow, Russian Federation; ${ }^{2}$ Research Centre for Medical Genetics, Moscow, Russian Federation; ${ }^{3}$ Sechenov First Moscow State Medical University, Moscow, Russian Federation; ${ }^{4}$ Bashlyaeva City Pediatric Hospital, Moscow, Russian Federation

Correspondence: Elena Zaklyazminskaya

Journal of Cardiothoracic Surgery 2017, 12(Suppl 1):010

\section{Objective}

Laminopathies are well-known and relatively common form of dilated cardiomyopathy (DCM) with cardiac conduction defects manifesting in adolescents and adults. The aim of this work is to characterize the clinical spectrum of the mutations found in the LMNA gene in Russian cohort of DCM patients.

Methods

Clinical examination and genetic testing was performed in accordance to Helsinki declaration. Clinical examination included echocardiography, cardiac MRI, resting ECG and Holter monitoring. DNAdiagnostic had included direct Sanger sequencing of the whole coding and adjacent regulatory areas of the LMNA gene.

Results

We had found 11 mutations in the LMNA gene in 12 index cases with DCM and wide range of the cardiac arrhythmias and conduction defects. In 7 families the clinical phenotype was classical for laminopathies: manifestation at the 2nd or 3rd decade, slowly progression of DCM, progressive conduction defects, and variable muscular abnormalities. Proband carrying mutation p. Glu372Asp had underwent heart transplantation at the age of 22 years. Two mutations (p.Glu84Lys and p.Glu635Asp) were found in pediatric patients. Heterozygous mutation p.Glu84Lys is associated with mild form of DCM and rhythm/conduction disturbances. When homozygous it produces fulminant form of DCM and characterizes by severe prognosis. Two homozygous p.Glu84Lys carriers (male, 2 y. $3 \mathrm{~m}$. old, and female, 2 y. $8 \mathrm{~m}$. old) died with the clinical signs of progressive heart failure. Heterozygous p.Glu635Asp mutation had risen de novo. The request from the couples was the genetic counseling and recurrent risk assessment for their expected pregnancy.

Conclusions

Mutations in the LMNA gene might mimic recessive inheritance and should be considered as a possible cause of fatal early-onset cardiomyopathy. A detailed understanding of the origin of DCM allows us to provide the most effective preventive reproductive strategies and clinical follow-up based on existing treatments. Study supported by RNF grant №16-15-10421

Consent for publication

Written informed consent to publish was obtained from the patients involved in this study.

\section{Topic: Pediatric Cardiology}

011

Quality of life in children with different pulmonary hypertension forms

Tatyana I. Ivanova-Razumova, Almira Baigalkanova, Aikerim Zhanatkyzy, Ludmila Mameganova, Aiman Tlepova

National Research Center for Cardiac Surgery, Astana, Kazakhstan

Correspondence: Tatyana I. Ivanova-Razumova

Journal of Cardiothoracic Surgery 2017, 12(Suppl 1):011

\section{Objective}

Quality of life is the main part of pulmonary hypertension patients follow-up. but now there are only occasional dates about this aspect in pediatric practice. we decide to evaluate quality of life in children with different forms of pulmonary hypertensions.

Methods

From January 2012 up to February 2017, 97 children with PH were hospitalized in our center. Median age - 9 years (range 2 - 18 years). Boys $42(43 \%)$, girls - 55 (57\%). PH was diagnosed according to ESC guidelines. We have used PedsQl for estimation physical, emotional, social and school functioning. Standard statistical measures of significance was used.

Results

Patients were divided for 4 groups: I - IPAH (12.1\%), II - postoperative PAH (25.6\%), III - Eisenmenger Syndrome (51.3), IV - PH due to lung disease (11\%). Total Qol score in I group was - 42,3 $\pm 5,6$ scores, in II - 40,4 $\pm 3,8$ scores, III - 60,78 $\pm 6,9$ scores, IV group - $68 \pm$ 4,9 scores. In all groups mean total Qol score was significantly lower than in healthy population (84.6 scores, $\mathrm{p}<0,001)$. By comparison 
between different groups lowest total Qol scores were find in I and II groups, difference was statistically valid for III and IV groups.

\section{Conclusions}

Between different groups lowest quality of life level was founded in patients with IPAH and in patients with post-operative pulmonary hypertension. Up to now question about necessity and advisability of surgical correction of borderline (operable/inoperable) left to right shunts CHD remains a big problem. We receive convincing evidence that quality of life in post-operative $\mathrm{PH}$ patients significantly low than in patients with Eisenmenger syndrome $(p<0,001)$. Further evaluation is required for determine optimal operability criteria.

\section{Topic: Pediatric Cardiology}

\section{2}

Structure and prevalence of congenital heart disease in children in the pavlodar region

O. S. Mukashev, G. D. Abilmazhinova, E. I. Khagay, I. O. Sarsembekova, D. K. Zhakipbayev

Pavlodar Regional Cardiological Center, Pavlodar, Kazakhstan

Correspondence: E. I. Khagay

Journal of Cardiothoracic Surgery 2017, 12(Suppl 1):012

\section{Objective}

To determine the structure and prevalence of CHD in children in our region by studying medical and demographic data.

Methods

A retrospective analysis was performed, medical documentation of children with CHD who received inpatient treatment at the center was analyzed, taking into account the main medical and demographic indicators (sex, maturity, age of identification of CHD) for 2016.

\section{Results}

During the study period, the our center received inpatient treatment with CHD and other cardiovascular diseases 264 children, including 138 children with CHD in our region, which was $52.3 \%$. Among the children with CHD, the proportion of girls was $56.5 \%$, boys - $42 \%$. Diagnosis of CHD in children was diagnosed in $36.9 \%$ in the maternity hospital, in $63 \%$ in the polyclinic. Up to 1 year of life CHD is exposed in $37.7 \%$ of cases, from $1-5$ years - $22.5 \%, 5-18$ years $-34 \%$. Half of the CHD was diagnosed early. Preterm infants among the CHD accounted for $13 \%$. Most of the children with CHD were born with normal body weight. VSD prevalence was $29.1 \%$, ASD - $21.3 \%$, PDA - 15\%, complex defects (tetralogy of Fallot, complete AVSD, TMA) $-8.7 \%$, other defects $-26 \%$.

\section{Conclusions}

Thus, CHD are one of the most widespread developmental anomalies all over the world, including in our region. Features of the current $\mathrm{CHD}$ at the present stage are the increase in their frequency, the increase in the specific gravity of complex and combined heart defects. The natural course of CHD is characterized by high mortality of children, especially at the first year of life. The untimely implementation of surgical correction of CHD in children in a number of cases is due to the low resolving power of ultrasound devices on the ground, and the lack of awareness of physicians about some rare vices.

\section{Topic: Pleuro-Pulmonary}

\section{3}

Minimally invasive surgical lung biopsy with local anesthesia and sedation in non-intubated patients with suspected idiopathic pulmonary fibrosis: preliminary results and patients satisfaction Roberto Cherchi', Laura Riva', Massimiliano Santoru', Roberto Perra², Roberto Allieri ${ }^{3}$, Matteo Pinna Susnik', Federico Palludo', Paolo A. Ferrari

'Department of Thoracic Surgery - P.O. A. Businco - AOBrotzu, Cagliari, Italy; ${ }^{2}$ Pneumology - P.O. Binaghi - ATS - ASSL, Cagliari, Italy; ${ }^{3}$ Radiology Service - P.O. Binaghi - ATS - ASSL, Cagliari, Italy

Correspondence: Roberto Cherchi

Journal of Cardiothoracic Surgery 2017, 12(Suppl 1):013

\section{Objective}

Exclusion of other known causes of interstitial lung disease (ILD) is a mandatory requirement to achieve diagnosis of idiopathic pulmonary fibrosis (IPF). It is best confirmed by the identification of usual interstitial pneumonia (UIP) on surgical lung biopsy, traditionally performed in video-assisted thoracic surgery (VATS) and general anesthesia (GA), associated with not negligible mortality and morbidity rates. Our center proposed to standardize a safe non-intubated VATS approach for SLB in patients with suspected IPF.

Methods

Twenty one awake VATS lung biopsy were performed under local anesthetic techniques, with or without consciousness sedation, in spontaneously breathing. Primary outcomes were technical feasibility, perioperative anesthetic issues, post-surgery complications and mortality within 30 days after procedures. We also considered as secondary outcomes the operative time of surgery and operating room (OR) stay, post-surgery length of stay (LOS), prolonged air leaks, diagnostic yield according to volume biopsy $\left(\mathrm{cm}^{3}\right)$ and patient numeric rating scale (NRS) of pain after surgery. Logistic regression and generalized linear models were applied to assess possible effects of aggregate risk factors to surgical outcomes.

Results

All the procedures had been carried out with VATS technique and sedation, without conversion to GA. Mean LOS was 1.42 days without prolonged air leaks evidence. There was no post-operative mortality within 30-days. Analgesia methods resulted in optimal feasibility with a mean NRS score of 0.95 . The mean OR stay and operative time were 116.84 minutes and 46.67 minutes respectively. The average biopsies volume was $3.3 \mathrm{~cm}^{3}$ with histological diagnosis achieved in all 21 patients.

Conclusions

A standardized non-intubated VATS approach with light sedation, spontaneous breathing and local wound anesthesia, appears to be a practice and safe solution with an excellent diagnostic yield and patients satisfaction. Larger studies are needed to identify the impact of aggregate risk factors over surgical outcomes in IPF patients.

\section{Topic: Pleuro-Pulmonary}

014

Sentinel node mapping in non-small cell lung cancer using intra operative radiotracer technique

Reza Bagheri, Susan Shafiei, Ramin Sadeghi, Vahid Reza Dabbagh Kakhki, Amir Hossein Jafarian, Reza Afghani, Davood Attaran, Reza Basiri,

Shahrzad M Lari

Mashhad University of Medical Sciences, Mashhad, Iran

Correspondence: Reza Bagheri

Journal of Cardiothoracic Surgery 2017, 12(Suppl 1):014

\section{Introduction}

Lymph node metastases are the most significant prognostic factor in localized non-small cell lung cancer (NSCLC). Identification of the first nodal drainage site may improve detection of metastatic nodes. Extended surgeries such as lobectomy or pneumonectomy with lymph node dissection are more acceptable for treatment. Sentinel node biopsy can be an alternative approach toward less invasive surgeries. In the current study, we evaluated accuracy of sentinel node mapping in 21 patients with NSCLC by intra-operative radiotracer techniques.

Method

After thoracotomy, before mobilizing the tumor, peritumoral injection of $2 \mathrm{mCi} / 0.4 \mathrm{~mL}$ Tc- $99 \mathrm{~m}$ - phytate was performed in tumor. After mobilization, the sentinel nodes were traced in hilar and mediastinal areas. Any lymph node with in vivo count twice the background was considered as sentinel node and sent for frozen section evaluation. All dissected nodes were evaluated by step sectioning, hematoxylin and eosin staining (H\&E). The variables were age, sex, kind of pathology, site of lesion, number of dissected sentinel nodes, number of sentinel nodes and site of sentinel nodes. 
Results

21 patients (male/female=15/6) were included in study with the age of $58.52 \pm 11.46$ years. Most common site for tumor was left lower lobe (LLL) (30.09\%). 11 patients had Squamous cell carcinoma (SCC) and 10 had Adeno carcinoma (AC). 120 lymph nodes were harvested with average of $5.71 \pm 2.9$ lymph nodes per patient. at least one sentinel node could be identified in all patients (detection rate of $95.2 \%$ ). Mean number of sentinel nodes per patient was $3.61 \pm 2$. Frozen section results showed $100 \%$ concordance with H\&E results.

Conclusion

Sentinel node mapping can be considered feasible and accurate for lymph node staging and NSCLC treatment.

Keywords

Sentinel node- non small cell lung cancer- radiotracer

\section{Topic: Pleuro-Pulmonary}

\section{5}

Ectopic adrenocorticotropic hormone syndrome: clinical features, diagnosis, treatment and long-term observation. Impact of bronchial carcinoid tumors

Vadim Krylov ${ }^{1}$, Ekaterina Dobreva ${ }^{2}$, Sergey Kharnas ${ }^{1}$, Vladimir Parshin ${ }^{1}$, Leonid Ippolitov' ${ }^{1}$ Nikolay Kuznetsov², Georgy Polunin ${ }^{1}$, Anna

Ananichuk'

${ }^{1}$ Sechenov University, Moscow, Russian Federation; ${ }^{2}$ Endocrinology

Research Centre, Moscow, Russian Federation

Correspondence: Vadim Krylov

Journal of Cardiothoracic Surgery 2017, 12(Suppl 1):015

\section{Objective}

Ectopic adrenocorticotropic hormone (ACTH-ectopic) syndrome (EAS) is a rare cause of ACTH-dependent endogenous hypercortisolism. The objective of this study was to analyze the clinical, biochemical, and radiological features, management, and treatment outcome of patients with EAS.

Methods

It was a retrospective case-record study of 47 patients with EAS. Clinical, biochemical, and radiological features and response to therapy and survival rate were measured.

Results

The median follow-up was 7 yrs. (range, 1-13 yrs.). None of the dynamic tests achieved $100 \%$ accuracy. Imaging correctly identified the lesion at first investigation in $80.9 \%$ of cases. Bronchial carcinoid tumors were the most common cause of EAS $(n=27 ; 57,5 \%)$, followed by other neuroendocrine tumors ( $n=11,23,4 \%)$. In $19.1 \%$ (9) of patients, the source of EAS was never found. Octreotide scintigraphy and whole-body venous sampling were of limited diagnostic value. Surgical attempt at curative resection was successful in $81 \%$ (38 out of 47 ) of all patients; $9(19.1 \%)$ responded generally well to bilateral adrenalectomy by vital indication. Tumor histology and the presence of distant metastases were the main predictors of overall survival $(P<0.05)$.

\section{Conclusions}

Bronchial carcinoid tumors is the main cause of ACTH-ectopic syndrome. No single test was capable of finding the source of EAS correctly. Despite a variety of tests and imaging studies for the correct diagnosis of the EAS, up to $19 \%$ of cases present as occult EAS syndrome. These cases require a prolonged follow-up, review, and repetition of diagnostic tests and scans, but, in severe cases, bilateral adrenalectomy is performed.

\section{Topic: Pleuro-Pulmonary}

016

Tracheal surgery complications

Aleksandr V. Starostin, Ovik S. Mirzoyan, Vladimir D. Parshin

I.M. Sechenov First Moscow State Medical University of Ministry of

Health, Moscow, Russian Federation

Correspondence: Ovik S. Mirzoyan

Journal of Cardiothoracic Surgery 2017, 12(Suppl 1):016

\section{Objective}

On the basis of studying of the immediate results to define factors of development of the adverse postoperative period and a mortality, and also to plan the relevant preventive activities.

Methods

During the period from 1963 to 20151128 patients with cicatricial stenosis of trachea are treated. The resection of trachea (RT) was executed $389(34,48 \%)$ the patient. Frequency of these operations has increased more than by 20 times in recent years. The staged reconstructive treatment (SRT) have transferred $667(59,13 \%)$ patients. In total them was produced 1157 operations. Endoscopic treatment was received by 72 patients $(6,38 \%)$. The main part of operations (more than 89\%) have been executed after 2001. In this regard patients were divided into 2 groups - from 1963 to 2000 and from 2001 to 2015 , respectively.

Results

The general frequency of complications was $6,8 \% .19$ people $(1,1 \%)$ died. After resection of trachea the frequency of complications was reduced from $32,2 \%$ in the first group to $14,6 \%$ - in the second. Later SRT frequency of complications was reduced from $10 \%$ in the first group to $2,2 \%$ - in the second. Mortality after RT was reduced more than by 30 times. In the first group of patients mortality was $20,3 \%$, and in the second - $0,6 \%$. Mortality after SRT was reduced from 1,3\% in first group to $0,2 \%$ in the second. The developed algorithm of postoperative treatment allows stopping most of developing complications with success. The positive tendency in safety of operations is the basis for extension of indications to one-stage radical treatment. Conclusions

In recent years the frequency of postoperative complications and a mortality managed to be reduced significantly that is the basis for more "aggressive" surgical tactics.

\section{Topic: Pleuro-Pulmonary}

017

A new classification of lung adenocarcinoma nodules guiding treatment

Zhangfan Mao, Jie Huang, Bo Wang, Ping Dong

Renmin Hospital, Wuhan University, Wuhan, China

Correspondence: Zhangfan Mao

Journal of Cardiothoracic Surgery 2017, 12(Suppl 1):017

\section{Background}

More and more small lung nodules were pathologically diagnosed as adenocarcinoma in China. These lung adenocarcinoma nodules have unique characteristics. They are commonly developed from pure GGN (ground glass nodules), to partially solid nodules and then solid nodules shown in chest $\mathrm{CT}$, and finally to adenocarcinoma diagnosed pathologically. This development is associated with corresponding pathological diagnoses, from AIS and MIA, and eventually to IA. The treatment strategies should be different. But because of the vague classification of these lung nodules, it is difficult for physicians to choose suitable treatment methods.

Methods

The lung adenocarcinoma nodules were classified according to the tumor development, and each class of nodules is corresponded to the chest CT imaging and pathological diagnoses.

Results

Firstly, according to the chest CT images, the lung adenocarcinoma nodules were divided into three categories: A. GGN; B. partial solid nodules; $C$. solid nodules.

The nodules in class A are divided into two categories: $\mathrm{A} 1$ and $\mathrm{A} 2$. A1 includes diameter $<8 \mathrm{~mm}$, peripheral no small vascular shadow, pathology is AIS, treatment is observation. A2 includes diameter $>8 \mathrm{~mm}$ or peripheral small vessels into the shadow, pathology is AIS or MIA, treatment is local resection.

The nodules of class B are divided into B1 and B2. B1 is defined as solid component $<25 \%$ and diameter of solid component $<5 \mathrm{~mm}$, pathology is MIA, treatment is local resection with lymph node sampling. B2 is defined as solid component $>25 \%$ or diameter of solid 
component $>5 \mathrm{~mm}$, pathology is IAC or MIA. Lobectomy and systematic lymph node dissection is warranted in IA.

All of the $C$ period solid nodules are associated with IAC pathologically. They are divided into $\mathrm{C} 1$ and $\mathrm{C} 2$ according to metastasis.

Conclusion

With this classification, we can determine the type of small nodules in the lung adenocarcinoma, and select the appropriate treatment.

\section{Topic: Pleuro-Pulmonary}

\section{8}

Surgical treatment of combined echinococcosis in children

Bakhytzhan Kerimbekovich Shirtaev, Mukhtar Magzumovich Sundetov, Ruslan Zhumakhanovich Kassenbayev, Denis Sergeevich Voronin,

Daniyor Rahmatulaevich Kurbanov, Nazira Zholdibayevna Erimova

National Scientific Center for Surgery, Almaty, Kazakhstan

Correspondence: Ruslan Zhumakhanovich Kassenbayev

Journal of Cardiothoracic Surgery 2017, 12(Suppl 1):018

\section{Objective}

To improve the results of surgical treatment of combined echinococcosis in children

Methods

In our clinic from 2001 to 2015 were treated 180 children with combined echinococcosis of the lungs and liver. The boys were $99(55 \%)$, girls - 81 (45\%).

Results

When performing combined operations, the following surgical tactics were followed: first, an echinococcectomy from the lung, followed by echinococcectomy from the liver. A staged surgical treatment was performed in $29(16.1 \%)$ of children with multiple complicated echinococcosis of the lungs and liver with an interval of 2-3 weeks up to 8 weeks. One-step sequential videothoracoscopic echinococcectomy and then laparotomy with liver echinococcectomy was performed in $46(25.5 \%)$ patients, no complications were observed. Indications for surgery were: a single lung cyst, single and multiple complicated and uncomplicated liver cysts. Analysis of postoperative complications in children with hydatid cysts of the lungs and liver shows that pulmonary-pleural complications in the form of postoperative pneumonia were observed in 6 (3.3\%) patients. Postoperative pneumonia is resolved in a conservative way. In $2(1.1 \%)$ patients, suppuration of a laparotomic postoperative wound was observed.

\section{Conclusions}

Videothoracoscopic echinococcectomy of the lung allowed to reduce the time of anesthetic and operational aggression, reduce the duration of the patient's stay in the hospital to $10-13$ days, which is 3-5 times less than with other procedures used for echinococcosis of the left lung and liver. The use of video-surgical echinococcectomy, characterized by its minimal invasiveness allows to increase the effectiveness of surgical treatment of combined echinococcosis of the lungs and liver in children.

\section{Topic: Pleuro-Pulmonary}

\section{9}

Catamenial pneumothorax and thoracic endometriosis an underdiagnosed disease

Kosmas Tsakiridis, Stamatis Arikas, Andreas Bakas

St Luke's Hospital, Thessaloniki, Greece

Correspondence: Kosmas Tsakiridis

Journal of Cardiothoracic Surgery 2017, 12(Suppl 1):019

\section{Objective}

Secondary spontaneous pneumothorax during menstruation is reported as catamenial pneumothorax. In many cases, the cause is thoracic endometriosis. According to many reviews, catamenial pneumothorax affects $3-6 \%$ of spontaneous pneumothorax while women who undergo surgery touch the significant percent of 25 $30 \%$.

Methods

Eight women were presented with recurrent catamenial pneumothorax. All of them had diaphragmatic perforations, which in one of the cases were of notable size with liver herniation. All women underwent atypical resection of lung parenchyma that appeared abnormal, all of them underwent perforation suturing and all of them had been through pleurodesis. VATS surgery was performed in seven patients and a Video-Assisted mini thoracotomy was performed in the patient with the liver herniation in order to suture a patch on the diaphragm.

Results

There were no deaths. The post-operative course was uneventful. Seven of the patients received hormonal therapy ( $\mathrm{GnRH}$ analogue) post-operatively. One patient had recurrence pneumothorax, six and a half months post-operatively. She received hormonal therapy for six months and had no further recurrences for a long period of five years.

Conclusions

Surgical treatment is the choice of treatment for catamenial pneumothorax. Highly suspicious rate and multi-disciplinary treatment, including hormonal therapy, may be beneficial to these women.

\section{Topic: Pleuro-Pulmonary}

020

Anatomical segmentectomy - single institution experience

Vytautas Nekrasas, Lars Møller

Department Cardiothoracic Surgery, Aalborg University Hospital, Aalborg, Denmark

Correspondence: Vytautas Nekrasas

Journal of Cardiothoracic Surgery 2017, 12(Suppl 1):020

\section{Objective}

There is a rising interest regarding anatomical segmental resections for early stage non-small cell lung cancer (NSCLC). In recent study we evaluated safety, postoperative outcomes for thoracotomy versus VATS in patients with poor lung function. Lobectomy is still the standard procedure for surgical treatment of lung cancer. However, in patients with poor lung function and in risk of post-lobectomy FEV1 or DLCO (diffusion capacity) below $30 \%$ of predicted, segmentectomy seems to be a safe choice for radical surgery.

Methods

We retrospectively analysed patients with poor lung function who underwent segmentectomy. RESULTS: From January 1996 to December 2016, 135 segmentectomies were performed on 130 consecutive patients. In this cohort patients presented with reduced FEV1, DLCO or both. Average preoperative FEV1 and DLCO was respectively $67 \%$ and $59 \%$ (range $30-118 \%$ ). Thoracotomy was performed in 43 cases, VATS in 87 cases, 16 was converted to thoracotomy. 5 patients underwent multisegmentectomy in different lobes. Chest tubes, on average, removed on postoperative day 5,9 for thoracotomy versus 3,3 in VATS. Hospital stay respectively 11,25 days and 6,4. Median follow up was 32 months. 21 patients died. Combined in hospital and 90-day mortality was 4. 17 late deaths occurred: 11 dissemination of lung cancer, 3 dissemination of other 
cancers, 3 lung-heart failure. 5-years survival for T1 NSCLC was $78 \%$. For T4 tumour (multiple tumours in same lung) no deaths in follow up period of 11-46 months. We did not observe a solitary case of local recurrence in the operated lobe.

\section{Conclusions}

Segmentectomy, performed by VATS or Thoracotomy, is a safe procedure with acceptable perioperative mortality and morbidity. It offers an opportunity for radical pulmonary resection in patients with poor lung function, multiple tumours in one or different lobes. In VATS group chest tubes removed earlier, shorter length of stay, and similar oncologic outcomes were observed.

\section{Topic: Pleuro-Pulmonary}

\section{1}

Recurrent pleural effusion complicating liver cirrhosis: period

January 2005 to January 2015

Fadil Gradica', Lutfi Lisha', Alma Cani ${ }^{1}$, Dhimitraq Argjiri ${ }^{1}$, Helidon Nino ${ }^{2}$, Elton Bollano ${ }^{3}$, Fahri Kokici', Ylber Vata', Donika Lala ${ }^{4}$, Leon Shpataraku', Dorjan Bozaxhiu', Fiqirete Gradica ${ }^{5}$

${ }^{1}$ University Hospital, Tirana, Albania; ${ }^{2}$ University Hospital "Mother

Theresa", Oncology University Hospital Tirana, Albania; ${ }^{3}$ University

Hospital " Mother Theresa' Visceral Surgery Department, Tirana, Albania;

${ }^{4}$ Public Stomatology Service, Tirana, Albania; ${ }^{5}$ Public Pharmacy Service, Tirana, Albania

Correspondence: Fadil Gradica

Journal of Cardiothoracic Surgery 2017, 12(Suppl 1):021

\section{Objective}

Pleural effusion (PE) is a rare complication of advanced liver cirrhosis, which may lead to an operation when uncontrolled. The purpose of this study was to evaluate the modality of the occurrence of pleural effusion and to describe its surgical management.

Methods

We studied 17 patients who were referred to the department of thoracic surgery because of massive and recurrent PE caused by liver cirrhosis. The PE was a transudate in 12 patients and an exudate in 5 . Talc pleurodesis was attempted in 5 patients, betadine pleurodesis 11, partial pleurectomy. Video assisted thoracoscopy was performed in 6 patients in whom the clinical condition permitted general anesthesia; the pleural cavity was entirely explored before pleurodesis. Chest tube drainage alone was performed in 11 patients who were unable to undergo general anesthesia.

Results

The PE was right-sided in 9 patients, left-sided in 5 , and bilateral in 3.Two patients had an early recurrence that was cured by complementary betadine pleurodesis. One patient presented with a right $\mathrm{PE}$; and one patient presented with a left PE. Of these, 3 patients died from multiorgan failure and septic shock. Two patients had a midterm recurrence.

\section{Conclusions}

Passage of ascites through diaphragmatic defects appears to be the main cause of PE complicating cirrhosis. Patients may benefit from betadine pleurodesis. Video assisted thoracoscopy pleurodesis is the technique of choice with consistent results. Repeated talc injection through the drain may prove useful for patients in poor clinical status.

\section{Topic: Pleuro-Pulmonary}

\section{2}

The rectus abdominis muscle flap - rare, but viable option for securing a bronchial stump fistula and basal pleural cavity obliteration in the management of complex infections after thoracic surgical procedures

Georgy Yankov, Anatoli V. Semkov, Danail Petrov

Thoracic Surgery Department, Saint Sophia University Hospital for

Pulmonary Diseases, Medical University, Sofia, Bulgaria

Correspondence: Anatoli V. Semkov

Journal of Cardiothoracic Surgery 2017, 12(Suppl 1):O22

\section{Objective}

To introduce the use of rectus abdominis muscle flap (RAMF) in the treatment of complex pleural infections after thoracic surgical procedures and to define the indications for its usage.

Methods

This approach was carried out in 4 debilitated patients with mean age 48.4 years. In two of them there was postpneumonectomy empyema (PPE) with bronchial stump fistula. They were operated on for MDR tuberculosis and severe late complications of chest trauma. The other two patients were with recalcitrant basal pleural cavity following pleurectomy and decortication for empyema. The operative access was posterolateral thoracotomy and there was previous laparotomy in all.

The RAMF was mobilized based on the superior epigastric vessels. The paramedian vertical incision was made and the anterior rectus sheath was opened, allowing exposure of the muscle. The rectus abdominis was raised from the posterior sheath and the inferior epigastric vessels were ligated. A special care was taken when the flap was turned towards the chest in order to avoid damaging of the vascular pedicle.

Results

In 2 patients RAMF was used to secure bronchial stump fistula and PPE was treated by accelerated Weder method. In the rest two it was used for obliteration of basal recalcificant residual cavity. The MDR patient willfully stopped her anti-TB medication and started intermittent fasting which led to relapse of bronchial fistula and empyema. The open window thoracotomy was performed for open management of empyema cavity. The patient died 2 years after the operation. The other three patients are alive and in excellent condition. Conclusion

The rectus RAMF is a viable option for securing a bronchial stump fistula and basal pleural cavity obliteration in the management of complex infections after thoracic surgical procedures. This method could be indicated when external thoracic muscles or omentum cannot be used due to different reasons.

\section{Topic: Pleuro-Pulmonary}

\section{3}

About classification of cicatrical tracheal stenosis

Alexey Parshin

I.M. Sechenov First Moscow State Medical University, Moscow, Russian

Federation

Journal of Cardiothoracic Surgery 2017, 12(Suppl 1):023

\section{Objective}

To improve the classification of cicatrical tracheal stenosis (CTS) taking into account the anatomic and clinical features of the patients.

Methods

Many classifications based on absolute values $(\mathrm{mm}, \mathrm{cm}$, amount of cartilaginous semirings) were offered. The length of the trachea (measured in $\mathrm{cm}$ ) and the amount of cartilaginous semirings, cannot determine the existent reality (that vary from 9 to $15 \mathrm{~cm}$, and $16-24$ accordingly). From 1963 to 20151128 patients with tracheal stenosis were cured. The patients were divided into 2 groups - from 1963 to 2000 (297) and from 2001 to 2015 (831). The classification of CTS is offered according to the stages of narrowing for every concrete patient: the 1st stage - less than on $1 / 3$ of the diameter of trachea, the 2nd stage - from $1 / 3$ to $1 / 2$, the 3 rd stage - from $1 / 2$ to $2 / 3$, the 4 th more than on $2 / 3$. According to the extension 5 stages are offered: the 1 st stage - limited a lesion less than $15 \%$ of the length of trachea, the 2 nd - medium - from 15 to $30 \%$, the 3rd - lengthy - from 30 to $60 \%$, the 4 th - total - more than $60 \%$, the 5 th - multifocal - different departments of air passage are engaged in process.

Results

After the resection of trachea with an anastomosis the frequency of complications decreased from $32,2 \%$ in the first group to $14,6 \%$ in the second. The lethality is diminished in 30 times from $12(20,3 \%)$ people of the first group to $2(0,6 \%)$ - in the second. The offered classification allowed to define the optimal plan of treatment of the 
patients with CTS, decreasing the amount of postoperative complications.

Conclusions

The offered classification can be used in clinical practice for the patients with CTS taking into account their anatomic features.

\section{Topic: Pleuro-Pulmonary}

\section{4}

Evaluation of lymph node dissection in VATS lobectomy versus lobectomy by thoracotomy

Konrad Pawelczyk, Piotr Blasiak, Marek Marciniak, Monika Szromek

Wroclaw Medical University, Wroclaw, Poland

Correspondence: Konrad Pawelczyk

Journal of Cardiothoracic Surgery 2017, 12(Suppl 1):024

\section{Objective}

VATS lobectomy is performed commonly in the treatment of early stage lung cancer. The adequacy of the lymph node resection achieved by VATS is disputable. The aim of the study is to compare the quality of lymph node dissection between VATS and open procedure.

Methods

Between Jan 2010 and Feb 2016209 consecutive open thoracotomy and 84 VATS lobectomies with systematic lymphadenectomy were performed in patients with lung cancer staged cT1a-2aNOMO. All the procedures were accomplished by one surgeon so the mode of lymphadenectomy, the dissection and the counting of lymph nodes were similar in all cases.

Results

Both groups were comparable regarding different clinical and pathological parameters. The mean pathologic tumor size was not significantly different in VATS $25.8 \pm 9.6 \mathrm{~mm}$ vs. thoracotomy $27.7 \pm 11.4 \mathrm{~mm}$, $\mathrm{p}=0.18$. In VATS approach, fewer lymph nodes were dissected in total $(19.9 \pm 8.2$ vs. $26 \pm 10 ; p<0.0001)$, in N1 $(8.3 \pm 5.4$ vs. $10.5 \pm 5.3, p=0.0006)$ and $\mathrm{N} 2$ compartment $(11.6 \pm 5.5$ vs. $15.5 \pm 7.9, \mathrm{p}=0.0001)$. In VATS group fewer lymph nodes were positive in total $(0.6 \pm 2.1$ vs. $1.6 \pm 3.5$, $\mathrm{p}=0.003)$ and in $\mathrm{N} 1$ compartment $(\mathrm{p}=0.003)$. Regarding the thoracotomy group $34 \%$ of the patients were upstaged to $\mathrm{pN} 1 / \mathrm{pN} 2$ compared with $16.6 \%$ in the VATS $(p=0.003)$. In VATS lobectomy group, fewer lymph nodes were dissected at the left side $(19.3 \pm 8.5$ vs. 22.2 $\pm 7.6 ; p=0.039)$ and the right side $(20.4 \pm 7.9$ vs. $28.6 \pm 10.7, p<0.001)$. There was no significant difference in 3-year survival between groups ( $84 \%$ VATS vs. $66 \%$ thoracotomy, $p=0.11$ ).

\section{Conclusions}

The experience with VATS lobectomy has been related with significantly less lymph nodes dissected and less patients upstaged to $\mathrm{N} 1$ or N2 disease compared with open thoracotomy. There was no 3-year survival difference in both groups. The mean number of nodes dissected during VATS lobectomy was relatively high however a particular attention should be placed on adequate lymph node dissection in this group to avoid missed N1/N2 disease.

\section{Topic: Vascullar}

\section{5}

Surgical treatment of patients with tumor thrombus in the right atrium

Uladzimir Andrushchuk', Youri Ostrovsky' Sergej Poliakov' ${ }^{2}$, Alexandr Rolevich², Liana Shestakova', Vladimir Zharkov ${ }^{2}$

${ }^{1}$ Belarus Cardiology Center, Minsk, Belarus; ${ }^{2}$ Belarus Oncology Center, Minsk, Belarus

Correspondence: Uladzimir Andrushchuk

Journal of Cardiothoracic Surgery 2017, 12(Suppl 1):025

\section{Objective}

To evaluate the experience of surgical procedures in patients with tumor thrombus in the right atrium.

Methods

From 2007 to 2016 it was operated ten patients with tumor thrombus in the right atrium, including six men and four women.
Renal cell carcinoma was diagnosed in seven, adrenocortical carcinoma in two, hepatocellular carcinoma in one patient. Among the complaints at the time of the first visit to a doctor prevailed dyspnea. Functional class NYHA heart failure was at the time of hospitalization 2.0 (2.0, 2.25). Surgical approaches in addition to median sternotomy: lymbo laparo-thoracotomy in the IX-the intercostal space in the four cases, the incision of the type "chevron" in three and one - median laparotomy incision. In one case, isolated lymbo laparo-thoracotomy was made in the IX-th intercostal space. Nine operations performed with cardiopulmonary bypass, seven of them under moderate hypothermia, two - in normothermia. One operation was performed using a temporary shunt. In three cases, it was performed pasty of retrohepatic portion of the inferior vena cava with kseno pericardial patch.

Results

Average time of myocardial ischemia was $47.6 \pm 5.5 \mathrm{~min}$, cardiopulmonary bypass time $148.7 \pm 17.1 \mathrm{~min}$, the total duration of the operation $509 \pm 44.7 \mathrm{~min}$. Total blood loss averaged $2579 \pm 699.4 \mathrm{ml}$, the mean duration of respiratory support $390 \pm 96.9 \mathrm{~min}$. Two patients noted one large hospital complications each, 30-days mortality - one patient. The mean follow $-21.9 \pm 5$ months. Total annual survival rate was $78,8 \pm 13,4 \%$, three-year - $52,5 \pm 13,6 \%$, median survival $-37 \pm 8.5$ months.

Conclusions

Radical excision "un bloc" of the primary tumor with tumor thrombus in the inferior vena cava and right atrium despite the technical complexity and the need for a multidisciplinary approach is the main method of treating patients with this pathology, increasing the life expectancy and relieves the symptom.

\section{Topic: Vascular}

026

Acute DeBakey type I aortic dissection: should arch and descending aortic intervention be recommended in the acute setting?

Elaine Teh', Simon Allen², Malcolm Dalrymple-Hay², James Kuo²,

Clinton Lloyd ${ }^{2}$, Adrian Marchbank², Michael J. Unsworth-White ${ }^{2}$

${ }^{1}$ University Hospitals Bristol NHS Foundation Trust, Bristol, UK; ${ }^{2}$ Plymouth Hospitals NHS Trust, Plymouth, UK

Correspondence: Elaine Teh

Journal of Cardiothoracic Surgery 2017, 12(Suppl 1):026

\section{Objective}

Acute Type A aortic dissection is a surgical emergency. Operative mortality is $20-30 \% .76 \%$ of our Type A patients have dissections into descending aorta (DeBakey Type I). In the era of the frozen elephant trunk and TEVAR, should surgeons manage just the proximal disease, or perform a more complex procedure to deal with distal disease in the acute setting? Advocates for aggressive complex intervention argue the theoretical risks of untreated dissection. However, the natural history of operated DeBakey Type I dissection is not fully understood. We therefore set out to review our experience and follow-up in these Type I patients to determine the outcome of persisting untreated distal disease.

Methods

A retrospective analysis of our operative and hospital database between September 1999 to January 2016 was performed, combined with scrutiny of the radiological evidence.

Results

96 patients with DeBakey Type I dissections underwent surgery. No interventions to the distal aorta were performed in the acute setting. $30 \mathrm{~d}$ or inpatient mortality was $28 \%$ in this extensive dissection group. 9 patients were non-resident in the region, hence no information regarding follow-up CT was available. 50 patients (71\%) had follow-up CT chest, results shown in Table 1. 1 patient underwent TEVAR but 2 were medically unfit for further intervention. 3-year and 5 -year survival are $66.6 \%$ and $57.2 \%$ respectively.

Conclusion

In conclusion, early mortality is high in this high-risk group. A small proportion $(6 \%)$ had significant distal disease progression requiring 
intervention. Only one underwent further treatment. CT follow-up needs to be carefully planned and scrutinized, but even allowing for this, our data suggests that arch and descending intervention in the acute setting would be overly aggressive for the majority of patients.

Table 1 (abstract 026). Outcomes of CT follow-up in patients with Acute DeBakey Type I aortic dissection

\begin{tabular}{ll}
\hline Results of CT & No of patients \\
Stable disease & 32 \\
Increased thrombus & 6 \\
Increased size of aorta & 10 \\
Increased dissection flap & 2 \\
Resolution/improvement in dissection flap & 3 \\
Complications with proximal anastomosis & 5 \\
\hline
\end{tabular}

\section{Topic: Vascular}

027

\section{Modern endovascular DVT therapy}

Alex Seredickiy ${ }^{1}$, Boris Sukovatykh², Vadim Muradian ${ }^{3}$, Andrew Azarov ${ }^{3}$, Michael Sukovatykh ${ }^{2}$

${ }^{1}$ Orel Regional Hospital, OGU, Orel, Russian Federation; ${ }^{2} \mathrm{KSMU}$, Kursk, Russian Federation; ${ }^{3}$ Orel Regional Hospital, Orel, Russian Federation Correspondence: Alex Seredickiy

Journal of Cardiothoracic Surgery 2017, 12(Suppl 1):027

\section{Purpose}

To compare results of treatment of DVT using medical and miniinvasive endovascular methods.

\section{Methods}

The analysis of 69 patients with acute DVT who were treated and investigated using laboratory, ultrasound, and phlebographic methods was carried out. The patients were randomized into two statistically identical groups. The first one consisting of 35 (50,7\%) patients were treated with a standard start therapy with heparin followed by warfarin administration. Patients of the second group consisting of $34(59,3 \%)$ had mini-invasive therapy. Of them 26 $(37,7 \%)$ patients had catheter-guided thrombolysis therapy using Urokinase. Another $8(11,6 \%)$ had endovascular thrombectomy Thrombolysis continued during 24-48 hours and was monitored radiologically. Endovascular thrombectomy was done using Aspirex Straub device. All patients were administered Rivaroxaban before and after the treatment.

The results were assessed based on phlebographic and ultrasound studies, and reduction of clinical syndromes.

Results

In $2(5,7 \%)$ patients during outpatient monitoring was diagnosed recurrent venous thromboembolism. The thromboembolic complications in the second group were not detected. The bleeding complications occurred in $8(22,9 \%)$ patients of the first and did not happen in the second group. Once the therapy had been completed the thrombus was recanalized in $23(65,7 \%)$ patients of the firs and $26(76,5 \%)$ patients of the second group respectively. Chronic venous insufficiency was absent or had a mild presentation in $23(65,7 \%)$ patients of the firs and 27 $(79,4 \%)$ patients of the second group respectively. Therefore, miniinvasive endovascular methods of DVT treatment can improve clinical results of treatment, reduce both the post-thrombotic syndrome and recurrent venous thromboembolism.

\section{Conclusion}

The combination of the Rivaroxaban therapy with endovascular methods in patients with DVT is safe and effective. It reduces clinical presentation of the chronic venous insufficiency and leads to better venous recanalization of deep veins.

\section{Topic: Vascular}

028

The first experience of reconstructive interventions on carotid arteries in patients with acute phase of ischemic stroke

Dmitriy Altman', Alexey Fokin², Vladimir Vladimirskiy ${ }^{1}$, Vladimir Ignatov' Alexey Baryshnikov ${ }^{1}$, Vadim Chernousov ${ }^{1}$, Anton Fedin', Pavel

Makovkin', Irina Rudakova', Georgii Treiger ${ }^{2}$

${ }^{1}$ Chelyabinsk Regional Hospital, Chelyabinsk, Russian Federation; ${ }^{2}$ South

Ural State Medical University, Chelyabinsk, Russian Federation

Correspondence: Vladimir Vladimirskiy

Journal of Cardiothoracic Surgery 2017, 12(Suppl 1):028

\section{Objective}

To assess the immediate results of reconstructive interventions on carotid arteries in the acute phase of ischemic stroke.

Methods

35 carotid endarterectomies (CEA) and 9 carotid artery stentings (CAS) were performed during 2016-2017 in 44 patients with acute stroke. The study included 9 women and 35 men aged from 38 to 88 years. Transient ischemic attack confirmed in 11 cases; ischemic stroke - in 32. All patients had associated pathology: coronary heart disease, hypertension, diabetes, obesity, etc. During the operations we used cerebral oximeter.

Results

One patient died (2.2\%) after eCEA. Autopsy verified an acute myocardial infarction (AMI). The indication for operation was the second cerebral stroke in the right hemisphere associated with $80 \%$ stenosis and coiling of the right internal carotid artery (ICA). In this case the operation of choice was CAS considering an accompanying pathology (aortic defect, diabetes mellitus), but due to severe tortuosity of the ICA it was impossible to perform. In one patient (2.2\%) perioperative stroke was developed. The diagnosis before surgery: repeated left middle cerebral artery (MCA) acute stoke. After 12 hours of onset of the disease eCEA was made. In the early postoperative period depression of consciousness to coma 1 was revealed. Patency of the carotid arteries up to the branches of the MCA was confirmed with the help of MSCT-AG. The degree of neurological deficit decreased during the therapy.

Conclusion

In the group of operated patients the index «AMI +stroke» was $7.1 \%$. These complications were registered in the first 3 months of 2016. After the creation of a multidisciplinary team included neurologist, vascular surgeon, cardiologist, anesthesiologist and endovascular surgeon severe complications weren't marked. Stroke frequency - 3.5\% - slightly higher than the recommended number for planned CEA.

\section{Topic: Vascular}

029

Delayed diagnosis and treatment of proximal post traumatic subclavian artery pseudoaneurysm

Hamidreza Davari

Tehran University of Medical Sciences, Tehran, Iran

Journal of Cardiothoracic Surgery 2017, 12(Suppl 1):029

\section{Objective}

Subclavian artery aneurysms (SAAs) are rare and may cause life and limb-threatening complications. Until 1980, most SAAs were related to TOS, infection or atherosclerosis. Afterwards, traumatic and 
iatrogenic causes were more frequently described. The gold standard of treatment is open surgery but there are changing profile of diagnosis and management by using endovascular stent.

Methods

Forty one year old lady had an accident 2 months ago with fracture of right mid clavicle and multiple ribs fracture. She had internal fixation of right clavicle. Because of hoarseness and progressive cough she went back to her physicians. Chest $\mathrm{x}$-rays were reviewed and a superior mediastinal mass was detected. CT angiography confirmed pseudoaneurysm of right subclavian artery. Due to the location of pseudoaneurysm and traumatic nature, open repair was done by partial median sternotomy which was extended to right supraclavicular area with partial claviculectomy. The paratracheal mass was opened with control of innominate artery and distal right subclavian artery. An interposition graft of ring Gore-Tex was placed between side of right innominate artery and end of distal subclavian artery with running closure of proximal right subclavian artery stump.

Results

The operation was uneventful but a tremendous bleeding from distal subclavian artery occurred during operation. The origins of internal thoracic and vertebral arteries could not be traced as were displaced by the pseudoaneurysm. The pseudoaneurysm was also noted to have transmural involvement and the anatomic 1st portion of subclavian artery was completely destroyed and it was not amenable to simple suture repair. The distal portion of anastomosis was done through the aneurysmal cuff.

\section{Conclusions}

Open repair can be difficult due to the overlying clavicle and close proximity of the brachial plexus. Endovascular stenting, coil embolization, thrombin injection and hybrid operation to these arterial regions have been shown to be alternatives to high risk surgery.

\section{Consent for publication}

Written informed consent to publish was obtained from the patients involved in this study.

\section{Topic: Vascular}

\section{0}

Composite PTFE-autologous saphenous vein graft to treat critical limb ischemia and single infrapopliteal vessel

Guiseppe Battaglia, Salvatore Alberto Turiano, Rosario Tringale, Ermanno Sabatino, Vincenzo Monaca

Ferrarotto Hospital, Catania, Italy

Correspondence: Salvatore Alberto Turiano

Journal of Cardiothoracic Surgery 2017, 12(Suppl 1):030

\section{Objectives}

The great saphenous vein (GSV) is the gold standard in below knee revascularization particularly when the target is a single tibial vessel. In cases of unavailability of the vein, were used composite graft heparin-bonded e-PTFE and saphenous vein, according the conclusions of the Italian Propaten Registry.

Methods

The authors retrospectively (Jan 2004 -Jan 2017) analyzed a group of patient candidates for single distal bypass and selected for the saphenous vein graft. When the GSV was only partially available, we used composite graft (Propaten + at least $10 \mathrm{~cm}$ of saphenous vein) and evaluated the results in the medium and long term. (Group A). The group A (50 pts) is compared with group of patients with GSV (Group B = $246 \mathrm{pts}$ ). Clinical data and the risk factors are statistically homogeneous in the two groups. The average length of GSV was 12 $\mathrm{cm}(10>30)$.

Results

At 30-days mortality, thrombosis and amputation are respectively 0 , $8,1 \%$ and $2 \%$ in the group $A ; 0,8 \%, 4,1 \%$ and $2,9 \%$ in the group $B$ : there are no statistically significant differences between the 2 groups.
The mean follow-up is 24 months. Primary patency at 6 years is $31 \%$ in group $A$ and $64 \%$ in group $B(p<0.02)$.

Conclusions

Obviously, in terms of primary patency the vein remains the conduit of choice, but the graft composites have proven a viable alternative solution than the only prosthetic graft. This is due to the fact that the factors that influence the patency are essentially the run-off and the homogeneity of the caliber at the level of the distal anastomosis: these 2 conditions are met by the use of the composite graft.

\section{Topic: Vascular}

031

The role of pentoxifylline and iloprost in prevention of ischemia-reperfusion: damages in experimental models of intestine ischemia-reperfusion in rats

Uğur Abakay, Sinan Soylu, Sabahattin Göksel, Bülent Saraç, Zeynep Deniz Şahin İnan, Atilla Kurt, Özge Korkmaz, Hüsnü Çağrı Genç, Öcal Berkan

Cumhuriyet University, Sivas, Turkey

Correspondence: Sabahattin Göksel

Journal of Cardiothoracic Surgery 2017, 12(Suppl 1):031

\section{Objective}

Intestinal ischemia occurs by partial or total occlusion of intestinal arterial blood flow and reperfusion occurs when blood flow is restored. Intestinal ischemia reperfusion (IIR) injury can lead to multiple organ failure and death. The aim of this study is to investigate the effect of pentoxifylline and lloprost administered after IIR injury following reperfusion.

Methods

Five groups of 25 male Wistar Albino rats weighing 250-300 g were divided into 5 groups each consisting of 5 subjects: control group $(n=5)$, Sham group $(n=5$, no RI), IR group $(n=5,45$ minutes ischemia, 120 minutes reperfusion), IR+Ptx group ( $n=5,45$ minutes ischemia following intraperitoneal $50 \mathrm{mg} / \mathrm{kg}$ pentoxifylline and 120 minutes reperfusion), IR+IL group ( $\mathrm{n}=5,45$ minutes ischemia following intraperitoneal $2 \mathrm{mcg} / \mathrm{kg}$ lloprost and 120 minutes reperfusion). At the end of the experiment, the ileum specimens were stained with hematoxylin eosin and histopathologically evaluated with Chiu score. Isometric contraction-relaxation responses were recorded by using in organ baths for contraction-relaxation responses. $p<0.05$ was considered statistically significant.

Results

Pentoxifylline provided significant improvement in response to histopathological and contraction-relaxation responses. Though lloprost provided recovery in reperfusion injury it wasn't statistically significant.

Conclusions

In light of these result, pentoxifylline may be promising in preventing small bowel ischemia-reperfusion injury. We conducted that further clinical and experimental studies for lloprost are needed.

\section{Topic: Vascular}

032

Our experience in applying endovasal methods of correction of critical lower limb ischemia in patients with infrainguinal arterial disease

Anastaia Artemova', Michael Ivanov', Nikolay Lukyanchikov²,

Alexandr Lipin ${ }^{3}$

${ }^{1}$ NWSMU named after I. I. Mechnikov, Saint Petersburg, Russian

Federation; ${ }^{2}$ I.M. Sechenov First Moscow State Medical University,

Moscow, Russian Federation; ${ }^{3}$ Limb Rescue Center "Saint Petersburg

State Hospital \#14", Saint Petersburg, Russian Federation

Correspondence: Anastaia Artemova

Journal of Cardiothoracic Surgery 2017, 12(Suppl 1):032 


\section{Objective}

Compare the results of endovascular and open surgical interventions for the correction of critical limb ischemia.

Methods

We analyzed direct treatment outcomes of 104 patients who underwent surgery on the femoropopliteal segment on the CLI. Patients were divided into 2 groups according to the type of arterial bed renovation: 63 patients who underwent endovasal interventions, composed the main group; 41 people which were performed endovasal interventions, composed the control group. We analyzed the duration of hospitalization and surgical intervention, intraoperative blood loss, blood pressure variability, the frequency of thrombosis of the operated segment and infectious complications.

Results

Duration of hospitalization: in the study group - 30,7 $\pm 14,3$ days, in the control group - $40,17 \pm 15,8$ days $(p<0,05)$. Duration of surgery was also less in patients of the main group (95, $1 \pm 57,2$ minutes) compared to controls $(214,0 \pm 90,9 \mathrm{~min} ; \mathrm{p}<0,05)$. Intraoperative blood loss in patients of the main group was $300 \pm 115 \mathrm{ml}$, which is significantly less than the control patients $-510 \pm 145 \mathrm{~mL}(p<0,05)$. The variability of systolic blood pressure during the first day after the surgery was greater in the control group $(32,1 \pm 7,2 \mathrm{mmHg} v / \mathrm{s} 29 \pm 3,1 \mathrm{mmHg} ; \mathrm{p}<0,05)$. Thrombosis of the operated segment was observed in 1 patient of the main group and in 4 patients of the control $(p<0,05)$. The development of infectious complications in the surgical area in patients of the main group were noted less frequently than in the control $(1 \mathrm{v} / \mathrm{s} 6 ; \mathrm{p}<0,01)$. As a result of developed complications in the study group were performed 2 low amputation, and in the control 1 low and 3 high amputation $(p<0,05)$

Conclusions

Endovasal intervention may be the method of choice in the treatment of patients with IAD on the background of critical ischemia

\section{Topic: Vascular}

033

- Assessing the impact of the sinus-saving modifications of eversion carotid endarterectomy on the stabilization of perioperative arterial hemodynamic

Alexey Fokin ${ }^{1}$, Georgii Treiger ${ }^{1}$, Vladimir Vladimirskiy ${ }^{2}$

'South Ural State Medical University, Chelyabinsk, Russian Federation;

${ }^{2}$ Chelyabinsk Regional Hospital, Chelyabinsk, Russian Federation

Correspondence: Georgii Treiger

Journal of Cardiothoracic Surgery 2017, 12(Suppl 1):033

\section{Objective}

Complications such as myocardial infarction, hyperperfusion syndrome and its consequences are serious threat postoperative carotid reconstructions. One of the main cause of these complications is variation of central hemodynamic especially with the tendency to hypertension. The aim of our study was to evaluate the effect of preserving the carotid sinus nerve during eversion carotid endarterectomy at preventing the development of hypertension. Our goal is to develop an operative technique that could let us avoid intersection of the carotid sinus nerves, which reduce the risk of complications thanks to a more manageable blood pressure, due to decreased sympathetic influence on the regulation of vascular tone. Methods

The research included 375 patients operated on carotid arteries in Chelyabinsk Regional Clinical Hospital since 2011 to 2017. Groups are even in age, sex, initial neurological and cardiac status and contralateral blood flow. The first group included 206 patients with eversion technique with the intersection of the carotid sinus nerves. The second group included 169 patients who had been used a modified technique, with saved carotid sinus nerves. On the 1st and 4th day after surgery the state of the autonomic regulation was assessed by analyzing heart rate variability.

Results

In the group of patients with saved carotid sinus nerve on the 1st day after surgery was more than noticeable decrease sympathetic influence on the rhythm, with a tendency to restore autonomic regulation on the 4 th day.

Conclusions

1. Obtained results show lower activity of the sympathetic and the higher activity of the parasympathetic system in the group with non-damaged carotid sinus nerves. 2. Carotid sinus-safe surgery decreases the risk of postoperative complications caused by arterial hypertension.

\section{Topic: Congenital}

034

Pulmonary autograft operations: 20 year experience

Alexander Romaniuk', Asya Avetyan', Illya Yemets ${ }^{2}$, Nadezhda Rudenko ${ }^{1}$

${ }^{1}$ P.L. Shupyk National Medical Academy of Postgraduate Education

(NMAPE), Kyiv, Ukraine; ${ }^{2}$ Ukrainian Children's Cardiac Center, Kyiv,

Ukraine

Correspondence: Alexander Romaniuk

Journal of Cardiothoracic Surgery 2017, 12(Suppl 1):O34

\section{Objective}

The paper presents results of the operation of aortic valve replacement by the own pulmonary valve - the pulmonary autograft operation in patients with aortic valve disease and the modifications of the surgical technique.

Methods

For 1996-2015, the pulmonary autograft operation was performed in 200 patients, the average age was $145 \pm 101.6$ months (1month - 54 years.). Indications for the operation were: aortic stenosis (AS) in 103 (51.5\%) patients, aortic insufficiency (Al) in $68(34 \%)$, combined aortic valve disease in $29(14.5 \%)$

Results

Hospital mortality was $6 \%$, the overall mortality $-8,9 \%$. The longterm follow-up period was $94.5 \pm 35.8$ months (3-156 months), $94 \%$ of patients were observed. Survival was $95.3 \%, 92.1 \%$ and $92.1 \%$ during 1,10 and 15 years of follow-up. The risk factors for mortality were: the age of patients under 1 year, the increase of the ischemic time and bypass, significantly reduced the risk with the use of surgical modifications. With neopulmonary valve, 52 interventions were performed in 64 patients (34.5\%). Freedom from reoperations was $91.8 \%, 87.4 \%, 85.1 \%$ at 5,10 and 15 years respectively, freedom from endovascular balloon interventions was $88.3 \%, 86.7 \%$ at 5 and 15 years, respectively. Freedom from all the reinterventions after the operation was $81.4 \%, 72.6 \%, 67.2 \%$ at 5,10 and 15 years.

Conclusions

The pulmonary autograft operation is an effective method of correction of the aortic valve disease, can be performed with low mortality and satisfactory long-term results. The use of a pulmonary valve as an aortic prosthesis provides growth of the neoaortic root, a low incidence of aortic valve dysfunction, and a low frequency of reoperations. The use of own modifications of the operation showed effectiveness, greatly improving the immediate and long-term results.

\section{Topic: Congenital}

035

Hybrid stenting for the right ventricle outflow tract in infants with critical obstruction and multiple congenital comorbidities

Pavel V. Teplov, Evgeniy V. Sahnov, Alexey S. llin, Valeriy A. Sakovich

Federal Center of Cardiovascular Surgery, Krasnoyarsk, Russian

Federation

Correspondence: Pavel V. Teplov

Journal of Cardiothoracic Surgery 2017, 12(Suppl 1):035

Objective

Hybrid stenting for the right ventricle outflow tract (RVOT) is one of the methods for RVOT critical obstruction palliative treatment in infants with multiple congenital comorbidities. 


\section{Methods}

Five infants were admitted to the Federal Center of Cardiovascular Surgery in Krasnoyarsk with critical obstruction of the RVOT and multiple malformations, including malformations of the respiratory system, digestive system, central nervous system, genetic malformations with a poor prognosis for survival. All infants were critically ill with constant infusion of Vazaprostan, 3 of them were on a ventilator. 3 patients congenital comorbidities were already surgically corrected, 3 had sepsis. 4 infants had ductus dependent hemodynamics with desaturation to $60 \%$. Stenting of RVOT was performed through median sternotomy and right ventricular free wall function. In the case of the pulmonary atresia, perforation of the membrane was performed with an obtuse needle under TEE control. Pulmonary artery valve diameter assessment was performing during contrast infusion into the RVOT. Metal stent size selection was based on pulmonary artery diameter $+1 \mathrm{~mm}$. In some cases, using of TEE or contrast agent were avoided due to severe comorbidities.

Results

All hybrid procedures have been performed successfully and increase of saturation in the range of $84 \%$ to $100 \%$. One infant required ECMO 2 days after the surgery due to low cardiac output. 2 months later, a systemic-pulmonary shunt was additionally required in two infants. Full correction of CHD was performed in 1 patient 6 month later.

\section{Conclusions}

The hybrid approach is an alternative option for critically ill newborns with multiple congenital malformations which may allow to survive to full correction of the CHD. It can be used instead of cardiopulmonary bypass and allows to survive in the neonatal period. It gives time for treatment (surgical correction) of comorbidity.

\section{Topic: Congenital}

\section{6}

- Surgical management of atrial septal defect device complications

Santosh Kumar

Meenakshi Mission Hospital and Research Centre, Madurai, India

Journal of Cardiothoracic Surgery 2017, 12(Suppl 1):036

\section{Background}

Atrial septal defect is a common congenital cardiac anomaly. Although surgical closure has been the traditional and gold standard treatment of choice, percutaneous device closure is gaining popularity in recent times because of the very short duration of stay, cosmetic advantage and relative avoidance of morbidity associated with surgery, nevertheless they are associated with some unavoidable complications making surgeons intervention mandatory.

\section{Methods}

Between May 2014 to June 2016, 6 patients has been referred to our center after transcatheter closure of ostium secundum type of atrial septal defects. Of them, 3 patients referred as emergency for device migration within $24 \mathrm{hrs}$ and 1 patient with device migration and Right atrial appendage puncture producing pericardial collection and cardiac tamponade. 1 patient referred with endocarditis of device done 6 months back and 1 patient with device done 1 year back eroding the aorta producing Aorto- RA fistula.

Results

The 3 patients with device migration underwent surgery and retrieval of device and pericardial patch closure of defect and 1 patient with device migration and RA perforation underwent emergency sternotomy with device retrieval and patch closure of defect. 1 patient with endocarditis of device underwent elective surgery with device retrieval, removal of vegetations from tricuspid valve, mitral valve replacement and pericardial patch closure of the defect. 1 patient with Aorto-RA fistula underwent device removal, closure of fistula with Dacron patch and pericardial patch closure of the defect. All patients are discharged with normal convalescence. Conclusion

Although the complications for Atrial septal defect devices are minor, sometimes they can be very disastrous leading to high mortality in a rapid transit of time, making surgeons intervention mandatory and emergency surgery with device retrieval and for the correction of original defect and other device related defects.

\section{Topic: Congenital}

037

- Our 4 year experience of pediatric cardiac surgery

Daulet Zhakipbayev, Oraz Mukashev, Adil Dyurzhanov, Timur

Raimkhanov, Renat Sarin, Yerlan Ordabayev, Arman Bayshin, Dauren Antikeyev, Assem Sarsekeyeva, Yelena Khagay, Zukhra Mendybayeva

Pavlodar Regional Cardiological Center, Pavlodar, Kazakhstan

Correspondence: Daulet Zhakipbayev

Journal of Cardiothoracic Surgery 2017, 12(Suppl 1):037

\section{Objective}

Analysis of immediate results of pediatric cardiac surgery unit (PCSU) in the regional cardiological center.

Methods

The population of our region is 750,000 people. About 100 newborns are born with congenital heart disease (CHD) annually. Our PCSU consists of 3 cardiosurgeons, 4 cardiologists, 1 interventional cardiologist, 2 reanimatologists, 1 neonatalogist and 1 perfusiologist. We have a 1 operational room and 1 cath lab, 6 cardiosurgical beds, 4 beds in ICU, 4 beds in rehabilitation and 2 cardiological beds. Since the opening of the center in January 2013 and until January 2017, we operated 362 children in operational room (ASD - 147, VSD - 135, PDA - 23, PAPVC - 12, CoAo - 9, BAV - 7, AVSD - 5, TOF - 5, TMA - 5 TAPVC - 5, SV - 3, DORV - 2, PA - 2, CTA - 1, EA - 1 repairs) and 106 children in cath lab (PDA - 40, ASD - 26 and MAPCA's - 1 occlusions, BVP of PS - 33 and AS - 2, BAS by Rashkind - 4). The age range was 1 day to 18 years. The weight range was $700 \mathrm{gr}$ to $67 \mathrm{~kg}$. The operations relate to the 1-3 categories of complexity according to the basic Aristotle scale.

Results

Overall mortality was 28 patients (6\%) mostly with critical CHD in neonatal period, complications were in 16 patients $(3,5 \%)$ such as complete AV blockade - 7, pneumothorax in the early postoperative period -3 , bleeding in the early postoperative period -2 , instability of sternum -2 , chylothorax -1 , thrombosis of the femoral artery - 1 . Conclusions

In this way, organization of PCSU in the regional cardiological center is a necessary and complex process, requiring maximum dedication from the medical staff and the administration of the center.

\section{Topic: Congenital}

\section{8}

New surgical technique in treatment of aneurysm sinus of valsalva

Khakimjon Abralov, Amonjon Alimov

Republican Specialized Center of Surgery Named after Academician V.

Vakhidov, Tashkent, Uzbekistan

Correspondence: Khakimjon Abralov

Journal of Cardiothoracic Surgery 2017, 12(Suppl 1):O38

\section{Background}

In the most cases of surgical repair of ruptured aneurysm sinus of Valsalva (RASV) it is quite difficult to distinguish healthy tissues from distorted one that is why in our department was designed new surgical technique which we would like to represent here.

Methods

From January 1, 1988, to December 30, 2016 - 65 patients with SVA underwent surgical repair in our center. There were 41 males $(63 \%)$ and 24 females $(37 \%)$, and their age ranged from 5 to 50 years (mean age $23 \pm 10$ years). The RSVA associated with a VSD was revealed in $46(70.76 \%)$ cases. In ten cases of RSVA associated with VSD, we have applied our original surgical technique of RSVA closure. We are believe, that it is desirable to maximize the amount of tissues available to support the annulus adjacent to the area of a VSD. That is why we don't cut off distorted tissues of RSVA, instead this we imbricate it like "a roll" by utilizing a running suture that 
begins at the distal part of the wind sock is then sewn at each turn of the wind sock until it is imbricated to the proximal part of the RSVA, forming as a result a solid and reliable upper edge of the VSD. Results

Hospital mortality was in 5 cases associated with simultaneous AV and root intervention. In new surgical technique series there is no death occurred. Postoperative echocardiography doesn't reveal any fistula.

\section{Conclusions}

We acknowledge that many aspects of this localized abnormality of one sinus of Valsalva share features and principles with more generalized aortic root disease. An appreciation of the principles of aortic root repair and consideration of etiological causes of localized forms of RSVA can be helpful in understanding and managing the spectrum of SVA and related anomalies.

\section{Topic: Congenital}

039

Long-term results of the transatrial-transpulmonary approach: are we doing better?

Elisabeth M. J. P. Mouws, Natasja MS de Groot, Ad J. J. C. Bogers

Erasmus Medical Center, Rotterdam, Netherlands

Correspondence: Ad J. J. C. Bogers

Journal of Cardiothoracic Surgery 2017, 12(Suppl 1):039

\section{Objective}

Surgical techniques for total correction of Tetralogy of Fallot (ToF) have changed from primary ventriculotomy to a transatrialtranspulmonary approach with the aim of limiting myocardial scarring and thereby reducing the risk of arrhythmias and ventricular deterioration. However, there still is a lack of data supporting this hypothesis.

Methods

A total of 85 ToF patients who underwent total correction via primary ventriculotomy $(\mathrm{N}=42)$ or via transatrial-transpulmonary approach $(\mathrm{N}=43)$ were included and categorized by ventricular incision in the free wall (FW) or limited to the right ventricular outflow tract (RVOT). Incidences of atrial fibrillation (AF), other supraventricular tachyarrhythmia (SVT), ventricular tachyarrhythmia (VT) and ventricular fibrillation (VF) were collected.

Results

Mean follow-up was $41 \pm 6(27-61)$ years. Total correction was performed via $\operatorname{RVOT}(\mathrm{N}=50(59 \%))$ or $\mathrm{FW}(\mathrm{N}=35(41 \%))$ incision and was valve-sparing (VS) in 27(32\%) patients (RVOT: 8(16\%); FW:19(54\%), $\mathrm{p}<0.001)$. Prior palliative shunting was performed in $43(51 \%)$ patients (RVOT:22(44\%); $\mathrm{FW}: 21(60 \%), \mathrm{p}=0.146)$. VS surgery resulted in a lower, yet still considerable, incidence of RV dysfunction (non-VS: $\mathrm{N}=48(83 \%)$; VS: $\mathrm{N}=16(59 \%)$, $\mathrm{p}=0.019)$. AF, SVT, VT and VF occurred in respectively 15(18\%), 35(41\%), $21(25 \%)$ and 4 patients (5\%). VT incidence was higher in those with FW incision (FW: $\mathrm{N}=14(40 \%) ; \mathrm{RVOT}: \mathrm{N}=7(14 \%), \mathrm{p}=0.006)$. Corrected for FW incision, RV dysfunction, LV dysfunction and VS surgery, only older age at correction was an independent predictor for $\mathrm{AF} /$ SVT(OR:1.13, $\mathrm{p}=0.035)$. Corrected for age at ToF correction, independent predictors for VT/VF were FW incision (OR:3.24, $p=0.031$ ) and RV dysfunction (OR:4.47, $\mathrm{p}=0.066$ ).

\section{Conclusions}

Aside from surgical approach, ventricular deterioration is a key factor in the development of life threatening arrhythmias in this population. Despite improvement in surgical techniques, ventricular dysfunction may present as an underexposed factor for long-term sequelae of ToF patients.

\section{Topic: Congenital}

\section{0}

- 15 year experience with Fontan operation

Vitali Pak, Luigi Arcieri, Vincenzo Poli, Daniyar Gilmanov, Bruno Murzi

Pediatric Cardiac Surgery Department, Ospedale del Cuore, Tuscany

Foundation Gabriele Monasterio, Massa, Italy

Correspondence: Vitali Pak

Journal of Cardiothoracic Surgery 2017, 12(Suppl 1):040

\section{Objectives}

Surgical approaches to single ventricle variants include fenestrated and non-fenestrated Fontan operations. This study compares outcomes of 115 consecutive Fontan operation with these modifications at a single center.

Methods

From January 2003 to March 2017115 patients underwent a modification of the Fontan procedure in which an extracardiac/ intracardiac total cavopulmonary connection. 65 pts were male, 50 female. Mean age was 4,4 years $(2,1-16,6) .49$ patients were fenestrated and 66 patients has non fenestration Fontan procedures. All patients received Gore-Tex prosthesis from $16-22 \mathrm{~mm}$ of size.

Results

There were no differences concerning age between fenestrated and non-fenestrated Fontan. The decision to fenestrate was based on various considerations as well, including preoperative status, hemodynamic data and anatomy of cardiac defect, whether intracardiac procedures were performed. There were no early hospital mortality. One patient died after 10 months after Fontan operation for acute thrombosis of prosthesis. No statistical differences were observed concerning ICU staying, duration of drainage and hospital staying between fenestrated and no fenestrated Fontan. All fenestrated Fontan are on anticoagulant therapy. There was one failing Fontan with associate plastic bronchitis that has been transplanted and one patients with protein loosing syndrome waiting for heart transplantation.

Conclusion

Proper selection of patients for modifications of the Fontan procedure resulted in excellent early and late survival with a low incidence of postoperative complications. Midterm functional outcomes were excellent.

\section{Topic: General Cardiology for Nurses and Allied Professionals}

\section{1}

Optimization of preparation for crimping of the delivery system of balloon-expanding aortic valves (experience of National Research Center for Cardiac Surgery)

Aigerim Sadykova, Gulmira Momanova, Akmaral Khazhymurat JSC National Research Center for Cardiac Surgery, Astana, Kazakhstan

Correspondence: Aigerim Sadykova

Journal of Cardiothoracic Surgery 2017, 12(Suppl 1):041

\section{Objective}

To optimize the development of the nursing pre-operative preparation plan for valve crimp.

Methods

Percutaneous implantation of the aortic valve requires coordinated operation of the entire operating team. Thereby, the quality of preoperative preparation requires analysis and optimization. In this regard, in the simulation room, two groups of nursing personnel performed preoperative valve assembly. The first group performed 
20 crepings, while the second group made 40 crepings. Subsequently, 131 balloon-expanding and self-expanding transcatheter aortic valves were implanted. The average age of the patients was $73 \pm 6$ years, $57 \%$ of male and $43 \%$ of female. In $71 \%$ of patients, the operation was performed under endotracheal anesthesia, in $29 \%$ of patients under local anesthesia. The transcathetero aortic valves were implanted by Edwards Sapien XT with a femoral access 36, an apical access of 19. Core Valve with a femoral access 42 , apical access 5, aortic access 2 . In 24 cases, the aortic valve Evolut R was implanted with a femoral access, in one case by transaorthal access in communication with pronounced atherosclerotic changes in the iliac, femoral and subclavian arteries.

Results

Timing of the operating protocols revealed that the average time of intraoperative creaming in the first group of training of nurses averaged $11.2 \pm 2.4 \mathrm{~min}$, whereas in the second group the same indicator was $6.1 \pm 1.8 \mathrm{~min}$. At the same time, in both groups, the assembly of the delivery system, delivery to the aortic position and extension were performed successfully.

Conclusion

The quality of preoperative preparation requires multiple repetitions of the operation stages in the simulation center. According to its own results, the optimal number is at least 40 crepings.

\section{Topic: Transplantation}

\section{2}

- Ex vivo allograft perfusion and conditioning for heart transplantation: single centre 1 year follow-up

Zhuldyz Nurmykhametova, Rymbay Kaliyev, Serik Bekbossynov,

Timur Lesbekov, Nurlan Smagulov, Svetlana Novikova, Ivan Vakhrushev, Muradym Murzagaliyev, Mahabbat Bekbossynova, Saltanat Jetybayeva,

Zhanibek Ashyrov, Linar Faizov, Zhadyra Duisenbina, Yuryi Pya

National Research Center for Cardiac Surgery, Astana, Kazakhstan

Correspondence: Zhuldyz Nurmykhametova

Journal of Cardiothoracic Surgery 2017, 12(Suppl 1):042

\section{Objective}

The Organ Care System (OCS) is the only clinical platform for ex-vivo perfusion of human donor hearts. We modified standard method of donor heart preservation using blood cardioplegia and conditioning (ultrafiltration, Levosimendan) during transport from the donor hospital to our Center. We reviewed our institutional experience to assess one-year outcomes of patients underwent heart transplantation managed with OCS.

Methods

Between 2014 and 2016, 37 heart transplant patients from a single center were operated using OCS with standard preservation (SC group) $n=12$ or blood cardioplegia and conditioning (BC group) $n=25$. Outcomes assessed included one-year survival, freedom from any-treated rejection (ATR), and non-fatal major cardiac events (NF-MACE).

Results

Preoperative risk factors were comparable in both groups. Results were similar for the BC group compared to the SC group: mean total warm ischemic time was 84.2 (SD 28) vs 86.9 (SD 8.4) minutes $(p=0.001)$. Mean ex-vivo perfusion time was 266.5 (SD 86.7) vs 260.4 (SD 88.4) minutes $(p=0.87)$. Mean venous lactate at the start of perfusion $(2.0 \mathrm{mmol} / \mathrm{l}$ vs. $3.2 \mathrm{mmol} / \mathrm{l})$ and at the end of perfusion $(3.7$ $\mathrm{mmol} / \mathrm{l}$ vs. $7.5 \mathrm{mmol} / \mathrm{l})$ were $(p=0.37)$ lower in the $B C$ group. Seven day tissue myocardial Doppler results were normal in both groups, except for 3 patients in SC group, who have right ventricular dysfunction. Median ICU stay 7 days (range 3 - 40) vs. 17 days (range 5 - 43) was significantly lower in BC group. There was no significant difference in the 1-year patients survival rate between the two groups (SC group $77.8 \%$ vs. BC group $87.2 \%, p=0.47$ ). Similarly, there was no in freedom from ATR, and NF-MACE.

\section{Conclusions}

Blood cardioplegia and conditioning is efficient and safe method for donor heart preservation and treatment in OCS.

\section{Topic: Transplantation}

\section{3}

Perioperative period in heart transplantation from donors with low left ventricle ejection fraction

Vitaly Poptsov, Ekaterina Spirina, Nadegda Koloskova, Elnur Aliev, Anastasiya Dogonascheva, Vitaly Pchelnikov, Vladislav Voronkov, Sergey Masyutin, Stanislav Ustin

Shumakov Federal Scientific Centre of Transplantology and Artificial

Organs, Moscow, Russian Federation

Correspondence: Vitaly Poptsov

Journal of Cardiothoracic Surgery 2017, 12(Suppl 1):043

\section{Objective}

Brain death donors with impaired systolic left ventricle (LV) function (LVEF $<50 \%)$ as the rule are not considered for heart transplantation (HT). However, some transplant teams have successful experience of HT from donors with low LVEF. Purpose of study was evaluated of early outcomes of HT performed from donors with LVEF $<50 \%$.

Methods

The study included 36 ( 29 men and 7 women, age 20 to 63 (45.5 \pm 12.5 ) years) recipients who received cardiac allograft from donors with LVEF $\leq 50 \%$. The urgency of HT was $1 A-B(n=14)$ and 2 status $(n=22)$ UNOS. $10(27,8 \%)$ patients needed peripheral VA ECMO.

Results

Heart donors ( 28 men and 8 women, 22 to $62(44.1 \pm 10.5)$ years) were the traumatic $(n=23)$ and non-traumatic $(n=13)$ brain damage. Maximal inotropic support was norepinephrine $550 \pm 142 \mathrm{ng} / \mathrm{kg} / \mathrm{min}$ $(\mathrm{n}=23)$ and dopamine $6.1 \pm 3.5 \mu \mathrm{g} / \mathrm{kg} / \mathrm{min}(\mathrm{n}=6)$. Laboratory parameters of the heart donor blood: $\mathrm{Hb} 11.8 \pm 0.8 \mathrm{~g} / \mathrm{l}$, total protein $60 \pm 21 \mathrm{~g} /$ I, $\mathrm{Na}^{+} 148 \pm 8 \mathrm{mmol} / \mathrm{l}$, troponin I $0.3 \pm 0.2 \mathrm{ng} / \mathrm{ml}$, CK-MB $98 \pm 18 \mathrm{U} / \mathrm{l}$. ECG of the donor's heart: right ventricle $2.7 \pm 0.2 \mathrm{~cm}$, LVEDV $134 \pm 9 \mathrm{ml}$, LVEF 22-49 (41.4 \pm 4.6$) \%$, LV wall thickness $1.3 \pm 0.3 \mathrm{~cm}$, diffuse LV hypokinesis $(n=33)$, regional dyskinesis $(n=3)$. Ischemic time was 159 $\pm 11 \mathrm{~min} .31(86.1 \%)$ had acceptable cardiac allograft function. $\mathrm{LVEF} \geq 60 \%$ was registered at $3.8 \pm 0.6$ days after HT. Primary graft failure treated by MCS (VAECMO $(n=5))$ was in $5(13.9 \%)$. ICU stay was $7.8 \pm 1.4$ days. 33 (91.3\%) recipients were discharged at home.

Conclusions

Own experience demonstrates the satisfactory results of $\mathrm{HT}$ from donors with $\mathrm{LVEF} \leq 50 \%$. In more cases LV systolic function of cardiac allograft quickly normalized in early period after HT.

\section{Topic: Transplantation}

\section{4}

Analyses of 30 lung transplant cases: initial single center experience from Turkey

Erdal Yekeler', Alkin Yazicioglu', Mahmut Subasi', Sinan Turkkan', Fatmanur Celik Basaran', Ibrahim Onur Alici ${ }^{1}$, Hija Yazicioglu', Sema Turan', Funda Demirag ${ }^{5}$, Omac Tufekcioglu' ${ }^{6}$, Umit Kervan 7 , Faruk Kalkan ${ }^{8}$

${ }^{1}$ Turkiye Yuksek Ihtisas Training and Research Hospital, Thoracic Surgery and Lung Transplantation Clinic, Ankara, Turkey; ${ }^{2}$ Suat Seren Training and Research Hospital, Department of Chest Diseases, Izmir, Turkey; ${ }^{3}$ Turkiye Yuksek Ihtisas Training and Research Hospital, Department of Anesthesiology, Ankara, Turkey; ${ }^{4}$ Turkiye Yuksek Ihtisas Training and Research Hospital, Anesthesiology and Intensive Care Unit, Ankara, Turkey; ${ }^{5}$ Ataturk Chest Disease and Thoracic Surgery Training and Research Hospital, Department of Pathology, Ankara, Turkey; ${ }^{6}$ Turkiye Yuksek Ihtisas Training and Research Hospital, Department of Cardiology, Ankara, Turkey; ${ }^{7}$ Turkiye Yuksek Ihtisas Training and Research Hospital, Department of Cardiovascular Surgery, Ankara, Turkey; ${ }^{8}$ Turkiye Yuksek Ihtisas Training and Research Hospital, Department of Immunology, Ankara, Turkey

Correspondence: Alkin Yazicioglu

Journal of Cardiothoracic Surgery 2017, 12(Suppl 1):044

\section{Objective}

Lung transplantation (LuTx) is a treatment option for end-stage pulmonary-insufficiency. 


\section{Methods}

From March 2013-March 2017, 30LuTx was performed.

Results

Of them, 28 (93.3\%) man, 2 (6.7\%) woman; mean age 46 years (22-62). COPD was common etiological factor $(n=11,36.7 \%)$ (Table 1). DoubleLuTx was performed in 26(86.7\%) cases; whereas, single LuTx in $4(13.3 \%)$ cases. Downsizing (middle lobectomy; lingulectomy or both) was performed to $10(33.3 \%$ ) cases. ECMO was supported $20(66.7 \%)$ cases; intraoperative ECMO was initiated to $18(60.0 \%)$ cases, central ECMO was the preferred type. Three patients (10.0\%) had revision due to bleeding after operation. Internal mammarial-artery, bronchial-artery, and oozing from chest-wall and diaphragm were the reasons. Induction medication (Alemtazumab or Basiliximab) was followed by tacrolimus and prednisolone in all cases. Mikofenolat-mofetil was added to medication of Basiliximab administrated cases. Nineteen cases (63.3\%) were weaned from mechanical ventilation in the first 48-hours; tracheostomy due to prolonged weaning was performed to six cases(20.0\%). Mean ICU stay was 12 days, and mean hospitalization was 34days. Five patients were deceased in the first three months after transplantation: two of them died at second and third postoperative day due to accelerating graft dysfunction and other one died at $23^{\text {rd }}$ day due to renal insufficiency and multi-organ failure. One patient deceased intraoperative; oozing, bleeding due to coagulopathy was the reason. One patient had a cerebrovascular accident and died at $6^{\text {th }}$ day. During long term follow-up, two patients died from BOS at $24^{\text {th }}$ and $29^{\text {th }}$ months. Surgical success was $82.2 \%$, conditional 1 -year survive was $93 \%$, conditional 3-year survive $70 \%$.

\section{Conclusions}

Owing to refined surgical technique, adequate perioperative support, and improved postoperative care, we believe LuTx can be performed with acceptable post-transplant mortality and satisfactory long-term outcomes in a low-volume center.

Table 1 (abstract 044). Analyses of 30 lung transplant cases

\begin{tabular}{|c|c|}
\hline & Distribution/Ratio \\
\hline $\begin{array}{l}\text { Etiological } \\
\text { factors }\end{array}$ & $\begin{array}{l}\operatorname{COPD}(n=11,36.7 \%) ; \text { IPF }(n=8,26.7 \%) \text {; Silicosis }(n=4,13.3 \%) ; \\
\text { Bronchiectasis }(n=2,6.7 \%) ; \text { Pulmonary Langerhans' cell } \\
\text { histiocytosis }(n=2,6.7 \%) ; \text { Pulmonary alveolar lipoproteinosis } \\
(n=1,3.3 \%) ; \text { Kartagener syndrome }(n=1,3.3 \%) ; a-1 \text { anti-trypsin } \\
\text { deficiency }(n=1,3.3 \%) ;\end{array}$ \\
\hline Ischemic-time & First lung: 292min (172-374); Second lung: 416min (300-510) \\
\hline $\begin{array}{l}\text { Induction } \\
\text { therapy }\end{array}$ & Alemtazumab ( $n=10,33.3 \%) ;$ Basiliximab $(n=20,66.7 \%)$ \\
\hline ECMO Support & $\begin{array}{l}\text { Intraoperative central }(n=17,56.7 \%) \text {; Intraoperative peripheral } \\
(n=1,3.3 \%) \text {; Postoperative peripheral }(n=11,36.7 \%)\end{array}$ \\
\hline Mean ICU Stay & 12 days \\
\hline $\begin{array}{l}\text { Mean } \\
\text { Hospitalization }\end{array}$ & 34 days \\
\hline
\end{tabular}

\section{Topic: Transplantation}

\section{5}

Mechanical circulatory support before and after heart

transplantation: impact on survival

Giuseppe M. Raffa, Gabriele Di Gesaro, Sergio Sciacca,

Giovanna Panarello, Francesco Clemenza, Michele Pilato

IRCCS-ISMETT (Istituto Mediterraneo per i Trapianti e Terapie ad

altaspecializzazione), Palermo, Italy

Correspondence: Giuseppe M. Raffa

Journal of Cardiothoracic Surgery 2017, 12(Suppl 1):045

\section{Objective}

To investigate the impact on survival of pre and post heart transplant (HT) use of mechanical circulatory support (MCS).

\section{Methods}

Data were collected retrospectively. ECMO was the MCS used to treat severe early graft failure (EGF). Log-rank test was used to compare
Kaplan-Meier survival curves. A p-value $<0.05$ was considered statistically significant.

Results

147 HT were done from 2004 to October 2016. The average male and female recipient's age was $50 \pm 13$ years and $47 \pm 14$ years, respectively. Percentage of use of MCS and IABP to bridge patients to $\mathrm{HT}$ were $16 \%$ and $7 \%$, respectively. MCS included: 8 HeartWare LVAD (5.4\%), 6 ECMO (4.1\%), 6 Levitronix BiVAD (4.1\%), 2 Thoratec LVAD (1.4\%) and 2 Levitronix LVAD (1.4\%). Hospital mortality was $14.3 \%$ (21pt). 1 -year survival was $82 \%$ and 5 -year survival was $75 \%$. Overall pre-transplant MCS was not correlated to worse posttransplant prognosis, $p=0.822$. Left sided MCS showed a worse survival compared to the no-MCS group $(p=0.045)$ and to the ECMO and BiVAD $(p=0.02)$. Severe primary EGF strongly impact the early mortality after heart transplantation $(p<0.001)$. Excluding the 90 day mortality, the survival between patients with EGF and those without is similar $(p=0.874)$. The risk of mortality increased almost 2 fold according each hour of ischemic time $[\mathrm{OR}=1.7,95 \% \mathrm{IC}, 1.1-2.7$, $\mathrm{p}=0.017]$.

Conclusions

ECMO because of severe EGF remains associated with worse posttransplant outcomes however, patients discharged after severe EGF, showed a long term survival comparable to those without EGF. A LVAD before HT seems to affect the hospital mortality.

\section{Topic: Transplantation}

046

Changes in dynamics of laboratory and echocardiography features after ventricular assist devices support before heart transplantation

Valeriya Krachak, Liana Shestakova, Dzmitry Krachak, Youry Ostrovsky RSPC Cardiology, Minsk, Belarus

Correspondence: Valeriya Krachak

Journal of Cardiothoracic Surgery 2017, 12(Suppl 1):046

\section{Objective}

More than 230 heart transplantations (HTx), including 19 operations of HTx after implantation of long-term ventricular assist devices (LVAD), have been performed between 2009-2016.The purpose of this study was to evaluate changes of laboratory and functional features in a group of 10 patients with the implanted axial and centrifugal LVAD.

Methods

All patients of this group were males, the mean age of the patients was $50,2 \pm 8,2$ years. The diagnosis ischemic cardiomyopathy (ICM) was made in $60 \%$ and dilated cardiomyopathy (DCM) - in $40 \%$ of cases. LVAD were implanted for 310,5 148,1 days (from 119 to 555 days) and then HTx was performed. $60 \%$ of LVAD implantations were associated with other cardiac surgical interventions such as coronary artery bypass surgery, tricuspid valve surgery, closure of patent foramen ovale.

Results

Baseline echo parameters before LVAD implantation were: left ventricular ejection fraction (LV EF) - 17,4 $\pm 4,4 \%$, right ventricular ejection fraction (RV EF) - 33,5 $\pm 8,3 \%$, pulmonary artery systolic pressure (PAPs) - 57,7 $\pm 3,7 \mathrm{mmHg}$ and baseline biochemical features: serum creatinine of blood $98 \pm 12,1 \mu \mathrm{mol} / \mathrm{l}$ and serum bilirubin of blood $28,8 \pm 14,2 \mathrm{mmol} / \mathrm{l}$. During mechanical support patients had positive dynamics of echo parameters before $\mathrm{HTx}$, i.e. LV EF increased to $30,8 \pm 12,3 \%(<0.05)$, RV EF - to $41,7 \pm 7,1 \%(<0.05)$ and PAPs decreased to $40,5 \pm 8,1 \mathrm{mmHg}(<0.05)$. Changes in biochemical features also included decrease in serum creatinine of blood to $93,3 \pm 16,8 \mu \mathrm{mol} / \mathrm{I}(>0.05)$ and serum bilirubin of blood to $14,7 \pm 4,4$ $\mathrm{mmol} / \mathrm{l}(>0.05)$. All of these patients had no hospital mortality.

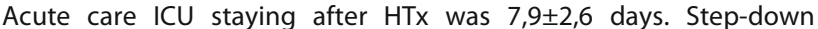
care staying - $25,3 \pm 14,4$ days.

Conclusions

LVAD used as "bridge to transplantation" and "bridge to transplant candidacy " allowed us to decrease PAH, MSOF and to performed HTx. 


\section{Topic: New Technology}

\section{7}

The effect of remote ischemic conditioning on myocardial damage during aortic valve replacement surgery

Dmitry Sevrukevitch, Vasily Sevrukevitch, Kiril Rubakhov, Veronika Barsukevich, Alexander Mrochek

Scientific and Practical Centre, Minsk, Belarus

Correspondence: Dmitry Sevrukevitch

Journal of Cardiothoracic Surgery 2017, 12(Suppl 1):047

\section{Objective}

The aim of this study was to investigate the cardioprotective effect of remote ischemic conditioning (RIC) on myocardial damage during aortic valve replacement surgery.

Methods

95 patients with aortic valve stenosis were selected to participate in the prospective randomized study. Patients were assigned to 3 groups: control group $(n=33)$, study group $1(n=32)$ and study group $2(n=30)$. All patients underwent the aortic valve repair surgery with cardiopulmonary bypass (CPB). In the study group 1 , before the operation, prior the induction of anesthesia, the patients underwent RIPC of the left upper limb. In the study group 2 patients, in addition to RIPC, also underwent remote ischemic postconditioning (RlpostC) with a similar protocol within 2 hours after the end of the CPB. Levels of high sensitive troponin I (hsTn I) in the blood were measured 2, 6, 24, 48, 72 hours after aortic unclamping. Also myocardial biopsy was taken and frozen immediately in liquid nitrogen. Micro-RNA-21 expression levels were measured in biopsies. The area under curve (AUC) of the hsTn I release in every group was evaluated.

Results

In the study group 1 the AUC of hsTn I was $9.7 \%$ lower than in the control group. The hsTn I differences were not significant ( $p>0.05)$, whereas Micro-RNA-21 showed statistically significant differences between controls and study group $1(p<0.05)$. However, in the group 2 , where patients underwent remote pre- and post-conditioning, there was a significant decrease in the AUC of hsTn I by $34.5 \%$ compared to the control group $(p<0.05)$.

Conclusions

The combination of remote ischemic preconditioning and remote ischemic postconditioning are effective additional cardioprotective methods for aortic valve replacement surgery.

\section{Topic: New Technology}

\section{8}

A warfarin free mechanical heart valve by design and material

Walter Dembitsky

Sharp Hospital, Del Mar, CA, USA

Journal of Cardiothoracic Surgery 2017, 12(Suppl 1):048

\section{Background}

Current mechanical valves were designed in the early 70 s with the assumption that damage to blood and subsequent activation of thrombosis occurs during the forward flow. New experimental, numerical and hematological studies have demonstrated that it is not the forward flow but the non-physiologic closing mode that is responsible for the need for life-long anticoagulation. Current tissue valves chemically treated are not living tissues but instead leathers. They elicit an immunologic response which is inherently thrombogenic. We developed a trileaflet mechanical heart valve with physiologic closing mode and immunologically inert bio-stable and blood compatible materials.

Methods

Projected Dynamic Valve Area (PDVA), Digital Particle Imaging Velocimetry (DPIV), Computational Fluid Dynamics (CFD) and
Platelet-Shear Flow Interaction (PSFI) diagnostic technologies were used to compare tissue valves (Carpentier Edward Perimount and Medtronic Intuity) and current mechanical valves (St-Jude Regent and Medtronic Open-Pivot) to the Lapeyre-Triflo tri-leaflet mechanical valve using a low density rigid polymer for the leaflets (Vesta Keep ${ }^{\circledR}$ PEEK, Evonik).

Results

The Lapeyre-Triflo mechanical valve demonstrates physiologic operating mode like tissue valves with no shape transition in the commissural pivoting spaces and no blood stasis in this critical area (the Achilles' heel of current mechanical valves). These physiologic hemodynamic profiles drastically differ from that of current bi-leaflet mechanical valves. Acute thrombogenicity of Vesta Keep ${ }^{\circledR}$ compare favorably with chemically fixed porcine and bovine collagen and carbon-coated surfaces. Validation of long-term structural performances is ongoing. They are equivalent so far to mechanical heart valves made of pyrolytic carbon and titanium.

Conclusion

The Lapeyre-Triflo Furtiva mechanical tri-leaflet design operates physiologically like tissue valves with low risk of occlusive thrombosis. It may function without Warfarin anticoagulation and serve the need for a huge population of patients worldwide needing valve replacement.

\section{Topic: New Technology}

\section{9}

Surgical management of deep sternal wound infection with longitudinal sternal reconstruction following heart surgery

Jeko M. Madjarov'1, Michael G. Katz², Joseph T. McGinn',

Svetozar Madzharov', Francis Robicsek ${ }^{1}$

${ }^{1}$ Sanger Heart \& Vascular Institute, Charlotte, NC, USA; ${ }^{2}$ Mount Sinai

School of Medicine, New York, NY, USA

Correspondence: Jeko M. Madjarov

Journal of Cardiothoracic Surgery 2017, 12(Suppl 1):049

\section{Objective}

Sternal wound complications are associated with increased morbidity and mortality, reaching 12 to $48 \%$. This study was conducted to determine whether longitudinal sternal fixation improves surgical outcome compared to conventional sternal closure.

Methods

We identified all high-risk patients receiving longitudinal sternal plating primary (group $1, n=342$ ), or for the treatment of deep sterna wound infection (group 2, n=16) between September 2010 and December 2016. We compared this cohort with matched population who received conventional closure $(n=266)$. High risk was defined as patients having 2 or more risk factors. Patients were evaluated using CT or MRI scans for sternal alignment, postoperative pain and pulmonary mechanics. We evaluated biomechanical sternal parameters as well.

Results

Mean number of risk factors were $2.4 \pm 0.04$ and no significantly different in the groups. Logistic regression analysis found two independent risk factors: obesity and diabetes. Coronary artery bypass graft surgery was performed in 449 patients $(71.9 \%)$, valve procedures in 152 patients (24.3\%). Of the patients plated prophylactically, and treated with deep sternal wound infection, 337 (98.5\%) and $14(87.5 \%)$ had no complications. Complications seen in these two groups were minor and sternal preservation was achieved in all cases. In control group with conventional closure the rate of sternal dehiscence, superficial sternal wound infections, and deep sternal wound infections were in $9(3.4 \%), 17(6.3 \%)$ and $10(3.75 \%)$ $(p<0.05)$ patients respectively. Results demonstrated better healing in group 1 and 2 than in control group $(3.9 \pm 0.09 ; 2.8 \pm 0.06$ vs $1.8 \pm 0.07, \mathrm{p}<0.001)$. Biomechanical analysis demonstrated significantly improvement of sternal stability in treatment groups. 


\section{Conclusions}

Longitudinal sternal fixation procedure significantly reduced morbidity and mortality with sternal preservation in high risk patients presenting with mediastinitis and sternal dehiscence.

\section{Topic: Lung Cancer}

\section{0}

- Pareto's cost/benefits estimation of a multicentre VATS lobectomy programme

Luca Bertolaccini ${ }^{1}$, Benedetta Bedetti ${ }^{2}$, Alessandro Pardolesi ${ }^{3}$,

Jury Brandolini $i^{3}$, Piergiorgio Solli ${ }^{3}$, Marco Scarci ${ }^{4}$

${ }^{1}$ Santa Maria delle Croci Hospital, Ravenna, Italy; ${ }^{2}$ Malteser Hospital,

Bonn, Germany; ${ }^{3}$ Morgagni-Pierantoni Hospital, Forlí, Italy; ${ }^{4}$ University

College London Hospitals, London, UK

Correspondence: Luca Bertolaccini

Journal of Cardiothoracic Surgery 2017, 12(Suppl 1):050

\section{Objectives}

Development and validation of a Pareto model to estimate the cost/ benefits ratio of video-assisted thoracoscopic (VATS) lobectomy programme in a multicentre setting.

Methods

Data from 379 patients who underwent a VATS lobectomy were collected in two high-volume thoracic surgery units and retrospectively analysed (January 2016 - March 2017). VATS conversions to thoracotomy were not included. All patients were treated by highly proficient VATS surgeons. One to three ports were used. All patients were managed according to standardised enhanced recovery pathways. Intraoperative and postoperative costs were retrieved from the Hospital Pharmacy Departments and included in the analysis. Medians were compared using the Kruskal-Wallis test, means were compared using a Student t-test allowing for unequal variance. Categorical variables were analysed using the $x^{2}$ test. To understand the relationship between costs and a VATS lobectomy programme, a series of regression models were constructed. Because of the nonnormal distribution of costs, a generalised linear model specifying a gamma distribution for values was used for the primary analysis. Definitive cost/benefit evaluation was conducted using Pareto optimal analysis. The analysis was performed by using $\mathrm{R}$ software version 3.4.0 (You Stupid Darkness).

Results

Average costs were $€ 7,500$ (range: $€ 5,000-15,000$ ). The variables of the multivariable regression analysis (dependent variable: total cost) which were associated with total costs after linear regression analysis are the number of purple staplers and the energy sealing devices used. The cost of disposables plays an important role in the total cost of VATS lobectomy, although the variation in the different types of lobectomy was not statistically significant. In the Pareto analysis, all fronts were convex, therefore, the VATS technique had a favourable multi-criteria analysis of cost/benefit ratio, with a good $\mathrm{Pa}$ reto optimum.

Conclusions

VATS lobectomy for lung cancer is associated with a favourable cost/ benefit ratio.

\section{Topic: Cardiac Surgery for Nurses and Allied} Professionals

\section{1}

The role of VAD coordinators in treatment and education of patients with implanted left ventricular mechanical assist device (hmii, Hm lii, Hw)

Arnur Zhambylov, Roman Salov JSC National Research Center for Cardiac Surgery, Astana, Kazakhstan

Correspondence: Arnur Zhambylov

Journal of Cardiothoracic Surgery 2017, 12(Suppl 1):051

\section{Objective}

To show the importance of a coordinator role in educating specifications of $V A D$ and providing treatment services during patients care pre- and post-VAD implantation.

Methods

Between November 2011 and December 2016 our Heart Centre managed 214 LVAD implantation for patients with drug resistant endstage heart failure. 191 of them were male and 23 of them were female. 97 patients have HM II, 62 patients have HM III, and 55 patients have HW implanted. The average age of the patients was $52,7+-$.

Results

Patients' vital signs and life expectancy after implantation is intimately connected with preventing postoperative complications. Thus, VAD coordinators' responsibilities include the following: To provide patients and their close relatives before and after surgery with full and basic information on using VAD, specifications in caring, and preventing possible complications. Besides, during planned outpatient acceptance following discharging a patient from the hospital, to ensure VAD occupational safety, and well-timed replacement of outdated equipment. To conduct diagnostic maneuvers (echocardiography, electrocardiography, 6 minute walk test, blood test, VAD parameters examination, dressing adjustment, and rating postoperative wound) with or without a cardiac professional and take part in regulating care process. The Centre enables each coordinator to help patients around the clock.

Conclusion

The role of VAD coordinators in preventing serious complications caused by VAD ("ascending" cable infection, pump thrombosis, cerebral intensive circulatory failure), providing psychological support of patients, increase quality of life are really important.

\section{Topic: Cardiac Surgery for Nurses and Allied Professionals}

052

The EU Sharps Directive

Ann Foline

Molnlycke Health Care, Göteborg, Sweden

Journal of Cardiothoracic Surgery 2017, 12(Suppl 1):052

\section{Objective}

The objective of the European sharps Directive 2010/32/EU is to achieve the safest possible working environment by preventing injuries caused by all medical sharps and protecting workers in the healthcare sector. The Directive applies to all workers in the healthcare sector, and all who are under the managerial authority of the employers.

Methods

The key strategy to prevent injuries is exposure prevention by eliminating and minimizing the risk of occupationally acquired injuries or infections. This is possible to achieve by a well-trained, adequately resourced and secure health service workforce.

To be able to prevent injuries or infections a risk assessment needs to be performed for all potential situations for injury or exposure to blood or other infectious material. This is done by identify how exposure can be eliminated together with considering possible alternative systems. The short list of measures to eliminate the risk of injuries with a sharp and to reduce the risk of exposure: effective disposal procedures for the handling of disposable sharps, eliminating the unnecessary use of sharps, correct use of personal protective equipment, provide medical devices incorporating safetyengineered protection mechanisms, the practice of recapping shall be banned immediately.

Conclusions

Some of the consequences in the daily practice for health care workers will be to minimize the risk by using traditional instruments with safer, modern equipment. Moreover safer equipment and 
instruments shall be used in areas that present a high risk of infection and/or injury and in the end all new measures needs to be monitored.

\section{Reference}

COUNCIL DIRECTIVE 2010/32/EU. implementing the Framework Agreement on prevention from sharp injuries in the hospital and healthcare sector concluded by HOSPEEM and EPSU. Official Journal of the European Union May 2010

\section{Topic: ECMO}

\section{3}

Extracorporeal membrane oxygenation program in National Research Center for Cardiac Surgery

Timur Lesbekov, Rymbay Kaliyev, Timur Kapyshev, Linar Faizov, Nurlan Smagulov, Zhanybek Ashyrov, Zhuldyz Nurmykhametova, Bauyrzhan Zhunussov, Tileuberdy Kyttybay, Nauryzbek Utin, Oralkhan Mikhailov, Pya Yuryi

National Research Center for Cardiac Surgery, Astana, Kazakhstan Correspondence: Timur Lesbekov

Journal of Cardiothoracic Surgery 2017, 12(Suppl 1):053

\section{Objective}

Extracorporeal membrane oxygenation (ECMO) is an established rescue therapy for severe respiratory failure, cardiogenic shock, and cardiac arrest refractory to conventional therapeutic modalities including ventilatory and high dose inotropic support. In 2011, we initiated the first ECMO program in Kazakhstan, and since 2013 our Center is a member of Extracorporeal Life Support Organization (ELSO). This paper describes the initial experience and early outcomes of applying ECMO in our Center.

\section{Methods}

We performed a retrospective analysis of 203 patients, to evaluate clinical outcomes after ECMO between May 2011 and September 2016. Of these, adult 141 patients at a median age of 47 (22-77) years old. Indication for VV ECMO was respiratory failure in 23 patients $(11 \%), 8$ of whom were pregnant women. Indication for VA ECMO were: postcardiotomy syndrome - $134(66 \%)$ patients, acute coronary syndrome - 7 patients (3.4\%), post-transplant heart graft failure - $19(9.3 \%)$, acute heart failure in $15(7.3 \%)$ patients. Logistic preoperative Euro Score II for patients with postcardiotomy syndrome was 7 (4-18). The primary outcome was all-cause mortality. The secondary outcomes were stroke, bleeding, sepsis.

Results

Hospital survival was $56 \%$, adult - $60 \%$, pediatric $-44 \%$, transported patients - 47\%. The secondary outcomes were stroke- $1.8 \%$, bleeding - $13.4 \%$, sepsis- $5.4 \%$. Complications on ECMO are very common and as expected it is associated with significant increase in morbidity and mortality.

\section{Conclusions}

Our results reflect findings from previous studies and ELSO registry. Further research in this direction will be helpful to understand outcomes in different clinical subgroups.

\section{Topic: ECMO}

\section{4}

- Worldwide aircraft ECMO transportation: experience at a German heart center

Thomas Strecker, Steffen Oehrlein, Michael Meyer, Michael Weyand,

Rene Tandler

Friedrich-Alexander University of Erlangen-Nuremberg, Erlangen, Germany

Correspondence: Thomas Strecker

Journal of Cardiothoracic Surgery 2017, 12(Suppl 1):054

\section{Background}

Extracorporeal membrane oxygenation (ECMO) is often the last resort for serious acute respiratory distress syndrome (ARDS) or acute life-threatening heart failure (HF) when all non-invasive treatment options have failed to improve the patient's pulmonary or cardiac condition. Because some patients are too sick to be transported over long distances to a specialized ECMO center, mobile ECMO teams have been developed and perform the transport with such patients.

Methods

We described our experience associated with international ECMO transportation by aircraft. In the last 14 months, eight patients were transported ( 5 male/3 female) on ECMO support or ECMO stand-by. The mean age for the 5 males was $60,0 \pm 18,3$ years (range 43-80 years), the mean age for the 3 females was $45,3 \pm 17,0$ years (range 26-58 years) at the time of ECMO implantation.

Results

The transport distances with ECMO support varied from $680 \mathrm{~km}$ airline distance (Zadar/Croatia to Erlangen/Germany) to $8.812 \mathrm{~km}$ airline distance (Bangkok/Thailand to Erlangen). Veno-venous ECMO was performed in five cases due to severe pneumonia or ARDS, in two cases veno-arterial ECMO was carried out due to acute myocardial infarction and giant-cell-myocarditis, one in ECMO stand-by with IABP support due to an acute rejection after heart transplantation. All ECMO's were installed through the Venae or Arteria femoralis, respectively. No serious major complications occurred during the transportation. Four patients were successful weaned from the ECMO system, two patients were bridge to a left ventricle assist device (LVAD), the remaining two patients are still in the hospital.

Conclusions

Worldwide aircraft transports on ECMO can be safely performed even over very long distances. The ECMO team should consist of a cardiac surgeon, a perfusionist, an anesthesiologist and a graduated nurse. To our experience the patient transfer on ECMO adds no significant risk of mortality to ECMO patients.

\section{Topic: ECMO}

\section{5}

Extracorporeal membrane oxygenation in lung transplantation

Alkin Yazicioglu', Mahmut Subasi', Dogan Emre Sert ${ }^{2}$, Umit Kervan², Ahmet Gungor', Sinan Turkkan', Fatmanur Celik Basaran', Ibrahim Onur Alici ${ }^{3}$, Hija Yazicioglu', Sema Turan ${ }^{5}$, Erdal Yekeler ${ }^{1}$

${ }^{1}$ Turkiye Yuksek Ihtisas Training and Research Hospital, Thoracic Surgery and Lung Transplantation Clinic, Ankara, Turkey; ${ }^{2}$ Turkiye Yuksek Ihtisas Training and Research Hospital, Department of Cardiovascular Surgery, Ankara, Turkey; ${ }^{3}$ Suat Seren Training and Research Hospital, Department of Chest Diseases, Izmir, Turkey; ${ }^{4}$ Turkiye Yuksek Ihtisas Training and Research Hospital, Department of Anesthesiology, Ankara, Turkey;

${ }^{5}$ Turkiye Yuksek Ihtisas Training and Research Hospital, Anesthesiology and Intensive Care Unit, Ankara, Turkey

Correspondence: Alkin Yazicioglu

Journal of Cardiothoracic Surgery 2017, 12(Suppl 1):055

\section{Objective}

Extracorporeal membrane-oxygenation (ECMO) is an irreplaceable component of lung transplantation (LuTx).

Methods

From March2013-March2017, 30LuTx was performed. Total of 20 patients $(66.7 \%)$ received ECMO, were enrolled.

Results

Of all patients, $18(90 \%)$ received ECMO intraoperatively (17 centralVA, 1 peripheral-VA) and two patients $(10 \%)$ received ECMO in the intensive care unit (ICU). Of 18 patients who received intraoperative ECMO, nine were transferred to the ICU with ECMO. Of these patients, eight received femoral $\mathrm{VA}-\mathrm{ECMO}$ and one received femoral vein to subclavian artery ECMO. Of eight patients who received 
femoral VA-ECMO, femoral-artery cannulation was switched to subclavian-artery in four patients. Advantage of subclavian-artery cannulation such as improved cerebral perfusion was the reason; cannulation was performed using a tubular vascular graft. Of 12 patients who were transferred to the ICU without ECMO support, two received ECMO during follow-up in the ICU. The mean follow-up with ECMO was 5 (range: 2 to 17) days in 11 patients who received ECMO during the ICU-stay.

\section{Conclusions}

Lung implantation with ECMO support has been one of the milestones of success in LuTx. This particularly allows better recovery from primary graft dysfunction period by decreasing elevated pulmonary pressure of the lungs which cold ischemia is yet resolved. Therefore, ECMO should be considered in all cases of LuTx with reversible causes. Of intraoperative ECMO applications, 94\% were central, and it is preferred to maintain improved cerebral circulation and protect recently implanted lung from high pulmonary pressure. Similarly, subclavian-artery cannulation has become increasingly preferred in peripheral VA-ECMO application and it provides improved cerebral circulation, despite technically being a more complicated procedure. In conclusion, ECMO should be irreplaceable for success and survival in the LuTx.

Table 1 (abstract 055). Analyses of ECMO applications in lung transplant cases

\begin{tabular}{|c|c|}
\hline & Distribution/Ratio $(n=20)$ \\
\hline Age & $44,9(22-62)$ \\
\hline Sex & $\mathrm{E}=18(90 \%) \mathrm{K}=2(10 \%)$ \\
\hline SPAP & $53 \mathrm{mmHg}(20-100)$ \\
\hline Pathology & $\begin{array}{l}\text { IPF }(n=8 ; 40 \%) \text { Silicosis }(n=4 ; 20 \%) \text { COPD }(n=4 ; 20 \%) \text { PLCH } \\
(n=2 ; 10 \%) \text { Bronchiectasis }(n=2 ; 10 \%)\end{array}$ \\
\hline $\begin{array}{l}\text { Double vs. Single } \\
\text { LuTx }\end{array}$ & DLuTx (n=16; 80\%) SLuTx $(n=4 ; 20 \%)$ \\
\hline Ischemia time & $\begin{array}{l}\text { First lung }=299 \min (175-374)(n=20) \text { Second lung }=424 \min \\
(300-510)(n=16)\end{array}$ \\
\hline ECMO support & $\begin{array}{l}\text { Intraoperative central }(n=17 ; 85 \%) \text { Intraoperative peripheral } \\
(n=1 ; 5 \%) \text { Postoperative peripheral }(n=11 ; 55 \%)\end{array}$ \\
\hline ICU stay & 17 days \\
\hline Hospitalization & 34 days \\
\hline
\end{tabular}

\section{Topic: Valvular Heart Disease}

\section{6}

- In-hospital outcomes of aortic valve replacement performed by trainees: a multi-centre study

Jennifer L. Whiteley', Kasra Shaikhrezai', Gwyn Beattie ${ }^{1}$, Karim Morcos ${ }^{1}$ Vipin Zamvar ${ }^{2}$, Graham Cooper ${ }^{3}$, Steven Hunter ${ }^{3}$, Geoffrey Berg ${ }^{1}$ ${ }^{1}$ Golden Jubilee National Hospital, Greater Glasgow, UK; 'Edinburgh Royal Infirmary, Edinburgh, UK; ${ }^{3}$ Sheffield Teaching Hospitals NHS Foundation Trust, Sheffield, UK

Correspondence: Jennifer L. Whiteley

Journal of Cardiothoracic Surgery 2017, 12(Suppl 1):056

\section{Objective}

Aortic valve replacement (AVR) is recommended to be preformed by cardiac surgery trainees in all training schemes of the United Kingdom. Public outcome report and uncertainty on safety of AVR for training are deemed to be obstacles for this. We retrospectively studied in-hospital outcome of isolated first-time AVR for degenerative disease and compared the results of consultants versus trainees. Methods

Between January 2010 and 2016, 2812 patients underwent isolated first-time AVR for degenerative disease with a stented valve pros- thesis in three teaching hospitals in the United Kingdom. Nonstented valves, concomitant procedures and endocarditis were excluded from the study.

Results

The mean age of the patients was $70.2+10.4$ and 1608 (57.1\%) were female. The mean logistic EuroSCORE was $6.4+5.3$ with $333(11.8 \%)$ cases performed as urgent in-house procedures. Bioprosthetic stented valves were implanted in $2196(78.0 \%)$ of patients. Total in hospital mortality was $1.8 \%$ (50 patients). Trainees performed 361 cases (12.8\%) with NTN and non-NTN) trainees performing 173 (6.1\%) and $188(6.7 \%)$ cases respectively. More patients with NYHA III-VI (916 (37.3\%) vs. $108(29.9 \%), p=0.006)$ and poor left ventricle function $(109(4.4 \%)$ vs. 7 (1.9\%), p=0.02) were operated by consultants versus trainees. Logistic EuroSCORE was similar between consultant and trainee groups $(6.4+5.6$ vs $5.6+4.6, \mathrm{p}=0.29)$. Cross clamp time $(66.2+25.7$ vs. $75.1+22.4$ minutes, $p=0.09)$, Severe patient-prosthesis mismatch (91 (3.7\%) vs. $15(4.1 \%), \mathrm{p}=0.65)$ and Reopening for bleeding (114 (4.6\%) vs. $21(5.8 \%) \mathrm{p}=0.35)$ did not significantly differ between the two groups. Mortality rate $(1.7 \%$ vs. $2.2 \%$, $\mathrm{p}=0.52)$ and post-operative cerebrovascular accident (42 (1.7\%) vs. 8 $(2.2 \%), p=0.52)$ were similar between the two groups.

Conclusions

Isolated first time AVR is a safe procedure for training and can be performed by trainees with similar results to those of consultants in selected patients.

\section{Topic: Valvular Heart Disease}

057

Trends in clinical practice of aortic valve replacement since the introduction of transcatheter aortic valve implantation: a northern ireland experience

Tinrui Toh, Umar Imran Hamid, Anne Gregg, Chris Austin,

Onyekwelu Nzewi, Colum Owens, Nicola Johnston, Ganesh Manoharan, Mark Spence, Reuben Jeganathan

Royal Victoria Hospital, Belfast, UK

Correspondence: Tinrui Toh

Journal of Cardiothoracic Surgery 2017, 12(Suppl 1):057

\section{Objective}

To evaluate changes in clinical practice of surgical aortic valve replacement (SAVR) with the introduction of transcatheter aortic valve replacement (TAVR) in Northern Ireland

Methods

We conducted a retrospective analysis on data collected from all patients who underwent SAVR and TAVR between 2008 and 2015 Patients who underwent emergency or salvage surgery, including those who underwent surgery for infective endocarditis were excluded from the analysis. TAVR and SAVR patients were compared for preoperative risk profile and short-term outcomes including inhospital mortality and postoperative length of stay.

Results

2152 SAVR and 517 TAVR were performed over the period. The number of SAVR procedures increased from 207 in 2008 to 338 in 2015, while the number of TAVR increased from 21 in 2008 to 100 in 2015. A reduction in SAVR and increase in TAVR was seen in octogenarian patients. Concomitant SAVRs decreased from 2013 onwards, in line with increasing number of TAVRs with preceding $\mathrm{PCl}$. Patients undergoing SAVR were younger than those undergoing TAVR (mean age $67.7 \pm 12.2$ vs. $81.8 \pm 6.6$ years) and had lower preoperative logistic EuroSCORE risk profiles (European System for Cardiac Operative Risk Evaluation), with a mean EuroSCORE of $8.2 \% \pm 9 \%$ vs. $19 \% \pm 12.6 \%$. In-hospital mortality decreased in both groups between 2008 and 2015, from $2.9 \%$ to $0.3 \%$ with SAVR and from $4.8 \%$ to $2.0 \%$ with TAVR. Postoperative length of stay also decreased in both groups from 13.7 to 12 days after SAVR and from 10.7 to 4.4 days after TAVR. 


\section{Conclusions}

These findings demonstrate that both TAVR and SAVR practice have increased, paralleled with improved hospital outcomes. The latter is likely the result of good patient selection due to a robust MDT process. We estimate an increase in the utilisation of TAVR in the coming future as a result of the increasingly ageing population witnessed over the last 3 years.

\section{Topic: Valvular Heart Disease}

\section{8}

Evaluation of the aortic valve leaflet reconstruction using autologous pericardium by 3D hologram created a novel workstation Visalius 3D

Takeo Tedoriya, Kenichi Kamiya, Tadamasa Miyauchi, Masaomi Fukuzumi Ageo Central General Hospital, Saitama, Japan

Correspondence: Takeo Tedoriya

Journal of Cardiothoracic Surgery 2017, 12(Suppl 1):058

\section{Background}

Aortic valve leaflet reconstruction using autologous pericardium (AVLR) has been performed at our institute for patients of the aortic valve disease with narrow aortic annuls or contra-indication of valve prosthesis. Our unique procedure consists of three-same-sized leaflets reconstruction referred from STJ diameter, with adjusting balanced neo-commissure as well as depth of nadir especially in non-coronary sinus of Valsalva. We evaluated AVLR procedure with a novel 3D image workstation, Visalius 3D which provides 3DHologram.

Methods

Enrolled patients planned for aortic valve surgery underwent contrast enhanced ECG-triggered cardiac computed tomography (CT). Axial images using a 264-row CT with slice thickness of $0.625 \mathrm{~mm}$ were obtained during mid-to-end diastole. Subtracted volume rendering $\mathrm{CT}$ data of aortic root were converted to stereolithography (STL) file in Visalius 3D. We assessed anatomical structure of the aortic valve, and in some particular cases we determined procedure for creating neo-commissure and offsetting of deviated nadir. The basic surgical technique of AVLR is as below; After the ascending aorta is opened transversely, STJ diameter is measured. Three pieces of leaflet of autologous pericardium treated by $0.6 \%$ glutaraldehyde for 6 minutes are tailored to original template referred by STJ diameter. Leaflets are sutured to the annulus by continuous stitching from the nadir to the new commissures. Finally, STJ is fixed by pericardial stripe as prevention of STJ dilatation.

Results

We present video clips of evaluation with 3D-Hologram assessment and surgical procedure of AVLR surgery. In all 44 cases, there was no mortality. One patient had AVR because of mild AR. The mean pressure-gradient after the surgery was $6.5 \mathrm{mmHg}$ with no development of AR during follow-up (mean 45 months).

Conclusions

Preoperative evaluation by the 3D-Hologram aortic root image had notably provides valuable information for adjustment of neocommissure and nadir in our AVLR surgery.

\section{Topic: Valvular Heart Disease}

\section{9}

Correction of narrow ostium of aorta during aortic valve

replacement in adults: choice of surgical method

Konstantin Vakulenko, Volodymyr Popov, Oleksandr Bolshak,

Vasyl Lazorishinets

National Institute of cardio-vascular surgery named after Amosov, Kiev,

Ukraine

Correspondence: Konstantin Vakulenko

Journal of Cardiothoracic Surgery 2017, 12(Suppl 1):059

\section{Background}

This investigation is to research possibilities of different method of surgical correction during aortic valve replacement (AVR) in patents (pts) with narrow ostium of aorta (NOA).

Methods

In analyzed group were included 165 pts with aortic valve disease $(\mathrm{n}=$ $142)$ and combined mitral-aortic diseases $(n=23)$ with NOA which were consecutive operated in Institute from 01.01. 1996 till 01.01.2017. AVR with reconstruction of ostium of aorta was performed in all cases by following methods: Konno's operation $(n=29)$ - group A, Nick's operation $(n=33)$ - group $B$, original method of reconstruction by posterior aortoplasty (Popov V.) ( $n=103)$ - group C.

Results

Hospital mortality (HM) (30 days) were: group A - 10,3\% ( $n=3 / 29)$, group $B-9,1 \%(n=3 / 33), C-6,8 \%(n=7 / 103)(p<0,05)$. At the last 21 operations in group C HM - $0 \%$. Reasons of deaths: group A - all heart failure, group B - brain damage $(n=1)$, bleeding $(n=1)$, MOF $(n=1)$, group $C$ - pneumonia $(n=2)$, acute colitis $(n=1)$, sepsis $(n=1)$, MOF $(n=2)$, brain damage $(n=1)$. In group $A$ at 7 years we had observed: survival rate $49.3 \%$, stability of good results occurred $23.3 \%$. The reasons of deaths: progressive heart failure (9), myocardial infarction $(n=1)$, arrhythmia $(n=1)$. In group $B$ at 7 years we had observed: survival rate $79.3 \%$, stability of good results occurred $67.3 \%$. The reasons of deaths: cancer $(n=1)$, arrhythmia $(n=2)$, ischemic disease $(n=1)$. In group $C$ at 7 years we had observed: survival rate $85.3 \%$, stability of good results occurred $75.3 \%$. The reasons of deaths: ischemic disease $(n=2)$, arrhythmia $(n=2)$, arterial hypertension $(n=1)$, sepsis $(n=1)$, pneumonia $(n=1)$.

\section{Conclusion}

Reconstruction of NOA during AVR by Nick's operation and proposed original method of posterior aortoplasty are effective interventions. Konno operation cannot be performed in adult patients.

\section{Topic: Valvular Heart Disease}

\section{0}

Cardiac remodeling after aortic valve implantation for aortic stenosis

Thomas Sénage, Justine Enée, Caroline Cueff, Nicolas Piriou,

Jean-christian Roussel, Thierry Le Tourneau

Thorax Institute, Nantes, France

Correspondence: Thomas Sénage

Journal of Cardiothoracic Surgery 2017, 12(Suppl 1):060

\section{Objective}

Reverse cardiac remodeling is an important objective of aortic valve replacement (AVR). Global longitudinal strain (GLS) allows a more sensitive evaluation of systolic function than usual left ventricle ejection fraction (LVEF). The aim of this study was to assess the cardiac remodeling of the 4 cardiac chambers 6 months after AVR.

Methods

The present study included patients with aortic stenosis scheduled for surgical AVR or percutaneous implantation (TAVI). Patients were separated in two groups according to baseline left ventricle (LV) GLS value (normal $\leq$ or altered $>-16 \%$ ). Patients with associated aortic, mitral or tricuspid regurgitation $(\geq 3 / 4)$ were excluded from analysis. Changes in LV GLS and cardiac morphology were evaluated 6 months after AVR.

\section{Results}

189 patients (37\% female, mean age $74 \pm 9$ years) were included, with 43 TAVI (22.8\%) and 13 mechanical AVR (6.9\%). The 6-month follow up was completed for all the patients. Preoperative mean LVEF and GLS were $63 \% \pm 10[28-83]$ and $-16 \pm 4 \%$ respectively. Patients with impaired GLS at baseline were more often diabetics and obese, and had higher NT-proBNP levels. At baseline, cardiac chambers remodeling was more pronounced, LVEF was moderately decreased $(58 \pm 11$ vs $66 \pm 7 \% ; p<0,01)$, and valvulo-arterial impedance (global afterload) 
was higher. In this sub-group, patients experienced a significant improvement in GLS at 6 months. Despite a significant decrease in pulmonary artery pressure, there was a significant alteration of right ventricle strain at 6 months.

\section{Conclusions}

Left ventricle presents a significant, positive, reverse remodeling after surgical or percutaneous AVR, especially in patients with altered GLS before procedure. This favourable LV remodeling contrast with the significant alteration of the right ventricle strain.

\section{Topic: Valvular Heart Disease}

\section{1}

Pathoanatomic findings and treatment during hypertrophic obstructive cardiomyopathy surgery: the role of mitral valve Giuseppe M. Raffa, Marco Turrisi, Giuseppe Romano, Michele Pilato IRCCS-ISMETT (Istituto Mediterraneo per i Trapianti e Terapie ad altaspecializzazione), Palermo, Italy

Correspondence: Giuseppe M. Raffa

Journal of Cardiothoracic Surgery 2017, 12(Suppl 1):061

\section{Objective}

To assess the role of the mitral valve apparatus (leaflets, chordae and papillary muscles, PM) in left ventricle outflow tract (LVOT) obstruction, and results of the surgical treatment for hypertrophic obstructive cardiomyopathy (HOCM).

\section{Methods}

Twenty-eight consecutive patients (58 11 years, 53\% female) undergoing HOCM surgery from 2007 to 2016 at our institute were retrospectively reviewed. End points included the involvement of the mitral valve in LVOT obstruction, mortality, and change in clinical and echocardiographic characteristics after HOCM surgery.

Results

Secondary chordae tendineae tractioning the anterior mitral leaflet to the interventricular septum, and systolic anterior motion were detected in $78 \%$ of the patients. Anomalous, hypertrophied, and fused PM with muscularis trabeculae hypertrophy were found in $50 \%, 25 \%$, and $35 \%$ of the patients, respectively. Four patients had posterior leaflet redundancy. Secondary chordae (92\%), PM, and muscularis trabeculae resection (71\%), and PM splitting and elongation (28\%) were added variably to septal myectomy (100\%). Nine procedures $(32 \%)$ on mitral valve leaflets were performed, involving 6 posterior and 3 anterior mitral leaflets. Long-term follow up was $4 \pm 2.8$ years. There was no hospital mortality, and NYHA was reduced from $3 \pm 0.5$ to $1 \pm 0.7$ ( $p<0.0001$ ), the LVOT gradient from 88 \pm 35 to $20 \pm 18 \mathrm{mmHg}(p<0.0001)$, mitral valve regurgitation from grade $3 \pm 1$ to $1 \pm 0.7(p<0.0001)$, and septum thickness from $18 \pm 3$ to $14 \pm 2 \mathrm{~mm}(p<0.0001)$.

\section{Conclusions}

The mitral valve apparatus with all its components contribute variably to LVOT dynamic obstruction thus, surgical correction in addition to extended myectomy is recommended to achieve the best outcome.

\section{Topic: Valvular Heart Disease}

\section{2}

Five year haemodynamic performance of the trifecta aortic prosthesis in octogenarians

Benjamin Adams, Prity Gupta, Mohamed Bashir, Amer Harkey,

Shirish Amberkar, Rakesh Uppal, Kulvinder Lall

Barts Heart Centre, London, UK

Correspondence: Benjamin Adams

Journal of Cardiothoracic Surgery 2017, 12(Suppl 1):062

\section{Objective}

The St. Jude Medical Trifecta aortic supra-annular bioprosthesis is regarded as the next generation in pericardial stented tissue valves. The unique design of tissue leaflets attached to the exterior of the valve stent provides unrivalled in-vivo mean gradients and haemodynamics. The aim of this prospective study was to evaluate midterm haemodynamic performance of valves implanted into patients over 80.

Methods

Ninety-one consecutive patients undergoing aortic valve replacement using the St. Jude Medical Trifecta valve at a single UK centre over a five-year period were included in this study. Patients undergoing concomitant cardiac procedures were included. All implanted valves were 19, 21, 23 and $25 \mathrm{~mm}$ in size. Assessment of haemodynamic function was carried out using transthoracic echocardiography pre-operatively and at follow-up, as well as transoesophageal echocardiography intra-operatively.

Results

The study population consisted of 91 patients (42 male, 49 female). Mean age was $82.8 \pm 2.49$ years. Implanted valve sizes were $19 \mathrm{~mm}$ $(n=8), 21 \mathrm{~mm}(n=39), 23 \mathrm{~mm}(n=32)$ and $25 \mathrm{~mm}(n=12)$. Overall mean post-operative pressure gradients were $9.51 \pm 8.29 \mathrm{mmHg}$ (mean) and $18.35 \mathrm{mmHg} \pm 15.1 \mathrm{mmHg}$ (peak). Subgroup mean post-operative pressure gradients were $13.56 \pm 5.22 \mathrm{mmHg}, 10.31 \pm 11.23 \mathrm{mmHg}$, $7.69 \pm 3.16 \mathrm{mmHg}$ and $5.41 \pm 1.48 \mathrm{mmHg}$, for the $19,21,23,25 \mathrm{~mm}$ cohort respectively. Overall mean post-operative left ventricular ejection fraction was $57 \pm 13.39 \%$. Overall mean effective orifice area was $1.73 \pm 0.53 \mathrm{~cm} 2$.

Conclusions

These results of our experience demonstrate excellent haemodynamic performance of the Trifecta bioprosthetic valve in octogenarians.

\section{Topic: Valvular Heart Disease}

063

Retrospective time series analysis on the use of standard biological aortic valve implantation vs TAVI

Beatrice Desirée Calabrese', Katy Pelagagge', Yermek Akhmetov²,

Piergiorgio Minotti ${ }^{1}$, Mihaela Dubinski ${ }^{1}$, Filippo Bartoccioni ${ }^{3}$

${ }^{1}$ HTAcamp, Viterbo, Italy; ${ }^{2}$ Astana Medical University, Astana, Kazakhstan;

${ }^{3}$ Asl Viterbo, Viterbo, Italy

Correspondence: Beatrice Desirée Calabrese

Journal of Cardiothoracic Surgery 2017, 12(Suppl 1):063

\section{Objective}

The aim of this study is to evaluate, within our hospital setting, the appropriate use of the TAVI procedure comparing them with the standard biological aortic valve implantation.

Methods

We first defined the trend of total stenotic AVR (ICD 9 code 35.21 ) in our hospital before and after the TAVI introduction. We used a monthly time series analysis to see how was this trend in time from January 2000 to 2012 and then we extend the monitoring to 2014. Then we use the same analysis to evaluate the trend of only standard biological valve implants.

Results

We statistically demonstrate that the trend of total AVR is increasing steadily in time but there are no significant angulations in the trend. The time series analysis of the trend of only standard biological valve implants showed that in the begging when only the interventional cardiology department started to use them there was no statistical modification of the trend. Surprisingly we discover that when the cardiac surgery department started to use the TAVI as well, a significant trend modification was in place. The standard biological valve implants decreased significantly.

Conclusions

The two techniques, following the international guidelines recommendations, should not interfere one another. They indeed should have two separated and independent target populations or eventually just a small overlap. We could aspect an increase of total number of patients operations due to the introduction of a target population but, instead, the TAVI usage did not increase significantly 
the arrival of new patients and we can see that the standard biological valve implants that were supposed to stay unmodified or increased due to the increase of attractiveness of this technique, did not occur. The decreasing trend of standard valve, negatively affected by the TAVI introduction, shows a probable inappropriate use of TAVI.

\section{Topic: Valvular Heart Disease}

064

Surgical aortic valve replacement in the elderly: a single center single surgeon retrospective series from Durban, South Africa

Robert Kleinloog, Darshan Reddy

Ethekwini Heart Center, Durban, South Africa

Correspondence: Robert Kleinloog

Journal of Cardiothoracic Surgery 2017, 12(Suppl 1):064

\section{Objective}

The number of conventional surgical aortic valve replacement procedures undertaken globally is progressively declining in the face of emerging technologies, including transcatheter aortic valve implantation and sutureless aortic valve implantation.

Methods

A retrospective chart review was made of all patients undergoing conventional aortic valve replacement, in isolation or in conjunction with other procedures, at a cardiac center in Durban, South Africa over a 5 year period from January 2011 to December 2016. All procedures were undertaken by a single surgeon and the perioperative care team remained constant for the duration of the study. Results

A total of 183 patients (61 women) underwent isolated aortic valve replacement in 59\% (108 patients), aortic valve replacement and coronary artery bypass grafting in 33\% (61 patients), and aortic valve replacement with concomitant mitral valve intervention in $8 \%(14$ patients). The median age of the group was 70.5 years and the indication for aortic valve replacement was aortic stenosis (94.8\%), mixed aortic valve disease (2.6\%) and aortic regurgitation (2.6\%). A bioprosthetic aortic valve was implanted in the vast majority of patients (97.8\%) and the 30 day survival following surgery was $94 \%$. Conclusions

Conventional aortic valve replacement, in isolation or with combined procedures, is a reproducible technique that may be undertaken with morbidity and mortality rates markedly lower than predicted outcomes scores. This technique should remain the standard against which the outcomes of emerging technologies are measured.

\section{Topic: Valvular Heart Disease}

\section{5}

CoreValve Evolut-r transcatheter aortic valve implantation experience of National Research Center for Cardiac Surgery Serik Alimbayev, Abdurashid Mussayev, Timur Lesbekov, Yuriy Klochan, Marat Aripov, Lazzat Abikeeva, Aigerim Sadykova, Rimbay Kaliyev, Yuriy Pya

National Research Center for Cardiac Surgery, Astana, Kazakhstan

Correspondence: Abdurashid Mussayev

Journal of Cardiothoracic Surgery 2017, 12(Suppl 1):065

\section{Objective}

The aim of our investigation is to show the short-term results of transcatheter implantation of self-expandable aortic valve CoreValve Evolut-R.

Methods

130 transcatheter aortic valves (balloon and self-expandable) have been implanted in "National Research Center for Cardiac Surgery" since 2012. CoreValve Evolut-R (Medtronic, Minneapolis, MN, USA) was performed in 30 patients with symptomatic severe aortic valve stenosis. The size distribution of the valves was: 23 valve sizes- $3 \%$;
$26-27 \% ; 29-63 \% ; 34-7 \%$. Mean age of patients was $74 \pm 16$ years, where $57 \%$ - men, $43 \%$ - women. An operation under general anesthesia was performed in $71 \%$ of patients, and under local anesthesia in $29 \%$ using ProStar XL and surgical cutting of femoral artery. In 29 cases aortic valve Evolut- $\mathrm{R}$ was implanted by femoral approach, in one case - by transaortic approach due to significant atherosclerotic lesion of iliac, femoral and subclavian arteries. The risk of traditional surgical intervention according to STS PROM $-7.2 \% \pm 3 \%$.

Results

Average duration of implantation procedure was $90 \pm 38$ minutes. Reimplantation of prosthesis was carried out in 10 cases due to deep or supre-annular implantation of aortic valve, in one case during the implantation of the 34 size of valve delivery system was broken after the 7 -th recapturing of the valve. In $43 \%$ of cases, post dilatation was performed due to hemodynamically significant paraprosthetic leak and incomplete disclosure of the prosthesis. Average hospital stay was $5 \pm 2$ days. In $10 \%$ of cases was implanted pacemaker.

Conclusions

Self-expanding prosthesis of aortic valve CoreValve Evolut $\mathrm{R}$ with EnVeoR $14 \mathrm{Fr}$ and $16 \mathrm{Fr}$ delivery system is an effective and relatively safe device (due to low-profile delivery system, opportunity to reimplant prosthesis and improved design of a valve) for treatment of patients with aortic valve stenosis of high and middle surgical risk.

\section{Topic: Valvular Heart Disease}

066

Is age a factor for refusal of aortic valve replacement surgery? The results from our center in patients over $\mathbf{8 0}$ years old

Tiago R. Velho, Hugo Ferreira, Catarina Carvalheiro, Nádia Junqueira, André Sena, Joana Silva, Nuno Guerra, Javier Gallego, Ângelo Nobre

Hospital de Santa Maria, Lisboa, Portugal

Correspondence: Tiago R. Velho

Journal of Cardiothoracic Surgery 2017, 12(Suppl 1):066

\section{Objective}

Aortic valve stenosis (AS) is the most common valvular pathology in the elderly. Surgical aortic valve replacement (AVR) remains the goldstandard of treatment for AS. However, emerging transcatheter aortic valve replacement (TAVI) has become an increasing alternative to surgery. In a recent survey from the European Society of Cardiology, $9.4 \%$ of the physicians stated that age was the main reason to indicate a TAVI instead of surgery.

Methods

Single-center retrospective study including 353 patients (149 patients over 80 years-old, compared to 204 patients between 60-69 yearsold) consecutively submitted to AVR between January 1, 2013, and December 31, 2016. Clinical data was collected and assessed from clinical files in all patients, and long-term survival was determined by Kaplan-Meier survival analysis.

Results

The demographic and clinical characteristics between the two groups were similar $(P>0.05)$. There were no significant differences in the survival between the two groups at 30 days $(96.57 \% 60-69$ yo vs. $96.64 \%>80 \mathrm{yo}), 12$ months $(89.57 \% 60-69 y o$ vs. $93.51 \%>80 \mathrm{yo})$ and 24 months (85.92\% $60-69 y$ o vs. $87.62 \%>80 y$ o) $\mathrm{P}>0.05$. The postoperative complication rates were similar between the two groups, excluding the rate of post-operative atrial fibrillation, higher in $>80$ years-old group $(29.06 \%$ vs. $17.28 \%, p=0.0147)$. ICU and average hospital length of stay was similar between the two groups $p>0.05$. In all patients EuroSCORE II was directly correlated to intensive care unit length of stay $(p=0.0044)$. Extracorporeal circulation time was directly correlated to invasive ventilation time $(p=0.0254)$ and to postoperative bleeding $(\mathrm{p}=0.0002)$.

Conclusions

Age is not a good indication to propose for TAVI, since survival rates are similar between the two groups, with similar post-operative complications. Post-operative atrial fibrillation is more common in 
patients over 80 years-old. In older patients lower periods of extracorporeal circulation much be achieved to reduce invasive ventilation time and post-operative bleeding, improving post-operative recovery.

\section{Topic: Cardiac Surgery}

\section{7}

Exstent, a completely new method of treatment of patients with Marfan syndrome

Jan Pirk

IKEM, Prague, Czech Republic

Journal of Cardiothoracic Surgery 2017, 12(Suppl 1):067

Marfan syndrome is the most common genetic disorder of connective tissue. One complication that threatens the lives of patients is progressive dilatation of the ascending aorta with development of aortic valve regurgitation or the emergence of dissection, often leading to sudden death. Until now these patients were operated only after dilatation of the ascending aorta causing hemodynamically significant regurgitation of the aortic valve and surgery consisted of the replacement of the ascending aorta and aortic valve, or valve sparing procedure. This new method is a preventive operation. The method involves creating a custom made external support of the root and the ascending portion of the aorta. Based on the CT examination prosthesis Exstent is created. The surgery is performed from the longitudinal median sternotomy without cardiopulmonary bypass. Entire aortic root is dissected to its origin from the left ventricle, ostia of the coronary arteries are encircled, the prosthesis is pulled underneath and fixed to the root and then sutured longitudinally. It is interesting that the prosthesis developed and as the world's first have it sewn on himself Mr. Tal Golesworthy 13 years ago in Oxford. Neither him nor the other 100 patients operated in this department with this disease had dilation or dissection throughout the study. It is because the prosthesis grows over time into the aortic wall thereby enforces it while maintaining the elastic properties of the wall. At our institute we have so far experience with operations in 8 patients, with good results. This operation moves the care of patients with Marfan syndrome to qualitatively higher level.

\section{Topic: Coronary Heart Disease}

\section{8}

Five-year results of surgical treatment patients with atrial fibrillation and coronary artery disease: prospective randomized study

Alexander Chernyavskiy, Yulia Kareva, Sardor Rakhmonov, Inessa Pak, Evgeny Pokushalov, Alexander Romanov

Novosibirsk Research Institute of Circulation Pathology, Novosibirsk, Russian Federation

Correspondence: Yulia Kareva

Journal of Cardiothoracic Surgery 2017, 12(Suppl 1):068

\section{Objective}

Assess the effectiveness of different methods of ablation of atrial fibrillation (AF) in patients with coronary heart disease in the long term.

Methods

In this study were included 297 patients, which were performed CABG combined with radiofrequency ablation of AF. There were 95 patients with paroxysmal AF (mean age $60,64 \pm 4,6$ years, duration $A F$ $39,24 \pm 6,2$ months), 104 - persistent $A F$ (mean age 62,92 $\pm 1,7$ years, duration AF $76,4 \pm 6,4$ months) and 98 - long-standing persistent AF (mean age $61,14 \pm 5,9$ years, duration $A F 77,13 \pm 5,9$ months). Patients were randomized into three groups: $C A B G$ and pulmonary vein isolation (CABG+PVI, $\mathrm{n}=97$ ), $C A B G$ and modified mini-maze procedure (CABG+MM, $n=103)$, and CABG with ablation ganglion plexus of left atrium (CABG+PG, $n=98)$. Patients with paroxysmal, persistent and long-standing persistent AF were included in each group. In 59,5\% patients received implantable loop recorders (ILRs). The study primary end point- freedom from AF 1 year postoperatively; secondary end point - long-term outcomes, quality of life, mortality, cardiovascular events.

Results

Hospital mortality was $2 \%$ (6 pts). The number of distal anastomoses and character additional procedures, the average time of stay in the intensive care unit, was not significantly different between groups. Freedom from $\mathrm{AF}$ in $\mathrm{CABG}+\mathrm{PVI}$ group in patients with paroxysmal $\mathrm{AF}$ was $88.2 \%$, in patients with persistent $-76.9 \%$, long-standing persistent38.7\%; in CABG+MM group - paroxysmal $\mathrm{AF}-83.3 \%$, persistent $-81.1 \%$, long-standing persistent $-48.6 \%$; in CABG+PG group -paroxysmal AF $73.9 \%$, persistent $-38.2 \%$, long-standing persistent $-33.2 \%$.

Conclusions

RFA of AF during CABG is a safe method of treatment of AF, provides a reasonable freedom from AF. In patients with paroxysmal AF are effective all methods of ablations, with persistent AF - are equally effective PVI and MM procedure, with a long persistent - only the MM procedure allows achieving satisfactory results. ILRs are an objective method for diagnosing arrhythmias.

\section{Topic: Coronary Heart Disease}

\section{9}

What is the affect of individual surgeon experience on risk adjusted survival rates in cardiac surgery?

Victoria Rizza, Umar Hamid, Glen Dunwoody, Alastair Graham

Royal Victoria Hospital, Belfast, Belfast, UK

Correspondence: Victoria Rizza

Journal of Cardiothoracic Surgery 2017, 12(Suppl 1):069

\section{Objective}

A consultant cardiac surgeon's practice may change with time; however it is uncertain whether this affects risk adjusted survival rate. The effect of numbers of cardiac operations done in UK cardiac surgery units on survival rates is not known.

Methods

The operation data performed by consultant cardiac surgeons in the UK and institutional volume was taken from public data on the SCTS website. The percentage of each operation done and risk-adjusted survival rates were recorded from 2011 to 2014. Specialist register entry date listed on the GMC website was used to estimate years of experience as a consultant cardiac surgeon. The influence of increasing years of consultant experience on type of practice and risk adjust survival rates was investigated. Number of operations done per unit was also tested using non parametric tests (SPSS).

Results

322 cardiothoracic UK surgeons were registered on the SCTS website. 71 were excluded due to the following: no surgeon level data, congenital heart disease, lack of GMC registration data. As time since registration of consultants increased, the percentage of CABG cases decreased significantly and percentages of MVR, MVR + CABG, AVR and Other cases increased significantly. Risk adjusted survival rate of surgeons was not correlated with surgical experience. Similarly surgeons who carried out $>400$ cases as compared to $<400$ cases had no significant difference in their outcome $(p=0.21)$. The risk adjusted survival rate of hospitals that did $>2500$ cases was $97.7 \%$ compared with $97.2 \%$ if $<2500$ cases done $(p=0.02$, Mann Whitney).

Conclusions

There is a change in type of practice as a cardiac surgeon progresses through their career, however, their risk adjusted survival rates are not affected by this. There is a small but measurable improvement in survival when larger units are compared to smaller ones. The reason for this merits further examination. 


\section{Topic: Coronary Heart Disease}

\section{0}

Bilateral internal mammary artery bypass grafting: extent of use and focus on sternal wound infection

Justine Ravaux', Tami Guennaoui' ${ }^{2}$, Christian Mélot ${ }^{3}$, Peter Schraverus ${ }^{1}$

${ }^{1}$ Grand Hôpital de Charleroi, Gilly, Belgium; ${ }^{2}$ Centres Hospitaliers

Jolimont ASBL, Haine Saint Paul, Belgium; ${ }^{3}$ Erasme University Hospital, Brussels, Belgium

Correspondence: Justine Ravaux

Journal of Cardiothoracic Surgery 2017, 12(Suppl 1):O70

\section{Objective}

Bilateral internal mammary arteries (BIMA) remain underused in coronary artery bypass grafting (CABG), especially in elderly, diabetics and obese patients. In this study, we aim to investigate in these high-risk patients the incidence of sternal wound infection, sternal instability, and re-intervention for bleeding.

Methods

A single-centre retrospective observational study was made in our centre Grand Hôpital de Charleroi, Gilly, BELGIUM. 319 patients undergoing CABG's from December 2011 to December 2015 were included. Three main outcome measures (incidence of sternal wound infection (SWI), sternal instability (SI), and re-intervention for bleeding (RIB) were investigated in obese vs non-obese patients, diabetic vs non diabetic patients, and elderly vs younger patients.

Results

Among the 319 patients, 14 have SWI, 11 have SI, and 6 have RIB. Incidence of death rate was: SWI: $2 / 14$ vs $17 / 305 p=0.178$; SI: $2 / 11$ vs $17 / 308 p=0.081 ;$ RIB: $2 / 6$ vs $17 / 313 p=0.004$. In obese $(n=113)$ vs non-obese $(n=206)$ patients, there was no difference for SWI $(p=$ $0.263)$, SI $(p=0.565)$, and RIB $(p=0.332)$. In diabetic $(n=118)$ vs non diabetic $(n=201)$ patients there was no difference for: SWI $(p=$ $0.642)$, SI $(p=0.497)$, and RIB $(p=0.298)$. In elderly $(n=62)$ vs younger $(n=257)$ patients, there was no difference for SWI $(p=0.619)$, SI $(p=0.915)$, and RIB $(p=0.385)$.

\section{Conclusions}

Obesity, age and diabetes treated by insulin (or not) do not seem to be risks factors for developing SWI, SI or RIB in patients receiving a CABG using BIMA. Nevertheless, mortality was higher in patients requiring RIB.

\section{Topic: Coronary Heart Disease}

\section{1}

Differential approach to prevent stroke during CABG procedure

Pavel Myalyuk, Andrey Marchenko

Federal Centre of Cardiovascular Surgery, Perm, Russian Federation

Correspondence: Pavel Myalyuk

Journal of Cardiothoracic Surgery 2017, 12(Suppl 1):071

\section{Objective}

For more than 50 years of existence, coronary bypass cultivate ways to reduce complications. Despite this, the incidence of cerebrovascular complications after revascularization remains within $0.8-4.2 \%$, according to different authors. The aim of this study is to develop and assess the effectiveness of the differentiated approach to the choice of optimal tactics coronary bypass surgery.

Methods

During the period from 01.01.2013 to 04.06 .2015 we performed 3454 coronary bypass grafting. The average age of $61.2 \pm 12.2$, men 2346 (67.9\%), obese in 1090 (31.6\%), diabetes was diagnosed in 281 (8.1\%) patients, permanent atrial fibrillation in $82(2.4 \%)$, left ventricular ejection fraction less than $30 \%$ in $31(0.9 \%)$, unstable angina in 370 (10.7\%). We divided the patients into 2 groups: 1 - 2689 patients, and 2 - 765 patients we used differentiated approach. In 1 group, patients underwent standard coronary artery bypass grafting. For Patients in 2 group we include into examination transesophageal echocardiography, CT and epiaortic ultrasound scanning. If the aorta is normal patients underwent standard coronary artery bypass grafting - 585 (76.4\%). Atheromatosis or plaques protruding into the lumen more than $5 \mathrm{~mm}$ or local calcinosis of aorta were detected in 190 patients. In all of them, we had changed the surgical tactics. Modifications applied: place of cannulation and place of clamping changing - $92(12 \%)$, «Single clamp» - $43(5.6 \%)$, «on pump beating »- 27 (3.5\%), «no-touch aorta» - 18 (2.3\%).

Results

Overall Hospital Mortality was $1.1 \%$ (38). Perioperative Stroke 33 (1.4\%) in Group 1, and 0 in Group 2.

Conclusions

Differentiated approach to surgical tactics choice, reduces the risk of neurological complications when atherosclerosis of the ascending aorta is detected after CABG.

\section{Topic: Coronary Heart Disease}

\section{2}

Effect of octopus tissue stabilizer on cardiac output during off pump coronary artery bypass graft surgery

Ganesh Kumar K Ammannaya

Lokmanya Tilak Municipal Medical College \& General Hospital, Mumbai, India

Journal of Cardiothoracic Surgery 2017, 12(Suppl 1):072

\section{Objective}

Significant hemodynamic derangements can occur during off-pump coronary artery bypass graft (OPCAB) surgery resulting from displacement of beating heart, necessitating conversion to on-pump surgery. We proposed to evaluate the alterations in hemodynamic parameters in patients during the course of anastomosis in $O P C A B$ surgery using octopus tissue stabilizer.

Methods

In 100 consecutive patients undergoing OPCAB surgery, hemodynamic variables including cardiac output (CO), heart rate (HR), mean arterial pressure (MAP) and central venous pressure (CVP) were recorded at baseline, during each coronary artery anastomosis at $2 \mathrm{~min}, 10 \mathrm{~min}$ and after release of Octopus tissue stabilizer.

Results

Cardiac output (CO) decreased significantly after target stabilization and during all coronary anastomoses $(5.42 \pm 1.1 \mathrm{~L} / \mathrm{min}$ at baseline, $4.26 \pm 1.02 \mathrm{~L} / \mathrm{min}$ at $2 \mathrm{~min}$ and $3.92 \pm 0.98 \mathrm{~L} / \mathrm{min}$ at $10 \mathrm{~min}$ ), with greatest decrease noted during obtuse marginal (OM) branch of left circumflex artery anastomosis $(3.67 \pm 0.86 \mathrm{~L} / \mathrm{min}$ at $2 \mathrm{~min} ; 3.38 \pm$ $0.78 \mathrm{~L} / \mathrm{min}$ at $10 \mathrm{~min}$ ). Inotropic drugs were required to maintain mean arterial pressure (MAP) $>60 \mathrm{mmHg}$ in 43 patients. While the incidence of bradycardia requiring inotropes was noted to be the highest (11 out of 16 patients) during left anterior descending (LAD) artery anastomosis, the fall in the MAP necessitating inotropes was most frequent during obtuse marginal (OM) artery anastomosis (32 patients). The central venous pressure was maintained without any significant changes during all coronary anastomoses.

\section{Conclusions}

During $O P C A B$ surgery using octopus for coronary target stabilization, CVP was maintained constantly, but CO decreased immediately after target stabilization and reduced further during all coronary artery anastomoses, and most during OM anastomosis. Incidence of bradycardia was highest during LAD anastomosis. The use of inotropes was most frequent during OM anastomosis. Careful monitoring and management of hemodynamic variables are of utmost importance to avoid conversion to on-pump surgery.

\section{Topic: Coronary Heart Disease}

073

Off-pump coronary artery bypass grafting through left-sided thoracotomy in the treatment of patients with coronary artery disease

Yuri Schneider

Federal Center of High Medical Technology, Kaliningrad, Russian Federation

Journal of Cardiothoracic Surgery 2017, 12(Suppl 1):073 


\section{Objective}

To present our experience off-pump coronary artery bypass grafting (CABG) through the left-sided thoracotomy.

\section{Methods}

During the period from 09.2012 to 04.2017 , in our center treated 439 patients with coronary artery disease who underwent CABG from left thoracotomy: 308 men (70.2\%) and 131 women (29.8\%). Age $66.7 \pm$ 15.5 years; the number of diseased coronary arteries - 2.2. 19 patients had a history of CABG, in 12 cases using the left internal thoracic artery (LITA). EuroSCORE II 3,8 \pm 3 ,7. Comorbidities- COPD, asthma, atherosclerosis, peripheral vascular disease, diabetes, obesity, CKD. Angina functional class- II-III, CHF - I-III of NYHA, EF $36 \pm 12.7 \%$. All patients underwent off pump CABG of the left anterior descending (LAD): in 209 patients with isolated LAD lesion, the rest as a stage hybrid intervention. In hybrid interventions the second stage were stenting 1 or 2 coronary arteries. In 427 cases the LITA was used, in 12 carried autovenous subclavian artery bypass surgery (LITA used in the previous surgery).

Results

2 patients died in early postoperative period, the reason was acute cardiovascular failure. There were no repeated coronary artery bypass grafting. The average time spent in the hospital 5,8 days. Rethoracotomy was done in $7(1.6 \%)$ cases after postoperative bleeding (LITA bed). Infectious complications of the postoperative wound were observed in $3(0.7 \%)$ women with body mass index more 35 with insulin-dependent diabetes mellitus. In 4 cases was stenting circumflex artery in the early postoperative period due to acute ischemic changes. The mean intraoperative blood loss $-150.0 \mathrm{ml}$.

Conclusions

Off-pump coronary artery bypass grafting through the left-sided thoracotomy is a surgical method with less injury, short hospitalization time and fast recovery, which can be used as the isolated lesion of the left artery descending, and multivessel disease as a stage of the hybrid intervention.

\section{Topic: Coronary Heart Disease}

\section{4}

\section{The "ZAMVAR" pericardial fold}

Vipin Zamvar

Royal Infirmary of Edinburgh, Edinburgh, UK

Journal of Cardiothoracic Surgery 2017, 12(Suppl 1):074

\section{Objective}

This paper describes a pericardial fold which has not yet been mentioned in the Anatomy or Surgery literature. It has been named the "ZAMVAR" pericardial fold. The surgical significance is described. Methods

The pericardium is made of two layers, the outer fibrous, and the inner serous. The serous pericardium is made up of two layers, the visceral pericardium which forms the outer layer of the heart; and the parietal layer which lines the undersurface of the fibrous pericardium. The visceral pericardium is reflected from the heart and great vessels to become continuous with the parietal pericardium. This paper describes a fold of the pericardium over the two left-sided pulmonary veins. The visceral pericardium over the left atrium imperceptibly becomes the parietal pericardium over the pulmonary veins. From the transition zone, the parietal pericardium folds back upon itself for a distance of 1 to $3 \mathrm{~mm}$; then courses over the undersurface of the fibrous pericardium. This folding back forms the "ZAMVAR" pericardial fold. Longitudinally it runs from just below the inferior margin of the left inferior pulmonary vein to just above the superior margin of the left superior pulmonary vein.(not present on right).

Results

Surgical significance: The "ZAMVAR" pericardial fold offers a perfect landing zone for the deep pericardial stitch taken to lift the heart up in Off-Pump Coronary Surgery. The deep pericardial stitch is passed through the "ZAMVAR" pericardial fold, over the inferior pulmonary vein. Conclusions

Combined with the Trendelenburg position, and lifting up the pericardium with the stitch in the "ZAMVAR" pericardial fold, all areas of the heart are made accessible for off-pump coronary revacularisation, with minimal or no haemodynamic consequences. Passing the stitch through the "ZAMVAR" pericardial fold affords protection against inadvertently passing the needle through the wall or lumen of the inferior vein which can cause bleeding.

\section{Topic: Coronary Heart Disease}

075

The "Zamvar" deep pericardial retraction stitch

Vipin Zamvar, Charilaos Koutsogiannidis

Royal Infirmary of Edinburgh, Edinburgh, UK

Correspondence: Vipin Zamvar; Charilaos Koutsogiannidis

Journal of Cardiothoracic Surgery 2017, 12(Suppl 1):075

\section{Objective}

Obtaining a still arteriotomy site, in a hemodynamically stable patient facilitates the construction of a perfect anastomosis, during off-pump CABG. Under these conditions the surgeon can perform an unhurried and excellent anastomosis. Many technical manoeuvres are required to facilitate this. One of the important ones is to use a deep pericardial retraction stitch which facilitates lifting up of the heart, and access to the posterior and inferior surface of the heart.

Many different types of retraction stitches have been described, including the LIMA stitch, and the stitch between the left and right inferior pulmonary veins. While experimenting with different positions for the pericardial retraction stitch, the author serendipitously realised that a stitch taken across the pericardial fold over the inferior pulmonary vein resulted in excellent hemodynamic stability, as well as very good exposure of the inferior and lateral surfaces of the heart. For the last 15 years, the author has used this stitch in over a 1000 off-pump CABG cases. Methods

This video demonstrates the anatomy of the pericardial fold (described as the ZAMVAR pericardial fold; Journal of Cardiothoracic Surgery 2017 Supplement 1) and the procedure of taking the deep pericardial retraction Zamvar stitch. Also shown are the echocardiographic images from simultaneous TOE, and the monitor showing the vital parameters. Also shown are video images of the position of the heart when the LAD and Diagonal arteries at the front of the heart, OM arteries near the base of the heart, and the PDA are stabilised; along with simultaneous echocardiographic, and monitor display of vital parameters.

Results

The video demonstrates hemodynamic stability during access of the different parts of the heart.

Conclusions

The presence of the pericardial fold running from the inferior edge of the left inferior pulmonary vein to the superior edge of the left superior pulmonary vein (also described as the Zamvar Pericardial fold; Journal of Cardiothoracic Surgery 2017 Supplement 1) facilitates the placement of the Zamvar pericardial stitch, which in turns facilitates easy exposure of the lateral and inferior surface of the heart, with minimal or no hemodynamic alterations. This is a very easy manoeuvre, and is much simpler to use compared to the other existing retraction stitches.

\section{Topic: Coronary Heart Disease}

076

Optimization of coronary artery grafting strategies based on long-term morphological and morphometrical assessment of coronary conduits

Grigore Tinica', Raluca Ozana Chistol', Diana Anghel', Mihail Enache', Cristina Furnica ${ }^{2}$

${ }^{1}$ Cardiovascular Diseases Institute, Iasi, Romania; ${ }^{2}$ University of Medicine and Pharmacy, lasi, Romania

Correspondence: Grigore Tinica

Journal of Cardiothoracic Surgery 2017, 12(Suppl 1):076

\section{Objective}

Completeness of revascularization is a factor conditioning intermediate and late survival. The choice of coronary conduits has 
been debated for decades with no consensus concerning the optimal grafting design in terms of graft type and surgical technique. The aim of the current study is to analyse our choice of coronary conduits and grafting strategy based on long-term follow-up data.

Methods

A single centre retrospective follow-up cohort study performed on 127 patients who have benefited from coronary artery bypass grafting (CABG) between 2000-2006 and those grafts were evaluated by coronary computed tomography angiography (CCTA) between 20132916 at $129.78 \pm 36.64$ months after CABG.

Results

There were analysed 399 grafts (mean $3.14 \pm 1 /$ patient) - 179 venous (SVG) and 200 arterial. For the right coronary territory, the highest patency rate was obtained with radial artery grafts (RAG) $-80.65 \%$, for circumflex territory with SVG (82.54\%) and for diagonal arteries with right internal thoracic artery $-92.86 \%$. Left internal thoracic artery (LITA) was anastomosed to the left anterior descending artery with a patency rate of $90.17 \%$ and unexplained occlusion (no competitive flow) only in $4.1 \%$. Identified risk factors for SVG occlusion were grafting to RCA, target vessel caliber $<1.5 \mathrm{~mm}$, female sex, family history and coronary endarterectomy. For arterial grafts, multisite artery disease, target vessel stenosis $<90 \%$, target vessel caliber $<1.5 \mathrm{~mm}, \mathrm{Y}$ anastomosis angle $>56^{\circ}$, and distal anastomosis angle $>60^{\circ}$ augmented occlusion risk. RAG registered a lower patency rate when anastomosed " $Y$ " to LITA (61.90\%) compared to the ascending aorta (85\%). SVG had a lower occlusion rate in sequential anastomosis compared to arterial grafts $(22.22 \%$ for endto-side anastomosis and 0\% for side-to-side versus 35\% for end-toside and $45 \%$ for side-to-side).

\section{Conclusions}

An optimal grafting design with favourable long-term patency can be imagined for each coronary territory based on morphological and morphometrical analysis of follow-up CCTA.

\section{Topic: Coronary Heart Disease}

\section{7}

Experience of using the "no-touch" technique harvesting a saphenous vein with surrounding tissue using an ultrasound harmonic scalpel

Aleksei Semagin ${ }^{1}$, Pavel Kuznetcov ${ }^{1}$, Evgeniy Ignatov ${ }^{1}$, Andrey Semagin ${ }^{2}$, Oleg Lukin ${ }^{1}$

${ }^{1}$ Federal State Institution, Chelyabinsk, Russian Federation; ${ }^{2}$ Regional

Clinical Cardiological Health Center, Samara, Russian Federation

Correspondence: Aleksei Semagin

Journal of Cardiothoracic Surgery 2017, 12(Suppl 1):077

\section{Background}

An angiographic evaluation of the patency rates of vein grafts harvesting using the "no-touch" technique with the use of an ultrasound harmonic scalpel in the short- and mid-term postoperative periods.

Methods

Coronarography was performed in patients with the coronary artery bypass surgery between 2006 and 2016. Angiographic examination was performed in 27 patients 12 months after the operation, in 50 patients at 36 months and in 16 patients after 60 months. A peculiarity of the group of patients who underwent coronary angiography after 1 year was that in 10 patients, LAD was cured by a vein graft, and 17 patients had intersystemic sequential grafting between the left and right coronary artery system.

Results

When coronary angiography was performed 1 year after surgery, all vein grafts were patency $(n=33)$, no anastomosis defects were detected, both the side-by-side anastomoses zone and the end-toside zone. In the period from 18 to 36 months, 95 functioning vein grafts were detected from 104 (91.35\%); within the period from 36 to 60 months, 23 functioning vein grafts out of 27 (85.19\%) were detected. During the entire follow-up period, the functional activity of vein grafts was $92.07 \%$ (151 of 164).

\section{Conclusions}

The results of the research showed that the application of the "notouch" technique provides excellent patency of vein grafts as soon as inter-system sequential grafting of RCA and LCA systems and bypassing LAD of LCA, and when used as linear grafts in the medium-long terms.

\section{Topic: Coronary Heart Disease}

078

Assessment of work capacity state of patients with ischaemic heart disease after CABG

Nurali Shakirov, Anar Tursynbekova, Yerkebulan Magauin,

Ainash Issayeva, Almaz Kiyabayev, Zeinat Ismailova

Sema Hospital, Almaty, Kazakhstan

Correspondence: Nurali Shakirov

Journal of Cardiothoracic Surgery 2017, 12(Suppl 1):078

\section{Objective}

Studying of the work capacity state in patients with IHD after CABG. Methods

The data of clinical, physical and instrumental examinations of 130 patients after coronary artery bypass grafting were analyzed for the period of 2016-2017 in Sema Hospital, 30 of them are observed in a line of dynamic case follow-up.

Men aged 30 to 62 years were examined. The overwhelming majority made up the persons aged 30 to 59 years. Patients distribution was as follows according to the work nature: physical work had performed 41 people, intellectual work - 42 and 17 had the driver's professions.

Results

The case follow-up results are evidenced about undoubted CABG efficiency, in 26 of 30 patients the clinical and functional stabilization was noted (absence of negative dynamics of clinical and ECG signs). It was established during the remote study of cardiovascular system clinical-functional state in patients after CABG that all the examined patients have ECG changes, the expressed changes are observed in $76 \%$ of patients, the scar changes in $61 \%$ of patients; violations of contractile myocardium function were noted in $47 \%$ of patients according to EchoCG, changes in the neuropsychic settings are fixed in all patients (100\%). Case follow-up showed a stabilization the clinicfunctional disorders (absence of negative dynamics of clinical and ECG signs) in $86 \%$ of patients after CABG.

\section{Conclusions}

1. The following factors affect the state of working ability of patients after CABG: the anamnesis duration, the periods after surgery, the initial myocardium condition, the completeness of myocardial revascularization, the personal features of this contingent, and the possibilities for rational employment.2. 38\% of patients used to begin work in the period from 6 months to 5 years after CABG when performing a full medical and social rehabilitation before and after surgery with the obligatory participation of a psychologist and psychotherapist.

\section{Topic: Oesophageal}

079

Esophageal cancer: surgery and precise prognosis

Oleg Kshivets

Clinic N1, Khimki, Russian Federation

Journal of Cardiothoracic Surgery 2017, 12(Suppl 1):079

\section{Objective}

We examined factors in terms of precise prognosis of 5-year survival (5YS) of esophageal cancer (EC) patients (ECP) (T1-4NO-2M0) after complete (R0) esophagogastrectomies (EG).

Methods

We analyzed data of 515 consecutive ECP (age $=56.3 \pm 8.9$ years; tumor size $=6.2 \pm 3.4 \mathrm{~cm}$ ) radically operated and monitored in 1975- 
2017 ( $m=380, f=135$; esophagogastrectomies (EG) Garlock=280, EG Lewis $=235$, combined EG with resection of pancreas, liver, diaphragm, aorta, VCS, colon transversum, lung, trachea, pericardium, splenectomy $=151$; adenocarcinoma $=292$, squamous $=213$, $\mathrm{mix}=10$; $\mathrm{T} 1=101, \mathrm{~T} 2=114, \mathrm{~T} 3=175, \mathrm{~T} 4=125 ; \mathrm{N} 0=248, \mathrm{~N} 1=69, \mathrm{~N} 2=198 ; \mathrm{G} 1=148$, $\mathrm{G} 2=125, \mathrm{G} 3=242$; early $\mathrm{EC}=82$, invasive $=433$; only surgery $=394$, adjuvant chemoimmunoradiotherapy-AT=121: 5 -FU+thymalin/taktivin +radiotherapy 45-50Gy). Multivariate Cox modeling, clustering, SEPATH, Monte Carlo, bootstrap and neural networks computing were used to determine any significant dependence.

Results

Overall life span (LS) was $1814.5 \pm 2225.6$ days and cumulative 5YS reached $48.8 \%$, 10 years $-42.3 \%, 20$ years $-31.7 \%$. 160 ECP lived more than 5 years $(\mathrm{LS}=4384.1 \pm 2474.1$ days), $89 \mathrm{ECP}$ - more than 10 years (LS=5913.1 \pm 2360.3 days). $224 \mathrm{ECP}$ died because of EC ( $(\mathrm{S}=629.2 \pm 320.1$ days). AT significantly improved $5 Y S(67.6 \%$ vs. $44.6 \%)(P=0.00008$ by log-rank test). Cox modeling displayed that $5 Y S$ of ECP significantly depended on: phase transition (PT) N0-N12 in terms of synergetics, cell ratio factors (ratio between cancer cellsCC and blood cells subpopulations), T, G, age, AT, localization, blood cells, prothrombin index, coagulation time, residual nitrogen, $\mathrm{RH}$ $(\mathrm{P}=0.000-0.026)$. Neural networks, genetic algorithm selection and bootstrap simulation revealed relationships between 5YS and healthy cells/CC (rank=1), PT early-invasive $\mathrm{EC}$, thrombocytes/CC, PT N0-N12, erythrocytes/CC, stick neutrophils/CC, segmented neutrophils/CC, lymphocytes/CC, leucocytes/CC, eosinophils/CC, monocytes/ CC. Correct prediction of 5 YS was $100 \%$ by neural networks computing (area ander ROC curve=1.0; error $=0.0$ ).

Conclusions

$5 Y S$ of ECP after radical procedures significantly depended on: 1) PT "early-invasive cancer"; 2) PT N0-N12; 3) Cell Ratio Factors; 4) blood cell circuit; 5) biochemical factors; 6) hemostasis system; 7) AT; 8) EC characteristics; 9) tumor localization; 10) anthropometric data.

\section{Topic: Oesophageal}

080

- Comparing hand-sewn and mechanical anastomosis in esophageal cancer patients undergo esophagectomy

Ali Ramouz, Seyed Ziaeddin Rasihashemi

Tabriz University of Medical Sciences, Tabriz, Iran

Correspondence: Ali Ramouz

Journal of Cardiothoracic Surgery 2017, 12(Suppl 1):080

\section{Objectives}

Despite current achievements, there are a controversy over the efficacy of mechanical stapler compared to hand-sewn technique in esophagogastric anastomosis in terms of morbidity and efficacy. Due to the discrepancies, the purpose of the present study is to compare the clinical outcomes of manual and mechanical anastomoses during esophagectomy for esophageal cancer.

Methods

A retrospective cohort study was conducted on 409 patients, candidate for esophagectomy for esophageal cancer during 2008 and 2013 at Tabriz University of Medical Sciences, Iran. Of these, 259 (63.3\%) patients were operated using manual anastomosis (handsewn technique) and 150 patients (36.7\%) were operated by mechanical anastomosis (stapled technique). All patients visited in the $2^{\text {nd }}$ week, $4^{\text {th }}$ month, $8^{\text {th }}$ month and $12^{\text {th }}$ month after operation and evaluated considering, post-operative complications including reflux, anastomotic stricture, dysphagia and anastomotic leakage.

Results

The mean operation time in the mechanical anastomosis group and manual group were 211.45 \pm 82.25 and $251.42 \pm 52.81 \mathrm{~min}$, respectively ( $P=0.023)$. Postoperatively, 38 (14.67\%) anastomotic leakage detected in the manual group compared to $8(5.3 \%)$ in the mechanical group $(P=0.002)$. In addition, comparing postoperative complications: reflux, anastomotic stricture, and need for esophageal dilatation were significantly less prevalent in the patients of the manual anastomosis group, respectively. $(P=0.021, P=0.029$, $\mathrm{P}=0.021$ ).

\section{Conclusions}

Using mechanical anastomosis not only reduces operation time, but also leads to lower anastomotic leakage and postoperative complications including: reflux, anastomotic stricture and need for esophageal dilatation.

Keywords

mechanical, esophagogastric anastomoses, esophageal cancer, handsewn, stapled

\section{1 - Independent VATS transdiaphragmatic thoracoabdominal approach for resection and reconstruction of lower esophageal cancer}

Hsing-Hsien

Wu

Tainan Municipal Hospital, Tainan, Taiwan

Journal of Cardiothoracic Surgery 2017, 12(Suppl 1):Hsing-Hsien

\section{Withdrawn}

082 - A battle against postoperative complication of esophagectomy

Yuequan

Jiang, Sr

Chongqing Cancer Institute, Chongqing, China

Journal of Cardiothoracic Surgery 2017, 12(Suppl 1):Yuequan

\section{Objectives}

Cervical anastomosis following esophagectomy is associated with a high incidence of postoperative complications. Predominant postoperative complications were anastomotic leakage, fibrous stricture, gastroesophageal reflux, pnuemonia and delayed gastric emptying time. We invented a series of new technique to prevent these complications.

Methods

These new techniques invented by ourselves include: 1 . Super narrow gastric tube with width smaller than $3 \mathrm{~cm}$. 2. A New technique for cervical gastroesophageal anastomosis. This new anastomotic technique was developed from a type of side-to-side anastomosis technique reported by Collard et al. The different aspect is that the stump of the anterior anastomotic part was pushed into the tubular stomach. This embedded part function as one way valve can prevent acid reflux.

Results

From November 2014 to March 2017, there were 278 patients underwent MIE with the series of new techniques. 5 patients lost follow-up. Only 2 patients died of postoperative pulmonary complications (0.73\%). 3 patients suffered anastomotic leakage (1.09\%). There were 32 patients had dysphagia symptoms, but radiograph showed that 2 patient had anastomotic stricture. No patients need balloon dilation. 27 patients had symptoms of acid regurgitation during supine time. 1 patient had gastric emptying time delay.

Conclusions

These series new modified techniques had significant reduce postoperative incidence of minimal invasive esophagectomy. It is an 
important breakthrough in esophagectomy and let the esophageal cancer surgery become a safe procedure.

Key words

esophageal cancer, MIE, Gastroesophageal Anastomosis, complications

\section{Topic: Mediastinal}

\section{3}

Thymectomy for nonthymomatous myasthenia gravis: comparison of video-assisted thoracoscopic and trans sternal thymectomy

Reza Bagheri, Reza Boostani, Ali Sadrizadeh, Maryam Salehi, Ali Rahnama, Vahab Azmounfar, Saeed Hakimian, Mohammad Baradaran Firoozabadi

Mashhad university of medical sciences, Mashhad, Iran

Correspondence: Reza Bagheri

Journal of Cardiothoracic Surgery 2017, 12(Suppl 1):083

\section{Background}

Thymectomy considered being as a standard procedure in treatment of all the steps of myasthenia gravis. VATS thymectomy is one of the minimally invasive procedure that now because of the short duration of hospitalization, reduced postoperative pain \& scarring after surgery have been replaced the traditional methods of surgery for patients with myasthenia gravis, but there are still differences \& concerns. The aim of this study is to compare outcomes of two different techniques of surgery, VATS vs. TS in the treatment of myasthenia gravis.

\section{Methods}

42 patients with myasthenia gravis \& lacks a thymus tumor were evaluated as pilot \& based on MGFA-classification \& drug consumption. Then, they randomly underwent two different techniques of surgery: VATS Vs. TS. Patients were evaluated based on preoperative \& postoperative variables.

Results

Length of stay in ICU \& hospitalization have been reduced in patients who underwent VATS thymectomy technique. Also duration of surgical procedure has been reduced significantly in these patients. These patients have less blood loss during surgery compared with TS group. The MGFA-postoperative status in VATS thymectomy revealed that the number of persons with complete stable remission was higher \& number of persons remained unchanged was lower.

\section{Conclusion}

VATS thymectomy is a safe $\&$ appropriate approach versus traditional methods such as TS thymectomy for patients with myasthenia gravis. This method has better results after surgery \& can be used as a minimally invasive alternative method instead of TS thymectomy. Keywords

VATS, Transsternal, Thymectomy, Myasthenia gravis

\section{Topic: Mediastinal}

\section{4}

Trachea sleeve resection after preliminary long-lasting stenting Dauren Amangeldiyev, Vladimir Parshin, Ovik Mirzoyan, Valeryi Parshin I.M. Sechenov First Moscow State Medical University, Moscow, Russian Federation

Correspondence: Dauren Amangeldiyev

Journal of Cardiothoracic Surgery 2017, 12(Suppl 1):084

\section{Objective}

Currently, trachea cicatrical stenosis (TCS) has iatrogenic etiology and appears after execution of artificial respirator. Variability of clinical status, degree and length of stenosis, as well as functional changes requires specification of indications to one-stage operations. From the one hand, pre-stenting of trachea permits to save a patient and prolong trachea sleeve resection (TSR) with anastomosis, to rehabilitate a resuscitation patient, but from the other hand, it is an adverse factor. Nowadays, treatment method of such patients is not defined. Aim of study: To improve the results of curative therapy of TCS patients after long-lasting stenting.

Methods

To study results of after-serial and one-stage operations in trachea stenosis, and on its basis to clarify indications to trachea sleeve resection.

Results

In Russian Scientific Surgery Center n.a. B.V. Petrovsky and First Moscow State Medical University n.a. Sechenov from 2000 to 2015 there were treated 831 of TCS patients. Trachea sleeve resection was executed for 330 patients (39.71\%), including 61 - after long-lasting stenting of trachea. Post-tracheostomic stenosis had in $n=51(83,6 \%)$, postintubation - in $n=9(14,7 \%)$ patients, in $n=1(1,6 \%)$ - postambustial. The reason for artificial respirator execution in $n=32$ $(52,4 \%)$ was a craniocerebral trauma. Previously, there was administered treatment concerning cicatrical stenosis of trachea for $n=43$ $(70,4 \%)$ patients. $\mathrm{N}=50(81,9 \%)$ patients had no complications after surgery. In $n=3(4,9 \%)$ cases there was found stomatitis, $n=2(3,2 \%)$ surgical sepsis, conservatively permitted. In $n=1(1,6 \%)$ patients thrombembolia of small pulmonary arteries, which required delivery of thrombolytic therapy, was developed. Death, severe complications, which required reoperation, were not found.

Conclusions

Trachea sleeve resection after preliminary stenting is possible and plenty safety.

\section{Topic: Mediastinal}

\section{5}

VATS mediastinal sentinel node biopsy reduces the need for systematic mediastinal lymphadenectomy in early stage NSCLC surgery

Nenad Ilic ${ }^{1}$, Josko Juricic ${ }^{1}$, Dragan Krnic ${ }^{1}$, Duje Orsulic ${ }^{1}$, Ivan Simundza ${ }^{1}$ Darko $\mathrm{llic}^{1}$, Nives Frleta $\mathrm{lic}^{2}$

${ }^{1}$ University Surgical Hospital, Split, Croatia; ${ }^{2}$ Policlinic Cito, Split, Croatia

Correspondence: Nenad llic

Journal of Cardiothoracic Surgery 2017, 12(Suppl 1):085

\section{Objective}

Systematic mediastinal lymphadenectomy is still essential for an adequate postoperative staging of NSCLC. We tried to investigate the controversial role of VATS sentinel node biopsy (SNB) in early stage non-small cell lung cancer (NSCLC) surgery.

Methods

A total of 72 patients with clinical T1NOMO NSCLC underwent VATS SN navigation lobectomy using Tc-99 labeled tin colloid followed by systematic mediastinal lymphadenectomy (SML) in three year time period (2012-2015). Mapping of the mediastinal lymph nodes by their number and station followed by histopathological evaluation was performed. Patients' data were statistically analyzed.

\section{Results}

Intraoperative SN was identified in $69(87 \%)$ of these patients with $92 \%$ of accuracy. We found lobe specific skip nodal metastases in 7 (10\%) patients resulting in upstaging. The incidence of ML metastases seemed to be more often in adenocarcinoma patients $(p<0.05)$, but skip nodal metastases showed higher rate in squamous cell carcinoma patients. Intraoperative frozen section was not confirmed accurate for detecting micrometastases in three (4\%) patients. Operative time was prolonged for 10 (8-25) minutes showing no difference in complication rate.

Conclusions

Procedure showed absolute safety and high accuracy. Our results indicated that VATS SN identification could reduce mediastinal lymph node dissection in early stage NSCLC. Further clinical studies should be carried out in order to prove that minimally invasive surgical procedures could be curative for T1NOMO NSCLC. 


\section{Topic: Mediastinal}

\section{6}

Effect of diaphragm plication on phrenic complication of nerve excision in thymoma patients with and without myasthenia gravis Reza Bagheri, Seyed ziaollah Haghi, Reza Afghani, Vahab Azmounfar, Saeed Hakimian, Mohammad Baradaran Firoozabadi, Negar Morovatdar Mashhad University of Medical Sciences, Mashhad, Iran

Correspondence: Reza Bagheri

Journal of Cardiothoracic Surgery 2017, 12(Suppl 1):086

\section{Background}

Thymoma is the most common tumor of the anterior mediastinum that the most effective treatment for it is complete removal of the thymus. In patients with advanced stage, phrenic nerve involvement can be seen that suggested treatment for these patients is unilateral phrenic excision \& diaphragm plication. However in patients with myasthenia gravis, there are concerns in relation to this method of treatment. The aim of this study is to evaluate the effects of plication of the diaphragm on complications of phrenic nerve excision in thymoma patients with \& without myasthenia gravis involving the phrenic nerve.

Methods

A retrospective cohort study was performed on 26 patients with thymoma that half of the patients had myasthenia gravis \& the other half where without myasthenia gravis. We performed diaphragm plication in 7 patients in each group with excision of phrenic nerve. Patients were evaluated based on preoperative \& postoperative variables.

Results

The patients' age $(P=0.943)$, sex $(P=0.999)$ blood loss during surgery $(P=0.919)$, need for transfusion during surgery $(P=0.999)$, short term complications $(P=0.186)$, need for tracheostomy $(P=0.27) \&$ mortality $(P=0.09)$ differences were not significant. But the average duration of ICU stay $(P=0.001)$ \& intubation in ICU $(P=0.001)$ in patients who had myasthenia gravis was more than patients without myasthenia gravis. However, these values were less in patients with myasthenia gravis \& diaphragm plication than patients with myasthenia gravis \& no diaphragm plication.

\section{Conclusions}

Excision of the phrenic nerve in patients with myasthenia gravis associated with thymoma \& phrenic nerve involvement is appropriate. Complication of this manner is increasing in ICU stay \& duration of intubation in ICU can be reduced by plication of diaphragm

Key words

Plication, Diaphragm, Phrenic nerve, Thymoma, Myasthenia gravis

\section{Topic: Minimally Invasive Cardiac}

\section{7}

Minimally invasive CABG with BITAS - a chronological review

Pradeep Nambiar', Sanjay Kumar ${ }^{2}$

${ }^{1}$ Moolchand Hospital, Gurgaron, India; ${ }^{2}$ Benares Hindu University,

Benares, India

Correspondence: Pradeep Nambiar

Journal of Cardiothoracic Surgery 2017, 12(Suppl 1):087

\section{Background}

Minimal access total arterial multivessel CABG using BITAs is being increasingly adopted and has demonstrated the myriad advantages of not only internal thoracic arteries but also the minimally invasive approach. In this approach harvesting of bilateral internal thoracic arteries (BITAS) are carried out under direct vision through a left mini-thoracotomy and the harvested BITAS are used for multi vessel revascularization as a LITA - RITA Y composite conduit. The aim was to review the chronological adoption and outcome of this novel technique

Methods

From August 2011 to December 2012, (Phase 1) 150 patients and from Jan 2013 to August 2016 (Phase 2) 669 patients underwent offpump minimally invasive multivessel CABG using BITAs, through a 2in left mini-thoracotomy incision.Both internal thoracic arteries were harvested under direct vision and complete revascularization was done using the LITA-RITAY composite conduit, followed by flow study of the grafts.

Results

150 patients had minimally invasive CABG in Phase 1 and 669 in phase 2. The mean number of grafts was 2.8. in phase 1 and 3.4 in phase 2 resulting in an overall mean of 3.1. EF was $40.5+/-5.2$. The mean hospital stay was 3.1 days in both phases. There was 1 mortality (0.6\%) and $5(0.7 \%)$ in phases 1 and $2.1(0.6 \%)$ patient and $3(0.4 \%)$ had an elective conversion to sternotomy in the respective phases. $4(0.5 \%)$ patients required reintervention angioplasty in phase 2.

Conclusions

Minimally invasive multivessel total arterial revascularization was done using the LITA - RITA Y composite conduit through a left minthoracotomy. The outcomes have been good in both phases and with a very low re-intervention rate. We feel, that this technique is a safe and reproducible option in coronary artery bypass grafting.

\section{Topic: Minimally Invasive Cardiac}

\section{8}

Transapical off-pump mitral valve repair with implantation of artificial chordae: single centre midterm results (case series of 78 patients)

Arturas Lipnevicius, Vilius Janusauskas, Agne Drasutiene, Viktorija Bleizgyte, Diana Zakarkaite, Rita Kramena, Sigita Aidietiene, Kestutis

Rucinskas, Audrius Aidietis

Vilnius University, Vilnius, Lithuania

Correspondence: Arturas Lipnevicius

Journal of Cardiothoracic Surgery 2017, 12(Suppl 1):088

\section{Objectives}

Transapical off-pump implantation of artificial mitral valve chordae using NeoChord DS1000 device is an alternative approach to treat severe mitral regurgitation. This study presents mid-term results after this procedure in a single center patients' cohort.

\section{Methodology}

From 2011 to 201678 patients underwent transapical mitral valve repair in our center. Patients were stratified into 4 anatomical types of mitral pathology: type A (15 patients) - isolated P2 prolapse, type B (43 patients) - P2 and adjacent segments disease, type C (16 patients) - single or bileaflet prolapse with pericommissural segments being involved, type D (4 patients) - isolated A2 prolapse. The mitral valve regurgitation more than $2+$ was considered to be a failure.

Results

Mean age of our patients was $59.5 \pm 12.8$ years, $67.9 \%$ - male. Preoperative median risk values according to STS score and EuroSCORE II were $0.47 \%(0.24 \%-0.74 \%)$ and $0.83 \%(0.67 \%-1.35 \%)$ respectively. Median duration of the surgery was 120min (110-146), postoperative ventilation - $4 \mathrm{~h}$ (2.5-5), length of ICU stay was $22 \mathrm{~h}$ (20-24), while postoperative hospital stay was 8 days (7-9). Median postoperative blood loss was 200ml (150-300), blood products were used in $6(7.7 \%)$ patients. Three (3.9\%) patients underwent re-exploration for bleeding. There were no stroke or wound infection. Transitory acute renal failure occurred in two cases. There was one early postoperative death. New atrial fibrillation was observed in $11.7 \%$ of the patients and $2.6 \%$ needed permanent pacemaker insertion. Mean follow-up time was $34 \pm 19$ 
months. Kaplan-Meier method was used to calculate estimated freedom from MR $>2+$ : it was $93 \%, 75 \%, 28 \%$ and $50 \%$ for types A, B, C and D respectively at 5 years' follow-up.

\section{Conclusion}

Transapical off-pump chordae implantation is feasible and safe procedure. Procedure is beneficial for type A and B groups of patients only, therefore patients should be carefully selected according to the mitral pathology.

\section{Topic: Minimally Invasive Cardiac}

\section{9}

Early and mid-term results of randomised trial MICSREVS - direct, indirect and intangible costs

Aliaksandr A. Ziankou', Mikalaj Laiko', Kyril Vykhrystsenka',

Vjacheslau Chuyashou', Aliaksandr Zhyhalkovich², Yuri Ostrovsky ${ }^{2}$

'Vitebsk Regional clinical hospital, Vitebsk State Medical University,

Vitebsk, Belarus; ${ }^{2}$ Republic Research-Practical Center "Cardiology", Minsk, Belarus

Correspondence: Aliaksandr A. Ziankou

Journal of Cardiothoracic Surgery 2017, 12(Suppl 1):089

\section{Objective}

We evaluated hospital and mid-term results of the prospective randomized controlled trial MICSREVS - Minimally Invasive Cardiac Surgery Revascularization Strategy (http://www.clinicaltrials.gov/show/NCT02047266) from the point of view of direct, indirect and intangible costs.

\section{Methods}

In accordance with the trial design, 150 patients were included divided into 3 groups of 50 people. In group I, the multivessel small thoracotomy coronary artery bypass grafting (MVST-CABG) strategy was directed to perform arterial revascularisation via a left minithoracotomy on the beating heart, using the aortic no-touch technique. In control groups II - off-pump (OPCABG) and III - onpump coronary artery bypass grafting (ONCABG), conventional surgery was performed via median sternotomy.

Results

MVST-CABG was associated with less perioperative blood loss, lower number of blood transfusions, shorter hospital length of stay, compared with other groups; less postoperative ventilation time and intensive care unit stay versus ONCAB group (p\&lt;0.05); fewer deep wound infections versus OPCABG patients (p\&lt;0.1). In treatment groups, the average direct cost (hospital) per patient were $\$ 2.732$, $\$ 3.454$ and $\$ 4.808$, respectively. Patients who visited a rehabilitation center stayed an average of $12.8 \pm 4.5$ days and incurred an average cost of $\$ 186.8$ per patient. The mean follow-up duration constituted $24.7 \pm 8.2$ months. Cumulative midterm survival and freedom from MACCE did not differ significantly between treatment groups (p\&gt;0.05). During the 1-year period after surgery an average of 87 $\pm 31,98 \pm 36$ and $117 \pm 47$ workdays per patient were lost, respectively. MVST-CABG patients demonstrated shorter time to return to full physical activity and greater improvement in 30-days physical health component (SF-36) of quality of life.

Conclusions

Application of the MVST-CABG leads to the reduction of the direct, indirect and intangible costs during the hospital period and after hospital discharge.

Table 1 (abstract 089). Operative Characteristics and Postoperative Results

\begin{tabular}{|c|c|c|c|c|c|}
\hline Characteristic & $\begin{array}{l}\text { MICS-CABG } \\
(n=50)\end{array}$ & $\begin{array}{l}\text { OPCABG } \\
(n=50)\end{array}$ & $\begin{array}{l}\text { ONCABG } \\
(n=50)\end{array}$ & $\begin{array}{l}\text { MVST- } \\
\text { CABG VS } \\
\text { OPCABG }\end{array}$ & $\begin{array}{l}\text { MVST- } \\
\text { CABG VS } \\
\text { ONCABG }\end{array}$ \\
\hline $\begin{array}{l}\text { Intraoperative blood } \\
\text { loss (ml) }\end{array}$ & $\begin{array}{l}250(162.5 \\
300)\end{array}$ & $\begin{array}{l}475(350 ; \\
587.5)\end{array}$ & $\begin{array}{l}400(300 ; \\
500)\end{array}$ & $\& \mid t ; 0.001$ & $\& \mid t ; 0.001$ \\
\hline \multirow{2}{*}{$\begin{array}{l}\text { First twenty-four hours } \\
\text { postoperative blood } \\
\text { loss (ml) }\end{array}$} & $\begin{array}{l}450(252.5 \\
587.5)\end{array}$ & $\begin{array}{l}575(450 ; \\
800)\end{array}$ & $\begin{array}{l}500(400 ; \\
800)\end{array}$ & 0.002 & 0.007 \\
\hline & $9(18.0)$ & $20(40.0)$ & $33(66.0)$ & 0.015 & $\& \mid t ; 0.001$ \\
\hline
\end{tabular}

Table 1 (abstract 089). Operative Characteristics and Postoperative Results (Continued)

\begin{tabular}{|c|c|c|c|c|c|}
\hline \multicolumn{6}{|l|}{$\begin{array}{l}\text { Transfusion of blood } \\
\text { and/or derivatives, n } \\
\text { (\%) }\end{array}$} \\
\hline $\begin{array}{l}\text { Postoperative } \\
\text { ventilation time (h) }\end{array}$ & $3.5(2.5 ; 5.0)$ & $\begin{array}{l}4.3(2.1 ; \\
6.0)\end{array}$ & $\begin{array}{l}5.3(3.5 ; \\
7.9)\end{array}$ & 0.399 & 0.007 \\
\hline $\begin{array}{l}\text { Intensive care unit stay } \\
\text { (h) }\end{array}$ & $\begin{array}{l}18(16.0 ; \\
20.8)\end{array}$ & $\begin{array}{l}\text { 18 (17.0; } \\
27.8)\end{array}$ & $\begin{array}{l}19.3 \\
(16.1 ; \\
43.6)\end{array}$ & 0.315 & 0.042 \\
\hline $\begin{array}{l}\text { Deep wound infection, } \\
\text { n (\%) }\end{array}$ & - & $3(6.0)$ & - & 0.079 & - \\
\hline $\begin{array}{l}\text { Postoperative length of } \\
\text { stay (d) }\end{array}$ & $12.0(9.3 ; 14.0)$ & $\begin{array}{l}14.0 \\
(13.0 ; \\
17.0)\end{array}$ & $\begin{array}{l}14.0 \\
(12.0 ; \\
17.8)\end{array}$ & $\& \mid t ; 0.001$ & 0.004 \\
\hline $\begin{array}{l}\text { Time to return to full } \\
\text { physical activity (d) }\end{array}$ & $14(7 ; 21)$ & $\begin{array}{l}56(42 ; \\
77)\end{array}$ & $\begin{array}{l}56(44 ; \\
79)\end{array}$ & $\& \mid t ; 0.001$ & $\& \mid t ; 0.001$ \\
\hline $\begin{array}{l}\text { Physical health } \\
\text { component SF-36 } \\
\text { Health Status Survey } \\
\text { quality of life }\end{array}$ & $\begin{array}{l}50.9(45,3 ; \\
52,8)\end{array}$ & $\begin{array}{l}47.3 \\
(44.9 ; \\
50.2)\end{array}$ & $\begin{array}{l}48.3 \\
(45.4 ; \\
50.5)\end{array}$ & 0.026 & 0.079 \\
\hline
\end{tabular}

Topic: Minimally Invasive Cardiac

090

Right anterior thoracotomy for aortic valve surgery: evolution of myocardial protection strategies

Arthur Martella, III, Atiq Rehman

Our Lady of Lourdes Medical Center, Camden, PA, USA

Correspondence: Arthur Martella, III

Journal of Cardiothoracic Surgery 2017, 12(Suppl 1):090

\section{Background}

Due to the growth of transcatheter techniques, patients needing aortic valve surgery(AVR) are more likely to have aortic insufficiency or require associated procedures. Interest in right anterior thoracotomy (RAT) AVR continues to grow. Improved exposure techniques and perfusion and myocardial protection strategies offer opportunity to manage more complex problems with this approach. Methods

We retrospectively reviewed all patients undergoing RAT AVR through a $4-8 \mathrm{~cm}$ skin incision at a single institution. From January 2014 to January 2017, 194 patients with aortic valve disease underwent RAT AVR. During the same period 255 patients underwent TAVR. Mean age was $67.4 \pm 10.0$ years, and $112(57.7 \%)$ were male.

Results

All patients received a bioprosthesis. Eight of the last 16 patients utilized minimal suture valves. Combined procedures included: 12 robotic LIMA to LAD, 7 SVG to RCA, 6 PVI, 8 LAA ligations, 3 MVRs, 4 TVRs. One patient required reoperation for bleeding. CBT was 84 min, and ACC was 73 min. In-hospital mortality was $1.5 \%$ (3/194) with no conversions to sternotomy. 38 patients had primary Al. Perfusion strategy: the first 24 patients utilized a groin incision for arterial and venous access. All further cases utilized percutaneous vacuumassisted venous drainage and central arterial cannulation was adopted in the subsequent 170 patients. Supplemental venous drainage was utilized with a floppy suction in 10 pts. Histadinetryptophan-ketoglutarate (HTK) was utilized in 94 percent of cases and Del Nido in the remaining. TAVR CT scans were utilized to determine the location of the incision.

Conclusions

Evolution of perfusion, myocardial protection and exposure techniques have allowed for safer management of more complex aortic disease. Management of more complex cardiac disease and patients with aortic insufficiency requires a patient-specific team strategy for exposure, perfusion access and myocardial protection. 


\section{Topic: Minimally Invasive Cardiac}

\section{1}

Aortic valve replacement through a right mini-thoracotomy: single center experience

Mauro Del Giglio, Elisa Mikus, Marco Panzavolta, Marco Paris, Diego Magnano, Simone Calvi

Maria Cecilia Hospital, Cotignola (RA), Italy

Correspondence: Elisa Mikus

Journal of Cardiothoracic Surgery 2017, 12(Suppl 1):091

\section{Objective}

Aortic valve replacement through a right mini-thoracotomy is a minimally invasive procedure developed during the last years. It is not frequently used because of a tiny operating field, limiting surgeon's view resulting in longer cardiopulmonary bypass and cross clamping times compared to the standard full sternotomy.

Methods

We retrospectively reviewed 488 patients (273 male $55.9 \%$ with median age of 75 ; range $16-93$ years) who received an aortic valve replacement between January 2010 and February 2017 through a right mini-thoracotomy In the first 45 patients the aortic cannulation and clamping were performed through the right minithoracotomy while a vacuum assisted venous drainage was obtained percutaneously through the groin. A total central arterial and venous cannulation was adopted in the last 443 patients. All patients received an aortic valve replacement with a pericardial bioprosthesis sutured using three 2-0 Prolene running sutures. Mean prosthesis size was $24.4 \mathrm{~mm}$

Results

Aortic replacement was performed through a 4 to $6 \mathrm{~cm}$ skin incision at the third intercostal space. One patient was a REDO case. Overall median cardiopulmonary bypass and aortic cross clamping time was respectively 54 minutes (range 25-121) and 42 minutes (range 16-134). Median ventilation time and intensive care stay were 7 and 44 hours. Patients transfused were $42 \%$. Hospital mortality was $1.4 \%$ (7/488).

\section{Conclusions}

This single center experience show that aortic valve replacement achieved through a right mini-thoracotomy is a safe procedure with excellent results. Thanks to a standardized technique cardiopulmonary bypass and cross-clamping times comparable with the standard can be obtained and peripheral cannulation avoided.

Advantages of this technique include early mobilization and rehabilitation, excellent aesthetic result and lower risk of wound complications

\section{Topic: Minimally Invasive Cardiac}

\section{2}

Cor-Knot, an automated knot-tying device: first experience in Asia

Evangelos Papadimas, Mohammad Armane Wadud, Peggy Hu,

Theo Kofidis

National University Hospital, Singapore, Singapore

Correspondence: Evangelos Papadimas

Journal of Cardiothoracic Surgery 2017, 12(Suppl 1):092

\section{Background}

This study compared the CPB, Cross-clamp and total operation time between minimally invasive and conventional cardiac surgery cases in which The Cor-Knot automated fastener (LSI SOLUTIONS, Victor, NY, USA) was used versus those in which a traditional knot pusher was used.

Methods

The Cor-Knot was used in a total of 30 cases, of which 20 were through median sternotomy, and 10 minimally invasive. The 30 patients who underwent surgery using this novel automated fastener
(January 2016-December 2016) were compared to 30 patients corrected with a traditional knot pusher (January 2015-December 2015). No significant differences regarding demographic data (age, gender, NYHA class, ejection fraction, BMI, cardiovascular risk factors) between the two groups were found. The two groups were compared for CPB time, Aortic Cross-clamp time, total OT time. Differences between groups were calculated with the Student's $t$-test. Overall significance is attained with a $p<0.05$.

Results

Transesophageal and transthoracic echocardiography at the end of operation and at discharge revealed no $(n=28)$ or mild $(n=2)$ residual regurgitation of the respective valve, without any evidence of ring dehiscence, paravalvular leaks and without any significant differences between the two groups. Cross-clamp time $(83.2 \pm 12.7$ vs. $98.4 \pm 13.8 ; p<0.01)$, CPB time $(131.7 \pm 21.7$ vs. $149.4 \pm 22.7$ $p<0.05)$ and operation time $(201.8 \pm 29.4$ vs. $225.2 \pm 27.02 ; p<0.01)$ were significantly reduced in the automated fastener group compared to the group using a traditional knot pusher.

Conclusions

The results of our study show that with the introduction of the CorKnot device to a diverse Asian population, cross-clamp time, CPB time and total operation time were significantly reduced compared to our control group. Time saved varied from 15 (cross-clamping) up to $18 \mathrm{~min}$ (operation). With an estimated $100 \mathrm{SGD} /$ minute operating theater time in our country, an average of at least 1000SGD is saved per case; thereby making this an efficient and cost-saving solution.

\section{Topic: Cardiac Surgery}

093

Bio-Bentall Procedure in Aortic Root Pathology Using both Full or Mini Sternotomy

Elisa Mikus, Simone Calvi, Marco Paris, Alberto Tripodi, Marco Panzavolta, Mauro Del Giglio

Maria Cecilia Hospital, Cotignola (RA), Italy

Correspondence: Elisa Mikus

Journal of Cardiothoracic Surgery 2017, 12(Suppl 1):093

\section{Objective}

Bentall and De Bono described a surgical technique to treat aortic root pathology. Alternative, less-common procedures, such as reimplantantion and remodelling, have been proposed in an effort to avoid anticoagulation therapy. We describe our experience with a biological conduit for aortic root pathology.

Methods

Between January 2010 and November 2015, 94 patients (84\% men; mean \pm standard deviation age $63.8 \pm 13.0$ years) underwent an isolated Bio-Bentall procedure at our institution, for an elective or urgent indication. None had previously undergone cardiac surgery. Patients were divided into those who underwent a minimally invasive approach through an upper ministernotomy and patients who underwent standard full sternotomy. Follow-up data up to 5 years were obtained retrospectively from hospital and office records and through telephone contacts. Overall survival curves after discharge were estimated using the Kaplan-Meier product-limit estimator and were compared using the log-rank test.

Results

Of the 94 patients, 45 (48\%) underwent a full sternotomy and 49 (52\%) underwent a minimally invasive approach through a ministernotomy. Both groups of patients showed similar results in terms of postoperative mortality and morbidity. Mean cardiopulmonary bypass $(97.4 \pm 35.2$ vs $115.3 \pm 44.5 ; \mathrm{P}=0.023)$ and cross-clamp $(82.8 \pm 29.4$ vs $97.7 \pm 38.2 ; \mathrm{P}=0.046)$ times were shorter in the minimally invasive group. During the early postoperative period, $51 \%$ of patients in both groups were free from complications. Two (4\%) patients in the full sternotomy group died in hospital versus none in the minimally invasive group $(P=0.22)$. The 
hospital mortality rate was $2 \%(2 / 94)$. Five-year overall survival after hospital discharge was $91 \%$ in the minimally invasive group and $89 \%$ in the full sternotomy group.

\section{Conclusions}

Our single centre experience shows that a biological prosthesis inserted into the Dacron graft for aortic root replacement has low mortality and good durability. In a selected population, minimally invasive surgery appears to have a role.

\section{Topic: New Technology}

\section{4}

Sutureless aortic valve implantation: first Asian experience

Jian Wei Tann', Jianye Chen ${ }^{2}$, Saideep Bose ${ }^{2}$, Duc Thang Vu²,

Theo Kofidis ${ }^{2}$

${ }^{1}$ Yong Loo Lin School of Medicine, National University of Singapore,

Singapore, Singapore; ${ }^{2}$ Department of Cardiac, Thoracic and Vascular

Surgery, National University Heart Centre, Singapore, Singapore

Correspondence: Jian Wei Tan

Journal of Cardiothoracic Surgery 2017, 12(Suppl 1):094

\section{Background}

Sutureless aortic valves have been shown in Western literature to be superior on some aspects compared to conventional aortic valves. This study aims to evaluate whether the sutureless valve is superior to the conventional valve after its first time use in a mixed Asian population.

Methods

A total of 50 patients who underwent aortic valve replacement, with or without coronary artery bypass graft surgery between January 2009 and December 2016 were included in this cohort study. 25 received a sutureless valve and 25 received a conventional valve. A propensity score analysis was performed, creating 25 matched pairs with similar preoperative characteristics. Operative time, patient outcomes and valvular performance were compared.

Results

Cardiopulmonary bypass ( $118 \pm 38$ vs $149 \pm 33$ minutes, $p<0.05)$ and aortic cross-clamp times ( $80 \pm 32$ vs $100 \pm 32$ minutes, $p<0.05$ ) were significantly shorter in the sutureless group. Patients in the sutureless group had higher post-operative albumin ( $34 \pm 4$ vs $30 \pm 5, p<0.05)$. The sutureless group had a shorter intensive care unit stay (3.8 \pm 2.0 vs $5.5 \pm 3.4$ days, $p<0.05)$ and hospital stay $(10.4 \pm 5.0$ vs $15.6 \pm 10.1$ days, $p<0.05)$. Post-operative patient outcomes were similar between the two groups. There were no cases of iatrogenic aortic dissection, stroke or valve degeneration. Mean aortic valve gradient at 1 month after surgery $(11.1 \pm 3.8$ vs $16.7 \pm 6.7 \mathrm{mmHg}, p<0.05)$ was lower in the sutureless group. Other clinical outcomes like ejection fraction ( $p$ $=0.314)$, left ventricular remodelling $(p=0.237)$ and valvular leaks $(p$ $=1.000$ ) were comparable between the two groups.

\section{Conclusions}

Sutureless aortic valves decrease the operative time, intensive care unit stay, hospital stay and post-operative mean aortic valve gradient. Patient outcomes are similar between the sutureless and conventional aortic valves.

\section{Topic: Perfusion}

\section{5}

Autotransfusion has an important effect on drug dosing in congenital cardiac surgery

Gerdien A. Zeilmaker-Roest, Annewil van Saet, Soma Bahmany,

Enno D. Wildschut, Dick Tibboel, Ad J.J.C. Bogers

Erasmus MC, Rotterdam, Netherlands

Correspondence: Gerdien A. Zeilmaker-Roest

Journal of Cardiothoracic Surgery 2017, 12(Suppl 1):095

\section{Objective}

Use of donor blood in congenital cardiac surgery increases the risk for post-operative morbidity and mortality. To reduce the need for allogenic blood transfusion a cell saver system is routinely used. Blood from the operation site is collected in a reservoir, processed, passed through a lipophilic filter and returned to the patient. Influence of this cell saver system on coagulation, fibrinolysis and inflammatory markers is known. To our knowledge no studies have been performed on the effects of autotransfusion on drug concentrations. A clinically relevant drug dose could potentially be returned to the patient through the auto-transfused blood, leading to unwanted drug reactions post-operatively. We aimed to measure drug concentrations in blood removed from the operation site and in the autotransfused blood to determine if a clinically relevant drug dose is returned to the patient.

Methods

The study was performed at the department of cardiothoracic surgery of a tertiary pediatric hospital. Blood samples were taken from 1, the reservoir, 2, after processing before the lipophilic filter, 3 , after processing after the lipophilic filter, 4 , the waste fluid. Samples were stored at -80 Celsius and drug concentration for Sufentanil, Propofol, Midazolam and Cefazoline were measured using LC-MS/MS. Results

Blood samples were taken from 19 cell saver runs in 18 patients, age 0-13 years. Drug concentrations in the reservoir were comparable to concomitant concentrations in the patient. For sufentanil $32 \%$ of drug concentration was retained from the reservoir in the autotransfused blood, for propofol 18\%, for midazolam $5 \%$ and for Cefazoline $4 \%$ respectively.

\section{Conclusions}

Depending on the drug, up to $32 \%$ of the drug concentration removed from the operation site is returned to the patient through autotransfusion, potentially causing unwanted drug reactions postoperatively. Additionally, influence of a cell saver system should be considered in pharmacological research during and after congenital cardiac surgery.

\section{Topic: Cardiac Surgery}

\section{6}

Elevated red cell distribution width as a predictive marker for development of systemic inflammatory response syndrome in patients undergoing cardiac surgery with extracorporeal circulation

Harsh S. Seth

Lokmanya Tilak Municipal Medical College, Mumbai, India

Journal of Cardiothoracic Surgery 2017, 12(Suppl 1):096

\section{Background}

Cardiac surgical operations involving extracorporeal circulation may develop severe inflammatory response (SIR). This severe inflammatory response syndrome (SIRS) is usually associated with poor outcome with no predictive marker. Red cell distribution width (RDW) is a routine hematological marker with a role in inflammation. We aim to determine the relationship between RDW and SIRS through our study.

Methods

A total of 1250 patients who underwent cardiac surgery with extracorporeal circulation were retrospectively analyzed out of which 26 fell into the SIRS criteria and 26 consecutive control patients were taken. RDW, Preoperative clinical data, operative time and postoperative data were compared between the SIRS and control group.

Results: The demographic profile of the patients was similar. RDW was significantly higher in the SIRS VS control group $(15.5 \pm 2.0$ VS $13.03 \pm 1.90$ ), respectively with $p$ value $<0.0001$. There was significant mortality in the SIRS group $20(76.92 \%)$ as compared to $2(7.6 \%)$ in control group with a $p$ value of $<0.005$. Multiple logistic regression analysis revealed that there was significant association with high RDW and development of SIRS after extracorporeal circulation, Odds ratio (OR for RDW levels exceeding 13.5\%; $95 \%$ confidence limits of 1.0-1.2; $\mathrm{p}<0.05)$.

\section{Conclusion}

Increased RDW was significantly associated with increased risk of SIRS after ECC. Thus RDW can act as a useful tool to predict SIRS in patients undergoing cardiac surgery with extracorporeal circulation. 
Hence more aggressive measures can be taken in patients with high RDW to prevent postoperative morbidity and mortality.

\section{Topic: Basic Sciences}

\section{7}

- Aortic allograft decellularization techniques efficacy: laboratory results

Mikalai Shchatsinka', Siarhei Spirydonau', Vitali Adzintsou',

Aliaksandr Shket ${ }^{1}$, Mikalai Yurkshtovich², Volha Yudzina ${ }^{3}$, Raisa Smaliakova ${ }^{4}$,

Alena Suboch ${ }^{4}$, Svetla Dryk ${ }^{5}$, Youry Ostrovsky ${ }^{1}$

${ }^{1}$ RSPC Cardiology, Minsk, Belarus; ${ }^{2}$ Belarusian State University, Minsk,

Belarus; ${ }^{3}$ City Clinical Pathologoanatomic Bureau, Minsk, Belarus; ${ }^{4}$ N.N.

Alexandrov National Cancer Centre, Minsk, Belarus; ${ }^{5}$ 9th City Clinical

Hospital, Minsk, Belarus

Correspondence: Mikalai Shchatsinka

Journal of Cardiothoracic Surgery 2017, 12(Suppl 1):097

\section{Objective}

To assess an efficacy of four aortic allograft decellularization techniques.

\section{Methods}

Aortic allografts were decellularized using deoxycholic acid 1\% (group $1, \mathrm{n}=10$ ), sodium deoxycholate $0.5 \%$, sodium dodecylsulfate $0.5 \%$ (group 2, $\mathrm{n}=10$ ), sodium deoxycholate $1 \%$, Triton $\mathrm{X}-1001 \%$ (group 3, $\mathrm{n}=10$ ), Triton $\mathrm{X}-100$ 3\%, EDTA $0.04 \%$ (group 4, $\mathrm{n}=10$ ). Valve specimens were subjected to histologic (H\&E, morphometry in 5 views) and histochemic (MSB, orcein) studies before and after decellularization; residual DNA content was assessed (spectrofluorometry at $460-600 \mathrm{~nm}$ ); mechanical testing was performed.

Results

According to histologic morphometry study, cell reduction in group 1 was $516(348-577)$ to $310(241-336)$ cells, $p<0,05$; in group $2-534$ (368-672) to 5 (2-5) cells, $\mathrm{p}<0,05$; in group 3 - 370 (367-447) to 191 (188-208) cells, $\mathrm{p}<0,05$; in group 4 - 229 (209-299) to 194 (132-200) cells, $p>0,05$ (Wilcoxon criteria). There was a statistically significant difference in cell reduction between groups 1 and $2(p<0.01)$ and between groups 2 and $3(p<0.01)$, with greatest reduction in group 2 (Mann-Whitney test). According to histochemic studies (MSB, orcein), connective tissue matrix was not affected in any of the groups. In group 2 it was found a statistically significant reduction of DNA content in aorta $(31,05$ vs $2,58 \mathrm{ng} / \mathrm{mg}, \mathrm{p}<0,01)$ and aortic valve leaflet $(39,7$ vs $1,36 \mathrm{ng} / \mathrm{mg}$, p $<0,05)$ after decellularization. Mechanical testing results of fresh, cryopreserved and decellularized allografts (group 2) were: Fmax - 31,3 $\pm 2,0 \mathrm{~N}, 33,4 \mathrm{~N}(29,4-37,3 \mathrm{~N})$, $33,1 \mathrm{~N}(18,2-38,9 \mathrm{~N})$, accordingly ( $p>0.05) ; \sigma-0,9 \mathrm{MPa}(0,7-1,2 \mathrm{MPa})$, $0,9 \mathrm{MPa}(0,8-1,1 \mathrm{MPa}), 0,9 \mathrm{MPa}(0,6-1,1 \mathrm{MPa})$, accordingly $(\mathrm{p}>0.05)$.

\section{Conclusions}

The most profound aortic allograft decellularization was achieved using sodium deoxycholate $0.5 \%$ and sodium dodecylsulfate $0.5 \%$, with statistically significant cell reduction and DNA reduction, without affecting connective tissue matrix and mechanical properties of the valve.

\section{Topic: Cardiac Surgery}

\section{8}

Predicting ascending aortic dilatation: differences between bicuspid aortic valve and tricuspid aortic valve patients

Giuseppe M. Raffa, Cesare Scardulla, Michele Pilato, Michele Pilato, Salvatore Pasta

IRCCS-ISMETT (Istituto Mediterraneo per i Trapianti e Terapie ad altaspecializzazione), Palermo, Italy

Correspondence: Giuseppe M. Raffa

Journal of Cardiothoracic Surgery 2017, 12(Suppl 1):098

\section{Objective}

The development of ascending aortic dilatation in patients with bicuspid aortic valve (BAV) is highly variable, and this makes surgical decision strategies particularly challenging. The purpose of this study was to identify new predictors that may help to stratify the risk of aortic dilatation in BAV patients, other the well-established aortic size. Methods

Using computational modeling, we compared both hemodynamic and structural parameters exerted on the ascending aortic wall of patients with either BAV $(n=21)$ or tricuspid aortic valve (TAV, $n=13)$ that were matched for age and aortic diameter. BAV phenotypes were stratified according to the leaflet fusion pattern and aortic shape.

Results

Systolic wall shear stress (WSS) of BAV patients was higher than TAV patients at sino-tubular junction $\left(6.8 \pm 3.3 \mathrm{~N} / \mathrm{m}^{2}\right.$ for BAV and $3.9 \pm 1.3$ $\mathrm{N} / \mathrm{m}^{2}$ for TAV, $\left.\mathrm{p}=0.006\right)$ and mid-ascending aorta $\left(9.8 \pm 3.3 \mathrm{~N} / \mathrm{m}^{2}\right.$ for $B A V$ and $7.1 \pm 2.3 \mathrm{~N} / \mathrm{m}^{2}$ for $\mathrm{TAV}, \mathrm{p}=0.040$ ). A statistically significant difference in BAV versus TAV was also observed for the intramural stress along the ascending aorta (e.g., $25.4 \pm 3.2 \mathrm{~N} / \mathrm{m}^{2}$ for BAV and $20.4 \pm 3.4 \mathrm{~N} / \mathrm{m}^{2}$ for TAV, $\left.\mathrm{p}<0.001\right)$ and pressure index $(0.329 \pm 0.107$ for $B A V$ and $0.223 \pm 0.139$ for TAV, $p=0.030$ ). Difference in the BAV phenotypes (i.e., BAV Type 1 versus BAV Type 2) and aortopathy (ie, isolated tubular versus aortic root dilatations) were associated to asymmetric WSS distributions in the right-anterior aortic wall and right-posterior aortic wall, respectively.

Conclusions

These findings suggest that valve-mediated hemodynamic and structural parameters are useful for the risk stratification of the severity of aortic dilatation in BAV.

\section{Topic: Cardiothoracic Radiology}

099

Multislice computed tomography angiography in the diagnosis of a coronary atherosclerosis in people of Kazakhstan

Elmira Yelshibayeva', Raushan Rakhimzhanova², Tairkhan Dautov'

${ }^{1}$ National Research Center for Cardiac Surgery, Astana, Kazakhstan;

${ }^{2}$ Medical University Astana, Astana, Kazakhstan

Correspondence: Elmira Yelshibayeva

Journal of Cardiothoracic Surgery 2017, 12(Suppl 1):099

\section{Objectives}

The study of ethnic and gender characteristics of coronary atherosclerosis in people of Kazakhstan using computer tomography. Methodology

Research carried out on Multislice computed tomography (MSCT) «Somatom Definition AS 64», Siemens Company, Germany, with prospective cardiac synchronization and reconstruction with $0.6 \mathrm{~mm}$ slice thickness. There were studied 2586 patients in period from September 2008 year to October of 2016 year with a primary diagnosis of coronary heart disease and patients without any complains on heart disease, the average age was 66.6 years. From all amount of patients atherosclerotic vascular changes were revealed in $2020(78.1 \%)$ and $566(21.8 \%)$ patients were healthy. Patients divided by gender characteristic: 2013 (77.8\%) men, $573(22.1 \%)$ women and by ethnicity: 1313 (65.2\%) Asian men, 700 (34.7\%) European men, 350 (61\%) Asian women, 223 (38.9\%) European women.

Results

In a retrospective analysis on a computed tomography angiography in 2020 (men-1447, women-573) patients with a primary diagnosis ischemic heart disease were identified single vessel disease in men: 600 (57.9\%) Asian and 300 (72.9\%) European; in women: 155 (67.6\%) Asian and $45(69.2 \%)$ European; multivessel disease - in men: 436 (42.0\%) Asian and 111 (27.0\%) European; in women 74 (32.3\%) Asian and 20 (30.7\%) European.

At the comparative analysis the frequency of the individual lesions of the coronary arteries revealed: the left coronary artery involved in the pathological process in 805 (77.7\%) Asian men and $112(27.2 \%)$ European men. The number of lesions in the right coronary artery 231 (22.2\%) Asian men and 299 (72.7\%) European men.

\section{Conclusion}

In the group of Asian men, the left coronary artery is more affected and multivessel lesions of CA with a stenosis of more than 50-75\% are detected. Among European men, the right coronary artery is 
more often involved and single-vessel lesions with a stenosis of 50$75 \%$ are detected.

\section{Topic: Heart Failure and Heart Muscle Disease}

\section{0}

The use of cardiac resynchronization pacing in patients with chronic heart failure

Bagdat Akhyt, Kulzida Koshumbayeva, Roin Rekvava, Kurmangazy Madaliyev, Sholpan Ismailova, Salim Berkinbayev Research Institute of Cardiology and Internal Diseases, Almaty, Kazakhstan

Correspondence: Bagdat Akhyt

Journal of Cardiothoracic Surgery 2017, 12(Suppl 1):0100

\section{Objective}

Aim of this study is to evaluate the clinical and haemodynamic efficiency of cardiac resynchronization therapy (CRT) in patients with chronic heart failure (CHF)

Methods

This follow-up study enrolled 70 adult patients undergoing cardiac resynchronization therapy device with a defibrillator (CRTD) function and cardiac resynchronization therapy device (CRTP) implantation. Inclusion criteria was presence of heart failure (CHF NYHA class III, IV) and left bundle branch block. We performed echocardiography to assess functional state of the heart at 6 and 12 months after CRTD or CRTP implantation.

\section{Results}

We observed significant increase in ejection fraction 12 months after CRTD or CRTP implantation ( $28.3 \pm 0.9 \%$ to $38.1 \pm 0.9 \%$ ( $p<0.001)$ ). There was no significant decrease in left ventricle end diastolic dimension $(6.8 \pm 0.1 \mathrm{~cm}$ to $6.5 \pm 0.1 \mathrm{~cm}(\mathrm{p}=0.068))$ and in pulmonary artery pressure decrease $(51.5 \pm 1.7 \mathrm{~mm} \mathrm{Hg}$. to $48.5 \pm 4.2 \mathrm{~mm} . \mathrm{Hg}$ $(\mathrm{p}=0.67))$. Intraventricular and interventricular delay decreased significantly during the observational period $(270.8 \pm 12.6 \mathrm{~ms}$ vs $183.8 \pm 20.3 \mathrm{~ms}, \mathrm{p}<0.001$ and $61.0 \pm 2.9 \mathrm{~ms}$ vs $29.7 \pm 5.6 \mathrm{~ms}, \mathrm{p}$ $<0.001$, respectively). There was no mortality in a given period of observation.

\section{Conclusions}

Cardiac resynchronizing therapy helps to achieve positive clinical and hemodynamic effect in patients with severe CHF.

\section{Topic: Cardiac Surgery}

\section{1}

The complex monitoring for diagnostic early heart rejection after transplantation

Makhabbat Bekbossynova, Yuriy Pya, Saltanat Jetibayeva, Gulzhan

Myrzahmetova, Saltanat Andossova, Murat Mukarov

National Research Center for Cardiac Surgery, Astana, Kazakhstan

Correspondence: Makhabbat Bekbossynova

Journal of Cardiothoracic Surgery 2017, 12(Suppl 1):0101

\section{Objective}

Endomyocardial biopsy is a standard diagnostic tool for detection of graft rejection. However, it is known, that endomyocardial invasive biopsy (EMB) is not safe and can entail serious complications. The aim of the study is to assess clinical and labor data with TD-indexes on revealing the heart rejection after transplantation.

\section{Methods}

Since August 2011 there were 48 heart transplantations performed (10 are women in the age of $41 \pm 13.8$ years from the donors of 42.5 \pm 13.5 years old. The $1^{\text {st }}$ group - 33 patients $(68.7 \%)$, which had undergone EMB and in $2^{\text {nd }}$ group - $15(31.2 \%)$ patients had not undergone EMB. All patients from both groups was performed of laboratory indicators (included CRP) and echocardiography (2D echo in a resting state, tissue Doppler (TDI) in a resting state (PW-systolic parameters $S$ velocity, myocardial strain) daily still the discharge and then in I, III, VI month.

Results

There were no significant differences evidenced in both groups (I/II groups) on basic results of leukocytes, lymphocytes and CRP analysis. One patient from the $2^{\text {nd }}$ group after 19 month after TX had nonspecific clinical symptoms (palpitation and general fatigue), high level CRP (1.94 mg/l) and abnormal tissue Doppler ( $S^{\prime}$ laterals $3.8 \mathrm{~cm} /$ c. S'medialis $3.9 \mathrm{~cm} / \mathrm{c}$. S'RV $3 \mathrm{~cm} / \mathrm{c}$.). And concentration of tacrolimus was low $(6.7 \mathrm{ng} / \mathrm{ml})$. We estimated this condition as graft rejection. After 10 days of timely administration of pulse therapy with solumedrol ( $1 \mathrm{~g}$ per day $\mathrm{i} / \mathrm{v})$ and increased concentration of tacrolimus $(13.8 \mathrm{ng} / \mathrm{ml})$ improvement of clinical status, CRP decreased $(0.1 \mathrm{mg} / \mathrm{l})$ and tissue Doppler had better (S'lateralis $8 \mathrm{~cm} / \mathrm{c}$. S'medialis $7 \mathrm{~cm} / \mathrm{c}$. S'RV $7 \mathrm{~cm} / \mathrm{c}$ ). Patient was discharged after recovery.

Conclusion

Thus, we confirm that clinic, CRP and tissue Doppler (PW-systolic parametres $S$ velocity) when patient has low concentration of tacrolimus can be used as non-invasive diagnostic tool of allograft rejection of patients after heart transplantation.

\section{Topic: Endovascular}

\section{2}

Complete coverage of the descending thoracic aorta during

endovascular repair of thoracoabdominal dissection is not associated with increased risk of spinal cord ischemia

Gilbert R. Schorlemmer

MountainStar Cardiovascular Surgery, Orem, UT, USA

Journal of Cardiothoracic Surgery 2017, 12(Suppl 1):0102

\section{Background}

In patients receiving endografts to treat thoracic aortic aneurysmal disease, length of aortic coverage is a risk factor for spinal cord ischemia (SCI). However, pathology specific studies of the length of aortic coverage as a risk factor for $\mathrm{SCl}$ have not been performed. A retrospective analysis of all patients in our network with complicated aortic dissections undergoing endovascular repair was undertaken to assess this risk.

\section{Methods}

The medical records and radiographic studies from a database of all aortic endovascular procedures performed from 2009 to 2015 were retrospectively reviewed. Postoperative length of graft coverage was measured from $\mathrm{CT}$ angiograms using centerline reconstructions. $\mathrm{SCl}$ was defined as transient or permanent lower extremity neurologic deficit without associated intracerebral hemispheric events.

Results

Thirty-eight consecutive patients with complicated aortic dissections had complete coverage of the descending thoracic aorta. The average length of covered descending thoracic aorta was $34 \mathrm{~cm}$. In some cases, abdominal and branch endografts were also used. Patency of flow in the left subclavian, mesenteric, renal and hypogastric arteries was aggressively preserved. No other attempt at prophylactic spinal cord protection was undertaken. Only one case of $\mathrm{SCl}$ (developing 24 hours postoperatively after mismanagement of postoperative blood pressure control) was reported. At 1 year, no further $\mathrm{SCl}$ occurred and complete false lumen obliteration in the descending thoracic aorta was achieved in all patients. There were no deaths, either acutely or during the follow up period.

Conclusions

Complete descending thoracic aortic coverage during endovascular repair of thoracoabdominal dissection was not associated with an increased risk of $\mathrm{SCl}$ as long as major aortic branch flow was maintained at an adequate perfusion pressure. Complete coverage with appropriately sized endografts is associated with false lumen obliteration which also does not increase the incidence of spinal cord ischemia. 


\section{Topic: Cardiac Surgery}

\section{3}

Clinical experience with amplatzer duct occluder ii additional sizes for transcatheter occlusion of PDAs in 127 cases

Fizuli Abasov' ${ }^{1}$ Pavel Gavora ${ }^{2}$, Osman Makhachev ${ }^{1}$, Manolis Pursanov ${ }^{3}$, Aydar Khamidullin ${ }^{4}$, Marat Bikmullin ${ }^{4}$, Madina Badrudinova',

Azamat Mamaev' ${ }^{1}$, Gadji Aliev', Akhmedkhan Shakhnavazov', Radis Garaev ${ }^{4}$, Marjam Magomedova', Dmitriy Bombin ${ }^{5}$

${ }^{1}$ Dagestan Center for Cardiology and Cardiovascular Surgery, Makhachkala, Russian Federation; ${ }^{2}$ Center for Cardiology and

Cardiovascular Surgery, Slovakia, Bratislava, Slovakia; ${ }^{3}$ A.N. Bakoulev Scientific Center for Cardiovascular Surgery, Moscow, Russian Federation; ${ }^{4}$ Children's Republican Clinical Hospital of the Ministry of Health of the Republic of Tatarstan, Kazan, Russian Federation; ${ }^{5}$ Rostov Regional Hospital, Rostov-on-Don, Russian Federation

Correspondence: Fizuli Abasov

Journal of Cardiothoracic Surgery 2017, 12(Suppl 1):0103

\section{Objective}

To present results of use of ADO ॥ AS for transcatheter closure of PDA.

\section{Methods}

From 2003 to 2016 transcatheter closure of PDA was performed in 1100 patients. In 330 cases (30\%) different PDA occluders were used, in $127(11.5 \%)$ ADO II AS. Median age of patients was 9 years (1 to 27 years). Male patients 52 (39\%), female - 75 (59\%). Transcatheter closure was indicated for patients with aortography results - blood shunt from aorta into pulmonary artery II-IV degree to LLOYD T.R. (1993) and PDA morphology types according to A. Krichenko et. al. (1989). Morphology of PDA was type $E$ in $76 \%$, type A in $18 \%$, type D in $6 \%$. Diameter of pulmonary end $1.7 \pm 0.3 \mathrm{~mm}$, aortic end $3,5 \pm 1.1$ $\mathrm{mm}$. Transfemoral arterial access was used with $4 \mathrm{~F}$ occluder delivery system. Only arterial access was used for all A-type patients. Closure efficacy was assessed by control aortography, and echocardiography in 24 hours after procedure.

\section{Results}

There was no hospital mortality. Complete closure was achieved in $99.2 \%$. One patient had residual shunt due to dislocation of occluder to the pulmonary artery. In two cases occluders migrated into pulmonary artery, both were successfully retrieved.

\section{Conclusions}

Transcatheter closure of PDA using ADO II AS is low-traumatic and effective. In type A PDA arterial access is preferable for ADO II AS, along with venous access for ADO I.

\section{Topic: Interventional Cardiology}

\section{4}

How many TAVI should be used in 1 year? Case study on an inappropriate use of TAVI

Filippo Bartoccioni ${ }^{1}$, Mihaela Dubinski ${ }^{2}$, Beatrice Desirée Calabrese ${ }^{2}$, Yermek Akhmetov ${ }^{3}$, Piergiorgio Minotti ${ }^{2}$, Katy Pelagagge ${ }^{2}$

${ }^{1}$ Asl Viterbo, Viterbo, Italy; ${ }^{2}$ Htacamp, Viterbo, Italy; ${ }^{3}$ Astana Medical University, Astana, Kazakhstan

Correspondence: Filippo Bartoccioni

Journal of Cardiothoracic Surgery 2017, 12(Suppl 1):0104

\section{Objective}

The aim of this study is to estimate the number of TAVI that should be implanted in one year following the natural trend of the pathology and the hospital base activity.

Methods

In 2008 the interventional cardiology unit started to use them and the following year the cardiac surgery department started as well. We collected the monthly consumption of biological aortic valve used in aortic stenosis from year January 2000 to 2012. We used the linear regression forecasting and the exponential regression forecasting technique based on the year consumption from year 2000 to 2008 and we extrapolated the forecasting for the following years. This forecasting lines show the standard biological valve consumption would be if the TAVI never arrived to our hospital. We compare the forecasting with the real usage of standard biological valves and the total consumption. We then calculated the "Appropriate Ratio" between "forecasted cases for standard procedure/(total implants - forecasted cases for standard procedure). This ratio indeed shows indirectly the appropriate usage of TAVI.

Results

The joinpoint analysis did not show a modification of the increasing slope of the total number of implants in our hospital. This shows that there was no significant modification on the total number of cases trend. The number of the standard procedures was at the same time decreasing below the forecasting lines. The difference between the forecasting lines and the real usage of standard procedure shows the inappropriate quantity of the TAVI procedure. The "Appropriate Ratio" in 2011 was 6.7 result of 140/(161-140).

Conclusions

Even if we cannot directly calculate the next year appropriate number of TAVI procedure. Binding the TAVI consumption to the standard procedure we can calculate the minimum appropriate ratio tailored to our institution.

\section{Topic: Transcatheter Procedures}

\section{5}

Conventional redo surgery vs catheter-based trans-apical

procedure in mitral paravalvular leak treatment

Aleksejus Zorinas', Vilius Janusauskas', Lina Puodziukaite', Rokas

Simakauskas', Giedrius Davidavicius', Valdas Bilkis', Rita Kramena², Diana

Zakarkaite', Rasa Cypiene' ${ }^{1}$, Robertas Stasys Samalavicius ${ }^{1}$, Kestutis

Rucinskas ${ }^{1}$, Audrius Aidietis ${ }^{1}$, Eustaquio Maria Onorato ${ }^{3}$

${ }^{1}$ Vilnius University, Vilnius, Lithuania; ${ }^{2}$ Rita Kramena, Vilnius, Lithuania;

${ }^{3}$ Cardiovascular Department, Humanitas Gavazzeni, Bergamo, Italy

Correspondence: Aleksejus Zorinas

Journal of Cardiothoracic Surgery 2017, 12(Suppl 1):0105

\section{Objective}

To the date efficacy and safety between surgical and catheter-based paravalvular mitral valve leak treatment is not well investigated. Objective of this study was to compare efficacy and safety between two treatment methods of mitral valve paravalvular leak.

Methods

A retrospective analysis of the patients treated for mitral paravalvular leak in year 2005-2017. A total of 79 patients underwent paravalvular leak treatment. Forty-nine patients underwent "conventional redo surgery". Thirty patients had catheter-based procedure (trans-apical approach). Among them 11 patients have been treated with "off label" product and 19 patients underwent defect closure with "purpose specific" device specifically designed for paravalvular leak closure.

Results

Patients were oldest in a group with "off label" closure device (71.1 \pm 6.2 years) vs $62 \pm 7.7$ in "conventional redo surgery" and $64.2 \pm 6.7$ in "purpose specific" group $(p=0.002)$. Anemia and chronic renal insufficiency was more common among in "off label" group of patients. Total mortality at 90 days after the procedure was $14 \%$ among the patients in "conventional redo surgery" group vs $18 \%$ in "off label" closure device group. None of the patients died in "purpose specific" device group. In "conventional redo surgery" group $3(6 \%)$ patients have suffered in hospital cerebrovascular event while none among the patients in catheter-based procedure cohort. Freedom from moderate or higher regurgitation thru the paravalvular leak was observed in 46 (92\%) patients in "conventional redo surgery" group vs $9(82 \%)$ in "off label" closure device group and $18(95 \%)$ in "purpose specific" group of patients $(p=0.358)$. 


\section{Conclusions}

Catheter-based closure of a paravalvular leak is not inferior to conventional redo surgery for mitral paravalvular leak. Patients treated with device specifically designed for paravalvular leak closure may have superior results over conventional surgery.

\section{Poster Abstracts}

P1

Kinetic of myocardial biomarkers at patients with and without renal dysfunction after cardiosurgical operations

Marina Charnaia, Inna Dementieva

Petrovsky National Research Center of Surgery, Moscow, Russian

Federation

Correspondence: Marina Charnaia

Journal of Cardiothoracic Surgery 2017, 12(Suppl 1):P1

Objective: to study dynamics of myocardial biomarkers at patients after coronary revascularization depending on renal function in the early postoperative period.

Methods:.Estimated levels of troponin $\mathrm{T}(\mathrm{TrT})$ and I (Trl), MB-CPC, myoglobin (Myo), and creatinine before, in 6, 12, 24, 48 and 72 hours after surgery. Calculated glomerular filtrations rate (GFR). Group $1(n=12)$ - patients with renal dysfunction (RD) in 1 day after surgery, group $2(n=15)$ - without RD. Counted maximum concentration (Cmax), achievement Cmax time (Tmax), the area under a curve "concentration-time" (AUC), Cmax/AUC, biomarker half-life (HL).

Results: In group $1 \mathrm{Cmax}$ for $\mathrm{Trl}$ and MB-CFC was maximal, but this parameter for TrT and Myo was approximately identical. Tmax for TrT and Trl in groups 1 and 2 was identical - 24 and 12 hours accordingly whereas for MB-CPC and Myo achievement of Tmax occurred faster 12 and 24 hours for MB-CPC and 6 and 12 hours for Myo in groups 1 and 2 respectively. Value AUC authentically changed only for Trl $111,4 \pm 10,6$ and $45,2 \pm 5,5 \mathrm{mkg}$ per hour/l for groups 1 and 2 respectively ( $p$ \&lt0,05). The relation of Smax/AuC was identical to Trl, MBCPC and Myo whereas for TrT in group 1 this indicator has appeared in 2 times more low, than in group 2. The HLP for TrT and Trl did not change for groups 1 and 2, and for MB-CPC and Myo - increased in group 1 in comparison with group 2, in a greater degree for Myo Conclusions: At patients with RD kinetic of biomarkers differs from that at patients without RD. It can be caused infringement of their renal clearance. Presence of RD considerably increases the HLP of MB-CPC and Myo, and also by infringement Trl kinetic, but not TrT.

P2

Qualitative cardiac fatty acid binding protein poc-test in an estimation of myocardium damages at cardiosurgical patients

Marina Charnaia, Inna Dementieva

Petrovsky National Research Center of Surgery, Moscow, Russian

Federation

Correspondence: Marina Charnaia

Journal of Cardiothoracic Surgery 2017, 12(Suppl 1):P2

Objective: To determine whether serum levels of cardiac type fatty acid binding protein (CFABP) are related to miocardium ischemia in patients undergoing cardiopulmonary bypass (CPB) surgery.

Methods: CFABP by qualitative express test (Russia) was measured in 40 patients: 10 - CABG with CPB, 10 - CABG with beating heart CPB, 10 - of-pump CABG, 10 - valve replacements with CPB before and after surgery, in 12 hours, for 2 and 3 days after surgery

Results: Before surgery the test was negative in all cases. In the end of operation in all cases the test has been regarded as positive. At patients after valve replacement surgery intensity of coloring of a test strip was considerably above, than after CABG. Depending on a kind $C A B G$ expressiveness of coloring increased in the following sequence: of-pump CPB \&lt beating heart CPB \&lt CPB. Only at 2 from 10 persons with CABG changes on an electrocardiogram were marked. The test remained positive at $50 \%$ of patients in 12 hours after operation, and at $40 \%$ from them changes on an electrocardiogram were marked. The same picture was and for 2 days after surgery. For 3 postoperative days the positive test was registered at $20 \%$ of patients against remaining changes on an electrocardiogram. At $80 \%$ of patients the test was negative, including one patient with a perioperative myocardium heart attack

Conclusions: Intensity of coloring of a test strip is more expressed at valve replacement surgery that is connected with a straight line myocardium «surgical trauma». At CABG the maximum intensity of coloring is marked at the operations with CPB. At electrocardiogramssigns of a myocardium heart attack after cardiac surgery the positive qualitative express test can serve as a method of an estimation of necrotic process dynamics east-font-family.

P3

Monitoring bloodstream infection of adult patient in cardiac intensive care unit

Nelya Bissenova, Aigerim Yergaliyeva

National Scientific Medical Research Center, Astana, Kazakhstan

Correspondence: Nelya Bissenova

Journal of Cardiothoracic Surgery 2017, 12(Suppl 1):P3

Objective: Bloodstream infections - the leading infectious complications in patients in intensive care unit (ICU), found in 15\% of patients with nosocomial infections and in $1 \%$ of all hospitalized patients. We aimed to study bacterial structure and antibiotic resistance blood stream infections from patients in cardiac ICU.

Methods: From January 2012 to September 2016788 blood samples from patients of cardiac ICU after cardiac surgery (surgery on the heart and major blood vessels) were included to prospective microbiologic study. Identification and susceptibility testing were performed using the Vitek 2 automated system.

Results: The rate of positive hemocultures was increased from $7.5 \%$ to $18.2 \%(p<0.05)$, in the mean of $16.6 \pm 1.3$. During study period 131 strains of 15 species were isolated. The prevalent microorganisms were Gram-negative (81.6\%), Gram-positive (16.1\%) and fungi (2.2\%). The most frequent pathogens were Klebsiella pneumoniae (19\%), Pseudomonas aeruginosa (16\%), Acinetobacter baumannii (16.7\%). ESBL-producing K.pneumoniae was found from $64.5 \%$, all isolates were susceptibility to carbapenems. Resistance rate of A.baumannii to piperacillin/tazobactam, to meropenem, to ceftazidime, to cefepime, to ciprofloxacin and to gentamicin were $100 \%, 80 \%, 94.1 \%$, $93.7 \%, 83.3 \%$ and $33.3 \%$. More than $40 \%$ of P.aeruginosa strains were resistance to cefepime, to ceftazidime $36.8 \%$, to meropenem $25 \%$, to piperacillin/tazobactam $23 \%$, to gentamicin $78.9 \%$.

Conclusions: Clinical sepsis syndrome is important from an epidemiological point of view. This leads to follow a strict policy on the use of antibiotics, the use of which should be based on local data.

P4

Monitoring of bloodstream infection of patients in pediatric cardiac intensive care unit

Nelya Bissenova, Aigerim Yergaliyeva

National Scientific Medical Research Center, Astana, Kazakhstan

Correspondence: Nelya Bissenova

Journal of Cardiothoracic Surgery 2017, 12(Suppl 1):P4

Objective: Patients from pediatric cardiac intensive care unit (ICU) may have different risk profile than those children's multidisciplinary ICU, which is caused by an early age of the patients, the severity of operations with cardiopulmonary bypass, as well as the frequent use of multiple invasive devices. We aimed to determine microbiologic spectrum of bloodstream infection in patients from pediatric cardiac ICU. Methods: From January 2012 to September 2016520 blood samples from patients of pediatric cardiac ICU (infants and children the first three years) after cardiac surgery (surgery on the heart and major blood vessels) were included to prospective microbiologic study. Identification and susceptibility testing were performed using the Vitek 2 automated system.

Results: During study period 133 isolates were collected. The rate of positive hemocultures was increased from $26.6 \%$ to $51.1 \%(p<0.01)$, 
in the mean of $25.5 \pm 1.9$. Gram-negatives were the most frequently isolated microorganisms, $60.1 \%(n=80)$, followed by fungi $24 \%$ $(n=32)$ and Gram-positives $23.3 \%(n=21)$. The most frequently pathogen Klebsiella pneumoniae - 34.5\% ( $n=46)$, Candida spp. 24\% ( $n=32)$, Burkholderia cepacia - $13.5 \%(n=18)$. ESBL-producing K.pneumoniae was found from $88.6 \%$ isolates. Resistance rate of 3 generation cephalosporins were (cefotaxime, ceftriaxone 100\%), ceftazidime $88.3 \%$, cefepime $91.1 \%$, piperacillin/tazobactam $60.8 \%$, amoxicillin/clavulanic acid $75 \%$, gentamicin $58.5 \%$, ciprofloxacin $20.9 \%$. All isolates of K.pneumoniae were susceptible to carbapenems and amikacin. The most common isolate from fungi was Candida albicans, resistance to amphotericin B was observed in $23.3 \%$, to fluconazole in $83.3 \%$, to clotrimazole in $34.4 \%$ strains.

Conclusions: Bloodstream infections in patients from pediatric ICU are major cause of morbidity and mortality. Further development of this nosology and strategies for the prevention, prevalence assessment and improvement of the quality of treatment, depending on the results of monitoring.

\section{P5}

Prevalence and antimicrobial resistance of pseudomonas aeruginosa isolates in pediatric cardiac intensive care unit Nelya Bissenova, Aigerim Yergaliyeva

National Scientific Medical Research Center, Astana, Kazakhstan Correspondence: Nelya Bissenova

Journal of Cardiothoracic Surgery 2017, 12(Suppl 1):P5

Objective: Pseudomonas aeruginosa is important cause of serious infections in newborns and young children that are associated with higher mortality. We aimed to determine dynamic of resistance rate of P.aeruginosa strains isolated from patient in pediatric cardiac intensive care unit.

Methods: A total of 264 strains of P.aeruginosa, isolated from clinical samples (blood, respiratory tract, wound samples) of 877 patients of pediatric cardiac ICU (infants and children the first three years) after cardiac surgery (surgery on the heart and major blood vessels) from January 2014 to September 2016 were included to the prospective microbiologic study. Identification and susceptibility testing were performed using the Vitek 2 automated system.

Results: During the study period the frequency of detection of P.aeruginosa strains from clinical samples increased from $5.7 \%$ in 2014 to $9.2 \%(p<0.05)$ in 2016 (from total number of isolates). The most isolates were allocated from respiratory tract (96.5\%). Resistance rate increased to meropenem from $37.7 \%$ in 2014 to $76.7 \%$ in 2016 ( $\mathrm{p}<0.001)$, to ceptazidime from $40.8 \%$ to $61.4 \%$ $(p<0.02)$, to cefepime from $29.3 \%$ to $61.4 \%(p<0.001)$, to ticarcillin/ clavulanic acid from $22.2 \%$ to $51.2 \%(p<0.01)$, to gentamicin from $25 \%$ to $60.5 \%(p<0.001)$, to ciprofloxacin from $10.4 \%$ to $50 \%$ $(p<0.001)$ respectively. The lowest resistance rate were to cefoperazone/sulbactam - 42.9\%; 95\% Cl 25.1-62.6

Conclusions: The results presented in this study have shown increasing resistance rates of $P$. aeruginosa to all antibiotics, especially to carbapenems. These suggest appropriate a strong antibiotic policy. Rapid and accurate reporting of antibiotic resistance is essential in therapeutic decisions.

\section{P6}

Results of cardiac surgery in patients aged 75 years and over Aktolkyn Uristemova, Yerzhan Khassen, Gabit Dildabayev, Dilmurat Nazhenov, Zeinat Ismailova

Sema Hospital, Almaty, Kazakhstan

Correspondence: Aktolkyn Uristemova

Journal of Cardiothoracic Surgery 2017, 12(Suppl 1):P6

The increase in the quality of life and the level of health care leads to an increase in life expectancy. This inevitably increases the number of patients of gerontological age having the cardiovascular system diseases and concomitant conditions.

Objective: To analyze cardiosurgery results in patients aged $75+$.
Methods: We have analyzed the data for 2015 and 2016, 33 patients aged 75 years and over were operated during this period.

All the gerontological patients had concomitant diseases: arterial hypertension in 27 patients, diabetes mellitus in 10 patients, respiratory pathology in 17 patients, heart arrhythmia in 5 patients, kidney disease in 14 patients, suffered acute cerebrovascular disorder in 2 patients, postinfarction cardiosclerosis in 19 patients, aortic aneurysm - 1, stenosis of internal carotid artery with hemodynamic disorders in 8 patients, malignant tumour in 1 patient, benign lesions in 4 patients, Parkinson's disease in 1 patient, obesity in 7 patients, ejection fraction below the norm for $30 \%$ in 2 patients, below for $35 \%$ in 2 patients, smokers -7 patients.

Two patients were operated on a beating heart with concomitant pathology, stenosis of the carotid artery above $90 \%$. Both patients were discharged without neurological deficiency.

Postsurgical complications: acute cerebrovascular disorders - 1 patient (stenosis of the carotid arteries at left $90 \%$, at right $100 \%$ ), respiratory failure - 2 patients, kidney failure with hemodialysis inducation - 1 patient, unstable hemodynamics - 5 patients, fatal outcome - 3 patients.

\section{Conclusions:}

1. Monitored anesthesia during cardiosurgery in gerontological patients, as well as a postoperative period in the intensive care unit requires an increased attention due to multiple concomitant diseases, and often patients of this category already have the suffered myocardial infarction.

2. Conduction of cardiosurgeriess on the beating heart in patients with stenosis of carotid arteries gives the good results

P7

The comparison between paravertebral and serratus anterior plane block for vats - pilot study

Darko Ilić, Nenad Ilić, Božena Ivančev, Toni Kljaković-Gašpić, Nikola Delić, Juričić Joško

University Hospital Centre Split, Split, Croatia

Correspondence: Darko llić

Journal of Cardiothoracic Surgery 2017, 12(Suppl 1):P7

\section{Objective:}

Video-assisted thoracic surgery (VATS) is emerging as the standard surgical procedure for lung surgery. However, there is no gold standard for regional analgesia for VATS. Serratus anterior plane block (SAPB) is a novel ultrasound guided thoracic wall nerve block reported recently. We performed SAPB for perioperative analgesia together with general anaesthesia in patients undergoing VATS surgery and compared it with PVB. The aim of this study was to assess the efficacy and safety of ultrasound guided serratus anterior plane block (SAPB) compared with paravertebral block for controlling acute pain during and 24 hours after VATS.

Methods:

Twenty patients scheduled for VATS under general anaesthesia were allocated randomly into 1 of 2 groups with 10 patients each. SAPB was performed before surgery with a single injection of $30 \mathrm{~mL}$ of $0.25 \%$ levobupivacaine. In the PVB group, single injection of $20 \mathrm{ml}$ of $0.25 \%$ levobupivacaine. Heart rate, mean arterial pressure, and the visual analog pain score (VAS) measurements were recorded for 24 hours. We also recorded consumption of Fentanyl during the surgery. Rescue analgesia using intravenous tramadol 50-100 mg, was administered if the VAS was $>5$.

Results:

Compared with preoperative values, the mean arterial pressure in the SAPB group did not change significantly in both groups $(p=$ 0.181 ). VAS scores and the total dose of tramadol consumed were comparable in the both groups. We recorded statistically higher consumption of Fentanyl during the surgery in SAPB group $(p<0,05)$. Conclusion:

The ultrasound guided SAPB appeared to be a safe and effective alternative for postoperative analgesia especially after VATS surgery. Serratus block provides effective regional anaesthesia, suitable for VATS surgery with easier to preform and with less complications. 
However, the higher consumption of opioids during the surgery indicates that PVB is superior in intraoperative analgesia.

\section{P8}

A multidisciplinary team approach in treatment of heart failure

Saltanat Dzetybayeva, Yuri Pya, Mahabbat Bekbossynova, Serik Bekbossynov, Saltanat Andossova, Svetlana Novikova, Timur Kapyshev

National Research Center for Cardiac Surgery, Astana, Kazakhstan

Correspondence: Saltanat Dzetybayeva

Journal of Cardiothoracic Surgery 2017, 12(Suppl 1):P8

Objective: Heart failure is a very common, disabling, and costly disease with a very difficult prognosis and poor quality of life. In Kazakhstan Heart Failure program started since 2011 with opening modern National Research Center for Cardiac Surgery in Astana. Our aim to illustrate our experience of multidisciplinary team approach in treatment of heart failure (HF) in our country.

Methods: Between November 2011 and April 2017 in our center was admitted 1768 patients with HF III-IV (NYHA), INTERMACS I-IV by multidisciplinary team which includes interventional cardiologist, cardiologist, anesthesiologist, cardiacsurgeon, psychologist, dietitian, pharmacist, VAD/transplant coordinators and nurses were analyzed clinical characteristics, laboratory indexes, CT scan, ECHO, catheterization parameters.

Results: In mostly cases 1043 (59\%) was recommended optimal medical therapy (OMT), 228 (12,95\%) underwent continuous-flow left ventricular assist device (CF-LVAD), $184(10 \%)$ implanted cardioverter - defibrillators (ICD) and 142 (8\%) CRTD, 122 (7,4\%) aortocoronary bypass surgery and $49(2,7 \%)$ heart transplantation was done.

Conclusion: An organised system of specialist heart failure care improves outcomes, including quality of life, the frequency and duration of follow up, and survival in patients with heart failure. A multidisciplinary team approach involving several professionals with their own expertise is important in attaining an optimal effect.

P9

One-step protocol of left ventricular reshaping and cell therapy in cases of ischemic dilative cardiomyopathy

Guglielmo Stefanelli', Fabrizio Pirro', Alina Olaru', Giuseppe Danniballe?', Paolo Giovanardi ${ }^{2}$

${ }^{1}$ Hesperia Hospital, Modena, Italy; ${ }^{2}$ University Hospitals, Modena, Italy Correspondence: Guglielmo Stefanelli

Journal of Cardiothoracic Surgery 2017, 12(Suppl 1):P9

Background: Aim of this study is to verify the potential advantages and benefits of a one step protocol of implant of bone-marrow derived autologous stem cells during surgical left ventricular restoration Methods : Between March,2007 and March,2013,30 patients affected by ischemic dilative cardiomyopathy received a surgical left ventricular restoration (SVR) at our unit. The patients were divided in two groups:16 patients were randomly assigned to receive Stem Cells Therapy (group A), 14 patients represented the control group (group B).The two groups were homogeneous in respect of age, gender, NYHA class, pre-operative mitral incompetence, and left ventricular size and volume.There was a significant difference as regards the EuroScore and Pre-operative Ejection Fraction, definitely worse in the group of patients who received stem cells therapy.

Results : All patients 30 days-in hospital mortality was 0 At last follow-up ejection fraction increased from $25,3 \%$ before surgery to $36,3 \%$ in group $A$, and from $31,8 \%$ to $45,6 \%$ in group $B$ ( $p>0,05)$. Reduction in LVEDD was $6 \%$ in group $A$, and $9 \%$ in group $B(p: n s)$ ESLVV decreased of $55 \%$ in group $A$ and $35 \%$ in group B,without statistical significance.Late cardiac mortality at 9 years follow-up was similar in the two groups of patients $(p=n s)$.No early or late adverse reaction or cases of infection were observed.
Conclusions : Patients affected by ischemic cardiomyopathy have a similar short and intermediate outcome after surgical ventricular restoration when a protocol of implant of autologous stem cell therapy is associated. However, observation at Pet-Scan of limited areas of myocardial tissue regeneration after cell therapy encourages the evolution and refinement of myocardial tissue engineering

P10

Association of gene polymorphisms with thrombosis and bleeding in patients with mechanical circulatory system

Madina Zhalbinova', Ainur Akilzhanova', Saule Rakhimova',

Makhabbat Bekbosynova², Saltanat Andossova ${ }^{2}$

'National Laboratory Astana, Nazarbayev University, Astana, Kazakhstan; ${ }^{2}$ National Research Center for Cardiac Surgery, National medical holding, Astana, Kazakhstan

Correspondence: Madina Zhalbinova

Journal of Cardiothoracic Surgery 2017, 12(Suppl 1):P10

Objective: Thromboembolism and gastrointestinal bleeding occurs often in patients with implanted left ventricular assist device (LVAD). The aim is to do genotyping of the genes encoding the stages of the blood clotting.

Methods: Venous blood samples were recruited from 100 patients with implanted LVADs. Patients were prescribed dose of the warfarin according to the clinical protocol of the Ministry of Healthcare of the Republic of Kazakhstan. The dose of the warfarin ranged from 0.625 to $8.125 \mathrm{mg} /$ day. According to the clinical characteristics patients divided into two groups: 1 . Uncomplicated group study - 79 patients (without bleedings and thrombosis); 2. Complicated group study- 21 (with bleeding, thrombosis). Genomic DNA was isolated using the PureLinkTM Genomic DNA Mini Kit (Invitrogen, UK). Genotyping of genes F5, F2 and ITGB3 was done by real-time polymerase chain reaction with TaqMan probes.

Results: The results of the genotyping showed that two groups of patients have only wild type of genotype G/G of F2 gene which is monomorphic SNP. Genotyping result of factor V Leiden (F5) showed wild type of genotype $(C / C)$ in 99 patients and in 1 patient heterozygote genotype C/T (control group). C/C genotype doesn't have risks to thrombosis, whereas heterozygote $\mathrm{C} / \mathrm{T}$ has risks to thrombosis. Patient with genotype $\mathrm{C} / \mathrm{T}$ was allocated into uncomplicated group according to the clinical data, but genotyping results represents opposite result - thrombosis risks. Statistical analyses of ITGB3 gene showed that heterozygote $C / T$ genotype is strongly associated to the risk of thrombosis (OR, 2.99; $95 \% \mathrm{Cl}, 1.05-$ 8.49; $\mathrm{P}=0.033$ ) and wild type of genotype $\mathrm{C} / \mathrm{C}$ in both groups.

Conclusions: Genotyping results showed that some patients of uncomplicated group have mutations to the risks of thrombosis. Patients need to be under control according to the genetic test results.

P11

Percutaneous driveline fracture following implantation of the left ventricular assist devices

Yuriy Pya, Serik Bekbossynov, Makhabbat Bekbossynova,

Assel Medressova, Saltanat Andossova, Saltanat Dzhetybayeva,

Muradym Murzagaliyev, Roman Salov

National Research Center for Cardiac Surgery, Astana, Kazakhstan

Correspondence: Assel Medressova

Journal of Cardiothoracic Surgery 2017, 12(Suppl 1):P11

Objective: Durability of the ventricular assist devices (VAD) percutaneous driveline is critical for the optimal long-term support of patients with end-stage heart failure. The purpose of this study was to evaluate the frequency of the driveline fracture and its effect on patient outcomes. 
Methods: In total, from November 2011 to April 2017, 226 patients underwent implantation of VAD in our Center. We use HeartMate II, CentriMag VAD и HeartMate 3 (St Jude Medical, Huntingdon, Cambridgeshire, UK), HeartWare HVAD (HeartWare International, Framingham MA, USA) assist devices. Median age of patients at VAD implantation was $50 \pm 13$ years $(9-76)$. There were $88,9 \%$ males $(n=201)$. Mean ejection fraction was $21,9 \pm 5,8 \% ; 44,69 \%(n=101)$ had an ischemic etiology, $38 \%$ of patients had idiopathic cardiomyopathy $(\mathrm{n}=86)$.

Results: Percutaneous driveline fracture was in 4 cases $(1,7 \%)$ with stopping of VAD function in 1 patient. In one case, the damage occurred by chance (on $18^{\text {th }}$ month of VAD support), in two patients - as a result of psychoorganic syndrome during the early postoperative period ( 4 and 8 days after operation), and in the forth case a cat was gnawing the driveline while the patient was asleep (on $14^{\text {th }}$ month after VAD implantation). In three cases cables were fully restored by engineer without any adverse effect on the further postoperative period. In one case VAD thrombosis occurred after driveline damage, and this patient is currently on the waiting list for heart transplantation.

Conclusions: These results indicate a small number of cases of the percutaneous driveline fracture following VAD implantation. Lifethreatening complications unlikely developed with timely full restoration of the continuity and function of the VAD driveline.

P12

Treatment of cardiopericardial echinnococos

Sokhib Mirzzakhmedov, Khamidulla Abdumadjidov, Khayrulla Buranov, Ilkhom Khodjakulov

Republican Scientific Center of Surgary named after V.Vakhidov,

Tashkent, Uzbekistan

Correspondence: Khamidulla Abdumadjidov

Journal of Cardiothoracic Surgery 2017, 12(Suppl 1):P12

Objective: Echinococcosis of the heart is extremely rare, only in 0.5$2 \%$ of patients.

Methods: 39 patients (100 \%) in RSCS operated apropos cardiopericardial echinococcus. Patients have been divided into 2 groups: I group - 27 patients $(69,2 \%)$ with the isolated echinococcus heart or a pericardium; II group12 patients $(30,8 \%)$ with combined echinococcus heart and other organs (liver, lungs). The average age composed of $20,7 \pm 2,7$. Male was - $12(30,8 \%)$, female - 27(69,2\%). Cardiopericardial echinococcus was diagnosed by X-ray of thorax, CT scan, transthoracic and transesophageal ECHO. Operative intervention in 30 cases $(80,3 \%)$ was performed by cardiopulmonary bypass (CPB-65,0 $\pm 7,6 \mathrm{~min}$ ) and cardioplegy (CP 45,3 $\pm 4,9 \mathrm{~min}$.).

Results: general hospital mortality has made 7,7 \% (3 patients). In I group mortality was observed in one case $(3,7 \%)$ because of spontaneous rupturing intense parasitic cyst in a heart cavity at the preoperative period. Postoperative mortality at patients of I group was not observed, in II group was 2 patients). In 1 cases multifocal defeat of heart, a pericardium, lungs and mediastinum took place. At the stage of opening the clinical picture of anaphylactic shock was observed which has led to a fatal outcome. In the second case at removal of echinococcus brings to damage of septal branches forward interventricular arteries with development of heart failure.

Conclusions: For timely revealing cardiopericardial echinococcus, a prominent aspect is performance $\mathrm{ECHO}$ of research to all patients with echinococcus - targets (lungs, a liver).Operative intervention an uncontested method of treatment at cardiopericardial echinococcus and echinococcectomy from heart is expedient for carrying out in the conditions of CPB and CP. At combined echinococcus of heart and bodies-targets (lungs, a liver) performance simultaneous operations is expedient: primarily it is necessary to carry out echinococcectomy from heart, then - from other organs (a liver, lungs).
P13

Cardiomyopathy and valvular dysplasia: accidental combination or related pathology

Sergey Dzemeshkevich

NRSC, Moscow, Russian Federation

Journal of Cardiothoracic Surgery 2017, 12(Suppl 1):P13

Objectives: The shortage of donor organs leads researchers to considering new solutions for the treatment of patients with cardiomyopathies and heart failure. One of the areas of scientific research is the development of organ-preserving techniques of surgical reversal cardiac remodeling. With this approach, the question of the etiology of cardiomyopathies becomes extremely important for the selection of the optimal therapeutic strategy.

Methodology: Since 2009, 55 surgeries to reverse cardiac remodeling have been performed in 31 patients with dilated cardiomyopathy (DCM) and 24 patients with obstructive hypertrophic cardiomyopathy (HCM) of a special diffused generalized type. To elucidate the etiology, molecular genetic study was performed in all the patients.

Results: In the DCM group, 29 patients were discharged from hospital; in the 5 year period after surgery one patient underwent a successful heart transplant. The patients with diffuse generalized HCM were also observed for 5 years and showed good results, despite a high prevalence of multisystem disorders: progressive myopathies, lysosomal storage diseases, and RASopathies were found in $32 \%$. However, the most unusual result of the analysis was the fact that $100 \%$ of the patients with DCM and HCM had a classical morphological confirmation of the myxomatous transformation of the mitral valve.

Conclusion: For the most part, DCM and HCM are genetically determined. The combination of this genesis of cardiomyomathies with genetically determined valvular dysplasia allows one to speak of a linked pathogenesis of the pathology and gives grounds for developing unified protocols of gene molecular therapy. The surgical treatment is also necessary as a condition for rapid normalization of intracardiac hemodynamics.

P14

Gastric perforation caused by a left ventricular assist device

Yuriy Pya, Serik Bekbossynov, Makhabbat Bekbossynova,

Saltanat Andossova, Saltanat Dzhetybayeva, Nurgul Konakbayeva,

Assel Medressova, Muradym Murzagaliyev, Svetlana Novikova

National Research Center for Cardiac Surgery, Astana, Kazakhstan

Correspondence: Assel Medressova

Journal of Cardiothoracic Surgery 2017, 12(Suppl 1):P14

Objective: This report describes a rare complication of gastric perforation caused of a left ventricular assist device (VAD).

Methods: From November 2011 to March 2017, 226 patients underwent implantation of 232 VADs at our Center. We use four devices for mechanically circulatory support - the HeartMate II, CentriMag VAD и HeartMate 3 (St Jude Medical, Huntingdon, Cambridgeshire, UK), HeartWare HVAD (HeartWare International, Framingham MA, USA). Two patients with HeartMate II left VAD had a pump thrombosis (due to low international normalized ratio) with gastric perforation $(0.88 \%)$ on $851^{\text {st }}$ and $1045^{\text {th }}$ days after the operation. Both patients complained on the sense of the heat in left subcostal area. Patients were admitted to our Center. In both cases on video assisted gastric endoscopy we found fistula of the great curvature with clear rounded shape, and the pump body was visualized through the stomach.

Results: Pumps were turned off. Both patients were underwent partial assist device explantation with suturing the stomach defect. In one case bleeding from the wound was developed on $23^{\text {rd }}$ day after the operation, and patient died. In the second case new gastric perforations were diagnosed on the $7^{\text {th }}$ day after the operation, and 
patient was transferred to the general surgery department of another hospital for the operation. This patient died at home probably due to infection.

Conclusions: These two reported cases show that gastric perforation is a rare, but serious complication of left VAD therapy and it should be kept in mind in the treatment of patients with VAD thrombosis.

P15

Primary Cardiac Angiosarcomas: A Review Of Three Cases

Thomas Strecker, Abbas Agaimy, Michael Weyand

Friedrich-Alexander-University of Erlangen-Nuemberg, Erlangen, Germany

Correspondence: Thomas Strecker

Journal of Cardiothoracic Surgery 2017, 12(Suppl 1):P15

\section{Withdrawn}

P16

Special aspects of ALCAPA in children of different age

Tatyana I. Ivanova-Razumova, Lyazzat Abikeeva, Almira Baigalkanova, Maira Uroganova, Aikerim Zhanatkyzy

National Research Center for Cardiac Surgery, Astana, Kazakhstan

Correspondence: Tatyana I. Ivanova-Razumova

Journal of Cardiothoracic Surgery 2017, 12(Suppl 1):P16

Objective: We decide to determine peculiarities of ALCAPA clinical course in different age groups of children.

Methods: Data's of 11 patients with ALCAPA were analyzed. Mean age of patients is $19 \pm 7$ months ( 1 month -7 years).Two groups were identified: I group - ALCAPA was diagnosed before the age of 2 years, II - patients with late manifestation. We evaluated them pre-, postoperatively and 3 month after. Statistica 6,0 was used for analyses.

Results: I group - 6 babies, mean age - $10 \pm 3,4$ months, II - 5 children, mean age $5 \pm 1.2 \mathrm{y}$. Initially in I group patients had CHF III IV (Ross), mean LV EF - 16 $\pm 5 \%$, mean pro-BNP level $-12000 \pm 2.500$ $\mathrm{pg} / \mathrm{ml}$. II group preoperatively - CHF FC II, mean LV EF - $53 \pm 4,5 \%$, pro-BNP level - $240 \pm 15 \mathrm{pg} / \mathrm{ml}$. In that group good collaterals were visualized by $\mathrm{ECHO}$ and angiogramm. Early postoperative period: in I group - EF LV - $19 \pm 2,3 \%$, pro-BNP level of $10,200 \pm 1250 \mathrm{pg} / \mathrm{ml}$ (the differences were not reliable). 2nd group, the LVEF and pro-BNP level remained practically unchanged. 3 month after in I group - LVEF-32 $\pm 3.5 \%(p<0.05)$, the level of pro-BNP decreased significantly - 4500 $\pm 860 \mathrm{pg} / \mathrm{ml}(\mathrm{p}<0.05)$. II group - LV EF, pro-BNP reached normal values.

Conclusions: clinical course and postoperative results for ALCAPA syndrome differ significantly for children of different age. ALCAPA among young children is characterized by the development of HF of high level of severity and slow recovery of myocardial contractility. A more favorable course of ALCAPA when detected in older age can be explained by the presence of collateral blood flow. Further study of the factors that contribute to the formation of collaterals represent great scientific interest.

P17

Modern tendencies in surgery of cicatricial stenosis of trachea

Vladimir Parshin, Zelimkhan Berikkhanov, Mikhail Rusakov,

Aleksey Parshin

I.M. Sechenov First Moscow State Medical University, Moscow, Russian Federation

Correspondence: Vladimir Parshin

Journal of Cardiothoracic Surgery 2017, 12(Suppl 1):P17
Objective: to improve results of treatment patients with cicatricial stenosis.

Methods: from 1963 to 2015 were operated 1128 patients with cicatricial stenosis of trachea, which were divided into two groups: the first - from 1963 to 2000 years (297 patients), the second - from 2001 to 2015 years (831 patients). Resection of the trachea with anastomosis were performed in 389 (34.48\%) patients. The frequency of this operation in the second group increased more than in 20 times. Staged operations with T-tube were performed in 667 (59.13\%) patients. Endoscopic treatment received in 72 (6.38\%) patients.

Results: the overall incidence of complications was 6.8\%. Died 19 patients $(1,1 \%)$. After the tracheal resection complications decreased from $32.2 \%$ in the first group to $14.6 \%$ in the second. Mortality reduced more than in 30 times: $12(20,3 \%)$ patients in the first group of patients to $2(0,6 \%)$ - in the second. The main reason of death was arrosive bleeding. Prevention of this complication has allowed to improve the immediate result. After staged operations 5 patients died. The main cause of death was also bleeding. In the second group of patients after the tracheal resection and staged operations with T-tube the frequency of postoperative complications and the mortality rate amounted to 14.6 and $2.2 \%, 0.6$ and $0.2 \%$, respectively. Conclusions: despite of larger operations size with tracheal stenosis, the rate of complications and mortality tends to decrease and this is the reason for a more aggressive surgical approach.

P18

Adrenalectomy for isolated adrenal metstases in operable patients with nsclc- two institutions study

Georgy Yankov', Anatoli V. Semkov ${ }^{1}$, Svetlana Shumarova², Danail Petrov ${ }^{\top}$

${ }^{1}$ Thoracic Surgery Department, Saint Sophia University Hospital for Pulmonary Diseases, Medical University, Sofia, Bulgaria; ${ }^{2}$ Depertment of Surgery, University Hospital Aleksanrdovska, Medical University Sofia, Sofia, Bulgaria

Correspondence: Anatoli V. Semkov

Journal of Cardiothoracic Surgery 2017, 12(Suppl 1):P18

Objective: To evaluate the outcome after resection of solitary adrenal metastases in operable NSCLC patients.

Methods: 11 patients- 7 males and 4 females, mean age 58,4 (43-67) years, underwent adrenalectomy for isolated NSCLC metastases- 6 synchronous and 5 metachronous. 4 of them were contralateralr and 7 ipsilateral.The patients were precisely assessed preoperativelyincluding PET- CT in 7 cases and mediastinoscopy in 3 of them. The pathoanatomically approved locoregional stage was IA in 2 patients, IB in 2, IIA and IIB in 3 and IIIA in 1 patient (single station N2 disease - N5). The histology was adenocarcinoma in 8 patients, squamous cell cancer in 2 and carcinosarcoma in 1 patient.

Results: One stage operation was performed in 1 patient by phrenotomy (left lower lobectomy and left adrenalectomy) and double stage in 10 . The lung resection was carried out first in 9 patients with double stage operation - 2 left lower lobectomies, 2 left pneumonectomies, 2 left upper lobectomies and 1 bilobectomy, followed by adrenalectomy via paracostal laparotomy in 3 and REA in 6 patients. In 1 patient REA was performed first, followed by right lower lobectomy. In 1 patient the first stage was left lower lobectomy, the second was left sided REA for metachronous metastasis, and polysegmental resection of the right lower lobe was performed after that for contralateral lung lesion. 6 patients are alive until the last follow up at the end of December 2016. The median survival at the other five patients is 26.1 (6-41) months. The postoperative period was uneventful for all patients. All of them 
underwent adjuvant chemotherapy, excluding one EGFR + patient, who underwent target therapy with Gefitinib.

Conclusions:

Long term survival is possible after resection of isolated NSCLC metastases in carefully selected patients with early locoregional stage.

\section{P19}

The transtracheal approach to the tracheoesophageal fistula

Valeriy Parshin, Vladimir Parshin, Juriy Terehov

I.M Sechenov First Moscow State Medical University, Moscow, Russian

Federation

Correspondence: Valeriy Parshin

Journal of Cardiothoracic Surgery 2017, 12(Suppl 1):P19

Objective: : to improve the variants of surgical treatment of acquired nonmalignant tracheoesophageal fistula (TEF) in adult patients.

Methods: the transtracheal approach means circular and transversal incision of trachea at the level of TEF. The feature of this approach is saving the area of membranous wall of trachea around the ostium of TEF. It allows to suture esophageal defect in 2 layers without narrowing the lumen. Vascularized muscle flap is fixed between trachea and esophagus to isolate them. Wholeness of air passage is restored by intertracheal anastomosis.

Results: during the period from 1963 to 2015123 patients with TEF were operated. $71(57,7 \%)$ had TEF in combination with cicatrical stenosis of trachea (CST). As an operative approach cervicotomy was used in $67,6 \%$ cases, cervicosternotomy- $16,2 \%$, posterior thoracotomy with resection of rib- $8.8 \%$, right lateral thoracotomy$4,4 \%$, cervicosternotomy in combination with laparotomy- 2,9\%. From 2012 to 201512 patients $(9,76 \%)$ with TEF were operated by transtracheal approach. 8 of them had TEF combined with CST. Good result were achieved in 11 patients $(91,7 \%)$ out of 12 . Complication such as anastomositis was observed in 2 cases, but it was managed by conservative therapy. $1(8,3 \%)$ had a recurrence of TEF associated with original bilateral pneumonia.

Conclusions: the transtracheal approach to the TEF is safe, provides good visualization and reliable closure esophageal defect, without narrowing the lumen, minimize the risk of damage recurrent laryngeal nerves. This method at the combination of TEF with the CST at the fistula level makes possible to heal the patients by one operation. Transtracheal approach is indicated in cases of sternotomy in the localization of TEF at the suprabifurcation level of trachea and also when performing right lateral thoracotomy is impossible for any reason.

\section{P20}

A rare cause of massive hemoptysis: obliteration of an atypical branch originating from the right coronary artery

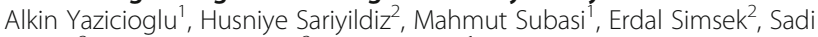
Kaplan², Omac Tufekcioglu³, Erdal Yekeler ${ }^{1}$

${ }^{1} T$ urkiye Yuksek Ihtisas Training and Research Hospital, Thoracic Surgery and Lung Transplantation Clinic, Ankara, Turkey; ${ }^{2}$ Turkiye Yuksek Ihtisas Training and Research Hospital, Department of Cardiovascular Surgery, Ankara, Turkey; ${ }^{3}$ Turkiye Yuksek Ihtisas Training and Research Hospital, Department of Cardiology, Ankara, Turkey

Correspondence: Alkin Yazicioglu

Journal of Cardiothoracic Surgery 2017, 12(Suppl 1):P20

Objective: Hemoptysis associated with necrosis of lung parenchyma supplied by abnormal vascular structure, is rare condition.

Methods: 43-year-old, female; underwent mitral-valve replacement (MVR).

Results: During preoperative cardiac-catheterization; a vessel branch originating from right coronary-artery (RCA) was observed to supply left-upper lobe(LUL). During MVR, the vessel branch originating from
RCA and supplying LUL was dissected and released, but was unable to be ligated. Patient suffered from massive-hemoptysis on postoperative day5 $(>600 \mathrm{~mL} /$ day). Thoracic $C T$ revealed a consolidated area in the posterior segment of the LUL; the etiology was considered to be associated with the vessel branch originating from RCA. Selective embolization was planned for this vessel branch. However, thrombosis was observed in this vessel during catheterization which was performed with the purpose of embolization. In this case, the etiology was realized to be thrombosis of the aberrant-artery and associated infarction in the LUL, and hemoptysis was secondary to this infarction.

Conclusions: Although lungs receive abnormal branches originating from a large number of arterial structures (i.e., subclavian, internal mammarian, vertebral), branches originating from the coronary arteries are rare. Abnormal vascular structures can play a role in the etiology of hemoptysis, although pulmonary infarction can also play a role in the etiology of hemoptysis. In our case, the LUL was supplied by the aberrant artery originating from the RCA. It was found that thrombosis occurred in the artery during manipulation to release artery during surgery. With the interruption of blood supply, necrosis and infarction occurred in the supplied lung area, and hemoptysis was associated with necrosis. In differential diagnosis, aberrant vessel itself was thought to cause massive hemoptysis. However, the lack of flow in aberrant vessel suggested another etiological cause. Hemoptysis associated with abnormal vascular structures has been frequently reported in literature; however, hemoptysis associated with obliteration of abnormal vascular structure was scarcely reported.

Consent for publication

Written informed consent to publish was obtained from the patients involved in this study.

P21

Uniportal video-assisted thoracoscopic surgery for pulmonary sequestration

Harish Mithiran Muthiah, Kingsfield Ong, Jai Ajitchandra Sule, John Kit

Chung Tam

National University Hospital, Singapore, Singapore

Correspondence: Kingsfield Ong

Journal of Cardiothoracic Surgery 2017, 12(Suppl 1):P21

Background Pulmonary sequestration is a rare congenital lung malformation where part of the lung has an aberrant blood supply without identifiable communication with the main bronchial tree. Wedge resection or lobectomy via a postero-lateral thoracotomy or video-assisted thoracoscopic surgery (VATS) are the main modalities of treatment.

Methods We report a case series of 3 consecutive patients who underwent Uniportal VATS for the treatment of (two intralobar and one extralobar respectively) pulmonary sequestration. The first patient was a 19-year-old male who presented with a recurrent chest infection and haemoptysis requiring hospital admission and antibiotics. The second was a 14-year-old male with tension pneumothorax and persistent pneumothorax after chest tube insertion. The third patient was a 44-year-old smoker with an incidental finding of a left retrocardiac opacity on chest $x$-ray. The aberrant artery was demonstrated on computed tomography of the chest and thoracoscopically in all patients. We performed a Uniportal VATS right lower lobe lobectomy, left lower lobe lobectomy and wedge resection of the left lower lobe respectively.

Results All patients were discharged within a week post-operatively. There was no re-intervention, complication or mortality in this study. They remained well at the 3-5 year follow-up review.

Conclusion This case series demonstrated reproducible and safe outcomes in the treatment of pulmonary sequestration with the Uniportal VATS approach. 


\section{Consent for publication}

Written informed consent to publish was obtained from the patients involved in this study.

\section{P22}

Surgery for diaphragmatic hernias and eventration with primary sutured closure and prosthetic reinforcement

Mikhail Khetagurov, Vladimir Parshin

MSMU, Moscow, Russian Federation

Correspondence: Mikhail Khetagurov

Journal of Cardiothoracic Surgery 2017, 12(Suppl 1):P22

\section{Objective:}

The anatomical location and important functional significance of the diaphragm explain the manifested interest in patients with diseases of the diaphragm of doctors of various directions. This study seeks to evaluate results regarding the repair of non-neoplastic disease of the diaphragm.

Methods: This study retrospectively investigated patients between may 1963 and march 2017 who underwent surgical treatment for non-neoplastic disease of the diaphragm. We recordered follow-up data and long-term effects by questioning patients by mail and telephone.

Results:

During the study period, 62 patients were operated (30 male and 32 female adults, mean-age 48,3 years). In this series, hernias of the weak phrenic zones and diaphragm $(n=27)$, eventration $(n=26)$ and posttraumatic diaphragm hernia $(n=9)$ were treated. The majority of patients had no complaints and disease was diagnosed on the X-ray examination. Rarely, the compression syndrome, caused by translocation of the abdominal organs in the thoracic cavity, was registered. Repair of traumatic defect and anatomically weak spots was performed using primary suture, with the exception of two cases of posttraumatic diaphragmatic hernia, when mesh prosthesis was used. In cases of the diaphragm eventration new diaphragm dome located on a normal level and consisting of two flaps and a mesh prosthesis between them was shaped. Postoperative complications $(8 / 60$ or $13,3 \%)$ were minimal, $1(1,7 \%)$ mortality was observed. No recurrences were observed.

Conclusions: With a traumatic defects and anatomically weak zones of the diaphragm, it is permissible to perform the primary sutured closure. In reconstruction of the diaphragm eventration, it is necessary to use reinforcing material.

P23

Combined lung resection in the combined treatment of resectable stage nonsmall-cell lung cancer

Marina Mironchenko, Andrey Lukin, Andrey Vazhenin, Evgeniy Mantcirev, Yakov Gnatyk, Rudolf Gatyatov, Sergey Timofeev, Egor Pecheritca,

Elizaveta Kolosova

Chelyabinsk regional oncology centre, Chelyabinsk, Russian Federation

Correspondence: Marina Mironchenko

Journal of Cardiothoracic Surgery 2017, 12(Suppl 1):P23

Objective: evaluate the role combined lung resection and adjuvant radiotherapy in improving survival rate in resectable stage nonsmallcell lung cancer.

Methods: Retrospective comparison of parallel groups was carried out. It is included 135 patiens with IIb, III stage NSCLC. Group Apatients being performed only pneumonectomy $(n=60)$, group Bpatients being undergo pneumonectomy and adjuvant radiotherapy $(\mathrm{n}=32)$. The group C - patients with combined lobectomy or combined bilobectomy $(n=24)$, and group $D$ - patients who were performed combined lobectomy or combined bilobectomy and adjuvant radiotherapy $(n=19)$. The mean age was $56+7,8$ years old. The stage Ilb occured more often and composet $48,1 \%$. The smallest percentage $(13,3 \%)$ was in stage Illb. The superior portion of the left lung was affected in 53 cases (39,3\%). The central cancer was seen at 102 patients $(75,6 \%)$. Histologic forms of squamous cell seems to prevail among the other forms (102 cases - 75,6\%). It has been performed 92 pneumonectomy $(68,1 \%), 39$ combined lobectomy $(28,9$ $\%)$. Among them was performed $25,2 \%$ expanded operations, $34,1 \%$ expanded and combined operations, 28,1\% - combined resections and $12,6 \%$ - typical pneumonectomy. The most often type of the combined operations was vasculoatrial - $51 \%$ (53 resections). Parietalodiaphragm resections were preformed less often - $13 \%$ (13 resections). There was preformed $36 \%$ of tracheobronhoplastycal resections.

Results: Postoperative Morbidity was $4,4 \%(p=0,049)$. Postoperative complications was $18,5 \%$. There was $14,8 \%$, after the pneumonectomy executing, and $3,7 \%$ after the perform of combined lobectomy $(p=0,03) .5$ years survival rate in group $A: 21,2 \%$. In group B: $25 \%$, In group C: $26,1 \%$, in group D: $26,3 \%(p>0,05)$.

Conclusions: The first choice for surgical treatment of patients with resectable stage II b and III Non-Small-Cell lung cancer is combined lobectomy.

P24

A primary pulmonary synovial sarcoma: a case of an uncommon tumor

Alkin Yazicioglu', Mahmut Subasi', Seda Yamak², Sinan Turkkan', Erdal Yekeler ${ }^{1}$

${ }^{1}$ Turkiye Yuksek Ihtisas Training and Research Hospital, Thoracic Surgery and Lung Transplantation Clinic, Ankara, Turkey; ${ }^{2}$ Turkiye Yuksek Ihtisas

Training and Research Hospital, Department of Pathology, Ankara, Turkey

Correspondence: Alkin Yazicioglu

Journal of Cardiothoracic Surgery 2017, 12(Suppl 1):P24

Objective: Synovial sarcoma is a soft tissue tumor which predominantly affects young adults. The tumor is usually diagnosed in the periarticular regions of extremities; while primary pulmonary synovial sarcoma (PPSS) is uncommon.

Methods: A 40-year-old male with incidentally detected left-lowerlobe mass was evaluated.

Results: The thoracic $C T$ revealed $6 \times 7 \times 8 \mathrm{~cm}$ solid mass with a suspicion of diaphragmatic invasion and SUVmax of the mass was calculated as 5.28 on PET-CT. The PET-CT revealed no other organ involvement except the lung. Left lower lobectomy with diaphragmatic resection and prolene mesh reconstruction was performed. Postoperative period was uneventful and he was discharged on day 10. The pathological examination was evaluated as a monophasic-type synovial sarcoma and a fluorescence in-situ hybridization(FISH) examination was planned. There were findings supporting the presence of translocation including SYT-gene. Bone scintigraphy was performed due to the possibility of primary bone focus. However, no findings were encountered in the other joints or bones. Based on these findings, the case was diagnosed as PPSS.

Conclusions: PPSS are uncommon tumors which represent $0.5 \%$ of all lung malignancies. This tumor has four subtypes, including monophasic-fibrous (spindle), monophasic-epithelial, biphasic, and poorly-differentiated. However, the monophasic subtype is the most common. Immunohistochemistry plays an important role for diagnosis; however, gold standard is genetic analyses. Synovial sarcoma is characterized by reciprocal chromosomal translocation $t(X: 18)(p 11.2: q 11.2)$, which results from the fusion of the SYT-gene on chromosome 18 to either of two genes, SSX1 and SSX2, on chromosome X. PPSS is an aggressive tumor with a poor prognosis. Poor prognostic factors include size ${ }^{\prime} 5 \mathrm{~cm}$, male sex, age'20years, extensive tumor necrosis, large number of mitotic-figures ( $>10 / 10$ highpowered fields), neurovascular-invasion, and SYT-SSX1 variant. Present treatment includes complete surgical resection, followed by chemotherapy and/or radiotherapy. Our case received both chemotherapy and radiotherapy and was still uneventful 31-months after the operation. 


\section{Consent for publication}

Written informed consent to publish was obtained from the patients involved in this study.

\section{P25}

Huge dilatation of the azygos vein (aneurysms of the azygos vein) mimicking mediastinal mass

Fadil Gradica', Lutfi Lisha' ${ }^{1}$ Dhimitraq Argjiri ${ }^{2}$, Alma Cani ${ }^{3}$, Flora Gradica ${ }^{4}$, Ajshe Lala ${ }^{4}$, Fahri Kokici ${ }^{5}$, Valbona Rexha ${ }^{4}$, Safet Beqiri ${ }^{6}$, Shqiptar

Demaci ${ }^{7}$, Agron Poniku', Saud Maliqii', Albert Leka ${ }^{8}$

"University Hospital "Shefqet Ndroqi" Thoracic Surgery Service, Tirane, Albania; ${ }^{2}$ University Hospital "Shefaet Ndrogi" Pneumology Service, Tirane, Albania; "University Hospital "Shefqet Ndroqi" Anestesie reanimation Service, Tirane, Albania; ${ }^{4}$ Public pharmacy service, Tirane, Albania; ${ }^{5}$ University Hospital "Shefqet Ndroqi" Anestesie reanimation Service, Tirane, Albania; ${ }^{6}$ Kerckhoff-Klinik GmbH, Bad Nauheim Germany, Germany; ${ }^{7}$ QKUK, Prishtine, Republic of Kosovo; ${ }^{8}$ Imagery privat Service Lezhe, Lezhe, Albania

Correspondence: Fadil Gradica

Journal of Cardiothoracic Surgery 2017, 12(Suppl 1):P25

Objective: We report a case of azygos vein varix mimicking a mediastinal mass in a patient with liver cirrhosis. Huge dilatacion of the azygos vein (Aneurysms of the azygos vein) are rare and can sometimes mimic a paratracheal or posterior mediastinal mass. It is important to confirm the diagnosis with radiologic tools before performing invasive procedures, which carry the risk of hemorrhage. Methods: The usual diagnosis of a mediastinal mass by mediastinoscopy or percutaneous fine-needle aspiration or biopsy is very hazardous if there is a venous varix. Noninvasive thoracic CT scanning is a safe and better choice for diagnosis. Here, we present a case in a 46-year-old man symptomatic patient of an increasing azygos vein aneurysm that mimicked a growing paratracheal mass and posterior mediastinal mass. Review of images obtained using various modalities, including contrast CT scanner without dynamic magnetic resonance image $(\mathrm{MRI})$, revealed that the image findings were suggestive of azygos vein huge dilatation of the azigos vein (aneurysm). Using this method, an exact diagnosis can be reached without resorting to invasive procedures or with mini-invassiv VATS thorax surgery. Results: We used VATS surgery for diagnosis confirmation converted in right minithoracotomy. The main causes of a dilated azygos vein include portal vein hypertension, obstruction of the superior vena cava, hypertension in the right-heart chamber, Budd-Chiari syndrome, hypervascular tumor draining into the azygos system, posttraumatic pseudoaneurysm, kinking of the aorta, and pregnancy.

Conclusions: In some cases, no definitive cause for Huge dilatacion of the azygos vein (Aneurysms of the azygos vein)is found only by imagery examination and invasiv investigation may have high risk for massive hemoragie complication.Some time we need to do VATS confirmation.

\section{Consent for publication}

Written informed consent to publish was obtained from the patients involved in this study.

\section{P26}

Chylotorax following lung transplantation in a case with pulmonary alveolar lipoproteinosis. is lymphatic canal ectasia related to disease pathogenesis?

Alkin Yazicioglu', Mahmut Subasi', Sinan Turkkan', Fatmanur Celik Basaran', Funda Demirag ${ }^{2}$, Sema Turan ${ }^{3}$, Erdal Yekeler

${ }^{1} T$ urkiye Yuksek Ihtisas Training and Research Hospital, Thoracic Surgery and Lung Transplantation Clinic, Ankara, Turkey; ${ }^{2}$ Ataturk Chest Disease and Thoracic Surgery Training and Research Hospital, Department of Pathology, Ankara, Turkey; ${ }^{3}$ Turkiye Yuksek Ihtisas Training and Research Hospital, Anesthesiology and Intensive Care Unit, Ankara, Turkey

Correspondence: Alkin Yazicioglu

Journal of Cardiothoracic Surgery 2017, 12(Suppl 1):P26
Objective: Pulmonary-alveolar-lipoproteinosis (PAL) is rare indication for lung transplantation(LuTx), and relationship between PAL and lymphatic system(LS) is unknown.

Methods: 39-year-old male, underwent bilateral-LuTx for PAL.

Results: After starting oral-intake at day-3, chylous drainage was observed from left-hemithorax (triglyceride: $322 \mathrm{mg} / \mathrm{dL}$ ). No adhesions have observed in mediastinum intraoperatively, no complication has occurred during surgery. Pathologic examination confirmed PAL; dilation, ectasia of the lymphatic ducts(LD) were observed during examination of LS at the level of main-bronchus. Conventional therapies failed during follow-up of chylothorax, he underwent right minithoracotomy and ductus-thoracicus mass-ligation at day-18. Drainage ceased, patient was switched to normal diet, discharged at day- 26 .

Conclusions: Surfactant (90\% lipids) is broken down in alveoli and aggregates are phagocytized by typell cells and macrophages. Typell cells are able to recycle the phagocytized aggregates, while macrophages only catabolize. This catabolic process is regulated by GM-CSF. A blockade in this pathway has been implicated in pathogenesis of PAL. Disruption in pathway prevents terminal differentiation of macrophages. Therefore, macrophages are unable to catabolize the phagocytized surfactant-fragments. Surfactant aggregates will inevitably accumulate in alveolar space with disruption of surfactant catabolism in macrophages. At this point, we consider that LS steps in, to remove lipids from alveoli and attempts to compensate. LS work to remove lipid load in alveoli; however, presence of vast amount of lipids results in dilation in LS and ectasia of the main pulmonary LD around main bronchus. We consider that these ectatic ducts were incised with incision of the main bronchus, resulted in chylothorax due to close proximity. There is no data in the literature regarding changes in pulmonary lymphatic tissues in patients with PAL. Dilation and ectasia of LD is associated with disease pathogenesis, and risk factor for chylothorax following LuTx. Careful dissection at hilar level and/or mass-ligation of ductus-thoracicus can be considered to avoid chylothorax complication.

Consent for publication

Written informed consent to publish was obtained from the patients involved in this study.

\section{P27}

Surgical resection and long-term disease-free survival in advanced lung adenocarcinoma after erlotinib downstaging: a report of two cases

Danail Petrov', Georgy Yankov', Anatoli V. Semkov', J. Mihailova²,

Elyuar Goranov'

${ }^{1}$ Thoracic Surgery Department, Saint Sophia University Hospital for

Pulmonary Diseases, Medical University, Sofia, Bulgaria; ${ }^{2}$ Medical

Oncology Ward, Military Medical Academy, Sofia, Bulgaria

Correspondence: Anatoli V. Semkov

Journal of Cardiothoracic Surgery 2017, 12(Suppl 1):P27

Objective. To assess the safety and survival benefit of surgery in initially inoperable lung adenocarcinoma patients who responded to first-line EGFR tyrosine kinase inhibitor (TKI) therapy with erlotinib.

Methods: Two female adenocarcinoma patients (P1 and P2) were initially staged as CT3N2M1b (malignant pleural effusion) and $c$ T2aN3M0. Pretreatment biopsies by bronchoscopy (P1) and scalene node biopsy (P2) harbored EGFR mutations: exon 21 L858R and exon 19 E746-T751insl deletions. The first-line treatment with erlotinib 150 $\mathrm{mg} /$ daily was administered in both patients. After ten (P1) and six (P2) months of treatment, imaging methods (FDG-PET/CT) and mediastinoscopy (P1) showed down-staging: cT2 bNOMO and CT1bNOMO.

Results: Extended right pneumonectomy (P1) (v. azygos resection) and left upper lobectomy (P2) were performed. Both postoperative periods were uneventful. On postoperative pathomorphology adenocarcinoma and EGFR mutations were identified. Adjuvant erlotinib treatment was initiated in both patients. Five month following surgery asymptomatic disease progression (osseous metastases) was observed in P1. At the end of the study she was 
with ECOG PS 1 and still on erlotinib (6 months). Initially erlotinib was applied for only 2 months in P2. Four months after treatment cessation $3 \mathrm{~cm}$ left supraclavicular mass (SUV-2.0) was diagnosed and resected. Adenocarcinoma involvement of 3 lymph nodes and EGFR mutation were confirmed. The patient continued on erlotinib and was monitored with MDCT scan every 3 months and a PET after 6 . No progression has been documented.

Conclusions: Treatment with erlotinib may lead to down-staging in initially inoperable patients with lung adenocarcinoma. Whether this therapy can be discontinued in such patients remains to be shown. The optimal duration of EGFR-TKI treatment, the timing of surgery, and the role of adjuvant EGFR-TKI therapy should be investigated as well.

\section{P28}

The risk of secondary metachronous malignant tumors after the treatment of lung cancer in men

Nasrulla Shanazarov ${ }^{1}$, Elena Shunko², Andrey Vazhenin ${ }^{3}$

${ }^{1}$ Medical Centre Hospital of the President affairs administration of the

Republic of Kazakhstan, Astana, Kazakhstan; ${ }^{2}$ Tyumen Medical University, Tyumen, Russian Federation; ${ }^{3}$ South Ural Medical University, Chelyabinsk, Russian Federation

Correspondence: Nasrulla Shanazarov

Journal of Cardiothoracic Surgery 2017, 12(Suppl 1):P28

Objective: Identification of the localization of the greatest risk of secondary metachronous malignant tumor after the treatment of lung cancer in men.

Methods: Archival data of the Chelyabinsk Regional Clinical Oncology Center of the treatment of 255 men with primary-multiple metachronous malignant tumors is analyzed. Group 1 comprised of 26 men after chemotherapy, group 2 - 82 men after radiotherapy, group 3 - 10 men after chemoradiotherapy, group $4-137$ men after surgical treatment of primary tumor.

Results: In the general structure of the localizations of primary tumor, lung cancer was presented: in the group $1-7.6 \%$, in the group $2-19.5 \%$, in the group $3-30.0 \%$, in the group $4-8.8 \%$. The secondary metachronous tumor was: in the group 1 - skin and digestive tumors $-50.0 \%\left(x^{2}=55.300, p=0.016\right)$; in the group 2 tumors of respiratory organs $-37.5 \%$, skin $-31.3 \%$, digestive and male genital organs $-12.5 \%$, urinary tracts $-6.3 \%\left(x^{2}=103.426 ; p=\right.$ $0.047)$; in the group 3 - tumors of the skin, respiratory and digestive organs - $33.3 \%\left(x^{2}=57.194, p=0.000\right)$; in the group 4 - skin tumors - $33.3 \%$, respiratory organs $-25 \%$ and digestion $-16.7 \%$, urinary tract, lymphoid tissue and thyroid gland $-8.3 \%\left(x^{2}=559,312, p=0.000\right)$. In all groups of men, after therapy of lung cancer, secondary metachronous tumors with localization in the skin, respiratory and digestive organs were most frequently developed.

Conclusions: For dispensary observation of men after the treatment of lung cancer, it is necessary to prescribe an additional examination of the above-mentioned organs and systems as a "risk zone" for the development of secondary metachronous tumor.

\section{P29}

The plombage of residual tb cavern using homolateral thoracic muscle flaps in the treatment of lung aspergilloma

Calin P. Tunea, Ioan A. Petrache, Gabriel V. Cozma, Voicu T. Voiculescu, Iris Miron, Dragos M. Brihac, Elisei M. Hasan, Ovidiu N. Burlacu

Spit Clinic Mun Urg Timisoara, Timisoara, Romania

Correspondence: Calin P. Tunea

Journal of Cardiothoracic Surgery 2017, 12(Suppl 1):P29

Objectives. Pulmonary aspergilloma (PA) following pulmonary tuberculosis may lead to massive and fatal hemoptysis. We compare different methods of surgical treatment emphasizing the plombage of the residual caverna with muscle flap

Methods: From 2004 until 2017 we treated surgically PA in 15 cases. We used wedge resections, (Group I), cavernostomy removing the micetoma and closing in a circular layer (Group II) or plombage with extrathoracic muscle flap (Group III).

Results: We operated 15 cases (M/F; in Group I was 4 patients, in Group II 6 patients, and 5 in Group III. The postoperative days for Group I was $8 \pm 2,5,12 \pm 3$ for Group II and $7 \pm 1$ for Group III. We used twice pectoralis major for anterior cavity and once the trapesisus, latissimus dorsi and serratus for the posterior localization. There were prolonged air leaks and postoperative hemorrhage as general complications in the 30 -postoperative day.

Conclusions Cavernostomy with muscle plombage, initially used only in poor-risk patients, may be extended as a rule for the surgically treatment of PA.

\section{P30}

Biopsy in diagnosis of interstitial lung diseases

R. A. Bakenova', M. M. Tussupbekova², Z. G. Bayturlin', A. I. Kolos ${ }^{3}$

${ }^{1} \mathrm{MCH}$ PAA RK, Astana, Kazakhstan; ${ }^{2} \mathrm{KSMU}$, Karaganda, Kazakhstan;

${ }^{3} \mathrm{NSMC}$, Astana, Kazakhstan

Journal of Cardiothoracic Surgery 2017, 12(Suppl 1):P30

Background: Diagnosis and effective treatment of interstitial lung diseases (ILD) remain one of the most difficult objectives of modern pulmonology

Aim: the assessment of the role of lung biopsy in the complex diagnosis and treatment of ILD.

Methods: Clinical examination, biochemical tests, microbiological and virological methods, spirometry, 6MWT, SaO2, arterial blood gases, ECG, DECG, HRCT, transthoracic puncture, endobronchial and miniinvasive thoracoscopic biopsy of 109 patients with ILD.

Results: There are 36 patients with sarcoidosis, 25 patients with ILF, 7 patients with LAM, 41 patients with IIP among the analyzed cases of ILD. $54(50 \%)$ biopsies of lungs, lymph nodes and skin were conducted. Miniinvasive thoracoscopic lung and pleural biopsy was used in 36 cases (66\%), transbronchial puncture biopsy - in 9 cases (17\%) and transthoracic puncture biopsy of lungs - in 7 cases (13\%). In 2 cases (4\%) of skin biopsy extrapulmonary lesion of sarcoidos is found. The distinctive clinical symptoms and CT-picture of the lungs allowed to diagnose ILF without lung biopsy in 20 cases (20\%). Pulmonary biopsy was performed in 11 out of 19 cases (53\%) of IIP, in sarcoidosis cases pulmonary biopsy was performed in 18 patients, which is $50 \%$. The lung biopsy allowed diagnosing orphan lung diseases and interstitial lung lesions in systemic connective tissue diseases.

Conclusion. The expediency and importance of conducting lungs and lymph nodes biopsy is dictated by the ability to verify the morphological variant of ILD more accurately and at earlier stages of the process, also to develop an adequate managing of patient, to determine the indications for performing pulmonary transplantation and to estimate the prognosis for patient.

P31

Emergency thoracotomy: experience of tertiary hospital in palestine

Moath Nairat, Ahmad Darwazah, Mohmad Lutfi, Hamad Madi, Mohmad Omar

Palestine medical complex, Ramallah, Palestinian Territory

Correspondence: Moath Nairat

Journal of Cardiothoracic Surgery 2017, 12(Suppl 1):P31

Chest trauma either penetrating or blunt consider as one of the leading cause of trauma mortality among young adult worldwide, Palestine considered as war zone have good experience in dealing with victims presented due to bullet injury in their chest, hereby we present our experience in manage victims with such cases, initially and upon arrival to emergency department the victim will be evaluated and managed according to advance trauma life support resuscitative protocol, the surgical approach could be ranged from simple insertion of chest tube to urgent thoracotomy to even 
Emergency room thoracotomy (ERT) which can be life saving in patients with penetrating injury. A protocol was established worldwide and adopted in our institution stating that ERT be performed for cases of cardiac tamponade secondary to penetrating chest trauma on patients with vital signs/mentation in the field or on arrival to the emergency room, also Emergency thoracotomy can be a lifesaving procedure in critically injured patients who present with no detectable pulse or blood pressure. This procedure is with limited benefit if cardiac electrical activity is absent, Best results are achieved in patients with penetrating chest injuries either to gunshot or stab wound and the worst results in those with isolated blunt chest injury with unwitnessed arrest. Survival is much better if patient taken directly to the operating room with ongoing cardiopulmonary resuscitation.In conclusion we believe that performing early thoracotomy in the ER is a life-saving measure for a substantial number of trauma patients who present to the ER.

Keywords: - emergency thoracotomy, cardiac tamponade, emergency room thoracotomy

\section{P32}

Internal carotid artery stenting prior to surgical myocardial

revascularisation

Vasiliy Masasin', Alex Fokin², Andrey Vishnaykov

${ }^{1}$ FSBE «Federal center for cardiovascular surgery», Chelaybinsk, Russian Federation; ${ }^{2}$ SBEF HPE SUSMU Ministry of Health of Russian Federation,

Chelaybinsk, Russian Federation

Correspondence: Vasiliy Masasin

Journal of Cardiothoracic Surgery 2017, 12(Suppl 1):P32

Objective: To estimate the immediate and short-term results of stenting of the internal carotid artery (ICA) performed in order to prevent perioperative stroke and its afteractions for patients undergoing open-heart surgery.

Methods: The results of treatment of 50 patients with concomitant carotid and coronary arteries disease has been analyzed, which were performed the staged reconstructive surgery on carotid and coronary arterial districts in the period from January 2011 to January 2017 in FCCS(Chelyabinsk). According to the Russian national guidelines, as the first step was performed CAS, followed by CABG. Among the patients were $45(95 \%)$ men and $5(5 \%)$ women, a mean age at the time of operation was 64 years. Exertional angina II FC on CCS was detected in $10(20 \%)$ of the patients, heart failure FC III - IV was detected in $40(80 \%)$ patients. Analysis of chronic cerebrovascular insufficiency in patients before surgery showed: asymptomatic course in $4(8 \%)$, transient ischemic attack in the previous history in $5(10 \%)$, encephalopathy in $45(95 \%)$, a history of stroke in $8(16 \%)$. When analyzing the data of coronary angiography, in $47(94 \%)$ patients had multivessel coronary artery disease. Unilateral significant carotid artery disease(CA) was detected in $34(68 \%)$, bilateral in 16(32\%).

Results: CAS technical success achieved in $100 \%$ of cases. The 49 patients after open-heart surgery were discharged with improvement. In one case, the patient after CABG led to perioperative MI, mesenteric thrombosis, multiple organ failure and death.

Conclusions: CAS as a stage of treatment before surgical myocardial revascularization in patients with hemodynamically significant stenosis of the ICA and the coronary arteries can reduce the frequency of ischemic coronary and cerebral circulation disturbances, thereby increasing overall survival.

\section{P33}

Safety and predictors of stroke and adverse outcome after carotid endarterectomy

Permyos Ruengsakulrach, Kosin Thupvong, Vitoon Pitiguagool, Piyapan Pamornsing, Siriwasan Akanitthapichat, Jamorn Udomkusonsri Bangkok Heart Hospital, Bangkok Hospital Group, Bangkok, Thailand

Correspondence: Permyos Ruengsakulrach

Journal of Cardiothoracic Surgery 2017, 12(Suppl 1):P33
Objective: To determine safety and predictors of postoperative stroke or adverse outcomes in patients undergoing carotid endarterectomy (CEA) for carotid artery stenosis.

Methods: Between September 2001 and March 2017, 144 consecutive carotid artery stenosis patients underwent CEA. Mean age was $69.0+/-9.0$ years. Male was 122 (77.8\%). Thirty four percent presented with stroke. Thirteen percent presented with transient ischemic attack (TIA) and $15.3 \%$ was asymptomatic. Nine percent was current smoker. Diabetes, hypertension, recent stroke (within 2 weeks) were found in $50 \%, 87.5 \%$ and $11.1 \%$, respectively. Degree of carotid stenosis at the operated site was $81.9 \pm 12.1 \%$. Contralateral stenosis of carotid artery stenosis, more than $50 \%$, was found in $42.3 \%$ of patients. Intraluminal shunt was used in $80.6 \%$ during procedure. Combined CEA and coronary artery bypass grafting (CABG) was performed in $13.9 \%$ of patients. All of the measured variables (age, gender, diabetes, hypertension, recent stroke, percent of carotid stenosis, contralateral carotid stenosis, combined CEA with CABG procedure, intraluminal shunt during (EA) were used in a multivariate logistic regression analysis to determine predictors of postoperative stroke or adverse outcomes.

Results: Thirty-day TIA and stroke rate were $0.7 \%(1 / 144)$ and $2.1 \%$ $(3 / 144)$, respectively. Postoperative delirium was found in $1.4 \%$ (2/ 144). Thirty-day mortality rate was $1.4 \%(2 / 144)$. Combined endpoint (delirium, TIA, Stroke, death) was $5.6 \%(8 / 144)$. The predictors of postoperative stroke or adverse outcomes (delirium, TIA and death) were gender $(P=0.036)$, percent of carotid stenosis $(P=0.013)$ and combined CEA with CABG procedure $(P=0.028)$.

Conclusions: Carotid endarterectomy is a safe for treatment of cerebrovascular insufficiency. Special care should be taken to avoid postoperative stroke or adverse outcomes in female patients, patients with high grade carotid artery stenosis and patients undergoing combined CEA with CABG.

P34

Carotid endoarterectomy. result comparison between direct closure and patch angioplasty. patch type analysis

Giuseppe Battaglia, Salvatore Alberto Turiano, Rosario Tringale,

Ermanno Sabatino, Vincenzo Monaca

Ferrarotto Hospital, Catania, Italy

Correspondence: Salvatore Alberto Turiano

Journal of Cardiothoracic Surgery 2017, 12(Suppl 1):P34

\section{Objectives}

Patch angioplasty in carotid endoarterectomy (CEA) according to many Authors reduces perioperatory stroke risk and early restenosis incidence. Some others, instead, avoid patch employment because of increased risk of wound infection and pseudo aneurysm formation. There is not mutual agreement regarding type of patch (A.V.S., Dacron, P.T.F.E.). We analyse our experience with all types of patch.

Methods

Between 2003 and 2017, we surgically treated 3220 patients suffering of internal carotid obstruction.

We decided the appropriate management in agreement with National Italian Guidelines.

419 patients had bilateral treatment, 65 patients had contralateral occlusion, 3064 patients had carotid patch angioplasty (2114 P.T.F.E., 672 A.S.V., 278 Dacron). We consider surgical technique, anaesthesia details and cerebral monitoring.

Results

We did not observe any significant difference related to patch type regarding: hospital mortality (respectively $0,3 \%-0,4 \%-0,4 \%$ ), permanent stroke $(0,4 \%-0,6 \%-0,7 \%)$, cardiac morbidity $(0,4 \%-$ $0,3 \%-0,3 \%)$, cranial nerve damage $(4,6 \%-6,4 \%-6,1 \%)$. Incidence of temporary cerebrovascular accident between patients who had P.T.F.E. and those who had A.S.V. patch was significant $(2,2 \%$ vs $0,7 \%$, $-p<0.05)$.

55 patients reported late restenosis (3 in Dacron group, 41 in P.T.F.E. group, 12 in the A.S.V. group), 48 cases were managed by stenting, 7 cases by open surgical treatment. 


\section{Conclusions}

Considerations of reported papers seem to favour use of patch in CEA because of reduced early death and stroke risk, and late restenosis incidence. Different type of stent does not influence hospital and late results.

\section{P35}

Infrapopliteal bypass in critical limb ischemia: long term results

analysis of peroneal versus tibial artery revascularization

Giuseppe Battaglia, Salvatore Alberto Turiano, Rosario Tringale,

Ermanno Sabatino, Nunzia Sinagra, Vincenzo Monaca

Ferrarotto Hospital, Catania, Italy

Correspondence: Salvatore Alberto Turiano

Journal of Cardiothoracic Surgery 2017, 12(Suppl 1):P35

\section{Objectives}

The purpose of the study is to evaluate the reliability of the peroneal artery when used as a single target vessel in patients with critical ischemia or highly disabling claudicatio.

Methods

From Jan 2003 to Sep 2016 we performed 250 revascularization procedures below knee on a single distal vessel: 121 on the posterior tibial (group TP), 87 anterior tibial (group TA) and $42(32 \mathrm{~m} / 10 \mathrm{f}$ mean age $73 \mathrm{yrs}$ ) on the peroneal artery (group PE).

40 patients were in critical ischemia and only one in chronic ischemia highly debilitating We used saphenous vein in 29 cases; in 13 cases we used the Propaten (1) or a composite graft propaten/ GSV (12) for partial or total not avalaible vein graft.

All bypass graft were extra- anatomic, with about $5 \mathrm{~cm}$ of bone peroneal resection.

We never had complications, early or late, resulting in bone resection Results

We had no hospital death. There were 4 early thrombosis, 3 VGS and 1 composite, treated unsuccessfully with embolectomy: two of them had already necrotic lesions distal. In two cases it was necessary the amputation.

There is no statistically significant difference related early thrombosis with group TA and TP.

All patients are discharged, unless contraindicated, with dual antiplatelet therapy.

The mean follow-up was 20 months (1 to 108 months).

There were 12 more graft thrombosis, 4 were amputated and 2 underwent to a redo bypass graft; 3 were treated successfully with embolectomy and/or thrombolysis, 3 were in pharmacologic treatment waiting for reoperation

The primary patency was $43 \%$ at 5 years, limb savage $81 \%$.

There is no statistically significant difference between the 3 group.

Conclusions

The results demonstrate how the peroneal artery is a good target vessel, for its ability to re-inhabiting the distal tracts of the tibialis vessel.

\section{P36}

Below knee bypass with heparin bonded graft: more than 10 years pratical experience

Giuseppe Battaglia, Salvatore Turiano, Rosario Tringale, Sara Catalfamo,

Ermanno Sabatino, Vincenzo Monaca

Ferrarotto Hospital, Catania, Italy

Correspondence: Salvatore Turiano

Journal of Cardiothoracic Surgery 2017, 12(Suppl 1):P36

\section{Objective}

To evaluate the results in patients with critical limb (CLI) treated with below knee bypass, performed with heparin bonded ePTFE conduit. Methods

In more than ten years of activity, we treated 615 patients reporting CLI. In 300 selected cases we performed below knee bypass employing heparin bonded prosthetic graft (HbePTFE;Propaten GoreTex ${ }^{\oplus}$, W.L. Gore \& Associates Inc, Flagstaff,AZ). Target vessels were: popliteal (219),tibio peroneal trunk (68), posterior tibial (9), anterior tibial (2), peroneal (2) arteries. At the beginning of our practice, we were forced to find an alternative graft in case of vein unavailability. Initial good results led us to widen prosthetic graft employment to patients whose veins had to be preserved for subsequent use (coronary or distal peripheral disease). Data regarding the abovementioned procedures were collected in a national multicentre registry with a linked database, in which the 7.5 cut off "Propaten Score" pointed out the increasing risk of graft thrombosis and guided conduit choice.

Results involved primary and secondary graft patency, limb salvage, amputation-free survival.

Results

Mean hospital stay 6 days.Hospital complications were: early graft thrombosis ( 8 cases $-2.7 \%$ ), A.K. amputation (5 cases $-1.7 \%$ ), wound infection ( 2 cases - 0.7\%), myocardial infarction ( 2 cases - $0.7 \%$ ) mortality rate $0.3 \%$ (one case). Mean follow up period was 42 months $(1-161)$. Primary graft patency was $50.21 \%$ at five and $38.44 \%$ at 12 years. Limb salvage respectively $90.68 \%$ and $87.66 \%$; whereas amputation-free survival $76.1 \%$ and $53.84 \%$.

Conclusions

Data from our retrospective study confirmed that the indexed HbePTFE graft provides satisfactory early and midterm results in patients undergoing surgical treatment for CLI.

The outcomes make this graft an excellent alternative to autologous saphenous vein not only when it is absent or unsuitable as conduit, but also when vein should be spared for successive bypass.

P37

The investigation of amiodarone, procainamide and sotalol's antiangiogenic effects in cam assay

Sabahattin Göksel ${ }^{1}$, Özge Korkmaz ${ }^{1}$, Ahmet Altun, Arif Pala², Bülent Saraç ${ }^{1}$ Öcal Berkan ${ }^{1}$

${ }^{1}$ Cumhuriyet Üniversitesi, Sivas, Turkey; ${ }^{2}$ Gölova Public Hospital,

Department of Emergency, Sivas, Turkey

Correspondence: Sabahattin Göksel

Journal of Cardiothoracic Surgery 2017, 12(Suppl 1):P37

Objective: Angiogenesis is a normal and vital process in growth and development, as well as in organ formation and physiological functions of granulation tissue. However, it is also a fundamental step in the transition of tumors from a benign state to a malignant state. We believe it is essential to understand the effect of the drugs used in cardiovascular interventions on angiogenesis. Therefore, we aimed to investigate the effects of antiarrhythmic drugs on angiogenesis by using chorioallantoic membrane model.

Methods: The antiangiogenic efficacies of amiodarone, procainamide and sotalol were examined in vivo in the chick chorioallantoic membrane model. Drug solutions are prepared in three different concentrations $\left(10^{-7}, 10^{-6}\right.$ and $\left.10^{-5} \mathrm{M}\right)$. For each set of experiment twenty fertilized eggs were used. The decrease of vessel formation is examined and scored according to previous literature.

Results: All antiarrhythmic drugs tested in the present study showed strong antiangiogenic effect in CAM model. While the strongest one was sotalol, procainamide showed significantly weaker effect when compared with other two.

Conclusions: Antiarrhythmic drugs are commonly used after coroner surgery. It is essential to know antiaritmic profiles of these drugs. Our study managed to create a small panel of antiarrhythmic drugs in terms of antiangiogenic properties. Sotalol seems to be a candidate agent to be used in situations where angiogenesis is preferable.

P38

Surgical treatment of popliteal arteriovenous fistula associated with aneurysm after penetrating gunshot to the lower limb

Moath Nairat, Ahmad Darwazah, Mohmad Lutfi, Morad Ganyem, Mohmad Omar

Palestine Medical Complex, Ramallah, Palestinian Territory

Correspondence: Moath Nairat

Journal of Cardiothoracic Surgery 2017, 12(Suppl 1):P38 
Repair of vascular injury after gunshot is generally an effective procedure with low rate of long term vascular complications. We report three young male patients with $\mathrm{A}-\mathrm{V}$ fistulae of the popliteal artery presented after gunshot injury.

The first 25-year-old had A-V fistula associated with left popliteal artery aneurysm developed 4 weeks after injury. The second 27 -yearold presented with isolated A-V fistula 2 weeks after repair of partial arterial injury and the third 21-year-old presented with femoropopliteal fistula after 7 years from gunshot injury.

These patients presented with popliteal and calf pain, bruit and thrill at the popliteal fossa. In addition, the third patient had sudden attack of chest pain, dyspnea and hemodynamic deterioration. Peripheral CT angiography confirmed the presence of fistula of the left popliteal artery associated with aneurysm, isolated fistula of the right popliteal artery and fistula of the right femoro-popliteal artery with aneurysmal formation together with pulmonary embolism as proved by $\mathrm{Ct}$ angiography of the chest for the three patients respectively.

During surgical exploration, the fistula between the popliteal artery and vein was isolated and repaired. Aneurysm was excised and the artery was repaired by interposition saphenous graft for the first case and direct repair for the second. Emergency pulmonary embolectomy was performed in the third patient, who had repair of fistula and excision of the aneurysm three days later. Operative and postoperative recovery was uneventful. Follow up showed complete recovery with no residual vascular insufficiency.

Key words: aneurysm, popliteal fossa, arterovenous fistula Consent for publication

Written informed consent to publish was obtained from the patients involved in this study.

P39

Impact of changes in the expression of metabolic of infectious complications in patients with peripheral atherosclerosis

Anastaia Artemova ${ }^{1}$, Michael Ivanov', Nikolay Lukyanchikov ${ }^{2}$

${ }^{1}$ NWSMU named after I. I. Mechnikov, Saint Petersburg, Russian

Federation; ${ }^{2}$ I.M. Sechenov First Moscow State Medical University,

Moscow, Russian Federation

Correspondence: Anastaia Artemova

Journal of Cardiothoracic Surgery 2017, 12(Suppl 1):P39

Introduction: The correction of metabolic disorders in patients with spread atherosclerosis may cause positive influences on the course of the postoperative period in people who came through reconstructive interventions on the main arteries.

Objective: The aim of the research was the observation of corrective risk circumstances of the development of infectious complications during the realization of rehabilitation algorithm in patients after reconstructions on the arterial vessels.

Methods: The observation after 57 patients with multifocal atherosclerosis who came through reconstructive interventions on the main arteries was placed in the base of the research. 34 of them were registered with the presence of the infection in the area of the operative intervention (main group). 23 operated patients formed the control group. The course features of hypertension, diabetes, lipid profile disorders, body mass changes, operative approach characteristics, nidus of autoinfection were analyzed.

Results: Decompensated diabetes was one of the main risk factors of the infection in the area of surgical intervention. The uncontrollable variation of hypertension also associated with the development of the infection $(p<0,05)$. The changes of the lipid profile negatively influenced the possibility of the infectious process $(p<0,05)$. Metabolic disorders caused the possibility of the infectious process in patients with the critical ischemia as far as they could $(p<0,05)$. Manifestations of the chronic kidney disease, as an element of metabolic disorders, made a supplementary contribution in the development of the infectious process $(p<0,05)$.

Conclusions: The rehabilitation algorithm of the disease management of spread atherosclerosis predispose the necessity of the influence on the corrective metabolic disorders before the execution of the reconstructive interventions on the major arteries.

\section{P40}

Staged surgical repair of pulmonary atresia with ventrical septal

defect and severe pulmonary arborization abnormalities

Aleksandr Morozov', Ruben Movsesian², Alexey Shikhranov²,

Evgeny Grekhov ${ }^{1}$, Aleksandr Latypov ${ }^{1}$, Andrey Tzytko²,

Gennady Chizhikov', Nikolay Antsygin'2, Vadim Lubomudrov ${ }^{3}$

${ }^{1}$ Federal Almazov North-West Medical Research Centre, St. Petersburg,

Russian Federation; ${ }^{2}$ Children's City Hospital №1, St. Petersburg, Russian

Federation; ${ }^{3}$ Chest Disease Hospital, Al-Kuwait, Kuwait

Correspondence: Aleksandr Morozov

Journal of Cardiothoracic Surgery 2017, 12(Suppl 1):P40

Objective: Surgical management of pulmonary atresia with ventrical septal defect and pulmonary arborization abnormalities is still a surgical challenge. The pulmonary arborization abnormalities in this complex cardiac malformation are characterized by hypoplastic or even absent native pulmonary arteries and multiple sources of pulmonary blood supply. We estimated our results of staged surgical treatment in such patients.

Methods: From 2000 to 2015, staged surgical repair was done in 28 patients with pulmonary atresia, ventrical septal defect and pulmonary arborization abnormalities. Severe hypoplastic pulmonary arteries were presented in 22 patients (median Nakata index 46,7 $\mathrm{mm} / \mathrm{m} 2$, range $13,1-135,4 \mathrm{~mm} / \mathrm{m} 2$ ), inconfluence or absence of native pulmonary artery was revealed in 6 patients. The pulmonary blood flow was provided by major aorto-pulmonary collateral artery (median 3, range 2-5). Initial surgical procedures were consisted of systemic-to-pulmonary shunt only, unifocalization and systemic-topulmonary shunt or palliative right ventricular outflow tract reconstruction, depending on the distribution of pulmonary blood flow. Results: Complete repair with ventricular septal defect closure was achieved in $10(36 \%)$ patients (median 2,5 surgical procedures, range 2-4). Unifocalization of the pulmonary blood flow was finished in 4 patients before the final surgical stage. Another 6 patients underwent complete repair and final unifocalization of the pulmonary blood flow at the same procedure. The pulmonary artery to the systemic blood pressure ratio after complete repair was 0.66 (range $0.44-1.00)$. Overall hospital mortality in staged group was $14,3 \%$ (3 patients died after staged procedures and 1 - after complete repair). Subsequently, dilation or/and stenting of pulmonary arteries was done in 3 patients, open redo surgery - in 1 patients.

Conclusions: Staged surgical approach can be applied in pulmonary atresia with ventricular septal defect and severe pulmonary arborization abnormalities as a way to reconstruction of pulmonary artery to prepare for complete correction with acceptable results.

\section{P41}

Aortic arch obstruction with ventricular septal defect: single- or two-stage strategy?

Nikolai Antsygin, Ruben Movsesian, Aleksei Shikhranov, Elena Michajlova, Andrey Tsytko, Dmitry Yamgurov

Children's City Hospital \#1, St. Petersburg, Russian Federation

Correspondence: Nikolai Antsygin

Journal of Cardiothoracic Surgery 2017, 12(Suppl 1):P41

Objective: We review our results during 10-years period with a singlestage or two-stage repair of these congenital malformations.

Methods: 66 patients underwent repair of AAO and isolated VSD. The cohort was divided into 2 groups: single-stage (group I); two-stage group (group II). Group 1 (49 babies) included 25 boys with median age $31 \pm 28$ days and mean weight 3,65 $\pm 1,1 \mathrm{~kg}$ ( 5 babies $<2,5 \mathrm{~kg}$ ); 15 patients with IAA and 34 with $C O A ; 7$ had a comorbid conditions. Group 2 (17 babies) included 6 boys with median age 10,8 $\pm 8,7 \mathrm{~d}$ and weight $3,25 \pm 1,07 \mathrm{~kg} ; 16$ babies had COA and 1 - IAA.; 6 babies had a comorbid conditions. 10 patients in group 2 had a multiple VSDs. In group I, all 
operation was performed using median sternotomy, deep hypothermic circulatory arrest was used in 33 (67\%). In 43 cases correction of AAO was made by direct anastomosis, in 5 babies - homograft patch and in 1 patient with autologous PA patch. In group II correction of AAO was made through the left thoracotomy with direct extended end-to-end anastomosis in 12 babies and in 5 babies, median sternotomy and bypass surgery was used. The second stage was performed on average time 7,6 months after previous surgery.

Results: postoperative ICU stay was 9 days in group I with mean inotropic support $75,4 \pm 44,8 \mathrm{~h}$ and 10,2 days with $53 \pm 23,1 \mathrm{~h}$ in group II. There were no deaths in each groups. Follow-up was 4,6 years. Four patients had a recurrent arch obstruction. One of them underwent reoperation and 3 patient endovascular intervention.

Conclusions: strategy of concomitant VSD closure at AAO repair does not result in worse discharge mortality. Two-stage repair is reasonable for critical ill babies with complex comorbid conditions and contraindications for bypass surgery.

P42

Surgical correction coarctation of the aorta with borderline size of the left ventricle in infants

Pavel V. Teplov, Alexey Sergeevich Ilin, Valeriy Anatol'evich Sakovich

Federal center of cardio-vascular surgery, Krasnoyarsk, Russian Federation

Correspondence: Pavel V. Teplov

Journal of Cardiothoracic Surgery 2017, 12(Suppl 1):P42

Objective: To assess the risks and the result of surgical correction of aortic coarctation combined with borderline size of the left ventricle. Methods: 47 newborn with coarctation of the aorta were divided into 2 groups. In group 1 patients with Z-score for AV and/or MV of -2.5 or less, in group 2 -normal size AV and MV. All patients operated in conditions of BP, deep hypothermia and arrest BP. Methods of reconstruction of the aortic arch were: an oblique anastomosis and plastic aortic arch patch. Material for the patch was xenopericardial, autopericardial and allografts.

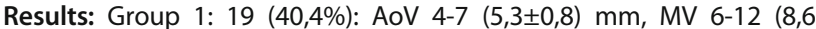
$\pm 1,4) \mathrm{mm}$, iEDV LV - 15-61,5 $(30,3 \pm 12,3) \mathrm{ml} / \mathrm{m} 2$. Hypoplasia of the aortic arch at $47.3 \%$. Group 2: 28 (59,6\%): AoV - 5.5-10 $(7,1 \pm 1,1) \mathrm{mm}$, MV - 8.4-13 $(10,2 \pm 1,2) \mathrm{mm}$, iEDV LV - 11,9-70 $(34,8 \pm 12,9) \mathrm{ml} / \mathrm{m} 2$. Hypoplasia of the aortic arch in $25 \%$. The duration of mechanical ventilation and stay in intensive care was, on average, higher in patients of group 1 at $37 \pm 47 \mathrm{~h}$ and $3.1 \pm 6$ days. The mortality rate was $15.8 \%$ in the first group and $7.4 \%$ in 2 . Follow-up: recoarctation in group 1 $21 \%$ in the $2-10.7 \%$. Aortic stenosis was observed in $26.3 \%$ only in group 1. OR: the risk of developing aortic stenosis in patients with hypoplasia of the AoV with hypoplasia of the aortic arch - 40.0 $[1.98 ; 807.10], p<0.0001$. The risk of recoarctation in patients using the patch from xenopericardial patch - 6.29 [1.14;34.57], $p=0.005$.

Conclusions: Patients with borderline size of the left ventricle and hypoplasia of the aortic arch are at risk for the development of aortic stenosis. Reconstruction of the aortic arch patch allows to avoid tension on the anastomosis and to form a sufficient size of the diameter of the aortic arch.

P43

Our experience of surgical treatment of fallot tetralogy in the children

Ulan E. Turusbekov, Samidin E. Shabyraliev, Taalai Z. Kudaiberdiev,

Zarema R. Zhakypova

Research Institute of Heart Surgery and Organ Transplantation, Bishkek, Kyrgyzstan

Correspondence: Ulan E. Turusbekov

Journal of Cardiothoracic Surgery 2017, 12(Suppl 1):P43
Objective: assessment of short-term outcomes for palliative correction of Fallot Tetralogy in the children, and reoperations rate after palliative procedures.

Methods: From 2012 to 2016, we performed 151 operations for Fallot Tetralogy (FT) (palliative and radical treatment). The age ranged from 3 months up to 52 months after birth (mean 24,2 $\pm 4,87$ months). Palliative procedure as first step of surgical treatment in FT was in 52 patients (34\%). Radical treatment in 99 patients, amongst which in $11(7,8 \%)$ it was a second step of surgical treatment $14-32$ months after first step. There was a tendency for reoperation in patients, whose radical treatment was after systemic-pulmonary

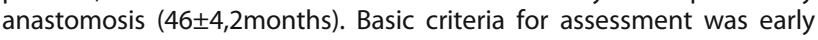
in-hospital mortality.

Results: 6 lethal cases after palliative treatment (11,5\%), and 5 lethal cases after radical treatment (5\%). This was due to severity of prior condition in this group (O2-saturation less than $70 \%$, concomitant pathologies and low body weight). Causes of adverse outcome were: uncontrolled infectious complications; in 1 case shunt-thrombosis; in 3 cases HF as a result of shunt hyperfunction. Echo assessed adequacy of treatment: $86 \%$ patients had lower PG of RV for $56 \%$ than initial, which was considered to be a good outcome. Partial recanalization noticed in $11 \%$ of patients.

Conclusions: Among 5 lethal cases after radical treatment only 1 was after anastomosis, which can be interpreted as positive effect of palliative procedures for children with FT and necessity of choosing of two-stage surgical tactic in dubious cases. There was a noticeable tendency of good hemodynamic results of primary correction of FT. In our study $2,3 \%$ of cases required reoperations after radical treatment.

P44

Permanent epicardial pacemaker implantation in pediatric age group

Santosh Kumar

Meenakshi Mission Hospital And Research Centre, Madurai, India Journal of Cardiothoracic Surgery 2017, 12(Suppl 1):P44

Objective : Permanent cardiac pacing in pediatric patients presents challenges related to small patient size, complex anatomy, electro physiologic abnormalities, and limited access to cardiac chambers. Epicardial pacing currently remains the conventional technique for infants and patients with complex congenital heart disease. However, epicardial pacemakers are less durable and have a higher incidence of complications as compared to endocardial pacemakers. The objective of this study is to analyze the midterm and long term outcome of epicardial permanent pacemaker implantation in children.

Methodology : Between 2011 and 2016, a total of 32 children received permanent epicardial pacemaker placement. Out of 32 patients, 15 are with congenital complete heart block (group 1) and 17 are post surgical iatrogenic $3^{\text {rd }}$ degree heart block (group 2).Bipolar leads was implanted in 20 pateints and unipolar leads in 12 patients.

Results; The pacing variables were noted at the time of implantation, 4days after implantation and there after every six months during follow up. 1 patient operated for vsd expired in hospital 10 days after implantation due to heart failure. 3 patients expired at home. 9 patients were lost contact. Out of 19 patients followed, all are free from lead/generator replacement, no change in parameters like amplitude, sensitivity, width. All patients are symptom free. 1 patient (post surgical) is completely pacing free for last 6 months.

Conclusion; Congenital complete heart block is detected early nowadays and increased PPI in recent years, due to developed medical technology and improved pulse generator and lead 
characteristics \& better results.Epicardial and bipolar leads is more preffered in younger children due to expected somatic growth, but the mode of implantation \& polarity should be optimized and individualized for every patient.

\section{P45}

Short term results of anatomical repair of double outlet right ventricle

Khakimjon Abralov, Amonjon Alimov, Murod Akhmedov

Republican Specialized Center of Surgery named after academician

V.Vakhidov, Tashkent, Uzbekistan

Correspondence: Khakimjon Abralov

Journal of Cardiothoracic Surgery 2017, 12(Suppl 1):P45

Background Double outlet right ventricle (DORV) - is one of the complex and challenging congenital heart defect. Prevalence of this CHD is rare - less than $1 \%$ of all CHDs. In some cases of heart disease, surgical tactics require anatomical or hemodynamic correction. In this message we would like to present immediate results of surgical anatomical correction DORV.

Methods In the Republican Specialized Center of Surgery named after academician V. Vakhidov from January 2008 to December 2016, 23 patients underwent a anatomical correction for the abovementioned heart disease. The age of patients ranged from 5 to 22 years (an average of $15.7+6.3$ years). Male patients were $17(73.9 \%)$ - and female 6 (26.1\%). Echocardiography and angiocardiography were routinely used in all patients to diagnose the defect. In 10 (43.4\%) cases was applied multislice computed tomography with contrast enhancement for specifying heart anatomy.

Results Anatomic surgical correction was represented by the following types of operations: in $21(86.7 \%)$ patients was performed intraventricular tunneling with transannular patch of the right ventricular outflow tract, in $3(13.0 \%)$ patients Rastelli procedure was performed. Hospital mortality was $2(8.6 \%)$ patients and was bounded with pulmonary vessel tree malformations. All survived patients had satisfactory postoperative result. Postoperative echocardiography doesn't revealed residual VSD, pressure gradient on RVOT varied from 5.5 to $18 \mathrm{~mm} \mathrm{Hg}$. The average stay in the intensive care unit was no more than 1.5 days. All patients were discharged within 7-10 postoperative days.

Conclusions Thus, despite the anatomical complexity, anatomical correction provides satisfactory immediate results. All survived patients shows objective and subjective improvement in the functional and somatic state with a leveling of the clinical picture of the defect after the operation. However, the presence of lethality requires a more detailed assessment of the pulmonary vessels.

\section{P46}

Immediate results of cavopulmonary anastomosis at the physiology of the single ventricle and ebshtein anomaly Khakimjon Abralov, Amonjon Alimov, Akmal Holmuradov

Republican Specialized Center of Surgery named after academician V.Vakhidov, Tashkent, Uzbekistan

Correspondence: Khakimjon Abralov

Journal of Cardiothoracic Surgery 2017, 12(Suppl 1):P46

Objective Cavopulmonary anastomosis is widely used type of stage surgical treatment in the physiology of the single ventricle and Ebstein's anomaly with severe atrialization of the right ventricle. The purpose of this report is to present the immediate results of bidirectional cavopulmonary anastomosis at single ventricle and the Ebstein anomaly.

Methods In the our department from January 2008 to March 2016 bidirectional cavo-pulmonary anastomosis was performed in 25 patients. Indication for this procedure was represented by the following pathologies: Ebstein's anomaly 9 (36.0\%), tricuspid valve atresia 13 $(52,0 \%)$, double outlet right ventricle with severe LV hypoplasia and pulmonary artery stenosis in $3(12,0 \%)$ patients. The age of patients ranged from 1 year to 18 years (an average of $8.5+5.3$ years). Male patients were $18(72,0 \%)$ - and female 7 (28.0\%). Echocardiography assessment was routinely used at all patients for specify the anatomy of CHD and its combinations. li some cases diagnostic program was extended by angiography.

Results Hospital mortality was $3(12,0 \%)$ of the patient. The lethal cases were associated by presence of distal stenosis of the pulmonary arterial tree. In 22 surviving patients, a significant improvement in the oxygen status was noted. Saturation by pulse oxymetry data improved on average from 78 to $86 \%$. The average stay in the intensive care unit was less then 1 day. All patients were discharged within 7 postoperative days.

Conclusion Proceeding from the foregoing, it should be noted that bi-directional cavopulmonary is a good and sometimes the only one alternative way at above-mentioned CHDs that improve the quality of life of patients. However, it should be noted that assessment of the state of pulmonary resistance requires careful study to select patients for this type of intervention.

P47

Comparative results of angiocardiography with intraoperative

diagnosis of aneuryms of sinus valsalva

Khakimjon Abralov, Amonjon Alimov

Republican Specialized Center of Surgery named after academician

V.Vakhidov, Tashkent, Uzbekistan

Correspondence: Khakimjon Abralov

Journal of Cardiothoracic Surgery 2017, 12(Suppl 1):P47

Background Aneurysm sinus of Valsalva (ASV) is a rare pathology and accounts for $0.1-3.5 \%$ of all congenital heart defects. Despite the wide spread of echocardiography in examination of anatomical and functional aspects of this pathology is some cases angiography is more beneficial method in diagnosis.

Methods Between from January 1, 1988, to December 30, 2016, 65 patients with SVA underwent surgical repair in our center. There were 41 males $(63 \%)$ and 24 females (37\%), and their age ranged from 5 to 50 years (mean age $23+10$ years, median 21 years). Echocardiography was routinely used to diagnose the defect in all patients. The rupture SVA (RSVA) associated with a VSD was revealed in $46(70.76 \%)$ cases. Aortic valve and aortic root surgical intervention were necessary in 22 patients. Out of them 34 (61.82\%) patients required an angiographic study to specify the components of the CHD and the degree of their severity.

Results Comparative correlation of results between angiography and perioperative evaluation were presented by the following data: RSVA was revealed in 12 from 15 (80.0\%), AV incompetence in 21 from 22 $(95,4 \%)$, VSD in 42 from 46 (91,3\%). Main advantages of angiography among other X-ray examination is the most accurate diagnosis of pulmonary hypertension, which is undoubtedly important in patients with Valsalva sinus rupture. So, according to angiography, pulmonary hypertension from all the examined patients was detected in 27 (100\%) cases.

Conclusions The angiography showed main advantages of this diagnostic method, which despite the disadvantages of visualization in comparison with CT and MRI is still more valuable in the functional and parametric evaluation of dynamic structures like AV incompetence and pulmonary hypertension. In comparison with other radiation diagnostic methods benefits of angiography limited by invasiveness of the research method limits its routine use in all patients.

\section{P48}

Short term results of anatomical correction double outlet right ventricle

Khakimjon Abralov, Jamshid Pirnazarov

Republican Specialized Center of Surgery named after academician

V.Vakhidov, Tashkent, Uzbekistan

Correspondence: Khakimjon Abralov

Journal of Cardiothoracic Surgery 2017, 12(Suppl 1):P48 
Background Double outlet right ventricle (DORV) - is a complex congenital heart defect. Prevalence of this heart malformation is less than $1 \%$ of all CHD. Depending on the intracardiac anatomy, total correction vary from intraventricular tunneling to Rastelli surgery.

The purpose of this message is represent short term result of surgical correction of DORV.

Methods In the department of surgery of congenital heart disease from 2008 to 2016 - 27 patients underwent surgical correction on the above mentioned heart disease. Age of patients ranged from 3 to 24 years (mean 12,7+6.3 years). Male patients were $18(66.6 \%)$ and female 9 (33.3\%). Echocardiography and angiography were routinely used in all patients to diagnose the defect. In 10 (37.1\%) cases to clarify the anatomy blemish held CT scan with contrast enhancement.

Results Anatomical correction represented by the following intervention types: in $24(88.8 \%)$ patients underwent intraventricular tunneling transannular plastic of outflow tract of the right ventriclet, in $3(11.1 \%)$ patients had performed Rastelli procedure Hospital mortality was $1(3.7 \%)$ patients. In 1 (3.7\%) cases death occurred in a patient after Rastelli procedure as a result of acute intrathoracic hemorrhage. In 23 surviving patients had satisfactory postoperative result. Average length of stay in the intensive care unit was no more than 1 to 3 days. All patients were discharged within 7-12 postoperative days.

Conclusions Thus, in spite of the different types of surgical correction in patients with double outlet right ventricle correction provides satisfactory results. In the postoperative control echocardiography, satisfactory functional echocardiographic parameters of the heart, all patients the gradient of the systolic pressure of the right ventricular outflow tract was less than 15 mm.Hg. The operated patients had objective and subjective improvement of the functional and physical.

\section{P49}

Hemodynamic correction at double outlet right ventricle

Khakimjon Abralov, Bunyod Akbarkhonov, Bekzod Kobiljonov,

Nodir Ibragimov

Republican Specialized Center of Surgery named after academician

V.Vakhidov, Tashkent, Uzbekistan

Correspondence: Khakimjon Abralov

Journal of Cardiothoracic Surgery 2017, 12(Suppl 1):P49

Background Double outlet right ventricular (DORV) is rare CHD with the prevalence less than 1\%..Hemodynamic correction (various types of cavo-pulmonary anastomosis) for DORV is the surgical treatment of pulmonary stenosis, malposition of great vessels with unbalanced ventricles (dominant right ventricle).

The aim of this study is presentation of the short term results of hemodynamic correction at DORV.

Methods From January 2000 by January 2016 for 14 patients underwent bidirectional cavopulmonary anastomois (CPA) with DORV. Age of patients varied from 3 to to 19 years (average 10.4 $\pm 1.1)$. Male $8(57,1 \%)$ patients - a female $6(42.8 \%)$ patients. All patients for diagnostics of CHD was used echocardiography and angiography examination. In $5(35,7 \%)$ cases for specifying the anatomy of CHD used CT scan with contrast.

Results End diastolic volume of LV varied from $5 \mathrm{ml}$ to $20 \mathrm{ml}$ (mean $9.2 \pm 0.7 \mathrm{ml}$ ), Nakata index of all patients was more than $153 \mathrm{~mm} 2 /$ $\mathrm{m} 2 . \mathrm{SpO} 2$ in the arterial blood ranged from $46 \%$ to $77 \%(64,7 \pm$ $2,9 \%)$. In $1(7.1 \%)$ case of 14 patients, there was an early postoperative period was noted respiratory failure. Hospital mortality doesn't occurred. At all patients was marked satisfactory postoperative result. Time in ICU varied from 16 hours to 82 hours (mean $26.3 \pm 4.1$ hours). SpO2 in the arterial blood was $83-91 \%$ $(86,2 \pm 3,4 \%)$.

Conclusions At patients with DORV and pulmonary stenosis on background of unbalanced ventricles, cavopulmonary anastomosis is more preferable than anatomical correction. Thus, hemodynamic correction provides satisfactory results short term result and enhancement quality of life.
P50

Short term result of surgical treatment of ebstein anomaly

Khakimjon Abralov, Nodir Ibragimov

Republican Specialized Center of Surgery named after academician

V.Vakhidov, Tashkent, Uzbekistan

Correspondence: Khakimjon Abralov

Journal of Cardiothoracic Surgery 2017, 12(Suppl 1):P50

Background Ebstein's anomaly with severe atrialization of the right ventricle one of the difficult for decision making cases in CHD practice. The purpose of this report is to present the short term result of surgical treatment of Ebstein anomaly

Methods From January 2012 to December 2016 in our department 23 patients with Ebstein anomaly underwent surgical treatment. Age of patients ranged from 4 to 19 years, (mean $11.6 \pm 3.4$ years). By the type of surgery 8 of $23(34.7 \%)$ patients underwent intervention. Out of them 3 patients underwent tricuspid valve replacement and 5 patients has been performed tricuspid valve repair by Danielson. Other $15(65.3 \%)$ patients underwent a one and a half ventricular repair: 11 patients underwent reconstruction of the tricuspid valve with cavopulmonary anastomosis and 4 patients underwent tricuspid valve prosthesis with cavopulmonary anastomosis.

Results Indications for the implementation of total correction were the following: RV EDV - 49 $\pm 8,2 \mathrm{ml}$; RV ejection fraction - 56 $\pm 4,9 \%$; LV ejection fraction $-58 \pm 3,6 \%$. Indications for a one and a half ventricle repair were the following: Cardio-Thoracic Ratio- $64 \pm 7,6$;Oxygen satur-

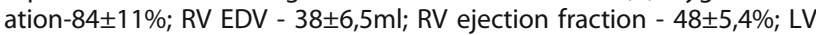
ejection fraction - 59 $\pm 8,1 \%$.All patients had ASD. 10 (43.5\%) patients had other CHD. Heart rhythm disturbances were detected in 2 (8.7\%) patients. Hospital mortality was in the group of children with one and a half ventricle repair and was $8.7 \%(n=2)$. In both cases, the cause of an unfavorable outcome was acute heart failure.

Conclusions The RV EDV is of paramount importance when choosing tactics for surgical treatment. 2. The presence or intensification of cyanosis is an indication for surgical treatment. 3. Severe concomitant pathology increases the risk of complicated course and the probability of a fatal outcome after surgery. 3 . Short term results showed that a total correction is more preferable.

P51

The case of successful surgical correction of coronary-left atrial fistula

Seitkhan D. Djozhibayev, Zhalil O. Sheishenov, Vladimir R. Muzayev, Zafar N. Rozbayev, Ikrom I. Mukhamedov

The Scientific-Clinical Center for Cardiosurgery and Transplantation,

Taraz, Kazakhstan

Correspondence: Zhalil O. Sheishenov

Journal of Cardiothoracic Surgery 2017, 12(Suppl 1):P51

Objective: Coronary artery fistula occurs both in isolated form and in combination with other congenital heart disease. The aim of this work was diagnosis and surgical treatment of a rare congenital coronary artery anomaly (coronary-left atrial fistula).

Methods: A 6-year-old boy, complaining of rapid fatigability, dyspnoea and palpitations upon little physical exercise (by indications of his mother), was hospitalized to our clinic. Based on the results of two-dimensional echocardiography, a coronary-left atrial fistula was diagnosed. In order to clarify the diagnosis and detail the individual angiographic findings, coronary angiography was performed. Coronary angiography results: The right type of coronary blood supply. LCA main is extended to $8 \mathrm{~mm}$, LAD - without peculiarities. CX - expanded to $6-7 \mathrm{~mm}$ at the proximal third, at the proximal and middle third border, the artery has a communication with the cavity of the left atrium, d-5 mm. Further on, the artery is of normal diameter-1.5 $\mathrm{mm}$. RCA without peculiarities.The suturing of a fistula from the inside of the left atrium under bypass and blood cardioplegia conditions were performed. During the operation, an enlarged CX and a fistula, flowing in between the mitral annulus of the anterior valve and the auricle of the left atrium, were found. The fistula was sutured with interrupted suture, 5.0 prolene. 
Results: The postoperative period progressed without any peculiarities. A month later, a planned check up was performed. During the check up, an improvement of patient's conditions was observed. According to Echocardiography data, there was no pathological discharge into the cavity of the left atrium, while the heart size decreased.

Conclusions: 1. Echocardiography allows establishing the correct diagnosis, nevertheless angiography (and CT) still remains an important auxiliary diagnostic method.2. The surgical technique should be individualized and depend on the anatomy of the vessel and the location of the shunt.

Consent for publication

Written informed consent to publish was obtained from the patients involved in this study.

\section{P52}

Use of bubble test in diagnostics of open foramen ovale

Himbatgul Joseph, Aigul Shokenova, Gulden Ospanova,

Saltanat Andossova, Saltanat Dzhetibayeva, Saule Kadirova

JSC "National Research Center for Cardiac Surgery", Astana, Kazakhstan

Correspondence: Himbatgul Joseph

Journal of Cardiothoracic Surgery 2017, 12(Suppl 1):P52

Objective: assessment of safety and simplicity of performance of "Bubble test" technique for identification of OFO and opportunities to perform the technique by nursing specialists under control of a doctor. According to data from information sources, rate of presence of open foramen ovale (OFO) in general population is about $25 \%$. One of frequent reasons of development of cryptogenic strokes is pathology. Where this category includes patients under 55 years of age. Almost $50 \%$ of patients with OFO are people suffering from migraine

Materials and methods: 200 patients have had transthoracic echocardiography for open foramen ovale since January 2017; 50 patients out of 200 had assumption of OFO relying on patients' complaints for frequent headache, some of them suffered from cryptogenic stroke. To identify the presence of OFO, transthoracic echocardiography was conducted in 4-chamber heart section, where interatrial septum is held vertically. The basis of Bubble test is introduction of saline solution intensely mixed with small quantities of blood of a patient that creates "bubbles" into peripheral vein. If there is OFO, one can observe their passage into left heart on the Valsalva test. Clinical state of the patient is estimated before and after the procedure. The technique was conducted by two nursing specialists (one performed echocardiography, the other - "Bubble test" procedure).

Results: There were 50 examined patients: 30 men and 20 women, 15 patients had a positive test. Average age is $44,42 \pm 15,98$ years. Complications and side symptoms were not identified during the test. Nursing specialists performed the technique independently in 15 cases under doctor's control.

Conclusion: Thus, the "Bubble test" procedure can be performed independently by a nursinf specialist in nursing who interprets the test result.

\section{P53}

Participation of a nursing specialist in conducting isolation of pulmonary veins entries by balloon cryoablation method

Aigerim Sadykova, Aliya Aykumbekova, Aliya Kanayeva,

Laura Yesmaganbetova

JSC "National Research Center for Cardiac Surgery", Astana, Kazakhstan

Correspondence: Aliya Aykumbekova

Journal of Cardiothoracic Surgery 2017, 12(Suppl 1):P53

Objective: Illustrate the importance of a nursing specialist in conducting ablation procedure.

Methods: "National Research Center for Cardiac Surgery" JSC has implemented balloon cryoablation procedure for isolation of pulmonary veins entries for the purpose of treatment of paroxysmal atrial fibrillation since February 2014. Over 150 procedures are performed every year for the purpose of isolation and re-isolation of pulmonary veins entries at our clinic. Preoperative preparation of expendable materials and apparatus management are included into duties of operating nurse. Operational field preparation and cover of sterile work surface are improved with installation of voltage and stimulation probes, transseptal puncture to access to left auricle. Delivery system Flexcath $12 \mathrm{Fr}$ is got through conductor to left auricle. When preparing balloon «Arctic Front Advance $28,23 \mathrm{~mm}$ », an important technique is to prevent air embolism. Injector for contrast agent delivery and diagnostic circular catheter "Achieve" is connected through «Y» connector. After passage of balloon catheter to left auricle, a nurse assists managing cryoconsole, inflating balloon and activating cryoablation by operator command. When the temperature of the balloon decreases according to indicators of cryoconsole lower than $-60{ }^{*} C$, a nurse stops cryoablation by force in connection with myocardial rupture. «Z»-shaped stitches are put on the site of puncture after procedure, taking into account high aspartate transaminase and large diameter of introducers.

Results: Thromboembolic complications and ruptures are closely associated with performance of duties by nurse in connection with important moment of preventive air embolism, which is directly belonged to nurse's duties. Timely break and delivery of cooling agent at reduction of temperature to $60 \mathrm{C}$.

Conclusion: Qualified assistance of a nurse in such comprehensive procedures is an important aspect. Coordinated work of the team allows to reduce time and risk of interventional maneuvers.

\section{P54}

\section{Computational technology as a teaching strategy in}

Cardiorespiratory Resuscitation (CPR)

Yolanda Dora M. Evora, Camila S. Sampaio, Anazilda C. Silva,

Andrea Bernardes

University of Sao Paulo, Ribeirão Preto, SP, Brazil

Correspondence: Yolanda Dora M. Evora

Journal of Cardiothoracic Surgery 2017, 12(Suppl 1):P54

Background: The Cardiopulmonary arrest is one of the most dreaded situations for health services professionals in Brazil. The diagnosis should be fast and accurate, and cardiopulmonary resuscitation (CPR) maneuvers should be started early in order to increase the chances of patient survival. Considering the difficulty that the health professional has in attending formal CPR training classes due to lack of time, computational technology is an efficient alternative for virtual education, since it facilitates the dissemination of knowledge at any time and place.

Objective: To develop a software as virtual learning environment (VLE) to provide Basic Life Support in cardiopulmonary resuscitation training.

Method: It was based on the instructional systems design ADDIE, which stands for analysis, design, development, implementation and evaluation, different stages to structure the teaching and learning planning. HTML (Hyper Text Markup Language), PHP (Personal Home Page) and CSS (Cascading Style Sheets) were used to develop the software, which enables interaction with content and can be accessed from platforms such as mobile phones or tablets.

Results: The VLE for CPR training was composed of texts based on bibliographic research, public domain websites, video with a laboratory simulation scenario and questions to assess the apprehension of context. Content was developed in modules composed of the following structures: module identification, objectives, content, links and references. Eight CPR specialists evaluated the software in order to verify if the virtual learning environment corresponded to their educational proposal and whether access was effective and efficient. The changes suggested were implemented and the VLE was released for free internet access, only in Portuguese language.

Conclusion: With the development of this study is expected to contribute to the training of health services professionals and available to use an innovative teaching strategy that can enhance 
learning, collaborating with the quality of care provided to the patient.

\section{P55}

Interleukin-6, 8 levels during ex vivo allograft perfusion for heart transplantation between blood cardioplegia and custodiol groups Zhuldyz Nurmykhametova, Rymbay Kaliyev, Mahabbat Bekbossynova, Serik Bekbossynov, Timur Lesbekov, Ivan Vakhrushev, Linar Faizov, Zhanybek Ashyrov, Yuriy Pya

National Research Center for Cardiac Surgery, Astana, Kazakhstan

Correspondence: Zhuldyz Nurmykhametova

Journal of Cardiothoracic Surgery 2017, 12(Suppl 1):P55

Objective: ILs has been shown to play important roles in a series of immunological and inflammatory responses. Interleukins (ILs) are a class of cytokines that are produced by leukocytes. For example, IL-6, 8 , and are common cytokines involved in inflammation. IL-6 is an anti-inflammatory cytokine which plays important roles in inducing acute phase reactions and controlling local or systemic acute inflammatory responses. This study aimed to evaluate concentration of IL-6, 8 during ex vivo allograft perfusion for heart transplantation with the use of two methods of preservation. In the PROCEED II trial, donor hearts were discarded because of rising lactate concentrations higher $4 \mathrm{mmol} / \mathrm{l}$. Modification of the initial reperfusion fluid, such as addition of Levosimendan and depletion of leukocyte with Ultrafiltration may have some possibility to restore the myocardial metabolism and to reduce lactate concentration resulting in successful heart transplantation.

Methods: $t$ test and their $95 \%$ confidence intervals $(95 \% \mathrm{Cls})$ were considered as effect estimates. A total of 15 ex vivo allografts for heart transplantation in group of blood cardioplegia and conditioning (BC) (Ultrafiltration, Levosimendan) ( $n=10)$, and standard Custodiol group (SC) $(n=5)$ were analyzed.

Results: Postoperative mean concentration of IL-6, 8 at 12 hours was significantly lower in BC group compared to SC group 1.3640 (SD

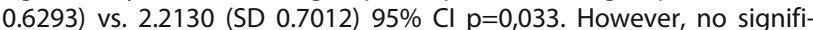
cant differences were found in preoperative concentrations of IL-6 and 8 between groups. In both groups, higher IL levels were associated with the lactate level more than $5.5 \mathrm{mmol} / \mathrm{l}$. Mean venous lactate at the start of perfusion $(2.0 \mathrm{mmol} / \mathrm{l}$ vs. $3.2 \mathrm{mmol} / \mathrm{l})$ and at the end of perfusion $(3.7 \mathrm{mmol} / \mathrm{l}$ vs. $7.5 \mathrm{mmol} / \mathrm{l})$ were lower in the $B C$ group $(p=0.37)$.

Conclusions: Lower IL-6, 8 and lactate levels were observed in BC group. Further research is needed to evaluate the clinical impact of these findings.

\section{P56}

A five-year experience in peripheral venoarterial extracorporeal membrane oxygenation as a method of mechanical circulatory support in potential heart transplant candidates

Vitaly Poptsov, Ekaterina Spirina, Nadegda Koloskova, Sergey Uschrenkov, Anastasiya Dogonascheva, Elnur Aliev

Shumakov Federal Scientific Centre of Transplantology and Artificial

Organs, Moscow, Russian Federation

Correspondence: Vitaly Poptsov

Journal of Cardiothoracic Surgery 2017, 12(Suppl 1):P56

Objective: Venoarterial extracorporeal membrane oxygenation (VAECMO) is one of methods of temporary mechanical circulatory support (MCS) prior heart transplantation (HT). This study presented own experience of peripheral VA ECMO for MCS at heart transplant candidates needed in urgent HT.

Methods: This study included 125 pts $(107$ (86\%) men and 18 (14\%) women, age from 12 to $72(43 \pm 1.2)$ yrs) treated with a peripheral VAECMO system installed within the period from 01.01.2011 - 31.12.2016. The indication for the start of its use was rapidly progressing congestive heart failure (CHF) of level 1 or 2 by the INTERMACS scale.

Results: The peripheral cannulation was used to perform VA ECMO in $100 \%(n=125)$. During VA ECMO extracorporeal blood flow rate ranged from 2.2 to $4.5(3.2 \pm 0.4 \mathrm{l} / \mathrm{min})$ or $1.6 \pm 0.2 \mathrm{l} / \mathrm{min} / \mathrm{m}^{2} .113$ (90.4\%) of 125 potential recipients underwent HT. The duration of VA ECMO prior to HT ( $n=113)$ was from 8 hours to $40(7.1 \pm 2.7)$ days. During of VA ECMO, 12 (9.6\%) of 125 potential recipients (11 male and 1 female, age from 21 to $63(40 \pm 4))$ died. In $2(16.7 \%)$ of 12 cases the cause of death was brain death associated with a thromboembolic cerebrovascular event. The majority of patients $(n=10,83.3 \%)$ died from progressive multiorgan failure and sepsis. Six of twelve patients presented clinical and chest Xray signs of acute unilateral $(n=2)$ or bilateral $(n=4)$ pneumonia.

Conclusions: Peripheral VA ECMO provides a successful bridge to HT in $90 \%$ of potential recipients who need pretransplant MCS. Beginning of VA ECMO before the development of pronounced hemodynamic, multiorgan and metabolic disorders creates the conditions for a successful use of MCS in heart transplant candidates.

\section{P57}

The continuous education of the regional clinical specialists who perform follow-up vad patients assessment

Roman Salov, Yury Pya, Serik Bekbossynov, Saltanat Dzhetybayeva, Saltanat Andossova

National Research Center for Cardiac Surgery, Astana, Kazakhstan

Correspondence: Roman Salov

Journal of Cardiothoracic Surgery 2017, 12(Suppl 1):P57

Background: Astana Center is the one center in the Republic of Kazakhstan which performs implantation of HVAD, HM II, HM3 assist devices. Astana center and regional centers across the country perform all selection process of patients and continued care. The VAD team from Astana provides training to staff from 15 regional hospitals across the country.

Methods: Distances between Astana center and regional centers are up to $2000 \mathrm{~km}$. Because patients are discharged home to regions which is long distant from the Astana center, education of a clinical specialist edgeable in VAD are essential. Cardiologists $(n=41)$, surgeons $(n=8)$ and nurses $(n=22)$ participate in education meetings at the Astana center. The educational meetings performing each year are aimed to provide sufficient knowledge of the patient's selection, to provide first-line-of-care in case of emergency and routine followup assessment.

Results: About 50 assist devices are implanted in the Astana Center annually and around 100 patients are supported per annum. In case of device troubleshooting, pump thrombosis, stroke, major bleeding and generalized infection, the VAD program provides air ambulance transport in case of necessity to return the patient to Astana center via plane or helicopter for advanced care. If patients are not stable for transport, VAD team provides advice to local clinical specialists on patient management. Over the past two years, we have seen a decrease in the number of cases when air ambulance was needed: 2013- 6 patients, 2014- 5 patients, 2015- 2 patients, 2016- 1 patient. Conclusions: Our experience with the continuous education of the regional clinical specialists has been positive. Long-term patient follow-up is necessary to confirm these preliminary findings.

\section{P58}

Lung transplantation for silicosis

Alkin Yazicioglu', Mahmut Subasi', Sinan Turkkan ${ }^{1}$, Fatmanur Celik Basaran?', Sema Turan ${ }^{2}$, Erdal Yekeler ${ }^{1}$

${ }^{1}$ Turkiye Yuksek Ihtisas Training and Research Hospital, Thoracic Surgery and Lung Transplantation Clinic, Ankara, Turkey; ${ }^{2}$ Turkiye Yuksek Ihtisas

Training and Research Hospital, Anesthesiology and Intensive Care Unit, Ankara, Turkey

Correspondence: Alkin Yazicioglu

Journal of Cardiothoracic Surgery 2017, 12(Suppl 1):P58

Objective: To emphasize importance of single lung transplantation(SLuTx) for silicosis patients.

Results: Case1: 29 year-old male with glass-sandblasting worker silicosis underwent SLuTx under assistance of central-ECMO. Ischemic 
time was $374 \mathrm{~min}$ and ECMO was successfully weaned after operation. He extubated 21 hours and discharged from ICU on day 8; hospitalization was 19 days. Case2: 27 year-old male with dental technician silicosis underwent double LuTx under the assistance of central-ECMO. Ischemic time was $339 \mathrm{~min}$ and $460 \mathrm{~min}$; ECMO was successfully weaned after operation. During ICU follow-up, a peripheral VA-ECMO was initiated at post-op $16^{\text {th }}$ hour due to hemodynamic instability and acidosis. However, bilateral infiltrations on the chest roentgenogram grew in time, hypercarbia, hypoxia, and hemodynamic instability worsened. Unfortunately, patient died on $2^{\text {nd }}$ day due to accelerating graft dysfunction. Case3: 29 year-old male with PTFE-sandblasting worker silicosis underwent SLUTx under the assistance of central-ECMO. Ischemic time was $365 \mathrm{~min}$ and ECMO was successfully weaned after operation. However, at postoperative $2^{\text {nd }}$ hour peripheral VA-ECMO was initiated due to hemodynamic instability and pulmonary edema. Peripheral ECMO was weaned at $2^{\text {nd }}$ day and he was weaned from mechanical ventilation at $4^{\text {th }}$ day after transplantation. He discharged from ICU on day 22; hospitalization was 36 days. Case4: 26 year-old with Erasmus syndrome (scleroderma+silicosis) underwent SLuTx without assistance of ECMO. His silicosis was due to marble worker silicosis and ischemic time was $360 \mathrm{~min}$. During ICU follow-up a peripheral VV-ECMO was initiated at post-op $4^{\text {th }}$ hour due to hemodynamic instability and graft dysfunction. However, during follow-up at ICU, a cerebrovascular accident was developed and patient was deceased on $6^{\text {th }}$ day.

Conclusions: Pleural thickening and dense pleural adhesions are serious problems in silicosis. If adhesions are not dense and lung releasing is completed within a short time, double lung transplantation may be performed. However, in cases of silicosis with dense adhesions, single lung transplant option should be evaluated. Consent for publication

Written informed consent to publish was obtained from the patients involved in this study.

\section{P59}

Extracorporeal membrane oxygenation for graft dysfunction after heart transplantation

Natalia Petrovich, Liana Shestakova, Roman Yarosh, Yuriy Ostrovsky Republican Scientific and Practical Center "Cardiology", Minsk, Belarus Correspondence: Natalia Petrovich

Journal of Cardiothoracic Surgery 2017, 12(Suppl 1):P59

Background: Graft dysfunction (GD) is a major risk factor for death within 30 days after heart transplantation. Despite the high risk of complications, veno-arterial extracorporeal membrane oxygenation (V- A ECMO) plays role of rescue in catastrophic conditions.

Methods: The study conducted a retrospective analysis of 14 heart recipients with GD requiring V-A ECMO rescue. In 35,7\% ( $n=5)$ ECMO was performed in case of the absence of a significant effect of intra-aortic balloon pump. Peripheral cannulation was used in $78.6 \%$, in three observations - central. Distal selective antegrade perfusion was implemented to prevent lower limb ischemia in $21,4 \%$.Three patients needed renal replacement therapy due to the expressed metabolic disorders and the kidney failure progression.

Results: In Belarus for the period 2009 - March 2017, 249 heart transplants were performed. GD developed in $5,6 \%$ of the heart's recipients. In $71.4 \%(n=10)$ the primary GD was observed, in $28,6 \%$ $(n=4)$ - the secondary. The ischemia time of the donor heart was $214.25 \pm 50.22 \mathrm{~min}$. Criteria for the VA ECMO initiation : LVEF $46.78 \pm$ $11.87 \%$, RVEF $37.38 \pm 10.81 \%$, Cl $1.58 \pm 0.16 \mathrm{l} / \mathrm{min} / \mathrm{m}^{2}$, CVP $11 \pm 5.94$ $\mathrm{mmHg}$; PCWP $11.5 \pm 6.24 \mathrm{mmHg}$. The duration of ECMO was $5.06 \pm$ 3.8 days. $50 \%$ of patients were successfully weaned from the assistance, 42,86\% were discharged. One patient underwent heart retransplant. The leading causes of death were sepsis, multiple organ failure and DIC. The one-year survival rate was $100 \%$.

Conclusions: VA ECMO is an effective method of restoring the function of the heart transplant, that maintains adequate hemodynamics, prevents the development of irreversible organ changes and prepares the recipient for retransplantation.
P60

Temporary mechanical circulatory support as a bridge to heart transplant

Natalia Petrovich, Liana Shestakova, Roman Yarosh, Dmitry Krachak, Marina Bushkevich, Yuri Ostrovsky

Republican Scientific and Practical Center "Cardiology", Minsk, Belarus

Correspondence: Natalia Petrovich

Journal of Cardiothoracic Surgery 2017, 12(Suppl 1):P60

Background: Heart transplantation (HT) is a golden standard for treating the terminal stage of chronic heart failure (CHF).Mechanical circulatory support (MCS) is used in potential high- risk recipients with refractory unstable hemodynamics and multiorgan dysfunction. It salvages time to complete a transplant evaluation.

Materials and methods: The study conducted a retrospective analysis of 11 patients with decompensation of $\mathrm{CHF}$, requiring temporary MCS. In $36,4 \%(n=4)$ the ventricular support system Centrimag Levitronix was used, in $63,6 \%(n=7)$ - peripheral extracorporeal membrane oxygenation in combination with decompression of left ventricle(LV). Selective antegrade perfusion was performed in 4 cases to prevent lower limbs ischemia. 45, 5\% (n $=5)$ needed renal replacement therapy, 9,1\% ( $n=1)$ - hepatic replacement therapy.

Results: In Belarus for the period 2009-March 2017249 heart transplants were performed. $11,6 \%,(n=29)$ followed temporary MCS (11 short-term and 18 long-term). The main pathology in $63,6 \%$ was dilated cardiomyopathy, in $36,4 \%$ - ischemic. The average duration of the MCS was $16,2 \pm 7,2$ days. In $100 \%$ there was a significant positive dynamic of biochemical indices, decrease in lactate by 5,4 times, reduction volume of left ventricle and increase of LVEF by $14,5 \%$, RVEF by $23,2 \%, \mathrm{Cl}$ by $43,6 \%$. Two patients $(18,2 \%)$ died due to complications, one patient was transferred to a long-term heart assist device. In 8 from 11 cases HT was performed. Hospital survival was $72,7 \%$. Conclusions: Temporary MCS effectively provides maintenance of vital functions, resolution of organ disorders, normalization of biochemical indices and greatly improves survival for patients with severe refractory hemodynamic instability awaiting $\mathrm{HT}$.

P61

Left ventricular assist device support in patient with marfan's syndrome: case report

Yuriy Pya, Serik Bekbossynov, Makhabbat Bekbossynova, Saltanat Andossova, Saltanat Dzhetybayeva, Lyazzat Abikeyeva, Assel Medressova, Svetlana Novikova, Ainur Tauyekelova, Yermagambet Kuatbayev National Research Center for Cardiac Surgery, Astana, Kazakhstan

Correspondence: Assel Medressova

Journal of Cardiothoracic Surgery 2017, 12(Suppl 1):P61

Objective: We report about left ventricular assist device (LVAD) implantation in patient with Marfan's syndrome.

Methods: Young man, 17 years old, was hospitalized to our Center and 29.06.2012 with dilated cardiomyopathy, Marfan's syndrome. He was on IV functional class of congestive heart failure (NYHA), INTERMACS 2. During the hospitalization he underwent two episodes of asystoly with successful cardiopulmonary resuscitation. Ejection fraction of the left ventricle was $15 \%$ on echocardiography, TAPSE (tricuspid annular plane systolic excursion) $-1.46 \mathrm{~cm}$. HeartMate II (St Jude Medical, Huntingdon, Cambridgeshire, UK) LVAD was implanted, and the patient was discharged to home.

Results: After the operation recurrent driveline infection was started. The patient underwent surgical debridement of the driveline exit site, cable repositioning. Sepsis developed in 33 months after LVAD implantation. Patient was hospitalized to our Center in severe condition with bleeding from the driveline exit site. HeartMate II LVAD was explanted, extracorporeal membrane oxygenation was set, and patient was included on the emergency waiting list for heart transplantation. In one month heart transplantation was performed, and the patient was discharged to home. Duration of the hospitalization was 11 months. 
Conclusions: Mechanical left ventricular support in patients with Marfan's syndrome is rare but possible. Such group of patients should be transplanted as soon as possible.

Consent for publication

Written informed consent to publish was obtained from the patients involved in this study.

\section{P62}

ST elevation following lung transplantation: tako-tsubo cardiomyopathy

Alkin Yazicioglu', Mahmut Subasi', Sinan Turkkan', Fatmanur Celik Basaran', Sema Turan², Halil Kisacik, Omac Tufekcioglu ${ }^{3}$, Erdal Yekeler ${ }^{1}$ ${ }^{1}$ Turkiye Yuksek Ihtisas Training and Research Hospital, Thoracic Surgery and Lung Transplantation Clinic, Ankara, Turkey; ${ }^{2}$ Turkiye Yuksek Ihtisas Training and Research Hospital, Anesthesiology and Intensive Care Unit, Ankara, Turkey; ${ }^{3}$ Turkiye Yuksek Ihtisas Training and Research Hospital, Department of Cardiology, Ankara, Turkey

Correspondence: Alkin Yazicioglu

Journal of Cardiothoracic Surgery 2017, 12(Suppl 1):P62

Objective: Tako-Tsubo cardiomyopathy (TTC) is a cardiac-syndrome characterized by temporary akinesia/dyskinesia in more than one segment of left-ventricle and dynamic ST/T changes on ECG. Condition is particularly important for differential diagnosis following lung transplantation (LuTx).

Methods: A 61-year-old male, history of end-stage lung-disease due to emphysema and previous stent implantation to circumflex artery was evaluated for LuTx.

Results: The patient had $65 \%$ left ventricular ejection-fraction(EF) and normal wall movements on echocardiography $(\mathrm{ECHO})$ during preoperative assessment. Mean pulmonary-artery pressure was $19 \mathrm{mmHg}$; pulmonary-artery-wedge pressure was $10 \mathrm{mmHg}$. After bilateral LuTx, he developed recent onset ST-elevation on the cardiac monitor at postoperative day-1. The ECG showed ST-elevation in inferior and lateral derivations. However, there was no reciprocal STdepression in other derivations. The patient then developed sustained ventricular tachycardia and returned to normal sinus rhythm after intravenous-amiodarone administration. Bedside $\mathrm{ECHO}$ revealed akinetic apical and midventricular segments and hypokinetic basal segments, and left ventricular EF was $30 \%$. The patient underwent emergency coronary angiography; however, there was no recent coronary pathology that would explain for ST changes. There was no increase in troponin or CK-MG levels explaining ST changes. During the follow-up period, ECG changes disappeared and disruption in left ventricular wall movements regressed, and EF was $60 \%$ at day- 15 . This clinical picture was interpreted as TTC.

Conclusions: In literature, there are only two reports of cases with TTC associated with LuTx. In a report by Michel-Cherqui et al., a case of TTC diagnosed before LuTx, has been reported. Ghandri et al. reported a case of TTC, who had underwent LuTx seven years before and had respiratory failure. Hemodynamic instability, hypercarbia, hypoxia were deemed responsible as stress factors. The current case has been the first case that manifested in early post-operative period. Surgical stress in the early period and hypotensive attack can be regarded as stress factors.

Consent for publication

Written informed consent to publish was obtained from the patients involved in this study.

\section{P63}

Four-year experience with the maze procedure for atrial fibrillation Georgy Antipov

Federal Center Of High Medical Technologies, Kaliningrad, Russian Federation

Journal of Cardiothoracic Surgery 2017, 12(Suppl 1):P63

Objective: To estimate midterm results of the Maze operation at treatment of atrial fibrillation (AF) in combination with other heart operations.
MethodS: From October, 2012 and December, 2016230 (6.1\%) patients (145 men; average age was $62 \pm 7,2$ years) underwent the maze procedure. Indications were: existence of $A F$, age no more than 70 years, inefficiency of antiarrhytmic therapy. Paroxysmal form of AF $43(18.7 \%)$ patients, persistent form - 44 (19.1\%), permanent - 143 (62.2\%). Maze III 205 (89.1\%) and Maze IV variants with isolated bipolar $12(5,2 \%)$ or cryoablation $13(5,6 \%)$ were performed. Concomitant procedures were: interventions on valves (136), ischemic heart disease (87) and others (7). Patients were followed up (19,6 $\pm 8,1$ months) and changes in rhythm, need for pacemaker implantation, and the incidence of CNS (central nervous system) complications were retrospectively studied.

Results: Hospital mortality was 3.4\% (7 patients). From not lethal complications at $9(3,9 \%)$ patients in the early postoperative period the bleeding which demanded a resternotomy was noted. At a hospital stage (till 30 days) restoration of a sinus rhythm is registered at 167 $(74,8 \%)$ patients, AF - $18(8,0 \%)$, SSSN - $23(10,3 \%)$, Pacemaker - 15 $(6,5 \%)$ pts. After 12 months $182(81.6 \%)$ pts were assessed. Sinus rhythm - $127(67,2 \%)$ pts, AF - 31 (16,4\%), pacemaker - 24 (12,7\%). 1year survival rate (Kaplan-Meier) after surgery was $96.4 \%$ for the entire group. In 9 cases re-RFA was performed with the restoration of the sinus rhythm.

Conclusions: The Maze operation allows to increase efficiency of surgical treatment of patients as with the combined and isolated pathology of the complicated AF. The combination of the Maze procedure to open operation on heart considerably doesn't influence number of complications and a lethality. The further assessment requires the analysis of the long-term results.

P64

Patients carrying two mutations in the genes of interest are at high risk of sudden cardiac death and rapid cardiomyopathy progression

Elena Zaklyazminskaya ${ }^{1}$, Anna Shestak', Margarita Polyak ${ }^{1}$

Leonid Makarov², Olga Blagova ${ }^{3}$, Sergey Dzemeshkevich ${ }^{\prime}$

${ }^{1}$ Petrovsky Russian research Centre of Surgery, Moscow, Russian

Federation; ${ }^{2}$ Federal Centre of Diagnostics, Treatment and Prophylaxis of Cardiac Arrhythmia in Children and Adolescents, Moscow, Russian

Federation; ${ }^{3}$ Sechenov First Moscow State Medical University, Moscow,

Russian Federation

Correspondence: Elena Zaklyazminskaya

Journal of Cardiothoracic Surgery 2017, 12(Suppl 1):P64

Objective: The most of the primary cardiomyopathies are monogenic but polyallelic disorders with autosomal dominant mode of inheritance. Molecular genetic test is the gold standard for diagnosis confirmation but the impact of particular mutation on risk assessment is still to be determined. The aim of the study is to evaluate the prevalence and clinical phenotype of those patients who carry two mutations in the genes responsible for inherited cardiomyopathies and channelopathies.

Methods: Clinical examination and genetic testing was performed in accordance to Helsinki declaration. Clinical examination included echo-cardiography, cardiac MRI, resting ECG and Holter monitoring. DNA-diagnostic had included NGS (lon Torrent) simultaneous sequencing of the target gene panel (11 genes for primary arrhythmia; 10 genes for HCM and LVNC; and 14 genes for ARVC), followed by Sanger sequencing of detected variants.

Results: We had found double-mutation carriers in 12 out of 232 probands (5.2\%) with cardiac arrhythmias (channelopathies). All of them had experienced multiple syncopes, 6 died suddenly before age of 20 y.o. despite anti-arrhythmic therapy. No double-hit mutations carriers were found in 45 ARVC patients but two mutations in the PKP2 and $D S C 2$ genes were detected in 1 apparently healthy SCD victim (14 y.o. boy). We had found 6 out of 130 (5\%) patients with HCM and/or LVNC with two mutations. Early manifestation, prominent cardiac remodeling, syncope and ventricular arrhythmia were the common features of these patients.

Conclusions: Two independent mutations in the genes encoding cardiac ion channels can be considered as an independent genetic 
risk factor of life-threatening arrhythmias. Patients carrying two mutations in the genes encoding sarcomeric and structural proteins also have a high risk of ventricular arrhythmias and rapid progression of cardiac remodeling. Careful primary prevention of SCD including early implantation of anti-arrhythmic devices should be considered for this particular group of patients.

\section{P65}

Impact of the right ventricular mid-septal pacing for left ventricular function in patients with permanent pacemaker implantation

Adil K. Baimbetov, Kanat A. Yergeshov, Kenzhebek A. Bizhanov Syzganov' National Scientific Centre of Surgery, Almaty, Kazakhstan Correspondence: Adil K. Baimbetov

Journal of Cardiothoracic Surgery 2017, 12(Suppl 1):P65

Aim: To compare the effect between the right ventricular apex (RVA) pacing and the right ventricular mid-septal (RVMS) pacing for left ventricular function and hemodynamics status in patients with bradyarrhythmias.

Methods: We observed 219 patients (age $59 \pm 19$ years, 137 men), who underwent permanent pacemaker implantation suffering from AV block III. We evaluated 122 patients with RVMS-pacing and 97 patients with RVA-pacing. Left atrial volume index, Pulsed-wave Doppler-derived mitral inflow indices, colour M-mode flow propagation velocities (Vp), Tissue Doppler measurements of systolic and diastolic (e') velocities at four mitral annular sites and mitral E/e' ratio, were assessed by transthoracic echocardiography, before and 6 month after implantation.

Results: Permanent RVA-pacing contributes to increase interventricular dyssynchrony and to increase pressure into the left atrium that lead to diastolic dysfunction of LV, as a result mechanical dyssynchrony caused by dyssynergia of systolic activation of walls LV. However, RVMS-pacing procedure did not lead to increase intraventricular dyssynchrony and to impairment of hemodynamics status of LV. Conclusion: This study reveals that permanent RVMS-pacing procedure contributes to less left ventricular dysfunction and mechanical dyssynchrony of walls LV compared with a conventional RVA-pacing procedure in patients with permanent pacemaker implantation. This abstract has already been published [1].

\section{Reference}

[1] Baimbetov AK; Iskakova BK; Moldabekov TK; Oshakbayev KP. The effect of the right ventricular mid-septal pacing for left ventricular function and hemodynamics status in patients with permanent pacemaker implantation. Pacing and Clinical Electrophysiology 2011;34(11):1307-61.

\section{P66}

The prevalence of atrial fibrillation in the almaty region of kazakhstan

Dinara Akpanova', Salim Berkinbayev' ${ }^{1}$, Dinara Ospanova ${ }^{2}$, Aisulu Musagaliyeva', Gulnara Dzhunusbekova'

${ }^{1}$ Scientific Research Institute of Cardiology and Internal Diseases, Almaty, Kazakhstan; ${ }^{2}$ Kazakh Medical University of Continuing Education, Almaty, Kazakhstan

Correspondence: Dinara Akpanova

Journal of Cardiothoracic Surgery 2017, 12(Suppl 1):P66

Objective: In recent years, Kazakhstan has seen increased incidence of cerebrovascular disease, and stroke in particular. Atrial fibrillation (AF) is the strong independent risk factor of stroke. Despite recommendations for anticoagulant therapy to patients with AF use of oral anticoagulants in real practice remains low.

Methods: The first step was to study the prevalence of AF in Kazakhstan. So, 1,500 adults aged over 18 years were interviewed in the Almaty region for the detection of AF. AF diagnosis was confirmed in the presence of the AF incident on ECG.The second step was a retrospective study of 162 case histories of patients with atrial fibrillation and acute stroke in stroke clinic in Almaty for the period from January to September 2015. Data were reported using frequencies and means, $\mathrm{p}$-value were calculated using X2-test.

Results: The prevalence of $\mathrm{AF}$ among the adult population in the Almaty region was $3.3 \%$. The mean age of participants was $47 \pm 11$ years, $69,9 \%$ was female. The prevalence of $\mathrm{AF}$ in participants aged $<40$ years was $2.9 \%$, in $40-49$ years group was $1.8 \%$, in $50-59$ years- $3 \%$, in adults aged $>60$ years $9.2 \%$. The prevalence of $\mathrm{AF}$ among the patients with acute stroke was $9.6 \%$. The mean age of patients with AF $70.9 \pm 0.82$ years, $60 \%$ was female. In patients with AF $80.9 \%$ was ischemic stroke, 8.9\%- transient ischemic attack, $10.5 \%$ hemorrhagic stroke. $37.7 \%$ patients with AF had prior stroke in anamnesis. The mortality in patients with atrial fibrillation and stroke was higher than in patients without AF (22.3\% vs $5.7 \%$; RR $3.6,95 \% \mathrm{Cl} 2.48 ; 5.08$ ). Only $8 \%$ patients with AF received oral anticoagulants before hospitalization

Conclusions: There are a high prevalence of AF among the adult population in Kazakhstan. At the same time the effectiveness of the stroke prevention in patients with atrial fibrillation remains unsatisfactory.

P67

Remote interrogation or in-clinic interrogation: benefits for icd follow-up

Anatoly A. Nechepurenko', Nikolay N. Ilov', Elena A. Kulikova', Mourad Moukhliss $^{2}$

${ }^{1}$ FCCVS (Astrakhan, Russia), Astrakhan, Russian Federation; ${ }^{2}$ Astrakhan

State Medical University (Astrakhan, Russia), Astrakhan, Russian

Federation

Correspondence: Anatoly A. Nechepurenko

Journal of Cardiothoracic Surgery 2017, 12(Suppl 1):P67

Objective: Remote Monitoring Systems (RMS) have showed its efficacy and feasibility in European trials. However, this technology is not widely spread in Russia. The purpose of this study was to compare two ICD follow up strategies: in-clinic interrogation (ICI) versus remote interrogation (RI) using RMS.

Methods: A total amount of 190 adult patients with a dual ICD (60\%) or CRT-D $(40 \%)$ were included in this retrospective, controlled, singlecenter clinical trial. They were divided into group 1 (100 pts using RMS) and group 2 (90 pts using $(\mathrm{Cl})$. Both groups had no significant differences in clinical and demographic characteristics. A period between scheduled transmissions/visits in clinic was 6 months. For a period of 6 months we analyzed 90 follow-up cards and about 500 RMS transmissions. Study end points included detection of arrhythmias, signs of leads failure and related events. A time from event occurrence to cardiologist's reaction on this event was calculated (TtR). Results: We did not estimate any difference in SVT (7\% vs 3\%, $p>0.01$ ) or VT detection rate ( $21 \%$ vs $23 \%, p>0.01)$ in studied groups. In 4 pts defibrillator lead failure was observed. In group 2 it led to inappropriate ICD therapy, meanwhile in group 1 (3 pts) it was early detected prior to shock. Sensing disorders ( $T$ and $R$ waves oversensing) were revealed in group 1 only ( 4 pts, $p<0.1)$. ICl was valuable due to antiarrhythmic therapy corrections which were made in $10 \%$ of pts from group 2 . TtR was significantly shorter in group 1 (7 days vs 65 days, $\mathrm{p}<0.05$ ).

Conclusions: $\mathrm{Rl}$ is safe and equal to $\mathrm{ICl}$ in efficacy of detection of arrhythmias and leads' failure. $\mathrm{RI}$ is superior to $\mathrm{ICl}$ in shorter TtR. It makes an opportunity to change individual therapeutic strategy without patient's visit in clinic.

P68

Our first outcome pulmonary vein isolation using second generation $28 \mathrm{~mm}$ cryo balloon

Adil K. Baimbetov, Kanat A. Yergeshov, Kenzhebek A. Bizhanov, llinara A. Yakupova

Syzganov' National Scientific Centre of Surgery, Almaty, Kazakhstan

Correspondence: Adil K. Baimbetov

Journal of Cardiothoracic Surgery 2017, 12(Suppl 1):P68 
Aim: to demonstrate our primary results after pulmonary vein cryoablation in patients with paroxysmal atrial fibrillation.

Materials and Methods: From 2014, in our electrophysiological laboratory, more than 200 ablation procedures of pulmonary vein isolation were carried in patients with paroxysmal atrial fibrillation. In selecting patients for cryoablation procedure patients with paroxysmal atrial fibrillation are taken with a condition of size of the left atrium is not more than $42 \mathrm{~mm}$ and not previously received any treatment. In order to avoid impairment of phrenic nerve, pacing through superior vena cava was carried out. At the end of isolation of each vein spiral mapping catheter was used to confirm the degree of isolation. The result showed that it is possible to achieve $95 \%$ of complete isolation in all the veins and $5 \%$ of incomplete isolation which is due to the anatomy of the left atrium and pulmonary veins. Results: During the procedure, significant complications were not observed. However, there was phrenic nerve palsy in 18 patients which evolved during isolation of right superior pulmonary vein. In 2 patients, the phrenic nerve palsy was maintained for 6 months, the rest went on their own within 1 month. In addition to that, return of AF was observed in $27 \%$ of patients who again underwent a RFA procedure using the navigation system with the construction of three-dimensional map of the left atrium.

Conclusion: The method of pulmonary vein isolation using cryoballoon of 2 nd generation is considered as highly effective and safe procedure in treatment of paroxysmal atrial fibrillation with anatomic non-dilated left atrium. This technique can be recommended not only for patients with paroxysmal, but also for persistent atrial fibrillation.

\section{P69}

Risk of arrhythmias manifestations in hemodialysis patients

Ulyana Kharlamova', Marina Mironchenko ${ }^{2}$

'South-Ural State Medical University, Chelyabinsk, Russian Federation;

${ }^{2}$ Chelyabinsk regional oncology centre, Chelyabinsk, Russian Federation

Correspondence: Ulyana Kharlamova

Journal of Cardiothoracic Surgery 2017, 12(Suppl 1):P69

Objective: Studying of predictors of arrhythmia's manifestations in hemodialysis patients

Methods: It is surveyed 215 hemodialysis patients during 5 years. To all patients were conducted clinical, laboratory, cardiovascular ultrasonic research.

Results: During follow-up in $15.8 \%$ of arrhythmia cases. With the arrhythmias was associated: hemodialysis-associated hypotension (OR 9,41; 95\% Cl [2.65- 14,24], $p=0,003)$; the presence of hyperparathyroidism (OR 3,48; $95 \% \mathrm{Cl}[0,97-12,51], \mathrm{p}=0,05)$; myocardial infarction in anamnesis (OR 10.08; 95\% Cl [2,58 - of 39.48], $\mathrm{p}=0,0009$ ); weight gain between hemodialysis sessions (OR 1,0028; $95 \% \mathrm{Cl}[1,0001-1,0055]$, $\mathrm{p}=0,03)$; age (OR 1.07; $95 \% \mathrm{Cl}[1,02-1,13], \mathrm{p}=0,006)$; the level of parathyroid hormone (OR 1,001; $95 \% \mathrm{Cl}[1,0002-1,002], \mathrm{p}=0,01)$; atherogenic coefficient (OR 1.81; 95\% Cl $[1.04-3,14]$, $p=0,03)$, Charlson comorbidity index (OR 2.34; $95 \% \mathrm{Cl}[1.64-3.35], p=0,0001)$; the size of the left atrium (OR 2.61; 95\% Cl $[1,11-6,11], p=0,02)$; pulse wave velocity (OR 4.52; 95\% Cl $[1.02-20,00], p=0,04)$.

Conclusions: During clinical, laboratory and instrumental studies in hemodialysis patients identified predictors of the manifestation of arrhythmias, which enables calculation of probability of their development.

\section{P70}

Small diameter vascular grafts made from electrospun

biodegradable polymers

Martin Kaláb', Jana Horáková², Petr Mikeš², Ales Šaman²,

Vladimir Lonský', Jiri Páral ${ }^{3}$, Věra Radochová ${ }^{3}$

${ }^{1}$ Department of Cardiosurgery, Faculty of Medicine and Dentistry, Palacky University Olomouc, Olomouc, Czech Republic; ${ }^{2}$ Technical University of Liberec, Faculty of Textile, Department of Nonwovens and Nanofibrous Materials, Liberec, Czech Republic; ${ }^{3}$ Faculty of Military Health Sciences, University of Defence, Hradec Králové, Hradec Králové, Czech Republic Correspondence: Martin Kaláb

Journal of Cardiothoracic Surgery 2017, 12(Suppl 1):P70
Objective: There is a pressing need to develop desirable small diameter vascular graft in some complicated cases. A promising approach is to develop off the shelf available graft composed of nanofibers that resemble the native extracellular matrix of native blood vessels. Therefore, biocompatible polymers have been electrospun and tested for its utilization as bypass grafting material. Methods: Vascular grafts, having the diameter between 1-6 mm, were produced by electrospinning. Biodegradable polyesters polycaprolactone and copolymer of polylactide-polycaprolactone were used for fabrication of prosthesis. Fibrous samples were visualized by scanning electrone microscopy and image analysis in order to characterize the resulting fibrous structure. Fibrous samples were tested in vitro with endothelial cells. Electrospun vascular grafts were implanted in animal models as a bypass of carotid artery (rabbits, pigs). The patency of the grafts was assessed by ultrasonography. Finally, the grafts with surrounding tissue were examined histologically.

Results: Biodegradable polymers were used for fabrication of tubular grafts composed of homogeneous fibers having about $1 \mu \mathrm{m}$ in diameter. Both used polymers in forms of microfibers promoted adhesion of endothelial cells in vitro. Preliminary results of in vivo tests showed patency of the grafts after a month of implantation. Prosthesis were infiltrated by cells indicating the ongoing healing process and regeneration of the tissue. However, histological assessment revealed fibrotic tissue surrounding the grafts. grafts with surrounding tissue were examined histologically.

Conclusions: Tubular scaffolds with diameter below $6 \mathrm{~mm}$ have been produced from polymers cytocompatible with endothelial cells. Prosthesis patency was confirmed by ultrasonography after a month making these materials promising for vascular tissue engineering. However, further modification of electrospun grafts will be considered. The financial support of this project is provided thanks to the Ministry of Health of the Czech Republic (Grant no: 15-29241A).

P71

HeartMate 3 assist device as destination therapy in end-stage heart failure patients

Yuriy Pya, Serik Bekbossynov, Makhabbat Bekbossynova, Assel Medressova, Saltanat Andossova, Saltanat Dzhetybayeva, Muradym Murzagaliyev, Svetlana Novikova

National Research Center for Cardiac Surgery, Astana, Kazakhstan

Correspondence: Assel Medressova

Journal of Cardiothoracic Surgery 2017, 12(Suppl 1):P71

Objective: Our center was one of the enrolling centers in the HeartMate 3 assist device CE Mark Study in 2014. Post-approval using of HeartMate 3 started in January 2015 first in the world in Kazakhstan. The objective of this report was to determine outcomes of patients with pre- and post-approval HeartMate 3 assist device. Methods: We analyzed data from 62 patients ( 8 patients were within the study and 54 patients - in the HeartMate 3 post-approval period). Most of them (96.7\%) are male. Mean age was $49 \pm 13$ years old. The median duration of support is 398 days. Maximum duration of support is 903 days (patient remains ongoing).

Results: The patients were a mix of "bridge to transplantation" (66\%) and "destination therapy" (34\%) patients. Patients were in INTERMACS profiles 3-4 in most of cases ( $n=60,96.7 \%)$. Currently 48 patients are ongoing with HeartMate 3 support, 6 transplanted and 7 expired. One patient was explanted on postimplant day 233 due to outflow-graft thrombosis secondary to infection, placed on extracorporeal membrane oxygenation, and died in 1 month. Kaplan-Meier survival estimates for patients with HeartMate 3 assist device were $95 \%$ after 1 month, 93\% after 6 and 12 months, $87 \%$ after 1 year and 6 months and $73 \%$ after 2 years. The main reason of death was the hemorrhagic stroke $(n=4,6.4 \%) .17$ patients $(27.4 \%)$ experienced right ventricular failure with setting right ventricular assist device only in 1 case (1.6\%), driveline infections was observed in 7 patients (11.3\%). There have been no hemolysis or pump thrombosis.

Conclusions: Overall experience with the HeartMate 3 has been positive with the high rates of survival. We didn't observed hemolysis or pump thrombosis. We hope that additional work with patients, 
their families, the local coordinators will help to improve the use of HeartMate 3 assist device.

\section{P72}

The Society of Thoracic Surgeons database application in a

European context: the first Italian experience

Giuseppe M. Raffa, Marco Turrisi, Michele Pilato

IRCCS-ISMETT (Istituto Mediterraneo per i Trapianti e Terapie ad

altaspecializzazione), Palermo, Italy

Correspondence: Giuseppe M. Raffa

Journal of Cardiothoracic Surgery 2017, 12(Suppl 1):P72

Objective: The Society of Thoracic Surgeons Adult Cardiac Surgery Database (STS ACSD) is globally recognized as the most robustly riskadjusted cardiac surgery registry, with over 6 million patients. Our institute became Italy's first STS ACSD participant in 2014. We sought to evaluate the impact of STS ACSD quality measurement on outcomes following 2 years of active participation.

Methods: From January 1, 2014 and to December 31, 2015, the quarterly outcome reports data in conjunction with STS quality benchmarks comparisons were shared among our surgeons and data management team as part of an IRB-approved quality improvement initiative.

Results: From January 1, 2014 to December 31, 2015, the available STS major composite operations (CABG, isolated AVR, AVR+CABG) were analyzed for 30-day or in-hospital mortality or the occurrence of any of 5 major morbidities (stroke, reoperation, renal failure, prolonged ventilation $>48 \mathrm{hrs}$, or deep sternal wound infection). There were 287 major composite operations performed in 2015 compared to 334 in 2014 ( $p=0.78$ ). Compared to 2014, we observed a significant reduction in the occurrence of any major morbidity in 2015 $(p=0.001)$, as well as slight decreases in post-operative length of stay. Reoperation for bleeding, prolonged ventilation and renal failure rate dropped from $1.8 \%, 8 \%$, and $4.1 \%$ to $1 \%, 3.1 \%$ and $1.3 \%$, respectively $(p=0.001)$. Process measure performance of preoperative betablockade and discharge beta-blocker, lipid lowering and antiplatelet medications for isolated CABG remained a challenge throughout the study period. Reductions in red blood cells transfusion rate were observed.

Conclusions: By utilizing our STS quality reports and benchmark data, institutional efforts and surgeon feedback resulted in significant improvement in overall morbidity and length of stay in the first 2-years of STS ACSD participation. The beneficial experience of international comparative effectiveness and quality improvement may assist other centers contemplating international STS ACSD participation.

\section{P73}

Using of bare metal stents in type b dissection treatment

Dmitry Sirota, Alexander Chernyavskiy, Maxim Lyashenko, Dmitry Khvan, Sergey Alsov

Siberian Biomedical Research Center Ministry of Health Russian

Federation, Novosibirsk, Russian Federation

Correspondence: Alexander Chernyavskiy

Journal of Cardiothoracic Surgery 2017, 12(Suppl 1):P73

Objective: Evaluation of the results of hybrid thoracoabdominal aortic repair.

Methods: From 2013 to 2016 twenty patients undergone hybrid thoracoabdominal aortic repair using bare metal stent Djumbodis. All patients had chronic aortic type B dissection. Procedure included conventional replacement of descending thoracic aorta and open abdominal aortic stenting at the level of the lower thoracic intercostal, visceral and renal arteries under X-ray control. There were $14(70 \%)$ men and и 6 (30\%) women. The average age was 50.3 \pm 8.9 (21-67) years. 6 (30\%) patients underwent procedure as second stage after conventional elephant trunk surgery. We used atrialfemoral bypass and cerebrospinal fluid drainage in all cases.

Results: The median time of operation was $356(337 ; 406) \mathrm{min}$. The mean time of stent placement was $8,1 \pm 2 \mathrm{~min}$. The median time in
ICU stay was $7(5 ; 9)$ days, the median time of artificial lung ventilation was $34(21 ; 53)$ hours. The average in-hospital stay was 25 \pm 6 days. All patients were performed CT-scan of thoracic and abdominal aorta at discharge, there wasn't any case of visceral malperfusion or permanent spinal cord injury. One case of postoperative mortality was due to lower limb malperfusion (5\%).

Conclusions: Hybrid thoracoabdominal aortic dissection repair using bare metal stents is feasible. This hybrid approach is time-saving technology, avoiding reimplantation of visceral and spinal arteries and decreasing complications, especially spinal cord injury.

P74

Percutaneous catheterization of the coronary sinus and the pulmonary artery in redo aortic surgery

Pietro Giorgio Malvindi, Vito Margari, Georgios Kounakis, Giuseppe Visicchio, Carmine Carbone, Domenico Paparella, Cataldo Labriola GVM Care \& Research, Ospedale Santa Maria, Bari, Italy

Correspondence: Pietro Giorgio Malvindi

Journal of Cardiothoracic Surgery 2017, 12(Suppl 1):P74

Background: Percutaneous catheterization of the coronary sinus (CS) and the pulmonary artery (PA) was conceived as a complement to mini-thoracotomy heart surgery in the late 1990s. We have reported the feasibility and the safety of these techniques in minimally invasive aortic valve surgery through either a $\mathrm{J}$ hemisternotomy and a right anterior thoracotomy. We describe the implementation of percutaneous catheterization of the coronary sinus and the pulmonary artery in the setting of redo aortic procedures.

Methods: During the period 02/2016-02/2017, 7 patients undergoing a redo aortic procedures received the percutaneous catheterization of the CS and the PA. Indications for reoperation were: Post surgical aortic false aneurysm $(n=3)$, prosthetic aortic valve endocarditis $(n=2)$, aortic valve stenosis in patients with previous mitral valve surgery $(n=1)$, TAVI retrieval $(n=1)$. Median age 70 [27 - 83] years. Male/Female 4/3. Indications for necklines: Proximal aortic false aneurysm/reducing tissue dissection $(n=3)$, planned ministernotomy for severe adhesions $(n=2)$, severe aortic regurgitation/expected difficulties in coronary ostia handling/patent LIMA-LAD $(n=2)$. The CS and PA cannulation was performed under TEE and pressure-curve monitoring.

Results: There were no complications associated with the cannulation of the CS and the PA. Cardioplegic arrest was successfully induced in all the cases by the initial retrograde dose of blood cardioplegia. The PA vent provided a dry and bloodless field throughout the procedure. The cardiac procedures: Aortic root replacement $(n=2)$, false aneurysm repair+aortic arch replacement $(n=1)$, aortic valve replacement $(n=4)$, were carried out without any technical problems. The postoperative course was uneventful in all the cases

Conclusions: Use of specific necklines for cardioplegia delivery and heart venting can ease conventional heart surgery. In our initial experience in the setting of redo aortic surgery and in patients presenting with infection, multiple sternotomies, severe adhesions, this strategy allowed for an effective cardiac protection and a satisfactory bloodless surgical field.

P75

Horizon scanning: how to assess valid tools in short period of time Katy Pelagagge ${ }^{1}$, Mihaela Dubinski ${ }^{1}$, Beatrice Desirée Calabrese ${ }^{1}$,

Yermek Akhmetov ${ }^{2}$, Piergiorgio Minotti ${ }^{1}$, Filippo Bartoccioni ${ }^{3}$

${ }^{1}$ HTAcamp, Viterbo, Italy; ${ }^{2}$ Astana Medical University, Astana, Kazakhstan; ${ }^{3}$ Asl Viterbo, Viterbo, Italy

Correspondence: Katy Pelagagge

Journal of Cardiothoracic Surgery 2017, 12(Suppl 1):P75

Objective: The aim of our study is to evaluate, in the shortest time possible, the beneficial potential effects on CVC sepsis related cases of the new experimental Guardian An-gel device (www.technologymedicine.com). 
Methods: The device was tested from may 2012 to August 2012 in all new cases of an intensive care unit (ICU). The data collection was monthly based from December 2006 to August 2012 for a total number of 68 consecutive months. All the new sepsis cases within the intensive care unit were been counted. A ratio was created "new sepsis cases/CVC implanted" and this ratio was created of each month. A time series analysis was done on the trend of this ratio though a Joinpoint regression analysis.

Results: The join point analysis highlighted a descended trend with only one statistical trend modification (Joinpoint) and this modification was located by the statistical software in May 2012 exactly the month when the device was starting to be used in the ICU. The slope trend went from -0.04 to $-6,90$ monthly percentage change. The statistical test was done with 4500 permutations and a P value of 0.0064444 and a Significant level of 0.0166667 . The number need to treat (NNT) was calculated as well and it was of 3,7.

Conclusions: The horizon scanning done with the Health Technology Assessment techniques showed and big change of sepsis cases in the ICU demonstrating the importance of this device in reducing the CVC sepsis cases. These results were proven in only few months thanks to the time series analysis and overcoming at the same time the ethic problems that often are present in this type of patients. This device will probably be a good investment in the clinical practice because we can actually save a sepsis case every 3,7 device used.

\section{P76}

Hemodynamics of plaque location in off-pump coronary bypass

Şanser Ates ${ }^{1}$, Tijen Alkan-Bozkaya', Gokcenur Oguz ${ }^{2}$, Kerem Pekkan³ Haldun Karagoz ${ }^{4}$

${ }^{1}$ Koç University Hospital, İstanbul, Turkey; ${ }^{2}$ Koç University Department of Mechanical Engineering, İstanbul, Turkey; ${ }^{3}$ Koç University, Department of Mechanical Engineering, Istanbul, Turkey; ${ }^{4}$ VKV.American Hospital,

Department of Cardiovascular Surgery, Istanbul, Turkey

Correspondence: Tijen Alkan-Bozkaya

Journal of Cardiothoracic Surgery 2017, 12(Suppl 1):P76

Background: For high-risk patients with advanced forms of atherosclerotic heart disease, off-pump coronary bypass surgery is procedure of choice. Plaque geometries vary considerably from patient to patient and and graft configurations are tailored for each patient to lead an optimal post-operative perfusion and graft hemodynamics. To our knowledge effect of plaque geometry on post-surgical flow has not been studied and may be important for the post-operative performance.

Methods: We aim to investigate the effect of different atheromatous plaques locations (lateralised/centralised or discrete stenosis) with same critical stenosis on post-surgical flow dynamics by calculating the flow fields through patient specific coronary artery tree with left internal mammary artery graft (LIMA). This approach resemble a virtual computational angiography leading quantitative results.

Patient specific anatomy of a 54 year-old patient with coronary heart disease is reconstructed and end-to-side anastomosis of LIMA graft is performed in our computational pre-surgical planning suite. Realistic lateralised and discrete stenosis geometries for scenarios of critical and non-critical stenosis ratios

(\%50 and \%90) are created based on real plaque geometries encountered in patients, inslico. High quality tetrahedral grids are created using Pointwise and 3D hemodynamic evaluation is achieved by solving steady, laminar, and incompressible Navier-Stokes equations using open source computational fluid dynamics software OpenFOAM.

Results: Preliminary simulation results suggests that the effect of plaque geometry has an important role in flow hemodynamics. The ideal graft patency that produces the minimum pressure gradient flow and wall shear stress pattern is achieved.
Conclusions: In future we may correlate post-surgical effect of coronary artery bypass grafting with different plaque geometries.

\section{P77}

Operation of surgical remodeling of the heart in multi-valve

rheumatic diseases

Feruz Turaev

RSCS, Tashkent, Uzbekistan

Journal of Cardiothoracic Surgery 2017, 12(Suppl 1):P77

Objective: The purpose is to present results of surgical remodeling at rheumatic multivalve heart diseases.

Methods: There were executed 54 operations. Male -10, female -44 , age $42,1 \pm 11,7$ years old. Mitral-tricuspid disease with enlarged Left Atrium - 23, Mitral-aortal-tricuspidal disease -19, mitral-aortal-tricuspidal disease with enlarged LA - 12. On MV there were following haemodynamic infringements: MS- 29, Mitral regurgitation-15, mitral restenosis -10 . On the aortal valve: AS-10, aortal disease without the accurate prevalence-21. On the tricuspidal valve: combined tricuspidal stenosis with prevalence of insufficiency - 48, TS-6. The term of Surgical Remodeling of the Heart we includ kinds of operations when 2 or 3 valves correction with left atrium reduction are done.

Results: Correction of 2 valves with atrioplastic of LA is executed at 23,3 valves - at 19, 3valves with atrioplastic LA-12. At correction of a pathology of valves following kinds of interventions are executed. There were done $24 \mathrm{MV}$ prosthetics with preservation of MV basic chords, at 30 - multicomponent MV plastic with basic back annuloplasty with two-layered stripe from a vascular artificial limb.

Aortal Valve reconstruction was done at 25 (by Carpentier -22, by ElKhury-3), at 25 prosthetics of AV. Annuloplasty of tricuspid valve was done at 38 by DeVega, by Doty - 16, 24 cases were taken further TV comissuroplastic. At 35 patients were done LA atrioplastic with one-stage suturing of the LA appendage (by Kawazoe-26, by "Mercedes" -7, by Sinatra-1), suturing of LA appendage -18 , thrombectomy from LA-10. Time of CPB was $140,3 \pm 35,5 \mathrm{~min}$, cross-clamp time $-105,8 \pm 24,3 \mathrm{~min}$. Hospital mortality $-1,85 \%(n=1)$.

Conclusions: Surgical remodeling at rheumatic multivalve heart diseases, including multicomponent correction 2 and 3 valves in a combination to one of reduction methods at left atrial, allows to restore normal functional indicators of heart with good nearest results.

P78

Experience in conducting waiting list for donor heart by nursing specialist on the base of one center in Kazakhstan

Zhadyra Duisenbina, Meruert Issupova, Gulzhan Myrzahmetova, Gulnur Nadirbekova, Saltanat Jetibayeva

JSC "National Research Center for Cardiac Surgery", Astana, Kazakhstan

Correspondence: Zhadyra Duisenbina

Journal of Cardiothoracic Surgery 2017, 12(Suppl 1):P78

Objective. Illustrating the experience in conducting waiting lists for donor heart by nursing specialist. Heart transplantation program in Kazakhstan was initiated in 2012 on the base of National Research Center for Cardiac Surgery (NRCCS), Astana. Our center is the only one in the country where waiting list is managed by nursing specialists. Monthly monitoring of recipients by nursing specialists begins from the moment of registration of a recipient in the waiting list. Taking into account large territory of the country and distance of regions, they conduct monitoring of every recipient. It includes 3 stages: online monitoring, ambulatory monitoring and periodic planned hospital admission.

Materials and techniques. 312 patients with chronic cardiac insufficiency in the age from 17 to 65 years have been registered since 2012. 
Results. Average age of patients in the waiting list is $37,8 \pm$ years There are 253 men (81.0\%) and 59 women (18.9\%). 48 patients (15.3\%) have been transplanted. An active waiting list includes 74 recipients (23.7\%). There are 109 patients (34.9\%) - recipients after LVAD implantation. Patients, who needed support of extracorporeal membrane oxygenation (EMO) - 3 (0.96\%). Recipients from the waiting list (due to deterioration in state) accepted for LVAD implantation with subsequent recurring inclusion into waiting list - 8 (2.5\%). 23 recipients (7.3\%) had following associated diseases: diabetes mellitus - 9 (2.8\%), Acute Cerebrovascular Event - 12 (3.8\%), Chronic Obstructive Pulmonary Disease - $2(0.64 \%)$, overweight - 28 (8.9\%), rejection of operation - 19 (6.08\%). 58 patients (18.5\%) have died. 23 patients $(7.3 \%)$ have been removed from the waiting list. Conclusion: Thus, maintaining the waiting list for donor heart by nursing specialists has demonstrated its effectiveness in the organizational approach to the heart transplantation, reducing participation of doctors in the process of preparation and maximizing their time for performing surgical procedures.

\section{P79}

An integrated approach to the system of prevention of infections associated with the healthcare in the conditions of the operational department

Olga Loktionova

Federal Biomedical Agency, Obninsk, Russian Federation

Journal of Cardiothoracic Surgery 2017, 12(Suppl 1):P79

The second half of the 20th century in surgery was marked by three important inventions:

laminar flow of ultrapure air, perioperative antibiotic prophylaxis and antibiotic therapy, minimally invasive surgery.

On the one hand, these inventions have made it possible to significantly reduce the number of postoperative complications. However, the successes achieved with the help of these inventions allowed us to doubt the necessity of observing the rules of asepsis, to weaken discipline and to neglect the rituals that were worked out by more than one generation of the surgical community.

It is unacceptable that neglect of discipline and rituals becomes a habit as it's almost impossible to win a habit. Therefore, it is necessary to form the right habits from the very beginning of the studing surgery.

It is alaso necessary to form a corporate culture of safety in the OR and create programs to prevent $\mathrm{BBI}$. Corporate culture implies an interaction of employers and employees: the employer is obliged to properly organize the working process, train the personnel, ensure the safety of the working environment. The employee must take care of his safety and be responsible for actions that threaten the safety of others, comply with the security orders and constantly improve the knowledge.

When forming the reporting procedure, the notification of undesirable events should be constructive, not repressive. The purpose is to obtain absolutely reliable information about the state of affairs that will allow making necessary changes to the security program and improving it.

\section{P80}

Endoscopic vein harvesting in coronary artery bypass surgery

Anar Khusainova, Gulnur Nadirbekova, Ermagambet Kuatbayev JSC "National Research Center for Cardiac Surgery", Astana, Kazakhstan Correspondence: Anar Khusainova

Journal of Cardiothoracic Surgery 2017, 12(Suppl 1):P80

Objective: Describe experience on conduction of endoscopic vena saphena magna harvesting for coronary artery bypass surgery.
Methods: 16 surgeries of mobilizing vena saphena magna were performed through mini-invasive access using endoscopic equipment AESCULAP and KARL STORZ within the period between 2016 and March, 2017. We studied the time and resources for preparing standard and endoscopic vein harvesting. At the earliest postoperative period, we evaluated frequency of postoperative complications at vein conduit harvesting and performance status of patients after surgery.

Results: The average age of patients was $62 \pm 12$. The followings were noted in the analysis of 16 surgeries of endoscopic vena saphena magna harvesting: formation of hematoma at the hip - 0, paresthesia 0 ; inflammatory conditions - 0 ; incompetence - 0 . No pain syndrome in autovenous intake. Time for graft harvesting was 20,5 9,5 minutes. In the first surgery, minimum set of surgical instruments consisting of $\sim 12$ items and suture material were used. In the second surgery, higher technological instrument (extractor for endoscopic vein harvesting, electric coagulator with bipolar mode) was used.

There was a significant cosmetic result in mini-invasive access (length of postoperative scar is no more than $2-3 \mathrm{~cm}$, in comparison with standard one, which is more than $40 \mathrm{~cm}$ ).

Patients with standard incision spend a lot of time and resources for binding and wound care in the postoperative period, while other group needs less time for rehabilitation.

Satisfaction rate of patients after endoscopic mobilization of vein was significantly higher in terms of both cosmetic result and decrease of pains in the postoperative period.

Conclusion: Despite of expenditure materials, endoscopic stand for vena saphena magna is efficient and safe method for preparation of autovenous intake for coronary bypass surgery and mini-invasive character of this method brings a minor frequency of complications in the postoperative period.

P81

Protecting the hands of surgical personnel from the point of view of evidence-based medicine

Iuliia Samoilova

Molnlycke Health Care Moscow Rep.office, Moscow, Russian Federation Journal of Cardiothoracic Surgery 2017, 12(Suppl 1):P81

A wide variety of medical products delivered to the medical market today dictates the need to form a correct approach to their choice. The requirements to the safety and effectiveness of medical devices are set out in state standards, but the standards can not consider the specifics of each medical procedure and contain only the most common basic requirements.

In this situation, the only way out is to evaluate the effectiveness and suitability of a medical device for a specific task in terms of evidence-based medicine, based on analysis of objective data of scientific research and practical experience. One of the most authoritative collections of scientific articles in medicine is the Cochrane library, which contains only proven scientific data free of commercial sponsorship.

The issue of protection of the hands of medical workers during surgery is given very serious attention in the world, and the Cochrane library contains a special section on the protective role of surgical gloves. The final review indicates that surgical gloves are damaged very often - from 17 to $34.7 \%$ of cases. At the same time, the use of double gloves leads to a significant reduction in the frequency of end-to-end injuries and a reduction in the incidence of blood on the hands of surgeons (from $13 \%$ to $2 \%$ of cases).

The use of double glove systems with color puncture indication provides an additional and higher level of protection. It is known that when using the usual single surgical gloves, about $77 \%$ of the punctures remain unnoticed. Puncture detection systems not only reduce the frequency of end-to-end injuries, but also allow you to 
quickly detect a puncture and take emergency measures to prevent infection.

\section{P82}

Timely use of a veno-arterial extracorporeal membrane

oxygenation improves survival in postcardiotomy heart failure

Roman Yarosh, Liana Shestakova, Dmitriy Krachack, Natalia Peatrovich, Yuriy Ostrovsky

Republican Scientific and Practical Center, Minsk, Belarus

Correspondence: Roman Yarosh

Journal of Cardiothoracic Surgery 2017, 12(Suppl 1):P82

Objective: Analysis of the results and effectiveness of VA ECMO in patients with postcardiotomy heart failure.

Methods: A retrospective single-center analysis of data from patients who received VA ECMO. We compared our experience with the ECMO for patients with postcardiotomy heart failure at two time periods: in the operating room (Group A) after unsuccessful weaning from cardiopulmonary bypass (CPB) and after transfer to the intensive care unit (Group B). Group A consisted of 31 patients (64.5\%), which could not be weaned from the $\mathrm{CPB}$, early initiation of VA ECMO performed in the operating room. Group B included 17 patients $(35.5 \%)$, requiring ECMO in the early postoperative period (1-6 days) in the intensive care unit.

Results: In 2010 - April 2016, 6359 was carried out open heart surgery with cardiopulmonary bypass. In $0.8 \%$ of cases $(n=48)$ developed postcardiotomy heart failure entailed initiation VA ECMO. In group A, survived ECLS - 54,8\% $(\mathrm{n}=17)$, survived to DC or transfer $-45,2 \%(n=14)$.In group $B-47,05 \%(n=8)$ and $29,4 \%(n=5)$, respectively.

Conclusions: Early use ECMO was in the first hours after the operations may increase the chance of eventual recovery.

\section{P83}

\section{Neurological complications after pediatric extracorporal} membrane oxygenation

Sergey Akhmetov', Dmitriy Gorbunov', Timur Lesbekov',

Tatyana Ivanova-Razumova', Shinar Mursalieva', Maya Zhumabaeva', Galimzhan Utegenov', Zhanna Rakish', Igor Tsoy', Ayzhan Ashirova', Tlektes Nashkenov', Olga Luksha²

${ }^{1}$ National Research Center for Cardiac Surgery, Astana, Kazakhstan; ${ }^{2}$ City Children's Hospital №1, Astana, Kazakhstan

Correspondence: Sergey Akhmetov

Journal of Cardiothoracic Surgery 2017, 12(Suppl 1):P83

Objective: The purpose of this study was to determine the frequency and nature of the neurological complications that occurred during the application of veno-arterial ECMO in children with CHD.

Methods: A retrospective analysis of VA-ECMO cases in the National Research Center for Cardiac Surgery at the period from 2011 to 2016 was performed. Statistical analysis of the obtained data was carried out using the $\mathrm{x} 2$ criterion for categorical variables and the Student's t-test for comparing mean values.

Results: The study included 57 cases of VA-ECMO in pediatric patients with CHD. In $39(68,5 \%)$ cases implantation was produced intraoperatively due to non-weaning from the CPB, 18 ECPR cases was performed. Survival before discharge was $40.4 \%$. In 18 (31.5\%) patients neurologic events was observed, including intracranial hemorrhage $(8.8 \%)$, stroke $(12.3 \%)$, epileptiform syndrome $(7 \%)$, anoxic CNS damage (1.7\%), brain death (1.7\%). In 7 (39\%) cases this complication occurred after ECPR. There was no significant difference between patients who underwent intraoperative ECMO implantation against ECPR ( $\left.p^{\prime} 0.05\right)$. The mean duration of ECMO in patients without neurologic events was $8.3 \pm 3.3$ days, in patients who had neurologic complications - $7.61 \pm 2.25$ days. There was no correlation between the duration of ECMO and the risk of neurological complications ( $p^{\prime} 0.05$ ). The mean duration of hospitalization in group with neurologic complications was $38,33 \pm 31,36$ days, in group without complications - $36,49 \pm 10,66$ days respectively. The analysis identified that development of neurological complications prolongs the period of hospitalization $(p=0.0009)$, however, it does not affect hospital mortality ( $\left.p^{\prime} 0.05\right)$.

Conclusion: The incidence of neurological complications in our study was not related to the indications for implantation and the duration of VA-ECMO. The development of neurological complications increased duration of hospitalization, but did not affect survival.

P84

External validation of the models in extracorporeal membrane oxygenator (ecmo) from developing country Puwadon Thitivaraporn, Saran Chiramongkol, Wirinda Chiravanich, Kanyarad Boonthim, Nopporn Pornpatrtanarak, Chanapong Kittayarak, Jule Namchaisiri, Seri Singhatanadgige, Pat Ongcharit, Vichai Benjacholamas

King Chulalongkorn Memorial Hospital, Bangkok, Thailand

Correspondence: Puwadon Thitivaraporn

Journal of Cardiothoracic Surgery 2017, 12(Suppl 1):P84

Objectives: This study was to describe and evaluate the proper situation and predicted outcomes of ECMO implantation in developing country and validate prior proposed models for prediction of mortality.

Methods: Data on ECMO used for cardiopulmonary support and eCPR were extracted. Demographic data included age, diagnosis, type of ECMO support, pre and post-ECMO parameters, postop complications and patient condition. We validated prior proposed 4 models using receiver operative characteristic curve and their area under the curve (AUC): 1) Respiratory Extracorporeal Membrane Oxygenator Surviving Prediction (RESP), 2) Extracorporeal membrane oxygenator network (ECMOnet) score for VV-ECMO, 3) Survival After Veno-arterial ECMO (SAVE) and 4) the Sequential Organ Failure Assessment (SOFA) Score for VA-ECMO. Associations between each of 4 models, 30-day and one-year survival were determined by hazard ratio and $95 \% \mathrm{Cl}$ calculated using Cox regression.

Results: 41 patients received ECMO, of which 23 were male at median age of $29.5 \pm 27.4$ year. Indication for ECMO were $6 / 41$ for respiratory support, $27 / 41$ for cardiac support and $8 / 41$ in e-CPR group which correspond with VV-ECMO $14.6 \%$ and VA-ECMO at $85.4 \%$. ECMO was successfully weaned off in $15 / 41$ while the survival to discharge rate was $12 / 41$. VV-ECMO survival rate was $3 / 6$ and VAECMO survival rate was $9 / 35$. Among survivor group, 4/12 patients needed ventricular assisted device (VAD) implantation (1/4 LVAD and 3/4 BiVADs). AUC of each model were $0.688,0.664,0.750$ and 0.625 for SOFA, SAVE, ECMOnet and RESP score, respectively. Only ECMOnet score of $\geq 7.5$ was associated with increased mortality at 30 days $(p=0.03)$ and 1 year $(p=0.03)$. Median ECMO duration was 125.5 hour and length of stay was 22.00 days.

Conclusion: From the 4 models, SOFA, SAVE and RESP were not associated with survival. However, ECMOnet score of $\geq 7.5$ was associated with increased mortality at 30 days and 1 year.

P85

Resuscitative capacity of ECMO in the cardiosurgical clinic

Stanislav V. Ibragimov, Sergey I. Belov, Vadim V. Pasyuga, Yuriy A. Shlakin, Dmitry G. Tarasov

Federal Center for Cardiovascular Surgery, Astrakhan, Russian Federation Correspondence: Stanislav V. Ibragimov

Journal of Cardiothoracic Surgery 2017, 12(Suppl 1):P85

Aim: To evaluate the results of using ECMO in adults and children in critical condition after cardiac surgery.

Methods: Over the 8 years of work, the Center has performed 30000 heart surgeries in adults and children. 151 patients required an ECMO procedure after surgery. The study presents the results of an 
analysis of this work performed in the intensive care unit in both an isolated version and as a component of cardiopulmonary resuscitation. Among the reasons that led to the procedure, the leading place is taken by perioperative damage and myocardial ischemia ( $55 \%$ of cases)

Results: Out of 151,78 patients $(51.65 \%)$ has the hemodynamics restored and the procedure was finished, 53 (67.94\%) of them were discharged from the clinic. Thus, the survival rate among patients receiving ECMO was $35.09 \%$. The causes of death included bleeding, heart failure, damage of the central nervous system, sepsis, MODS.

Conclusions: Inclusion of ECMO in the protocol of resuscitation measures allows to improve their result. The readiness of the staff for a quick start of the procedure makes it possible to initiate a repetitive surgical intervention and is a condition for achieving a positive resuscitation result.

\section{P86}

Cardiogénicic shock as debut of undiagnosed pheochromocytoma: ECMO, time to think

Míreia Fernández, José Ramón Echevarría, Lucía Pañeda, Miriam Blanco, Nuria Arce, Salvatore Di Stefano, Enrique Fulquet, Christian Ortega,

Manuel Fernández

Hospital Clínico Universitario de Valladolid, Valladolid, Spain

Correspondence: Lucía Pañeda

Journal of Cardiothoracic Surgery 2017, 12(Suppl 1):P86

\section{Withdrawn}

P87

Stability of the chest wall - the basic condition of successful therapy of sternotomy dehiscence; a 15 years analysis

Martin Kaláb ${ }^{1}$, Jan Karkoška ${ }^{2}$, Milan Kaminek ${ }^{3}$, Petr Šantavy ${ }^{1}$ 'Department of Cardiosurgery, Faculty of Medicine and Dentistry, Palacky University Olomouc, Olomouc, Czech Republic; ${ }^{2}$ National Cell and Tissue Centre, Brno, Czech Republic; ${ }^{3}$ Department of Nucler Medicine, Faculty of Medicine and Dentistry, Palacky University Olomouc, Olomouc, Czech Republic

Correspondence: Martin Kaláb

Journal of Cardiothoracic Surgery 2017, 12(Suppl 1):P87

Objective: Sternotomy dehiscence represents serious complication of cardiosurgery operation. Stability of the chest wall plays the key role. AO osteosynthesis, including the allogeneic bone graft transplantation in post-sternotomy defects, represents modern therapeutic approach.

Methods: In the retrospective analysis of the 15-years period, we compared methods of the re-cerclage and $\mathrm{AO}$ osteosynthesis technique in the reconstruction of the chest wall, also with the respect to the wound infect therapy evolution (closed wound drainage, CWD, and negative pressure wound therapy, NPWT). The efficiency of the allogeneic bone graft transplantation was compared with the group patients, with the residual chest wall instability, earlier technically insoluble. Fischer test, Student t-test, Mann-whitney $\mathrm{U}$ test and Shapiro-Wilk test were used in the statistical analysis.

Results: Comparing the following groups and sub-groups, AO osteosynthesis total $(n=42)$ and Re-cerclage total $(n=69)$, AO osteosynthesis/NPWT ( $n=39)$ and Re-cerclage/NPWT $(n=43)$, AO osteosynthesis $(n=39)$ and Re-cerclage/CWD ( $n=26)$, next statistically significant results, always with the advantage to the $\mathrm{AO}$ osteosynthesis technique, were described: lower hospital mortality, lower incidence of the first re-osteosynthesis failure, lower incidence of the first soft tissue reclosure failure, lower average index of re-osteosynthesis trials, lower average index of soft tissue re-closure trials, lower incidence of residual chest wall instability.
Conclusions: Using the $\mathrm{AO}$ osteosynthesis technique, including the allogeneic bone graft transplantation, the high index of the chest wall stability, decrease of mortality and reduction of next complications were reached.

P88

Which provocation test is more reliable for the clinical diagnosis of thoracic outlet syndrome?

Selcuk Kose', Murat Kara ${ }^{2}$, Atilay Buker ${ }^{3}$, Simay Kara ${ }^{4}$

${ }^{1}$ Bakirkoy Research and Training Hospital, Istanbul, Turkey; ${ }^{2}$ Canakkale

University School of Medicine, Canakkale, Turkey; ${ }^{3}$ Istanbul Research and

Training Hospital, Istanbul, Turkey; ${ }^{4}$ Acibadem University School of

Medicine, Istanbul, Turkey

Correspondence: Murat Kara

Journal of Cardiothoracic Surgery 2017, 12(Suppl 1):P88

Objective: Thoracic outlet syndrome is a clinical condition without any definitive clinical diagnostic tests. Provocation tests in the physical examination, cervical $\mathrm{x}$-rays, Doppler ultrasonography and electromyography might be used for the clinical diagnosis. However, symptomatic patients may even undergo operations without any positive laboratory findings. Provocation tests such as Adson, Halsted, and Wright are of particular significance in this regard, however the most reliable test for the clinical diagnosis remains unclear.

Methods: A total 102 patients, 15 (14.7\%) male and 87 (85.3\%) female with a mean age of $38.7 \pm 10.1$ underwent operation with the diagnosis of thoracic outlet syndrome. A total of 127 examinations were done with Doppler ultrasonography in these patients being bilateral in 25 cases. The patients were evaluated for the diameter and peak systolic velocity of the subclavian artery on neutral position and after provocation tests.

Results: The diameter of subclavian artery on neutral position was $6.3 \pm 0.8 \mathrm{~mm}$. The mean diameter of subclavian artery after Adson, Halsted, and Wright tests were $4.8 \pm 1.3 \mathrm{~mm}(\mathrm{p}<0.0001), 5.3 \pm 1.2$ $\mathrm{mm}(\mathrm{p}<0.0001), 5.4 \pm 1.1 \mathrm{~mm}(\mathrm{p}<0.0001)$, respectively. The peak systolic velocity of subclavian artery was $71.4 \pm 27.8 \mathrm{~cm} / \mathrm{sec}$ on neutral position. The mean peak systolic velocity of subclavian artery after Adson, Halsted, and Wright tests were $85.9 \pm 44.6(p<0.0001)$, $83.3 \pm 41.1(p<0.0001), 88.8 \pm 47.0(p<0.0001)$, respectively.

Conclusions: Adson, Halsted and Wright tests make a significant pressure on the subclavian artery. However, Adson test makes comparably more significant differences regarding the means of the diameter and the peak systolic velocity of the subclavian artery.

P89

Thoracic metastasis from breast cancer

Kosmas Tsakiridis, Stamatis Arikas, Konstantina Triantafillopoulou, Andreas Bakas

St Luke's Hospital, Thessaloniki, Greece

Correspondence: Kosmas Tsakiridis

Journal of Cardiothoracic Surgery 2017, 12(Suppl 1):P89

Objective: After breast cancer surgery, there is a recurrence rate of $30 \%$ of the patients in the first five years. This means that $36 \%$ of them have local recurrence, $58 \%$ have distant metastasis and $6 \%$ had a combination of the two.

Methods: Twelve women had thoracic metastasis. Ten of them had pulmonary metastasis and the rest had thoracic wall metastasis. The mean age was $63,8 \pm 9,9$ years. The disease free interval lasted for about 130,2 $\pm 96,7$ months. Two of the women had 30 and 10 year periods of disease free interval respectively. All of them underwent complete excision of one to five metastases.

Results: There were no deaths. The post-operative course was uneventful. Three of them died of widespread disease, two had bone 
metastasis and the rest remained stable according to their follow-up routines.

Conclusions: When the medical treatment fails or when there is a metastasis that can be removed then the surgical excision is achievable with very good results. R0 excision, extended disease free interval and fewer numbers of metastases (the ideal is one) provide this specific group of patients with a higher survival rate.

P90

Effectiveness of the modified ultrafiltration of blood on the state of the myocardium in children with congenital heart defects during surgery and in the early postperfusion period

Nurlan Baizhigitov', Tolegen Yegemberdiyev ${ }^{2}$, Kairat Kuatbekov', Anar Sepbayeva ${ }^{1}$

${ }^{1}$ Center for Perinatology and Pediatric Cardiosurgery, Almaty, Kazakhstan; ${ }^{2}$ Kazakh National Medical University, Almaty, Kazakhstan

Correspondence: Nurlan Baizhigitov

Journal of Cardiothoracic Surgery 2017, 12(Suppl 1):P90

Background: To study the effect of postoperative ultrafiltration of blood on the state of the myocardium in children with congenital heart defects during surgery and in the early postperfusion period. Most researchers agree that the observed by us increase in the general edema and contracture damage in the myocardium in the early post-perfusion period is the morphological criteria of the development of reperfusion injuries.

Methods: During 2016, biopsies of the right atrial appendage were taken from 150 children with septal heart defects during their surgical correction. After stopping the cardiopulmonary bypass (CB), all patients underwent the modified ultrafiltration (MUF) of blood by taking it from the arterial cannula and returning after the MUF directly to the right atrium via the trunk. Ultrafiltration volumes are calculated. Biopsies were taken at different stages of operation: before CB was connected, during myocardial ischemia, after the removal of clamp from aorta but before MUF, and 15 minutes after MUF. Histological methods (including coloring) were used along with light and polarization microscopy.

Results: Biopsies taken at the first stage showed changes in the form of vacuolular and granular dystrophy, and small contracture damages of cardiomyocytes. At the second stage, the changes continued to increase. After removing the clamp from aorta at the third stage, the intercellular edema increased even more, and contracture changes reached second and third degrees. Moreover, there was hyperrelaxation of cardiomyocytes which was not observed earlier. Biopsies taken after MUF showed that edema and contracture damage to the myocardium significantly decreased, hyperrelaxation of cardiomyocytes practically disappeared, myocardium structure restored.

Conclusions: The procedure of MUF immediately after stopping $C B$ prevents the development of edema, contracture damage and ischemic reperfusion changes in the myocardium. In further studies, it will be necessary to make comparisons with patients who have not undergone the MUF of blood.

P91

The average duration of cardiopulmonary bypass and cross-clamp at "save operations" and "significant operations"

Dauren A. Antikeyev, Oraz S. Mukashev, Adil A. Dyurzhanov,

Sagit B. Imangazinov, Bauyrzhan B. Tlemyssov, Aigul M. Nurgalieva,

Almas M. Antikeyev, Elena Yu. Kochengina

Pavlodar Regional Cardiological Center, Pavlodar, Kazakhstan

Correspondence: Dauren A. Antikeyev

Journal of Cardiothoracic Surgery 2017, 12(Suppl 1):P91
Objective:

To convert the qualitative concepts of "safe operation" and "significant operation" into quantitative indicators by showing duration of cardiopulmonary bypass (CPB) and cross-clamp (CC) time. Methods:

We studied 542 cases of adult patients operated on the heart in 2016. The first group included 310 cases with cardioplegia delivered by pressure-bag, the second group included 232 cases with using set (cardioplegia delivery system). Based on the perfusion protocols, the maximum and the minimum values, average duration of CPB and cross-clamp, standard deviation were determined.

Results:

$57.2 \%$ was operations with pressure-bag, $42.8 \%$ - with set. In the first group, the maximum duration of CPB T $\mathrm{CPB}$ max was $162 \mathrm{~min}$, the minimum $\mathrm{T}_{\mathrm{CPB} \text { min }} 30 \mathrm{~min}$, the average duration of CPB $\mathrm{T}_{\mathrm{CPB} \text { aver }} 72.94 \pm 18.26 \mathrm{~min}$; $\mathrm{T}_{\mathrm{CC} \max } 115 \mathrm{~min}, \mathrm{~T}_{\mathrm{CC} \text { min }} 15 \mathrm{~min}$, the average duration of cross-clamp $\mathrm{T}_{\mathrm{CC}}$ iver $44.48 \pm 14.97 \mathrm{~min}$. In the second group $\mathrm{T}_{\text {CPBmax }}$ was $287 \mathrm{~min}$, $\mathrm{T}_{\mathrm{CPB} \text { min }}$ 44min, $T_{\text {CPBaver }} 110.4 \pm 32.84 \mathrm{~min}$; $\mathrm{T}_{\text {CCmax }} 194 \mathrm{~min}, \mathrm{~T}_{\mathrm{CC} \text { min }} 13 \mathrm{~min}, \mathrm{~T}_{\text {CCaver }}$ $73.65 \pm 27.77 \mathrm{~min}$. Comparative analysis showed that the using of set allowed to increase the indicators to the following values: $\mathrm{T}_{\mathrm{CPBmax}}$ $287 \min (>77.16 \%), \quad \mathrm{T}_{\text {CPBmin }} 44 \mathrm{~min} \quad(>46.67 \%), \mathrm{T}_{\text {CPBaver }} 110.4 \mathrm{~min}$ $(>51.36 \%) ; \mathrm{T}_{\text {CCmax }} 194 \mathrm{~min}(>68.70 \%), \mathrm{T}_{\text {CCaver }} 73.65 \mathrm{~min}$ (>65.58\%). In the cardiosurgical clinic of Pavlodar at $57.2 \%$ of cases cardioplegia was delivered by pressure-bag, $42.8 \%$ - by set. In opinion of the cardiac surgeons, the "safe operation's" average duration of CPB and CC was $72.94 \mathrm{~min}$ and $44.48 \mathrm{~min}$, respectively.

Conclusions:

Operations with average duration of CPB $110.4 \mathrm{~min}$ and $73.65 \mathrm{~min}$ of cross-clamp were considered as complex and "significant operations". The differences between two groups in mortality are low, insignificant. In addition, this approach of cardiac surgeons together with perfusionists to choose the method of cardioplegia, which depends on the complexity of operation, results to $\$ 40000$ of economic benefit for 542 cases.

P92

Effect of modified ultrafiltration of blood on the immune response of body in children

Nurlan Baizhigitov ${ }^{1}$, Kairat Kuatbekov ${ }^{1}$, Alibek Mereke²,

Mukhtar Kulimbet ${ }^{2}$

${ }^{1}$ Center for Perinatology and Pediatric Cardiosurgery, Almaty, Kazakhstan;

${ }^{2}$ Scientific Research Institute of Fundamental and Applied Medicine,

Almaty, Kazakhstan

Correspondence: Nurlan Baizhigitov

Journal of Cardiothoracic Surgery 2017, 12(Suppl 1):P92

Background: This paper deals with the scientific analysis of the immune response during cardiopulmonary bypass. Methods of ultrafiltration (UV) and modified ultrafiltration (MUF) have found application in the practice of surgical interventions with the use of artificial circulation (IC) more than 20 years ago and remain relevant at the present time.

Methods: Authors conducted a retrospective analysis of the cases of children born with congenital heart disease from 2011 to 2016.

Results: Before the operation, the plasma levels of TNF in the group I was within physiological limits and was $13.4 \pm 2.24 \mathrm{pg} / \mathrm{ml}$. In group $\mathrm{II}$, at this stage, the figure was $11.5 \pm 0.02 \mathrm{pg} / \mathrm{ml}$. In step 2 to the study, during the CPB, no significant changes in the levels of TNF in the group I did not happen and the scale of it was $16.4 \pm 2.61 \mathrm{pg} / \mathrm{ml}$. In group II, at this stage the figure is also not changed significantly and amounted to $14.9 \pm 0.28 \mathrm{pg} / \mathrm{ml}$. Phase 3 after UF in Group I, there was a significant change levels of TNF, which have dropped to $9.9 \pm 0.84 \mathrm{pg} / \mathrm{ml}$. Group II was contrary significant increase up to 18.94 
$\pm 2.15 \mathrm{pg} / \mathrm{ml}$. Stage 4 studies, 3 hours after the operation, there was a speaker in relation to the plasma concentrations of TNF - its value was significantly decreased to $7.1 \pm 5.26 \mathrm{pg} / \mathrm{ml}$.

Conclusions: These data suggest that ultrafiltration of blood can effectively eliminate unrelated cell receptors and plasma protein cytokines. The use of UV and MUF methods allows controlled removal of excess fluid from the bloodstream, reducing the likelihood of edema development. Removal of inflammatory mediators from the bloodstream reduces the emergence of the syndrome of a systemic inflammatory response.

P93

Modified ultrafiltration and ultrafiltration when correcting children heart failure

Nurlan Baizhigitov, Kairat Kuatbekov, Abai Surtai, Berkin Turkulov, Gennadi Nigai

Center for Perinatology and Pediatric Cardiosurgery, Almaty, Kazakhstan Correspondence: Nurlan Baizhigitov

Journal of Cardiothoracic Surgery 2017, 12(Suppl 1):P93

Background: To justify the use of ultrafiltration (U) and modified ultrafiltration $(M U)$ methods for the balance of fluid during artificial circulation (AC) in children.

Methods: Conducted statistical analysis of the protocols of artificial circulation in children weighing up to $10 \mathrm{~kg}$, operated in the department of pediatric cardiosurgery of the Center of Perinatology and Pediatric Heart Surgery, Almaty city in 2016, using U and MU methods. Protocols for statistical processing were divided into 3 groups. Group 1 - perfusion protocols without using $U$ and $M U$ methods (15 people). Group 2 - perfusion protocols using $U$ (27 persons). Group 3- perfusion protocols using MU (8 patients). The difference in the volume of the injected and withdrawn liquid during the AC time $(\mathrm{ml} / \mathrm{kg} / \mathrm{min})$ was calculated, followed by a statistical analysis of the data. During the time interval during which the fluid balance was calculated, the AC time was taken in minutes.

Results: The fluid balance in Group 1 was $0.48 \pm 0.096 \mathrm{ml} / \mathrm{kg} / \mathrm{min}$; The balance of fluid in group 2 was $-0.12 \pm 0.062 \mathrm{ml} / \mathrm{kg} / \mathrm{min}$; The liquid balance in 3 was $0.46 \pm 0.068 \mathrm{ml} / \mathrm{kg} / \mathrm{min}$; The fluid balance in groups 2 and 3 is statistically significantly different from the fluid balance in group $1 \mathrm{p}<0.05$. There are also differences between groups 2 and $3 p<0.05$.

Conclusions: Comparison of the liquid balance during the AC time indicates the prevalence of the negative indicator in group 3 . The results of statistical analyzes shows the effectiveness of the use of $U$ and $\mathrm{MU}$ for the purpose of correcting the degree of hemodilution by removing excess fluid from the bloodstream.

\section{P94}

Application of modified ultrafiltration for prevention and treatment of edema in children when correcting congenital heart diseases

Nurlan Baizhigitov' ${ }^{1}$ Tolegen Yegemberdiyev², Abai Mereke³, Mukhtar Kulimbet ${ }^{3}$

${ }^{1}$ Center for Perinatology and Pediatric Cardiosurgery, Almaty, Kazakhstan; ${ }^{2}$ Kazakh National Medical University, Almaty, Kazakhstan; ${ }^{3}$ Scientific Research Institute of Fundamental and Applied Medicine, Almaty, Kazakhstan

Correspondence: Nurlan Baizhigitov

Journal of Cardiothoracic Surgery 2017, 12(Suppl 1):P94

Background: To study the effectiveness of modified ultrafiltration of blood to reduce extravasal edema in children operated under conditions of artificial circulation (AC). The use of AC in pediatric surgery, especially in early childhood, leads to accumulation of fluid in the extravasal space. The most promising method for the prevention and treatment of extravasal edema is MUF, the clinical use of which began in 1991.
Methods: Two groups of patients were examined: the main group 15 people (mean age $3.6 \pm 0.71$ years), control group - 12 people (mean age $7.1 \pm 1.04$ years). All the children of the main group were followed by MUF after the AC. The amount of total extracellular (extravasal) extravascular water of the body (OVVO) was controlled by the bioelectroimpedance method.

Results: In both groups, the postoperative period was compared. In the control group, there was an increase in OVVO with a maximum in the first 6 hours after the operation (from $84.8 \pm 3.1$ to $99.3 \pm$ $4.4 \%$ of body weight). Normalization of the index was on the 2 nd day after the operation.

Conclusions: Low mass, hemodilution, duration of myocardial ischemia and hypothermia increased the amount of edematous water. In the main group, the increase in extravasal water was significantly lower (from $90 \pm 0.5$ to $95 \pm 0.7 \%$ of body weight). Normalization occurred in the 1st day.

P95

Efficiency of myocardial protection by single-dose of "custodiol" in small children with aortic cross-clamp time more than 100 minutes D. T. Loginov, O. Y. Kornoukhov, V. N. Ilyin

Filatov Children's Hospital, Moscow, Russian Federation

Correspondence: D. T. Loginov

Journal of Cardiothoracic Surgery 2017, 12(Suppl 1):P95

Background. Clinical techniques of cardioplegia in children vary significantly from different surgical teams. Most of them prefer multiple-doses of infusion. We evaluated an efficiency of myocardial protection by single-dose of Custodiol infusion with prolonged aortic cross-clamp time.

Patients and methods. 62 patients underwent surgical procedures of complex CHD repair with a median weight of $7.3(3.1-13.4) \mathrm{kg}$ and an age of 355 (30-1433) days. The main group of observations had aortic cross-clamp time $\geq 100$ minutes (mean $=115 \mathrm{~min}, \mathrm{n}=25$ ) and the second group - less than 100 minutes (mean=83 $\mathrm{min}, \mathrm{n}=37$ ). The complexity of the surgical procedures in the main group was higher than in the second one according to Aristotle Basic Score (9.3 \pm 1.18 and $8.0 \pm 1.13$, respectively, $p=, 006$ ) and Comprehensive Score $(11.4 \pm 3,14$ and $8.9 \pm 1.83$, respectively, $p=, 004)$. Total induction volume of Custodiol $40-50 \mathrm{~mL} / \mathrm{kg}$ was infused into the aortic root during 7 minutes and was combined with systemic hypothermia $28-32^{\circ} \mathrm{C}$. Biochemical markers of myocardial damage (CK-MB, Myoglobin, Tnl) were evaluated before and 18 hours after the surgery. Venous pO2, sO2, lactate level, "vasoactive-inotropic score" (VIS) were measured at control points 0,6 and 18 hours after the operation.

Results. Hospital survival rate was $100 \%$. Biochemical markers of myocardial injury before and 18 hours after the surgery did not differ significantly between groups. Patients of the main group had slightly more inotropic support 18 hours after surgery in comparison with the second group: VIS $5.0 \pm 3.4$ and $3.4 \pm 2.2$, respectively $(p=, 03)$. There were no differences in levels of $\mathrm{pvO} 2, \mathrm{SvO} 2$ and blood lactate at all stages in both groups.

Conclusion. These data demonstrate satisfactory protection of the myocardium by single-dose Custodiol infusion in pediatric patients with an aortic cross-clamp time about 115 minutes.

P96

The ATS Medtronic 3F stentless aortic valve: an excellent prosthesis unappreciated and misunderstood. a single surgeon experience with 220 cases

Guglielmo Stefanelli', Fabrizio Pirro', Alina Olaru', Antonio Mingiano' Mariassunta Telesca ' Luca Weltert ${ }^{2}$

${ }^{1}$ Hesperia Hospital, Modena, Italy; ${ }^{2}$ European Hospital, Rome, Italy

Correspondence: Guglielmo Stefanelli

Journal of Cardiothoracic Surgery 2017, 12(Suppl 1):P96 
Background: Aim of this study was to evaluate the clinical and hemodynamic results after 220 consecutive implants of Medtronic $3 \mathrm{~F}^{\oplus}$ stentless equine pericardial aortic valve, and to confirm its ease and reproducibility of implantation.

Methods: Between March 2007 and August,2015 220 consecutive patients affected by aortic valve disease received a $3 \mathrm{~F}$ valve at our unit by a single surgeon. The size ranged between 21 and 29, with prevalence of size 23 and 25 .Mean age at operation was $73,8 \pm 8,5$ years. $58,7 \%$ of patients were males, the mean logistic EuroScore was $9,76 \pm 6,3$ and $51,8 \%$ of patients received concomitant procedures. For isolated valve replacement the mean ECC time was $90^{\prime} \pm 10^{\prime}$, Cross Clamp time $72^{\prime} \pm 8^{\prime}$.In 31 pts the aortic prosthesis was included in a Dacron tube straight graft for a Bentall operation.

Results: Early mortality in isolated AVR was 1,3\% and 1,8\% in the entire group. There have been 15 late all-causes deaths(10-non cardiac), with a survival of $89 \%$ at 9 yrs. $91 \%$ of patients were in NYHA class I or II at last control. Actuarial freedom from reoperation due to structural deterioration was $100 \%$ at 5 years, $96 \%$ at 8 years.Freedom from endocarditis and thromboembolic events at 9 years were $95 \%$ and $100 \%$ respectively. Mean aortic pressure gradient measured by echo was $11,5 \mathrm{mmHg}$ at hospital discharge and $10,4 \mathrm{mmHg}$ at the time of follow-up. Patient-prosthesis mismatch was absent in $90 \%$ of patients, moderate in $10 \%$.

Conclusions: The $3 \mathrm{~F}$ valve is an interesting new generation, user friendly stentless aortic valve substitute with excellent hemodynamics, resistance to infections, durability and freedom from structural deterioration at 9 yrs follow-up. Unfortunately, for many reasons mostly related to business strategies this prosthesis has not obtained a great diffusion among cardiac surgeons.

\section{P97}

Clinical and hemodynamic results of correction aortic valve disease in elderly patients: stentless and stented biological prosthesis Denis Demidov, Dmitry Astapov, Alexander Bogachev-Prokofiev, Sergey Zheleznov, Alexander Karaskov, Alexander Afanasyev E.N. Meshalkin Siberian Federal Biomedical Research Center, Ministry of Health Care of Russian Feder, Novosibirsk, Russian Federation Correspondence: Denis Demidov

Journal of Cardiothoracic Surgery 2017, 12(Suppl 1):P97

Objective: The growing number of biological valve replacement were performed in different age-subset patients in last decades. The aim of our study was to assess clinical and hemodynamic results in patients who scheduled to aortic valve replacement with stentless biological prosthesis. The use of a stentless biological prosthesis is intended to reduce the residual prosthetic gradient and to improve cardiac hemodynamics.

Methods: from 2011 to 2015114 patients were enrolled in a prospective randomized study. Group I include 57 patients with stentless biological prosthesis, group II - 57 patients with stented xenopericardial prosthesis. The primary endpoint was the value of the peak prosthesis gradient at five years after surgery, secondary points - incidence of valve dysfunction and late survival. The mean age was 71,5 \pm 4 (65-84). Mean left ventricle ejection fraction was 65.5 $(40-86) \%$. Mean preoperative Log.Euroscore was $5,4 \pm 1,3(1,4-12,4)$. Results: Mean follow-up were 42,07 $\pm 10,24$ and 44,2 $\pm 13,75$ months in group I and II, respectively $(p=0,219)$. Peak prosthesis aortic gradient were $16,3 \pm 4,4$ (8-29) and $24,7 \pm 4,7(14,4-34) \mathrm{mm} \mathrm{Hg}$, respectively $(p=0,006)$. Mean orifice area were $2,14 \pm 0,16(1,67-2,41)$ and $2,12 \pm 0,21$ $(1,77-2,7) \mathrm{cm} 2$, respectively $(p=0,342)$. The effective orifice area were $1,31 \pm 0,11(1,17-1,62)$ and $1,27 \pm 0,18(1,09-2,13) \mathrm{cm} 2 / \mathrm{m} 2$, respectively $(p=0,071)$. There are no significant differences in incidence of postoperative complications (neurological and cardiac events). There were no explant, valve thrombosis or dysfunctions during follow-up. The cumulative survival did not significantly differ between the groups (logrank test, $p=0,226)$ : 5 -years survival were $96 \%$ (90\%; 100\%), and $90 \%(81 \% ; 99 \%)$, respectively.

Conclusions: stentless xenopericardial biological prostheses demonstrate better hemodynamics in elderly patients with comparable 5-years clinical results.

P98

Medium term performance of the St Jude Trifecta aortic pericardial valve

Giuseppe Petrone, Giancarlo Sportelli, Luca Sandrelli, Paolo Pisani,

Giulio Tessitore, Ascanio Graniero, Guido Costantino, Mario Fabbrocini Heart Centre, City of Alessandria - Policlinico di Monza, Alessandria, Italy

Correspondence: Giuseppe Petrone

Journal of Cardiothoracic Surgery 2017, 12(Suppl 1):P98

\section{Introduction}

The aim of the study was to evaluate the midterm performance of the Trifecta bioprosthesis.

Methods

One hundred sixty-seven patients underwent aortic valve replacement (AVR) with the Trifecta valve between October 2013 and December 2016 in our institution. 72 patients were female and 95 were male $(56.8 \%)$. Mean age was $73.09 \pm 6.68$ (range $48-88$ years). Indications for aortic valve replacement surgery included stenosis in 87 patients $(52.1 \%)$, regurgitation in 51 patients $(30.5 \%)$, and mixed pathology in 29 patients (17.4\%). The mean preoperative pressure gradient was $52 \pm 16$ (range $22-83 \mathrm{mmHg}$ ), and the mean aortic valve area was $0.81 \pm 0.31$.

Results

In our series, $13.2 \%$ of patients received a $19-\mathrm{mm}, 40.1 \%$ had a $21-$ $\mathrm{mm}$ and $46.7 \%$ had a $23-\mathrm{mm}$. There were no intraoperative deaths. Three late deaths occured during the in-hospital stay. There were 7 thromboembolic events, including 2 strokes and 5 reversible neurologic events. Follow-up was complete among survivors. The average mean transvalvular gradient (mTVG) at 18 months was 9.5, 10.2 and $11.8 \mathrm{mmHg}$ (sizes 19 to 23 , respectively). There was no statistically significant difference between average mTVGs measured immediately after implantation versus at the 18 months follow-up in the overall population. The average effective orifice area at 18 months was $1.46,1.65$ and $1.83 \mathrm{~cm} 2$ (sizes 19-23, respectively). There were no instances of severe patient-prosthesis mismatch. No prosthesis dislocation, endocarditis, valve thrombosis or relevant aortic regurgitation was observed at follow-up. Complete left ventricular mass regression was observed in 89 patients $(53.2 \%$, patients with pure aortic stenosis or a combination of aortic stenosis and trace/mild aortic regurgitation) by 1 year postoperatively.

Conclusions

The study demonstrates that the Trifecta valve has a low complication rate and it confirms the good clinical outcomes and brilliant haemodynamic performance at a medium term follow-up.

\section{P99}

Stentless composite biological aortic valved conduit for bentall operation

Guglielmo Stefanelli', Fabrizio Pirro', Alina Olaru', Davide Trevisan', Luigi Steffanon', Luca Weltert ${ }^{2}$

${ }^{1}$ Hesperia Hospital, Modena, Italy; ${ }^{2}$ European Hospital, Rome, Italy

Correspondence: Guglielmo Stefanelli

Journal of Cardiothoracic Surgery 2017, 12(Suppl 1):P99

Background: Aim of this study was to verify the feasibility and easiness of construction, implantation and the hemodynamic behaviour of a woven tubular prosthesis containing a $3 \mathrm{~F}$ stentless valve for Bentall operation. 
Methods: Between April 2010 and August, 2015, 30 patients underwent Bentall operation, using a $3 \mathrm{~F}^{\oplus}$ stentless valve included in a Hemashield ${ }^{\otimes}$ straight woven tube graft. Mean age was 66.3 yrs.Mean ischemic time was 108', mean CPB time 123'. In the last 21 cases the bioroot was pre-assembled before clamping the aorta, with the size of implant obtained from precise intraoperative transesophageal echographic measurements, and the mean cross clamping time dropped to $88^{\prime}$.The valve, one size smaller than the tube, was secured inside the graft by three 4-0 polypropylene sutures at $120^{\circ}$. Same stitches were used to fix the valve commissural tabs to the graft wall.Proximal anastomosis to the aortic root by $\mathrm{U}$ stitches, coronary arteries reimplantation and distal anastomosis by 5-0 polypropylene on teflon felt completed the procedure.

Results: Early 30 days-in hospital mortality was $0 \%$. One patient died one year after surgery for a non-cardiac cause. The immediate postoperative transesophageal Echo showed in all cases a physiological movement of the valve leaflets whit images of linear and smooth flow and a mean pressure gradient of $8 \mathrm{mmHg}$. At last follow (range: 2 months- 7 years) all patients were in NYHA class I. Hemodynamic performance was unchanged with a peak pressure gradient of 13 $\mathrm{mmHg}$ and a mean pressure gradient of $9 \mathrm{mmHg}$.

Conclusions: The $3 \mathrm{~F}$ composite stentless bioroot represents a valid option for biological Bentall operation. We emphasize the importance of using a straight tube in order to keep the commissural tabs of $3 \mathrm{~F}$ valve in a vertical alignment, avoiding any leaflet distortion.

\section{P100}

Surgical results for infective endocarditis

Chan-young $\mathrm{Na}^{1}$, Taesik Kim²

${ }^{1}$ Keimyung University, Daegu, Republic of Korea; ${ }^{2}$ Korea University, Seoul, Republic of Korea

Correspondence: Chan-young $\mathrm{Na}$

Journal of Cardiothoracic Surgery 2017, 12(Suppl 1):P100

Objective: Infective endocarditis is a serious, life-threatening disease with significant morbidity and mortality. We evaluated the early-andlong-term postoperative outcomes of native valve and prosthetic valve infective endocarditis.

Methods: We retrospectively reviewed 72 patients $(25$ male, and 47 female, mean age $=51$ years, ranging from 17 to 80 years old) with proven infective native $(n=53)$ or prosthetic valve $(n=19)$ endocarditis who underwent heart valve surgery between December 1999 and August 2016 by a single surgeon.Regarding 53-native vale infective endocarditis, Twenty-six patients (49\%) underwent aortic (19 replacements, 5 homograft, and 1 repair and Bentall, respectively), $26(49 \%)$ mitral (17 repairs and 9 replacements), 6(11\%), tricuspid (4 repairs and 2 replacements) and 2 (4\%) pulmonic (1 repair/ replacement, respectively).Regarding 19 prosthetic valve infective endocarditis, redo-operation was performed from 2 months to 252 months postoperatively, ten patients $(53 \%)$ underwent redo-mitral, 5 (26\%) redo-aortic. $3(16 \%)$ redo- aortic and mitral, and $1(5 \%)$ redoBentall procedure.

Results: Overall hospital mortality was $2.7 \%(n=2)$, There was no operative mortality in native valve infective endocarditis and 2 (10.5\%) mortality in prosthetic valve infective endocarditis. Causes of early death were sepsis with multiple organ failure and intracranial hemorrhage, respectively. During the follow-up from 1 to 199months (mean=78 months), four (5.5\%) patients were performed redocardiac surgery (tricuspid valve replacement and coronary bypass surgery, respectively in native valve infective endocarditis, and aortic valve repair and redo-mitral valve replacement, respectively in prosthetic valve endocarditis)

Conclusions: We report satisfactory early-and-long-term results in native and prosthetic valve infective endocarditis. However, the outcomes are worse in prosthetic valve endocarditis. Thus we recommend aggressive surgery in native valve and prosthetic valve endocarditis.

P101

Implantation of the aortic root into the prosthesis - a new

convolution of the aortic surgery: from complex to simple

Alexander Chernyavskiy, Dmitry Khvan, Dmitry Sirota, Sergey Alsov, Maxim Lyashenko

Siberian Biomedical Research Center Ministry of Health Russia

Federation, Novosibirask, Russian Federation

Correspondence: Alexander Chernyavskiy

Journal of Cardiothoracic Surgery 2017, 12(Suppl 1):P101

\section{Objectives}

The Florida Sleeve technique was introduced as simplified aortic valve-sparing procedure. The aim of this study was to evaluate midterm results of modified Florida Sleeve technique in patients with aortic root aneurysm and concomitant aortic insufficiency.

Methodology

The study included all patients undergoing modified Florida sleeve procedure since 2011 to 2016 . The original Florida Sleeve technique was made without dissecting coronary arteries and repairing the graft under them. The primary end-point was increase in aortic insufficiency of grade $\geq 2+$ at 5 years.

Results

A total of 35 patients were enrolled. Mean age was $56 \pm 13$ years. Mean aortic annulus diameter was $27 \pm 2 \mathrm{~mm}$, sinuses Valsalva diameter was $54 \pm 7 \mathrm{~mm}$ and sinotubular junction diameter $52 \pm 7 \mathrm{~mm}$. Median aortic insufficiency was 3(interquartile range [IQR], 2;3). Cardiopulmonary bypass time and aortic cross-clamping time were $161([\mathrm{IQR}], 142 ; 198) \mathrm{min}$ and $120([\mathrm{IQR}], 105 ; 140) \mathrm{min}$. At 5 years of follow-up survival was $83.9 \%$. At 5 years freedom from aortic insufficiency more than $2+$ degree was $92.1 \%$. The median degree of aortic regurgitation at follow-up was $1([\mathrm{lQR}], 1 ; 1)$. Mean aortic annulus diameter was $26 \pm 1 \mathrm{~mm}$. Median sinuses Valsalva diameter was $43([\mathrm{IQR}], 38 ; 47) \mathrm{mm}$ and sinotubular junction diameter was $31([\mathrm{IQR}], 29 ; 32) \mathrm{mm}$. All follow-up data were collected by common closing date. Clinical follow-up was $100 \%$ complete and median follow-up period was 29 ([IQR],11;47) months.

Conclusion

Modified Florida Sleeve technique demonstrates acceptable survival and freedom from aortic valve insufficiency. It allows to expect good results in further follow-up and a broader usage of simplified aortic root reimplantation technique.

P102

Mitral valve replacement for left ventriculomegaly: is translocation of papillary muscles of anterior leaflet necessary?

Volodymyr Popov, Igor Zhekov, Kateryna Pukas, Elena Trembovetskaya National Institute of Cardio-Vascular Surgery Named After Amosov, Kiev, Ukraine

Correspondence: Volodymyr Popov

Journal of Cardiothoracic Surgery 2017, 12(Suppl 1):P102

Aim To determine the possibilities of correction of mitral valve disease (MVD) at left ventriculomegalia (LVM) (end-diastolic volume of the left ventricle $\geq 300 \mathrm{ml}$ ) by original method of translocation of papillary muscles of anterior leaflet (TPMAL).

Material. There were $85(62,1 \%)$ males and $52(37,9 \%)$ females with isolated MVD and combination with LVM were operated at period 2000-2016 yy in Institute..All pts was divided at 3 groups:1) group A (229 pts) - mitral valve replacement (MVR) + preserving of posterior leaflet of MV; group B (59 pts) MVR + preserving of posterior leaflet of MV + original method of TPMAL (island technique); group C (247 pts) only isolated MVR without preservation of MV's structure. 
Results. Data of echo for group A: end-diastolic volume (EDV) (ml): preoperative (PRE) - 331,4 $\pm 19,1$, postoperative period (11 - th day) (POST) - 247,2 $\pm 21,7$, remote period (RP) - 175,5 $\pm 23,2$; ejection fraction of LV (EFLV): PRE - 0,43 $\pm 0,03$, POST - 0,51 $\pm 0,04, R P-0,53 \pm 0,04$. Data of echo for group B: EDV (ml): PRE - 331,2 $\pm 29,1$, POST - 185,8 $\pm 23,1, \mathrm{RP}-$ $163,5 \pm 17,2$; EFLV: PRE $-0,43 \pm 0,05$, POST $-0,51 \pm 0,03, \mathrm{RP}-0,57 \pm$ 0,03. Data of echo for group C: EDV (ml): PRE - 337,4 $\pm 30,9$, POST $279,2 \pm 31,5, \mathrm{RP}-221,5 \pm 27,8$; EFLV: PRE - 0,42 $\pm 0,04$, POST - 0,44 \pm $0,03, \mathrm{RP}-0,46 \pm 0,03$.

Conclusion. Correction of MVD with preservation of valve apparatus of MV especially with original method of TPMAL (island technique) is highly-efficient intervention for pts with LVM and associated with good LV's morphometry at all postoperative period in compare with group $C(p<0,05)$.

\section{P103}

Midterm haemodynamic performance of the st. jude medical trifecta valve in the small aortic annulus: is there really a need for aortic root enlargement surgery?

Benjamin Adams, Prity Gupta, Amer Harkey, Mohamad Bashir, Shirish Ambekar, Rakesh Uppal, Kulvinder Lall

Barts Heart Centre, London, UK

Correspondence: Benjamin Adams

Journal of Cardiothoracic Surgery 2017, 12(Suppl 1):P103

Objective: The St. Jude Medical Trifecta aortic supra-annular bioprosthesis is regarded as the next generation in pericardial stented tissue valves. The unique design of tissue leaflets attached to the exterior of the valve stent provides unrivalled in-vivo mean gradients and hemodynamics. The aim of this prospective study was to evaluate the midterm hemodynamic performance of the Trifecta valve in patients with a small aortic annulus (Sizes $19 \mathrm{~mm} \& 21 \mathrm{~mm}$ ).

Methods: One hundred and forty two consecutive patients undergoing aortic valve replacement using the St. Jude Medical Trifecta valve (Sizes $19 \mathrm{~mm} \& 21 \mathrm{~mm}$ ) at a single UK centre over a thirty-eight month period were included in this study. Patients undergoing concomitant cardiac procedures were included. Assessment of hemodynamic function was carried out using transthoracic echocardiography pre-operatively and at follow-up, as well as transesophageal echocardiography intra-operatively.

Results: The study population consisted of 142 patients. Mean age was $78.7 \pm 10.4$ years. Implanted valve sizes were $19 \mathrm{~mm}(n=26)$ and $21 \mathrm{~mm}(\mathrm{n}=116)$. Subgroups mean post-operative pressure gradients were $11.4 \pm 3.6 \mathrm{mmHg}, 10.7 \pm 6.3 \mathrm{mmHg} 19 \mathrm{~mm} \& 21 \mathrm{~mm}$ cohorts respectively. Overall mean post-operative left ventricular ejection fraction was $53.2 \pm 9.8 \%$.

Conclusions: Clinicians have traditionally been reluctant to insert 19 and $21 \mathrm{~mm}$ valves due to patient prosthesis mismatch and have resorted to aortic root enlargement surgery with associated increase in risk. Our experience demonstrate excellent haemodynamic performance of the Trifecta bioprosthetic valve in the small aortic annulus. Should longer-term results continue to show persistently low gradients in the smaller annulus, clinicians should carefully consider the necessity for aortic root enlargement surgery.

\section{P104}

\section{Cardiac surgery in Marfan syndrome}

Chan-young $\mathrm{Na}^{1}$, Taesik Kim²

${ }^{1}$ Keimyung University, Daegu, Republic of Korea; ${ }^{2}$ Korea University, Seoul, Republic of Korea

Correspondence: Chan-young $\mathrm{Na}$

Journal of Cardiothoracic Surgery 2017, 12(Suppl 1):P104
Objective: Marfan syndrome is characterized by variable clinical manifestations, including skeletal, ocular, and cardiovascular abnormalities. Ascending aortic aneurysm ensuing dissection and rupture is the main life-threatening cardiovascular manifestations of Marfan syndrome. The aim of this study was to evaluate the operative results of patients with Marfan syndrome who underwent aortic root surgery

Methods: Author retrospectively analyzed the data of 72 operations in 68 patients with Marfan syndrome who underwent aortic root surgery between Jan. 1996 and Aug. 2014.

Results: Among the 68 patients, 16 patients (24\%) had family history. There were 55 patients who had aortic root replacement surgery, 17 patients who had aortic root sparing surgery. Aortic root replacement surgery included 52 button type aortic root replacement, and 3 Cabrol type aortic root replacement. Aortic root sparing procedure included 7 root remodeling procedure, 9 root reimplantation procedure and 1 other type aortic root sparing procedure. Combined procedures included 9 mitral valve surgery, 8 coronary artery bypass surgery, 2 total aortic arch replacement and 1 femorofemoral bypass surgery. There were one early death. During follow up period, 4 patients needed redo aortic root surgery included 3 recurrent aortic regurgitation and 1 endocarditis

Conclusions: Aortic root surgery in Marfan patients can be performed with good results. Close follow up of patients undergoing surgery is important.

\section{P105}

What variables predictive mortality in redo mitral valve? 20-year experience of a single surgeon

Daniel T. K. S. Almeida, Filipe T. K. S. Almeida, Rui M. S. Almeida

Assis Gurgacz University, Cascavel, Brazil

Correspondence: Rui M. S. Almeida

Journal of Cardiothoracic Surgery 2017, 12(Suppl 1):P105

Objective: Mitral valve diseases are one of the most prevalent heart valve diseases in the world. As an ideal valve substitute has not beenachieved, the great majority of patients will have their prostheses replaced once or more times, during their lifetime. Authors evaluated a consecutive series of mitral valve reoperations, performed by the same surgeon, and identifying the variables, predicting mitral valve mortality, using multivariate analysis.

Methods: A retrospective analysis, February 1993 to January 2015, of 107 patients, undergoing mitral valve reoperation was performed. The demographics included pre-operative clinical and ecochardiografic data, trans and post-operative variables. Data was analyzed in a multivariate analysis frame, to target, which would predict hospital mortality. The patients were divided according to the logistic EuroScore into two groups (0-9,9\% and $10 \%$ and above).

Results: The mean age was $48,00 \pm 15,18$ (8-76 years), being $71,96 \%$ females. In $70,09 \%$ biological valves were used and in the other cases the valve was replaced by a mechanical one $(23,36 \%)$ or repair was performed $(6,54 \%)$. Associated procedures were performed concomitantly in $9,35 \%$, being concomitant aortic valve replacement performed in $50 \%$ and CABG in 30\%. The left atrial appendage was closed in all cases were atrial fibrillation was the basic rhythm $(56,07 \%)$. The mean logistic EuroScore was $8,10 \%$. The mean length of stay in ITU was $3,00 \pm 6,15$ and in hospital $7,00 \pm 10,43$ days. The overall mortality was $14,02 \%$, mainly on the high EuroScore group, and on those patients with more than one reoperation.

Conclusions: The statistical analysis identified age, NYHA class and pulmonary hypertension as preoperative primary predictors for mortality. The statistical analysis identified age, NYHA class and pulmonary hypertension as preoperative primary predictors for 
mortality. The authors conclude that the reoperation can be performed safely on the technical point and the result depends on the patient pre-operative conditions.

\section{P106}

Diagnostics of persistent sepsis in patients with infective

endocarditis after surgical intervention

Natallia Feaktsistava, Ann Lukashevich, Natallia Kazaeva,

Svetlana Sudjaeva, Volga Sudjaeva

RSPC, Minsk, Belarus

Correspondence: Natallia Feaktsistava

Journal of Cardiothoracic Surgery 2017, 12(Suppl 1):P106

Objective: to study the role of early diagnostic of persistent sepsis in patients with infective endocarditis (IE) after surgical intervention in the early post-operative outcomes

Methods: we included 55 patients in the active phase IE $(43.4 \pm 13.6$ years). Blood sampling was performed before operation, on the $1 \mathrm{st}$, 7th days after. If in the blood plasma the content of procalcitonin (PCT) on the 1st and/or the 7th day did not differ from the initial one or increased $\leq 2.5$ times - the absence of persistence was diagnosed, increasing in PCT $>2.5$ times $\leq 5$ times - a moderate severity of sepsis persistence, increasing in PCT $>5$ times - a high severity of sepsis persistence. Patients were divided in three groups: group I - the absence of sepsis persistence $(n=13)$, group II with moderate severity sepsis persistence $(n=10)$, group III - high sepsis severity $(n=10)$.

Results: Before the operation, there were no statistically significant differences $(H=1.3, p=0.05)$. On the 1 st day there was a statistical difference the level of PCT in the groups ( $H=20.3, p=0.00001)$. On the 7th day, significant differences in the groups were found $(H=3.5$, $p=0.2)$. The level of PCT was statistically higher in groups II and III ( $R$ $=18.8, \mathrm{p} \leq 0.05$ and $\mathrm{R}=25.4, \mathrm{p} \leq 0.00001)$, compared with group $\mathrm{I}$. There were no differences between groups II and III ( $p>0.05)$. The PCT level in the groups on the 1st day has a direct strong statistically significant correlation of sepsis persistence degree in the subacute period after the operation $(r=+0.81 ; p \leq 0.05)$.

Conclusions: Dynamics of PCT level after valvular correction of IE makes it possible to conduct early diagnosis of the degree of sepsis persistence in order to initiate its treatment.

\section{P107}

Early experience after sutureless versus biological aortic valve replacement in high risk patients

Ibrahim M. Yassin ${ }^{1}$, Farouk M. Oueida ${ }^{2}$, Khaled Al Khamees ${ }^{2}$

Hatem Al Taher, Sherif Al Nosairy', Ali Bayoumi ${ }^{3}$, Abdul-Waheed Butt ${ }^{3}$, Baher N. Nashy ${ }^{5}$, Mustafa Al Refaei $^{6}$, Khalid Eskander $^{2}$

${ }^{1}$ Cardio-thoracic Surgery Department, Tanta University Hospitals, Tanta, 31111, Egypt; ${ }^{2}$ Cardiac Surgery Department, Saud al Babtin Cardiac Center, Al Dammam, Saudi Arabia; ${ }^{3}$ Anaesthesia Department, Saud al-Babtin Cardiac Center SBCC, Al Dammam, Saudi Arabia; ${ }^{4}$ Laboratory Department, Saud Al Babtin Cardiac Center SBCC, Al Dammam, Saudi Arabia; ${ }^{5}$ Cardiology Department, Zagazig University, Zagazig, Egypt; ${ }^{6}$ Cardiology Department, Saud al-Babtin Cardiac Center SBCC, Al Dammam, Saudi Arabia

Correspondence: Ibrahim M. Yassin

Journal of Cardiothoracic Surgery 2017, 12(Suppl 1):P107

Objective: We thought to evaluate our recent experience with one of the sutureless valves in the aortic position

Methods: Retrospective analysis of two randomized Groups (25patients) of stable, intermediate-high risk patients candidate for pure AVR. (Groupl) (Sutureless - AVR) (mean age of $69.8 \pm 5.5$ years, mean EuroSCORE II $9.6 \pm 4.3 \%$ ) versus a propenisity matched similar group (Groupll) (Biological -AVR) (mean age of $67.4 \pm 5.4$ years mean EuroSCORE II $8.2 \pm 3.9 \%)$

Results: Significant difference $(p<0.001)$ in Aortic cross-clamp time ( $35 \pm 13.8$ vs. $52 \pm 20.4 \mathrm{~min}$.) and cardiopulmonary bypass times $(59 \pm 21.1$ vs. $68 \pm 34.8 \mathrm{~min}$.). No significant differences in postoperative arrhythmias and pacemaker implantation (16\% vs. $20 \%)$ and $(8 \%$ vs.12\%). Significant difference $(p<0.01)$ in the required blood transfusion $(1.4 \pm 1.3$ vs. $2.1 \pm 1.8$ units), intubation time (7.2 \pm 3.6 vs. $12 \pm 6.8$ hours) and Intensive Care Unit stay ( $1.7 \pm 0.9$ vs. $2.8 \pm 1.9$ days). No significant difference in the total length of stay $(6.7 \pm 2.3$ vs. $7.4 \pm 2.1$ days). Significant difference in the mean prosthesis size $(23.9 \pm 1.9$ vs. $22.1 \pm 1.1 \mathrm{~mm}, p<0.01)$. Significant better hemodynamic performance in (Groupl) $(16.2 \pm 9.8$ vs. $21 \pm$ 8.5 and $8.4 \pm 6$ vs. $12 \pm 4.9 \mathrm{mmHg}, p<0.01$ )for the maximum and mean gradient respectively. The percentage of patients with mild to moderate paravalvular leak was statistically higher in Group I $(60 \%$ vs. $20 \% p<0.000)$ which sealed gradually during the first 3monthes. Significant difference in the (\%) decrease of the platelet count which is highly significant in postoperative day one $(67.2 \pm 15.8$ vs. $31.6 \pm 18.5, p<0.000)$. It gradually become insignificant within $(6.3 \pm 2.9$ days $)$.

Conclusions: The sutureless bioprosthesis seems as a good alternative in patients with a high-risk profile. It minimizes the operative time, surgical trauma and risk.

P108

Universal chordal-sparing mitral valve replacement: 20 years of clinical experience

Sergey Dzemeshkevich

NRSC, Moscow, Russian Federation

Journal of Cardiothoracic Surgery 2017, 12(Suppl 1):P108

Objectives: The purpose of the present study is to investigate the possibilities and evaluate the outcomes of a original procedure of universal chordal-sparing mitral valve replacement in patients with dysplasia, mitral insufficiency and rheumatic mitral stenosis.

Methodology: The technique of universal chordal-sparing mitral valve replacement presupposes the preservation of four strut chords with four small segments of leaflets that are fixed on the anterior and posterior semicircles of the fibrous ring of the mitral valve. Such distribution and fixation of the preserved chords are thought to ensure a uniform motion of the plane of the mitral orifice.

Results: During the period from September 1998 to September 2016, we performed 508 surgeries of universal chordal-sparing mitral valve replacement. 238 patients had dysplasia of the mitral valve leaflets (isolated myxomatous transformation of the leaflets with predominantly multiple chordal rupture, dysplasia and mitral failure combined with dilated and hypertrophic cardiomyopathy); and 270 patients had fibrotic rheumatic changes and calcified leaflets with predominant valve stenosis. Isolated chordal-sparing mitral valve replacement was performed in $23.3 \%$ of patients. Hospital mortality in the whole group including patients with dilated cardiomyopathy who underwent reverse cardiac remodeling surgeries was $3.7 \%$, mortality in patients who underwent isolated replacement was $1.1 \%$. Conclusion: The technique of universal chordal-sparing mitral valve replacement ensures complete preservation of annulopapillary chordal attachment, regardless of the type of mitral valve disease (whether it is mitral stenosis or mitral regurgitation). As for the functional effect, this procedure is identical to plastic reconstruction of the mitral valve.

P109

Early and late outcome of dialysis dependent patients after cardiac surgery

Sergey Leontyev, Piroze Davierwala, Lisa Gaube, Martin Misfeld

Heart Center Leipzig, Leipzig, Germany

Correspondence: Sergey Leontyev

Journal of Cardiothoracic Surgery 2017, 12(Suppl 1):P109

Objective: The aim of this study was to evaluate the preoperative predictors of in-hospital and medium-term mortality in patients with dialysis-dependent chronic renal failure (DD CRF) undergoing cardiac surgery procedures.

Methods: 483 consecutive patients with DD CRF underwent cardiac surgical procedures. Mean age was $65 \pm 11$ years, $32.3 \%$ were female. Combined surgical procedure (CABG with valve surgery) were 
necessary in $20.3 \%$ patients. Isolated coronary artery bypass graft (CABG), or isolated valve surgeries were performed in $39.8 \%$ and 32.3 $6 \%$, of patients, respectively. Urgent or emergency surgery was performed in $49.3 \%$ of patients. Endocarditis was an indication for surgery in $11 \%$ of patients.

Results: The in-hospital mortality was $15.3 \%$. The most common postoperative complication were respiratory failure, gastrointestinal complications, low cardiac output, stroke and sepsis and occurred in $25.7 \%, 12.4 \%, 11.8 \%, 5.6 \%$ and $5.2 \%$ of patients, respectively. The multivariante analysis indentified combined mitral and aortic valve pathology (OR3.7,95\%Cl 1.5-9, $\mathrm{p}=0.003)$, chronic obstructive pulmonary disease (OR2.6, 95\% $\mathrm{Cl} 1.1-5.9, \mathrm{p}=0.03)$, peripheral vascular disease (OR 2.3, 95\%Cl 1.04-4, $\mathrm{p}=0.003$ ), left ventricular ejection fraction $<30 \%$ (OR2.9, 95\% Cl 1.3-6.4, $\mathrm{p}=0.008$ ) and active endocarditis (OR2.2, $95 \% \mathrm{Cl} 1.04-4.6, \mathrm{p}=0.04)$ as the independent predictors of in-hospital mortality.

The independent predictors of medium-term mortality were previous cerebrovascular accident, active endocarditis, prior cardiac surgery and combined aortic/mitral valve pathology. The estimated 2-, 4- and 6-year survival was $50.1 \pm 2 \%, 34.3 \pm 2 \%$, and $20.3 \pm 2 \%$, respectively. Conclusions: Patients with DD CRF undergoing cardiac surgery have a high perioperative and medium-term mortality, particularly in the presence of combined aortic and mitral valve pathology, active endocarditis and poor left ventricular function.

\section{P110}

Treatment of poststenotic aneurysms of ascending aorta: choice of correction's method

Volodymyr Popov, Ivan Kravchenko, Vitaliy Kravchenko,

Oleksandr Bolshak, Vasyl Lazorishinets

National Institute of Cardio-Vascular Surgery named after Amosov, Kiev, Ukraine

Correspondence: Volodymyr Popov

Journal of Cardiothoracic Surgery 2017, 12(Suppl 1):P110

Purpose. To research possibilities of surgical treatment of poststenotic aneurysms of ascending aorta (PAAA) by different methods.

Methods. During 2000-2016 yy 581 patients (pts) with aortic stenoses (AS) and PAAA were consecutively operated in Institute. The average age of pts was $61,5 \pm 7,4(18-71)$ yy. At all group $25(4,3 \%)$ pts were in II NYHA class, $274(47,2 \%)$ pts were in III NYHA class and 282 $(48,5 \%)$ pts in IV. The following operations were performed: aortic valve replacement $(A V R)+$ original method of wrapping tape operation (WTO) of AA (Popov V.) - 226 (38,9\%) pts (group A), AVR + Robischek's operation - $237(40,8 \%)$ pts (group B), Benthal's $(n=94)$ and Wheat's $(n=9)$ operations - $118(20,3 \%)$ pts (group C).

Results. Hospital mortality were $0,8 \%$ in group $A, 1,6 \%$ in group $B$ and $3,1 \%$ in group $C(p<0.05)$. Staying in ICU was $49,3 \pm 6,2$ hours (group A), 54,2 $\pm 7,5$ hours (group B) and 77,4 $\pm 9,2$ hours (group C) $(p<0.05)$. Actuarial survival at 9 years after operation was occured in group A - 91,2\% ( $n=224)$, in group B - 88,3\% $(n=206)$, and group C $79,7 \%(n=101)(p<0.05)$. Echo examination of diameter of $A A$ for group A $(\mathrm{cm})$ : preoperative (PRE) $4,7 \pm 0,5$, postoperative (POST) (6-7 days) $3,8 \pm 0,3$, remote period (RP) $4,0 \pm 0,4$; for group $B$ : preoperative $5,0 \pm 0,5$, postoperative $-4,0 \pm 0,4$, remote period $4,1 \pm 0,3$ and for group C: preoperative $5,9 \pm 0,7$, postoperative $-3,4 \pm 0,3$, remote period 3,5 $\pm 0,3$.

Conclusion. On the basis of our experience we recommend the expedient original method of wrapping tape operation for moderate forms of AAA (diameter of AA till 5,5 cm) during AVR. Reconstruction of AA for PAAA by WTO is safe, chipper and prevent aneurysm formation at $A A$ at the remote period.
P111

Results of morrow procedure at patients without intervention on the mitral valve at hypertrophic obstructive cardiomyopathy Khakimjon Abralov, Miraziz Mirsaidov

Republican Specialized Center of Surgery named after academician V.Vakhidov, Tashkent, Uzbekistan

Correspondence: Khakimjon Abralov

Journal of Cardiothoracic Surgery 2017, 12(Suppl 1):P111

Background According to studies, concomitant mitral valve surgery was required in $11-20 \%$ of all surgeries at patients with hypertrophic obstructive cardiomyopathy. In this message we would like to represent results of the extended myectomy without interventions on the mitral valve at patients with hypertrophic obstructive cardiomyopathy who did not have an abnormality of the mitral valve apparatus. Methods We retrospectively analyzed 22 patients who undergoing extended myectomy from January 2005 to December 2016. There were 14 men (63.6\%) and 8 women (36.3\%). Patients age ranged from 6 to 41 years (mean $22.26 \pm 9.86$ ). Functional class by NYHA: $3(13.6 \%)$ patients was in class II, $17(77.3 \%)$ patients were in class III and 2 were $(9.1 \%)$ in class IV. In all patients underwent Morrow procedure. The duration of cardiopulmonary bypass in this cases varied from 31 to 79 minutes averaging $(49.2 \pm 13.64 \mathrm{~min})$. Occlusion of the aorta varied from 22 to 68 minutes, on average ( $37 \pm 13.48$ $\mathrm{min})$. The duration of stay in the ICU ranged from 1 to 3 days, averaging $(2.22 \pm 0.67)$. Patients were divided into two groups, depending on the degree of preoperative mitral regurgitation. One group had regurgitation I, and the other had moderate or severe regurgitation on the mitral valve.

Results A significant reduction in the symptoms of the disease and an improvement in the quality of life was observed in all cases of surgical treatment. The gradient of the systolic pressure on LVOT decreased on average from $106.7 \pm 43.08 \mathrm{~mm} \mathrm{Hg}$ to $40.6 \pm 23.64$ $\mathrm{mm} \mathrm{Hg}$. One (4.76\%) patient after surgery had complite AV block, when implantation of permanent pacemakerwas required.

Conclusions Concomitant intervention on the mitral valve are not necessary for patients with hypertrophic obstructive cardiomyopathy and mitral regurgitation caused by systolic anterior motion of themitral valve.

P112

Surgical treatment of severe infective valve endocarditis. single-centre experience

Mikhail Nuzhdin, Sr. ${ }^{1}$, Aleksey Fokin², Denis Loganenko', Yuriy Malinovskiy', Pavel Vereshagin' 'Vitaliy Pupkov', Alik Serazhitdinov' Garik Asatryan ${ }^{1}$

${ }^{1}$ Chelyabinsk Regional Clinical Hospital. Department of Cardiac Surgery, Chelyabinsk, Russian Federation; ${ }^{2}$ South Ural State Medical University,

Chelyabinsk, Russian Federation

Correspondence: Mikhail Nuzhdin, Sr.

Journal of Cardiothoracic Surgery 2017, 12(Suppl 1):P112

Objective: We represented the profile and outcome of patients with infective endocarditis (IE) operated on in a single department over a period of 8 years.

Methods: Between 2009 and 2016, a total of 326 patients with IE underwent surgery. Of these, 55 (16.9\%) had mitral IE, 97 (29,7\%)aortic IE, 120 (36,8\%)-tricuspid IE and $54(16,6 \%)$ had multiple valve IE. Root involvement was in $26(8 \%)$ pts. Blood culture positive IE was in $68,7 \%$. The most frequent microorganisms were St.aureus, Enterococcus spp., Streptococcus spp. Hepatitis C was found in $30,6 \%$ and HIV in $8,9 \%$. IVDA-associated IE was in $30,6 \%$ pts. 212 $(65 \%)$ had to be operated upon due to severe congestive heart failure, $17,1 \%$ due to uncontrolled infection and severe valve 
regurgitation. Perioperative, operative characteristics, early postoperative results, long-term survival and freedom from IE recurrence were analysed. Follow-up (maximum: 7.5 years) was completed in 62.8\% pts. Results: Overall in-hospital mortality was $2.4 \%$. For valve replacement we used either mechanical or stented biological prosthesis. In cases of aortic root involvement complete debridement and pericardium patch reconstruction was performed. In postoperative complications profile were heart failure $(35,2 \%)$, respiratory $(10,7 \%)$, renal failure $(1,5 \%)$, neurological $(1,8 \%)$, sepsis $(0,6 \%)$, wound infection $(1,8 \%)$, need for pacemaker implantation (3,3\%). The 30-day, 1-, 5- year survival was $97.5 \%, 88.6 \pm 2.7 \%$ and $67.4 \pm 3.5 \%$. The poor 1 -year and long-term survival were strongly associated with IVDA, HIV pts. Predictors of early mortality were emergency operation, multiple valve replacement, severe multiorgan damage. Freedom from re-operation due to recurrent IE at 1-and 5 years was $87.5 \pm 5.6 \% 79,3 \pm 4,7 \%$.

Conclusions: Surgery for IE continues to be challenging despite improvements in pre- and postoperative care. The in-hospital mortality in such patients is seen to be appropriate in our study. High longterm mortality is associated with preoperative patient's profile and risk factors.

\section{P113}

Mitral valve replacement with small cavity of left ventricle

Kateryna Pukas, Svitlana Andrievskaya, Volodymyr Popov,

Vasyl Lazorishinets

National Institute Of Cardio-Vascular Surgery named after Amosov, Kiev, Ukraine

Correspondence: Kateryna Pukas

Journal of Cardiothoracic Surgery 2017, 12(Suppl 1):P113

Aim. To determine significance of patient-prosthesis mismatch (PPM) (indexed effective orifice area $<1,2 \mathrm{~cm}^{2} / \mathrm{m}^{2}$ ) after isolated mitral valve replacement(MVR) in pts with small cavity of left ventricle (SCLV) (end-diastolic volume (EDV) $\leq 75 \mathrm{ml}$ ) during hospital period.

Materials. 1811 adult patients (pts) with isolated mitral valve disease MVR were operated in Institute from 01.01.2000 till 01.01.2007. There were $127(7,0 \%)$ pts with SCLV. There were $48(37,8 \%)$ males and $79(62,2 \%)$ females. $110(86,6 \%)$ pts belonged to IV NYHA class of heart failure, $17(13,4 \%)$ - to III class. Following prostheses were implanted: bileaflet (Saint Jude, Carbomedics, On-X, Edwards-Mira) $(n=88)$ and monodisc as Alcarbon's type (MIKS, LIKS) $(n=40)$. Following prosthesis sizes were used: $23 \mathrm{~mm}(n=1), 25 \mathrm{~mm}(n=74), 26 \mathrm{~mm}$ $(\mathrm{n}=3), 27 \mathrm{~mm}(\mathrm{n}=49)$.

Results. Hospital mortality $(H M)$ was $5,5 \%(n=7)$. It was higher in cases with $27 \mathrm{~mm}$ size of implanted prosthesis $-8,2 \%(n=4 / 49)$, than other group - 3,8\% $(n=3 / 78)(p<0,01)$. PPM (indexed effective orifice area $\left.=1,03 \pm 0,03 \mathrm{~cm}^{2} / \mathrm{m}^{2}\right)$ were marked in $21(16,5 \%)$ pts with BSA $>1,75 \mathrm{~m}^{2}$ and size of prothesis $25 \mathrm{~mm}$ but theren't influence on HM. But heart failure and PPM were marked in $5(3,9 \%)$ pts with $B S A>1,75 \mathrm{~m}^{2}$, size of prothesis $25 \mathrm{~mm}$ and cavity of LV (EDV $\left.\leq 50 \mathrm{ml}\right)$. In these cases implantation of $25 \mathrm{~mm}$ prosthesis is expedient, but for pts with EDV $\leq 50 \mathrm{ml}$ and $B S A>1,75 \mathrm{~m}^{2}$ it may lead for significant PPM (indexed effective orifice area $=0,93 \pm 0,02 \mathrm{~cm}^{2} / \mathrm{m}^{2}$ ) and heart failure. During 5 postoperative days volume of left ventricle increasing on $15 \%$. During this period inotropic support (dobutamin $4,0-5,0 \mathrm{mcrg} / \mathrm{min} / \mathrm{kg}$ ) is necessary. $23 \mathrm{~mm}$ prosthesis may be used in pts with body mass $\leq 45 \mathrm{~kg}\left(\mathrm{BSA}<1,5 \mathrm{~m}^{2}\right)$.

Conclusion. Pts with SCLV are in group of higher risk for operation and increasing risk of PPM.

\section{P114}

Modified method of implanting neochords in degenerative mitral valve repair

Murat Mukharyamov, Roin Dzhordzhikiya, Rustem Khairullin,

Ildar Vagizov, Ildar Abdulyanov, Artur Kaipov

MKDC Kazan, Kazan, Russian Federation

Correspondence: Murat Mukharyamov

Journal of Cardiothoracic Surgery 2017, 12(Suppl 1):P114
Objective. The aim of the current study is to analyze the results of neochords implantation for anterior and/or posterior leaflet prolapse with excessive tissue plication with the PTFE suture.

Methods. 48 patients operated between 2012 and 2016 were retrospectively included in the study. The mean age was $54 \pm 14$ years, 29 were males. All patients had severe degenerative mitral valve regurgitation due to Barlow syndrome or "forme fruste" kind of degenerative lesions. All operations were performed via median sternotomy with cardiopulmonary bypass and cardioplegia. The CV-5 PTFE suture was passed twice through the fibrous tip of the papillary muscle corresponding to the prolapsing segments. Arms of the suture were then passed through both sides of the areas between normal and prolapsed segments at a width slightly less than the diseased segment. Two arms were tied down with the single or double knots to achieve annular plane and to plicate and pleat excessive tissue into the ventricular site. Annuloplasty ring (Medlng Rigid-AM) was sized according to the final anterior leaflet area and implanted in a standard fashion.

Results. The mean cross-clamp and cardiopulmonary bypass times were $72 \pm 14$ and $84 \pm 11$ minutes respectively. There were no perioperative mortality or major morbidity observed. 2 cases (4\%) required second cardioplegia and valve replacement due to the unsatisfactory echocardiographic result. 2 cases (4\%) of moderate residual regurgitation were left untreated because of general patient related considerations. The $82 \%$ of the group were available for the follow up. The freedom from the recurrent mitral insufficiency in the long term is $92 \%$ with the mean follow up period of $33 \pm 14$ months. Conclusions. Plication of the prolapsing segment with the two arms of the single PTFE artificial chord suture is the reproducible, simple and reliable method to correct both excessive height and width of the posterior and/or anterior leaflet.

\section{P115}

Mitral valve prolapse correction with pre-measured gore-tex loops Pavel Kakhktsyan, Irma Tsiskaridze, Leonid Spirin, Garik Aleksanyan,

Alexander Vavilov, Ivan Scopin

Bakulev NSPCCVS, Moscow, Russian Federation

Correspondence: Pavel Kakhktsyan

Journal of Cardiothoracic Surgery 2017, 12(Suppl 1):P115

Objective: We assessed the early and late outcomes for the loop technique in patients with mitral valve (MV) prolapse.

Methods: A total of 118 patients (74 male) underwent MV repair with Gore-Tex loops for severe mitral regurgitation (MR). The mean age was $50,6 \pm 13,7$, the mean preoperative LVEF was $63,6 \pm 7,0 \%$, the mean NYHA functional class was 2,7 $\pm 0,5$, the mean logistic EuroSCORE $-3,0 \pm 2,6$. Posterior mitral leaflet (PML) prolapse only was diagnosed in 24 patients, anterior leaflet (AML) prolapse only in 19 patients, both leaflet (BL) prolapse - in the 75 patients. The 90 $(76,3 \%)$ patients had concomitant procedures.

Results: All patients received the loop technique and the ring annuloplasty, with a mean ring size $32,8 \pm 2,3 \mathrm{~mm}$. In 30 patients loops were used for PML only, in 39 patients for AML only, and in 49 patients for BL. PML resection and sliding annuloplasty was performed in $23,7 \%$ and $13,6 \%$ of cases, respectively. No patient died early postoperatively. Predischarge echocardiography revealed trace or mild MR in $100 \%$ patients. The mean MR grade decreased from $3,24 \pm 0,45$ at baseline to $0,62 \pm 0,37$ before discharge. There was 2 cases of severe recurrent MR for 81 months in this series, MV systolic anterior motion was not observed. The mean coaptation lengths among all patients were 10,1 $\pm 2,57 \mathrm{~mm}$ and 9,57 $\pm 2,69 \mathrm{~mm}$, the mean coaptation depth $-5,0 \pm 2,5 \mathrm{~mm}$ and $4,95 \pm 2,1 \mathrm{~mm}$, the mean MV effective orifice area $-3,88 \pm 1,08 \mathrm{~mm} 2$ and 4,0 $\pm 0,92 \mathrm{~mm} 2$ in the early and late periods of observation, respectively. Reoperation free actuarial survival for 81 months in this series were $98,31 \%$.

Conclusions: MV repair with premeasured Gore-Tex loops shows excellent early and late results for mitral leaflet prolapse. 
P116

Long-term effectiveness of minimally-invasive xenograft

bioprosthesis in isolated mitral valve replacement - does the age matter?

Jens Garbade, Piroze Davierwala, Joerg Seeburger, Anna Meyer,

Martin Misfeld, Friedrich-Wilhelm Mohr, Sven Lehmann

Heart Center Leipzig, Leipzig, Germany

Correspondence: Jens Garbade

Journal of Cardiothoracic Surgery 2017, 12(Suppl 1):P116

Objective: Structural alteration of biological prosthesis in mitral position is one major limitation leading to potentially reoperation. For this reason mechanical valve is the first choice for MV replacement in younger patients. The study aim was to evaluate the structural event free outcome after isolated mitral valve (Epic stented porcine xenograft, St. Jude Medical) implantation in a very young population using minimally invasive surgical Approach (EpicBioMiniMV).

Methods: Between 2001 and 2016 a total of 357 patients underwent isolated EpicBioMiniMV with a mean age of $70.9 \pm 9.3$ years. Of these, 14 patients ( 7 male) were operated with a mean age of $43.3 \pm 6.8$ years. The indication for EpicBioMiniMV was severe symptomatic MV dysfunction. The decision for bioprosthesis was an underlying MV pathology not amenable for MV repair and the patient's wish to prefer a biological valve. In two patients a previous MV repair failed. The mean ejection fraction was $53.2 \pm 11.2 \%$ and the mean logistic EuroSCORE predicted risk of mortality was $5.9 \pm 5.5 \%$. The mean follow-up for this young patient cohort was $3.32 \pm 3.9$ years.

Results: Relevant preoperative patient characteristics were: severe pulmonary hypertension $(n=4)$, dialysis $(n=3)$, coronary artery disease $(n=2)$, neurological dysfunction $(n=4)$ and endocarditis $(n=3)$. The EpicBioMiniMV procedure was performed uneventful without conversion to full sternotomie. The duration of CPB was $133 \pm 59 \mathrm{~min}$ and cross clamp time was $72 \pm 49 \mathrm{~min}$. The postoperative course was uneventful. Only one patient died after 1.51 years and this was not valve related. The 10-year survival for this young subgroup was 90.0 \pm 9.5 and no patient received a mitral valve related re-operation (in comparison to total number of 357 patients: 10-year survival 66.8 \pm 4.3 and freedom from MV related re-intervention $98.1 \pm 1.2 \%$ ).

Conclusions: The Epic-Xenograft Bioprosthesis in isolated mitral valve replacement shows a very promising long-term outcome with a very low rate of structural bioprosthesis related degeneration even in younger patients.

\section{P117}

Persistent pulmonary hypertension after mitral valve replacement for mitral stenosis with moderate-to-severe pulmonary hypertension -analysis of associated clinical and hemodynamic factors

Usha Parvathy, Alexander Georegevich Feybuschevich

MPS Hopsital, Moscow, Russian Federation

Correspondence: Usha Parvathy

Journal of Cardiothoracic Surgery 2017, 12(Suppl 1):P117

Objective: Pulmonary Hypertension( $\mathrm{PAH})$, complicating mitral stenosis(MS), shows variability in its post-intervention regression; normalizing in some, and with various levels of non-regression in others. The issue of persistent-PAH has received limited attention, with few reports after balloon-valvotomy, and patient-prosthesis-mismatch after mitral-valve replacement(MVR). The study compares the pre, intra and postoperative variables among patients with normalised pulmonary artery pressure(PAP)/near-normal PAP, and residual-PAH at one-year post-MVR for MS with moderate-to-severe PH (preop-PAP $>50 \mathrm{mmHg}$ ); thereby identify factors related to persistent-PAH.
Methods: 55 patients who underwent MVR for significant-MS with $\mathrm{PH}(\mathrm{PAP}>50 \mathrm{mmHg})$ were divided based on their 1-year postoperativePAP as: I-normalised( $<30 \mathrm{mmHg}, \mathrm{n}=18)$, II-near-normal( $36-40 \mathrm{mmHg}$, $\mathrm{n}=17)$, III-residual-PH( $>40 \mathrm{mmHg} ; \mathrm{n}=20)$. Their demographic, preoperative (clinical, hemodynamic), intraoperative, and postoperative(clinical, hemodynamic, outcome) variables were compared. Univariate analysis followed by multivariate-analysis identified the determiningfactors of residual-PAH.

Results: The demographics were not greatly different. The baseline clinical and hemodynamic parameters were comparatively worse in Gp-III; with a higher-functional-class (III:IV) 15:3vs 15:2vs 11:9(p $<0.05)$, strikingly higher-PAP: 60.42 vs.62.64 vs.87.04 $p<0.01$; greater prevalence of severe-PAH $17(85 \%)$, cardiomegaly, right-atrial(RA), right-ventricular(RV)( $55 \%$ vs $58 \%$ vs $70 \%)$, pulmonary artery(PA) dilatations ( $p$ 0.04), and moderate-to-severe tricuspid regurgitation(TR)( $p$ $0.04)\}$. Calcified-valves $(p<0.05)$, and higher supportive-need (inotropic, ventilator, ICU duration\} substantiated their advanced-disease. Postoperative(follow-up) persistence of hemodynamic-derangements was greater, with RV and PA sizes, TR, and functional-disability attaining significance $(p<0.05)$. Multivariate analysis showed preop-PAP, TR and RVenlargements as significant predictors of residual-PAH.

Conclusions: Patients with persistent-PAH(post-MVR) represent advanced-disease shown by their preoperative significant functionaldisability, hemodynamic-derangements, valve-morphology, higher postoperative morbidity, and postoperative persisting-derangements, possibly indicating the extent of remodeling, and its nonresolution; stressing the need for close follow-up, and also early surgery before $\mathrm{PAH}$ becomes established. Preop-PAP stands an important confounding-variable that affects the postoperative hemodynamics and course.

P118

The role of LA's diameter in renewal of sinus rhythm during mitral valve replacement

Volodymyr Popov, Kateryna Pukas, Victoria Roy, Elena Trembovetskaya National Institute of cardio-vascular surgery named after Amosov, Kiev,

Ukraine

Correspondence: Volodymyr Popov

Journal of Cardiothoracic Surgery 2017, 12(Suppl 1):P118

Purpose of investigation is to research possibilities of intraoperative renewal of sinus rhythm during mitral valve replacement (MVR).

Methods And Materials. There were 111 (42,5\%) males and 150 (57,5\%). females from 01.01.2009 to 01.01.2017..Average age of patients was $59,3 \pm 7,3$ yy. $89(34,1 \%)$ patients belonged to III NYHA class, $172(65,9 \%)$ patients - to IV class. Reduction of left atrium's (LA) dilatation was occurred in $139(53,2 \%)$ pts by 3 methods: paraannular plasty of LA (62 pts),triangular plasty of LA (original method) (47 pts) and arch plasty of LA (original method) (30 pts) including ligation $(n=27)$ and resection of LA's auricle $(n=112)$ in both groups. Operations of left Maze - III-box $(n=120)$ and Maze - IVbox $(n=141)$ were performed in all cases by radio-frequency method + sew-tecnique.

Results. Among 261 patients 5 patient died on a hospital stage (hospital mortality-1,9\%) because of pneumonia $(n=1)$, brain damage $(n=1)$, MOF $(n=3)$.Sinus rhythm renewed after taking clamp from aorta in $84,8 \%$, at discharging was registered on ECG in $80,2 \%$. In LA's plasty group $(n=139)$ diameter of LA were decreased at postoperative period: $63,7 \pm 1,8$ (before), 49,3 $\pm 0,9$ (after), 51,5 $\pm 0,4$ (remote period). Renewal of sinus rhythm in group pts with LA's plasty was higher, than in alternative group: $85,6 \%(n=119 / 139)$ and $73,7 \%$ $(n=90 / 122)(p<0,01) .241(93,2 \%)$ pts were followed during 5 years after operation. In group with renewed sinus rhythm it was retained: 
1 year- 97,2\%, 2 year- 96,1\%, 3 year- 94,7\%, 4 year- 93,1\%, 5 year$92,0 \%$,

Conclusion. MVR with concomitant operation Maze-III,IV allows renew sinus rhythm on a hospital stage and stabilize it during remote period after operation. Left atrium's plasty with reduction of diameter of LA less than $50 \mathrm{~mm}$ is important factor for sinus rhythm renewal.

\section{P119}

Epic $^{T m}$ xenograft mitral valve replacement: long-term follow-up

Sven Lehmann, Khalil Jawad, Alexandro Hoyer, Stefan Feder,

Martin Misfeld, Jens Garbade

Heart Center Leipzig, Leipzig, Germany

Correspondence: Sven Lehmann

Journal of Cardiothoracic Surgery 2017, 12(Suppl 1):P119

Objective: Aim of this study was to evaluate the results after stented porcine xenograft implantation (Epic ${ }^{T M}$, SJM, St.Paul, MN, USA) with Linx $^{\mathrm{TM}}$ anticalcification treatment in mitral valve position for elderly patients.

Methods: A total of 1342 patients receiving mitral replacement between 11/2001 and 12/2016 were evaluated. All Patients having elective or emergency indications were included. Outcome was assessed by reviewing the prospectively acquired hospital database results as well as regular follow-up information by annual written interview.

Results: Patient age was $74.0 \pm 7$ years, body mass index was $26.3 \pm 5$ $\mathrm{kg} / \mathrm{m}^{2}, 41.8 \%$ were male, active endocarditis was in $20.8 \%$ indication for valve surgery, left ventricular ejection fraction was $56.0 \pm 14 \%$, glomerular filtration rate was $57.9 \pm 24 \%$, dialysis in $5.4 \%$ and the logistic EUROSCORE predicted risk for mortality was $21.0 \pm 20 \%$, respectively. Additional surgical procedures were aortic valve replacement in $277(44.4 \%)$ patients, aortic valve conduit in $20(3.2 \%)$ patients, CABG in $176(28.2 \%)$ patients, cryoablation in $148(23.7 \%)$ patients, tricuspid valve repair in $167(26.8 \%)$ and surgery on the thoracic aorta in $42(6.7 \%)$ patients. Mean aortic cross clamp time was $100 \pm 48 \mathrm{~min}$ and bypass time was $162 \pm 83 \mathrm{~min}$. Mean follow-up was 3.2 \pm 3.0 years (Range 0-12.3 years). Freedom from endocarditis after 10 year was $94.5 \pm 3.1 \%$. Freedom for valve reoperation was 86.5 $\pm 4.3 \%$ after 10 years. Freedom from thrombembolic event was 88.5 $\pm 3.3 \%$ after 10 years with a significant higher event rate in female patients ( $p=0.048) .30$ day survival rate was $79.1 \pm 1.6 \%, 1$ year survival rate was $62.0 \pm 1.9 \%$ and 10 year survival rate was $30.0 \pm 3.7 \%$, respectively.

Conclusions: $\mathrm{Epic}^{\mathrm{TM}}$ stented porcine xenograft is associated with acceptable survival, freedom from valve related complication and freedom from valve reintervention in mitral Position.

\section{P120}

The efficiency of left atrial surgical ablation when mitral valve prosthetics in patients with atrial fibrillation

Marat Sungatullin', Ildar Abdulyanov', Ildar Vagizov ${ }^{2}$

${ }^{1}$ Kazan State Medical Academy, Kazan, Russian Federation; ${ }^{2}$ Interregional Clinical and Diagnostics Center, Kazan, Russian Federation

Correspondence: Marat Sungatullin

Journal of Cardiothoracic Surgery 2017, 12(Suppl 1):P120

Background: A retrospective analysis of the efficiency of carrying out an electrical isolation of the left pulmonary veins' entries (PVE), the left atrium (LA) through the method of radiofrequency ablation (RFA) in patients with persistent left atrial fibrillation in the course of mitral stenosis treatment.

Methods: The study included 302 patients: the first group include mitral valve replacement and electrical isolation of PVE by radiofrequency energy under the conditions of cardiopulmonary bypass (CPB) and was comprised of 152 patients (50,3\%). The second group covered only mitral valve replacement under the conditions of CPB including 150 (49,7\%).

The female gender was the predominant one -172 patients $(57,2 \%)$. The average age of the patients was $58 \pm 7$ years.
All the patients demonstrated persistent atrial fibrillation (AF) with a duration of $22 \pm 8$ months. All the patients belonged to II-III CHF NYHA.

The electrical isolation procedure was performed by linear lesions using a bipolar clamp and unipolar pen in the LA. All patients underwent an obliteration of the auricle of the left atrium.

In the postoperative period, the patients in the first group took amiodarone for up to 6 months.

The efficiency of the method was evaluated by means of the following studies: echocardiography, electrocardiography, Holter ECG monitoring.

Results: The first group: during the postoperative period, the sinus rhythm (SR) was restored in $112(73,6 \%)$ patients whereas in the second group it was restored in $64(42,6 \%)$ patients, $p \leq 0.05$.

After 3 years, the follow-up completeness was $74,8 \%$ (226 patients). In the first group, SR was preserved in 76 cases $(71 \%)$, in the second group, the SR was preserved in 55 cases (46\%), $\mathrm{p} \leq 0.05$.

Conclusions: Left atrial surgical ablation with the help of radiofrequency energy is an effective method of treating $\mathrm{AF}$ in patients with prosthetic mitral valve.

P121

Correction of ischemic mitral regurgitation. 4-year experience

Georgy Antipov

Federal Center of High Medical Technologies, Kaliningrad, Russian

Federation

Journal of Cardiothoracic Surgery 2017, 12(Suppl 1):P121

Objective: to estimate results of mitral valve repair in ischemic mitral regurgitation patients.

Methods: From January, 2013 and December, 2016203 (7.5\% of CABG) procedures at patients with ischemic mitral regurgitation of the II-IV degree were executed. Average age was $66.2 \pm 11.3$ years (135 men). Indications were: $\mathrm{Ml}>2$ degree, annular dilatation $>37$ $\mathrm{mm}$, coaptation depth $>6 \mathrm{~mm}$, coaptation length $<5 \mathrm{~mm}$, tented area $>1.6 \mathrm{~cm}^{2}$. Acute IMR had 4(2\%) patients, chronic IMR 199(98\%) pts. MV replacement - 13(6.4\%) pts, MV repair - 190(93.6\%) pts. Performing MV repair we used synthetic band 4.5 to $5.0 \mathrm{~cm}$ long, depending on diameter of $\mathrm{MV}$ ( $<40 \mathrm{~mm}$ and $>40 \mathrm{~mm}$ according to) and rigid ring diameter 28 and $30 \mathrm{~mm}$ depending on diameter of MV also. Concomitant procedures were: CABG 203(100\%), other valves reconstructions/replacements/Maze procedure 159(84.2\%), LV reconstructions $25(13.7 \%)$, papillary muscle approximation $21(11.5 \%)$. In 59(30.6\%) patients Alfieri stitch was added. Mean follow-up period was 13.5 months, recurrence of MR estimated as mild and severe degree.

Results: The hospital mortality was $4.4 \%$ (9 patients). 3 patients required mitral valve replacement after inadequate repair. In hospital stay was 14,4 $\pm 6,9$ days. MR 0-1 degree at discharge was in 104(56.6\%) pts, 1-2 - 60(36.1\%) pts, 2 - 12 (6.0\%) pts, 3 - 5(2.5\%) pts. During follow-up period recurrence of mild and severe MVR were in $19.5 \%$ of patients. 3 patients undergo redo surgery in 15, 18 and 23 months after first procedure.

Conclusions: Use of a ring or a synthetic band for correction of ischemic mitral insufficiency effective and safe, connected with the low frequency of intraoperative and postoperative complications. The maximum number of recurrences of MVR after repair occurs in the first year of observations (14\%), then this index slightly increases.

P122

Five year experience of haemodynamic performance of the St Jude Medical Trifecta aortic bioprosthesis

Benjamin Adams, Prity Gupta, Amer Harkey, Mohamad Bashir,

Shirish Ambekar, Rakesh Uppal, Kulvinder Lall

Barts Heart Centre, London, UK

Correspondence: Benjamin Adams

Journal of Cardiothoracic Surgery 2017, 12(Suppl 1):P122 
Objective: The St. Jude Medical Trifecta aortic supra-annular bioprosthesis is regarded as the next generation in pericardial stented tissue valves. The unique design of tissue leaflets attached to the exterior of the valve stent provides unrivalled in-vivo mean gradients and haemodynamics. The aim of this prospective study was to evaluate the five-year haemodynamic performance of the Trifecta valve.

Methods: Four hundred and nineteen consecutive patients undergoing aortic valve replacement using the St. Jude Medical Trifecta valve at a single UK centre over a five-year period were included in this study. Patients undergoing concomitant cardiac procedures were included. All implanted valves were 19, 21, 23, 25, 27 \& $29 \mathrm{~mm}$ in size. Assessment of haemodynamic function was carried out using transthoracic echocardiography pre-operatively and at follow-up, as well as transoesophageal echocardiography intraoperatively.

Results: The study population consisted of 419 patients ( 245 male, 174 female). Mean age was $72.7 \pm 9.3$ years. Implanted valve sizes were $19 \mathrm{~mm}(\mathrm{n}=27), 21 \mathrm{~mm}(\mathrm{n}=124), 23 \mathrm{~mm} \quad(n=147), 25 \mathrm{~mm} \quad(n=95), 27 \mathrm{~mm}$ $(n=13)$ and $29 \mathrm{~mm}(n=1)$. Overall mean post-operative pressure gradients were $9.27 \pm 5.3 \mathrm{mmHg}$ (mean) and $17.5 \pm 9.45 \mathrm{mmHg}$ (peak). Subgroups mean post-operative pressure gradients were $12.5 \pm 3.9 \mathrm{mmHg}$, $10.5 \pm 7.4 \mathrm{mmHg}, \quad 8.8 \pm 4.3 \mathrm{mmHg}, \quad 8.0 \pm 3.7 \mathrm{mmHg}, 7.5 \pm 3.7 \mathrm{mmHg}, \quad 6.86$ $\pm 0 \mathrm{mmHg}$, for the $19,21,23,25,27$ and $29 \mathrm{~mm}$ cohort respectively. Overall mean post-operative left ventricular ejection fraction was 55.1 $\pm 12.1 \%$. Overall mean effective orifice area was $1.81 \pm 0.55 \mathrm{~cm}^{2}$.

Conclusions: These results of our experience demonstrate excellent haemodynamic performance of the Trifecta bioprosthetic valve.

\section{P123}

Outcomes of Sutureless Aortic Valve Replacement (SU-AVR) in moderate-to-high risk patients with unexplained postoperative thrombocytopenia

Puwadon Thitivaraporn, Saran Chiramongkol, Chanapong Kittayarak, Nopporn Pornpatrtanarak, Jule Namchaisiri, Seri Singhatanadgige, Pat Ongcharit, Vichai Benjacholamas

King Chulalongkorn Memorial Hospital, Bangkok, Thailand

Correspondence: Puwadon Thitivaraporn

Journal of Cardiothoracic Surgery 2017, 12(Suppl 1):P123

Objectives: The aim of this study was to compare perioperative and postoperative outcomes of first series SU-AVR and AVR in developing country.

Methods: We conducted a retrospective study of SU-AVR in moderate-to-high risk patients (pts). Data of those underwent AVR or SU-AVR from 2013 to 2016 were obtained. A 1:1 propensity matching with sex, age and Society of Thoracic Surgeons (STS) score. Preoperative and post-operative echocardiograms were obtained. Primary outcome was 30-day mortality. Secondary outcomes were perioperative outcomes and complications. Demographics and outcomes of interest were compared using Fisher's exact and t-tests.

Results: 277 pts in both AVR and SU-AVR groups. There are 10 patients (5 were male, median age of 81.5 years) undergone SU-AVR with propensity compared to AVR. Sutureless valve were successfully implanted in $9 / 10$ pts. The median STS score in SU-AVR group was 5.77 (2.1-79) vs 5.81 (2.5-34.8) in AVR group. The median Cardiopulmonary bypass(CPB) time was $120 \mathrm{~min}$ (48-276) in SU-AVR vs 148 min (103-261) in AVR, $p=0.61$. The median cross-clamp duration was $93.5 \mathrm{~min}$ (37-157) in SU-AVR vs $124 \mathrm{~min}$ (73-168) in AVR, $\mathrm{p}=0.14$ Postoperative echocardiogram demonstrated impressive outcomes in SU-AVR group, defined as reduced mean pressure gradient from 53.1 to $12 \mathrm{mmHg}$ without left ventricular impairment. 30-day mortality was $20 \%$ in both SU-AVR and matched AVR group. All patient in SUAVR developed postoperative thrombocytopenia. Platelets level decreased from $225 * 10^{3} / \mu \mathrm{l}$ preoperatively to $94.5,54.5,50.1$ at postoperative day (POD) 1, 2 and 3 compared with 135.5, 93.4, 91.8 in AVR group ( $p=0.04,0.16$ and 0.20 , respectively). The requirement of platelets transfusion was higher in SU-AVR group compared to AVR (LPPC 14.5 units vs 4units) ( $p=0.04)$.
Conclusion: There was no difference in 30-day mortality among moderate-to-high risk SU-AVR vs AVR. Despite SU-AVR was associated with favorable CPB and clamp time, SU-AVR found related to postoperative thrombocytopenia.

P124

Two dimension echocardiographic evaluation of left ventricular diastolic function after beating heart Closed Mitral Valvotomy (CMV) in rheumatic mitral stenosis

Kumar Rahul

King Georges Medical University, Lucknow, India

Journal of Cardiothoracic Surgery 2017, 12(Suppl 1):P124

Background: Diastolic dysfunction is seen in patients of rheumatic severe mitral stenosis. This study investigates the effects of left ventricular diastolic function on outcome in patients with rheumatic mitral stenosis undergoing closed mitral valvotomy.

Methods: This is a single centre two year comparative study consisting of 60 patients. We evaluated preoperative and post operative transthoracic Two D echocardiographic parameters of diastolic function and compared both data to evaluate improvement of diastolic function.

Statistical analysis: The statistical analyses were performed on SPSS (Statistical Package for Social Sciences). A two-sided ( $\mathrm{fi}=2$ ) $\mathrm{p}$ value less than $0.05(p<0.05)$ was considered statistically significant.

Result: Maximum patients were in New York heart association (NYHA) grade 3. Wilkins score ranged from 6 to 10 . Mitral valve area (MVA) increased from $0.77 \pm 0.13$ to $2.32 \pm 0.26$, Ejection fraction (EF) increased from $61.38 \pm 4.61$ to $64.79 \pm 3.22$, Deceleration time, (DT $\mathrm{ms}$ ) decreased from $231.55 \pm 49.31$ to $168.28 \pm 14.30$, E/A ratio reverted to $1.70 \pm 0.54$ from $0.89 \pm 0.39$. Total ejection isovolume (TEI) index improved from $0.50 \pm 0.03$ to $0.39 \pm 0.06$, Mitral inflow propagation velocity, (MIPV cm/sec) increased from $47.28 \pm 3.71$ to $57.86 \pm 3.19$. Peri-operative and follow up was uneventful

Conclusion: Surgical closed mitral valvotomy produce excellent and comparable early hemodynamic improvement, significant improvement in clinical stage of disease and improvement in diastolic function. It remains a simple, safe, and effective means of treating mitral stenosis in regions where socioeconomic changes have not yet reduced the incidence of rheumatic heart disease and where resources for its treatment are limited there is still a place for this procedure.

\section{P125}

Comparison of different types of aortic valve bioprosthesis in patients with narrow fibrous ring

Ihar Andraloits, Vadim Shumavets, Alexandr Shket, Irina Grinchuk, Svetlana Kurganovich, Elena Lysjonok, Natalia Uss, Youry Ostrovski Belarus Centre Cardiology, Minsk, Belarus

Correspondence: Ihar Andraloits

Journal of Cardiothoracic Surgery 2017, 12(Suppl 1):P125

Objective: Evaluation of hospital results of replacement of the aortic valve with a bioprosthesis in patients with a narrow fibrous ring of the aortic valve.

Methods: The study included 293 patients (mean age 69,7 \pm 6,7years) with valve's landing sizes of 18-19, 20-21 and 22-23 mm were continuously included in the study. Isolated or combined with correction of co-morbidities aortic valve replacement with different types of bioprosthesis was performed. St. Jude Epic (126 pts), Carpentier-Edwards (C-E) PERIMOUNT Magna (92 pts), Sorin Soprano Armonia (32 pts), Medtronic Mosaic (23 pts) and BioValsalva valved conduit (11 pts) were used. Directly hospital results were analyzed depending on the type and size of a bioprosthesis.

Results: Hospital mortality was $3,85 \%$. A significant reduction in aortic peak and medium transvalvular gradient $(p<0,0001)$, as well as an increase in effective opening area (EOA) of aortic valve were 
noted for all types of bioprosthesis. No significant differences in effective opening area between the same sizes of the studied a bioprosthesis were revealed. Moderate degree of "prosthesis-patient mismatch" (PPM) in the postoperative period were observed in 33,8 $\%$ of patients, severe - in $6,34 \%$, and no difference between bioprothesis were found $(p=0,551)$. However, hemodynamic parameters in patients with severe PPM meet value of moderate stenosis of the aortic valve

Conclusions: The implanted bioprostheses are comparable on hemodynamic parameters and don't concede each other in the early postoperative period. The implantation of the aortic bioprosthesis even with small but adequate diameter reduces the pressure gradient, increases the EOA of aortic valve. We noted significant difference between expected and observed frequency of "prosthesispatient mismatch" phenomena for all implanted types of small size bioprosthesis.

\section{P126}

Patch augmentation of the anterior leaflet in type 3a carpentier mitral valve disease

Giuseppe M. Raffa, Marco Turrisi, Giuseppe Romano, Michele Pilato IRCCS-ISMETT (Istituto Mediterraneo per i Trapianti e Terapie ad altaspecializzazione), Palermo, Italy

Correspondence: Giuseppe M. Raffa

Journal of Cardiothoracic Surgery 2017, 12(Suppl 1):P126

Objective: To asses the anterior leaflet repair with patch augmentation for type III mitral valve disease.

Methods: From September 2014 to December 2015, 8 patients (6 affected by rheumatic and 2 affected by post irradiation mitral valve regurgitation (MR) underwent anterior leaflet augmentation at our Institute. The mean age was $59 \pm 11$ years and $25 \%$ were male. All patients presented with symptomatic severe MR and the mean EF was $62 \pm 13 \%$. Fixed bovine pericardium for intra-cardiac use was used in 7 cases. Repair of rheumatic or post radiation valvulopathy must address five key pathoanatomical aspects: commissural fusion, subvalvular restriction, leaflet immobility, coaptation depth and overall mitral orifice. A two-stitch technique utilizing 4-0 or 5-0 monofilament polypropylene suture is preferred to avoid purse-stringing as the defect is patched with the pericardial graft. Posterior band anuloplasty was performed in all cases but one.

Results: There were no operative 30-day mortalities. All patients left the operating room with trace to no MR without stenosis and transmitral gradients $<5 \mathrm{mmHg}$. Post operative complications included: pleural effusion $(n=2)$, Atrial fibrillation $(n=2), P M$ insertion $(n=1)$ and acute renal failure requiring dialysis $(n=1)$. All patients demonstrated clinical improvements (NYHA I-II) following repair. No mitral stenosis nor significant regurgitation was revealed at pre discharge ETT. Follow-up was complete in both groups. No patients required reoperation and the overall survival was $100 \%$. No cardiovascular events were recorded but persistent $\mathrm{AF}$ in one patient requiring electrical cardioversion.Midterm follow-up echocardiography results did non demonstrate residual MR in five patients and trivial in three.All patients had a mean gradient $<8 \mathrm{mmHg}$ at follow up. The mean $\mathrm{EF}$ at follow up was $58 \pm 4 \%$. Patients were anticoagulated for 3 months following repair.

Conclusions: Anterior leaflet augmentation is a valuable option to treat post inflammatory mitral valvulopathy allowing for oversize annuloplasty and mitigation of mitral valve stenosis.

\section{P127}

Single center experience with cross clamp technique for aortic dissection

Mauro Del Giglio, Simone Calvi, Marco Paris, Alberto Tripodi, Marco Pagliaro, Elisa Mikus

Maria Cecilia Hospital, Cotignola (RA), Italy

Correspondence: Elisa Mikus

Journal of Cardiothoracic Surgery 2017, 12(Suppl 1):P127
Objective: Standard surgical therapy of type A dissection consists of replacing the ascending aorta and, if necessary, the aortic root and or aortic arch with resection of the entry tear. The debate regarding the best surgical approach is still open. The purpose of this study is to evaluate clinical outcome after surgery for acute type A dissection limited to ascending aorta replacement with distal clamping in normothermic cardiopulmonary bypass.

Methods: Between January 2010 and March 2016, 154 consecutive patients underwent surgical operation for type $A$ aortic dissection at our Institution. This study is focused on 103 of them operated using standard aortic normothermic cross clamp technique, as the aortic arch was not involved. There were $69(67 \%)$ male with a mean age of 63.3 \pm 12.9 years (range: $31-84$ years). Major morbidity, operative mortality and two-year actuarial survival were analysed.

Results: Emergency operations were performed in all patients. An aortic repair involving the aortic root was necessary in 23 patients ( ${ }^{\circ}$ 7 treated with valve sparing and 16 with a valved conduit). Overall median cardiopulmonary bypass time was 114 (IQR 88-146) minutes and aortic cross clamp time was 87 (IQR 63-111) minutes. Median intensive care and hospital stay were 4 and 9 days respectively. Inhospital mortality was $7 \%$. The 2 -yr overall survival after discharge was $87 \%$.

Conclusions: This study shows that type A aortic dissection in selected patients can be treated with aortic cross clamp avoiding hypothermic arrest or moderate hypothermia with cerebral perfusion. Results are encouraging and the follow-up suggests that it could be considered a good alternative.

P128

Myocardial protection for isolated aortic valve replacement: is retrograde applying of cardioplegia is better?

Volodymyr Popov, Anatoliy Hripachenko, Alex Gurtovenko,

Bogdan Gumenjuk, Lina Tihonenko

National Institute of cardio-vascular surgery named after Amosov, Kiev,

Ukraine

Correspondence: Volodymyr Popov

Journal of Cardiothoracic Surgery 2017, 12(Suppl 1):P128

Objective. To present analysis of retrograde crystalloid cardioplegia during isolated aortic valve replacement (AVR).

Materials And Methods. During 2009-2016 y 415 patients (pts) - 236 $(56,9 \%)$ males, $179(43,1 \%)$ female.Average age was $57,5 \pm 8,6$ yy. The following applying of cardioplegia (Custadiol) were performed by: antegrade $47(11,3 \%)$ pts (group A), ante-retrograde $89(21,4 \%)$ pts (group B), retrograde - $279(67,3 \%)$ pts (group C) ways. Systemic hypothermia 32-34 C, cardiopulmonary bypass, retrograde cardioplegic solution (Custadiol) (in dose $20 \mathrm{ml} / \mathrm{kg}$ ) + external cooling of myocardium were occured in all pts. Average time of improvement of

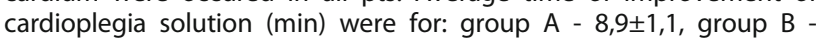
$12,7 \pm 1,3$, group $C-22,5 \pm 2,9$. Average cross-clamping time (min) were for: group A - 83,2 $\pm 9,1$, group B - 77,4 48,7 , group C - $69,5 \pm 7,6$ $(p<0,05)$ and reperfusion time $(\mathrm{min})$ were for: group $A-29,8 \pm 4,9$, group B - 26,3 $\pm 4,2$, group C - 23,5 $\pm 3,7(p<0,05)$.

Results. Hospital mortality was for group A $2,1 \%(n=1 / 47)$., group B $2,2 \%(n=2 / 89)$, group $C-1,1 \%(n=3 / 279)(p<0,05)$. Average doses of dobutamin $(1,0-3,0 \mathrm{mcrg} / \mathrm{min} / \mathrm{kg})$ were marked for: group A - 56,3 $\pm 7,1$ hours, group $B-51,1 \pm 6,4$ hours, group $C-43,2 \pm 5,8$ hours ( $p$ $<0,05)$. Average level of MB KFK $(\mathrm{U} / \mathrm{L})$ at 2-td postoperative day were occured for: group A - 95,4 $\pm 10,6$, group B - 83,5 $\pm 9,7$, group C - 77,3 $\pm 9,1$ ( $p<0,05$ ). Duration of stay on artificial lung ventilation (hours)

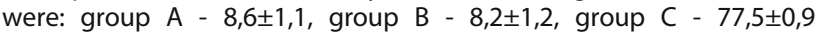
$(p>0,05)$. Absence of applying donor blood product during all postoperative period for group A - 32,2\%, group B - 34,6\%, group C $35,1 \%(p>0,05)$.

Conclusion. Improved myocardial protection in cases with isolated AVD by using retrograde crystalloid cardioplegic solution (Custadiol) (group $C$ ) lead to better results than in group $A$ and $B(p<0,05)$. 
P129

The remote results after mitral valve replacement

Kateryna Pukas, Vasyl Lazorishinets

National Institute of Cardio-Vascular Surgery named after Amosov, Kiev,

Ukraine

Correspondence: Kateryna Pukas

Journal of Cardiothoracic Surgery 2017, 12(Suppl 1):P129

The aim of the research is to study the characteristics of a remote period after mitral valve replacement (MVR)

Material And Methods. In the analyzed group included 569 patients discharged after the MVR at the Institute for the period 2005-2006. This represented $96.7 \%$ of discharged on hospital stage. The men were $268(47.1 \%), 301$ women (52.9\%). The age of patients ranged from 39 to 71 years (mean, $53.1+7.5$ years). 49 (8.6\%) patients treated to class II of NYHA classification, 179 (31.4\%) patients belonged to class III of NYHA classification and 341 (60.0\%) patients class IV. Atrial fibrillation was observed in 532 (93.4\%) patients. Concomitant tricuspid malformation was observed in $135(23.7 \%)$ patients. Previous heart surgery (closed commissurotomy) had 129 (21.4\%) patients. Mechanical prostheses implanted only: double-leaf (Saint Jude, Carbomedics, ATS, Edwards-Mira, On-X) (482 patients) and mono-leaf (87 patients).

Results. After 10 years after surgery survival rate was $69.4 \%$, stable good results occurred in $67.2 \%$, no trombembolic complications were observed in $79.7 \%$. Sinus rhythm was observed in 31 (5.4\%) patients. The main risk factors at remotr period were: IV functional class, atrial fibrillation, left atriomegaly (diameter of $6.0 \mathrm{~cm}$ or more), ejection fraction less than 0.45 , high pulmonary hypertension, left ventriculomegaly (ESVI > $95 \mathrm{ml} / \mathrm{m} . q)$, progressive ischemic heart disease, monodisc prosthesis.

Conclusion. A good result of the operation in the remote period is observed in most cases with the implantation of double-leaf models (except Edwards-Mira), operated in II-III functional class and with sinus rhythm. Reduction element of the left atrium to the physiological norms, ear resection are important factors for the restoration of sinus rhythm and to reduce the risk of trombembolic complications.

\section{P130}

Triangular plasty of left atrium for atriomegaly during mitral valve replacement

Kateryna Pukas, Bogdan Gumenjuk, Svitlana Andrievskaya,

Volodymyr Popov

National Institute of Cardio-Vascular Surgery named after Amosov, Kiev, Ukraine

Correspondence: Kateryna Pukas

Journal of Cardiothoracic Surgery 2017, 12(Suppl 1):P130

Objective. To determined possibillities of left atrium (LA)'s reduction by original method of triangular plasty of LA (TPLA) during mitral valve replacement (MVR) for isolated mitral valve disease (MVD).

Methods. During 2005 - 2015 yy. 705 adult patients (pts) with MVD and LA's atriomegaly (diameter of LA $>60 \mathrm{~mm}$ ) average 71,7 $\pm 1,8$ were operated at Institute. There were $428(60,7 \%)$ in IY NYHA class and $277(39,3 \%)$ in III class. All data divided at 2 groups: group A TPLA + ligation of LA's auriculum was 128 pts and group B - 577 pts only MVR without LA's plasty or ligation's auriculum.

Results. The hospital mortality were: in group A - 1,6\% ( $n=2 / 128)$ and in group $B-2,6 \%(n=15 / 577)(p<0,05)$. At the remote period (average was 7,2 $\pm 0,7$ yy) $651(93,5 \%)$ pts were followed-up. Data of echo for group A: diameter of LA (mm) - preoperative (PRE) - 71,4 $\pm 1,4$, postoperative (POST) - 51,6 $\pm 0,8$, remote period (RP) - 52,2 $\pm 0,7$; ejection fraction of LV (EFLV): PRE - 0,52 $\pm 0,05$, POST - 0,55 $\pm 0,04$, RP $0,58 \pm 0,02$. Data of echo for group B were: diameter of LA (mm): PRE$71,3 \pm 1,5$, POST $-69,3 \pm 1,8, \mathrm{RP}-78,1 \pm 1,8$; EFLV: PRE - 0,53 $\pm 0,04$, POST - 0,54 $\pm 0,05$, RP - 0,47 $\pm 0,04$. At remote period thrombembolic events and heart failure were marked respectively: in group A -1,7\% and $2,9 \%$ and in group $B-7,5 \%$ and $27,2 \%(p<0,05)$. Sinus rhythm was marked in group $A-3,5 \%$ and in group $B-0,0 \%(p<0,05)$.
Conclusion. The original method of TPLA was allowing to improve better clinical results at group $A$ than in $B(p<0,05)$.

P131

Cardiopulmonary bypass during pregnancy: fetal and maternal outcomes

Zhanybek Ashyrov, Rymbay Kaliyev, Timur Lesbekov, Zhuldyz

Nurmykhametova, Daniil Shustov, Linar Faizov, Pya Yuriy

National Research Center for Cardiac Surgery, Astana, Kazakhstan

Correspondence: Zhanybek Ashyrov

Journal of Cardiothoracic Surgery 2017, 12(Suppl 1):P131

Objective: Cardiac surgery carried out on cardiopulmonary bypass (CPB) during pregnancy is associated with potentially increased fetal and maternal mortality. The CPB is associated with utero-placental hypoperfusion due to a number of factors, which may translate into low fetal cardiac output, hypoxia and even death. The study aim was to evaluate outcomes in females who underwent open-heart surgery with CPB during pregnancy.

Methods: Between 2012 and 2015, 8 pregnant women (mean age 32 \pm 5 years; mean gestational age $25 \pm 8$ weeks) underwent urgent cardiac surgery using CPB. The preoperative diagnosis included valve dysfunction in 8 women (two aortic, six mitral). Seven patients underwent valve replacement, and one - Bentall procedure. One patient was in the first trimester, one in the second trimester, and six in the third trimester. Maternal variables such as age, gestational age, cardiac diagnosis, surgical details, morbidity and mortality, and type of delivery, as well as fetal variables including low birth weight, prematurity, and fetal malformation were retrospectively analyzed.

Results: The CPB time was $110.3 \pm 57.1 \mathrm{~min}$, the aortic cross-clamp time was $54.3 \pm 27.2 \mathrm{~min}$., duration of operation was $180 \pm 35$. Perfusion pressure was $68 \pm 8$, and core temperature during CPB was $34 \pm 2$. There was no in-hospital maternal mortality, but one case of fetal mortality (gestation age - 28 weeks) in patient who required 7 defibrillations during reperfusion period. No congenital abnormalities in neonates were diagnosed.

Conclusions: The durations of CPB and aortic cross-clamping, highflow, high-pressure, normothermic bypass and continuous fetal and uterine monitoring allowed cardiac surgery to be performed with the greatest control of risk in the pregnant patients.

P132

Surgical treatment results of patients with an aortic aneurysm in combination with bicuspid aortic valve by David technique

Vladimir Dikolayev, Saule Narakaeva, Aidos Erpashov, Kenzhebek Altenov, Sabitkhan Pernekhanov, Adilzhan Albazarov

JSC NSMC, Astana, Kazakhstan

Correspondence: Vladimir Dikolayev

Journal of Cardiothoracic Surgery 2017, 12(Suppl 1):P132

Objective: The aim of this study is to estimate short-term results of surgical treatment of patients with an aortic aneurysm in combination with bicuspid aortic valve by David technique (BAV).

Methods: 13 patients with an aortic aneurysm in combination with BAV were operated by David technique in the cardiothoracic department of the National Scientific Medical Center (NSMC) from 2013 to 2016.

Results: Patients' average age was 33,71 \pm 5,903. Transthoracic echocardiography (Echo) was used as preoperative examination. The average Echo value of the patients during the preoperative period the Ejection Fraction (EF) of the left ventricle was $58,57 \pm 2,571 \%$, End Diastolic Volume (EDV) of the left ventricle was 142,8571 \pm $28,3158 \mathrm{ml}$, and the degree of the aortic valve insufficiency was $2,2857 \pm 0,4738$. Surgical treatment was carried out with the usage of the blood cardioplegia. Cardiopulmonary bypass time was 139,57 \pm 13,659 minutes, cardiac arrest time was 103,14 $\pm 8,210$ minutes. Early postoperative transthoracic Echo was carried out for control purpose. Echo results revealed that postoperative $\mathrm{EF}$ of the left ventricle was $61,714 \pm 2,360 \%(p=0,2492)$, EDV of the left ventricle was $118,714 \pm$ 
$56,094 \mathrm{ml}(p=0,0566)$, the degree of the aortic valve insufficiency was $0,857 \pm 1,069(p=0,0353)$, and the aortic valve gradient was $5,8571 \pm$ $1,2234 \mathrm{~mm} \mathrm{Hg}$. Complications in early postoperative period were observed in 2 out of 13 (15,3\%) patients (bleeding and AV-blockade). Hospital mortality cases were not observed.

Conclusions: Surgical treatment of the patients with an aortic aneurysm in combination with BAV by using David technique allows not only to achieve satisfactory results in the early postoperative period, but also to eliminate the patients who need in acceptance of anticoagulants which is one of the main advantages of the valvesparing surgery.

\section{P133}

Abnormalities in extracellular matrix microstructure in infective endocarditis: the role of underlying heart disease or the influence of infection

Sergey Kovalev ${ }^{1}$, Dmitry Griaznov ${ }^{1}$, Andrey Bulynin ${ }^{1}$, Alexander Kinshov ${ }^{2}$ ${ }^{1}$ Voronezh Regional Clinical Hospital, Voronezh, Russian Federation; ${ }^{2}$ Voronezh Burdenko State Medical University, Voronezh, Russian Federation

Correspondence: Dmitry Griaznov

Journal of Cardiothoracic Surgery 2017, 12(Suppl 1):P133

\section{Objective:}

The major structural components of native heart valves extracellular matrix are collagen-I (C-I) and collagen-III (C-III) fibers. Disbalance of these proteins is described in bicuspid aortic valve (AV) and mitral valve (MV) prolapse. The aim of study - to evaluate the influence of IE on C-I and C-III expression.

Methods:

60 native valve IE patients and 28 patients with non-infective valve disease, all undergone surgery, were studied. Immunohistochemistry of heart valve tissue was performed by using Anti-Collagen I and Anti-Collagen III antibodies. Protein expression was estimated via digital calculation of optical density of stained specimens.

Results:

Inside the control group next results were found: there were no differences in the level of $\mathrm{C}-\mathrm{I}$ and $\mathrm{C}-\mathrm{III}$ between MV and AV $\left(p_{\mathrm{C}_{1}}=0.378, p_{\mathrm{C} 3}=0.486\right)$. At the same time, tricuspid valve (TV) specimens both in control and in IE groups showed significantly lower rates of $\mathrm{C}-\mathrm{I}$ and $\mathrm{C}-\mathrm{III}$ expression ( $\mathrm{p}<0.0001$ in all comparisons). Studying subgroups with underlying heart disease: cases with calcified aortic stenosis (CAS) and with IE-complicated CAS gave statistically less levels of both collagen types $\left(p_{\mathrm{C} 1}=0.003 ; p_{\mathrm{C}_{3}}=0.019 ; p_{\mathrm{C} 1 \mathrm{E}}<0.0001\right.$ and $\mathrm{p}_{\text {C3IE }}<0.0001$ ).

The results of search of deviations specified by IE were: comparing general IE and general control groups no differences in C-I expression were identified, and the level of C-III in IE group was significantly lower $(p<0.0001)$. The studying of collagen expression in acute IE has revealed decrease of both $C-I$ and $C-I I I(p<0.0001)$. In subacute IE the level of $C-I$ was higher $(p<0.0001)$ and the level of $C$-III was lower than in control group $(p=0.001)$.

Conclusions:

The localization of valve lesion and the underlying heart disease take an influence on ECM proteins distribution in IE. The main IE hallmark was the decrease of C-III. The results can provide useful information for understanding connective tissue dysfunctions in IE patients.

\section{P134}

The remote results after isolated aortic valve replacement

Konstantin Vakulenko, Volodymyr Popov

National Institute of Cardio-Vascular Surgery named after Amosov, Kiev, Ukraine

Correspondence: Konstantin Vakulenko

Journal of Cardiothoracic Surgery 2017, 12(Suppl 1):P134
Aim. The purpose of the research is to analyzed the characteristics of a remote period after isolated aortic valve replacement (AVR)

Material And Methods. In the analyzed group included 1354 patients discharged after isolated AVR at the Institute for the period 2006-2008. This represented $94.7 \%$ of discharged on hospital stage. There were $783(57.8 \%)$ men, women $571(42.8 \%)$. The age of patients ranged from 20 to 72 years (mean 52,7 \pm 9.4 years). By NYHA classification there were followed-up: II class 98 (7.3\%) patients, III class 544 (40.2\%) patients and IV class 711 (52.5\%) patients. Atrial fibrillation was observed in 21 (1.5\%) patients. Only mechanical prostheses were implanted: (Saint Jude, On-X,Carbomedics, ATS). Concomitant CABG was observed in 93 (5.0\%) patients.

Results. Average followed-up at remote period $9.4 \pm 0.7$ yy At 10 years we had observed: survival rate was $81.3 \%$, stability of good results was occurred $57.3 \%$, freedom from thrombembolic complications were observed in $95.3 \%$, freedom from reoperations was observed in $97.1 \%$. Reoperations were occured: thomboses (panus) of aortic prostheses $(n=2)$, prosthetic endocardytis $(n=3)$. Atrial fibrillation was marked in 50 (3.7\%) patients. A-V blocade was occured in $48(3.5 \%)$ patients. The main risk factors for remote period: IV functional class, atrial fibrillation, left atriomegaly (diameter of atrium $6.0 \mathrm{~cm}$ or more), ejection fraction less than 0.4 , high pulmonary hypertension (PSP $>70 \mathrm{~mm} . \mathrm{Hg}$ ), left ventriculomegaly (ESVI $>95 \mathrm{ml} / \mathrm{m} . q)$, progressive ischemic heart disease. Conclusion. At the remote period good results of the operation by mechanical aortic prostheses was observed in the most cases. Operation should be better perform in II-III functional class, with sinus rhythm and good myocardial contractility.

\section{P135}

Edge-to-Edge procedure as an alternative to quadrangolar resection of isolated prolapse of posterior mitral leaflet

Carlo Fucci, Giuseppe DeCicco, Andrea Bortolami, Gerardo DiMatteo, Leonardo Latini, Aldo Prencipe, Tommaso Aquino, Giuseppe Coletti

Civic Hospital, Brescia, Italy

Correspondence: Carlo Fucci

Journal of Cardiothoracic Surgery 2017, 12(Suppl 1):P135

Objective: Quadrangolar resection (QR) represent the gold standard technique to correct central scallop prolapse/flail of posterior mitral leaflet. Particular condition, however may impede or discourage its application. We report our experience with the application of the edge-to-edge (ETE) technique in these cases.

Methods: From January 1999 to January 201589 patients (53 male, mean age 56+/-14.56) were submitted to ETE in consideration of the following factors: need for excessive posterior leaflet resection in 45 patients, calcification or fibrosis of posterior annulus in 20 and hypertrophic/small left ventricle with high risk for perioperative SAM in 24. Annular remodellig was performed in all with complete ring. Results: There were no hospital death. Freedom from reoperation was $97 \%$ and survival $95 \%$. Echocardiographic examination revealed no residual mitral insufficiency in 40 patients, mild in 36 and moderate in 13 . The mean transvalvular gradient was $3.37+/-1.66$ and the mean mitral valve area was 3.06+/-063.

Conclusions: In our experience, ETE technique represents a valuable option for the correction of isolate prolapse of the posterior mitral leaflet and should be particularly considered when standard QR may nor be applicable or not advisable.

\section{P136}

Our experience in radiofrequescy ablation during open heart surgery

Darkhan Suigenbayev, Ermagambet Kuatbayev, Timur Lesbekov,

Azamat Kurmalayev, Galiya Turganbayeva

National Research Center for Cardiac Surgery, Astana, Kazakhstan

Correspondence: Darkhan Suigenbayev

Journal of Cardiothoracic Surgery 2017, 12(Suppl 1):P136 
Background: To assess the results of the surgical radiofrequency ablation (RFA) for atrial fibrillation (AF) treatment during open heart surgery.

Methods: 239 surgical radiofrequency ablations were performed from October 2011 to January 2017. Cardiopulmonary bypass was used in all cases. In $98(41 \%)$ patients left atrial monopolar RFA were performed. Cava-tricuspid isthmus (CTI) ablation was added in 73 $(30,5 \%)$ patients. Transmural bipolar RFA of the pulmonary veins was performed in $68(28,5 \%)$ patients. RFA was performed during mitral or aortic valves replacement/repair, ascending aorta replacement, congenital heart defect corrections in adults, coronary artery bypass grafting. Patients were divided into 3 groups: A - left atrial RFA; B left atrial and CTI RFA; C - bipolar RFA.

Results: There was no hospital mortality. $80 \%$ of patients converted to sinus rhythm after the operation. $192(79 \%)$ patients preserved sinus rhythm during post-operative period and after discharge in early post-operative period. AF persisted in $43(19 \%)$ patients; 4 (2\%) patients required a pacemaker implantation.

Conclusion: RFA is an effective and safe method for comprehended treatment of $\mathrm{AF}$ at open heart surgeries. In the majority of cases patients have long-term persistent $\mathrm{AF}$, which indicates the delayed detection of the latter. The most successful outcomes were in patients with paroxysmal and persistent AF types.

\section{P137}

Results of Cox-Maze V procedure

Agzam Aldeshev, Zhanar Nurbay, Galymzhan Sultanov,

Yerbol Kudabayev

Scientific Research Institute of the Cardiology and Internal Diseases, Almaty, Kazakhstan

Correspondence: Agzam Aldeshev

Journal of Cardiothoracic Surgery 2017, 12(Suppl 1):P137

Objective: To assess the effectiveness and measure long-term results of Cox-Maze $\mathrm{V}$ procedure performed for atrial fibrillation in combination with prosthetics of mitral valve.

Methods: In 2015-2016 fifteen patients underwent Cox-Maze V procedure for atrial fibrillation performed by the Department of cardiac surgery of the Scientific Research Institute of Cardiology and Internal Diseases. The average age of the patients is 51 years (from 42 to 69 ); 9 male $(60 \%)$ and 6 female patients (40\%). Every Cox-Maze $V$ procedure is performed in conjunction with mitral valve prosthetics. Twelve patients $(80 \%)$ underwent tricuspid valve plastic simultaneously. Two patients were diagnosed with paroxysmal form of atrial fibrillation $(13.3 \%)$, and thirteen patients with constant form of atrial fibrillation (86.7\%).

Results: At discharge, thirteen patients (86.7\%) were in sinus rhythm one patient $(6.7 \%)$ had atrial fibrillation, and one patient had permanent pacemaker implantation in early postoperative period due to the remaining AV-nodal rhythm. During 6 months after the procedure, all patients were treated with cordarone in a typical dose. After 6 and 12 months period after procedure all 13 patients (100\%) preserved the sinus rhythm. Two years later, 11 patients $(84.6 \%)$ preserved the sinus rhythm. None of the patients had thromboembolic complications. In the long-term, all patients were classified as NYHA classes I and II.

Conclusions: Cox-Maze V procedure in combination with mitral valve prosthetics is effective, able to maintain sinus rhythm for a long time, allows to avoid complications of atrial fibrillation and significantly improves the quality of life of patients. Despite the development of various modifications of the Cox-Maze procedure, it is advisable to use this procedure in patients with atrial fibrillation refractory to antiarrhythmic therapy and catheter ablation.
P138

Concomitant atrioventricular valves surgery during aortic valve replacement in patients with aortic stenosis

Vladislav Podpalov, Eugeni Gorbachev, Mikalay Yudo, Yauheny Kastsyukovich, Vladimir Silchenko, Kiryl Rubakhov, Vasily Sevrukevitch, Yuri Ostrovsky Republican Scientific and Practical Center of Cardiology, Minsk, Belarus Correspondence: Vladislav Podpalov

Journal of Cardiothoracic Surgery 2017, 12(Suppl 1):P138

Objective: To analyze influence of atrioventricular valves surgery on 5 -year mortality in patients underwent aortic valve replacement (AVR) for Aortic Stenosis.

Methods: Among all patients admitted to the Clinic (2005-2007) 288 patients operated for aortic stenosis with secondary atrioventricular valves regurgitation were included in prospective non-randomized study. Using exclusion criteria (infective endocarditis, cancer, mitral stenosis) 152 patients were selected and divided into 2 groups: $1^{\text {st }}$ group - 68 patients underwent isolated AVR; $2^{\text {nd }}$ group - 84 patients with double/triple valve surgery. Folow-up period was 5 years.

Results: The average age of 152 patients was $57,61( \pm 9,81)$ years, $65,8 \%$ of men. Mean EuroSCORE value was $4,7 \pm 2,4$; NYHA functional class III was in $73,3 \%$ of patients. The average LVEF was $48,15( \pm 14,52) \%$. Groups had different mitral regurgitation grade: $2+$ in the $1^{\text {st }}$ group versus $3-4+$ in the $2^{\text {nd }}$ group $(p<0,001)$. Concomitant CABG was performed according to ESC/EACTS guidelines in $20(29,4 \%)$ patients from the $1^{\text {st }}$ group and $19(22,6 \%)$ patients from the $2^{\text {nd }}$ group. 5-year mortality rate in the $1^{\text {st }}$ group was $20,0 \%$ in patients with CABG versus $22,9 \%$ patients without CABG (no difference: $p>0,05$ ), in the $2^{\text {nd }}$ group - $26,3 \%$ and $10,8 \%$ respectively (tendency to difference: $p<0,1) .5$-year mortality rate wasn't significantly higher in patients underwent multiple valve surgery as compared with isolated AVR in case of CABG $(p>0,05)$. Using multivariate regression model after adjustment for age and sex no significant relationship was found between 5-year mortality and concomitant CABG $(p>0,05)$. In patients without CABG there was a tendency to significant increase in 5 -year mortality rate in the $1^{\text {st }}$ group as compared with the $2^{\text {nd }}(p<0,1)$.

Conclusions: Concomitant atrioventricular valves surgery in patients with Aortic Stenosis complicated with atrioventricular valves insufficiency should be recommend as compared with isolated AVR because of better 5 -year outcome after surgery.

P139

Surgical tactics for treatment of infectious endocarditis with multivalvular affection

Sokhib Mirzzakhmedov, Khamidulla Abdumajidov, Khayrulla Buranov, Ikrom Mirhodjaev

Republican Scientific Center of Surgary named after V.Vakhidov, Tashkent, Uzbekistan

Correspondence: Sokhib Mirzzakhmedov

Journal of Cardiothoracic Surgery 2017, 12(Suppl 1):P139

Objective: to analyze the results of surgical treatment of Infective endocarditis(IE).

Methods: 138 patients with IE were investigated during the period of 2001-2016 at the department of surgery for acquired heart diseases in RSCS named after acad. V. Vakhidov. The average age of patients was $32,76+1,6$ years old (from 12 to 68 years old). Males were $85(54,5 \%)$ and females - 71(45,5\%). The diagnosis was based on the Dukes criterion. The patients are divided into 2 groups: $1^{\text {st }}$ group is consisted of $89(57,4 \%)$ patients, in which staged surgical treatment has been performed; mechanical sanitation of infective focus of the valve, implantation of prosthesis cuff on which antibiotics and 
hyperthermic perfusions have been processed. $2^{\text {nd }}$ group is consisted of $67(42,6 \%)$ patients, on which the surgical treatment is performed on traditional methods without using of abovementioned techniques. Results: The hospital mortality was $6,9 \%(7$ patients). In the 1 st group $(n-89)$ the mortality was observed in $5,1 \%$ patients in the 2 nd group it was $9,3 \%$. The most important reasons of mortality were septic complications, heart failure, as well. After analyzing the causes of mortality on groups, it was defined that the acute heart failure in patients of the 1st group has been revealed in $2 / 3$ cases, and so, in the 2 nd group, the progressive infection has been prevaluated that was revealed in 3/4 dead patients.Among the reasons of non-lethal complications, there were also comlications related to pyoseptic processes. In patiens of 1st group, medicoprophylactic measures allowed significantly reduce the rate of pyoseptic complications which are consisted of $20,6 \%$ as compared with 2 nd group $(47,5 \%)$.

Conclusions: application of medioprophylactic measures in surgical tactics of valvular infective endocarditis allowed to reduce the hospital mortality from $9,3 \%$ to $5,1 \%$, and also, the rate of infective complications - from $47,5 \%$ to $20,6 \%$.

P140

Clinical experience of original bileaflet heart valve prosthesis with antipannus construction Planics-A in aortic and mitral valve replacement

Vadim Shumavets ${ }^{1}$, Alexandr Shket ${ }^{1}$, Ihar Andraloits ${ }^{1}$, Andrey Moskalenko ${ }^{2}$, Svetlana Kurganovich', Nalalja Uss', Alena Lisjonok', Yuory Ostrovski ${ }^{1}$

'Brlarus Centre Cardiology, Minsk, Belarus; 'Brlarus Trade "Belmashpribor", Minsk, Belarus

Correspondence: Vadim Shumavets

Journal of Cardiothoracic Surgery 2017, 12(Suppl 1):P140

Background: Pannus formation on mechanical heart valve prosthesis and related valve dysfunction are still factors affected result of heart valve replacement. We present the initial 1-year follow-up results of aortic and mitral valve replacement with a new bileaflet mechanical heart valve prosthesis with anti-pannus construction "Planics A".

Methods: The aortic (23 patients) or mitral (18 patients) valve replacement (patients age from 29 to 74 years) were conducted in a routine daily manner. $43 \%$ of the patients had the class III or IV of NYHA. Aortic valves with landing sizes $21-27 \mathrm{~mm}$ and mitral valves with landing sizes $27-33 \mathrm{~mm}$ were used. The hospital and 1-year results were analyzed.

Results: There were no prosthesis-related complications or structural valve deterioration during the period of observation. The effective orifice area of Planics-A during hospital stay was $1,53 \pm 0,21 \mathrm{~cm} 2$ to $2,43 \pm 0,15 \mathrm{~cm} 2$ for aortic prosthesis valves and from $3,5 \mathrm{~cm} 2$ to 3,7 $\mathrm{cm} 2$ for mitral valves. The average transmitral gradient was not more than $6,33 \pm 0,58 \mathrm{~mm} \mathrm{Hg}$. A signifi cant reduction (up to $48 \%$ ) of aortic peak and medium transvalvular gradient of aortic valve $(p<0,015)$ was noted during the mid-term observation. The total LV mass regression $(15-26 \% ; p=0,020)$ and the index of LV mass regression (14$29 \%, p=0,031)$ were revealed in patients operated on severe aortic stenosis. The EOA for mitral prosthesis valves Planics-A were stable without decrease during the mid-term observation. The hemodynamic parameters of domestic valves Planics-A were comparable to foreign prostheses.

Conclusions: The implantation of the aortic prosthesis with an adequate diameter reduces the pressure gradient, increases the EOA of aortic valve and results in regression of LV myocardial mass. Our 1-year experience shows that the new valve "Planics $\mathrm{A}$ " with anti-pannus construction has an excellent intermediate-term durability, and it is characterized by substantial echocardiographic improvement.
P141

Surgery management of infective valve endocarditis in 83 patients operated in a university public hospital

Paulo Roberto B. Evora, Solange Bassetto, Lafaiete Alves Junior,

Gustavo F. Silva, Luiza S. Mateus, Alfredo José Rodrigues,

Walter Vilella A. Vicente

Ribeirão Preto Medical School, University of São Paulo, Ribeirão Preto, SP, Brazil

Correspondence: Paulo Roberto B. Evora

Journal of Cardiothoracic Surgery 2017, 12(Suppl 1):P141

Introduction. Infective endocarditis is a life-threatening condition that frequently necessitates heart surgery intervention on native or valve prosthesis, many times with an ongoing systemic inflammatory response.

Objective. This retrospective investigation aimed to review the surgical experience management of valve heart infective endocarditis (IE).

Method. Patient's medical database evaluation including a cohort of 86 patients operated on a university public hospital.

Results. A) Demographic and mortality data: 1) The cohort study included 86 patients operated from 2000 to 2016; 2) Gender (62 men/72\%; 24 female/28\%); 3) Prevalent age was from 20 to 40 years; 4) There was no difference in the global mortality incidence: $(n=33 /$ $37 \%), 5)$ Mortality was higher in males $(n=26 / 78 \%)$ than in females $(\mathrm{n}=7 / 22 \%) ; \mathrm{B})$ Endocarditis classification: Acute and subacute ( $\mathrm{n}=$ 78/94\%), Subacute unspecified valve $(n=5 / 6 \%)$; C) Microbiology: Streptococcus and staphylococcus were the most common organisms; D) Urgent surgery $(n=11 / 12 \%)$; Elective surgery $(n=75 /$ $88 \%)$; E) Number of valves affected $(n=96)$ F) Type of prosthesis: mechanical $(n=25 / 35 \%)$ biological $(n=44 / 65 \%)$ F) Surgery: Prosthesis $(n=67 / 80 \%)$ Valvuloplasty $(n=16 / 20 \%)$.

Conclusion. Endocarditis surgery is always a high-risk one, mainly related to patient's clinical and hemodynamic status and microbiological factors. These results are quite similar to the worldwide published experience, revealing tendencies for older men increased the incidence and the increased preference for tissue valves.

P142

New structural, chemical, and immunoenzymatic investigations of cryopreserved aortic valve allografts

Roman J. Pfitzner', Dorota Barecka², Maciej Pawlikowski ${ }^{3}$,

Magdalena Kopytek², Lucyna Rudnicka-Sosin², Elzbieta Majewska²,

Marta Mazur ${ }^{2}$, Zdzisława Marcinkowska ${ }^{2}$

'Jagiellonian University, Cracow, Poland; ${ }^{2} J o h n$ Paul II Hospital, Cracow,

Poland; ${ }^{3} \mathrm{AGH}$ University of Science and Technology, Cracow, Poland

Correspondence: Roman J. Pfitzner

Journal of Cardiothoracic Surgery 2017, 12(Suppl 1):P142

Objective: Inspite of excellent results of clinical use of aortic valve allografts (A), some issues should be soluted.

Methods: 23 A, harvested during forensic autopsies (19M, age 20-51, average 32,5) were proceeded: $20 \mathrm{~A}$ were cryopreserved in DMSO\&RPMI solution under $-160 \mathrm{C}$ for 1-15 years, and 3 controls were stored in calf serum at $+4 \mathrm{C}$ up to 2 weeks. Classical histology, digital microscopy (DM), scanning electron microscopy (SEM), energy dispersion X-ray spectrometry (EDS); PECAM1 and CD34 immunoenzymatic tests were used.

Results: All A were macroscopically normal. The rewarmed tissues were a little more elastic than fresh. Microscopy presented normal structure of leaflets, but massive loss of endothelium: on the surface of $5 \mathrm{~mm}$ long scraps were found in average 59 cells. DM and SEM showed generally normal collagen fibers. In 5 specimens were found 
local collagenic micrograins <30 microns. Small deformities (empty spaces, superficial plates), probably influenced by freezingdefreezing, occured in 4 preparations. EDS detected somewhat elevated calcium amount in 1 specimen (51y) only. The mean protein concentrations of PECAM1 in cryopreserved and fresh A were 1,9 and $2,0 \mathrm{ng} / \mathrm{ml}$, while the CD34 concentrations were $0,8 \mathrm{vs.} 0,6 \mathrm{ng} / \mathrm{ml}$. Additionally, clinical and echocardiographic status of frozen A, implanted in 65 pts, was satisfactory, without evidence of calcifications, except $1 \mathrm{pt}$, who needed 2 reoperations because fresh, and then frozen A degenaration, both after 7 years.

Conclusions: The "fresh" and cryopreservation technologies of A preparation did not influence significantly the basic properties of leaflets, however, both induced massive endothelial decellularization. It correlated with no endothelial cell activation, and no signs of inflammation. The findings were independent to donors' age and sex; technology and time of storage. There were no evident differences between long term stored $\mathrm{A}$ and these preserved during obligatory 5 years period.

\section{P143}

Surgical treatment of cardiac echinococcosis

Victor Ivanov, Liubov Ivanova, Tatiana Nikityuk, lashar Aidamirov Russian Research Center of Surgery, Moscow, Russian Federation

Correspondence: Victor Ivanov

Journal of Cardiothoracic Surgery 2017, 12(Suppl 1):P143

Objective: Cardiac involvement of cystic echinococcosis does not exceed $3 \%$ of all sites infested by echinococci. The left ventricle (LV) was affected 2-3 times more often than the right ventricle and accounted for $55-60 \%$ of all observations of cardiac echinococcosis (CE). In most cases, the left ventricular cysts were localized in the subepicardial region and the right ventricular cysts in subendocardial region. The rupture of cyst remains one of the most frequent and terrible complications of CE. More often CEwas found in combination with echinococcosis of lungs, liver and brain. Isolated cases of cardiac echinococcosiswere rare. The clinical presentation of CE shows no pathognomonic symptoms based on which a possible diagnosis can be established. To show the peculiar features of surgical treatment of cardiac echinococcosis (CE).

Methods: Examination and treatment were carried out on 8 patients (5 men, 3 women), aged 20-60 years. The clinical manifestations of CE are non-specific.

Results: Often the disease simulates coronary heart disease and signs of coronary disease were recorded on ECG. The diagnosis CE was establishedusing radiography, echocardiography, CT, coupled with serological tests. Isolated CE was diagnosed in 3 patients. In other patients, echinococcal cysts were located in the brain, lungs, liver and in gluteal region in addition to heart. All the patients were successfully operated on with cardiopulmonary bypass.

Conclusions: The rare localization of echinococcal cysts in the heart shows the parasite's aggressiveness and is an evidence of its unusual morphology. The accuracy in diagnosis of CE is achieved by combining echocardiography, CT and serum samples. Due to high risk of rupture of the cyst, surgery with cardiopulmonary bypass remains the preferred form of treatment. The surgical tactics requires an individual approachin each case. Treatment of patients with CE should be integrated with the mandatory use of postoperative antirelapse chemotherapy.

\section{P144}

Reconstruction of the isolated posterior leaflet prolapse with loop technique

Pavel Kakhktsyan, Irma Tsiskaridze, Tigran Asatryan, Alexander Vavilov, Dovran Kakabayev, Ivan Scopin

Bakulev NSPCCVS, Moscow, Russian Federation

Correspondence: Pavel Kakhktsyan

Journal of Cardiothoracic Surgery 2017, 12(Suppl 1):P144
Objective: We assessed the early and midterm outcomes for the reconstruction of the isolated posterior mitral leaflet (PML) prolapse with loop technique.

Methods: Between 2010 and 2016, 23 patients underwent a prolapsing posterior leaflet (PPL) repair for severe mitral regurgitation (MR) with loop technique for the isolated posterior leaflet prolapse. The 17 $(73,9 \%)$ patients had concomitant procedures. Patient demographics were as follows: mean age, 50,6 $\pm 13,7$ years; male, $12(52,2 \%)$; NYHA functional class, 2,7 $\pm 0,5$; logistic EuroSCORE, 3,0 $\pm 2,6$. PML resection was performed in $21,7 \%$ of cases, sliding annuloplasty - in $17,4 \%$ of patients.

Results: No patients died postoperatively. Survival and freedom from reoperation at 6 years was $100 \%$. At echocardiographic follow-up, 13 patients $(56,5 \%)$ showed no or trace MR, $10(43,5 \%)$ - mild MR. The mean coaptation lengths among all patients were $9,75 \pm 2,83 \mathrm{~mm}$ and $10,64 \pm 2,09 \mathrm{~mm}$, the mean coaptation depth $-4,17 \pm 1,87 \mathrm{~mm}$ and $4,18 \pm 2,15 \mathrm{~mm}$, the mean mitral valve effective orifice area $3,55 \pm 0,82 \mathrm{~mm} 2$ and $3,75 \pm 0,67 \mathrm{~mm} 2$ in the early and midterm periods of observation, respectively. Posterior coaptation distance at unresection approach amounted of 10,05 $\pm 2,7 \mathrm{~mm}$, at resection approach $-11,56 \pm 2,92 \mathrm{~mm}(p=0,216)$. The mean mitral valve effective orifice area at unresection approach $(3,6 \pm 0,33 \mathrm{~cm} 2)$ was greater than at resection approach $(3,51 \pm 1,19 \mathrm{~cm} 2)(p=0,796)$ but not significant in this series.

Conclusions: PPL repair using pre-measured Gore-Tex loops aims for restoration of physiologic leaflet motion, creation of an adequately sized mitral orifice with a sufficient line of leaflet coaptation, and stabilization of the mitral annulus.

P145

Can ultrasonic valve decalcification be alternative to replacement for aortic valve stenosis with small annulus?

Borys Todurov', Andrey Markovets', Oleg Zelenchuk², Natali Volodkina²,

Vitaly Demyanchuk'

${ }^{1}$ Kyiv Heart Institute, Kyiv, Ukraine: ${ }^{2}$ P.L. Shupyk National Medical

Academy of Postgraduate Education, Kyiv, Ukraine

Correspondence: Vitaly Demyanchuk

Journal of Cardiothoracic Surgery 2017, 12(Suppl 1):P145

Objective: Valve repair is superior to valve replacement in terms of postoperative risk. There has been increasing interest in valvesparing surgery to treat pathology of the aortic valve. At the same time, there is lack of studies concerning valve repair for calcified aortic valve stenosis. The aim of study is to present the hemodynamic outcomes of the relief aortic valve stenosis by means of ultrasonic valve decalcification.

Methods: Between January 2014 and January 2017, 16 patients (mean age, 70.2 \pm 7.4 years) with aortic valve stenosis underwent ultrasonic valve decalcification. Preoperative transthoracic echocardiography revealed aortic ring diameter $19 \pm 1.1 \mathrm{~mm}$, aortic valve peak systolic pressure gradient as $102 \pm 20.5 \mathrm{~mm} \mathrm{Hg}$ (mean gradient - $66 \pm 0.8 \mathrm{~mm} \mathrm{Hg}$ ) and AVA as $0.80 \pm 0.03 \mathrm{~cm}^{2}$ (index AVA $-0.40 \pm 0.01 \mathrm{~cm}^{2} / \mathrm{m}^{2}$ ). The operative technique consisted of standard nonpulsative cardiopulmonary bypass. Aortic valve debridement was performed under direct vision using irrigation/aspiration probe of modern ultrasound machine. Ultrasonic intermittent applications were used in of approximately 10 seconds until the calcium was removed, avoiding damage to the valve and restoring the cusps mobility.

Results: There were no hospital mortality in study group. After procedure, peak systolic pressure gradient was reduced to $25 \pm 11 \mathrm{~mm}$ $\mathrm{Hg}$ (mean - $12 \pm 5.1 \mathrm{~mm} \mathrm{Hg}$ ) and AVA was increased to $1.9 \pm 0.2 \mathrm{~cm}^{2}$ (index AVA - $1.0 \pm 0.13 \mathrm{~cm}^{2} / \mathrm{m}^{2}$ ). Postoperative follow-up at 7 months showed the peak systolic pressure gradient as $22 \pm 9.7 \mathrm{~mm} \mathrm{Hg}$ (mean $11 \pm 3 \mathrm{~mm} \mathrm{Hg}$ ).

Conclusions: We believe ultrasonic decalcification can be considered as a type of aortic valve repair for treatment of elderly patients with aortic valve stenosis with small annulus. But long-term follow-up 
study, in a big group of patients, on the results of this technique is necessary.

\section{P146}

Myocardial protection for isolated mitral valve replacement: is retrograde applying of cardioplegia is better?

Volodymyr Popov, Alex Gurtovenko, Bogdan Gumenjuk

National Institute of Cardio-Vascular Surgery named after Amosov, Kiev, Ukraine

Correspondence: Volodymyr Popov

Journal of Cardiothoracic Surgery 2017, 12(Suppl 1):P146

Objective. To present analysis of retrograde crystalloid cardioplegia during isolated mitral valve replacement (MVR).

Materials And Methods. During 2009-2016 y 529 patients (pts) with isolated pathology of mitral valve disease (MVD) were operated by MVR. NYHA class in all group were followings: II class - 28 (5,4\%), III class - $229(43,3 \%)$, IV class - $272(51,3 \%)$ ptc. The following applying of cardioplegia (Custadiol) were performed by: antegrade $84(15,8 \%)$ pts (group A), ante-retrograde 66 (12,5\%) pts (group B), retrograde $379(71,7 \%)$ pts (group C) ways. Systemic hypothermia 32-34 C, cardiopulmonary bypass, retrograde cardioplegic solution (Custadiol) (in dose $20 \mathrm{ml} / \mathrm{kg}$ ) + external cooling of myocardium were occured in all pts. Average time of improvement of cardioplegia solution (min) were for: group A - 9,8 $\pm 0,9$, group B - 12,8 $\pm 1,5$, group C - 20,2 $\pm 2,7(p<0,05)$. Average cross-clamping time ( $\mathrm{min})$ were for: group $A-$ $69,8 \pm 7,9$, group $B-64,8 \pm 7,5$, group $C-52,7 \pm 6,4(p<0,05)$ and reper-

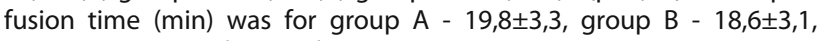
group C - 16,1 $\pm 2,5(p>0,05)$.

Results. Hospital mortality was for group A 3,5\% ( $n=3 / 84)$., group B $3,0 \%(n=2 / 66)$, group $C-1,1 \%(n=4 / 379)(p<0,05)$. Average doses of dobutamin (1,0-3,0 mcrg $/ \mathrm{min} / \mathrm{kg})$ were marked for: group A - 54,8 $\pm 7,2$ hours, group $B-49,1 \pm 5,7$ hours, group $C-41,5 \pm 5,4$ hours $(p<0,05)$. Average level of MB KFK (U/L) at 2-td postoperative day were occured

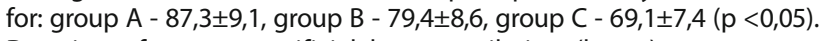
Duration of stay on artificial lung ventilation (hours) were: group A - 8,7 $\pm 0,9$, group B $-8,1 \pm 0,7$, group C - 7,5 $\pm 0,5$ ( $p>0,05)$. Conclusion. Improved myocardial protection in cases with isolated MVD by using retrograde crystalloid cardioplegic solution (Custadiol) (group $C$ ) lead to better results than in group $A$ and $B(p<0,05)$.

P147

Evaluating the safety of surgical ablation when mitral valve prosthetics

Ildar Abdulyanov', Marat Sungatullin', Ildar Vagizov²

${ }^{1}$ Kazan State Medical Academy, Kazan, Russian Federation; ${ }^{2}$ Interregional

Clinical and Diagnostics Center, Kazan, Russian Federation

Correspondence: Ildar Abdulyanov

Journal of Cardiothoracic Surgery 2017, 12(Suppl 1):P147

Background: A retrospective analysis of the safety of carrying out an electrical isolation of the pulmonary veins through the method of radiofrequency ablation (RFA) in patients when mitral valve prosthetics.

Methods: The retrospective analysis included 302 patients: the first group include patients mitral valve replacement and RFA of the left atrium (entry of the left pulmonary veins, the walls of the left atrium (LA) and the auricle of the LA) under the cardiopulmonary bypass (CPB) was comprised of 152 patients (50,3\%). The second group covered only those who had undergone mitral valve replacement under the conditions of CPB including 150 (49.7 per cent).

The female gender was the predominant one - 172 patients $(57,2 \%)$. The average age of the patients was $58 \pm 7$ years.

All the patients demonstrated persistent atrial fibrillation (AF) with a duration of $22 \pm 8$ months. All the patients belonged to II-III CHF
NYHA. The EuroScore II in the first group was $8 \pm 3.1 \%$ and $6.9 \pm 4.5 \%$ in the second group.

In $152(50,3 \%)$ cases, the RFA procedure was performed by a bipolar clamp and a unipolar pen produced. The procedure was performed on a stopped heart.

Results: The first group demonstrated a marked increase in the duration of aortic clamp resulting from the RFA: $72.8 \pm 6,6$ minutes in comparison with $58,3 \pm 8,8$ minutes in the second group.

No specific intraoperative and postoperative complications were marked in the first group. The implantation of a permanent cardiac pacemaker in both groups was statistically equivalent.

The mortality rate in the first group - 1,3\%, the second group $-1,97 \%$. Conclusions: Non-fatal complications and surgical mortality in both groups had no statistical differences. RFA prolongs the aortic clamp time without the risk of aggravating complications and mortality. Surgical ablation with mitral valve replacement represent a safe means of treatment of atrial fibrillation.

P148

The alternative strategy of revascularization for coronary arteries disease with concomitant valve correction

Pavel Kakhktsyan, Irma Tsiskaridze, Alexander Vavilov,

Khachatur Paronyan, Alim Otarov, Ivan Scopin

Bakulev NSPCCVS, Moscow, Russian Federation

Correspondence: Pavel Kakhktsyan

Journal of Cardiothoracic Surgery 2017, 12(Suppl 1):P148

Objective: To optimize the results of surgical treatment of patients with the heart valve desaese in conjunction with coronary artery disease by identifying the strategy of revascularization.

Methods: A total of 108 patients (78 male) underwent valve replacement or repair with concomitant coronary artery revascularization. The mean age was $62,2 \pm 6,7$. There are 69 aortic, 25 mitral and 13 combined mitral and aortic valves diseased patients. In all cases we utilized on-pump beating coronary arteries revascularization followed by valve correction performed in the standard technique. Indications for beating heart surgery were the volume of surgery which involves prolonged aortic clamping, severe concomitant diseases. The exception criteria's - a small coronary artery initial diameter less than $2 \mathrm{~mm}$, diffuse coronary artery calcification.

Results: Mean bypass and cross-clamp time were $167 \pm 28$ and $68 \pm 15$ minutes, respectively. Mortality rate was $4,6 \%$ ( 3 in the aortic, 2 in the mitral valves groups). Acute heart failure was in 3 patients with mitral valve pathology.

Conclusions: Beating heart on-pump coronary surgery due to changing the balance of oxygen delivery and consumption may have a favorable effect on the performance of the myocardium after cardioplegia, which reflected in the low rate of the heart failure and mortality.

P149

The role of Aortic Valve Effective Orifice Area in essessment of the time for surgery in patients with Aortic Stenosis complicated with atrioventricular valves insufficiency

Vladislav Podpalov, Svetlana Kurganovich, Kiryl Rubakhov,

Vasily Sevrukevitch, Yuri Ostrovsky

Republican Scientific and Practical Center of Cardiology, Minsk, Belarus

Correspondence: Vladislav Podpalov

Journal of Cardiothoracic Surgery 2017, 12(Suppl 1):P149

Objective: To analyze influence of Aortic Valve Effective Orifice Area (AV EOA) on 5-year mortality as index for surgery in patients with Aortic Stenosis (AS) and atrioventricular valves insufficiency.

Methods: Among all patients admitted to the Clinic during 20052007. 415 patients with AS complicated with secondary mitral and 
tricuspidal regurgitation were included in the study. Using exclusion criteria (infective endocarditis, cancer, mitral stenosis) finally 198 patients were included: 152 patients underwent Aortic Valve replacement (AVR) adding repair of atrioventricular valves; 46 patients were not operated for different reasons. Patients were divided into 3 subgroups according to the EOA values: 1st subgroup has EOA of 0,3$0,79 \mathrm{~cm}^{2}$ (15 unoperated and 50 operated patients); 2 nd subgroup $0,8-0,99 \mathrm{~cm}^{2}$ (16 unoperated and 44 operated patients); $3 \mathrm{~d}$ subgroup - 1,0-1,3 $\mathrm{cm}^{2}$ (15 unoperated and 58 operated patients). Follow-up period was 5 years.

Results: The average age of 198 patients was $59.16( \pm 11,19)$ years, the proportion of men contained $67 \%$ (133 persons). The majority of patients were in the $3 \mathrm{~d}$ NYHA Heart Failure functional class $(73,3 \%)$. The average left ventricular ejection fraction was $47.76( \pm 14,22) \%$. Subgroups were similar by these parameters $(p<0,05) .5$-year mortality rate in unoperated patients increased with the decrease in AV EOA value $\left(x^{2}=12,1, p<0,01\right)$. For operated patients such tendency wasn't observed $\left(X^{2}=1,2, P>0,05\right)$. 5-year mortality after operation with adjustment for age and sex in patients either from the 1st group or from the 2nd group wasn't significantly different in comparison with the patients from the $3 d$ group (OR=1,4, 95\% C.I. for $\operatorname{Exp}(B) 0,44-4,39, P>0,05$, and $O R=1,26,95 \%$ C.I. for $\operatorname{Exp}(B) 0,36-4,34$, $\mathrm{P}>0,05$, relatively).

Conclusions: Long-term results after AVR have no significant relationship with AV EOA size in AS with atrioventricular valves insufficiency. The indication of time for surgery needs to be individually predicted in this category of patients.

\section{P150}

Saint Jude mechanical valve for isolated mitral stenoses complicated massive thromboses of left atrium

Volodymyr Popov, Volodymyr Mnishenko, Kateryna Pukas, Bogdan

Gumenjuk, Svitlana Andrievskaya

National Institute of cardio-vascular surgery named after Amosov, Kiev,

Ukraine

Correspondence: Volodymyr Popov

Journal of Cardiothoracic Surgery 2017, 12(Suppl 1):P150

Objective. To analyzed main problems in surgery of mitral valve diseases (MVD) complicated by left atrium's massive thromboses (LAMT) (thromboses more than $1 / 3$ of left atrium's volume).

Materials and Methods. There were male 147 (43.7\%) and females 189 (56.3\%) from 01.01.1984 till 01.01.2015. The average age was 59.2 $\pm 5.2 \mathrm{yy}$. Only mechanical valves were used. All pts was devided on 2 groups; group A $(n=162)$ maternal thrombotic basement was removal together with all thrombotic masses out of LA, group B $(n=174)$ maternal thrombotic basement wasn't removal.

Results. At all period of experience thromboembolic events were marked: group A - 1.8\% ( $n=3 / 162)$ (lethal $=0)$, remote period $3.6 \%(n=5 / 140)$ (lethal $-1.4 \%)$, group $B-5.8 \%(n=10 / 174)$ (lethal$3.4 \%)$, remote period $-13.3 \%(n=19 / 142)$ (lethal $-9.2 \%)(p<0.05)$. At all period of experience thromboembolic events were marked: MVR in $4.1 \%(n=12 / 294)$, (lethal $-1.0 \%)$, remote period $-8.9 \%(n=$ $22 / 245)$ (lethal $-5.7 \%)$ and during OMC $2.4 \%(n=1 / 42)$, (lethal -0$)$, remote period $-5.4 \%(n=2 / 37)$ (lethal $-2.7 \%)(p<0,05) .282(95.5 \%$ alive) pts was followed-up at the remote period (average $16.2 \pm 3.1$ yy). At all period of experience thromboembolic events were marked for bileaflet prosthesis: Saint Jude $-1.5 \%$ (lethal $=0,7 \%$ ), remote period $-3,6 \%$ (lethal $-0.9 \%$ ), Carbomedics $-4.3 \%$ (lethal $-2.7 \%$ ), remote period $-6.5 \%$ (lethal $-2.2 \%$ ), On-X $-2,4 \%$ (lethal $=2.3 \%$ ), remote period $-4,2 \%$ (lethal - 0$)$, group ATS $(n=32$ pts) $-6.2 \%$ (lethal $=$ $3.1 \%)$, remote period $-9.3 \%$ (lethal $-6.3 \%)(p<0.05)$.

Conclusion. Thromboembolic events at postoperative period is specific complicated factor for LAMT. Maternal thrombotic basement must be removed with all thrombotic masses in all pts.
P151

Long term outcome of mitral valve repair versus mitral valve replacement

Grigore Tinica' ${ }^{1}$, Mihail Enache', Diana Anghel' ${ }^{1}$, Victor Prisacari ${ }^{1}$

Raluca Ozana Chistol', Cristina Furnica ${ }^{2}$

${ }^{1}$ Cardiovascular Diseases Institute, lasi, Romania; ${ }^{2}$ University of Medicine and Pharmacy, lasi, Romania

Correspondence: Grigore Tinica

Journal of Cardiothoracic Surgery 2017, 12(Suppl 1):P151

Objective Current guidelines offer a general framework for the management of mitral valve (MV) disease without offering evidencebased indications on whether to repair or to replace a MV according to subjacent lesions. The aim of the study was to conduct a longterm follow-up evaluation of MV repair results compared to MV replacement in terms of cardiac function, morbidity and survival.

Methods The authors performed a single center retrospective study on 681 patients that underwent MV surgery between 2002-2014 and were divided in 2 groups, group I consisting in 196 patients (28.78\%) with MV repair, and group II in 485 patients (71.22\%) with MV replacement. 91 patients $(13.36 \%)$ were evaluated every 6-12 months for $\geq 48$ months postoperatively. Perioperative and follow-up data were assessed in terms of prognosis and survival.

Results MV repair was preferred in male patients (2.06:1 M:F ratio) and MV replacement in female patients (0.72:1 ratio). Mean age and NYHA class registered no significant difference between the groups. MV repair was performed mostly in patients with mitral regurgitation (56.63\%) and type I/II Carpentier lesions (66\%) due to degenerative processes, papillary muscle rupture, endocarditis. In rheumatismal disease and extensive calcifications, MV prosthesis was preferred. Within 30 days mortality was similar in the two groups $(1.02 \% \mathrm{MV}$ repair, $1.03 \% \mathrm{MV}$ replacement). Long-term mortality rate was $4.12 \%$ (8 cases) for MV replacement and 5.62\% (27 cases) for MV repair at 63.7 months. At 48 months, $68.57 \%$ of MV repair patients had no or mild regurgitation and $88.57 \%$ were reevaluated in NYHA class I/II. The independent predictors of mortality were concomitant CABG, renal and liver failure, LVEF $<35 \%$, age $>73$ years.

Conclusions The current study proves favorable short and long-term prognosis in MV repair, thus making it the procedure of choice whenever the anatomy of the mitral apparatus and functional status allows it.

P152

Narrow ostium of aorta for aortic stenoses: is posterior aortoplasty necessary during aortic valve replacement?

Konstantin Vakulenko, Volodymyr Popov, Oleksandr Bolshak

National Institute of cardio-vascular surgery named after Amosov, Kiev, Ukraine

Correspondence: Konstantin Vakulenko

Journal of Cardiothoracic Surgery 2017, 12(Suppl 1):P152

Purpose of this investigation is to research possibilities of original method of posterior aortoplasty (PA) during aortic valve replacement (AVR) in patents (pts) with narrow ostium of aorta (NOA).

Materials And Methods. There were 464 (56.2\%) males and 361 (43.8\%) females in average age $57.5 \pm 8.4$ yy. $315(38.2 \%)$ pts belonged to III NYHA class and $510(61.8 \%)$ - to IV from 01.01. 2010 till 01.01.2017..Operations were performed by following methods: group $A$ - AVR+ original method of reconstruction by PV (Popov V.) ( $n=89)$; group B - AVR with model 21 $\mathrm{mm}(\mathrm{n}=379)$; group C- AVR with model $19 \mathrm{~mm}(\mathrm{n}=357)$.

Results. Indexed effective orifice area $\left(\mathrm{cm}^{2} / \mathrm{m}^{2}\right)$ and peak gradient on aortic prosthesis (mm Hg)(PGAP) were marked for: group A - 0.95 \pm 0.03 $(P G A P=22.3 \pm 2.7 \mathrm{~mm} \mathrm{Hg}) ;$ group $B=0.88 \pm 0.03(P G A P-26.3 \pm 3.8)$ group $C-0.82 \pm 0.03(P G A P=35.3 \pm 5.2)(p<0.05)$. In group $A(n=83)$ survival rate $83.4 \%$ and stability of good results $63.5 \%$ were observed at 7 years after operation. In group B $(n=343)$ survival rate $78.3 \%$ and 
stability of good results $23.3 \%$ were marked at 7 years after operation. In group C $(n=327)$ survival rate $49.3 \%$ and stability of good results $23.3 \%$ were occured at 7 years after operation. At remote period indexed effective orifice area $\left(\mathrm{cm}^{2} / \mathrm{m}^{2}\right)$ and PGAP $(\mathrm{mm} \mathrm{Hg})$ were marked for: group $A-0.92 \pm 0.03(P G A P=21.3 \pm 2.3)$; group $B-0.84 \pm 0.04(P G A P=$ $29.3 \pm 3.9)$; group $C-0.78 \pm 0.04(P G A P=42.3 \pm 4.7)(p<0.05)$.

Conclusion. Reconstruction of NOA during AVR by proposed original method of posterior aortoplasty is effective intervention especially at remote period in group $A$.

\section{P153}

8 year experience of surgical treatment of ascending aorta aneurysms

Khakimjon Abralov, Umidjon Murotov

Republican Specialized Center of Surgery named after academician

V.Vakhidov, Tashkent, Uzbekistan

Correspondence: Khakimjon Abralov

Journal of Cardiothoracic Surgery 2017, 12(Suppl 1):P153

Background Diagnosis and surgical repair of aneurysms of the ascending aorta is one of the hardest parts of modern cardiovascular surgery. Among $66.6 \%$ of the observations there is perform standard one-stage operation - Bentall procedure. We have experience of surgical treatment ascending aorta aneurism associated with coarctation and would like to present it.

Methods Between from January 1, 2009, to January 1, 2017 at Republican Specialized Center of Surgery - 41 patients with aneurysm of ascending part of aorta underwent Bentall procedure. There were 26 males $(63 \%)$ and 15 females (37\%), and their age ranged from 25 to 65 years (mean age $31.6 \pm 0.2$ years). Etiology of aneurysms was presented by: Marfan syndrome at 24 (58.5\%) athereosclerosois $(34.1 \%)$ and 3 patients $(7.3 \%)$ with degenerative lesions of the aorta. At 12(29.2\%) patients aneurysms was complicated by aortic intimae dissection (type II by De Bakey); other 18(43.9\%) operated patients without aortic dissection. Bicuspid aortic valve was found in $8(19.5 \%)$ cases. In $3(7.3 \%)$ cases aneurysm was associated with coarctation of the aorta in typical place - in that cases has been chosen the tactics of the two-step repair - the first step performed resection of coarctation with conduit replacement, and 10-12 days after first step was performed Bentall procedure with excellent result.

Results Hospital mortality consisted 4 (9.7\%). Hospital mortality was caused by: acute heart failure, restrict postoperative bleeding and multiple organ failure.

Conclusions Despite on occurred complications and technical difficulties, Bentall procedure provide adequate radicalism of repair. Bentall procedure shows excellent short and long-term patients survival and freedom from reoperation. That is why it can be recommended in wide etiological spectrum of aneurism even in combined lesions of aorta including coarctation of aorta.

\section{P154}

\section{Application of biocor bovine patch for posterior aortoplasty}

during aortic valve replacement

Konstantin Vakulenko, Oleksandr Bolshak, Svitlana Andrievskaya,

Volodymyr Popov

National Institute of cardio-vascular surgery named after Amosov, Kiev,

Ukraine

Correspondence: Konstantin Vakulenko

Journal of Cardiothoracic Surgery 2017, 12(Suppl 1):P154

Purpose of this investigation is to research possibilities of application of biocor bovine patch by original method of posterior aortoplasty (PA) during AVR in patents (pts) with narrow ostium of aorta (NOA).

Materials And Methods. There were included 105 pts with aortic (n $=84)$ and combined mitral-aortic diseases $(n=21)$ with NOA which were consecutive operated in Institute from 01.05. 2009 till 01.01.2017. There were $63(60.0 \%)$ males and $42(40.0 \%)$ females and average age $63,7 \pm 5,9$ yy. 33 (31.5\%) pts belonged to III NYHA class,
$72(68.5 \%)$ - to IV. AVR with PA was performed in all cases by original method (Popov V.) in which aorta's incision was made in the middle of non-coronary leaflet, and then into central fibrous body of right trigone on depth 7-8 $\mathrm{mm}$. Patch (sizes $3 \times 4 \mathrm{~cm}$ ) was replaced at the area of the basement of noncoronary sinus, fibrous annulus, ascending aorta. The following patches were used: Vascutek's $(n=57)$ (group A), autopericardial $(n=15)$ (group B), bovine biocor SJM ( $n=33$ ) (group C). Bileaflet prosthesis (Saint Jude Medical, On-X, Carbomedics,) were used in sizes: 21 ( $n=9$ pts), 23 ( $n=84$ pts), 25 ( $n=10$ pts), 27 ( $n=2$ ts).

Results. Among 105 operated died 7 pts at hospital period (30 days) (hospital mortality $-6.7 \%$ ). Absence of using donor product during all hospital period were: $8.8 \%$ (group A), 6.6\% (group B) and 15.2\% (group C) $(p<0.05)$. Staying in ICU were: $63.3 \pm 7.2$ hours (group A), $72.2 \pm 8.5$ hours (group B) and $57.4 \pm 7.2$ hours (group C) $(p<0.05)$. Reexploration were: $3.5 \%$ (group $A), 6.7 \%$ (group $B$ ) and $0.0 \%$ (group $C$ ) ( $<<0.05$ ).

Conclusion. Reconstruction of NOA by bovine patch during AVR by proposed original method of posterior aortoplasty is effective intervention.

P155

Prevention sick sinus syndrome in patients with the fragmentation operation in left atrium for correction of isolated mitral valve disease

llya Leoshko, Volodymyr Popov

National Institute of cardio-vascular surgery named after Amosov, Kiev,

Ukraine

Correspondence: llya Leoshko

Journal of Cardiothoracic Surgery 2017, 12(Suppl 1):P155

Objective. The aim of the study is to study the possibilities of the proposed technique of the auto pericardial nutrient-foot to improve the results of sinus rhythm restoration in mitral valve replacement (MVR) and prevention of sick sinus syndrome.

Methods. From January 1, 2013 to May 1, 2017 men were 111 (42.5\%), women - 150 (57.5\%). In 255 patients was performed MVP: 1) Preserving posterior mitral valve leaf (214 patients), 2) Preserving posterior mitral valve leaf + translocation papillary muscles in the front wing cut the fibrous ring of the left AV opening (39 patients), 3) Full preservation of the anterior and posterior valves of the mitral valve (2 patients). In 33 patients (study group) was supplemented implantation operation in the zone sinus of auto pericardial nutrientfoot.

Results. In the comparison group (248 patents) the mitral valve replacement (MVR) in combination with Operation Labyrinth In lowfrequency mode allows us to successfully restore the correct rhythm in $76.2 \%$ of cases at the hospital stage and stabilize it within six months or a year after the operation. However, considering the sick sinus syndrome 4 pacemakers (1.5\%) were implanted in the postoperative period. At discharge, the recovery of sinus rhythm in the group of patients with PL plastic was higher than in the alternative group: $85.6 \%(n=119 / 139)$ i $73.7 \%(n=90 / 122)(p<0.05)$. In the comparison group, the sinus rhythm recovered immediately after the operation in $30(92.5 \%)$ patients, with an ECG discharge recorded in $30(92.5 \%)$ and in 2 years was noted in $80.0 \%$ of cases.

Conclusion. The proposed method of implantation and implemented application of a nutrient-foot from auto pericardium the zone sinus simple in execution, no traumatic and simultaneously provides a remarkable clinical results in early and remote postoperative period.

P156

Clinical experience of the Scientific Research Institute of the Cardiology and Internal Diseases in performing Bentall De Bono procedure

Agzam Aldeshev, Zhanar Nurbay, Yerbol Kudabayev,

Galymzhan Sultanov

Scientific Research Institute of the Cardiology and Internal Diseases,

Almaty, Kazakhstan

Correspondence: Agzam Aldeshev

Journal of Cardiothoracic Surgery 2017, 12(Suppl 1):P156 
Objective: To present results of performing Bentall De Bono procedure in patients with aneurysm of the ascending aorta at 6 and 12 months after the procedure.

Methods: Results of correction of aneurysm of the ascending aorta in 17 patients performed from 2015 for 2017 by the Department of Cardiac surgery are analyzed. Patients 15 - 78 years (average 46.5). Men: $14(82.4 \%)$, women - $3(17.6 \%)$. All patients had a failure of the aortal valve 2-3. Aetiology: atherosclerosis - 6 (35.3\%), infectious endocarditis - $3(17.7 \%)$, bicuspid aortic valve - $1(6 \%)$, degenerative diseases - 7 (41.2\%), including Marfan's Syndrome - 3 (17.6\%). Isolated Bentall De Bono procedure was performed in 12, combined Bentall De Bono procedure with a CABG was performed in 2, with mitral valve repair and replacement was performed by 1.16 operations - planned order, 1 - emergency. In all the cases was performed Aortic Valved Graft was used.

Results: The average length of the Bentall De Bono procedure is 3.5 hours. The cardiopulmonary bypass time averaged $135 \pm 26 \mathrm{~min}$, crossclamping of an aorta is $95 \pm 20 \mathrm{~min}$. Drainage losses averaged $469 \pm 115 \mathrm{ml}$. Bleedings in the postoperative period were not observed. 15 patients were extubated in the first days after operation. Two patients had following complications: 1) respiratory failure (prolonged pulmonary ventilation), 2) acute heart failure (central veno-arterial ECMO was used, successfully disconnected on the 4th day). A postoperative lethality averaged $11.7 \%$ (2 patients out of 17). Without hospital mortality, patient survival over 12 months is $100 \%$ (15).

Conclusions: Bentall De Bono procedure is a radical method of correction in patients with aneurysm of the ascending aorta with good immediate results and high rate of freedom from reoperation.

\section{P157}

Arch plasty of left atrium for moderate dilatation of left atrium: is it necessary?

Volodymyr Popov, Bogdan Gumenuk, Svitlana Andrievskaya,

Kateryna Pukas

National Institute of cardio-vascular surgery named after Amosov, Kiev, Ukraine

\section{Correspondence: Volodymyr Popov}

Journal of Cardiothoracic Surgery 2017, 12(Suppl 1):P157

Objective. To determined possibillities of left atrium (LA)'s reduction by original method of arch plasty of LA (APLA) during mitral valve replacement (MVR) for isolated mitral valve disease (MVD).

Methods. During 2005 - 2016 yy. 454 adult patients (pts) with MVD and LA's moderate dilatation of LA (diameter of LA $50<60$ ) average $57.1 \pm 0.5 \mathrm{~mm}$ were operated at Institute. There were $239(52.7 \%)$ in IY NYHA class, $186(41.0 \%)$ in III class and $29(6.3 \%)$ in II class. All data divided at 2 groups: group A - APLA + ligation of LA's auriculum was 117 pts and group B - 337 pts only MVR without LA's plasty or ligation's auriculum were performed.

Results. Sinus rhythm was restored at discharge: group A - 21.6\% and group $B-5.2 \%(p<0.05)$. At the remote period (average was $9.3 \pm$ 1.4 yy) 437 (93.2\%) pts were followed-up. Data of echo for group $A$ were: diameter of LA (mm) - preoperative (PRE) - $57.9 \pm 0.7$, postoperative (POST) - $48.3 \pm 0.4$, remote period (RP) $-49.5 \pm 0.4$; ejection fraction of LV (EFLV): PRE $-0.54 \pm 0.03$, POST $-0.57 \pm 0.03, R P-0.59$ \pm 0.02 . At the remote period were marked absence of thromboembolic events and $\mathrm{HF}$ and sinus rhythm was occured in $13.5 \%$ pts. Data of echo for group B were: diameter of LA (mm): PRE- $57.4 \pm 0.5$, POST $-55.2 \pm 0.8, \mathrm{RP}-62.2 \pm 1.1$; EFLV: PRE $-0.54 \pm 0.04$, POST $0.55 \pm 0.03, \mathrm{RP}-0.53 \pm 0.05$. Thrombembolic events and HF were marked at remote period respectively $-4.5 \%$ and $13.2 \%$.

Conclusion. The original method of APLA was allowing to improve better clinical results at group $A$ than in $B$ during all postoperative period $(p<0.05)$.
P158

Surgical treatment of patients with three valve heart diseases

Khamidulla Abdumadjidov, Khayrulla Buranov, Ilkhom Khodjakulov, Ilkhom Mirhodjaev

Republican Scientific Center of Surgary named after V.Vakhidov,

Tashkent, Uzbekistan

Correspondence: Khamidulla Abdumadjidov

Journal of Cardiothoracic Surgery 2017, 12(Suppl 1):P158

Objective: To analyze the results of surgical treatment of three-valve heart defects.

Methods: Because of three-valvular heart disease, 106 (100\%) operations were performed. Main method for diagnosing valvular defects was echocardiography. Patients older than 40 years underwent coronary angiography. The average age was $47.6 \pm 9.3$ years. There were $45.9 \%$ of men, $54.4 \%$ of women. The etiological factor was the rheumatic process - in $61.5 \%$ of patients; Infectious endocarditis (IE) $18.2 \%$. Pulmonary hypertension as a complicating factor was detected in $35(32.7 \%)$ patients. $22(20.7 \%)$ patients, previously operated for mitral stenosis, closed mitral commissurotomy. The time of CPB was $145.1 \pm 62.3 \mathrm{~min}$, the time of $\mathrm{CP}$ was $105.9 \pm 31.9 \mathrm{~min}$ at a temperature of $31.8 \pm 1.6^{\circ}$. It should be noted that in $82 \%$ ( 87 patients) preserved all or part of the posterior flap of the mitral valve. Results: of 106 operated, in-hospital patients died was 5 (4.7\%). The main cause of death was acute heart failure (AHF), which was observed in 3 patients, and was $60 \%$ mortality in the structure. Among other reasons for an adverse outcome marked acute renal and hepatic failure (1), acute cerebrovascular insufficiency (1). In the structure of non-fatal complications (18.8\%) dominated: heart failure - 12 cases (1 1.3\%); hypoxic encephalopathy - 3 (2, 8\%); ventricular systole and extras - 4 (3.8\%); Complete atrioventricular block, require implantation of permanent pacemaker $1(0.9 \%)$ case.

Conclusions: Currently, with the improvement of diagnosis, surgical treatment and surgery and early postoperative period may be difficult to conduct the correction of defects multivalve with sufficiently good results. According to our research: Postoperative hospital mortality at three-valvular heart disease was $4.7 \%$ and was the main cause of mortality acute heart failure. In the postoperative period in $18.8 \%$ of patients observed nonlethal complications.

P159

Surgical correction of multiple valves complicated by thrombosis

Khamidulla Abdumadjidov, Khayrulla Buranov, Ikrom Mirhodjaev,

Ilkhom Khodjakulov

Republican Scientific Center of Surgary named after V.Vakhidov,

Tashkent, Uzbekistan

Correspondence: Khamidulla Abdumadjidov

Journal of Cardiothoracic Surgery 2017, 12(Suppl 1):P159

Objective: Modern cardiac surgery can successfully perform correction of multi-valvular heart disease with thrombosis, with good immediate and long-term results. The choice of tactics for surgical correction of this category of patients is important.

Methods: 137 patients operated during the last 5 years by CPB and $\mathrm{CP}$, with three-valve defects, patients were complicated by thrombosis $36(26.3 \%)$. The age of patients ranged from 12 to 74 years $(36.7+9.4)$. The women were $-67.4 \%$ and men $-32.6 \%$, in the diagnosis perform ECHO, CT scan, for patient over 40 years old - coronary angiography, and intraoperative transesophageal echocardiography. Results: In patients performed mitral-aortic prosthesis with tricuspid plastics by deVega was $51.6 \%$, MV prosthesis with aortic and tricuspid valves was $17.6 \%$, aortic prosthesis with mitral and tricuspid valve plastics $13.4 \%$, and also the plastic of the three valves (mitral, aortic, tricuspid) - in $18.3 \%$. Mortality was $7.4 \%$. In our practice, in the overwhelming majority of cases (up to $83 \%$ ), we managed to partially or 
completely save the chordopapillary system of the posterior flap of the mitral valve. Lethality analysis confirms that in most (more than $50 \%$ ) cases, the cause of the adverse outcome was acute heart failure. Against the background of heart failure, hepatic-renal failure developed in $25 \%$ of cases, in $18 \%$ - the cause of death was an acute disorder of cerebral circulation, in the remaining $17 \%$ - sepsis.

Conclusions: When performing a three-valve correction complicated by atrial thrombosis, it is necessary to perform thrombectomy from left atrium with plastics of left atrium. If possible, preference should be given to valve-preserving reconstructive surgery on the heart valves, which we performed in $18.3 \%$ of cases. In order to prevent acute heart failure, it is necessary to preserve the chordopapillary system of the posterior flip of mitral valve.

\section{P160}

Surgical treatment of ischemic mitral regurgitation

Alexander Vavilov, Pavel Kakhktsyan, Irma Tsiskaridze, Inessa Slivneva, Mikhail Latyshev, Ivan Scopin

Bakulev NSPCCVS, Moscow, Russian Federation

Correspondence: Alexander Vavilov

Journal of Cardiothoracic Surgery 2017, 12(Suppl 1):P160

Objective: The analysis of immediate and mid-term results of surgical treatment in patients with CAD and concomitant MR of an ischemic genesis.

Methods: Research included 60 patients with CAD aged from 49 to 74 years operated from 2010 to 2016. Most part of patients had a dilatation of left ventricle (LVEDVI - 100.1 $\pm 27.0 \mathrm{ml} / \mathrm{m}^{2}$, LVESVI -49.2 $\pm 18.3 \mathrm{ml} / \mathrm{m}^{2}$ ), 12 from them had LVEDD more than $6.5 \mathrm{~cm}$. MV annulodilatation made $40 \pm 4.3 \mathrm{~mm}$. 22 patients had symptoms of LV systolic dysfunction (EF $<50 \%$ ) against a significant MR. 54 patients had the severe MR. The accompanying pulmonary hypertension was taped in 47 cases, 13 from them had the high degree. 40 patients had previously MI. In all cases, CABG and MV repair was performed.

Results: In 55 cases, with the aim of over-correction was implanted semi-rigid annuloplasty ring 26 to 32 size. After surgery, regurgitation of MV was minimal ( $n=47)$, in 12 patients it was reduced to 1 degree. Hospital mortality rate was $6 \%(n=4)$.

Conclusions: IMR can be primary (type II Carpentier) and secondary (type IIIb). True IMR is considered as a chronic process resulting from myocardial damage is directly dependent on LV dysfunction and remodeling. The type of reconstructive intervention on the MV and subvalvular structures in patients with IMR depends on the anatomy of all structures of the MV and functional status of the myocardium.

\section{P161}

Long-term results of plastic correction of mitral insufficiency with violating the integrity of chordal-papillary continuation

Volodymyr Popov, Oleksandr Bolshak, Volodymyr Mnishenko, Vasyl Lazorishinets

National Institute of cardio-vascular surgery named after Amosov, Kiev, Ukraine

Correspondence: Volodymyr Popov

Journal of Cardiothoracic Surgery 2017, 12(Suppl 1):P161

Purpose: analysis of long-term results of surgical treatment of $\mathrm{Ml}$ in violation integrity chordal-papillary apparatus using plastic methods of correction.

Material and methods. During the period from 01.01.2006 to 31.12.2015 years were operated 201 patients.The number of men were $144(71.6 \%)$, women - 57 (28.4\%). Supporting ring and semiring for mitral anuloplastic were used in 178 cases (88.5\%), suture anuloplastic used in 15 patients (7.5\%), 8 patients $(4.0 \%)$ anuloplastic not been applied. Formation of artificial chordae made in 21 cases (10.4\%), translocation chords - 4 (2.0\%).

Results. In the remote period studied the results of 192 patients (95.5\% of the total issued). Five patients (2.6\%) in the remote period reoperation done on $\mathrm{MV}$ (MV replacement $-4, \mathrm{MV}$ replastic -1$)$, 4 of them - about significant $\mathrm{Ml}$, in 1 - on infective endocarditis operated $\mathrm{MV}$. The main reason the unsatisfactory results was MV dysfunction (81.2\%) (high gradient associated with the formation of moderate stenosis, regurgitation of moderate or more). The causes of deaths were: sudden death - $33.3 \%$, progressive cardiovascular failure $22.2 \%$, infective endocarditis MV $-11.1 \%$ other $-33.3 \%$. Unsatisfactory results and fatalities in lesions received only the anterior leaflet in 7 cases out of $18(38.9 \%)$, only the posterior leaflet -33 of $178(18.5 \%)$, both leaflets -1 in $5(20.0 \%)(p<0.05)$.

Conclusions. This document presents the long-term results of 201 operation for $\mathrm{Ml}$ that have been made by plastic correction techniques for the period 2006-2015. In the long term results were studied $95.5 \%$ of patients were discharged. Unsatisfied results were often caused by dysfunction of the MV. The causes of deaths, mainly by progressive cardiovascular failure and sudden death. In the remote period marked only 1 of thromboembolic complications, which have not resulted in a fatal outcome.

P162

Results of surgical mitral valve repairs in progressive its insufficiency

Seitkhan Joshibyev, Berik Bolatbekov, Almaz Begdildayev, Baglan

Turtabayev, Auez Jakelov, Aziz Azkhojayev

Research Clinical Center of Cardiosergery and Transplantology, Taraz, Kazakhstan

Correspondence: Berik Bolatbekov

Journal of Cardiothoracic Surgery 2017, 12(Suppl 1):P162

Objective: To estimate results of a surgical mitral valve repairs by type of technique.

Methods: There were analyzed 51 patients after mitral valve insufficiency repairs. Average age were $55.8 \pm 7$ years. The mitral valve repair was performed: 1) suture annuloplasty technique at $33(64 \%)$ cases; from them 1A) on the basis of posterior cusp with using neochordas in 16(31\%), on the basis of posterior cusp with a segmentary resection of the cusp in 10 (19\%) pts, and 1B) commissural with neochordas 7 (14\%). 2) annuloplasty with usage of a rigid rings $28(36 \%)$ cases; from them with neochordas 11 (22\%) cases.

Results: Dynamics of the end-diastolic size and ejection fraction of left ventricle after (1) suture annuloplasty mitral repair were: before $59.1 \pm 7.8 \mathrm{~cm}$ and $47 \pm 4.3 \%$ respectively, after operation: $53.7 \pm 6.5$ $\mathrm{mm}$ and $51 \pm 5 \%$, in 1 year of $54 \pm 10 \mathrm{~mm}$ and $50 \pm 7 \%$, in 2 years of $58.7 \pm 9.1 \mathrm{~mm}$ and $44 \pm 7 \%$, in 3 years of $64.3 \pm 7.4 \mathrm{~mm}$ and $41 \pm 5 \%$. Whereas data's after (2) annuloplasty with usage of a rigid rings were: before $61.2 \pm 7.4 \mathrm{~mm}$ and $51 \pm 7 \%$, after operation: $57.4 \pm 5.4 \mathrm{~mm}$ and $51 \pm 8 \%$, in 1 year of $51 \pm 5,7 \mathrm{~mm}$ and $56 \pm 4 \%$, in 2 years of $52.3 \pm 3.2$ $\mathrm{mm}$ and $61 \pm 5 \%$, in 3 years of $55 \pm 2,1 \mathrm{~mm}$ and $64 \pm 8 \%$.

$6(12 \%)$ patients after suture annuloplasty needed a reoperation with a further prosthesis of the mitral valve, and only for 1 patient after an annuloplasty with using of a rigid ring. There was no in-hospital mortality. Long-term mortality was $2(4 \%)$ patients. One died in 1.5 years after suture annuloplasty due to ischemic stroke; the second in 2 years after operation of an annuloplasty with using of a rigid ring, but the cause is unknown.

Conclusions: Mitral valve annuloplasty with using rigid ring is safer for patients and is more preferable.

P163

Comparison of long-term results of methods of surgical correction of mitral insufficiency with violating the integrity of chordal-papillary continuation

Volodymyr Popov, Oleksandr Bolshak, Volodymyr Mnishenko, Elena Trembovetskaya, Elena Horoshkovataya, Anatoliy Rudenko National Institute of cardio-vascular surgery named after Amosov, Kiev, Ukraine

Correspondence: Volodymyr Popov

Journal of Cardiothoracic Surgery 2017, 12(Suppl 1):P163 
Purpose. Analysis of long-term results of surgical correction of mitral insufficiency with violation of the integrity chordal-papillary apparatus using different methods of correction.

Material and methods. All patients were divided into two groups: with mitral valve repair (group A - 201 patients); with mitral valve replacement (MVR) (group B - 319 patients) from period 01.01.2006 to 31.12 .2015 . Total men were $367(70.6 \%)(144-71.6 \%$ in group A and $223-69.9 \%$ in group B $(P>0.05))$ women - $153(29.4 \%)(57-28.4$ $\%$ in group $A$ and $96-30.1 \%$ in group $B(p>0.05)$ ). In addition to correcting mitral insufficiency, patients in both groups of observations related surgical procedures performed, $136(67.7 \%)$ patients in group A and $171(53.6 \%)$ in group B ( $<<0.05)$.

Results. In the late period studied the results of 487 patients $(93.8 \%$ of the total issued). In group A - 192 patients (95.5\% of all issued) in group B - 295 (92.8\%). Good results were obtained in $24.0 \%$ of patients in group $A$ and $24.7 \%$ in group $B(P>0.05)$, satisfied $-55.2 \%$ and $56.3 \%$ respectively ( $p>0.05$ ), poor $-16,6 \%$ and $8.1 \%$, respectively $(p<0.05)$, died $-4.7 \%$ and $10.8 \%$, respectively $(p<0.05)$. The prevalent cause of deaths in group $B$ were thromboembolic complications $(40.6 \%)$, in group A in $33.3 \%$ of cases of sudden death was observed.

Conclusions. Non satisfied results among patients of group A were due mainly dysfunction of the mitral valve, in group $B$ thromboembolic complications due to the disturbances and difficulties anticoagulation monitoring, and advanced cardiovascular failure. The causes of deaths in the remote period in patients with MVR often have been thromboembolic complications and progressive cardiovascular failure. Patients with plastic interventions on the mitral valve causes deaths, mainly by progressive cardiovascular failure and sudden death.

\section{P164}

The use of photodynamic therapy in the palliative treatment in stenotic central lung cancer

Rudolf Gatyatov

Chelyabinsk Regional Clinical Oncology Center, Chelyabinsk, Russian Federation

Journal of Cardiothoracic Surgery 2017, 12(Suppl 1):P164

The objective of the research was to investigate the effectiveness photodynamic therapy (PDT) stenotic central lung cancer.

Methods. The study included 122 patients with with central lung cancer. Within the framework of the present study evaluated the effect of photodynamic (PDT) therapy on median survival in patients with central lung cancer. The study included 41 patients with central squamous cell lung cancer stage IV, not previously treated special methods of tumor treatment. Patients were divided into two groups: group 1 received photodynamic therapy with palliative chemotherapy, the standard scheme, 2 receiving only palliative chemotherapy. PDT was performed before the start of chemotherapy with photosensitizers chlorine of "Photolon" and "Radachlorin".

Results. More than $80 \%$ of the patients with central lung cancer noted a decrease or disappearance of previously presented complaints: dyspnea, cough, hemoptysis; $16 \%$ - did not find any changes after PDT. X-ray and endoscopic control showed positive dynamics in the form of recanalization of the lumen of the bronchi, atelectasis and reduced ventilation abnormalities in $72 \%$ of patients with central lung cancer. Vital capacity (VC) increased by $13.35 \%$, forced expiration volume in 1 second (FEV1) - by $18.4 \%$. The median survival in group 1 was 13.31 months in group $2-8.09$ months $(p=$ 0.038). The toxicity of the treatment in patients of both groups did not differ.

Conclusions: Thus, PDT is effective in the treatment of malignant stenosing tumors of bronchi. The method does not involve significant technical difficulties, has expressed palliative efficient, and safe.
P165

Selected images of diaphragm and intercostal muscle using 3D-CT

Abulaiti Abudurezake, Terumasa Morita, Takuya Mori, Atsushi Amano

Juntendo University, Tokyo, Japan

Correspondence: Abulaiti Abudurezake

Journal of Cardiothoracic Surgery 2017, 12(Suppl 1):P165

Background - Mechanical ventilation with a mechanical ventilator (MV) is indispensable for surgery performed under general anesthesia. In animal experiments, atrophy was induced in the diaphragm's muscle fibers as persistent passive ventilation of the diaphragm by MV lasted over 18 hours. Evaluation of the diaphragm and intercostal muscles is done only by measuring the muscle thickness using ultrasonic examination. This time, 3D-CT was used for the first time in the world to accurately evaluate the morphology and volume of the diaphragm and intercostal muscle in the world. Methods - CT imaging of the entire chest is performed using multidetector $\mathrm{CT}$, the diaphragm and the intercostal muscle are selectively extracted and reconstructed using a work station manufactured by PixSpace, and the volume is measured.

Results - 1) The irregular and membrane-like diaphragm targeted for selective extraction is from the other surrounding tissues, and the intercostal muscles which are thin membrane like muscles are ribs with internal and external intercostal muscles as muscle groups. It was possible to separate from surrounding muscle.

2) The volumes obtained from selected reconstructed images were $199.0 \mathrm{ml}$ in the diaphragm and $434.4 \mathrm{ml}$ in the right intercostal muscle. Conclusions - 1) The anatomical examination of the diaphragm and the intercostal muscle is mostly in the autopsy case, there has not been reported in the living body using the 3D-CT yet, the measurement of the volume of the diaphragm and the intercostal muscle is considered to be the world first.

2) The measured value is a reference value, but clinical evaluation of respiratory muscles becomes possible using the data of chest CT.

3) Although respiratory muscles are directly impaired by intercostal thoracotomy or diaphragm incision in coronary artery bypass surgery, it can be used to study the influence and countermeasure.

P166

The role of 64slice cardiac computed tomography in diagnostics of Transposition of the Great Arteries

Farkhat Bayembayev, Dautov Tairkhan, Duysenbayeva Bakhit, Yelshibayeva El'mira, Kondibayev Maxat, Zhampiisova Azhar

National Research Center for Cardiac Surgery, Astana, Kazakhstan

Correspondence: Farkhat Bayembayev

Journal of Cardiothoracic Surgery 2017, 12(Suppl 1):P166

Objective: The ability of 64slice cardiac computed tomography(CT) of the diagnostics of the Transposition of Great Arteries(TGA) was evaluated. In this observational study we aimed to identify the sensitivity and specificity of CT as compared to echocardiography $(\mathrm{ECHO})$ and invasive cardiac catheterization.

Methods: The retrospective study was performed at National Research Center for Cardiac Surgery in Astana,Kazakhstan, within October 2011-December 2016. 1280 patients with congenital heart disease underwent CTA, ninety-nine among them expected to have TGA (age 0-30). Cardiac CT was performed using the 64slice scanner without prospective ECGsynchronization. Some patients experienced invasive cardiac angiography. The potential of CT to detect and distinguish both types was analyzed by performing and comparing different studies in sequence. Gender ratio presents predominance of $54(60 \%)$ males over $36(40 \%)$ females.

Results: MSCT correctly detected TGA in 90 patients $(90.9 \%)$ out of 99 in whom surgeon or invasive cardiac catheterization had identified 
TGA, with a sensitivity, specificity and negative predictive value of $100 \%$. 9 cases of false negative results were received, which were confirmed on ECHO subsequently. Results of $\mathrm{CT}$ and $\mathrm{ECHO}$ matched in $85(95 \%)$ patients. 68 patients $(76 \%)$ had DTGA and $22(24 \%)$ patients had LTGA only. The majority of patients have been identified under the age of 1 month. 83(92\%) patients from 90 were operated, but in 17(18\%) from them died. In all 90 patients TGA combined with different $C H D$, such as: Patent ductus arteriosus(PDA) in 52 patients(57.8\%); Ventricular Septal Defect(VSD) in 39 patients(43.3\%); Atrial Septal Defect(ASD) in 35(38.8\%); Single ventricle(SV) in 26(28.8\%); Patent foramen ovale(PFO) in 24(26.6\%); Atrioventricular septal defect(AVSD) in 12(13.3\%); Single atrium(SA) in 7(7.7\%); Anomalous pulmonary venous drainage(APVD) in 7(10\%); Interrupted aortic arch(IAA) in 3 cases(3.3\%).

Conclusions: 64slice Cardiac CT showed high level in diagnostics TGA, with sensitivity of $93.75 \%$ and specificity of $99.34 \%$. CT also provides detailed information on the types of TGA and combined CHD.

\section{P167}

The diagnostic possibilities of CT angiography in the diagnosis of total anomalous pulmonary venous connection

Umissalima Khassanova, Tairkhan Dautov, Alena Em, Aigerim Suigembayeva

National Research Center for Cardiac Surgery, Astana, Kazakhstan

Correspondence: Umissalima Khassanova

Journal of Cardiothoracic Surgery 2017, 12(Suppl 1):P167

Aim: To study the possibilities of $C T$ angiography in the diagnosis of total anomalous pulmonary venous connection (TAPVC).

Materials and methods: The study was conducted in period from October 2011 to December 2016 and included 1280 patients expected to have congenital heart disease(CHD), in 53(4.14\%) from them total anomalous pulmonary venous connection were identified. $\mathrm{CT}$ angiography in infants were performed with medical sedation.

Results: We analyzed the results of examination in 53 patients with total anomalous pulmonary venous connection, in 30(56.6\%) patients a supracardial form(type I) were revealed, in $11(20.8 \%)$ patients an intracardial form(type II), in 7(13.2\%) patients an infracardial form (type III), and in 5(9.4\%) patients a mixed form (type IV) were founded.

TAPVC was diagnosed in 29(54.72\%) patients who are under 1 month, in $17(32.07 \%)$ patients from 1 to 6 months, in 5(9.43\%) patients from 6 months to 1 year, in 2(3.77\%) patients older than 1 year.

In our cases, TAPVC was combined with: ASD in 32(60.37\%), VSD 14(26.41\%), single ventricle - 12(22.64\%) and AVSD - 13(24.52\%) patients. In 17(32.07\%) patients with total anomalous pulmonary venous connection, dilatation of the right heart and pulmonary hypertension developed in $5(9.43 \%)$ patients.

The results of echocardiography matched with $\mathrm{CT}$ angiography only in 36 patients(67.92\%). 45(84.9\%) patients from 53 were operated, with lethal outcome in 19(35.84\%) cases.

CT angiography was characterized with high informatively parameters in the diagnosis of TAPVC: sensitivity - $95.1 \%$, specificity $99.8 \%$, accuracy $-99.7 \%$.

Conclusions: CT angiography is a highly informative and minimally invasive method of diagnosis of total anomalous pulmonary venous connection, which in some cases could completely substitute invasive techniques such as a cardiac catheterization and angiocardiography.

\section{P168}

Possibilities of computed tomography in the detection of complications after LVAD implantation in patients with heart failure III-IV degree

Azhar Zhampiissova, Dautov Tairkhan, Yelshibayeva El'mira, Duysenbayeva Bakhit, Kondibayev Maxat, Eskaliev Askar National Research Center for Cardiac Surgery, Astana, Kazakhstan Correspondence: Azhar Zhampiissova

Journal of Cardiothoracic Surgery 2017, 12(Suppl 1):P168
Objective: Left ventricular assist devices (LVADs) have become an increasingly beneficial option for patients with heart failure, especially in light of the insufficient availability of donor hearts. LVADs have been used effectively in end-stage heart failure as a bridge to heart transplantation, as destination therapy for those ineligible for transplantation. However LVAD has some specific complications. We aimed to analyze the CT findings of complications in patients after LVAD transplantation.

Methods: The study was conducted within the period from November 2011 to December 2016, retrospective analysis of CT examinations of 218 patients after LVAD implantation was performed, 92 patients were identified with complications, mean age was 50,3 ; 196 patients of them were men (89.6\%), 22 women (10.3\%). Examinations were performed on MSCT «Somatom Definition AS 64», "Siemens», Germany, with a prospective ECG synchronization and slice thickness reconstruction of a $0.6 \mathrm{~mm}$. We used the standard patient packing lying on his back, with intravenous bolus of $4 \mathrm{ml} / \mathrm{s}$ using automatic CT Injector.

Results: We analyzed the results of 92 patients' examinations with various complications, of which $32(34.7 \%)$ patients showed ischemic type of stroke, in $18(19.6 \%)$ showed hemorrhagic type of stroke, 20 (21.7\%) pump thrombosis, $52(56.5 \%)$ patients with infection and 2 patients with sternum instability. Pump thrombosis: Heart Ware 7 (7.6\%), Heart Matell -12 (13\%), Heart matelll-1 (1\%). Infection: Heart Ware $12(13 \%)$, Heart Matell -38 (41.3\%); Heart matelll-2 (2.1\%). Ischemic type of stroke- Heart Ware 15 (16.3\%), Heart Matell -17 (18.5\%), Heart matell- 0 . Hemorrhagic type of stroke: Heart Ware 8 (8.7\%), Heart Matell -10 (10.9\%); Heart matelll-0.

Conclusions: Thus, MSCT is a highly informative and minimally invasive method of investigation which allows to identify complication after LVAD implantation such as stroke, infection, pump thrombosis.

P169

Magnetic resonance imaging in the diagnosis of hypertrophic cardiomyopathy

Zhaina Kassymova, Tairkhan Dautov, Elmira Elshibaeva, Askar Eskaliev,

Maksat Kondybayev, Azhar Zhampiisova

NNCS, Astana, Kazakhstan

Correspondence: Zhaina Kassymova

Journal of Cardiothoracic Surgery 2017, 12(Suppl 1):P169

Objective: The study of the possibility of magnetic resonance imaging in the diagnosis of hypertrophic cardiomyopathy.

Methods: The retrospective study was performed at National Cardiac Surgery Center in Astana, Kazakhstan, within October 2011 December 2016. 74 patients with a diagnosis of cardiomyopathy (CM) were examined, and in 41 (55.45\%) patients hypertrophic cardiomyopathy (HCM) was detected, often in females $23(56.1 \%)$, male sex was 18 (43.9\%). The studies were performed on a magnetic resonance tomograph "Magnetom Avanto" of 1.5T, SIEMENS. Subsequent postprocessing handling of the received data was carried out on the workstations "Multi Modality" using special programs for image processing.

Results: We analyzed the results of an MRI examination of the heart in patients with hypertrophic cardiomyopathy, 23 of them $(56.1 \%)$ had an obstructive form, $18(43.9 \%)$ had a non-obstructive form. $\mathrm{HCM}$ was diagnosed in $22(53.65 \%)$ patients until the age of 25 years and in $19(46.35 \%)$ patients from 25 years and above. In our observations, HCM was combined with: mitral valve insufficiency in 11 (26.82\%) patients, aortic valve insufficiency - $5(12.18 \%)$ patients, tricuspid valve insufficiency - 2 (4.87\%) patients, open oval window - 2 $(4.87 \%)$ patients, the defect of the interventricular septum was 1 $(2.43 \%)$ of the patient. Magnetic resonance imaging was characterized by high information content of parameters in the diagnosis of HCM: sensitivity $90 \%$, specificity $94.4 \%$, accuracy $-90.5 \%$.

Conclusions: Thus, MRI is a highly effective method for estimating and morphological and functional changes in hypertrophic cardiomyopathy. 
P170

What should be done in patients with carotid artery disease to be submitted to CABG?

Filipe T. K. S. Almeida, Daniel T. K. S. Almeida, Rui M. Almeida

Assis Gurgacz University, Cascavel, Brazil

Correspondence: Rui M. Almeida

Journal of Cardiothoracic Surgery 2017, 12(Suppl 1):P170

Background: Patients that have important coronary artery disease and indication to coronary artery bypass graft (CABG), often have important carotid stenosis.

The objective of this study is to find out if pre-operative carotid artery screening is important in patients who will undergo CABG.

Methods: Between January 2010 and March 2017, 532 patients were submitted to CABG, being 346 above 50 years. From this group 127 were submitted to carotid screening before surgery due to having more than 50 years, history of stroke, transient ischemic attack, carotid bruit or extensive vascular disease. The male sex was predominant in $62.20 \%$ and the mean age was 68 years. The patients were divided in three groups, according to the stenosis: mild or no $(\mathrm{G} 1)$, moderate (G2) or severe (G3). In patients with moderate lesions an analysis of the carotid plaque was performed to see if ulcerations were present and to what extent.

Results: Hundred and five patients (72.44\%) were in G1, 13 (10.23\%) in G2 and nine (7.09\%) in G3. All patients from G3 and seven from G2 were submitted to open (31.25\%) or endovascular surgery (68.75\%). Seven of the patients from the endovascular surgical group had CABG performed before, and all the others after treatment of the carotid lesions. There were no strokes in these patients. In the all-overall group of 127 , the stroke incidence of $3.94 \%$, none of them with unsolved sequel. This incidence was higher than that of the 532 patients

Conclusions: The authors conclude that is important to screen pre CABG, the carotid system and

to treat them according to their severity, in patients with a history of stroke, transient ischemic attack or carotid bruit and those with extensive vascular disease.

\section{P171}

The use of two internal thoracic artery as the gold standard of the coronary bypass surgery

Yuri Schneider, Aleksei Cherkes, Tsoi Viktor, Shilenko Pavel

Federal Center of High Medical Technologies Ministry of Health of the

Russian Federation, Kaliningrad, Russian Federation

Correspondence: Aleksei Cherkes

Journal of Cardiothoracic Surgery 2017, 12(Suppl 1):P171

Objective: CABG is the preferred method of myocardial revascularization in patients with multifocal coronary artery disease. Using the LITA and the GSV is still considered the gold standard for CABG. Most surgeons still don't want to use two internal thoracic artery because they believe this process is technically more difficult, time-consuming and associated with a higher risk of infectious complications.

Methods: Since 09.2012 in our clinic performed 1286 operations isolated CABG using two ITA. We made an analysis of the results of these operations on several criteria: patient's age, type of perfusion during surgery, the number of conduits, the volume of blood loss $(\mathrm{ml})$, hospital mortality, perioperative myocardial infarction, resternotomy about bleeding, the frequency of infectious complications from the sternum (minor infection, mediastinitis), the incidence of stroke.

Results: The average age of patients was 65.5 years. $541(42.1 \%)$ patients underwent OPCAB, $288(22.4 \%)$ patients underwent beatingheart on-pump grafting and 457 (35.5\%) patients - on-pump grafting and cardioplegia. The average number of grafts -2.9. In all groups was observed low incidence of myocardial infarction in the early postoperative period $(0.5 \%)$. The average volume of intraoperative blood loss was $470 \mathrm{ml}$. Infectious complications of the sternum was observed in $11.2 \%$. It was performed $23(1.8 \%)$ resternotomy. Stroke was observed in $2(0.16 \%)$ patients. There was $4(0.3 \%)$ lethal outcome. Within three years under the supervision there were 370 patients. Total mortality was $4.9 \%$. Freedom from angina and cardiac events $-82.3 \%$. Needed a redo-CABG was 0 . Need for cardiac interventions (PTCA or stent) was 11 (3.1\%).

Conclusions: Isolated CABG using 2 ITA immediately after surgery accompanied by low incidence of myocardial infarction, resternotomy about bleeding, infectious complications of the sternum, intraoperative blood loss, need for transfusion of blood components, neurological complications and good follow-up (3 years) results.

P172

Do triclosan-coated sutures reduce leg wound surgical site infections following coronary artery bypass grafting?

Roi Glam', Liran Shani', Bojan Biocina², Tomislav Kopjarr ${ }^{2}$, Kari Teittinen ${ }^{3}$, Keren Bitton-Worms', Gil Bolotin ${ }^{1}$

${ }^{1}$ Cardiac surgery, Rambam Health Care Campus and Faculty of Medicine, Technion-Israel Institute of Technology, Haifa, Israel; ${ }^{2}$ University of Zagreb School of Medicine, Department of Cardiac Surgery, University Hospital Center Zagreb, Zagreb, Croatia; ${ }^{3}$ Department of Vascular Surgery, Helsinki University Central Hospital, Helsinki, Finland

Correspondence: Roi Glam

Journal of Cardiothoracic Surgery 2017, 12(Suppl 1):P172

Objective: Surgical site infections (SSIs) account for 38\% of nosocomial infections among surgical patients. The incidence of infection at saphenous vein harvest site has been reported to range from $1 \%$ to $24 \%$. The suture knot, which has been hypothesized to be the main site of bacterial colonization in the wound, led to the development of triclosan-coated sutures which has been used as an effective antimicrobial agent for more than 3 decades. The purpose of this study was to determine whether or not Triclosan-coated sutures would reduce wound SSIs following saphenous vein harvesting. Methods: A prospective, randomized, double-blind, multi-center study. Patients underwent elective or urgent CABG in two major Centers (Israel and Croatia), were included in the study between 2011 and 2015. Pre-, Intra- and a post-operative assessment data were registered prospectively. All leg wounds were inspected by a cardiac surgeon daily, from post-operative day one until discharge and in the clinic approximately 15 and 45 days post-surgery. The wounds were evaluated according to the ASEPSIS score and the CDC criteria. The surgeon who inspected the wound was blinded to the group allocation.

Results: A total of 344 patients were randomized in this study. 315 Patients completed 45 days of follow-up. The overall incidence of SSI was $16.7 \%$ in the triclosan group and $17.6 \%$ in the control group, $\mathrm{P}=0.81$. There was no difference between the groups related to the use of antibiotics for leg wound SSIs, $17 \%$ in the triclosan group and $19 \%$ in the control group, $\mathrm{P}=0.67$.

Conclusions: Triclosan coated sutures were not found to reduce surgical site infections following open saphenous vein harvesting. Moreover, the study did not find any statistically significant advantage to using triclosan-coated sutures in the subgroups. These results contradict former studies which demonstrate benefit to triclosan-coated sutures in cardiac and general surgery.

\section{P173}

Short-term outcomes of off-pump coronary artery bypass grafting in patients with acute myocardial infarction with st-segment elevation

Daniil Borisov, Aleksandr Zotov, Aleksandr Troitskiy, Robert Khabazov, Sergei Vachev

Federal research Clinical center for specialized types of health care and medical technologies, Moscow, Russian Federation

Correspondence: Daniil Borisov

Journal of Cardiothoracic Surgery 2017, 12(Suppl 1):P173

Objective: This study aimed to compare the results of off-pump coronary artery bypass grafting (OPCABG) and on-pump coronary artery 
bypass (ONCAB) in patients with acute myocardial infarction with STSegment Elevation (STEMI).

\section{Methods:}

This was a retrospective analysis of prospectively gathered data of 148 patients with acute STEMI, treated with CABG in our hospital from January, 2013 to February, 2017. Patients were divided into two groups. The first group included $96(65 \%)$ patients receiving ONCAB. The second group included $52(35 \%)$ patients treated with OPCABG. The mean age for the first group was $57.9 \pm 8.3$ years and for the second group it was $59.4 \pm 7.4$ years. All patients underwent CABG due to multi-vessel coronary artery disease. 82 patients $(85 \%)$ in the first group and 37 patients (71\%) in the second group had triple vessel coronary artery disease. 24 (25\%) patients in the first group and $17(33 \%)$ patients in the second group had left main coronary artery disease. Mean EuroSCORE for the first group was $7.0 \pm 2.7$ versus 6.8 \pm 2.6 for the second group.

Results:

The mean number of distal anastomoses was $3.2 \pm 0.8$ in the ONCAB group and for the OPCABG group it was $2.6 \pm 0.8(p<0.001)$. The mean operating time was $4.1 \pm 0.7$ hours for ONCAB and $3.1 \pm 0.6$ hours for OPGABG $(p<0.001)$. In the first group, three patients $(3.1 \%)$ died owing to a myocardial infarction, one patient $(1 \%)$ died owing to a multiple organ dysfunction syndrome. In the second group, one patient (1.9\%) died owing to a myocardial infarction. The mean duration of mechanical ventilation after surgery and length of stay in the intensive care unit were significantly shorter in the second group $(p=0.03$, and $p=0.02$, respectively). The postoperative blood loss was significantly lower in the OPCABG group than in the ONCAB group $(p<0.01)$.

Conclusions: OPCABG in patients with STEMI can be performed with good results.

\section{P174}

Intramyocardial implantation of autologous bone marrow cells treated with erythropoietin as an additional method of myocardial revascularization (6-month results)

Alexey Fomichev, Alexander Chernyavskiy, Julia Kareva, Alexandra

Tarkova, Olga Poveschenko, Nikita Nikitin, Stanislav Minin

Siberian Biomedical Research Center Ministry of Health Russian

Federation, Novosibirsk, Russian Federation

Correspondence: Alexey Fomichev

Journal of Cardiothoracic Surgery 2017, 12(Suppl 1):P174

Objective: According to a series of studies bone marrow cells (BMC) therapy during ischemic heart disease (IHD) surgery has shown mixed results. BMC preconditioning using different growth factors becomes the promising direction of cell therapy. Experiments with laboratory animals have shown that the erythropoietin using leads to the myocardial ischemia zone restriction and facilitates the neoangiogenesis.

Methods:

The half-yearly results of erythropoietin treated BMC implantation in cases of the distal coronary lesions (30 patient - BMC-group) were estimated. The control group consists of 30 patients with diffuse and distal coronary disease (artery with distal lesion was not shunted). In addition, the phenotype and functional properties of implanted cells were estimated by the flow cytofluometry.

Results: According to perfusion scintigraphy, the stable perfusion defect (SPD) before surgery in BMC group was $9.1 \% \pm 2.7 \%$, and $7.4 \% \pm 1.9 \%$ after six months ( $p=0.047)$, SPD in the control group decreased from $8.8 \pm 1.7 \%$ to $7.9 \pm 1.7 \%(p=0.07)$. However, there is a moderate improvement of the left ventricle functional parameters, more significant in the main group. Left ventricle end diastolic volume (LVEDV) decreased from $114 \pm 38$ preoperatively to $98 \pm$ $34 \mathrm{ml}$ after six months $(p=0.048)$, left ventricular ejection fraction (LVEF) increased from $55 \% \pm 17$ to $61 \pm 25 \%$ ( $p=0.041)$. According to the flow cytometry BMC with erythropoietin increases CD34 + pool of cells at different stages of differentiation, initiates cell retention in the phase of rest and initial growth, decreases the number of cells at the early stage of apoptosis and reduces the proliferative capacity of the cells.
Conclusions: Preparation of autologous bone marrow cells by erythropoietin treatment improves BMC functional properties. Intramyocardial implantation of BMC treated with erythropoietin improves myocardial perfusion and function in the affected area.

\section{Acknowledgements}

This work was supported by a Russian Scientific Foundation grant (project number 16-15-00057).

P175

The possibilities of using two internal thoracic arteries to achieve complete autoarterial myocardial revascularization

Armen Martirosyan, Igor Zhbanov, Vadim Uryuzhnikov, Irakli Kiladze, Nariman Galimov, Georgy Revishvili, Boris Shabalkin

Petrovsky Russian Research Center of Surgery (Moscow), Moscow, Russian Federation

Correspondence: Armen Martirosyan

Journal of Cardiothoracic Surgery 2017, 12(Suppl 1):P175

Objectives: to present and analyze our results of multiple coronary bypass grafting using both internal mammary arteries.

Methods. Total number of 759 patients (2009-2016) underwent bilateral mammary artery coronary bypass grafting. Mean age of the patients was $58.5 \pm 8.5(36-81)$, there were $635(83.7 \%)$ male patients. ECG Q and QS waves were detected in 463(61.3\%) cases, mean LVEF was $44.6 \pm 7.1 \%$.

Results. The interventions were performed with CPB and pharmacological cardioplegia in 91 (12.9\%) patients, on beating heart and CPB - in 288 (38.1\%) and off-pump - in 380 (49\%) cases. In most of the cases $(622(82 \%))$ both internal mammary arteries were used as in situ grafts and only in $18 \%$ of cases a composite mammary artery bypass using a T-graft was performed. Revascularization index was $3.02 \pm 0.95$. And autarterial revascularization index using both IMA was $2.46 \pm 0.59$. There was difference observed in the autarterial revascularization index using T-graft compared with in situ method $(2.6 \pm 0.6$ versus $2.4 \pm 0.5(p=0.016))$. In $432(57.2 \%)$ cases additionally we used autovenous graft, in $39(5.8 \%)$ - radial artery graft. The number of autovenous grafts was higher (354(57\%) vs $56(41 \%)$, $\mathrm{p}=0.01$ in cases when IMA in situ was used. Sequential bypass with mammary artery was performed in $311(41.2 \%)$ patients. In-hospital mortality and perioperative $\mathrm{MI}$ rate was $0 \%$. CHF requiring dopamine $>5 \mathrm{mcg} / \mathrm{kg} / \mathrm{min}$ was observed in $5.8 \%$ of patients, there was no case of IABP, resternotomy for bleeding - in $2.2 \%$ of cases.

Conclusion. Bilateral mammary artery multiple coronary bypass grafting is a modern and safe method of myocardial revascularization. It does not increase the risk of perioperative complications and should be considered the method of choice in the majority of patients with ischemic heart disease, undergoing surgical treatment.

P176

Surgical revascularization in acute coronary syndrome

Milenko I. Rosic, Aleksandar Redžek, Živojin Jonjev, Bogdan Okiljević, Aleksandar M. Milosavljevic

Institute of Cardiovascular Diseases, Sremska Kamenica, Sremska Kamenica, Serbia

Correspondence: Milenko I. Rosic

Journal of Cardiothoracic Surgery 2017, 12(Suppl 1):P176

Introduction: Patients in acute coronary syndrome represent a higher risk group for surgical intervention, and there is still controversy among cardiac surgeons about the time of their surgical treatment.

Objective: The objective of this work is to determine the postoperative mortality in patients suffering from acute coronary syndrome who were operated in our Clinic and to evaluate all the complications that lead to it.

Methods: At the Clinic for Cardiovascular Surgery in Sremska Kamenica, in the period from 2013 to 2016, a total of 2,175 patients with coronary artery disease were operated. Of the total number of operated patients, 335 patients were in acute coronary syndrome. All 
patients operated in acute coronary syndrome were divided into three groups. The first group (patients with unstable angina), the second group (NSTEMI) and the third group (STEMI) patients. The main parameter that was followed was a thirty-day postoperative mortality. Other parameters of interest were analyzed as well: duration of mechanical ventilation, intra-aortic balloon pump support, kidney failure (dialysis), stroke, revisions due to bleeding, the length of stay in the intensive care unit, the length of hospital stay and cardiopulmonary resuscitation.

Results: Of the total number of operated patients $(\mathrm{N}=335)$ in acute coronary syndrome, 194 patients were with unstable angina (58\%), 83 patients with NSTEMI (24\%) and 58 patients with STEMI (17.3\%). Post-operative mortality in groups: the first group $\mathrm{N}=4$ patients (2\%), the second group $\mathrm{N}=4$ patients $(4.82 \%)$ and the third group $\mathrm{N}=2$ patients (3.45\%). A total 30 -day postoperative mortality is $2.9 \%$.

Conclusion: Surgical revascularization in patients suffering from acute coronary syndrome, can be carried out smoothly with very good results and should not be avoided.

\section{P177}

Short-term outcomes of off-pump coronary artery bypass grafting in patients aged over 70 years

Aleksandr Zotov, Aleksandr Troitskiy, Robert Khabazov, Sergei Vachev,

Daniil Borisov

Federal research Clinical center for specialized types of health care and medical technologies, Moscow, Russian Federation

Correspondence: Daniil Borisov

Journal of Cardiothoracic Surgery 2017, 12(Suppl 1):P177

Objective: This study aimed to examine the safety and applicability of off-pump coronary artery bypass grafting (OPCABG) compared with on-pump coronary artery bypass (ONCAB) in patients aged over 70 years.

Methods: Clinical data were reviewed for 152 patients with stable angina (class III and IV) receiving CABG at our center between January, 2013 and February, 2017. The patients were divided into two groups. The first group included 49 (32\%) patients receiving ONCAB at our center (ONCAB group). The second group included $103(68 \%)$ patients (OPCABG group). The mean age for the first group was $74.9 \pm 4.2$ years and for the second group it was $76.5 \pm 3.9$ years $(p=0.02)$. All patients underwent CABG due to multi-vessel coronary artery disease (SyntaxScore for ONCAB group was $35.1 \pm 0.9$ and for OPCABG group it was $34.2 \pm 1.8(\mathrm{p}=0.001))$. Patients with unstable angina were excluded from our study.

Results: The mean number of grafted vessels in the ONCAB group was $3.1 \pm 0.4$ and for the OPCABG group it was $2.9 \pm 0.7(p=0.07)$. The mean duration of mechanical ventilation after surgery was $8.9 \pm 4.6$ hours for the ONCAB group versus 5.9 \pm 2.1 hours for the OPCABG group $(p<0.0001)$. Patients of the first group had significantly higher need of inotropic support postoperatively than patients of the second group (16 $(33 \%)$ versus $3(6.8 \%), p<0.0001)$. The mean intensive care unit stay was $25.8 \pm 8.9$ hours in the first group and $16.1 \pm 5.6$ hours in the second group $(p<0.0001)$. The mean hospital stay was significantly higher in the first group $(14.4 \pm 2.9$ days versus $9.2 \pm 2.1$ days, $p<0.0001)$. Need for blood transfusion was also significantly higher in the ONCAB group (28 $(57 \%)$ versus $21(20.4 \%), p<0.0001)$. During the study one patient (ONCAB group) died due to multiple organ failure.

Conclusions: OPCABG is safe and effective procedure in elderly patients with good short-term outcomes.

\section{P178}

Efficacy and safety of surgical treatment of coronary artery disease in combination with carotid endarterectomy

Vadim Uryuzhnikov, Sr., Igor Zhbanov, Irakli Kiladze, Armen Martirosyan, Georgy Revishvili, Boris Shabalkin

Petrovsky National Research Center of Surgery, Moscow, Russian Federation

Correspondence: Vadim Uryuzhnikov, Sr.

Journal of Cardiothoracic Surgery 2017, 12(Suppl 1):P178
Purpose. To assess the results of one-stage surgical treatment of patients with CAD with concomitant carotid arteries (CA) lesion.

Methods. In the department of CAD surgery 60 patients underwent carotid endarterectomy (CEA) simultaneously with CABG and valvular heart disease correction (11/18.3\%). Men-46 (76.7\%) patients, women-14 (23.3\%) patients, the average age was $63.3 \pm 15$ years. 30 (50.0\%) patients had bilateral, hemodynamically significant CA lesions, $20(33 \%)$ had diffuse encephalopathy, 5 patients $(8.3 \%)$ had previously suffered acute cerebrovascular accident (CVA), $36(60.0 \%)$ patients suffered Q-positive MI. $16(26.7 \%)$ patients with lesion of the left coronary artery trunk, remaining 44 patients (73.3\%) - a threevessel lesion of the coronary bed. Left ventricular ejection fraction less than 0.4 detected in 11 (18.3\%) patients. On-Pump CABG performed to $23(43.3 \%)$ without aorta cross clamping and $13(21.6 \%)$ with cardioplegia; OPCAB performed to $24(40.0 \%)$ patients. Along with $C A B G$ and CEA mitral annuloplasty with a support ring were made to $8(13.3 \%)$ patients - aortic valve prosthesis (AVP) - to 3 (5.6\%) patients.

Results. Postoperative mortality was $0 \%$. There were no perioperative Ml. There wasn't a need for IABP. In 1 (1.6\%) patient after CABG and AVP developed a persistent third-degree atrioventricular block, which required the implantation of a dual-chamber pacemaker, $1(1.6 \%)$ patient - diffuse encephalopathy as psychosis, 1 (1.6\%) patient gastrointestinal bleeding. The ICU time was $1.2 \pm 0.5$ days, hospital time $-15.9 \pm 2.9$ days.

Conclusions. One-stage operation of CABG and CEA is a safe and effective surgical intervention in cases with CAD with concomitant $C A$ lesion. By virtue of this, the performance of such operation is possible and permissible in the cases of hemodynamically significant unilateral lesion and is absolutely indicated in the cases of bilateral lesion of the CA.

P179

Original 3D-geometric ring for mitral valve repair in patient with ischemic mitral regurgitation

Vadim Shumavets', Alexandr Shket', Ihar Andraloits', Irina Grinchuk',

Svetlana Kurganovich', Nalalja Uss', Andrey Moskalenko², Yuory

Ostrovski'

'Brlarus Centre Cardiology, Minsk, Belarus; ${ }^{2 B e l a r u s ~ T r a d e ~ " E l e c t r o n m a s h ", ~}$ Minsk, Belarus

Correspondence: Vadim Shumavets

Journal of Cardiothoracic Surgery 2017, 12(Suppl 1):P179

Objective: To assess the results of the new original 3D-adaptive ring "Plancor- $A$ " for the treatment of functional ischemic mitral regurgitation.

Methods: Mitral valve repair with original 3D-adaptive ring combined with CABG for treatment of ischemic MR was performed on 46 (8 females, 38 males) consecutive patients. Six cases were redo operation. Most of pts (73\%) were in the New York Heart Association (NYHA) class III-IV. Tricuspid valve repair (16 pts), atrial fibrillation ablation (18 pts), and left-ventricular (LV) reconstruction (2 pts) were performed concomitantly.

Results: One pts died. All survivors underwent clinical and echocardiographic follow-up at discharge and at 1 year. Mean hospital stay $11+/-2$ days. Following mitral annuloplasty, mitral regurgitation decreased from $3.2+/-0.4$ to $0.5+/-0.54$ ( $p$-value $<0.0001$ ). Only two pts had mild MR, and no one had moderate MR The Echo examination showed a mean MV area of 3.1+/-0.36 cm2 (range 2.3-4.1 $\mathrm{cm} 2$ ) and a mean transmitral diastolic gradient of $4.6+/-2.2 \mathrm{mmHg}$. Both LV end-diastolic and end-systolic volumes indexed significantly decreased with concomitant improvement of local contractility index. But LV systolic and diastolic dimentions, LV EF and systolic pulmonary artery pressure was unchanged $(p>0.05)$. Mitral valve tenting area, annulus area, septo-lateral distance at the level of A2-P2 and intercommissural annular distance decreased significantly ( $p$-value $<0.05$ ) after "Plancor-A"- 3D ring implantation.

Conclusion: The new "Plancor-A " 3D-adaptive ring is effective in relieving IMR in most of the pts. Recurrence rate of severe IMR in 1-year follow-up consist $4.6 \%$. These geometric changes after mitral 
valve annuloplasty combined with coronary revascularization were associated with the improvement in the NYHA class. Clinically significant mitral stenosis was not detected during post-op examination.

\section{P180}

Off-pump surgery in coronary endarterectomy patients

Kosmas Tsakiridis, Stamatis Arikas, Konstantina Triantafillopoulou, Andreas Bakas

St Luke's Hospital, Thessaloniki, Greece

Correspondence: Kosmas Tsakiridis

Journal of Cardiothoracic Surgery 2017, 12(Suppl 1):P180

Objective: Endarterectomy in coronary arteries sometimes is a devastating and unexpected fact when we perform Coronary Artery Bypass Graft. The aim of the presentation is to review our experience in endarterectomy performed without cardio-pulmonary bypass.

Methods: During the last five years, we have performed off-pump coronary endarterectomy on twelve patients. Left Anterior Descending (LAD) Endarterectomy was performed on seven patients, while one was performed in the branches of the Circumflex (Cx) artery and the rest four endarterectomies were performed on the Right Coronary Artery (RCA).

Results: There were no deaths and none of the procedures were converted to on-pump operations. All endarterectomies and by-pass grafts were performed on a beating heart. All patients were completely revascularized. Left Interior Thoracic Artery (LITA) was used on the LAD and the Saphenous Vain Grafts (SVG) were used for the rest (Cx and RCA). All patients received double anti-platelet therapy and they were checked for any symptom one year after the surgery. The follow-up was performed with clinical examination and stress-echo. None of the patients had signs of graft dysfunction. All of the patients NYHA and angina status was decreased significantly.

Conclusions: Endarterectomy in Off-pump Coronary Bypass Surgery can be achieved successfully. There is no need for conversion to onpump. The surgeon needs to be experienced in order for the patient to benefit the most by the use of complete revascularization in the off-pump technique.

\section{P181}

Single aortic cross-clamp against partial occluding aortic clamp

Assel Tleuova, Murat Raikhanov, Oraz Mukashev, Adil Dyurzhanov (PhD), Vitali Berezutski, Kamshat Kakimova, Daulet Zhakipbayev

Pavlodar Regional Cardiological Center, Pavlodar, Kazakhstan

Correspondence: Murat Raikhanov

Journal of Cardiothoracic Surgery 2017, 12(Suppl 1):P181

Objective: Analysis of immediate results of single aortic cross-clamp and partial occluding aortic clamp technigues during coronary artery bypass graft (CABG).

Methods: Neurological complications in CABG are associated with embolism of brain arteries. In most cases, embolism is associated with manipulation on the aorta. The application of distal and proximal anastomoses on a single cross-clamp of the aorta reduces the risk of embolism and neurologic complications in CABG. In the period from January 2016 to January 2017 were operated 452 patients with ischemic cardiac disease: 282 using single aortic crossclamp technique and 244 using partial occluding aortic clamp technique. The age range was 43 to 83 years. NYHA functional class was II-III. 75 was urgent and 377 was elective. 312 were men and 140 women.

Results: In the group with single aortic cross-clamp technique was not any complications but in the group with partial occluding aortic clamp technique mortality was 2 cases, and 3 patients from 244 had ischemic stroke. The cross clamp time was more, but the results of ECG and ECHO and postoperational period have no difference.

Conclusions: Performance of CABG on the single aortic cross-clamp significantly reduces the risk of development ischemic stroke and lethality in the early postoperative period.
P182

Comparative analysis of the effectiveness of complicated forms of ind after surgical treatment of LV aneurysm

Agzam Aldeshev, Yerbol Kudabayev, Zhanar Nurbay,

Galymzhan Sultanov

Scientific Research Institute of the Cardiology and Internal Diseases,

Almaty, Kazakhstan

Correspondence: Agzam Aldeshev

Journal of Cardiothoracic Surgery 2017, 12(Suppl 1):P182

Objective: Complex evaluation of the effectiveness of surgical methods for the treatment of IHD complicated by LV aneurysms in the early postoperative period.

Methods: In the Institute of the Cardiology from 2015 to 2017, 36 patients underwent surgery for IHD with post-infarction LV aneurysm. The male (97.2\%), against the female- (2.8\%). Mean LV EF in the preoperative period is $39.97 \%$. The following types of LV reconstruction were performed: CABG and plastic LV by V.Dor by in 18 patients $(50 \%)$, by D.Cooley in $3(8.3 \%)$, plastic LV in modification by L.Menicanty-15 (41.6\%). At revision of LV in 17 patients (47.22\%), thrombomasses were revealed. The average size is $5.1 \pm 2.3 \mathrm{~cm}$. The index of myocardial revascularization is 2.75 . The average duration of the operation is $235 \mathrm{~min}$. Average CPB time $143.61 \mathrm{~min}$, cross $96.72 \mathrm{~min}$. 1 mortality.

Results: as a result of performed operations, postoperative mean LV $\mathrm{EF}$ in the early postoperative period increased from $39.97 \%$ to $44.54 \%$ : by Cooley from $39.33 \%$ to $40.33 \%$, by Dor from $43 \%$ to $44.47 \%$, by Menicanty from $36.53 \%$ to $45.61 \%$. Mean LV EDV decreased from $199.16 \mathrm{ml}$ to $151.1 \mathrm{ml}$. Of them by the Dor method by $33.28 \mathrm{ml}$, by Cooley by $12.67 \mathrm{ml}$, by Menicanty by $72.44 \mathrm{ml}$. Average bed-day: for Cooley 11days, for Dor 9.72 days, for Menicanty 8.6days. The duration of the operation: by Cooley $230 \mathrm{~min}$, by Menicanty $219.4 \mathrm{~min}$, by Dor $214.4 \mathrm{~min}$. The mean IR time by Cooley is $122.66 \mathrm{~min}$, by Menicanty $138.46 \mathrm{~min}$, by Dor $151.38 \mathrm{~min}$. Time of cross: by Dor $102.5 \mathrm{~min}$, by Menicanty $93 \mathrm{~min}$, by Cooley $80.66 \mathrm{~min}$.

Conclusions: The method by Menicanty is more preferable, taking into account the best characteristics, which makes it possible to use this technique by choice, as proved and effective.

P183

Feasibility of OPCAB in high risk patients

Islam Sharipov, Rustam Yarbekov, Muslim Mustaev, Abdulla Ismatov, Sandjar Omonov, Tokhir Vakhidov

Republican Specialized Centre of Cardiology, Tashkent, Uzbekistan

Correspondence: Islam Sharipov

Journal of Cardiothoracic Surgery 2017, 12(Suppl 1):P183

Objective: Results of off-pump coronary artery bypass surgery (OPCAB) in low-risk patients are encouraging but little is known about feasibility of OPCAB in high-risk patients. The aim of study was to assess feasibility of OPCAB in high-risk patients with ischemic heart disease.

Methods: A comparative analysis of the results of OPCAB in 255 patients operated on at our centre from April 2015 to March 2017 was done. The patients were divided into two groups according to the additive EuroScore: group I, 102 high-risk patients (EuroScore $\geq 5$ ) (38.5\%) and group II, 153 low-risk patients (EuroScore < 5) (61.5\%). Group I patients had older mean age $(62.85 \pm 7.4$ vs $56.31 \pm 7.0$ yrs, $p<0.001)$, more female patients $(35.3 \%$ vs $7.4 \%, p=0.002)$, and LVEF $<45 \%(23.5 \%$ vs $9.3 \%, p=0.06)$. Mean addEuroScore in group I was $6.38 \pm 1.4$ (logEuroScore $7.1 \pm 4.7 \%$ ), while in group II patients addEuroScore was $2.17 \pm 1.3$ (logEuroScore $1.8 \pm 0.7 \%$ ), $p<0.001$.

Results: The mean quantity of grafts in patients of group I was 2.9 \pm 0.6 (range, 1 to 4 grafts) and $3.07 \pm 0.7$ in group II (1 to 5 grafts) $\mathrm{p}=0.316$. The mean duration of operations in group I and II was $266.3 \pm 10.9$ mins and $254.6 \pm 12.2$ mins, respectively $(p=0.625)$. Inotropic support was required intraoperatively and immediately after surgery in $51.5 \%$ of patients of group I and in $24.5 \%$ of patients of group II $(p=0.021)$. The mean ventilation time was in the group I 
was $6.9 \pm 4.5$ hours, while in group $\|-4.9 \pm 1.9$ hours $(p=0.01)$. The mean ICU stay was $28.2 \pm 4.1$ hours in group I and $21.3 \pm 2.1$ hours in group $\|(p=0.07)$. There was 1 mortality $(0.98 \%)$ in group I and 2 cases $(1.3 \%)$ in group II $(p=0.316)$.

Conclusions: Although more inotropic support, ventilation and longer ICU time were required in high-risk patients, the overall results showed no statistical difference between the groups. Hence, OPCAB is a feasible option in high-risk patients.

P184

Intracoronary use of levosimendan during surgical repair of acute coronary syndrome

Galymzhan N. Sarzhanov

Almaty City Heart Center, Almaty, Kazakhstan

Journal of Cardiothoracic Surgery 2017, 12(Suppl 1):P184

Objective: is to study the efficacy of intraoperative application of levosimendan in the surgical treatment of ACS.

The analysis of 22 emergency operations of coronary artery bypass grafting in acute coronary syndrome, performed in the Almaty City Heart Center for 12 months.

Methods: levosimendan was used in two ways: before operation as a preconditioning tool and combination: before operation and intraoperative intracoronary administration. Intracoronary administration was performed in two ways: into the aorta root (5 cases) and into the vein graft (3 cases) at a dose of $2.5 \mathrm{mg}$ of clear solution. Group 1(14 patients), group 2(8 patients), 18 male, 4 female.

10 patients were operated on-pump, 12 off-pump. In 9 cases, myocardial revascularization was performed using 2 graft, in 7 cases - 3 graft and in 6 cases 1 graft. Mammarocoronary anastomosis was used in all cases. On average, 2.04 arteria, were bypassed per each single operation.

Results: Surgery time was 3 hours, on average. Once in the ICU, all patients were extubated in the nearest three hours post-op, from cardiotonic support withdrew in the nearest post-op. A follow-up echocardiography, two days post-op, demonstrated a mean of $10 \%$ increase in LV ejection fraction in all patients. Recovery was uneventful in all patients and they were discharged on day 7 post-op.

Conclusions: Intracoronary bolus administration of levosimendan in isolated form and in combination with intravenous infusion of the drug gives comparable results with traditional use as preoperative preparation. The proposed method has a great advantage in cases of surgical treatment for vital indications caused by cardiogenic shock, allowing the use of the most active surgical strategy.

\section{P185}

Off pump coronary artery bypass grafting and outcomes

Hasan Uncu, Hacı Ali Ucak

Adana Numune Training and Research Hospital, Adana, Turkey

Correspondence: Hasan Uncu

Journal of Cardiothoracic Surgery 2017, 12(Suppl 1):P185

Objectives: In recent years, coronary bypass operations have also been increased which parallel to the increase of coronary artery disease. Although surgical applications are often performed under the cardiopulmonary bypass (CPB), nowadays off pump coronary artery bypass (OPCAB) technique is widely used primarily high risk patient with the increase in surgical skills for it. In this lecture, we submit to results of OPCAB cases in our clinic.

Methodology: In Adana Numune Education and Training Hospital, 832 patients that performed OPCAB between January 2004 and February 2016 were included to study. Patients with heavily calcified aorta and impaired renal function are evaluated primarly for OPCAB. While patients were selected, anatomic factors like nonintramyocardial LAD, absence of heavily calcified target coronary artery and diameter of coronary not less than $1.5 \mathrm{~mm}$ were taken into consideration. Patients that were not fulfilling these criteria were operated with the help of CPB so they were not included the study. 519 patients were male, 313 were female. Diabetes mellitus was detected in 203 patients, hypertension was 285, chronic obstructive pulmonary disease was 118 . Average of age was $58.9 \pm 10.5$.

Results: Average of length of stay in intensive care unit was $1.2 \pm 0.5$ and average of blood transfusion requirement was $1.3 \pm 1.0$. One vessel bypass was performed to 365 patients, two vessels bypass were 385 and triple bypass were 82 . In postoperative early period, 9 patients were died.

Conclusion: Mortality and morbidity rate of OPCAB procedure was lower than on pump operations. However applicability of procedure is required satisfactory surgical experience and appropriate coronary anatomy. Requirement of blood transfusion, hospital and intensive care unit stay decrease with OPCAB procedure for selected patient group. In our clinic, OPCAB procedure is performed low morbidity and mortality rates.

P186

Multivessal OPCAB through right anterior thoracotomy with single lung: a rare case scenario

Gaurav Goel, Abhishek N Raychura

Sterling Hospital, Vadodara, India

Correspondence: Gaurav Goel

Journal of Cardiothoracic Surgery 2017, 12(Suppl 1):P186

Objective: Case report - Multi vessel OPCAB through right anterior thoracotomy in patient with single lung physiology.

Methods: 68 years male, hypertensive, diabetic presented with ACS. CAG showed double vessel disease not amenable to PCI. CXR and CECT scan showed extreme shifting of mediastinum to right hemithorax with almost complete collapse/destruction of right lung, ascending aorta near right lateral chest wall. Off pump CABG was planned through right anterior thoracotomy under GA. Detailed preoperative evaluation, workup, PFT and baseline ABG were done. Apart from routine cardiac surgery monitors, disposable defibrillator pads were applied. GA was given in routine manner. Intubation done with armoured ET tube, with fibre optic bronchoscope kept ready in case of need for left single lung ventilation. CVC cannulation was right IJV under USG guidance. right groin was kept ready painted and drepped for emergency CPB. Right anterior thoracotomy was done through 4th intercostal space with excision of anterior 5th rib. RIMA harvesting tried, but could not be completed due to fibrosis and adhesions in upper part. saphenous venous grafts to distal LAD and early PDA were done Off pump and proximal anastomosis were done to aorta. patient was shifted to CSICU and extubated after overnight ventilation. Postoperative analgesia was through continuous iv infusion of fentanyl and paracetamol intermittently. Results: Multi vessel OPCAB was done successfully through unconventional right anterior thoracotomy. Patient was extubated on 1 st postoperative day and shifted to ward on 3rd day, discharged in stable condition on 6th day. Three month followup of patient is complete with patient remaining symptom free.

Conclusions: OPCAB through right anterior thoracotomy is a very rare case scenario. It was necessary in our case due to extreme non anatomical position of heart and great vessels on right side. It was done successfully with complete revascularisation with any complication.

Consent for publication

Written informed consent to publish was obtained from the patients involved in this study.

P187

Minimally invasive direct coronary artery bypass (MIDCAB) - safety assessment in own material

Jacek Piatek, Janusz Konstanty - Kalandyk, Anna Kędziora, Jerzy Sadowski, Bogusław Kapelak

John Paul II Hospital, Krakow, Poland

Correspondence: Jacek Piatek

Journal of Cardiothoracic Surgery 2017, 12(Suppl 1):P187

Objective: Introduction: Minimally invasive direct coronary artery bypass (MIDCAB) allows achieving similar safety and efficacy, with 
markedly reduced post-operative length of stay when compared to conventional surgical revascularization. Despite promising results, a small number of minimally invasive procedures are performed in Poland. The aim of the study is to assess short- and long-term outcome of MIDCAB revascularization in order to evaluate the safety of the procedure.

Methods: Retrospective observational study analyzing 38 consecutive patients who underwent MIDCAB procedure between 2014 to 2016 in the Department of Cardiovascular Surgery and Transplantology at the John Paul II Hospital, Kraków. Perioperative data was obtained from patient medical records and the median follow-up period valued 17.3 months.

Results:

No postoperative deaths and only 1 case of postoperative myocardial infarction were observed. Throughout the follow-up period, the survival rate and freedom from MACCE rate valued $100 \%$, with only 1 case of repeated revascularization.

Conclusions: Minimally invasive revascularization is a safe procedure which can be performed with excellent short- and long-term outcome in low-risk patients.

\section{P188}

Bimammarocoronary bypass on a beating heart

Adil Aitmukhanov, Alen Kuanyshbekov

Central clinical hospital JSC, Almaty, Kazakhstan

Correspondence: Alen Kuanyshbekov

Journal of Cardiothoracic Surgery 2017, 12(Suppl 1):P188

Purpose of the study: study of results bimammarocoronary bypass surgery on a beating heart.

Methods: the study included 141 patients operated for the period of 2014-2017. The mean age was 60.4 years old. Male patients prevailed and were $109(77 \%)$, female - $32(23 \%)$. Disease: Q-wave myocardial infarction - $19(13,4 \%)$, infarction without Q-wave - $20(14,1 \%)$, unstable angina - $14(10 \%)$, stable angina - $88(62.4 \%)$. The fraction of cardiac output before the surgery was $48.3 \%$. EUROSCORE II - 1.73 . During surgery, Medistim Ultrasound Diagnostic Device and Medtronic autolog autotransfusion system were used.

Results: the scheme of the surgery: in situ bimammarocoronary bypass surgery - $64(45.3 \%)$, and in situ bimammarocoronary bypass on 3rd artery - $33(23.4 \%)$, arterial conduit on 2nd artery - 10 (7\%), surgery with Y-shaped arterial conduit on 3rd artery - $12(8.5 \%)$, Yshaped arterial conduit on 4th artery - $6(4.2 \%)$. bimammarocoronary bypass surgery + autovenous one - 16 (11.3\%). The number of shunt in one patient was 2.5. The pulsation index (Medistim) is 1.8, the average fraction of cardiac output after surgery was $49.8 \%$, autoreinfusion was carried out for $76(54 \%)$. In 3 (2.1\%) cases due to the high $(>5) \mathrm{Pl}$, anastomoses were applied with satisfactory control values of $\mathrm{PI}$ (up to 3 ). In 3 (2.1\%) cases, repeated surgery for instability of the sternum was carried out. Intra-aortic balloon counterpulsation was carried out in 6 cases (4.2 percent). Re-exploration due to the bleeding was carried out 1 time (0.7 percent). Postoperative mortality was 2 (1.4\%).

Conclusions: Revascularization of myocardium with arterial conduits on a beating heart was significantly less accompanied by the development of acute heart failure, acute renal failure and the need for blood hemotransfusion.

\section{P189}

Surgical treatment

Pavel Myalyuk, Andrey Marchenko, Aleksey Vronsky

Federal centre of cardiovascular surgery, Perm, Russian Federatio

Correspondence: Pavel Myalyuk

Journal of Cardiothoracic Surgery 2017, 12(Suppl 1):P189

Objective: According to the multicenter randomized SYNTAX study (Synergy between PCI with TAXUS drug-eluting stent and Cardiac Surgery), the risk of perioperative ischemic stroke in CABG is $2.2 \%$. In patient with hemodynamically significant carotid involvement risk rises to $14 \%$. Purpose: To present the experience of surgical treatment of patients with multifocal atherosclerosis based on a differential approach to the selection of the stage and sequence of operations in the carotid and coronary arteries

Methods: During the period from 01/01/2015 - 01/01/2017 in "Federal Centre of Cardiovacular Surgery n.a. S.G. Sukhanov», Perm we performed 387 operations of carotid endarterectomy. 232 patients also underwent myocardial revascularization (60\%). The average age of patients is $63 \pm 12.1$ years. Depending on the extent of the carotid and coronary lesion, the tactics of treatment were chosen. 83 patients (36\%) were operated simultaneously and 149 (64\%) patients underwent stage treatment. In case of massive multifocal atherosclerotic lesion, multistage hybrid interventions were performed, including stenting of vertebral arteries, open carotid artery surgery, myocardial revascularization, stenting or carotidsubclavian anastomosis in patients with Still-syndrome and revascularization of the lower extremities by open or endovascular methods. Results: In a group with simultaneous surgical correction, no cases of perioperative ischemic stroke, myocardial infarction or lethality were recorded. In the group of two stages patients, there was 1 case of myocardial infarction after CEA (0.6\%) and 1 case of ischemic stroke after CABG $(0.6 \%)$, which ended in a lethal outcome.

Conclusions: Simultaneous surgical correction of lesions of coronary and brachiocephalic arteries does not increase the risk of postoperative complications in comparison with stage treatment. The proposed approach to the choice of treatment methods for combined lesions of the carotid and coronary arteries based on the differential approach is safe and allows to adequately eliminate the lesions.

\section{P190}

Carotid artery disease and stroke after CABG

Alexandre C. Hueb, Samuel V. Barbosa, Rafael D. Abrantes,

Miguelângelo C. Junior, Mauricio L. Guerrieri, Vinícius C. Furquim, Elias Kallas University Vale do Sapucaí, Pouso alegre, Brazil

Correspondence: Alexandre C. Hueb

Journal of Cardiothoracic Surgery 2017, 12(Suppl 1):P190

Objective: The objective of this study was to analyze whether unilateral or bilateral advanced carotid disease interferes in the neurological evolution in the postoperative period of patients submitted to CABG.

Methods: We analyzed 30 patients submitted to CABG on pump with carotid disease greater than $70 \%$ and 20 with lesion lower than $70 \%$. Was evaluated in the postoperative cognitive alterations, Patients were analyzed on the fifth postoperative day, in relation to the cognitive evaluation with questionnaire of questions and memorization. We check for stroke and cranial tomography.

Results: The mean age was 65 years, with $75 \%$ males, mean CPB time was 63 minutes and mean extubation time was 9.54 hours There were no deaths in this series. We observe that patients with bilateral carotid lesions greater than $70 \%$ had more mental confusion and longer extubation time, without indeterminable tomographic lesion. There was no relation between unilateral lesion greater or less than $70 \%$. There were 4 patients who did crossover to CABG off pump due to advanced aortic plaques, with no postoperative brain injury.

Conclusions: Bilateral advanced carotid lesion has worse neurological prognosis in CABG postoperative period.

P191

OPCAB in patients with acute coronary syndrome

Islam Sharipov, Rustam Yarbekov, Muslim Mustaev, Abdulla Ismatov, Sandjar Omonov, Tokhir Vakhidov

Republican Specialized Centre of Cardiology, Tashkent, Uzbekistan

Correspondence: Islam Sharipov

Journal of Cardiothoracic Surgery 2017, 12(Suppl 1):P191 
Objective: It is well-known that traditional coronary artery bypass surgery (CABG) in patients with acute coronary syndrome (ACS) is associated with higher risk of complications and mortality. The aim of the study was to assess feasibility of OPCAB in patients with ACS.

Methods: A comparative analysis of OPCAB results in 265 patients operated on at our centre from April 2015 to March 2017 was done. The patients were divided into two groups, 82 patients with ACS (30.9\%) and 183 patients with stable angina (69.1\%). Both groups were similar by age, gender and other parameters. Twenty-eight patients of the $1^{\text {st }}$ group $(34.2 \%)$ and sixty-four patients of the $2^{\text {nd }}$ group (35\%) had left main coronary artery disease.

Results: The mean quantity of grafts in patients of the $1^{\text {st }}$ group was 2.9 (range, 1 to 4 grafts) and 3.05 in the $2^{\text {nd }}$ group (range, 1 to 4 grafts). The mean duration of the operations in the $1^{\text {st }}$ and $2^{\text {nd }}$ group was $266.3 \pm 10.9$ mins and $254.6 \pm 12.2$ mins, respectively $(p=0.625)$. Inotropic support was required intraoperatively and after surgery in 12 patients of the $1^{\text {st }}$ group $(14.6 \%)$ and in 14 patients of the $2^{\text {nd }}$ group $(7.7 \%)(p=0.09)$. The mean ICU stay was $18.2 \pm 4.1$ hours in the $1^{\text {st }}$ group and $19.3 \pm 2.1$ hours in the $2^{\text {nd }}$ group. There was no significant difference in quantity of non-fatal complications occurred in 8 patients $(9.8 \%)$ of the $1^{\text {st }}$ and 16 patients $(8.7 \%)$ of the $2^{\text {nd }}$ group $(\mathrm{p}=0.333)$. Mortality was 1 patient $(1.2 \%)$ in the $1^{\text {st }}$ group and 2 patients $(1.09 \%)$ in the $2^{\text {nd }}$ group $(p=0.625)$.

Conclusions: Although the inotropic support was required more often in patients with ACS, the overall results of OPCAB in our patients showed no statistical difference between the groups. This supports the view that OPCAB is an acceptable option in patients with ACS.

\section{P192}

Our experience of ischemic heart disease treatment by total arterial revascularization (tar) in the regional cardiologic centre in Pavlodar

Murat Raikhanov, Vltaly Berezuckiy, Adil Dyurzhanov, Asel Tleyova

Regional Cardiologic Centre in Pavlodar, Pavlodar, Kazakhstan

Correspondence: Murat Raikhanov

Journal of Cardiothoracic Surgery 2017, 12(Suppl 1):P192

Objective: Analyses of the results of ischemic heart disease treatment by total arterial revascularization.

Methods: The graft patency determines prognosis in coronary artery bypass grafting (CABG). Many reports documented superior patencies and prognosis when multiple arterial grafts are used. Saphenous vein grafts (SVG) begin to fail with intimal hypertrophy and then atheroma after 5 years, with patency rates of $50 \%$ to $60 \%$ at 10 years, and $<30 \%$ at 15 years. In contrast, left internal thoracic artery (LITA) patency is $>95 \%$ at 10 years and $>90 \%$ at 20 years. Using of arterial grafts actually in patients with multiple concomitant diseases such as diabetes mellitus, obesity, renal dysfunction and also elderly patients, reoperation of which accompanied with high risk. In the period from October 2015 till April 2017 provided 156 operations by (TAR). There were used grafts: radial artery (RA), (LITA) and right internal thoracic artery (RITA), the last one also used like free arterial graft. Patients who have 2 coronary vessels diseased bilateral internal thoracic artery (BITA) revascularization were used in $90 \%$. Patients who have 3 and more coronary vessels diseased (RITA) used like free arterial graft for sequential. We are prefer (RA) +(LITA) grafting than (BITA) in patients BSA $>2.3 \mathrm{~m} 2$ (Mass body index $>40 \mathrm{~kg} \backslash \mathrm{m} 2$ ). The average age of the patients was $60 \pm 7.5$ years, from which $67.1 \%(102)$ were male, and $33.9 \%(54)$ were female.

Results: Early post operation period patients feel better, free of dyspnea and chest pain. Approximately after 1 month after operation we saw increasement of left ventricular ejection fraction. - We had some post operational complications in 5 patients $(7.8 \%)$. Two of them had sternal wound infection(3.12\%), 3 patients had post operational bleeding.
Conclusions: Thus the results of (TAR) is good. Due to arterial grafts have better patencies and clinical results it can be provided and used more widely.

\section{P193}

Differences in demography \& revascularization strategy in young adults with acute coronary syndrome (ACS) and stable angina (SA) Fuad Z. Abdullayev, Sr. ', Rashad M. Makhmudov' ${ }^{2}$ Imadaddin M. Bagirov', "lgar D. Geybatov ${ }^{1}$, Larisa S. Shikhiyeva ${ }^{1}$

${ }^{1}$ Topchibashev Research Centre of Surgery, Baku, Azerbaijan; ${ }^{2}$ Central

Hospital of Oil Workers, Baku, Azerbaijan

Correspondence: Fuad Z. Abdullayev, Sr.

Journal of Cardiothoracic Surgery 2017, 12(Suppl 1):P193

Objective: To verify features of demography and revascularization of the myocardium in young adults with ACS and SA.

Material: Enrolled 126 pts $27-40$ years old( $38.5 \pm 0.3)$, of them 19(15.1\%) -younger 35years(32.5 \pm 0.6$)$. 64.3\% pts admitted with $S A$ of them $60.5 \%$ - with early Ml; $35.7 \%$ pts - with ACS, including $37.8 \%$ - with early MI. CABG performed in $62 \%$ pts, PCI - in 38\% pts. In ACS vs.SA groups $53.5 \%$ vs. $46.5 \%$ pts underwent $\mathrm{PCl}$. In $\mathrm{SA}$ vs.ACS groups $71.4 \%$ vs. $28.6 \%$ pts underwent CABG. On-pump CABG done in $82.9 \%$ pts; OPCAB - in $17.1 \%$ pts.

Results: Pts with ACS differed from SA group with prevalence of pts younger 35 years old in $20 \%$ vs. $12.3 \%$; 1VD in $46.7 \%$ vs. $30.9 \%$; one risk predictor in $66.7 \%$ vs. $30.8 \%$. Pts with SA differed from ACS group by early $\mathrm{MI}$ in $60.5 \%$ vs.37.8\%; $3 \mathrm{VD}$ in $40.7 \%$ vs. $22.7 \%$; $\geq 2-3$ risk predictors in $69.2 \%$ vs. $30.8 \%$.

ACS group differed with prevalence of PCl; SA group - with CABG. $\mathrm{PCl}$ ratio in ACS vs. SA groups 1.15:1, with target 1 CA in $78.3 \%$ vs. $55 \%$ pts; 2 CA - in $13 \%$ vs.45\%. CABG ratio in SA vs.ACS groups $2.5: 1$, with $\mathrm{CABG}(\mathrm{x} 1)$ in $28 \%$ vs. $35 \%$; $\mathrm{CABG}(\mathrm{x} 2)$ in $20 \%$ vs. $25 \%$; $\mathrm{CABG}(\mathrm{x} 3)$ in $52 \%$ vs. $40 \%$.

$80 \%$ pts with SA manifested with on 48hours «door-to-CABG»: $52 \%$ pts underwent CABG on 24hours; $28 \%$ - 24 to 48 hours; $20 \%$ - more 48 hours. ACS group differed with more 48 hours «door-to-CABG» in $65 \%$ pts; on 48 hours in $35 \%$ pts.

Conclusion: 1.Young adults with ACS differed with prevalence of $\mathrm{PCl}$; delay $C A B G$; twofold rate of re-interventions, and threefold rate of $\mathrm{CABG}$ after early $\mathrm{PCl} ; 2$.Young adults with $\mathrm{SA}$ differed with prevalence of $C A B G(x 3)$, and on 24-48hours «door-to-CABG».

\section{P194}

Comparison of two different ventricular closing techniques in repair of left ventricular aneurysm

Eldaniz Aliyev, Arzu Aliyeva

Scientific centre of surgery by Acad. M.Topchubashov Baku, Azerbaijan, Baku, Azerbaijan

Correspondence: Eldaniz Aliyev

Journal of Cardiothoracic Surgery 2017, 12(Suppl 1):P194

Methods: There has been surgical operation of left ventricular between January 1997 - February 2009 among 138 patients in average age around 70 years old by the DOR technique in our clinic. 73 of 138 DOR made patients have been reached or have got information and those patients were included in the study. After putting patch to ventricular, patients group $A(n=36)$ whose ventriculotomy closed by epicardial, and whereas the patients group $B(n=37)$ who closed with teflon felt. Clinical findings of 19 of the patients in group A (52.8\%) NYHA class 1-2, 14 (38.4\%) class 3-4. And clinical findings of the 19 patients in group B (51.4\%) class 1 to 2 , other $18(48.6 \%)$ had class 3 to 4 . X-clamp time in patients in group $B$ is usually less than 80 minutes. 23 of patients in group $A(63.9 \%)$ and 7 patients in group B (18.9\%) on the X-clamp time was over than 80 minutes $(p<0.001)$. 
Results: 1 out of 37 patients in group B perioperative, and one died due to low cardiac output in postoperative period in intensive care. 5 of the patients in group A (13.9\%), 11 of the patients in group B (29.7\%) needed adrenaline support in the postoperative period in the intensive care.

Conclusions: Factors associated with mortality at univariate analysis: the patient's age over 70 years, existence of diabetes mellitus, 30\% and below ejection fraction, the end diastolic diameter of the left ventricle is $55 \mathrm{~mm}$ and over, postoperative low cardiac output growth, respiratory and drainage has been identified as being 1000 cc and over.

Conducted multivariate analysis between factors associated with mortality in univariate analysis. Accordingly, surgical technique, in postoperative development of low cardiac output, existence of diabetes as an independent risk factor comes into the foreground.

\section{P195}

Treatment of patients with coexistent carotid and coronary artery disease: our attitude

Giuseppe Battaglia, Salvatore Alberto Turiano, Rosario Tringale, Vincenzo Monaca

Ferrarotto Hospital, Catania, Italy

Correspondence: Salvatore Alberto Turiano

Journal of Cardiothoracic Surgery 2017, 12(Suppl 1):P195

\section{Objective}

The $35 \%$ of patients undergoing coronary artery bypass grafting (CAB) have significative carotid artery disease. The incidence of major neurologic events in patients undergoing $C A B$ (cerebral hypoperfusion, carotid embolic events, microembolism frombypass circuit) ranges from $1 \%$ to $5 \%$. Finally, $10 \%$ of patients operated $C A B$ undergo the next 10 years to CEA and vice versa. Therefore, in patients with evidence or suspicion of ischemic cardiopathy undergoing CEA is indicated exercise test and/or myocardial scintigraphy stress or dipyridamole. In patients undergoing cardiac surgery is indicated preoperative Doppler ultrasound examination of the TSA. In the years $80-90$ spread the combined procedure of CAB + $E A C$, in the belief that the aggregate risks was reduced: the results were not satisfactory so the beginning of the 2000 s has established the concept of the procedure "staged" according to the pathology prominent, reserving the combined procedure in selected cases.

Lastly we analyzed the influence of carotid stenting procedures in this approach.

Methods

The authors analyze their first experience, from 1990 to 2003, about 112 consecutive pts underwent combined procedure (Group A). The Group B includes 70 patients, treated between 2004 and 2016.: 56 $B A C+C E A$ for unstable angina or left main stenosis with high risk carotid patient (neurologic symptoms, severe bilateral stenosis, contralateral occlusion); $11 \mathrm{BAC}>\mathrm{CEA} / \mathrm{CAS}$ for unstable angina with low-risk carotid patients; 3 CEA/CAS > BAC for high risk carotid patient and stable angina.

Results

In the group A operative mortality and morbility were higher than the single events in the $B$ group $(p=0.07)$.

Conclusions

Currently, no consensus exists regarding the optimal treatment strategy for patients with concomitant severe coronary and carotid disease. Surgery planning should take into account clinical status and prevalent symptoms in each patient (individualized approach) in order to minimize mortality and morbidity risk.

\section{P196}

Results on "off pump" in patients older than $\mathbf{7 0}$ years

A. A. Aytmukhanov, G. B. Yakupov

АО УДП ЦКБ РК, Almaty, Kazakhstan

Correspondence: A. A. Aytmukhanov; G. B. Yakupov Journal of Cardiothoracic Surgery 2017, 12(Suppl 1):P196
Objective: To study the results on "off pump" in patients older than 70 years.

Methods: The research was based on the observations of the examination and treatment of 142 patients within the period of 2013-2017. The average age of the patients was 75.7 years old, and $54 \%$ of the them were males. According to the type of concomitant diseases the patients were classified as following: myocardial infarction with Q - 8(5.6\%), without Q - 31 (21.8\%), unstable angina - 45 $(31.7 \%)$, stable angina - $58(40.8 \%)$ The risk stratification was calculated according to a EuroScore model $-3.7 \%$. Average stay of patients in the hospital 6.9 days. The patients were divided into three groups according to the age: $70-75$ years old - 124 patients (87.3\%), 76-80 years old - 16 patients (11.2\%), 81 and above -2 patients (1.4\%).

Results: All operations were performed: $12(8.4 \%)$ of them were in LAD artery, 17(11.9\%) - Bi maracaronary shunting, 32(22.5\%) - two vessel bypass, $74(52.1 \%)$ - three vessel bypass, 7 (4.9\%) - four vessel bypass. Shunting coefficient is 2.7. The ultrasonic index PI of the shuntwas on the average - 2.3 Intra-aortic balloon pulsation was established- 7(4.9\%) Reinfusion of blood was applied - 115(80.9\%) Mortality was 3 patients (2.11\%)

Conclusions: This work shows the possibility of prompt treatment of patients older than 70 years with good postoperative results.

P197

Changes in CABG patients' profiles at the Almaty cardiology center Alibek Mereke', Azamat Merekenov², Mukhtar Kulimbet ${ }^{1}$,

Toregen Egemberdiyev ${ }^{1}$

${ }^{1}$ Kazakh National Medical University, Almaty, Kazakhstan; ${ }^{2}$ Kazakh Russian Medical University, Almaty, Kazakhstan

Correspondence: Alibek Mereke

Journal of Cardiothoracic Surgery 2017, 12(Suppl 1):P197

Objective. To compare coronary artery bypass grafting (CABG) patients' profiles between two groups with a 5-year interval.

Methods. This was a cross-sectional study of 285 patients who had undergone CABG surgery from January 1 to September 30, 2009, and 2015 at the Almaty Cardiology Center, Kazakhstan (N: 55 vs. 230). Demographic characteristics, clinical profile, and surgical volume were obtained by retrospective review of medical records. Descriptive statistics were performed to characterize patients' demographics. Independent t-tests and Wilcoxon signed rank tests were used for continuous variables; whereas, Chi-square and Fisher's exact tests were used for categorical data.

Results. Patients operated in 2015 had a higher mean (SD) age (61.71 (8.03) vs. $56.07(9.84)$ years, $p<0.05)$, more retired group patients ( 45.65 vs. $27.27 \%, p<0.05$ ) with severe heart diseases (NYHA IIA: 88.7 vs. $54.55 \%, p<0.05)$, but with fewer cases of respiratory $(9.09$ vs. $0.87 \%, p<0.05)$ and cerebrovascular pathologies (6.97 vs. $18.18 \%$, $\mathrm{p}<0.05)$ than in the second group. There were significant improvements in decreasing operative and perfusion time in 2015 compared to 2009 (160.7 (37.04) vs. 203.5 (52.28) min; 79.26 (19.53) vs. 90.85 (32.8) $\mathrm{min}$ ). Surgeons have significantly increased Off-Pump Bypass Surgery compared to 2009 (47.83 vs. 3.64\%).

Conclusion. This study demonstrated that the recently admitted CABG group was older with better health conditions. Domestic cardiac surgeons have shown an improvement in provided medical care. The long-term survival and quality of life of post-surgery patients are needed to better understand the quality of provided medical care.

P198

Modern opportunities the therapy of acute heart failure

Asel H. Isabekova, Salim F. Berkinbaev, Gulnara A. Dzhunusbekova,

Aisulu T. Musagalieva

Scientific and Research Institute of Cardiology and Internal Diseases,

Almaty, Kazakhstan

Correspondence: Asel H. Isabekova

Journal of Cardiothoracic Surgery 2017, 12(Suppl 1):P198 
Objective: Evaluate the effectiveness of Levosimendan (Simdaks company Orion Pharma, Finland) in acute myocardial infarction with ST-segment elevation in patients with acute heart failure (AHF) compared with standard therapy.

Methods: The study included 50 patients. With DOS - 30 patients (18 men and 12 women). Comparison group were 20 patients with AHF to standard therapy.

Results: According to the study design in patients with AHF, which arose after 48 hours with acute myocardial infarction (AMI) with STsegment elevation after percutaneous intervention (PCI) was performed infusion of Levosimendan. Mean systolic pulmonary artery pressure (SBP) after infusion of Levosimendan increased by $28.3 \%$, and by the end of follow-up was $108.7 \pm 19.21$, as compared with the control group above $27.6 \%, p<0.05$. We obtained similar data by indices of diastolic blood pressure DBP, because in a group of Levosimendan on DBP was 3.3\% higher than the control group. Heart rate (HR) at 48 hours after infusion of levosimendan decreased by $12.8 \%$. Refractive end-systolic and end-diastolic dimensions have not undergone significant changes in both groups, and an average of Levosimendan group decreased by $15.3 \%$ in the control group by $13.3 \%$. End-systolic and end-diastolic volumes were not significantly changed, but however, in the group of Levosimendan 3.2\% reduced data rates compared to the control group. Significantly increased ejection fraction after 48 hours from the start of the infusion, and during the whole observation period, these indicators were stable $43.9 \pm 17.2 \%$, which is $29.8 \%$ higher than the benchmarks.

Conclusions: Treatment of Levosimendan resulted in more rapid transition to a phase compensation of heart failure, showed improvement in hemodynamic parameters inotropic function, significant reduction of maladaptive remodeling, improve the level of compared to the standard dos.

\section{P199}

Association between anemia and ejection fraction among patients with heart failure

Amina Rakisheva', Salim Berkinbayev', Aisulu Mussagaliyeva', Baurzhan

Zhussupov $^{2}$

'Scientific and research Institute of cardiology and internak diseases, Almaty, Kazakhstan; ${ }^{2}$ Kazakh National Medical University named after S. Asfendiyarov, Almaty, Kazakhstan

Correspondence: Amina Rakisheva

Journal of Cardiothoracic Surgery 2017, 12(Suppl 1):P199

Background: HF has an epidemic value, associated with mortality, morbidity. Co-morbidities such as anemia play an important role in progression of $\mathrm{HF}$ and its prognosis. The prevalence of anemia in $\mathrm{HF}$ patients ranges from $10 \%$ to $>50 \%$. The purpose was to assess the prevalence and association of anemia and REF in hospitalized HF patients.

Methods. We conducted a cross-sectional study among 565 patients admitted for HF from 1st January to 31st December 2014. Anemia was defined according WHO definition. An EF less than 40 percent was considered as HF-REF.

Results. There were 395 men (70\%) and 170 women (30\%). M \pm SD of age were 60.8 and 12 years respectively. The prevalence of anemia was $21.8 \%$. The number of patients with $\mathrm{EF}<30 \%$ was $75(13.4 \%)$; LVEF between 30 and 39,9\% - 166 (29.6\%); LVEF $\geq 40 \%-320(57 \%)$. In females, anemia and REF have the same prevalence trends. The peak of anemia and REF among HF female patients was identified at age less than 50 (54\% and 58\% respectively) and declined till 70 and then rose. In males, the prevalence of anemia steadily increased by age, while HF-REF was tended to decline till age 70. We constructed contingency tables of anemia and EF level in gender-age groups. The group that demonstrated significant association between anaemia and EF was males 50-59. Thus, the proportion of patients with
LVEF $<30 \%$ was significantly higher among anaemic patients as compared to patients without anaemia $(p \leq 0.006)$.

Conclusion. Prevalence of anaemia and REF in HF patients significantly vary by age and gender. Adjusting for these variables, we did not see an evidence that anaemia and REF are associated except males 50-59. This group doesn't have or has less chance to have known anaemia risk factors as elderly age, female sex and renal insufficiency.

P200

Right ventricular failure in patients with left ventricular assist device: single center experience

Saltanat Andossova, Yuriy Pya, Makhabbat Bekbossynova,

Saltanat Dzhetibayeva, Roman Salov, Serik Bekbossynov,

Muradym Murzagaliyev, Svetlana Novikova, Assel Medressova,

Timur Kapishev, Nurlan Smagulov, Ainur Taukelova

JSC "National Research Center for Cardiac Surgery", Astana, Kazakhstan

Correspondence: Saltanat Andossova

Journal of Cardiothoracic Surgery 2017, 12(Suppl 1):P200

Objective: This study evaluated right ventricular failure (RVF) predictors of development after ventricular assist device (LVAD) implantation.

Methods: We reviewed 225 consecutive LVAD patients: 81 cases $(35,5 \%)$ before LVAD had biventricular failure (INTERMACS I-III). RVF was defined as the need for a subsequent right ventricular assist device (RVAD), $\geq 14$ days of intravenous inotropes/pulmonary vasodilators, or both, high total bilirubin, CVP $\geq 18$ according to INTERMACS definition. 45 (20\%) RVF patients were identified. Outcome parameters included preoperative dates, early ( $\leq 30$ day) mortality, incidence of re-operation for bleeding, length of stay in intensive care unit (ICU), comparison of patients with moderate to severe tricuspid regurgitation (TR) who underwent or not tricuspid valve repair.

Results: 45 patients (20\%) developed RVF after LVAD implantation, 9 (20\%) patients died during 1 month, $6(10 \%)$ required RVAD support. RVF patients had preoperative longer duration of HF $(31,5 \pm 24,2$ vs $24,3 \pm 19,5$ month, $p<0,001)$, low level of albumin $(36,1 \pm 4,56$ vs 38,3 $\pm 18,9, \quad p<0,0001)$, high level of NT-pro BNP $(8843,6 \pm 11845,5 \mathrm{vs}$ $7287,19 \pm 4824,9, p<0,0003)$, high level of bilirubin $(1,6 \pm 0,8$ vs 0,77 $\pm 0,65, p<0,001)$, and preoperative ratio of central venous pressure (CVP) to pulmonary capillary wedge pressure $(0,41 \pm 0,18$ vs $0,63 \pm 0,3$, $p<0,0001)$, higher rates of re-operation for bleeding $(351,51 \pm 253$ vs $338,88 \pm 141,96 \mathrm{ml}, \mathrm{p}<0,0006)$, greater ICU LOS $(11,73 \pm 11,1 \mathrm{vs} 6,64$ $\pm 6,40, p<0,0001)$. Repair of TV was done 9 patient because of severe $\mathrm{TR}$, in 5 of them RVF were ongoing.

Conclusion: RVF patients had higher early mortality rate. Longer duration of HF, poor nutrition status, high level of bilirubin and ratio CVP/PCWP were preoperative significant predictors for RVF development in short term period. They had higher rates of reoperating for bleeding and greater ICU LOS. The benefits from concomitant repair of TV due to severe TR is unclear because of small group size.

P201

Distribution characteristics of HLA antigens in patients with dilated cardiomyopathy

Indira Ramilyeva', Zhandos Burkitbayev ${ }^{1}$, Sania Abdrakhmanova'

Aida Turganbekova', Dana Baimukasheva', Eugene Zhiburt ${ }^{2}$

${ }^{1}$ Scientific-Production Center of Transfusiology, Astana, Kazakhstan;

${ }^{2}$ National Medical and Surgical Center named after N.I. Pirogov of

Ministry of Health of the Russian Federation, Moscow, Russian

Federation

Correspondence: Indira Ramilyeva

Journal of Cardiothoracic Surgery 2017, 12(Suppl 1):P201 


\section{Objective}

Associations between Human Leukocyte Antigen (HLA) genes and the development of dilated cardiomyopathy (DCM) have never been studied in Kazakhstan. Hence, it is important to study the characteristics of HLA alleles and conduct research to study the connection between the development of this disease and HLA genes. Therefore, the aim of this study was to investigate the rate of frequency of HLA class I (HLA-A, B) and class II (HLA-DRB1*) genes among healthy blood donors and patients with dilated cardiomyopathy, residing in Kazakhstan.

Methods

The study enrolled 3850 participants: 3621 healthy blood donors considered as a control group (of them HLA-A - 3621; B - 3607; CW 3582; DRB1-3595; DQB1-3576) and 229 patients with DCM (of them HLA-A - 229; B - 229; DRB1 - 206). The HLA-typing method was consisted of three stages: DNA isolation, amplification, detection. Lowresolution HLA-typing (HLA-A, B, C, DRB1, DQB1) for both groups was performed by polymerase chain reaction (PCR) method using a set from Protrans (Protrans, Germany).

Results

The analysis revealed a distribution profile of HLA system in patients with heart failure in the Kazakh population. The alleles positively associated with the development of heart failure were HLA-A *34, *66; HLA-B*58; HLA-DRB1*12. In addition, the following HLA-A*03, ${ }^{*} 25,{ }^{*} 32$; HLA-B*42, ${ }^{*} 59$; HLA- DRB $1{ }^{*} 01,{ }^{*} 15$ alleles might have a protective role in the development of cardiac abnormalities.

Conclusions

In conclusion, four alleles at the HLA-A, HLA-B and HLA-DRB1* loci appear to be associated with heart failure development and seven alleles at the same loci appear to be associated with heart abnormality protection within the Kazakh population. Furthermore, this study adds useful information to study a variety of diseases associated with HLA genes including dilated cardiomyopathy and other cardiac abnormalities.

\section{P202}

The diagnostic possibility of tissue doppler for myocardial dysfunction of patients in post-tranplant follow up after heart transplantation

Saltanat Dzetybayeva, Mahabbat Bekbossynova, Marat Aripov,

Myrat Mukarov, Saltanat Andossova, Gulzhan Myrzahmetova

National Research Center for Cardiac Surgery, Astana, Kazakhstan

Correspondence: Saltanat Dzetybayeva

Journal of Cardiothoracic Surgery 2017, 12(Suppl 1):P202

Objective: We would like to describe our follow up experience on Tissue Doppler (TD) as a non-invasive diagnostic to for early myocardial dysfunction after transplantation.

Aim of research: to assess revealing of TD-indexes of myocardial dysfunction after transplantation.

Materials and methods: Since August 2011 there were 48 heart transplantations performed (in the age of $41 \pm 13.8$ years from the donors of $42.5 \pm 13.5$ years old). All patients every day before discharging, during 1, 3, 6, 12 mounts had follow up or undergone echocardiography (2D echo in a resting state, tissue Doppler (TDI) (PW-systolic parameters S velocity, myocardial strain).

Results: In average TD indexes were the following: $S$ lateralis-10.2 \pm 1.4 , S medialis $8.9 \pm 1.2$, S basal RV $9.9 \pm 1.5$. After 12 months in one patient there were next indicate: S' laterals $3.8 \mathrm{~cm} / \mathrm{c}$. S'medialis 3.9 $\mathrm{cm} / \mathrm{c}$. S'RV $3 \mathrm{~cm} / \mathrm{c}$. After 10 days of timely administration of pulse therapy S'lateralis $8 \mathrm{~cm} / \mathrm{c}$. S'medialis $7 \mathrm{~cm} / \mathrm{c}$. S'RV $7 \mathrm{~cm} / \mathrm{c}$, were acquired with improvement of clinical status and further, patient was discharged after recovery.

Conclusion: Thus, TD indexes can reveal the myocardial dysfunction (PW-systolic parametres S velocity), and can be useful for diagnostic tool. However, which requires further study.
P203

Using cardiopulmonary bypass in patients with locally advanced chest tumors. results of surgical treatment of $\mathbf{4 5}$ patients Stanislav Cherniavskii ${ }^{1}$, Jurii Belov², Vladimir Parshin' ${ }^{1}$, Roman Komarov ${ }^{1}$ Ovik Mirzoyan

${ }^{1}$ First MSMU IM Sechenov, Moscow, Russian Federation; ${ }^{2}$ RSCS BV

Petrovsky, Moscow, Russian Federation

Correspondence: Stanislav Cherniavskii

Journal of Cardiothoracic Surgery 2017, 12(Suppl 1):P203

Improvement surgical techniques led to the expansion of the indications for performing simultaneous operations in cancer patients with invasion of great vessels. Despite appearing in the literature reports of extensive operations combined with resection of the affected vessels, some surgeons still classify certain tumors of the chest as the unresectable. Such intervention could hardly have spread among practicing surgeons because of the complexity of their implementation. In this regard, the development of new surgical techniques in patients with tumors of the chest with the invasion of the main blood vessels and heart is actual.

Objective: To evaluate the immediate and ling-term results of surgical treatment of patients with locally advanced tumors of the chest with the invasion of the great vessels.

Methods: 1. Clinical examination 2. Instrumental methods of examination 3. Histological examination of surgical specimens 4. Statistical analysis of results.

Results: in the period from 2002 was operated 45 patients with great vessels lesions. All divided into 2 groups (radical-23 and palliative22). Hospital mortality in 1 group was $17,4 \%$, in 2 group - $0 \%$. 5 -years survival rate was higher in 1 group - 25\% (median survival - 43,8 month), against 2 group it was - $2 \%$ (median survival - 24,9 month).

Conclusions: We believe that the widespread opinion among surgeons about the impossibility and inappropriate performance of the combined operations in a small number of patients with T-4 lung cancer with invasion great vessels of at least exaggerated. Such operations are conducted jointly oncology and heart surgery teams, today can be seen as the only possibility to achieve recovery and prolong the life of cancer patients.

P204

Application of endoscopic experience ultrasound ministers for diagnostics of peripheral lung tumors

Konstantin Kulaev, Andrey Vazhenin, Andrey Lukin, Marina Mironchenko, Konstantin Zuikov, Ildar Yusupov

Chelyabinsk regional oncology centre, Chelyabinsk, Russian Federation

Correspondence: Konstantin Kulaev

Journal of Cardiothoracic Surgery 2017, 12(Suppl 1):P204

Objective: The objective of this study was to evaluate the effectiveness of Endobronchial Ultrasound Bronchoscopy (EBUS), as a complement to bronchoscopy, for the search and verification of peripheral neoplasms of the lungs.

Methods: In 2015, on an outpatient basis, 279 bronchoscopies using EBUS were performed in patients with suspected malignant neoplasm of the lungs. We used an ultrathin $20-\mathrm{MHz}$ ultrasonic probe. Indications for EBUS, absence of visualization of the neoplasm with normal bronchoscopy and changes in the neoplasm were more than $2 \mathrm{~cm}$. Among them: men - 198 (71\%), the average age of patients was 67 years \pm 8.3 years; Women - $81(29 \%)$, the average age was 67 years \pm 8.7 years. Before the ultrasound examination of the lungs, all the patients, along with lung radiography, performed a CT scan or positron emission tomography.

Results: Visualization of the pathological focus was achieved in $170(60.9 \%)$ patients, the percentage of successful morphological verification in 113 (66.4\%). The most frequent histological form of the tumor was squamous cell carcinoma, diagnosed in 38 
patients or in $33.7 \%$ of cases. Second in frequency was non-small cell lung cancer, which was detected in 25 patients or in $22.2 \%$ of cases. And in 19 patients, adenocarcinoma was diagnosed, which was $16.8 \%$ of the total number of verifications received. Small cell carcinoma was verified in 15 patients, which was $13.2 \%$. Other forms of malignant neoplasms of the lung (neuroendocrine cancer, spindle cell tumor, large cell carcinoma and bronchoalveolar carcinoma) were verified in 5 patients, which amounted to $4.4 \%$. Metastases in the lungs were verified in 11 patients $(9.7 \%)$ of the total number of verifications received.

Conclusions: The use of EBUS with the condition of ultrasound imaging of the neoplasm of $66.4 \%$ allows verification of the process at the outpatient stage.

\section{P205}

CT guided microcoil localization followed by fluoroscopically guided uniportal VATS resection of small lung lesions, a single center experience

L. I. Yue, John Kit-Chung Tam, Bernard Boon Kee Wee, Harish Mithiran Muthiah

National University Health System, Singapore, Singapore

Correspondence: L. I. Yue

Journal of Cardiothoracic Surgery 2017, 12(Suppl 1):P205

\section{Objective:}

As cancer screening and surveillance becomes more popular, physicians nowadays are facing more cases of small pulmonary nodules picked up in CT scans. Uniportal video assisted thoracoscopic surgery (UVATS) is the standard of care for such cases in our institution. CT guided microcoil localization can aid surgeons in performing UVATS resections for non-palpable lung nodules.

Methods:

We performed retrospective analysis of cases of CT guided microcoil localization followed by fluoroscopically guided UVATS resection of small lung nodules in our institution from March 2015 to Feb 2017.

Results:

CT guided microcoil localization followed by fluoroscopically guided UVATS resection were performed in 13 patients, 9 are male $(69.2 \%)$ Patients' median age was 63 , mean age was 60.77 . The lung lesions included $8(61.5 \%)$ solid nodules, 3(23.1\%) ground glass opacities (GGO), and $2(15.4 \%)$ cavitatory nodules. 10 patients (76.9\%) had treated malignancy of other sites. 9 patients $(69.2 \%)$ underwent wedge resection of the nodule, $3(23.1 \%)$ underwent segmentectomy, $1(7.7 \%)$ underwent completion lobectomy after wedge resection as intra-operative frozen section showed adenocarcinoma of the lung. There were 2 cases of postoperative air leak, both resolved spontaneously within 3 days. There were 2 cases of postoperative pneumonia requiring IV antibiotic treatment. There was one case of pneumothorax after microcoil insertion which required immediate chest tube insertion in the radiology department. There was one case UVATS had to be converted to open thoracotomy because the microcoil was not identifiable intraoperatively through fluoroscopy. Postoperative stay was typically between 2-10 days, (median 2 days, Average 3.6 days). All patients obtained pathological diagnosis of the lung lesion from UVTAS surgery.

Conclusions:

CT guided microcoil localization followed by fluoroscopically guided UVATS resection is safe. It can provide minimally invasive diagnosis and treatment for small lung lesions. Care should be taken when inserting microcoil to avoid its complications.

\section{P206}

Artificial ventilation in tracheo-bronchial surgery - a view from the present to the past, 1963 - 2017

Margarita Vyzhigina, Vladimir Parshin, Armen Bunyatyan, Viktor Titov, Aleksander Alekseev

Sechenov University, Moscow, Russian Federation

Correspondence: Margarita Vyzhigina

Journal of Cardiothoracic Surgery 2017, 12(Suppl 1):P206
Objective: The guarantee of successful dynamic development of tracheobronchial surgery (TBS) for more than 50 years was determined by the human factor and technical support. Global importance in anesthetic protection in TBS belongs to the methods of Respiratory Support (RS) and the adaptation of anesthesia technologies to them.

Methods: Since 1963 we treated more than 2,000 patients with diseases of the trachea and major bronchi. The success of anesthesia during the analyzed period is associated with four major technologies: a twochannel system "shunt-breathing" (1963); Intravenous anesthesia (1975); High-frequency jet ventilation (1983); apneic oxygenation (2014). We studied the possibilities and limitations of the basic RS in TBS in the context of evidence-based medicine.

Results: At the beginning, TBS was more dramatic and less reliable, but always inevitably necessary. Comparative expertise and systematization of approaches in assessing the effectiveness and relevance of RS technologies for TBS provide a basis not only for summing up the results, but also for considering development prospects. We determined leading factors of each RS technique based on gas exchange, hemodynamic and metabolic stability, allowing to expanding TBS capabilities and improving its results. The modern strategy of RS and patient safety, and the highest level of surgical techniques, remove from the agenda the concept of inoperability according to concomitant pathology and level of stenosis. Intraoperative monitoring is recognized as the most important safety factor for RS in TBS.

Conclusions: All used techniques of RS, anesthesia and monitoring in TBS are relevant and can be used with considering of individual possibilities and limitations. We offer to discuss a personalized strategy of development of RS in TBS.

\section{P207}

Robot-assisted surgery for nononcological chest diseases: four years' experience

Piotr Yablonskii, Grigorii Kudriashov, Igor Vasilev, Andrey Kozak, Armen Avetisyan, Galina Marfina, Olga Sokolova

Saint-Petersburg State Research Institute of Phthisiopulmonology, Saint-

Petersburg, Russian Federation

Correspondence: Piotr Yablonskii

Journal of Cardiothoracic Surgery 2017, 12(Suppl 1):P207

Objective: Robot-assisted thoracoscopic surgery (RATS) is current method, which appeared to replace VATS. Many papers present its efficacy for lung cancer. We collected all cases with nononcological chest diseases, which were underwent robotic surgery during last four years.

Methods: Between 2013 and 2017, 85 patients with chest benign diseases were selected for RATS by multidisciplinary team. There were 60 patients with pulmonary tuberculosis, 19 - with mediastinal masses, three - with bronchiectasis, two - with interlobar pulmonary sequestration and one patient with chronic lung abscess.

Results: 66 lobectomy and 19 operations for mediastinal masses were performed with DaVinci Si surgical system. Tuberculosis cases associated with high rate of pleural and hilar adhesions (65\%). Closure of additional vessel from descending aorta was feature of lobectomies for pulmonary sequestrations. Overall operative time was 90-515 min for lobectomy and 85-360 min for resection of mediastinal masses. Three lobectomies were associated with conversion to open surgery due to hilar adhesions (including traction gap of the pulmonary artery between A2 and A6 during fissureless lobectomy; and injury of left pulmonary artery during isolation of left upper lobe bronchus) and pleural adhesions. All patients were discharged on time. The intraoperative blood loss was less than $200 \mathrm{ml}$ in both cases. One converted case was justified of adhesions on the neck and large size of mediastinal mass. Eight (9\%) serious surgical complications were recorded: gastrointestinal bleeding, hematoma of the right liver lobe, exacerbation of COPD, prolonged air leak, pleural effusion and seroma in the area of assistant port. $75 \%$ of complications were during the learning curve. 
Conclusions: RATS - is effective and safety approach for nononcological chest diseases. Carefully selection of patients with mediastinal masses can reduce rate of converted cases. Vascular injures during RATS for inflammatory diseases associated with low blood loss and stable hemostasis with robotic tools.

\section{P208}

Minimally invasive treatment complicated forms of lung cancer

Vladislav Severgin, Sr. ${ }^{1}$, Pavel Shipulin ${ }^{2}$, Olena Tronina ${ }^{1}$

${ }^{1}$ Odessa National Medical University, Odessa, Ukraine; ${ }^{2}$ Odessa Regional

Clinical Hospital, Odessa, Ukraine

Correspondence: Vladislav Severgin, $\mathrm{Sr}$.

Journal of Cardiothoracic Surgery 2017, 12(Suppl 1):P208

Objective: Lung cancer is one of the leading and challenged diseases in oncology, due progression of the disease and its complications such as lung bleedings and lung atelectasis caused by tumor obstruction of bronchi.

Methods: We present complex of palliative methods for the treatment and management of generalized lung cancer forms and its complications. These methods includes roentgen endovascular embolization of bronchial arteries, followed by radiofrequency ablation (RFA) and recanalization of the bronchial stenosis by the tumor.

Results: These methods were applied in 43 patients with the age group from 61 to 79 years. The patients were diagnosed with lung cancer stage III-IV. In this group of patients radical operative treatment was not possible due to the tumor progression accompanied with lung bleeding and other low functional implications. All patients had lung bleeding with blood loss of $100-300 \mathrm{ml}$ per day, which is a contraindication for palliative chemotherapy. Along with that, patients had lung atelectasis due to tumor obstruction of mail bronchus or lobar bronchi. Hemostatic effect seen in 40 patients immediately, others had to undergo a repeated embolization of the intercostal arteries, additionally vascularizing the tumor. Recanalization of the bronchial stenosis was achieved in 18 patients, which improved the quality of life. In future, the patients were recommended to undergo chemotherapy or radiotherapy depending on the morphological character of the tumor. In addition, one patient pneumonectomy was performed, and another - an upper lobectomy. For 6 months, 5 patients died, 2 of them from the ongoing bleeding, 3 - from the process generalization.

Conclusions: The use of the proposed method in the treatment of neoplastic stenosis of the trachea and bronchi leads to a positive effect, which is reflected in fewer complications, reducing the recurrence of hemoptysis and longer maintaining patency of the bronchi.

\section{P209}

Cardio-thoracic surgery: the new approach in the organization of the highly specialized medical care

Maksat Zhanturganov, Adilzhan Albazarov, Anatoliy Kolos

JSC NSMC, Astana, Kazakhstan

Correspondence: Maksat Zhanturganov

Journal of Cardiothoracic Surgery 2017, 12(Suppl 1):P209

Objective: To show the achievement of the cardiothoracic department as a part of scientific center.

Methods: The analysis of efficiency of using of cardiac surgery technologies in thoracic surgery.

Results: The priority direction of public health services in Kazakhstanis is improvement of the highly specialized medical care (HSMC). Over the last 10 years the medical cluster in Astana was created and equipped by modern equipment and highly specialized employees. The greatest development has got the cardiothoracic direction owing to, about 1000 cardiac surgery procedures on heart, main vessels and mediastinum are carried out. Using cardiac surgery technologies in thoracic procedures expanded the opportunities in providing operative care to patients with the different forms lung cancer, tumors, cysts of a mediastinum, intrathoracic localization parasitic diseases and congenital pathology of the bronchopulmonary system. Development and deployment of simultaneous cardiothoracic operations is also important direction that is actual for elderly patients suffering from the ischemic heart disease and the combined oncopathology. These patients are being refused in surgery by oncologists because of ischemic heart disease risk and cardiac surgeons did not operate these patients in order to possible tumor dissemination during cardiopulmonary bypass. Cardiothoracic department with the staff of cardiothoracic surgeons, perfusionists, specialized intensive care unit, where quality of cardiovascular and respiratory systems are being continuously monitored, has given a chance to make possible to perform simultaneous procedures.

Conclusions: Thus, integration of the cardiac and thoracic surgery has increased working effectiveness of HSMC in JSC NSMC. For the first time in Kazakhstan has got a positive experience of effective lung cancer surgical treatment and prevention of morbidity in patients with serious cardiac pathology.

P210

Coronary fistulas and shunts in terms of local hemodynamics

Andrey V. Tsokolov' ${ }^{1}$. Dmitrii A. Lushchenkov ${ }^{2}$

${ }^{1}$ Federal State Agency «1409 Clinical hospital of the Baltic Sea fleet», Kaliningrad, Russian Federation; ${ }^{2}$ Hospital Maternoinfantil - Universitat de Barcelona Sant Joan de Deu, Barcelona, Spain

Correspondence: Andrey V. Tsokolov

Journal of Cardiothoracic Surgery 2017, 12(Suppl 1):P210

Objective: Data on coronary fistulas (CF) available in the literature is non-systemic. No article is specified to explain an abnormal variance of speed rates (Vmax) and barometric gradient (Gmax) registerable in case of such pathology.

Methods: As follows from the analysis of literature and native data we made a conclusion that the imbalance between the registered Vmax and Gmax in the CF projection with the estimates expected taking into account the cardiac chambers and coronary arteries (CA) pressure indicators most commonly must be caused by embryogenesis disturbance. Against this background of abnormalities "sinusoids" are formed by CA extended terminals type proceeding to cardiac chambers, often in the midst of remaining myocardium cancellation due to dividing of CA to segments of 1-2-3 order, pressure in distal segments of coronary flow from $60-90 \mathrm{~mm}$ $\mathrm{Hg}$ to $18-28 \mathrm{~mm} \mathrm{Hg}$. Maximum reduction of flow speed and pressure in "sinusoids" leads to even higher pressure differential between CA and cardiac chamber, up to $4-6 \mathrm{~mm} \mathrm{Hg}$. Such CF, in terms of mechanism of its development and local hemodynamic characteristics of flows, and to avoid confusion among doctors, it is better to describe genuine CF.

Results: Higher Vmax and Gmax (up to $44-51 \mathrm{~mm} \mathrm{Hg}$ ) more often may be classified as CF/shunts or to CF in proximal compartment of the coronary stream. Unlike genuine $\mathrm{CF}$, the first ones should be classified as secondary/postsurgical CF/shunts. Reasonability of such division on "genuine" and "secondary" CF is determines by complication probability as well as patient surveillance, physical activity level prescriptions. Conclusions: Thus, with account of haemodynamic features of pathologic flows, registered between CA and cardiac chambers, practically it is reasonable to divide them into: 1) genuine $\mathrm{CF}_{\text {, }}$ predominantly with diastolic flows, and either low (distal), or high (proximal) Vmax/Gmax, and 2) secondary (mostly postsurgical) fistula/shunts, with high Vmax/Gmax. 
P211

Molecular gene analysis of hereditary predisposition to arterial hypertension and ischemic insulating in Kazakhstan

Aliya Dzholdasbekova', A. Dzhusipov², N. Baitasov ${ }^{3}$

${ }^{1}$ Medical Centre Hospital of the President affairs administration of the Republic of Kazakhstan, Astana, Kazakhstan; ${ }^{2}$ Scientific Research Institute for Cardiology and Internal Diseases, Almaty, Kazakhstan; ${ }^{3}$ Scientific Research Institute for Cardiology and Internal Diseases, Almaty, Kazakhstan

Correspondence: Aliya Dzholdasbekova

Journal of Cardiothoracic Surgery 2017, 12(Suppl 1):P211

Objective: Investigate the relationship between polymorphisms of the main genes-candidates and predisposition to the development of arterial hypertension and ischemic stroke in Kazakh nationality.

Methods: The patients were. 64 patients aged $30-55$ years in the anamnesis had the transferred ischemic stroke. The study conducted with polymerase chain reaction (PCR) using oligonucleotide primers. Results: Among the frequency distribution of genotypes and alleles of genes: 4a/4b NOS3, C677T MTHFR, G/A FGB in patients with arterial hypertension and relatively healthy persons, no significant differences were found except 4G/5G PAI-1, Lys198Asn END genes. So, with the frequency distribution, the genotype $5 \mathrm{G} 5 \mathrm{G}$ and allele $5 \mathrm{G}$ of the polymorphism of the PAl-1 gene predominated in patients with arterial hypertension compared to healthy individuals, and this difference was significant $(P<0.05)$. When comparing the frequency distribution of the genotype and the allele of the END gene between groups of patients with arterial hypertension and healthy individuals of Kazakh nationality, it was found that among patients the frequency of the AsnAsn genotype and the Asn alleles is significantly higher than the frequency of this genotype and alleles in the group of healthy individuals $(P<0.05)$.Mutant genotypes of the $5 \mathrm{G} / 5 \mathrm{G}$ PAl- 1 gene, $\Pi$ genotypes of the MTHFR gene and the AA genotypes of the FGB gene are found exclusively in patients with a history of ischemic stroke $(P=0.001)$.

Conclusions: The polymorphisms of the PAI-1 and END genes are molecular genetic markers of predisposition to the development arterial hypertension, and the presence of the mutant alleles of the MTHFR, FGB and PAl-1 genes increases the risk of ischemic stroke in patients with hypertonia and with a hereditary burden.

\section{P212}

\section{Case history of obstinate subfebrilitate in patient with the} pacemaker

Andrey V. Tsokolov' ${ }^{1}$ Dmitrii A. Lushchenkov²

${ }^{1}$ Federal State Agency «1409 Clinical hospital of the Baltic Sea fleet», Kaliningrad, Russian Federation; ${ }^{2}$ Hospital Maternoinfantil - Universitat de Barcelona Sant Joan de Deu, Barcelona, Spain

Correspondence: Andrey V. Tsokolov

Journal of Cardiothoracic Surgery 2017, 12(Suppl 1):P212

Objective: Pulmonary artery thromboembolia (PATE) is one of the most severe complications of pacing treatment of patients with rhythm and cardiac conduction disorders. References witness that in $15 \%$ cases the apical pacing is accompanied by the development of PATE, which usually has the latent from. The situation is complicated with the additional fact that thrombosis is possible on an electrode as well. Moreover, the formation of vegetation on pacemaker electrodes is possible. That is why the presence of additional echo signals on electrodes cannot be interpreted unambiguously. This case history lends evidence.

Results: In patient A, against the background of clinical PATE and recurrent multisegmental abscess forming pneumonia, clinical septicemia was observed for 2 months, at a poor effect of the combined antibacterial therapy conducted. A transthoracic and transesophageal echocardiography study, the pacemaker electrode thickened to $6-8 \mathrm{~mm}$ was visualized in the right atrium, and in the right ventricle to $12.1 \mathrm{~mm}$, with indistinct contours from of loose medium-dense depositions, with their minimal mobility. With regard of obstinate subfibrilitet and septicemia, the Echo pattern could not be considered unambiguously as the electrode thrombosis. The possibility of electrode endocarditis remained. As the Vena Cava Filter was earlier installed in a patient, the source of thromboembols into the pulmonary artery could be pacemaker electrodes. In 6 months, at the repeated Echo, electrodes had normal diameter (2.1-2.6 mm). Against the background of thrombolytic therapy, the situation with PATE was solved. With regard of dynamics of changing "thickness/ structure" of electrodes in the right compartments of heart, the changed detected earlier were "post factum" interpreted by us as loose mobile thrombotic masses.

Conclusions: This case history shows the need of doctors cautious attitude to the possible development of thrombosis of intracardiac electrodes, keeping in mind infective endocarditis and the need of timely diagnosing the latter.

P213

Management of delayed esophageal perforations following anterior cervical spinal surgery with applied hardware

Georgy Yankov ${ }^{1}$, Vladimir Stanoev ${ }^{1}$, Hristo Hristov², Anatoli V. Semkov' Danail Petrov ${ }^{1}$

${ }^{1}$ Thoracic Surgery Department, Saint Sophia University Hospital for Pulmonary Diseases, Medical University, Sofia, Bulgaria; "Saint Ivan Rilsky" University Hospital, Medical University, Sofia, Bulgaria, Sofia, Bulgaria

Correspondence: Anatoli V. Semkov

Journal of Cardiothoracic Surgery 2017, 12(Suppl 1):P213

Methods: Two patients (P1 and P2) with delayed esophageal perforations following anterior cervical spine surgery for trauma with hardware applied are presented.

Results: P1 initially underwent closure of the perforation by general surgeon. Postoperatively paraspinal abscesses were formed, treated by incisions. The cervical esophageal fistula was again unsuccessfully repaired by a neurosurgeon. In our department massive adhesions were debrided, the plate and screws were removed through a left cervicotomy. A 5-mm esophageal fistula was closed by an absorbable running suture with left infrathyreoid muscle flap reinforcement. Three cutaneous fistulas and three smaller paraspinal abscesses were excised. Uneventful postoperative course. P1 recovered normal deglutition and is without complaints 14 month following last operation. P2 underwent primary closure of esophageal perforation in our department with split left sternocleidomastoideus flap. The anterior hardware was not removed due to fear of spinal instability following previous C-6 corporectomy for trauma. After seven months P2 presented to us with symptoms of neck pain, swelling, fevers and dysphagia. The cervical incisions were performed with symptoms' relief. A posterior stabilization and C3-C7 fusion was applied by neurosurgeon. Seven days after this procedure anterior plate, screws and cage were removed, and $5 \mathrm{~mm}$ esophageal fistula was repaired by interrupted sutures and remaining part of left sternocleidomastoideus muscle by combined surgical team. One month later P1was again with symptoms of neck pain, swelling and fever. Two cervical incisions were carried out for open wound healing, which were closed after 10 days. The oesophageal perforation healed with no recurrent fistula and complaints for a follow up period of 6 months.

Conclusions: The interdisciplinary approach is essential in the treatment of this type of patients. The anterior hardware should be removed obligatory. If the patient does not have evidence of fusion at the time of presentation, then posterior cervical instrumentation is a viable alternative. 
P214

Modified ileocolic conduit and colo-jejunal Roux-en $Y$ for esophageal and stomach replacement after two subsequent complicated laparoscopic bariatric surgery

Hamidreza Davari

Tehran University of Medical Sciencws, Tehran, Iran

Journal of Cardiothoracic Surgery 2017, 12(Suppl 1):P214

Objective: Nowadays more patients undergo bariatric surgery, it is not unusual to see some postoperative complications which are needed to manage by a thoracic surgeon.

Methods: Forty-six years was referred for esophageal replacement. Five years ago she had Laproscopic gastric banding (LGB) with late ring erosion complication. She underwent 2 nd laproscopic sleeve gasterctomy (LSG). Within 3 months' post op; she had occasional nausea, vomiting and tachycardia. She was managed by conservative treatment and total parenteral nutrition. CT scan with contrast study showed hiatal herniation. She had left thoracotomy which was showed confine leakage and hiatal herniation. She had esophagectomy, extended proximal gastrectomy, splenectomy and repair diaphragmatic defect with jejunostomy. After 2 months of jejunal feeding and build up, midline laparotomy was done. There was only small remnant of pyloric stomach with serous fluid in left upper quadrant and severe dense adhesion around left splenic flexure. used right colon base on left colic artery. I found accessory middle colic artery. An ileocolic conduit base on accessory middle and left colic artery was prepared. Retrosternal cervical esophago- ileocolic conduit was done with left hemimanubrectomy. Distal anastomosis was completed by colo-jejunal Roux-en Y. lleo-colic anastomosis was performed and a new jejunostomy tube for early feeding was placed. Results: The operation was uneventful. Jejunostomy tube feeding was started on day 3 post op. She had barium swallowing and no evidence of leakage on day 10.

Conclusions: Laparoscopic gastric banding is obsolete but still complication can be seen. The choice for $2^{\text {nd }}$ operation is laparoscopic gastric by pass and not LSG. Bariatric surgery needs good experience and doing the right operation for the right patient. Early detection and treatment remain pivotal principles in the management of Gl leaks and may play a role in reducing subsequent morbidity and mortality.

\section{Consent for publication}

Written informed consent to publish was obtained from the patients involved in this study.

\section{P215}

Stroke in cardiosurgery expirience of Pavlodar

Almas M. Antikeev, Askar M. Abiltayev, Oraz S. Mukashev, Adil A.

Dyurzhanov, Darkhan S. Dairov, Handulla H. Nakipov, N T. Shakisheva

Pavlodar Regional Cardiological Center, Pavlodar, Kazakhstan

Correspondence: Almas M. Antikeev

Journal of Cardiothoracic Surgery 2017, 12(Suppl 1):P215

Objective: to present two years results of stroke after operation on the heart.

Methods: from 2015 to 2016 years, 1188 patients operated on the heart, 20 patients has Stroke after operation $(\mathrm{m}-60 \%, \mathrm{f}-40 \%)$, age 39 $71(59.65 \pm 10.65)$ years.

Results: according to ultrasound dopplerography investigation of vessels and angiography in $35 \%$ (7 patients) we fined stenosis of carotid artery and s-shaped vertebral arteries, at $40 \%$ (8 patients) we don't find any changes in arteries, $25 \%$ (5 patients) we don't examined. At this patients in $70 \%$ (14 patients) associated diseases was arterial hypertension $3^{\text {rd }}$ degree, in 30\% (6 patients) diabetes mellitus was find, disturbances of heart rhythm occurs in $20 \%$ (4 patients) of cases. The coronary artery bypass was performed in $55 \%$ of cases, correction of valves pathology at $20 \%$, in $25 \%$ of cases combined operation was performed. At 10\% (2 patients) of cases cause of stroke was thrombosis of common and internal carotid arteries. Localization of stroke in $65 \%$ cases was in basin of the middle cerebral artery, more often from the right sight. In all cases was ischemic stroke, during operation $65 \%$, after operation $35 \%$. The mortality in hospital is $10 \%$ ( 2 patients). The total index for stroke after surgery is $1.7 \%$.

Conclusions: main part of stroke begins during operation. Not in all cases stenosis of carotid artery cause Stroke. Most of patients suffer from arterial hypertension and diabetes mellitus. The percentage of Stroke after surgery correspond to other cardiosurgical clinics. Basing on the results, we can assume that ischemic stroke mostly have hypoxic genesis and not thromboembolic.

\section{P216}

Multivalve heart disease - interventional treatment strategy

Abdurashid Mussayev, Serik Alimbayev, Timur Lesbekov, Lazzat Abikeeva, Saule Kadirova, Marat Aripov, Aigerim Sadykova, Aidyn Kuanyshbek, Yuriy Pya

National Research Center for Cardiac Surgery, Astana, Kazakhstan

Correspondence: Abdurashid Mussayev

Journal of Cardiothoracic Surgery 2017, 12(Suppl 1):P216

\section{Withdrawn}

\section{P217}

Mahaim fibers accessory pathways: electrocardiographic exercise testing

Andrey V. Tsokolov', Dmitrii A. Lushchenkov²

${ }^{1}$ Federal State Agency «1409 Clinical hospital of the Baltic Sea fleet», Kaliningrad, Russian Federation; ${ }^{2}$ Hospital Maternoinfantil - Universitat de Barcelona Sant Joan de Deu, Barcelona, Spain

Correspondence: Andrey V. Tsokolov

Journal of Cardiothoracic Surgery 2017, 12(Suppl 1):P217

Objective: Information about Mahaim fibers (MF) accessory pathways (AP), in contrast to atrioventricular (AV) pathways in terms of the exercise and electrophysiological testing to date is fragmentary.

Methods: The most common difficulties in ECG-diagnostics of MF are caused by the absence of initial QRS-complex expansion which is typical for WPW-syndrome due to delta-wave and PQ-interval reduction. Such patients are often diagnosticated with a wrong diagnosis of "myocardial infarction". As comparative data on such AP options are not given in literature available, and electrocardiographic indices of the persons with MF are non-systemic and not completely presented in view of exercise testing (as well as drug testing), we have tried to eliminate this gap in diagnostics.

Results: In contrast to Bundle of Kent AP at which the QRS-complex expansion or delta-wave occurrence/increase can be observed in association with heart rate (HR) increase, in case of MF such tachydependent QRS expansion is not completely observed! QRS-complex length remains unchanged. As an example, the results of ergometric exercise testing of a male patient, age 22 are presented. The results of Holter monitoring are similar to ergometric testing results. Principal differences between MF and Bundle of Kent AP besides exercise testing are registered in case of drug testing. During Adenosine triphosphate solution intravenous bolus injection under MF presence the following indices are notified: 1) sinoatrial (SA)-node rate increase with consequent reduction; 2 ) full temporary functional physiological AV-block; 3) no atrium pulses propagation to ventricles due to AP anatomic features, and 4) no QRS-complex expansion.

Conclusions: The main MF criteria, using male patient's data as an example, all the criteria listed below and moreover combined should be considered: - Left bundle branch block; - QRS $\leq 150 \mathrm{~ms}$; - Alpha- 
angle from 0 to -75 degrees - R>S V4IV5; - No changes in QRScomplex shape and length under HR change.

\section{P218}

Our experience of catheter ablation in patients with WPW syndrome

Adil K. Baimbetov, Kanat A. Yergeshov, Kenzhebek A. Bizhanov, llinara A. Yakupova

Syzganov' National Scientific centre of Surgery, Almaty, Kazakhstan

Correspondence: Adil K. Baimbetov

Journal of Cardiothoracic Surgery 2017, 12(Suppl 1):P218

Aims: to present results of radiofrequency ablation (RFA) in patients with WPW syndrome.

Material and methods. From 2014 to 2016, we performed electrophysiological study (EPS) and RFA on 119 consecutive patients with different types of WPW syndrome. Patients age ranged from 3 to 62 years (mean $29.5 \pm 14.5$ yrs). 88 patients $(73.9 \%)$ were diagnosed with manifest WPW syndrome, 24 (20.2\%) had intermittent WPW syndrome, 7 (5.9\%) and had concealed form. Patients had 0.5 to 25 years' (mean 18.4 \pm 8.5 yrs) history of tachycardia episodes. The left side APs (accessory pathways) were accessed via the left femoral artery. Radiofrequency ablation of the AP then would start with the following parameters: $\mathrm{P}=42.1 \pm 5.2 \mathrm{Wt} ; \mathrm{T}=$ $55 \pm 3.7^{\circ} \mathrm{C} ; \mathrm{I}=155.3 \pm 55.7 \mathrm{Ohm}$, duration $60 \mathrm{sec}$, each session. Conduction over an AP typically terminated after 5 to 10 seconds of initial application of RFA. Post-ablation follow-up EPS was then done using overdriving and programmed pacing to see if tachycardia could be induced; $0.5 \mathrm{mg} / \mathrm{kg}$ body weight ATP (adenosine) would be injected to rule out persistence of an AP.

Results. In two patients (1.7\%) tachycardia returned next morning postop. In one, a repeat RFA successfully terminated the AP conduction. In other patient, a repeat EPS revealed para-Hisian AP. The overall success rate of RFA procedure in WPW patients was as high as $97.5 \%$ (without repeat intervention). In a patient with recurrence of tachycardia, the repeat RFA helped completely eliminate the AP. No other perioperative complication was reported.

Conclusion. Radiofrequency ablation is an effective, definitive and relatively safe treatment option for patients with the WPW syndrome. Success rate of the procedure is close to $98 \%$ and relieves the patients of a life-long use of antiarrhythmic drugs.

P219

Treatment of postoperative mediastinitis in cardiac surgery patients

Vyacheslav Potapov, Evgeny Kokhan, Oleg Asanov

Military medical academy named after S.M.Kirov, Moscow, Russian

Federation

Correspondence: Vyacheslav Potapov

Journal of Cardiothoracic Surgery 2017, 12(Suppl 1):P219

Background Deep sternal wound infection (DSWI) after open heart surgery is a surgical complication with a high mortality rate, long period of hospitalization, the need for repeated surgical procedures and high cost of treatment. Objective: to analyze the results of surgical treatment of patients with DSWI in a multi-hospital.

Methods In the period from January 2010 to January 2017 in the purulent surgery department of 3 Central military clinical hospital named after AA Vishnevsky were treated 60 patients with infectious complications sternotomy wounds (ICSW), men - $98.3 \%$ and women $1.7 \%$, respectively. The average age of patients was 65.2 years (range 45 to 81 years).

Results According to the classification used R. M. El Oakley et al. (1996), DSWI identified 52 patients. Most of the patients (51 or $85 \%)$ the treatment was surgical. In connection with relapsing DSWI in 20 patients during the follow-up required repeat surgical intervention.

From September 2015, was selected a prospective group at the time of publication 20 patients with DSWI, in which treatment is applied modern method of vacuum drainage of wounds (VAC therapy) In this prospective group decreased the recurrence rate from $27.3 \%$ to $14.8 \%$ compared with retrospective results of treatment of patients without the use of VAC therapy.

Of all patients treated died $6(10 \%)$ patients. Three, severe sepsis with multiple organ failure. One patient from acute transmural myocardial infarction \& one from an extensive ischemic stroke. In a prospective group died, one patient with decompensation of diabetes, the increase of acute renal failure on the background of diabetic nephropathy.

Conclusions Treatment of DSWI should be early, comprehensive, sequential inclusion of the methods of local effects on wound process.

Our observations confirm the positive role of the method of VACtherapy in reducing the number of repeated surgical interventions with DSWI.

\section{P220}

An anterior mediastinal, giant, thymic neuroendocrine carcinoma

Alkin Yazicioglu', Seda Yamak ${ }^{2}$, Mahmut Subasi ${ }^{1}$, Fatmanur Celik Basaran ${ }^{1}$, Erdal Yekeler

${ }^{1}$ Turkiye Yuksek Ihtisas Training and Research Hospital, Thoracic Surgery and Lung Transplantation Clinic, Ankara, Turkey; ${ }^{2}$ Turkiye Yuksek Ihtisas Training and Research Hospital, Department of Pathology, Ankara, Turkey

Correspondence: Alkin Yazicioglu

Journal of Cardiothoracic Surgery 2017, 12(Suppl 1):P220

Objective: Thymic neuroendocrine carcinomas (NECs) are rare malignant tumors with poor prognosis, originating from Kulchitsky-cells. They have tendency of local invasion, distant metastasis, and constitute $2-4 \%$ of mediastinal tumors.

Methods: 13-year-old male was referred with complaint of swelling in anterior chest-wall.

Results: On evaluation, longitudinal, giant, $16 \times 10 \times 9 \mathrm{~cm}$ mass was observed in the anterior-mediastinum. Based on transmediastinalbiopsy results and normal urological examination findings, an undifferentiated carcinoma was reported. Surgical excision was planned; sternotomy and collar incision with mediastinal mass excision, pericardial graft reconstruction and left-upper-lobe wedgeresection performed. The mass was closely adjacent to superior vena cava and azygos-vein medially. It was also adjacent to arcus-aorta, descending-aorta, left subclavian-artery, left common carotid-artery, and left jugular-vein superiorly and laterally without invasion. Brachiocephalic vein was passed across the mass transversally. The mass was excised from all vascular structures, including the brachiocephalic vein. The mass, which was spread from the posterior to pericardium, was excised with pericardium and pericardial reconstruction was performed. Wedge-resection was applied to left-upper-lobe of lungs for invasion; mass was totally excised. Left phrenic-nerve was excised due to being left in mass, and diaphragm-plication-surgey was performed. The pathological examination was reported as an undifferentiated NECs, and it was considered as Masaoka-Stage3 and T3N1M0. Chemotherapy and radiotherapy were used adjuvantly.

Conclusions: According to the histopathological examinations, thymic NECs show positivity's for at least two methods of synaptophysin, chromogranin, and CD56 immunohistochemical staining. In our case, there were expressions for synaptophysin and chromogranin. These tumors can reach to large sizes, and metastases may develop even after years of total excision. Adjuvant chemotherapy is recommended following surgery, and radiotherapy should be added to the treatment to prevent localrecurrences. Patients should be closely followed for metastasis and recurrence. Our case received chemotherapy and radiotherapy following complete excision, and is still under medical-oncology follow-up with survival of 33-months.

Consent for publication

Written informed consent to publish was obtained from the patients involved in this study. 
P222

Migration of thymic cysts

Alkin Yazicioglu', Serhat Koca², Mahmut Subasi', Nesrin Turhan ${ }^{3}$, Yavuz Fuat Yilmaz ${ }^{4}$, Erdal Yekeler'

${ }^{1}$ Turkiye Yuksek Ihtisas Training and Research Hospital, Thoracic Surgery and Lung Transplantation Clinic, Ankara, Turkey; ${ }^{2}$ Turkiye Yuksek Ihtisas Training and Research Hospital, Department of Pediatric Cardiology, Ankara, Turkey; ${ }^{3}$ Turkiye Yuksek Ihtisas Training and Research Hospital, Department of Pathology, Ankara, Turkey; ${ }^{4}$ Numune Training and Research Hospital, Department of Ear, Nose, and Throat Surgery, Ankara, Turkey

Correspondence: Alkin Yazicioglu

Journal of Cardiothoracic Surgery 2017, 12(Suppl 1):P222

Objective: Thymus begins development from the third-cleft and progresses along the thymopharyngeal pathway. Thymic cysts are thought to develop from the cells present in this pathway. Although rare, congenital thymic cysts have a cervical or thoracic component.

Methods: Medical records of 2 patients were analyzed.

Results: First case was 30-month-old girl, hospitalized with complaint of mass in the neck, enlarging within the past one-year. Cystic mass lesion measuring $43 \times 126 \times 30 \mathrm{~mm}$ in size, beginning in the left submandibular region and extending to the anterior mediastinum and the vicinity of aortic arch and left main pulmonary artery, was detected by thoracic-CT. Cervicothoracic MRI revealed the cyst was displaced the left jugular vein on the posteromedial. The mass was excised with sternotomy using a left cervical incision. The histopathological examination revealed unilocular-thymic cyst. Second case was 25-year-old male, examined after the detection of cystic mass lesion measuring $6 \times 9 \times 7 \mathrm{~cm}$ in size, located in the right paracardial-region on thoracic CT. After right mini-thoracotomy, mass which was in close vicinity to the pericardium was detached. When the lesion was dissected, it was revealed that the lesion was adherent in a restricted region of the pericardium and cyst showed a superiomedial extension towards the mediastinum with a stalk of approximately $6 \mathrm{~cm}$ at the apex. The intact cyst was resected and it was diagnosed as a thymic cyst.

Conclusions: Thymic cysts are divided into two categories as congenital and acquired. The latter cysts are multilocular and congenital cysts can be unilocular or multilocular. Congenital thymic cysts are filled with unilocular cystic-fluid and possess a thin wall. Upon the histopathological examination, the presence of Hassall's corpuscles in congenital thymic cysts is diagnostic. Migration to the cervical or thoracic regions is quite rare and its treatment is en-bloc excision. The risk of recurrence is quite rare, compared to the complete excision.

Consent for publication

Consent to publish was obtained from the patient included in this study.

\section{P223}

\section{Pericardial infected cyst}

Fadil Gradica', Lutfi Lisha', Alma Cani ${ }^{2}$, Dhimitraq Argjiri ${ }^{3}$, Fahri Kokici ${ }^{2}$, Danjela Xhemalaj ${ }^{4}$, Laureta Bica ${ }^{5}$, Laureta Karaulli ${ }^{6}$, Ylber Vata ${ }^{7}$, Zamira Arifaj $^{8}$, Sali Gradica ${ }^{9}$, Flora Gradica ${ }^{10}$

'University Hospital "Shefqet Ndroqi" Thoracic Surgery Service, Tirane, Albania; "University Hospital "Shefqet Ndroqi" Anestesiology reanimation Service, Tirane, Albania; ${ }^{3}$ University Hospital "Shefqet Ndroqi" Pneumology Service, Tirane, Albania; ${ }^{4}$ University Hospital "Shefqet Ndroqi" Anatomopathology Service, Tirane, Albania; ${ }^{5}$ University Hospital "Shefqet Ndroqi' Kardiology Service, Tirane, Albania; ${ }^{6}$ University Hospital "shefqet Ndroqi' Pneumology Service, Tirane, Albania; ' University Hospital "Shefget Ndroqi" Visceral Surgery Service, Tirane, Albania; ${ }^{8}$ Medical Faculty of Science Technice Tirana Albania, Tirane, Albania; ${ }^{9}$ Public pharmacy service, Kamez,Tirane, Albania; ${ }^{10}$ Public pharmacy service, Tirane, Albania

Correspondence: Fadil Gradica

Journal of Cardiothoracic Surgery 2017, 12(Suppl 1):P223
Objective: Pericardial infected cysts are rare lesions. These benign cysts are located in the middle mediastinum. The incidence of pericardial cysts is about 1 per 100,000 (1). Pericardial cysts are uncommon benign congenital lesions in the middle mediastinum.

Methods: We present a 56 -year-old woman who was referred to the emergency department with dyspnea and persistent non productive cough,chest pain,temperature 39,2 celsius degree. In physical exam, no abnormality was found. Her past medical history was normal. On CT -scan was revealed a cystic formation of the antero-superior mediastinum. No pericardial effusion was found.

Results: The patient underwent surgery. After lateral anterior dexter toracotomy, a huge cyst measuring approximately $7 \times 6 \times 5 \mathrm{~cm}$ in diameters was found on the right side and outside the pericardium that was totally excised. After 7 days, the patient was discharged and pathologic report confirmed preoperative diagnosis of pericardial cyst.One month later developed pericarditis.

Conclusions: Morbidity and mortality are very low. Surgery has been demonstrated as the only definitive curative treatment (1)

Key words: Pericardial cyst, pericardial effusion, pleural effusion, mediastinal cyst.

Consent for publication

Written informed consent to publish was obtained from the patients involved in this study.

\section{P224}

Surgical treatment of a tracheal squamous cell carcinoma

Alkin Yazicioglu, Mahmut Subasi, Ahmet Güngör, Fatmanur Celik

Basaran, Erdal Yekeler

Turkiye Yuksek Ihtisas Training and Research Hospital, Thoracic Surgery and Lung Transplantation Clinic, Ankara, Turkey

Correspondence: Alkin Yazicioglu

Journal of Cardiothoracic Surgery 2017, 12(Suppl 1):P224

Objective: Primary neoplasms of trachea are uncommon malignancies which account for about $0.2 \%$ of all respiratory tract malignant lesions. Most common histologic types are squamous-cell carcinomas (SCCs) and adenoid cystic carcinomas (ACCs).

Methods: A 69-year-old male patient was referred with a complaint of cough.

Results: Fiberoptic bronchoscopy showed a polypoid mass lesion of $1 \mathrm{~cm}$ in the tracheal membranous area, $7 \mathrm{~cm}$ distal from the cord vocals and $6 \mathrm{~cm}$ proximal to the carina. Bronchoscopic biopsy confirmed a SCC. An esophageal SCC was considered in the differential diagnosis; however, thoracic CT and esophagoscopy did not show any sign for an esophageal carcinoma. Therefore, a surgical excision with right thoracotomy was planned, and tracheal segment excision $(2.1 \mathrm{~cm})$ with an end-to-end anastomosis was performed. No postoperative complication was observed. The chest tube was removed on day2, and the patient was discharged on day 6 .

Conclusions: Squamous-cell carcinomas of traches usually originate from the tracheal membranous face, and show an ulcerativemorphology. The prevalence peaks at the sixth and seventh decades of life, and is closely related to smoking. In the study by Gaissert et al., tracheal SCC cases were compared with tracheal ACC cases, and they found that SCC cases were mostly male with a smoking and alcohol use history, diagnosed with hemoptysis in general, and tender to have other organ cancer. Our case had no complains of hemoptysis and have no previous cancer diagnosis, however, he had a 60 pack/year cigarette-history. In the aforementioned study, complete surgical excision and negative surgical margins were found to be the most effective parameters for survival. Surgical excision is recommended to improve quality of life and ensure long-term survival. Five- and 10 -year survival rates are $40-50 \%$ and $20-30 \%$, respectively in the surgically treated tracheal primer SCC cases. At six months, our case is still under follow-up with no complaints. 


\section{Consent for publication}

Written informed consent to publish was obtained from the patients involved in this study.

\section{P225}

Pericardial benign cystic mesothelioma

Alkin Yazicioglu', Mahmut Subasi ${ }^{1}$, Neslihan Inci Zengin², Ahmet Gungor ${ }^{1}$, Sinan Turkkan ${ }^{1}$, Erdal Yekeler ${ }^{1}$

${ }^{1}$ Turkiye Yuksek Ihtisas Training and Research Hospital, Thoracic Surgery and Lung Transplantation Clinic, Ankara, Turkey; ${ }^{2}$ Turkiye Yuksek Ihtisas Training and Research Hospital, Department of Pathology, Ankara, Turkey

Correspondence: Alkin Yazicioglu

Journal of Cardiothoracic Surgery 2017, 12(Suppl 1):P225

Objective: Benign cystic mesotheliomas (BCMs) are also known as multilocular mesothelial inclusion-cysts, frequently observed in females, localized at pelvic peritoneum. It is rarely seen in thoracic and mediastinal areas, and there are a few cases in the literature.

Methods: 28-year old female, presented with cough. On chest x-ray, a mass with defined margins was detected.

Results: Thoracic CT revealed cystic lesion, $8 \times 6 \times 6 \mathrm{~cm}$ in left hemithorax next to pericardium. Preliminary diagnosis was pericardial cyst, and excision with VATS was planned. A thin-walled, transparent cyst was detected upon entry. The findings suggested a pericardial cyst, and cyst wall was completely excised. Drainage tube was removed on day2, and she was discharged on day4. On pathological examination; 4-5 cysts adjacent to one-side of the fibroadipose tissue, the largest 1,2 cm diameter, containing transparentliquid, covered in a distinguishable whitish-membrane, appearing as adjacent bubbles, were detected. In addition, there was a larger cyst $(8 \mathrm{~cm})$ comprising one chamber. Surfaces of both large cyst and cyst comprising adjacent bubbles were covered in a single layer of mostly squamous mesothelial cells. Immunohistochemical analysis; epithelial mesothelial cells layering the inner part of the cyst were calretinin(+)/CD31(-), endothelial cells of the vessel wall were calreti$\operatorname{nin}(-) / C D 31(+)$.

Conclusions: $\mathrm{BCMs}$ are benign pathologies frequently originating from peritoneum, and rarely seen in mediastinum. There are three cases in the literature with pleural-origin, and two cases with pericardial-origin. The proliferation rate is very slow with a low potential of malignant transformation. Metastatic disease has not been reported; however, close follow-up is recommended, as these lesions have a potential for local recurrence. There is no standard protocol for the treatment of this cystic neoplasia, and the World Health Organization (WHO) classification is still controversial. We recommend surgical excision and close follow-up in these cases. Our case is still in her postoperative first year without any recurrence.

\section{Consent for publication}

Written informed consent to publish was obtained from the patients involved in this study.

\section{P226}

Infantile mediastinal desmoid fibromatosis

Alkin Yazicioglu' ${ }^{1}$, Mahmut Subasi ${ }^{1}$, Nesrin Turhan ${ }^{2}$, Ahmet Gungor ${ }^{1}$

Erdal Yekeler ${ }^{1}$

${ }^{1}$ Turkiye Yuksek Ihtisas Training and Research Hospital, Thoracic Surgery and Lung Transplantation Clinic, Ankara, Turkey; ${ }^{2}$ Turkiye Yuksek Ihtisas Training and Research Hospital, Department of Pathology, Ankara,

Turkey

Correspondence: Alkin Yazicioglu

Journal of Cardiothoracic Surgery 2017, 12(Suppl 1):P226

Objective: Desmoid fibromatosis is rare, locally-aggressive benign tumor. While it is very uncommon in infantile age and in mediastinum, there are a small number of pediatric-cases.

Methods: 19-month-old male baby was admitted with fatigue and shortness of respiration.
Results: Thoracic CT revealed solid mass of $14 \times 10 \times 8 \mathrm{~cm}$ with defined margins, containing heterogeneous densities, which compresses the heart towards the left in the anterior mediastinum. Patient with a normal urological evaluation underwent transthoracic-biopsy; however, not diagnostic. Subsequently, en-bloc excision was performed via sternotomy and clamshell incision. A solid, grey-white colored mass with a shiny cross-section was detected in the gross examination. Intercrossing bundles and a well-defined tumor consisting of dense collagenous stroma were detected in the microscopic examination and tumor comprised fusiform cells with vesicular chromatin, elongated nucleus and eosinophilic cytoplasm was found. Vimentin and $\beta$-catenin staining pattern was diffused, and smooth muscle actin staining pattern was focal in the immunohistochemical staining. CD34, S-100, and desmin staining were not observed. Ki-67 proliferation index was low (3\%) and mitosis number was one. Based on these findings, desmoid-type fibromatosis was reported.

Conclusions: Although desmoid fibromatosis can be seen in any localization, it most frequently involves subcutaneous tissues, extremities, and abdomen. It is usually seen in adults and is very rare in children and in the mediastinum. There are less than 10 cases with mediastinal localization and pediatric occurrence. To distinguish from lymphoma, germ cell tumors and thymic pathologies, definitive diagnosis should be performed, as the mass is localized to the anterior mediastinum in pediatric cases. It can reach large sizes asymptomatically, and it is rare, locally invasive fibroblasticproliferation with low metastatic potential. Complete surgicalexcision is recommended; however, recurrence can be seen. Due to the risk of recurrence, close follow-up is recommended. Chemotherapy and radiotherapy can be also useful in reducing risk of local recurrence.

Consent for publication

Written informed consent to publish was obtained from the patients involved in this study.

P227

Diseases of mediastinum, inducing heart pathology

Taalai Z. Kudaiberdiev', Alymkadyr S. Beishenaliev², Gulzada A. Imanalieva', Azat A. Togochuev

${ }^{1}$ Research Institute of Heart Surgery and Organ Transplantation, Bishkek, Kyrgyzstan; ${ }^{2}$ Kyrgyz State Medical Academy, Bishkek, Kyrgyzstan

Correspondence: Taalai Z. Kudaiberdiev

Journal of Cardiothoracic Surgery 2017, 12(Suppl 1):P227

Objective: To determine etiologic factors of heart pathology in diseases of mediastinum.

Methods: Presented 75 cases of different diseases of mediastinum, that were reasons of pathologic changes in the heart. Amongst those were 62 patients with primary lymphatic complex in tuberculous lesion of lymphatic vessels in mediastinum, 8 patients were with lymphomas of mediastinum, and 2 of them were with Hodgkin's lymphomas. 2 patients were with malignant thymomas and 1 was with tumour of trachea. In all cases diagnosis was verified by cytologic and histologic studies, with X-ray and MSCT, and Echo of the heart and vessels.

Results: patients with tuberculous lesion of mediastinal lymphatics had developed in 47 cases exudative-fibrinous and in 15casesconstrictive pericarditis. Lypmhomas of mediastinum had led to exudative $(n-6)$ and constrictive (n-2) pericarditis. In malignant thymomas there were observed intergrowth into pericardium (constrictive-type) with thrombosis of the venae cavae in 1 case. Patients with trachea's tumour had developed intergrowth of tumour into pericardium with secondary exudative-hemorragic pericarditis. All patients undergone combined surgical treatment with pericardial drainage in 54 cases and in 19 cases pericardectomy, with consequential anti-tuberculous, chemo and radiotherapy.

Conclusions: Heart is positioned in mediastinum and is an anatomical part of it, has direct connection with the other organs of it, therefore pathology of any other organ of mediastinum often can lead to heart's lesion, requiring surgical treatment. 
P228

Videothoracoscopy in the diagnosis and treatment of thymoma

Bakhytzhan Kerimbekovich Shirtaev, Mukhtar Magzumovich Sundetov, Ruslan Zhumakhanovich Kassenbayev, Denis Sergeevich Voronin, Nazira Zholdibayevna Erimova, Daniyor Rahmatulaevich Kurbanov National Scientific Center for Surgery, Almaty, Kazakhstan Correspondence: Ruslan Zhumakhanovich Kassenbayev Journal of Cardiothoracic Surgery 2017, 12(Suppl 1):P228

Background: Minimally invasive surgery is increasingly introduced in the surgical management of thymoma.

Objective: to determine the effectiveness of videothoracoscopy in the diagnosis and treatment of thymoma.

Methods: Since 2010, in our clinic 27 videothoracoscopies were performed in patients with thymoma. The age of the patients ranged from 8 months to 64 years. The number of women and men was the same. In 5 patients, thymoma was combined with myasthenia. Videotoracoscopy was performed under general anesthesia with one-lung ventilation.

Results: Videotoracoscopy on the right side was performed in 18 patients, 6 patients were performed on the left side. The choice of approach was determined by the localization of thymoma. In 3 cases, the operation was completed with a videothoracoscopic biopsy due to the prevalence of the malignant process. 19 patients underwent VATS thymectomy. The remaining 5 patients required a conversion. In 3 cases this was due to the germination of the tumor into the adjacent lung. These patients were performed thoracotomy, thymectomy with a resection of the lung. In 1 patient during the video-thoracoscopy was established that thymoma germinated the lung root and pericardium, therefore, thoracotomy, thymectomy with pneumonectomy and resection of the pericardium part was performed. The cause of conversion in 1 case was profuse bleeding from a damaged vessel. There were no lethal outcomes in the early postoperative period. In three patients developed a myasthenic crisis, which managed to be stopped in intensive care unit.

Conclusions: Videothoracoscopy allows to determine the localization and spread of the tumor, morphologically verify thymoma and avoid unnecessary thoracotomy. VATS thymectomy is an effective method of surgical treatment and reducing the duration of post-operative hospital period.

\section{P229}

Odontogenic mediastinitis, grim complication of oral infections, a single center's experience

Calin Tunea, Ioan A. Petrache, Gabriel V. Cozma, Iris Miron, Voicu T.

Voiculescu, Vasile Gaborean, Brindusa Laitin, Ovidiu N. Burlacu

Spit Clinic Mun Urg Timisoara, Timisoara, Romania

Correspondence: Calin Tunea

Journal of Cardiothoracic Surgery 2017, 12(Suppl 1):P229

\section{Objectives}

Poor oral hygiene is a risk factor for a potential deadly disease acute necrotic mediastinitis. Eastern european hospitals still have a high rate of adimissions for patients with this disease originated in odontogenic infections. We present the experience of our center in dealing with this patient cathegory.

\section{Methods}

For this study we reviewed the charts and operating logs of 24 patients, on a sixteen year interval. We included in our statistics the patients with odontogenic DNM only. We performed emergency cervicotomy and mediastinal drainage in all cases, with repeated daily lavage. We performed thoracotomy in 10 cases, 8 of which at submission, with 21 pleural drainages.

Results

Complications occured in $79,16 \%$ of the cases - ARDS, MSOF, hemorhage, empyema, purulent pericarditis, extended chest wall cellulitis, tracheal fistulas, cutaneous defects, vasculitis that required future surgical care. Mortality recorded was $29,16 \%$, with a mean hospital stay of 4.7 weeks, $80 \%$ of admission time was ICU care.

\section{Conclusions}

Odontogenic DNM is a very dangerous condition, with high mortality rate, mainly due to bacterial polymorphism and delayed diagnosis; unless aggresive surgical treatment associated with antibiotherapy is started promptly, the outcome is grim. Improvement in dental care of patients is mandatory to minimize DNM frequency.

\section{P230}

Early clinical and angiographical outcomes of delayed percutaneous coronary interventions in acute coronary syndrome Semen Chevgun, Bektur Daniyarov, Ildar Abdyldaev Institute of Cardiac Surgery and Organs Transplantation, Bishkek, Kyrgyzstan

Correspondence: Semen Chevgun

Journal of Cardiothoracic Surgery 2017, 12(Suppl 1):P230

Objective: In Kyrgyzstan due to economical difficulties percutaneous coronary interventions $(\mathrm{PCl})$ in patients (Pts) with acute coronary syndrome (ACS) are often performed with delay. Purpose of the study was to assess early clinical, electrocardigraphic and functional outcomes of PCl's performed with time delay from symptoms onset (TD).

Methods: Consecutive patients with ACS with ST elevation (STEMI) undergone $\mathrm{PCl}$ (34 Pts, "acute PCl"group) were included. Control group consisted of 39 STEMI Pts with medical therapy (MT) to include thrombolysis. We analyzed: maximal single lead ST elevation (STE-max, mm); percent reduction of STEmax on ECG from baseline to post $\mathrm{PCl}$ on third day or from initiation of therapy to third day (STE-rec); residual ST elevation in worst lead post PCl or therapy regardless of baseline elevation (ST-res) and analyzed in 3 cathegories - $<1 \mathrm{~mm} ; 1$ to $<2 \mathrm{~mm}$ and $\geq 2 \mathrm{~mm}$.

Results: Median TD in PCl group was $89.4 \pm 17.5$ hours and 5.3 hours in MT group. In PCl group STE-max was $2.83+1.07$ at baseline and $0.90+0.20 \mathrm{~mm}$ on third day post $\mathrm{PCl}(\mathrm{p}=0.02)$; STE-rec was $68 \%$ in $\mathrm{PCl}$ group and $39 \%$ in MT group $(p<0.01)$. Left ventricular ejection fraction (EF) in $\mathrm{PCl}$ group was $47.3+12.0 \%$ baseline and $52.8+10.1 \%$ post $\mathrm{PCl}(\mathrm{p}<0.05)$; in MT group it was $44.3+10.2 \%$ and $46.0+6.2 \%$ respectively $(p>0.05)$. In the subgroup of patients with ST-res $>2 \mathrm{~mm}$ there was no significant increase in EF post $\mathrm{PCl}$ and substantial greater proportion of patients with ST-res $>2 \mathrm{~mm}$ in MT group. Early mortality was $5.9 \%$ in $\mathrm{PCl}$ group and $17.9 \%$ in MT group.

Conclusions: Present study shows that delayed PCl in Pts with STEMI in the environment with economical difficulties and shortage of up to date antithrombotic therapy could still be superior to medical therapy. Long term results require further analysis.

\section{P231}

Occurrence and treatment of resistant form of arterial

hypertension in patients with renal artery stenosis

Vadim K. Seisembekov, Timur K. Seisembekov, Almas T. Begdildayev,

Elizaveta A. Dyisenbiyeva, Vladimir R. Muzayev, Seitkhan D. Djozhibayev

The Scientific-Clinical Center for Cardiosurgery and Transplantation,

Taraz, Kazakhstan

Correspondence: Vadim K. Seisembekov

Journal of Cardiothoracic Surgery 2017, 12(Suppl 1):P231

Objective: Resistant hypertension is an increasingly common medical problem and patients with this disease are at a high risk of cardiovascular events. The aim of the study was to determine the role and occurrence frequency of hemodynamically significant renal artery stenoses in patients (of Zhambyl region population, Kazakhstan) with resistant form of arterial hypertension. Additional aim of the study was to confirm the treatment efficiency of renal artery stenting for the treatment of resistant hypertension.

Methods: From 2015 through 2017, due to a resistant form of arterial hypertension, 181 patients with resistant hypertension were referred to our clinic. All patients underwent renal artery angiography.

Results: Renal artery angiography detected relative hemodynamically significant stenoses above $75 \%$ in 12 patients. In 9 cases, (75\%), bare 
metal renal stenting (Palmatz Blue and Hipocampus), and in 3cases (25\%), coronary drug elusion stenting (Orsiro and Resolute Integrity) were performed. Systolic blood pressure and diastolic blood pressure in $10(80 \%)$ patients showed a steady decrease from $192+/-14 \mathrm{~mm}$ $\mathrm{Hg}$ to $127+/-20 \mathrm{~mm} \mathrm{Hg}$ and from $130+/-7 \mathrm{~mm} \mathrm{Hg}$ to $96+/-10$ $\mathrm{mm} \mathrm{Hg}$, respectively. At the same time, 2 patients demonstrated no decrease in blood pressure, for which, 1 month later, the denervation of renal arteries (from both sides) was performed, upon which a steady positive result was achieved.

Conclusions: In our study, we have successfully demonstrated that renal artery stenting along with denervation of renal arteries in patients with resistant form of resistant hypertension leads to improved conditions. We showed that Zhambyl region population has $6.6 \%$ frequency occurrence of renal artery stenosis.We concluded that this technique can be an effective treatment way for resistant hypertension as it is localized procedure, minimally invasive, and has no systemic side-effects with very short recovery times.

\section{P232}

Single center expirience in usage of the ultrahigh pressure balloon catheters in dilatation of rigid vessel stenosis in patients with congenital heart disease

Manolis Pursanov, Michail Chiaurelly, Andrey Sobolev, Ulugbek Atajanov A.N. Bakoulev Scientific Center for Cardiovascular Surgery, Moscow, Russian Federation

Correspondence: Manolis Pursanov

Journal of Cardiothoracic Surgery 2017, 12(Suppl 1):P232

Introduction of the ultrahigh pressure balloons (UHPB), such as ATLAS GOLD and CONQUEST made by BARD firm (USA), which can withstand pressure up to 26-30 atm. allows to manage a rigid vessel stenosis in patients (pts) with various congenital heart diseases (CHD) in a new way of percutaneous transluminal balloon angioplasty (PTBA).

Aim. to exam our experience in usage of UHPB in patients with CHD and rigid vessel stenosis in various vascular systems.

Materials and methods. During the period from April 2015 until April 2017 in 66 pts were preformed PTBA of 84 segments of various vessel segments at age from 6 month to 32 years (10.34 \pm 5.57 years) and weight from $4.6 \mathrm{~kg}$ to $96 \mathrm{~kg}(33.8 \pm 28.2 \mathrm{~kg})$. In $39(59.1 \%)$ patients were previously performed ineffective repeated PTA (12-16 atm). Balloon dilatation using UHPB were preformed in 53 pts with pulmonary artery stenosis (71 segments); in 7 pts with aortic isthmus coarctation; 5 patient with stenosis of right ventricle- pulmonary artery conduit; in 1 patient with superior vena cava (SVC) stenosis. Pulmonary artery PTBA were performed after right ventricle outflow tract reconstruction in 23 patients due to tetralogy of Fallot, in 22 after radical surgery in complex CHD, in 4 after bidirectional cavopulmonary anastomosis.

Results. Usage of the UHPB were examined according to a full balloon expansion. In $98.6 \%$ of all cases (70 of 71 segments) of PTBA full expansion to the nominal volume were achieved. Only in 1 case $(1.4 \%)$ we were unable to achieve full expansion of the UHP due to fracture of previously implanted pulmonary artery stent. We've registered no complications associated with usage of UHPB.

Conclusion. Usage of UHPB is an effective and safe method of managing rigid stenosis of various vascular systems, allowing to eliminate stenosis in $98.6 \%$ of cases.

\section{P233}

Left atrial appendage occlusion to prevent stroke in patients with atrial fibrillation

Adil K. Baimbetov, Kanat A. Yergeshov, Kenzhebek A. Bizhanov Syzganov' National Scientific centre of Surgery, Almaty, Kazakhstan Correspondence: Adil K. Baimbetov

Journal of Cardiothoracic Surgery 2017, 12(Suppl 1):P233

Aim and Objective: To demonstrate our experience of the left atrial appendage occlusion in patients with atrial fibrillation with a high risk of thromboembolic and hemorrhagic complications.
Materials and Methods: From 2014, in our center we have implanted 50 devices -Amplatzer Cardiac Plug for the patients with different forms of atrial fibrillation. The indications for the procedure were a high risk of thromboembolic complications according to $\mathrm{CHA}_{2} \mathrm{DS}_{2}$-VASC $\leq 2$ points and hemorrhagic complications according to HASBLED $\leq 3$ points. Despite the risk of bleeding, all patients before surgery received warfarin to achieve a target INR. However, 2 days before the surgery warfarin canceled due to carrying out TEE monitoring to exclude the presence of thrombus in the left atrial appendage. In order to select the appropriate size of the device the size of left atrial appendage was measured using TEE and angiography. This was followed by the closure of the left atrial appendage using occluder through the transseptal puncture.

Results: All the procedures were successful. 2-3 months after surgery all the patients underwent TEE control, confirming the localization of device on the original site. In 1 patient, there has been a dislocation of the occluder from the left atrial appendage into the cavity of the left chambers of the heart which on the 2 nd day fallen to the thoracic aorta. After verification of deployed occluder site we were able to extract it from the cavity of aorta through endovascular access by a puncture of the left femoral artery. During extraction procedure special device- "traps" were utilized. All patients assigned to aspirin alone.

Conclusion: Our experience has shown high efficacy and safety of LAA occlusion with occlude device that can be recommended as a non-pharmacological method of stroke prevention in patients with atrial fibrillation with a high risk of thromboembolic and hemorrhagic complications.

\section{P234}

Immediate and long-term outcomes of stent implantation for coarctation or recoarctation of aorta

Manolis Pursanov, Bagrat Alekyan, Vladimir Podzolkov, Gulamzhan Dadabayev, Alexander Novak

A.N. Bakoulev Scientific Center for Cardiovascular Surgery, Moscow, Russian Federation

Correspondence: Manolis Pursanov

Journal of Cardiothoracic Surgery 2017, 12(Suppl 1):P234

Objective: To evaluate the effectiveness of stent implantation for coarctation or recoarctation of aorta based upon an assessment of immediate and long-term outcomes.

Methods: We have gathered experience in stenting of 92 patients aged 3 to 53 years ( $25.4 \pm 16.1$ years) and weighing from 15 to 108 $\mathrm{kg}(54,8 \pm 20.1 \mathrm{~kg})$ with aortic coarctation (CoA) and recoarctation (reCoA). CoA stenting was performed in 61 patients $(66.3 \%)$; reCoA in 31 patients (33.7\%). ReCoA developed after previous endovascular treatment in $16(51.6 \%)$ cases, and in 15 (48.4\%) - after surgical correction.

Results: 96 stents were implanted in 92 patients for the elimination of aortic isthmus stenosis. After stenting, systolic pressure gradient (SPG) in the isthmus decreased from $49.4 \pm$ 15.2 to $6.7 \pm 1.3 \mathrm{mmHg}$ on average ( $p<0.001)$; cross-sectional area of stenosis increased from $79.4 \pm 11.5$ to $138.2 \pm 35.2 \mathrm{~mm}^{2}$ $(p<0.001)$. The result of stenting was qualified as good (SPG <10 $\mathrm{mmHg}$ ) in $88(95.7 \%)$ patients, and as satisfactory (SPG from 15 to $40 \mathrm{mmHg}$ ) - in $4(4.3 \%)$ patients. Complications occurred in 6 $(6.6 \%)$ cases. During long-term period (from 6 months to 12 years) $62(69.3 \%)$ patients were examined: $40(64.5 \%)$ patients after CoA stenting and $22(35.5 \%)$ - after reCoA stenting. Improvement in general state and increase of exercise tolerance occurred in all patients in the long-term. State of 53 (85.5\%) patients was qualified as good and in 9 (14.5\%) patients - as satisfactory by reason of SPG increase $(n=8)$ and paracoarction aneurysm formation ( $n=1)$, which required repeated intervention in 9 cases.

Conclusions:

CoA and reCoA stenting results in SPG decrease in stenosis area and blood pressure normalization and is followed by a minimum number of complications. 
P235

Results of percutaneous coronary intervention in patients after coronary artery bypass grafting

Karen Petrosian, Leo Bockeria, Boris Mkrtychyan

Bakoulev SCCVS, Moscow, Russian Federation

Correspondence: Karen Petrosian

Journal of Cardiothoracic Surgery 2017, 12(Suppl 1):P235

Objective: To study immediate and long-term results of percutaneous coronary interventions $(\mathrm{PCl})$ in patients after coronary bypass graft surgery $(C A B G)$, taking into account the data of intraoperative angiography.

Methods: We analyzed the immediate results of $\mathrm{PCl}$ in 82 patients and long-term results in $35(42,7 \%)$ patients due to the dysfunction previously imposed grafts, who underwent CABG and intraoperative angiography (IA) from 2009 to 2016. Sixty-six patients were imposed 190 (2,3 0,8 in average for one patient) grafts. PCl were performed due to graft dysfunction which were revealed intraoperatively, during hospital stay and/or longterm period after $C A B G$, also in patients with incomplete revascularization or with progressive atherosclerosis in long-term period after CABG ta of intraoperative angiography.

Results: During intraoperative angiography, dysfunction was found in 59 (31.1\%) of 190 grafts (41 of 82 patients, respectively), and in the period from 1 to 64 months after CABG dysfunction was observed in 84 (44.2\%) grafts in 53 patients. Fifteen (18.3 grafts) of 82 patients underwent $\mathrm{PCl}$ on a hospital stage after CABG. PCI (of grafts and arteries both) was successful in $79(96.3 \%)$ patients. One patient $(1.2 \%)$ had residual stenosis equal to $30 \%$ due to extensive coronary bed calcification. In two patients (2.4\%) recanalization of the right coronary artery failed due to remote occlusive lesion. On a hospital stage we registered one lethal case (1.2\%). The clinical success was about $96.3 \%$, angiographic success was $95.1 \%$.

Long-term patency results we examined in $35(42.7 \%)$ patients. In 3 (8.6\%) patients of 35 stent occlusion was registered. In $2(5.7 \%)$ secondary $\mathrm{PCl}$ was performed and after one year on control angiography no signs of restenosis were registered.

Conclusions: It is necessary to perform intraoperative revascularization in patients with stenosis of grafts and/or anastomosis more than $50 \%$ which were revealed during intraoperative angiography and affect coronary hemodynamics.

\section{P236}

Transcatheter closure of postinfarction ventricular septal defect using the ASD occluders

Serik Alimbayev, Abdurashid Mussayev, Marat Aripov, Ermagambet Kuatbayev, Timur Lesbekov, Ivan Vakhrushev, Saule Kadirova, Kaysar Alimbayev, Gaukhar Amreeva, Eric Otarbayev

National Research Center for Cardiac Surgery, Astana, Kazakhstan

Correspondence: Abdurashid Mussayev

Journal of Cardiothoracic Surgery 2017, 12(Suppl 1):P236

Objective: We reviewed our experience at the National Research Center for Cardiac Surgery with percutaneous closure of postinfarction ventricular septal defects (PVSD) using the ASD occluder. PVSD occurs in $0.2 \%$ of myocardial infarcts and associated with high mortality and morbidity

Methods: Six patients with postinfarction VSD were treated for the period from November 2014 to April 2017. The mean age was $63 \pm 10$ year, $67 \%$ of the patients are females. For all patients who was treated percutaneously we used ASD occludes. Two patients underwent the closure in the acute phase (within 6 days from the infarct); the remaining patients underwent closure on day 12-67 after the diagnosis of the infarct.

Results: Half of the patients were in cardiogenic shock, and two of them was supported by an intraaortic balloon pump before the procedure and underwent primary percutaneous coronary intervention. One patient after PVSD closing was put on veno-arterial extracorporeal membrane oxygenation. The mean of the left ventricular ejection fraction was $42 \pm 12 \%$, and Qp/Qs averaged from 2.1 to 3.7 (mean 2.8 \pm 0.6 ). PVSD localization was apical for all patients. The diameter of PIVS was from $9 \mathrm{~mm}$ to $22 \mathrm{~mm}$ (mean diameter 15 \pm 6 ). After PVSD correction a moderate to large residual shunts were found in $33 \%$ of the patients. Flve patients were treated with a percutaneous ASD occluder devices by femoral approach, one by jugular vein and the 30 -day mortality was $17 \%$.

Conclusions: According to our data, PVSD closure with ASD occluders is effective and safe treatment. ASD occluder can be used as an alternative choice for PVSD closure.

P237

Single centre expirience: hybrid stenting of the pulmonary artery in patients with congenital heart disiase

Manolis Pursanov, Bagrat Alekyan, Konstantin Shatalov, Andrey Sobolev, Michail Chiaurelly, Alexander Kim, Irina Arnautova, Sherkhan Tursunov

A.N. Bakoulev Scientific Center for Cardiovascular Surgery, Moscow,

Russian Federation

Correspondence: Manolis Pursanov

Journal of Cardiothoracic Surgery 2017, 12(Suppl 1):P237

Objective: To determine the possibility of intraoperational hybrid stenting of the pulmonary artery (PA) stenting in surgical correction of complex congenital heart disease (CHD).

Methods: Hybrid stenting of the PA were performed in 15 patients with various $C H D$, age from 11 months to 19 years $(6,3+5,9$ years) and weight from $5,4 \mathrm{~kg}$ to $68 \mathrm{~kg}(21,3+21,4 \mathrm{~kg})$. Stenting of the pulmonary artery were performed: in 6 patients during hemodynamic correction of the CHD; in 2 patients during surgical correction of the tetralogy of Fallor; 6 after radical surgery of the complex CHD; in 1 patient during surgical closure of ASD in combination with left PA hypoplasia. Hybrid stenting we performed on schedule in 11 cases, and as an emergency procedure in 4 cases. In 7 cases stenting of the left PA we preformed though right pulmonary access. In 4 cases main access site of operation was pulmonary trunk, in 1 case- apex of the heart and in 2 case we used a vain access, in 1 case we performed bifurcation stenting throughout RVOT using two delivery systems for right and left PA. Balloon predilation was performed in 9 cases. In 14 cases we preformed angiography controlled stenting, in 1under visual observations.

Results: In 14 out of 15 patients stent was implanted into the obstruction zone (right or left PA, pulmonary trunk). In 1 case the procedure was complicated by stent dislocation and surgical removal was required. There was one lethal cause in early postoperative period due to increasing polyorganic insufficiency. In other patients no complications were observed in early postoperative period. Hybrid stenting of pulmonary artery allowed to eliminate stenosis in all the cased.

Conclusions: Intraoperational hybrid stenting is effective and reliably safe method of management pulmonary artery stenosis during openheart surgery of the congenital heart disease.

\section{P238}

Transcatheter closure of LVAD inflow cannula after partial myocardial recovery

Yuriy Pya, Abdurashid Mussayev, Serik Alimbayev, Serik Bekbosynov, Asel Medresova, Saltanat Andosova, Saltanat Dzhetybayeva, Muradym Murzagaliyev, Gulmira Momanova

National Research Center for Cardiac Surgery, Astana, Kazakhstan

Correspondence: Abdurashid Mussayev

Journal of Cardiothoracic Surgery 2017, 12(Suppl 1):P238

\section{Withdrawn}

\section{P239}

Transapical implantation of a balloon expandable valve in a failed mitral bioprosthesis in a pregnant woman

Yuriy Pya' ${ }^{1}$, Timur Lesbekov', Serik Alimbayev ${ }^{1}$, Abdurashid Mussayev' Aidyn Kuanyshbek', Lazzat Abikeeva', Marat Aripov', Aigerim Sadykova', Gulmira Rapilbekova², Baurzhan Zhunusov ${ }^{1}$

${ }^{1}$ National Research Center for Cardiac Surgery, Astana, Kazakhstan;

¿Univercity medical center, Astana, Kazakhstan

Correspondence: Abdurashid Mussayev

Journal of Cardiothoracic Surgery 2017, 12(Suppl 1):P239 
Objective: Redo surgery for failed mitral bioprosthesis is associated with high risk of complications both for patient and for fetus. In this setting "Valve in valve" implantation (T-VIV) could be an attractive treatment alternative. We report a case with the T-VIV in a pregnant woman with a failed mitral bioprosthesis.

Methods: Redo surgery for failed mitral bioprosthesis is associated with high risk of complications both for patient and for fetus. In this setting, T-VIV could be an attractive treatment alternative.

Results: Postoperatively, echocardiography revealed good mitral hemodynamic parameters. Transvalvular pressure gradients significantly decreased (maximal pressure gradient, $13 \mathrm{~mm}$. Hg; mean pressure gradient $7 \mathrm{~mm} \mathrm{Hg}$ ). The fetus after procedure was alive. The patient was discharged home in 5 days with NYHA functional class II.

Conclusions: This case demonstrate the feasibility and efficacy of transapical implantation of the Edwards SAPIENXT XT valve into a failed mitral valve bioprosthesis. This technique could be used to improve treatment options in such difficult cases.

\section{P240}

Influence of insulin resistance on the development of cardiovascular diseases in individuals of the Kazakh population Valeriy Benberin ${ }^{1}$, Tamara Voshenkova ${ }^{2}$, Nasrulla Shanazarov², Temirkhan Kulkhan, Sr. ${ }^{2}$

${ }^{1}$ Medical Center of the Presidential Affairs Administration of the Republic of Kazakhstan, Astana, Kazakhstan; ${ }^{2}$ Scientific Research Institute on Gerontology and Antiaging Medicine, Astana, Kazakhstan

Correspondence: Temirkhan Kulkhan, Sr.

Journal of Cardiothoracic Surgery 2017, 12(Suppl 1):P240

Objective: Investigate the biological basis of the body's susceptibility to insulin resistance that can be the basis for developing new strategies of cardiovascular diseases prevention and treatment in individuals of the Kazakh population.

Methods: The research was conducted on the basis of the RSE "Medical Center Hospital of the Presidential Affairs Administration of the Republic of Kazakhstan" in 2016-2017. In the study (n-456) and control (n-438) groups, a comparative analysis was conducted to determine the biochemical parameters of the fats and carbohydrates metabolism. The patients with verified insulin resistance were selected to the study group, and the polyclinic patients who is attending regular preventive examinations were included to the comparison group.

Results: There were comparative analysis between moleculargenetic markers and development of insulin resistance, ensuring the formation of diabetes in a second type in individuals of the European and Kazakh populations, carried out. We found influence on the development diabetes type 2 and cardiovascular complications from metabolic syndrome's elements, such as abdominal obesity, lipid imbalance in blood serum, hypertension and insulin dysfunction in midterm perspective: in 385 patients $(84.4 \%)$ in the study group and in 73 patients $(16.6 \%)$ in the control group $(p<0,05)$. The subsequent stages of our research can be carry out in molecular genetic studies using Chip-sequencing. Conclusions: The study showed that the assessment of genetic risk increases the possibility of using high-precision drug effects in the early development of insulin resistance. To compensate the effect of genetic variants in insulin resistances development on the manifestation of cardiovascular complications of type 2 diabetes, for each patient needs personalized approach for correcting the parameters of the metabolic syndrome and insulin dysfunction.
P241

The role of hyperlipidemia in the development of arterial hypertension in individuals of the Kazakh population Valeriy Benberin', Tamara Voshenkova², Amir Akhetov ${ }^{3}$, Nasrulla Shanazarov ${ }^{2}$, Temirkhan Kulkhan, Sr. ${ }^{2}$

${ }^{1}$ Medical Center of the Presidential Affairs Administration of the Republic of Kazakhstan, Astana, Kazakhstan; ${ }^{2}$ Scientific Research Institute on Gerontology and Antiaging Medicine, Astana, Kazakhstan; ${ }^{3}$ Medical Center Hospital of the Presidential Affairs Administration of the Republic of Kazakhstan, Astana, Kazakhstan

Correspondence: Temirkhan Kulkhan, Sr.

Journal of Cardiothoracic Surgery 2017, 12(Suppl 1):P241

Objective: Investigation of the prognostic significance of the serum lipid level in individuals of the Kazakh population for primary and secondary prevention of arterial hypertension.

Methods: The research was conducted on the basis of the RSE "Medical Center Hospital of the Presidential Affairs Administration of the Republic of Kazakhstan" in 2016. A comparative analysis of biochemical parameters of fat metabolism (total cholesterol, low density lipoprotein cholesterol, high-density lipoprotein cholesterol, triglycerides) was performed in study (n-180) and control (n-168) groups. The study group were included individuals of the Kazakh population aged 18-39 years with arterial prehypertension, in the control group - persons who comparable in nationality, sex, age, body mass index, but with normal blood pressure. Blood pressure levels were determined in accordance with the North American classification of 2003 (JNC-VII).

Results: A reliable relationship between the presence of hypercholesterolemia and arterial prehypertension was revealed, as well as a reliable correlation between the level of total cholesterol ( $p$ $=0.01)$, low-density lipoprotein cholesterol $(p<0.01)$ and systolic blood pressure level. According to nonlinear binary logistic regression, the level of total cholesterol is defined as a significant risk factor $(p=0.039)$. Since the patients of the study group showed a significant level of presence of relatives of the first line with cardiovascular diseases ( $p<0.01)$, the next stage would be the conducting of molecular genetic studies using Chip-sequencing. Evaluation of the main types of metabolism in 180 men under the age of 40 years with arterial prehypertension made it possible to define the predictive value of determining the level of total cholesterol.

Conclusions: The study would be continued with the aim of adjusting the molecular and genetic approaches in primary and secondary prevention of hypertension in individuals of the Kazakh population.

\section{P242}

Effect of the chronic metabolic acidosis on rat thoracic aorta reactivity

Paulo Roberto B. Evora, Willian Marcio da Silva, Agnes Afrodite S.

Albuquerque, Gustavo S. Barros, Luiza S. Mateus, Vitor T. Sarni, Adriana

Paula S. Schiavetto, Andrea Carla Celotto

Ribeirão Preto Medical School, University of São Paulo, SP, Brazil, Ribeirão Preto, SP, Brazi

Correspondence: Paulo Roberto B. Evora

Journal of Cardiothoracic Surgery 2017, 12(Suppl 1):P242

Introduction: More than one century there is some knowledge that the extracellular $\mathrm{pH}$ has promoted changes in the vascular tonus, which affects the circulation and the blood pressure control. The acid-base disorders are common in the medical practice and can vary from an acidosis or simple alkalosis to a mixed, complex and potentially fatal disorder. The metabolic acidosis occurs because the nonvolatile acids increase under conditions such as kidney disease, 
sepsis, and diabetes, commonly faced on heart surgery under cardiopulmonary bypass.

Objective: To investigate the effects of the chronic metabolic acidosis (CMA) on rat aorta reactivity as well as the mechanisms involved in this response.

Methodology: The CMA was induced by $\mathrm{NH} 4 \mathrm{Cl} 0.5 \mathrm{M}$ ad libitum + $0.02 \mathrm{M}$ by gavage during 10 days. The assessed ventilatory parameters were respiratory frequency (fR), tidal volume (Vt) and pulmonary ventilation (VE). The studies of the vascular reactivity were carried out by a dose-response curve to acetylcholine (ACh), phenylephrine (Phe), endotelina1 (ET-1) and angiotensin II (Ang II), in the aorta with and without endothelium, in absence and presence of the L-NAME.

Results: The acidosis induced by $\mathrm{NH} 4 \mathrm{Cl}$ reduced the $\mathrm{pH}$ to 7.17 (7.39 control), with levels of bicarbonate about 9.8 (21.9 control). As for the ventilatory parameters, there was an increase of the Vt on the second day of the treatment, which leads to an increase in the VE. In the studies of the vascular reactivity, the CMA have not changed the response to the Ach and ET-1, however, the vasoconstriction induced by Ang II and Phe were reduced after CMA, and this was restored by L-NAME.

Conclusion: The effect of the CMA on the vascular reactivity seems to be agonist-selective and the nitric oxide is involved in this response.

\section{P243}

Warfarin genotype polymorphisms in kazakhstani patients with mechanical circulatory support - left ventricular assist devices Ainur Akilzhanova', Madina Zhalbinova', Saule Rakhimova', Saltanat Andosova², Bagdat Abdirova², Zhaxybay Zhumadilov', Mahabbat Bekbosynova $^{2}$

${ }^{1}$ National Laboratory Astana,Center for Life Sciences, Nazarbayev University, Astana, Kazakhstan; ${ }^{2}$ National Scientific Cardiac Surgery Center, Astana, Kazakhstan

Correspondence: Ainur Akilzhanova

Journal of Cardiothoracic Surgery 2017, 12(Suppl 1):P243

Objective. Left ventricular assist devices (LVADs) have dramatically increased the survival of adults with end-stage systolic heart failure. However, rates of bleeding and thromboembolism remain high. Warfarin is a commonly used anticoagulant with a narrow therapeutic index and high rate of significant adverse reactions from both overand under-dosing. Several studies have shown that pharmacogenomic variants help predict stable warfarin dosing. Polymorphisms for VKORC1 and CYP2C9 are associated with increased warfarin sensitivity. The prevalence of these polymorphisms in patients with mechanical circulatory support (MCS) - LVADs is unknown.

Methods. Polymorphisms for VKORC1 and CYP2C9 were determined in 100 patients undergoing MCS surgery - implantation of LVADs at single clinic - National Research Center for Cardiac Surgery, Astana, Kazakhstan from December 2011 to July 2016. Postoperative warfarin dose, international normalized ratio (INR), and bleeding events were measured until discharge, 6 months, or composite end point (inhospital MCS recovery, heart transplant, or death).

Results. A total of $32.0 \%(32 / 100)$ of patients with implanted LVADs had at least one polymorphism: VKORC1 (14.0\%), CYP2C9*2 (15.0\%), CYP2C9*2 and VKORC1 (3.0\%). At discharge or before composite end point, patients with any polymorphism received a lower mean warfarin dosage than patients having no polymorphism $(3.2 \pm 1.5$ vs. $5.5 \pm 3.7 \mathrm{mg}, p=0.015)$ and achieved a similar mean INR $(2.20 \pm 0.67$ vs. $2.19 \pm 0.69, p=0.96)$. There was no significant difference in bleeding rates within 6 months or before composite end point $(6.13$ vs. 8.02 events/patient-year, $p=0.13$ ).

Conclusions. One or more polymorphisms for VKORC1 or CYP2C9 (associated with warfarin sensitivity) were found in 32.0\% of MCS/ LVAD patients. By using a warfarin genotype-guided approach, LVAD patients with polymorphisms received a lower warfarin dosage to achieve a similar INR, with similar bleeding rates, in comparison with no polymorphisms. A warfarin genotype-guided approach avoided excessive anticoagulation and its attendant bleeding risks.
P244

Minimally invasive direct-vision right minithoracotomy technique for mitral valve repair

Zhyhalkovich Aliaksandr, Ladygin Pavel, Chesnov Yury, Pyzhyk Roman, Bushkevich Marina, Piatrovich Natallia, Dydyshko Leonid, Ostrovsky Yury Republican Scientific and Practical Center of Cardiology, Minsk, Belarus Correspondence: Zhyhalkovich Aliaksandr

Journal of Cardiothoracic Surgery 2017, 12(Suppl 1):P244

Objective: Minimally Invasive right minithoracotomy technique for mitral valve pathology has become an alternative to the median sternotomy approach. We report a single-center experience with direct-vision minithoracotomy technique for mitral valve repair (DVMTMVR).

Methods: From September 2013 to March 2017 we performed 70 operations using DVMTMVR. It concerned 30 males and 40 females with the average age $47.4 \pm 11.6$ years (range $21-68$ years). All patients had prevalent mitral insufficiency (ERO $0.4 \pm 0.12 \mathrm{~cm} 2$, RV 50 $\pm 12 \mathrm{ml}$ ) due to the primary prolapse of posterior leaflet in 45 , anterior leaflet prolapse in 10 and bileaflet prolapse in 15 cases. 12 (17\%) patients were in NYHA III. We used a right minithoracotomy in fourth intercostal space 5 to $7 \mathrm{~cm}, \mathrm{CPB}$ was established with femoral artery and vein $(n=54$, vacuum assisted), right internal jugular vein $(n=10)$ or direct aortic $(n=6)$ cannulations. Mitral ring was implanted in all patients, e-PTFE neo-chordae implantation - $14(20 \%)$, resection of the leaflet - $40(57 \%)$, edge-to-edge repair - $7(10 \%)$, sliding plasty $-10(14 \%)$, plication of the leaflet - $16(23 \%)$.Concomitant procedures were MAZE procedure $(n=2)$, interatrial defect closure $(n=3)$, tricuspid valve repair $(n=3)$

Results: Mean CPB time and x-clamp time were $120 \pm 20.76$ and 84,5 $\pm 14,8$ min respectively. There were no in-hospital mortality, no major neurological events and no conversion to sternotomy. We had one rethoracotomy for bleeding and reversible ischemia of the right foot in two cases. We achieved good results of mitral valve repair in operating room in all cases. In follow-up (mean 16 months) we observed no or trivial MR in $60(86 \%)$ pts and mild MR (1+) in $10(14 \%)$ pts. There were no late deaths.

Conclusions: Minimally invasive direct-vision right minithoracotomy is a safe and reproducible technique for mitral valve repair with low rate of peri-procedural complications and good mid-term results.

P245

Our 3 year experience of minimally invasive perventricular off pump ventricular septal defect occlusion

Timur Raimkhanov, Oraz Mukashev, Adil Dyurzhanov, Daulet

Zhakipbayev, Yerlan Ordabayev, Zhanna Beisetayeva, Assem Sarsekeyeva, Yelena Khagay

Pavlodar Regional Cardiological Center, Pavlodar, Kazakhstan

Correspondence: Timur Raimkhanov

Journal of Cardiothoracic Surgery 2017, 12(Suppl 1):P245

Objective: analysis of immediate results of minimally invasive perventricular off pump ventricular septal defect (VSD) occlusion.

Methods: In the period from March 2014 to March 2017 were performed 29 operations according to this method in patients aged from 1,5 to 25 years old, weighing from $7 \mathrm{~kg}$ to $70 \mathrm{~kg}$ with different types of localization (perimembranous - 14, subaortal - 8, aneurysmatic - 4, muscular - 2, residual - 1) and dimensions (from 4 $\mathrm{mm}$ to $7 \mathrm{~mm}$ ) of defects. The calculated pressure in the pulmonary artery varied from 25 to $45 \mathrm{~mm} \mathrm{Hg}$. The $Q \mathrm{p} / \mathrm{Qs}$ ratio averaged 1.5. In the lower third of the sternum, a $3-4 \mathrm{~cm}$ incision is made and a Ushaped suture is placed on the anterior wall of the right ventricle in the epicenter of systolic tremor. Under transesophageal echocardiography control the right ventricle was punctured and the defect was closed with a special occluder that was $1 \mathrm{~mm}$ larger than the defect size.

Results: The closure of VSD according to this method was achieved in 27 patients $(93 \%)$, the duration of the operation was 50 minutes to 2.5 hours, intraoperative blood loss - 10 to $30 \mathrm{ml}$, lung ventilation - 2 to 3 hours, stay in the ICU for 3 to 12 hours, absence of 
cardiotonic support and blood transfusion, absence of heart valves regurgitation, removal of drainage for 1 day after surgery, duration of postoperative hospital stay from 3 to 5 days.

Conclusions: This technique of closing the VSD is a highly effective procedure, accompanied by minimal trauma and no consequences after cardiopulmonary bypass, in contrast to the classical technique. The period of hospitalization and rehabilitation are shortened; a good cosmetic effect is achieved.

P246

The use of mini-thoracotomy in the correction of septal heart defects in children and adolescents

Zhalil O. Sheishenov, Seitkhan D. Djozhibayev, Ikrom I. Mukhamedov, Serzhan K. Kuramys, Rakhat A. Aidarova, Erbol B. Ustemirov

The Scientific-Clinical Center for Cardiosurgery and Transplantation, Taraz, Kazakhstan

Correspondence: Zhalil O. Sheishenov

Journal of Cardiothoracic Surgery 2017, 12(Suppl 1):P246

Objective: The gold standard for open heart surgery is considered to be a full sternotomy incision. With the development of modern technology, improvement of anesthetic and perfusion protection allowing bypassing certain negative effects after the surgery, a full sternotomy access with minimal invasion of the heart is gaining an colossal attention within medical community. The aim of work was to show the results of ASD and VSD correction in children and adolescents using the right-sided anterior mini-thoracotomy.

Methods: From time period between 2010 and 2016, the centre implemented 280 ASD and VSD corrections. Importantly, patients with subpulmonic VSD and synastosis of the cartilaginous part of the ribs have been excluded. The cannulation of the vessels was central, i.e. ascending aorta and SVC through the wound, while IVC via a stab wound to the lateral surface of the chest.

Results: Deterioration of the surgical field view and complications in wound manipulation were not reported. In children weighing up to $20 \mathrm{~kg}$, the internal thoracic artery and the cartilaginous part of the rib retained in all cases. In patients weighing 20 to $40 \mathrm{~kg}$, depending on the availability of the ascending aorta, the stepwise-like cartilaginous part of the overlying rib, in some cases, was intersected with retaining intrathoracic artery. The length of the cutaneous incision varied from 4 to $7 \mathrm{~cm}$.

Conclusions: From our experience, the use of an anterior right-sided mini-thoracotomy allows safe and adequate septal defect correction of the heart. Moreover, in all patients, minimization of blood loss, reductions in pain level and time of mechanical ventilation were achieved. In addition, the duration of hospitalization showed a significant reduction. Finally, all patients and their relatives emphasized on experiencing an excellent cosmetic effect from these surgeries.

\section{P247}

The initial experience of Totally Thoracoscopic Closure for Atrial

Septal Defect on perfused beating hearts

Agzam Aldeshev, Galymzhan Sultanov, Zhanar Nurbay, Yerbol

Kudabayev

Scientific Research Institute of the Cardiology and Internal Diseases,

Almaty, Kazakhstan

Correspondence: Agzam Aldeshev

Journal of Cardiothoracic Surgery 2017, 12(Suppl 1):P247

Objective: To assess the results of totally thoracoscopic closure for atrial septal defect.

Methods: In 2017 totally thoracoscopic closure for atrial septal defect on perfused beating hearts is performed in 7 patients. All patients were female, average age is $27 \pm 8$. All patients had atrial septal defect without the upper or bottom edges of the septum. Average pressure in pulmonary artery averaged $35.7 \pm 12 \mathrm{~mm} \mathrm{Hg}$.

Results: In the early postoperative period no complications were recorded. Duration of the operation is less than 5 hours. On average the cardiopulmonary bypass time is $196 \pm 85 \mathrm{~min}$. Drainage losses averaged $253 \pm 120 \mathrm{ml}$. Patients were extubated in first 4 hours. Early activation of patients in the first 12 hours after operation is noted. Postoperative hospital stay is 5 days. This category of patients didn't need rehabilitation. The control echocardiography has not shown any residual shunt.

Conclusions: Totally thoracoscopic closure for atrial septal defect is a miniinvasive surgery, allowing keeping a thorax framework with positive impact on rehabilitation of the patient and his/her early activation.

P248

Totally Thoracoscopic Surgery of congenital septal defects: our first experience

Seitkhan Joshibyev', Ikromzhon Mukhamedov², Berik Bolatbekov ${ }^{3}$, Zhalil Sheishenov ${ }^{3}$, Serjan Kuramys ${ }^{3}$, Daulet Saparaliev ${ }^{3}$, Almas Alibekov ${ }^{3}$, Kulmariyam Sadykbergenova ${ }^{3}$, Dauren Narbayev ${ }^{\prime}$, Tolegen Egemberdiev ${ }^{1}$ ${ }^{1}$ Asfendiyarov Kazakh National Medical University, Almaty, Kazakhstan; ${ }^{2}$ International Kazakh-Turkish University, Turkestan, Kazakhstan; ${ }^{3}$ Research clinical center of cardiac surgery and transplantology, Taraz, Kazakhstan

Correspondence: Ikromzhon Mukhamedov

Journal of Cardiothoracic Surgery 2017, 12(Suppl 1):P248

Objective: Since improvements achieved in both endoscopic instruments and surgical skills, Totally Thoracoscopic Surgery (TTS), has been extensively used in clinical practice. We have demonstrated our first TTS's experience using three ports and endoscopy technology for the closures of simple congenital septal defects of the heart. The objective of the given work was to assess the new method's effectiveness and its safeness.

Methods: From the August, 2015, our centre has begun to perform TTSs, carrying out cardiac surgery for 2 types of simple congenital septal defects: closures of the atrial septal defects (ASD) was performed in $47(78 \%)$ cases, and closures of ventricular septal defects (VSD) of the heart was performed in $13(22 \%)$ patients. The average age of patients was $26 \pm 17$ years and the average weight was $49 \pm 23 \mathrm{~kg}$. Closure of the ASD was performed on the beating heart, while VSD closure carried out with aortic cross clamp. Cardiopulmonary bypass (CPB) was made by peripherally cannulation: Femoralis artery and vein + Jugular vein.

RESULTS: All operations were successful as there were no hospital mortality or major complications during observed and rehabilitation period. The mean duration of operation time was $252 \pm 51$ minutes and $249 \pm 26$ minutes for ASD and VSD respectively, at the same time, CPB times for ASD and VSD were $59 \pm 18$ and $88 \pm 20$ minutes, correspondingly. Aortic Cross clamp time during VSD closures were $70 \pm 14 \mathrm{~min}$. Moreover, the duration of stay in intensive care unit (ICU) was $16 \pm 5$ hrs, while the mechanical ventilation time and postoperative hospital stay were $172 \pm 130$ minutes and $6.4 \pm 2.1$ days, respectively. Follow-up transthoracic echocardiography before discharge showed no residual shunts in all patients.

Conclusions: We believe that the thoracoscopic closures of congenital septal defects using $T \mathrm{TS}$ is a safe and feasible procedure, presenting a high degree of satisfaction for cosmetic effect.

P249

Conventional and minimally invasive approaches in the surgery of mitral valve dysplasia

Dmitrii Anatolyevich Kondratyev, Dmitrii Yuryevich Kozmin, Dmitrii Sergeevich Tungusov, Sergeii Alexeevich Makeev, Soslan Taisumovich Enginoev, Yliya Borisovna Martyanova, Igor lonovich Chernov, Dmitrii Georgievich Tarasov

Federal Centre Cariovascular surgery, Astrakhan, Russian Federation

Correspondence: Dmitrii Anatolyevich Kondratyev

Journal of Cardiothoracic Surgery 2017, 12(Suppl 1):P249

Objective: Minimally invasive mitral surgery in contrast to traditional sternotomy guarantees less surgical trauma, faster rehabilitation and good cosmetic effect. The purpose of the study was to compare the results of surgical treatment of degenerative mitral valve disease 
through the minimally invasive approaches and conventional sternotomy.

Methods: During 2009 - 2015 we have prospectively collected cohort of 207 patients who underwent surgery of myxomatous mitral valve. The mean age was $53.8 \pm 8.7$ years. The cohort contained 107 males (52\%). All the patients had preserved LV EF $(60 \pm 5 \%)$ and were in III and IV NYHA class. Atrial fibrillation was registered in 77 pts (37\%). Anterior leaflet prolapse had 39 pts (18.8\%), posterior leaflet prolapse 108 pts (52\%), dual leaflet prolapse 60 pts (29\%). Mitral valve repair was performed in 157 pts (75.9\%) and mitral valve replacement in 50 pts (24\%). In 22 (44\%) cases biological valve was implanted. Tricuspid valve was repaired in 54 pts (26\%). The patients were dived in to two groups. The mitral valve surgery was performed through the minimally invasive approach in 90 pts ( $1^{\text {st }}$ group) and through the conventional sternotomy in 117 pts $\left(2^{\text {nd }}\right.$ group). The $1^{\text {st }}$ group consisted of $11(\%)$ minsternotomies and 79 (\%) minithoracotomies. Comparative analysis included bypass time, cross-clamp time, ventilation time, ICU and hospital stay. The blood loss, stroke, wound infections pericarditis and tamponade and mortality rate were compared. Results: The bypass time was longer in the $1^{\text {st }}$ group. There was no significant difference in cross-clamp time. Minimally invasive approach was associated with shorter ventilation time, blood loss and ICU stay. However difference in hospital stay wasn't observed as the early and late mortality.

Conclusions: Surgical treatment of degenerative mitral valve disease through the minimal invasive has advantages but long term results are almost identical.

\section{P250}

Minimally invasive coronary artery bypass grafting surgery increases postoperative haemoglobin levels and reduces postoperative blood transfusion

Sherlyn Yen Yu Tham?', Yi Jie Chew ${ }^{1}$, Siew Pang Chan², Jianye Chen³, Lowell Choong Kiat Leow ${ }^{3}$, Theodoros Kofidis ${ }^{3}$

${ }^{1}$ National University of Singapore, Yong Loo Lin School of Medicine, Singapore, Singapore; ${ }^{2}$ Cardiovascular Research Institute, National University of Singapore, Yong Loo Lin School of Medicine, Singapore, Singapore; ${ }^{3}$ Department of Cardiac, Thoracic and Vascular Surgery, National University Heart Centre Singapore, Singapore, Singapore Correspondence: Sherlyn Yen Yu Tham

Journal of Cardiothoracic Surgery 2017, 12(Suppl 1):P250

Objective: Lower postoperative haemoglobin has been associated with poorer postoperative outcomes and greater need for postoperative blood transfusions. This study compares immediate postoperative haemoglobin levels and postoperative transfusion between minimally invasive cardiac surgery (MICS) coronary artery bypass graft (CABG) and conventional CABG surgery.

Methods: A retrospective study was conducted on all patients who underwent conventional and MICS CABG performed by a single surgical team in our Heart Centre between 2013 to 2016. A total of 307 patients (conventional: 260, MICS: 47) from a mixed Asian population were recruited. Their demographics, preoperative, intraoperative and postoperative variables were collected. A generalized structural equation model (gSEM) was applied for data analysis and all statistical tests were performed with $95 \%$ confidence interval.

Results: There was no significant difference in preoperative profiles between the two groups (i.e. Charlsons Comorbidity index, BMI and preoperative blood tests). Patients who had undergone MICS had a significantly higher average postoperative haemoglobin level (coefficient: $1.23 \mathrm{~g} / \mathrm{dL}$; $95 \%$ C.I.: 0.74-1.73; p-value <0.001), after adjusting with preoperative haemoglobin level. The results showed that with every unit increase in postoperative haemoglobin, the odds of requiring postoperative transfusion decreases by $30 \%$, confirming an indirect relationship between MICS and postoperative transfusion (adjusted odds ratio: $0.70,95 \%$ C.I. : $0.60-0.81 ; \mathrm{p}<0.001$ ), after adjusting with the type of operation. The odds of having postoperative transfusion was 9\% lower in the MICS group (adjusted odds ratio: 0.91, 95\% C.I.: 0.45- $-1.84 ;$ p:0.800). Platelet levels were similar between the two groups (coefficient: $1.69 \times 10^{9} / \mathrm{L} ; 95 \%$ C.I.: $-13.47-$ 16.86; p:0.827).

Conclusions: In summary, MICS approach had significantly higher postoperative haemoglobin levels and an indirect effect in reducing postoperative transfusion as compared with the conventional approach. MICS leads to improved patient outcomes and should be strongly considered in patients who are eligible for both MICS and conventional CABG surgeries.

P251

Thoracoscopic maze procedure with tourniquet ligation of left atrial appendage

Yuriy Shneider, Pavel Shilenko, Men De Tsoy, Aleksey Cherkes, Anastasiya Sigareva, Elena Khudenkih

FGBU FCHMT, Kaliningrad, Russian Federation

Correspondence: Pavel Shilenko

Journal of Cardiothoracic Surgery 2017, 12(Suppl 1):P251

objective: the totally thoracoscopic maze procedure for the treatment of atrial fibrillation is a highly effective method of treatment atrial fibrillation. Effective method of stroke prevention is exclusion left atrial appendage. This research shows results of thoracoscopic radiofrequency ablation atrial fibrillation and efficiency of tourniquet technique ligation of left atrial appendage.

methods: from January 2015 to April 201746 patients were made totally thoracoscopic bilateral pulmonary vein isolation, roof and floor lesions and additional lines. In 28 cases, for exclude left atrial appendage, we have made tourniquet ligation of left atrial appendage. $44(95.6 \%)$ patients were with persistent atrial fibrillation. $4(4.4 \%)$ with paroxysmal. 7 (15.2\%) were made earlier endocardial ablation of pulmonary veins. Was made periodic holter monitoring for determing freedom from atrial fibrillation. Was made cardiac computed tomography for determing effectiveness of exclusion left atrial appendage.

results: by April 2017 we have got 90-days results of freedom from atrial fibrillation from 31 patients. Rhythm of 12 patients were evaluated after year. After 90 days 3 patients had a recurrence atrial fibrillation (9.6\%). After year sinus rhythm was marked in $83.4 \%$ cases. 2 patients with recurrence atrial fibrillation were made catheter endocardial ablation, such as a variant of hybrid tactics of treatment. Positive result was confirmed in all 28 cases Tourniquet ligation of left atrial appendage. There was a pneumothorax in 2 cases such as complication of the surgery.

conclusions: the totally thoracoscopic maze procedure is highly effective method of treatment atrial fibrillation with high security surgery intervention. There was a low percent of postoperative complications. Tourniquet technique ligation is an effective and safe method of exclude left atrial appendage.

P252

Four year single center experience on minimal invasive surgery

Azamat Kurmalayev, Ermagambet Kuatbayev, Darkhan Suigenbayev, Mirzabek Mahmudov, Shaimurat Tulegenov, Gaukhar Amreeva, Zhanibek Ashirov

National Research Center for Cardiac Surgery, Astana, Kazakhstan

Correspondence: Azamat Kurmalayev

Journal of Cardiothoracic Surgery 2017, 12(Suppl 1):P252

Background: The objective of this prospective cohort observational study was to assess in-hospital mortality, bypass time and morbidity in all patients undergoing MICS at our Center.

Methods: Starting from January 2013 and December 2016 JSC "NRCCS" in Astana performed 229 operations through minimally invasive access using thoracoscopic video equipment. All of 229 patients, 158 were female, 71 were male patients. The age distribution was between 18 and 72. Mean NYHA functional class 2.18 \pm 0.8$)$. Mean ejection fraction $48 \pm 11$. Pulmonary hypertension (PAP $>30 \mathrm{mmHg}, \mathrm{n}=169$ [74\%]). The $82(36 \%)$ patients underwent with ASD, $64(30 \%)$ patients primarily underwent Mitral valve repair (14 (22\%) 
Annuloplasty, 31 (48\%) Chordoplasty, 16 (25\%) Posterior leaflet resection and sliding valvuloplasty, 2 (3\%) augmentation, 1 (2\%) Cleft closure) and $51(22 \%)$ underwent Mitral valve replacement (26 (51\%) biological and 25 (49\%) mechanical valve), 3 (1.3\%) patients underwent with VSD, 16 (7\%) patients underwent tricuspid valve repair and prosthesis, 3 (1.3\%) ASD and tricuspid valve repair, 4 (1.7\%) aortic valve replacement, 5 (2.1\%) patients underwent fenestration and drainage of the pericardium. In 4 cases, of mitral valve surgery monopolar radiofrequency ablation of the left atrium. Results: There was no hospital mortality. Rethoracotomy for bleeding from intercostal arteries was made in one case. In one case we have stroke, ischemic type. Conversion to sternotomy was made in 4 patients because of adhesive pericardium. After surgery hospital stay was 4-5 days. The average time of cardiopulmonary bypass and aortic clamping was $135.4 \pm 15 \mathrm{~min} / 65.8 \pm 5$ minutes respectively.

Conclusions: Minimaly invasive access without sternotomy reduces the duration of hospital stay after surgery. It is associated with very low rates of conversion to a conventional sternotomy and mortality. MICS using thoracoscopic equipment with cardiopulmonary bypass demonstrates that is a feasible method that can be performed safely and effectively, especially in the hands of experienced surgeons.

P253

Periareolar incision in minimally invasive mitral valve surgery

Vito Margari ${ }^{1}$, Pietro Giorgio Malvindi ${ }^{1}$, Florinda Mastro ${ }^{2}$, Georgios

Kounakis ${ }^{1}$, Giuseppe Visicchio ${ }^{1}$, Cataldo Labriola' ${ }^{1}$, Carmine Carbone', Domenico Paparella ${ }^{2}$

${ }^{1}$ GVM Care \& Research, Ospedale Santa Maria, Bari, Italy; ${ }^{2}$ Universita' degli Studi di Bari, Bari, Italy

Correspondence: Vito Margari

Journal of Cardiothoracic Surgery 2017, 12(Suppl 1):P253

Background: Several approaches have been reported in small thoracotomy cardiac surgery. The periareolar approach has been used for years in plastic surgery especially for breast augmentation. We described our initial experience with a periareolar approach in small thoracotomy mitral valve surgery.

Methods: From 02/2015-02/2017, 94 patients underwent mitral valve surgery through a small right thoracotomy. In eleven cases a 3-5 cm periareolar incision was performed. Age (median/range): 66 [48-81] years. Male/Female: 8/3. LVEF (median/range): 55 [45-70]\%. PAPs $>45$ mmHg: 5/11 patients. Etiology: degenerative 8 pts/DCM functional 1 pt/Rheumatic 2 pts. Preoperative AF: 5/11 patients. The periareolar area was incised at the superior margin in case of thoracotomy at the $3^{\text {rd }}$ intercostal space; the incision of the inferior margin allows for a thoracotomy at the $4^{\text {th }}$ space. The lateral side was preserved in order to avoid any injury to the sensorial innervation. Before the wound closure, we resected the skin margins stretched by the retractor.

Results: A satisfactory exposure was achieved in all the patients. Nine patients underwent isolated mitral valve repair. Associated procedures were: tricuspid valve annuloplasty $(n=1)$, AF ablation $(n=1)$, ASD closure $(n=1)$. Patients with rheumatic disease underwent mitral valve replacement. The postoperative course was uneventful in 10 cases, one patient sustained cerebral stroke on day 4 after the operation. At 1-month follow-up there were no wound complications. No alteration of the nipple-areolar sensitivity was reported. The cosmetic result was satisfactory in all the cases.

Conclusions: The periareolar incision provides a satisfactory surgical exposure while respecting the esthetic and the function of the mammary gland. This approach is better indicated in male patients and in female with a small breast not allowing for a submammary incision. Video assistance is mandatory in order to have perfect visualization trough the small access.

Publisher's Note

Springer Nature remains neutral with regard to jurisdictional claims in published maps and institutional affiliations.

\footnotetext{
Submit your next manuscript to BioMed Central and we will help you at every step:

- We accept pre-submission inquiries

- Our selector tool helps you to find the most relevant journal

- We provide round the clock customer support

- Convenient online submission

- Thorough peer review

- Inclusion in PubMed and all major indexing services

- Maximum visibility for your research
}

Submit your manuscript at www.biomedcentral.com/submit
BioMed Central 